Vladimir M. Danilov

\title{
Non-stationarity of Open Star Clusters
}

Monograph

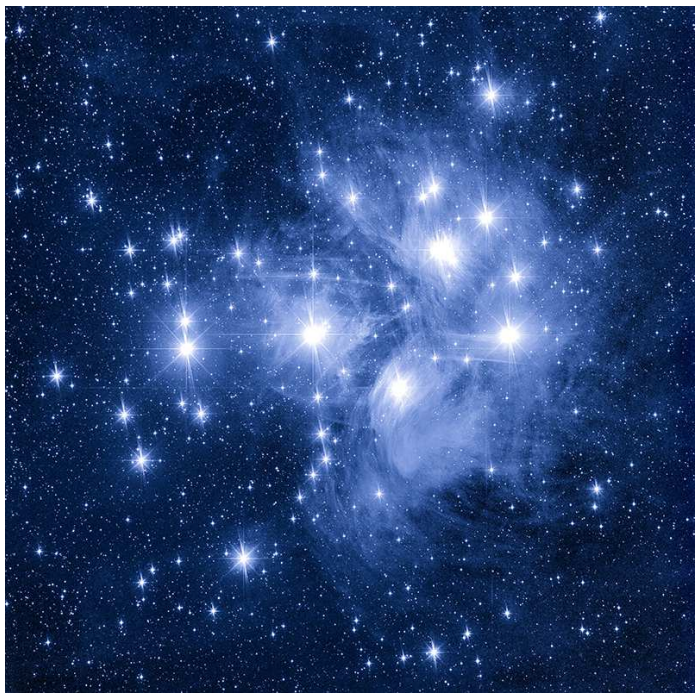





\section{Vladimir M. Danilov}

\section{Non-stationarity of \\ Open Star Clusters \\ Monograph}

Ekaterinburg

Ural University Press

2021 
UDK 524.4

D183

Translation from Russian by E. Shabalina and A. F. Seleznev

Reviewers:

B. P. Kondratyev, Doctor of Science, Professor (MSU)

N. V. Emelyanov, Doctor of Science, Head of the Section of Celestial Mechanics SAI MSU;

V. E. Zharov, Doctor of Science, Professor, Head of the Department of Celestial Mechanics SAI MSU;

Yu. V. Khachay, Doctor of Science, Professor (Geophysics Institute of the

Ural Branch of the Russian Academy of Sciences)

\section{Danilov, V. M.}

D183 Non-stationarity of Open Star Clusters : monograph / V. M. Danilov ; [trans. of E. Shabalina and A. F. Seleznev] ; Ministry of science and higher education of the Russian Federation. - Ekaterinburg : Ural University Press, 2021. - 352 p. - ISBN 978-5-7996-3173-4. - Text : electronic.

ISBN 978-5-7996-3173-4

DOI 10.15826/B978-5-7996-3173-4

The monograph poses issues related to the study of the non-stationarity of open star clusters (OSCs), starting with an analysis of the properties of the trajectories of individual stars to the study of collective motion of stars. A discussion of the dynamics of correlations and wave processes in such clusters is presented. The mechanisms of the dynamic evolution of OSCs, the gravitational instability of OSC nuclei, the spectra of frequencies and wavenumbers for oscillations of numerical models of OSCs, astrophysical applications of the results of studies of the dynamics of OSCs are considered.

We address the monograph to academic researchers (astronomers and physicists) who are interested in astrophysics, the dynamics of stellar systems, and $\mathrm{PhD}$ and senior students of relevant specialities.

UDK 524.4

ISBN 978-5-7996-3173-4

(C) Данилов В. М., 2015

(C) Shabalina E. , Seleznev A.F., translation to English, 2021

(C) Mironov K. V., cover photo, 2011

(C) English Edition, typography Ural University Press, 2021 


\section{Foreword}

We live in the era of the Gaia satellite and the expectation of a revolution in the study of stellar systems. Its precise astrometry provides us with a sharp snapshot of the actual 3D structure of several nearby star clusters, while kinematics offer insights on their evolution. Detailed theory has to be developed, to interpret this wealth of data, and to cast light on processes like star formation and dynamical evolution of star clusters. The focus of this book is on open, or Galactic, star clusters, which populated the disc of the Milky Way, and are typically composed of less than 100 up to a few 1000 stars. Many of them survive little time and dissolve fast into the general Galactic field. Most (and extremely valuable) work has been done in the past in this field, essentially thanks to the efforts of Sverre Aarseth and collaborators, and the various releases of his $\mathrm{N}$-body code. This book summarises another, independent and original, effort. The author, Vladimir M. Danilov, from Ural Federal University in Russia, has spent his scientific career to develop analytical and numerical methods to study open cluster dynamical evolution. The focus is on non-stationarity, which effectively evokes the idea of time evolution, sudden changes, phase mixing, re-organisation of trajectories of individual stars, collective processes, and so forth. Open clusters are lively entities, rapidly evolving and highly sub-structured. Most of the results of this life-long studies are included in papers published in Russian journals which, unfortunately, got little attention by foreign researchers. This comprehensive book, translated from the original in Russian, contains the most important results of Danilov work with a lot of details, and is intended for researchers in Galactic dynamics and N-body methods. It is not an easy book, but it offers to everybody the opportunity to hear a different voice, to consult an independent opinion from another world.

Padova, July 2020

Giovanni Carraro 



\section{Acknowledgements}

Author expresses his gratitude to Miss E. Shabalina, who performed a huge work to translate to English a very complicated Russian text, and to Dr. Anton F. Seleznev, who finished and edited the translation and prepared the layout of the book.

The work of V.M.D. was supported by the Ministry of Science and Higher Education of the Russian Federation, FEUZ-2020-0030 and also by the Act No. 211 of the Government of the Russian Federation, agreement No. 02. A03.21.0006. 



\section{Contents}

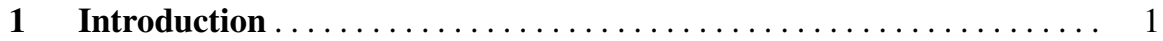

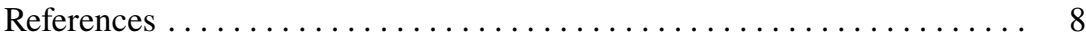

2 Motion of Halo Stars in the Numerical Dynamical Models of Open

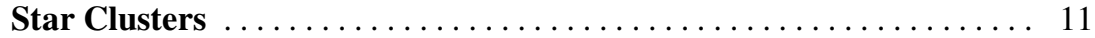

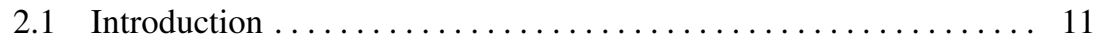

2.2 Description of OSC Models ........................ 12

2.3 Small Parameter in the Equations of Stellar Motion ........... 16

2.4 Stellar Motion Frequencies in OSC Models ................ 19

2.5 Conclusions ..................................... 21

References ................................... 22

3 Properties of Stellar Trajectories in Numerical Dynamical Models

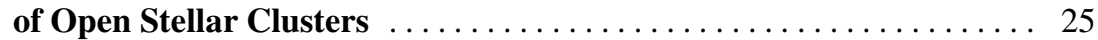

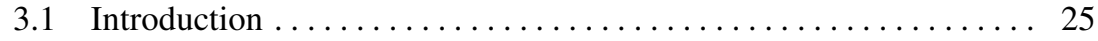

3.2 Basic Formulas and Notations ..................... 28

3.3 The Lyapunov Times and Oscillation Periods of Stellar Trajectories 29

3.4 Conclusions ...................................... 41

References ...................................... 42

4 Dynamic Models of the Stellar Motion at the Periphery of Open

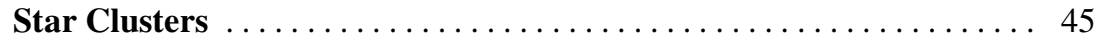

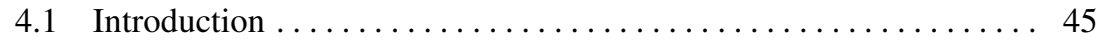

4.2 Equations and Integrals of Stellar Motion $\ldots \ldots \ldots \ldots \ldots \ldots \ldots . \ldots 7$

4.3 Stellar Motion in the Plane $(\xi, \eta) \ldots \ldots \ldots \ldots \ldots \ldots \ldots \ldots \ldots \ldots$

4.4 Phase Density Function Depending on the Integrals $J_{1}$ and $J_{2} \ldots \ldots 56$

4.5 Distribution of the Stellar Velocities in the Case of Three-Integral

PDF ....................................... 62

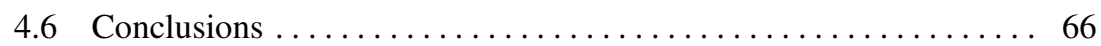

References ................................... 67 
5 Investigation of the Stellar Trajectories in the Two-Point Model of

Open Star Cluster . . . . . . . . . . . . . . . . . . . . . . . . . 69

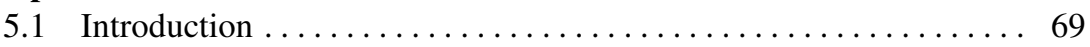

5.2 Two-Point Model of Non-Isolated Star Cluster … . . . . . . . . . . 73

5.3 Calculation of Separatrix ........................ 77

5.4 Canonical Form of Equations in Variations .............. 78

5.5 Construction of Poincaré Sections .................. 81

5.6 Discussion of The Properties of Stellar Trajectories ........... . 84

5.7 Conclusions ................................... 89

References ................................. 90

6 Time Scales of Dynamic Evolution Mechanisms of Open Star Clusters 93

6.1 Introduction ................................ 93

6.2 Description of OSC Models ....................... 96

6.3 Basic Calculation Techniques .................... 98

6.4 Changes in the Parameters of Stellar Velocity Distribution with

Time. Instability of Phase Density to Small Initial Perturbations... . 100

6.5 Conclusions ...................................... 110

References ................................... 111

7 Analysis of Density Oscillations in Models of Open Star Clusters . . . 115

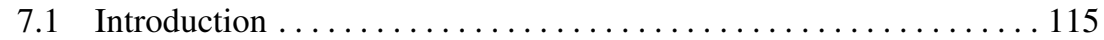

7.2 Oscillations of a Homogeneous Triaxial Gravitating Ellipsoid . . . . 116

7.3 Nonhomological Oscillations of $H$-Ellipsoid . ............. 121

7.4 Three-Integral Equilibrium Function of Phase Density ........... 123

7.5 The Boltzmann Equation ........................... 125

7.6 Instability of Phase Density Oscillations in the Cores of OSC Models. Estimates of Parameters and Consequences of Such

Oscillations ....................................... 129

7.7 Conclusions ........................................ 134

References ....................................... 136

8 On Phase Density Oscillations at the Centers of Six Open Star

Clusters ........................................... 137

8.1 Introduction . . . . . . . . . . . . . . . . . . . . . . . . . 137

8.2 Structural Parameters of Six OSC .................... 138

8.3 Structural and Dynamic Parameters of Six OSC Models ......... 145

8.4 Dynamic Parameters of Six OSC and Two OSC Models .......... 148

8.5 Analysis of Dependencies Between the Parameters of Unstable Oscillations of Phase Density in the Centers of the Considered OSCs and Cluster Models ............................. 153

8.6 Conclusions . . . . . . . . . . . . . . . . . . . . . . . . . . 159

References .................................... 160 


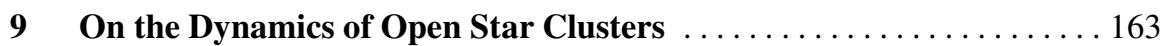

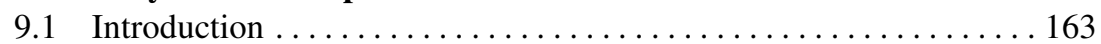

9.2 Stellar Velocity Dispersion in the OSC Models ... . . . . . . . . . . 165

9.3 Instability Parameters of OSC and their Models . . . . . . . . . . . 170

9.4 Structure of Regions of the PD Oscillation Instability in the Centers

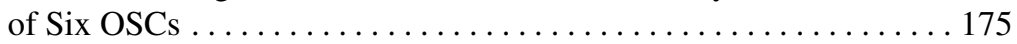

9.5 Conclusions ............................... 182

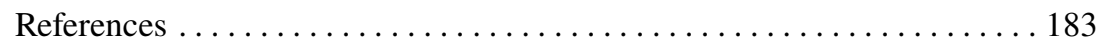

10 Parameters of Nonstationarity of Open Star Clusters . . . . . . . . . . 185

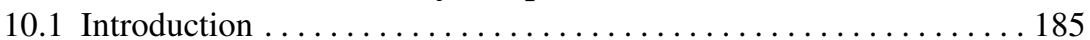

10.2 Contrast of Densities in the OSC Cores . . . . . . . . . . . . . . . . 187

10.3 Homologous Oscillations of the OSC Models . . . . . . . . . . . . . 190

10.4 Oscillations in the Cores of OSC and OSC Models . . . . . . . . . . . . . 197

10.5 Dynamic Dispersions of Stellar Velocities in OSC . . . . . . . . . . 205

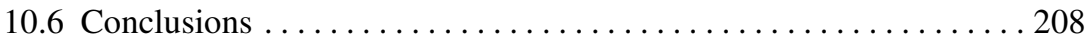

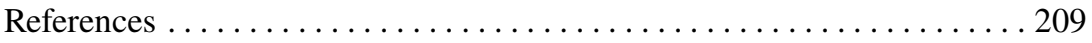

11 On the Dynamics of Correlations in Models of Open Star Clusters . . 213

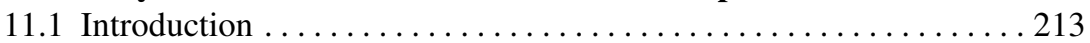

11.2 Times and Radii of Correlations in OSC Models . . . . . . . . . 215

11.3 Distributions of Correlations ... . . . . . . . . . . . . . . . 224

11.4 Fluxes of Correlations in the Space of $y_{f} \ldots \ldots \ldots \ldots \ldots \ldots 28$

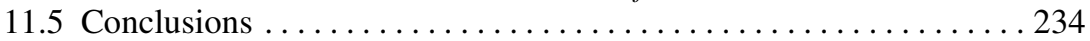

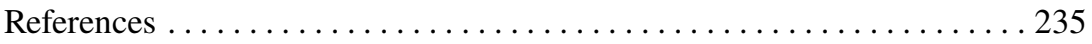

12 Correlations, Spectra, and Instability of Phase-Density Oscillations in Models of Open Star Clusters . . . . . . . . . . . . . . . . . . . . . . . 239

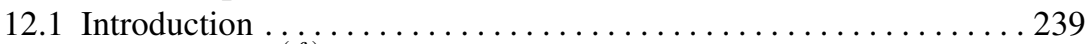

12.2 Estimates of $\tau_{c}^{(f)}, v_{f}, v_{r}, v_{v}$ in the OSC Models $\ldots \ldots \ldots \ldots \ldots 243$

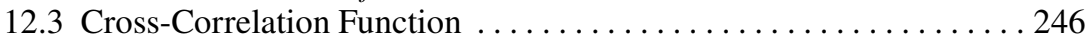

12.4 Frequency Spectra and Dispersion Curves . . . . . . . . . . . 248

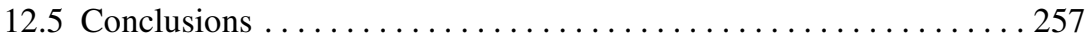

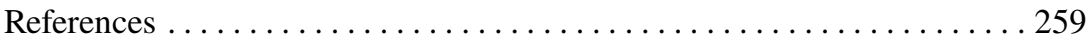

13 Smoothing of Force Functions and Oscillation Spectra of Open Star Cluster Model ............................. 263

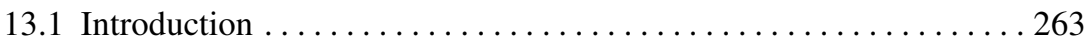

13.2 On a Connection Between the Parameters of the OSC Model and $\epsilon .268$

13.3 Cross-Correlation Functions . . . . . . . . . . . . . . . 270

13.4 Frequency Spectra and Dispersion Curves . . . . . . . . . . . . . 272

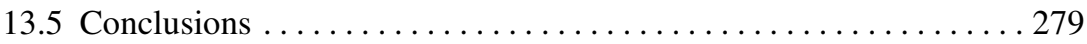

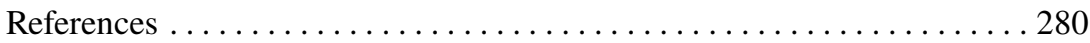


14 Spectra of Wave Numbers of Oscillations in OSC Models . . . . . . . . 283

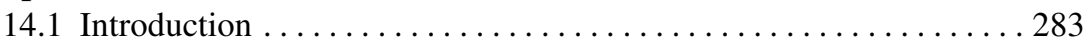

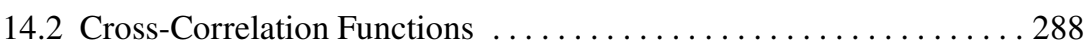

14.3 Spectra of Wave Numbers . . . . . . . . . . . . . . . . . . . . . . . . . . 290

14.4 Dependencies of $S(k)$ Spectra of OSC Model 1 on $\epsilon \ldots \ldots . . . .296$

14.5 Conclusions . . . . . . . . . . . . . . . . . . . . . . . . . 299

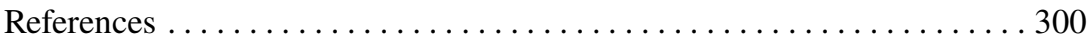

15 Dynamics of the Coronas of Open Star Clusters . . . . . . . . . . . . 303

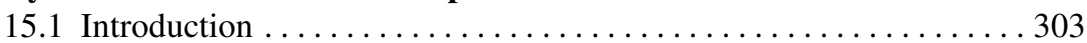

15.2 Constructing Coronas of OSC models . . . . . . . . . . . . . . 307

15.3 Parameters of Coronas of the OSC Models. Mechanisms of Corona Formation and Rate of Dissipation of Stars from Coronas of the

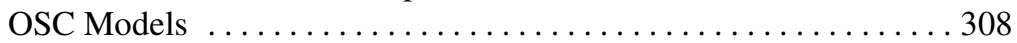

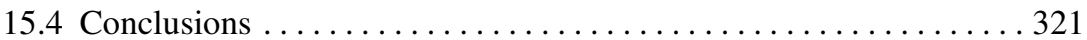

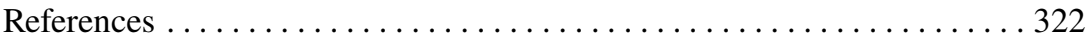

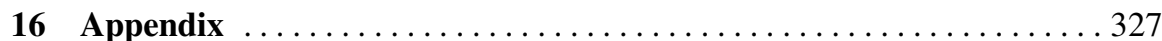

16.1 Description of OSC Models . . . . . . . . . . . . . . . . . . . 327

16.2 Statistical comparison of OSC models . . . . . . . . . . . . . . 330

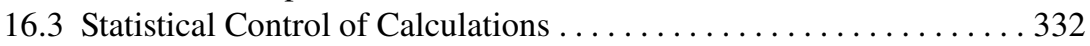

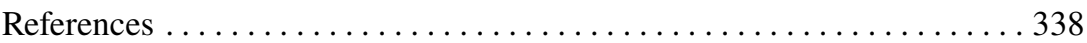




\title{
Acronyms
}

\author{
BS Binary Star \\ CBS Close Binary Star \\ CR3BP Circular Restricted Three-Body Problem \\ CZVS Critical Zero Velocities Surface \\ DSO Divergence of Stellar Orbits \\ FFT Fast Fourier Transform \\ FLI Fast Lyapunov Indicator \\ GD Gross-Dynamic \\ GMC Giant Molecular Cloud \\ GSC Gas-Star Complex \\ KDE Kernel Density Estimator \\ MC Molecular Cloud \\ MLCE Maximum Lyapunov Characteristic Exponent \\ OSC Open Star Cluster \\ PCS Phase Coordinates of the Stars \\ PD Phase Density \\ PDF Phase Density Function \\ PS Phase Space \\ QSS Quasi-Stationary State \\ SC Sinusoidal Component \\ SS Single Star \\ ZAMS Zero-Age Main Sequence \\ ZVS Zero-Velocity Surface
}





\title{
Chapter 1 \\ Introduction
}

\begin{abstract}
In the Introduction we briefly discuss the modern status of the problem of an analysis of the dynamical state of the open star clusters. The formulation of the problems necessary for such analysis is presented. Also, we list the most important results connected with this analysis and described in the present book.
\end{abstract}

Open star clusters (OSC) are traditionally used by researchers in testing emerging theories, hypotheses, assumptions about the physical and dynamic evolution of stars and star clusters. Until the early 80's of the last century the OSC were mainly considered as objects existing in the state close to virial equilibrium. In order to theoretically describe the OSC structure, the quasistationary (and even stationary) OSC models were used which did not take into account the possibility of significant deviation of OSC from the stationarity in a regular field. However, structural features of young and old OSC (distortion of the shape of cluster cores, splitting of cores, a mismatch of centers of density distributions of subsystems of stars with different masses in clusters, presence of step-like structures in the density profiles of clusters, etc.) found back in 1950-1960'scan not be explained without invoking the hypothesis of non-stationarity of clusters in regular field. It assumes an explicit time-dependence of the phase density functions (that is, a partial derivative of the phase density function is not equal to zero; see Chapters 4 and 7 of Binney and Tremaine (2008)). Completed in recent years, theoretical researches and numerical experiments on the modeling of a dynamics of non-isolated systems of $N$ gravitating point masses showed that in clusters with a low density of the matter (close to the critical one in the field of external forces), the action of gravitational instability can lead to the development of a non-stationarity in the regular field and even to the loss of a significant part of stars during several intersections of the cluster by a star.

In the framework of numerical experiments on the modeling of a dynamics of OSC, it was revealed that the velocity dispersion of stars in cluster cores (cold cores) was insufficiently high for the equilibrium state, and the dispersion of velocities of stars was increasing along with the distance from the center of the cluster, which indicates the lack of equilibrium and a non-stationarity of OSC (Danilov and Putkov, 2012a). In the models of OSC (Danilov and Dorogavtseva, 2008), density and 
regular field oscillations develop rapidly and spontaneously, and do not decay during 5-10 times of violent relaxation $\tau_{v r}$. Theoretical description of the phase density oscillations in cores of ellipsoidal OSC models was performed by Danilov (2008). Danilov (2010) showed the instability of the natural oscillations of the phase density in the centers of six OSC models and several real OSC and obtained the theoretical estimates of the periods and the growth increments of the homological oscillations of the phase density. Danilov and Putkov (2012b) determined the parameters of density, potential, and phase density waves in OSC models by the data on correlations for the values of the radius-vector absolute values, velocities, specific energies of stars, densities, and phase densities. They discovered the signs of formation of polarization clouds, when a series of distances between the stars is reached; the dominant flux of correlations from the region of strong correlations to the weak correlation region is detected, which leads to the appearance of kinetic energy flux to the center of the cluster. Danilov and Putkov (2012b, 2013b) revealed a number of indications of the formation of a weak turbulence in the motions of the core stars in the OSC model with the greatest degree of non-stationarity in a regular field.

We emphasize that the average values of the matter density in the OSC are usually determined inaccurately and depend to a large extent on the observer's estimate of the cluster radius. To analyze the observed manifestations of non-stationary nature of OSC, it is necessary to substantially refine the data on the dimensions, average and critical densities of the clusters in an external force field. In order to proceed with the analysis of the observable manifestations of non-stationary nature of the OSC, it is necessary to develop methods for estimating the degree of clusters' non-stationarity, taking into account the action of the most important factors in the clusters (low peculiar velocities of the stars and small matter densities in the OSC, the encounters of the cluster-member stars, presence of external force fields).

In this connection, the mass application of the statistical methods to the evaluation of the OSC sizes and the number of stars in clusters against the field star density fluctuations, as well as the development of means to assess the reality of existence of certain structural features of OSC (Danilov et al., 1985; Danilov and Seleznev, 1994), turned out to be very timely. The study of OSC structure using the method of significance levels (Danilov et al., 1985; Danilov and Seleznev, 1994) showed that the stars in young clusters are on average located deeper beneath the tidal surface in the Galactic force field than stars in old OSC. When analysing this phenomenon, it is necessary to take into account the joint effect on the young OSC of the force fields of the Galaxy and the gas-star complexes (GSC), in which these clusters are formed. An important place in this analysis belongs to the investigation of the stability of OSC in external force fields. Such studies provide information on the tidal size of OSCs and on certain characteristics of GSC containing these clusters (Danilov and Seleznev, 1995). An important role in the study of the OSC dynamics is also played by the observational data obtained by Danilov and Seleznev (1994) on the characteristics of the haloes and the cores of these clusters.

Reliable observational data on the OSC structure makes it possible to formulate and solve a number of theoretical problems: 
1. Study of a dynamics of the OSC non-stationary in the regular field. Modeling of the dynamics of non-stationary OSC with a low density of matter and fast decay processes (during several crossings of a cluster by a star) in the presence of external force fields.

2. Development of methods for estimating the degree of non-stationarity of the OSC with taking into account the action of the most important mechanisms in the clusters (gravitational instability of cold OSC cores, small densities of the mass and number of stars in OSC, the presence of external force fields). Analysis of the observed manifestations of the non-stationarity of the OSCs in the structure of clusters.

3. Theoretical and numerical-experimental description of the dynamics of cores and external regions of a non-isolated OSC. Analysis of the features of gravitational instability development in the cores of OSC and separation of the main resonances in the motion of stars at the periphery of OSCs. Construction of a method for extracting the coronas in numerical dynamic OSC models. Investigation of the corona dynamics in OSCs and analysis of causes for the existence of coronas in the observed OSC.

4. Correlation and spectral analysis of phase density and potential oscillations in the numerical dynamic OSC models. Identification of the features of spectra and dispersion curves of oscillations in such cluster models. Analysis of mechanisms for the formation of various regions of oscillation spectra in OSC models.

5. Estimates of the non-stationarity parameters of a number of open star clusters (contrast of densities in the cluster core, dispersion of stellar velocities obtained with taking into account the effect of the external field of the Galaxy and non-stationarity of the cluster, periods of oscillations in the cluster and cluster core, etc.).

The most important new results, estimates and conclusions described in this book can be summarized as follows:

1. For a number of models of non-stationary OSC, data on time dependencies of the dispersions of stellar velocities in three mutually perpendicular directions in the halo and the cluster core are given and discussed in Chapter 6. During the dynamic evolution of OSC models, the values of the velocity dispersion undergo oscillations which do not decay during 5-10 times of violent relaxation $\tau_{v r}$. We discuss the estimates of the synchronization time $t_{s}$ of rotation of considered OSC models with their motion around the center of the Galaxy. Depending on the parameters of the OSC models, synchronization times are $t_{s} \simeq(5-27) \tau_{v r}$. The mechanisms of a synchronization of the considered models are discussed. A prominent role of the tidal friction in the decay of such systems in the Galactic field is noted. Estimates of the time of the formation of a spherical distribution of stellar velocities in the clusters' models are $t_{\sigma} \simeq(6-25) \tau_{v r}$. The effect of instability in the motion of cluster stars on the formation of a spherical distribution of stellar velocities in the considered OSC models is discussed. We detect a tendency toward weakening of the dependency of the coarse-grained phase density of the cluster on the effect of small initial perturbations of the phase coordinates of stars in the cores of the cluster models at the time points which are $\sim 5$ times longer than the violent relaxation time (these results were obtained in a collaboration with L.V. Dorogavtseva). 
2. Chapter 8 discusses instability of the natural oscillations of the phase density in the centers of six OSCs. The boundaries of the regions of instability of such oscillations in the space of the mean mass density of the cluster core are indicated. Estimates of a number of dynamic parameters (potentials, dispersion of stellar velocities, mass density, periods of phase density oscillations, the time of the development of the instability of such oscillations, etc.) are considered for the central parts of these clusters and for several numerical dynamic OSC models. Equation is derived for estimating the dynamic mass of a cluster, taking into account influence of the external field of the Galaxy and the non-stationary nature of the cluster. Taking into account the influence of these effects leads to a decrease in the dynamic mass of the cluster in comparison with the virial mass for an isolated cluster. Astrophysical applications of these results are discussed.

3. In Chapter 9, we consider stellar kinematics in OSC models. Central regions of these models are quite "cold". This leads to gravitational instability in the cores of clusters and their models. In the cluster models, the temporary virialization is noted, in which a significant part of the energy of cluster oscillations temporarily passes into kinetic energy of peculiar star motions. Duration of this stage can reach $\sim 10^{8}$ years. We discuss the reasons of the temporary virialization of clusters, as well as instability of the natural oscillations of phase density in the centers of six clusters and six cluster models. In addition to the known regions of unstable oscillations of phase density, several new regions are noted. An approach of clusters and their models to a state of stable equilibrium is possible both with decreasing and with increasing density of the cluster core. The structure of the regions of instability of phasedensity oscillations in the centers of six clusters is discussed. Resonance curves are presented for the amplitudes of steady-state oscillations of the phase density at the center of the cluster NGC 6705. An analysis of the structure of instability regions in clusters indicates significant rates of loss of oscillation energy in clusters under the action of relaxation effects. With the increase of the distance of the cluster from the Galactic center, the instability increments and the widths of the instability regions decrease. An important role in the formation of instability regions can be played by resonances between frequency of the orbital motion of the cluster in the Galaxy and frequencies of the natural oscillations of the phase density at the centers of the considered clusters.

4. Chapter 11 discusses the two-time and two-point (two-particle) correlations for a number of parameters of stellar motions, as well as for the density and phase density of the OSC models in the vicinity of these stars, obtained from correlation data. Estimates of the time and radius of a correlation in the spaces of the indicated parameters are given. We consider distributions of two-point correlations over distances between stars in the spaces of coordinates and stellar velocities. We also discuss the estimates of the parameters of density waves, potential, and phase density in the models of star clusters obtained from the correlation data. Analysis of the fine structure of the two-point correlations in the space of mutual distances between the stars indicates the formation of polarization clouds when a series of distances between the stars is reached. The distributions of the correlations of the phase density values and the dynamics of such distributions are discussed. Dispersions of 
such distributions depend strongly on the presence of wide "wings" of distributions (that is, on the presence of strong correlations in the system). These dispersions are considered as a measure of the correlation of phase density fluctuations in the cluster models. Increase of correlations with time for $50 \%$ of the considered models of star clusters is noted. Fluxes of correlations of the phase density values are discussed. The dominant flux of correlations from the region of strong correlations to the region of weak correlations is detected. Such a flow leads to the appearance of kinetic energy flux to the centre of cluster. We give the estimates of the rate of a heating for the cores of cluster models by this flux. We also note the signs of a weak turbulence in the stellar motions in the core of the cluster model with the greatest degree of non-stationarity in a regular field (the results were obtained in a collaboration with S.I. Putkov).

5. Chapter 12 discusses application of correlation and spectral analysis of phase density oscillations in the study of a dynamic evolution of OSC models. We present the results of calculations of two-time correlations and cross- correlation functions for fluctuations $\delta f$ of the phase density of cluster models. Estimates of the correlation time $\tau_{c}$ of the phase density oscillations, which were obtained from the data on two-time correlations, range from 0.1 to 1 violent relaxation time of the model $\tau_{v r}$. Average phase velocities $v_{f}$ of propagation of such oscillations in the cluster models, which were obtained from the data on $\tau_{c}$ and the radii $r_{c}$ of two-particle correlations of the oscillations $\delta f$, are 2-20 times less than the mean square velocities of the stars in the cluster core. Fourier transformation of cross-correlation functions is used to calculate power spectra and dispersion curves of phase density oscillations. The presence of known unstable oscillations of the phase density associated with the homological oscillations of cluster cores is confirmed. A number of new unstable oscillations of the phase density in these models is noted (up to 32-41 pairs of oscillations with different complex conjugate frequencies in each model). The rise time of the amplitudes of such oscillations in $e$ times is $(0.4-10) \tau_{v r}$, the phases of such oscillations are distributed fairly evenly. We discuss the astrophysical applications of these results, namely the irregular structure of open star clusters, the formation and decay of quasistationary states in such clusters (the results were obtained in a collaboration with S.I. Putkov).

6. Chapter 13 considers application of the correlation and spectral analysis of the phase density and potential oscillations in the model of open star cluster at different values of the smoothing parameter $\epsilon$ of force functions in the equations of cluster stars' motion. We discuss the results of calculations of cross-correlation functions for the fluctuations of the potential $U$ and the phase density $f$ of cluster model for different distances from its center. In order to calculate the power spectra and dispersion curves of the oscillations of $U$ and $f$ values, we use the Fourier transform of the cross-correlation functions. The oscillation spectrum of $U$ is simpler in comparison with the oscillation spectrum of $f$. The most powerful $U$ oscillations are related to $f$ oscillations, and are located at low frequencies $v<3 / \tau_{v r}$; at medium and high frequencies $\left(v>3 / \tau_{v r}\right)$, contribution of $U$ oscillations to $f$ oscillations is small or zero. We note a number of unstable oscillations of $U$ in the core of the cluster model (up to 30 pairs of oscillations with different complex conjugate 
frequencies). We discuss the dependencies of the spectra and dispersion curves of $f$ and $U$ oscillations on $\epsilon$. A "repeatability" (a significant correlation) of the spectra at certain $\epsilon$ values and the instability of the dispersion curve shape to small changes in $\epsilon$ are noted. Furthermore, Chapter 13 discusses astrophysical applications of these results (which were obtained in a collaboration with S.I. Putkov):

- a decay in the cluster core of the $f$ oscillation wave traveling from the periphery of the cluster to its center onto several waves with frequencies commensurate with the frequency of the external (tidal) action;

- emission and reflection of the waves of $f$ and $U$ oscillations near the boundary of the cluster core;

- possible discreteness of the wavelengths and phases of $f$ and $U$ oscillations in the cluster model.

7. Chapter 14 discusses the results of investigation of the cross- correlation functions for the phase-density $f$ fluctuations of cluster models and for the moduli of stellar velocities $v$. The spatial Fourier transform of the cross-correlation functions with the zero time shift is used to calculate the spectra of wave numbers of $f$ and $v$ oscillations. The spectrum of the wave numbers for $f$ oscillations turned out to be simpler in comparison with the spectrum of the wave numbers for $v$ oscillations. The most powerful $f$ and $v$ oscillations are located in the region of small values of the wave number $k$ (and large wavelengths $\lambda>(0.91-1.25) \mathrm{pc}$ ). A significant contribution to the average power of $f$ and $v$ oscillations is made by homologous oscillations of the clusters. We discuss the dependencies of the spectra of wave numbers of $f$ and $v$ oscillations on $\epsilon$ in cluster model 1 which has the greatest degree of non-stationarity. Such spectra change noticeably less with changes in the $\epsilon$, in comparison with the frequency spectra of $f$ oscillations. An increase in $\epsilon$ less affects the spectra of wave numbers of $f$ oscillations than the spectra of wave numbers of $v$ oscillations. With increasing $\epsilon$, the slope of the wave number spectrum increases on average, that is caused by a change in the structure of the cluster model along the distance from its center. We discuss the astrophysical applications of these results (which were obtained in a collaboration with S.I. Putkov):

- the difference between the spectra obtained and the Kolmogorov spectrum;

- the discreteness of the wavelengths of $v$ oscillations in the cluster models;

- the widths of the spectra of the most powerful oscillations of the models;

- the estimates of the tidal radii of the clusters; etc.

8. Chapter 3 discusses the results of the study of stellar trajectories in the OSC models, which are non-stationary in a regular field. We give the estimates of the maximum characteristic Lyapunov exponent $\lambda$ of stellar trajectories in OSC models. Average $\lambda$ values in the considered OSC models are $\bar{\lambda} \simeq 1$ / Myr. Cluster cores are the regions of increased stochasticity, halos are the regions of more ordered stellar motions. $\bar{\lambda}$ grows with the increasing density of the cluster model, and the sizes of the region of high stochasticity in the cluster core increase. The "adhesion" of the trajectory of a star in the phase space to a region with a given $\lambda$ value is noted. We use a Fourier analysis of the stellar trajectories in the considered OSC models. We 
give the distributions of the stellar trajectories by the periods of stellar motions with the largest values of the power spectrum. In the distributions of stellar trajectories by the periods of the most significant oscillations, we have marked the "peaks" with the periods corresponding to (or close to) the periods commensurate with the oscillation period of the regular field of the system. Features of the distributions of stellar trajectories by the periods of the most significant oscillations and the reasons for the formation of these features in the considered OSC models are discussed (the results were obtained in a collaboration with E.V. Leskov).

9. In Chapter 5, a two-point model of a non-isolated star cluster with a circular orbit in the Galactic plane is considered. The equations of stellar motion are linearized in the neighborhood of a nonsimple point on the critical surface of zero velocities (CSZV) and in the vicinity of the point under CSZV on a trajectory with a stellar "energy" that is less than the critical one. We give the formulas for the eigenvalues and eigenvectors of these equations. We note the instability of two nonsimple points located on the CSZV and numerically obtain the separatrix that connects these points to each other. For the trajectories located under the CSZV, the moduli of eigenvalues of the linearized equations of motion increase with a decreasing stellar "energy" and a decreasing maximum distance of the trajectory from the center of the cluster mass. This leads to an increase in the numerical estimates of the maximum Lyapunov characteristic exponent (MLCE) of the trajectories located closer to the center of the cluster mass. The properties of the stellar trajectories are discussed using Poincare sections and MLCE. A number of periodic orbits is noted for different values of the stellar "energy". Properties of the trajectories in the neighborhood of these periodic orbits are discussed. Almost all the considered stellar trajectories are stochastic; the degree of stochasticity increases with a decreasing stellar "energy". We mark the regions with different degrees of stochasticity on Poincaré maps (the results were obtained in a collaboration with O.A. Chernova).

10. In Chapter 2 we record the equations of stellar motion at the periphery of OSC; these equations contain small $\mu$ parameter. $\mu$ values are given for six numerical dynamic OSC models. A general analytical solution of the equations of stellar motion is given for $\mu=0$. We use the method of successive approximations and investigate the frequencies of stellar motions at the periphery of OSC by the first order expansion by $\mu$ of the solutions of the stellar motion equations. Application of the obtained results is discussed.

11. Chapter 4 considers a model of a homogeneous gravitating ellipsoidal star cluster moving along a circular orbit around the Galactic center. Three independent isolating integrals of stellar motion are written for this model. Analysis of the features of star cluster motion is made, taking into account the influence of these three integrals of motion. The periphery of the considered cluster model is dominated by the retrograde motion of the stars, and stellar velocity distribution is elongated along the direction of cluster motion. We write an expression for the phase density function which depends on two integrals of motion and give the distribution of stellar velocities in the case of a three-integral phase density function. Applications of the obtained results are discussed. 
12. Chapter 15 examines the method for extracting coronas in OSC models. The method uses stellar trajectories that do not exceed the limits of the coronas during the time intervals $t$ comparable to the average lifetime $\tau$ of such clusters. For six numerical models of clusters we constructed the models of coronas and determined the direction and character of their dynamic evolution. In the coronas, retrograde stellar motions predominate. We note the formation of density and phase density distributions close to equilibrium ones in the interval of distances of stars from the cluster center from one to three tidal radii of the cluster despite the signs of the dynamical instability of the coronas (small densities in comparison with the critical density, and accelerated expansion of the coronas). We give the approximations for the phase density of the corona and the cluster by the distributions depending on three arguments (parameters of stellar motion in the Lindblad's System of Rotating Coordinates). This temporal equilibrium of the corona is due to the balance of the number of stars that move to the corona from the central regions of the cluster and leave to the corona periphery or beyond. The signs of the gravitational coupling of the corona stars up to the distances of four tidal radii from the cluster center are noted (the presence of a large number of the corona stars in the Galactic plane with the mean motion close to the periodic retrograde motions; (91-99)\% of the corona stars satisfy the criterion of the gravitational coupling (Ross et al., 1997) on the time intervals of the order of the cluster lifetime). We estimate the dissipation rate of the corona stars to be from 0.03 to 0.23 of the number of corona stars during the time of violent relaxation of the cluster at $t \geq \tau$ (these results were obtained in a collaboration with S.I. Putkov and A.F. Seleznev).

13. Chapter 10 discusses the estimates of a number of dynamic parameters of 103 OSC (contrast of densities in the cluster core; dispersion of stellar velocities obtained with taking into account the influence on the cluster of the external field of the Galaxy and the non-stationarity of the cluster; periods of oscillations of the cluster and cluster core, etc.). We give the analytic solutions of the equations of gross dynamics for simple cluster models. These solutions are used to estimate the values characterizing the degree of cluster non-stationarity (an amplitude of the oscillations of the virial coefficient of the cluster; an amplitude of the oscillations of the radius of the cluster core; etc.). Astrophysical application of these results are discussed (the results were obtained in a collaboration with S.I. Putkov).

\section{References}

Binney, J., Tremaine, S.: Galactic Dynamics, Second edition. Princeton University Press, Princeton, Oxford (2008). ISBN: 9780691130279

Danilov, V.M.: Analysis of density fluctuations in models of open clusters. Astron. Reports 52, 888-899 (2008). doi:10.1134/S1063772908110036

Danilov, V.M.: Phase-Density Fluctuations at the Centers of Six Open Clusters. Astron. Reports 54, 514-527 (2010). doi:10.1134/S1063772910060053 
Danilov, V.M., Dorogavtseva, L.V.: Timescales for mechanisms for the dynamical evolution of open star clusters. Astron. Reports 52, 467-478 (2008). doi:10.1134/S1063772908060048

Danilov, V.M., Matkin, N.V., Pylskaya, O.P.: Statistical Boundaries for Open Clusters. Soviet Astron. 29, 621-625 (1985).

Danilov, V.M., Putkov, S.I.: Non-stationarity parameters of open clusters. Astron. Reports 56, 609-622 (2012a). doi:10.1134/S106377291208001X

Danilov, V.M., Putkov, S.I.: The dynamics of correlations in open-star cluster models. Astron. Reports 56, 623-637 (2012b). doi:10.1134/S1063772912080021

Danilov, V.M., Putkov, S.I.: Correlations, spectra and instability of phase-space density fluctuations in open-cluster models. Astrophys. Bull. 68, 154-168 (2013b). doi:10.1134/S199034131302003X

Danilov, V.M., Seleznev, A.F.: The catalogue of structural and dynamical characteristics of $103 \mathrm{OCl}$ and the first results of its investigations. Astron. Astrophys. Trans. 6, 85-156 (1994). doi:10.1080/10556799408232061

Danilov, V.M., Seleznev, A.F.: Estimates of geometric and dynamic parameters of star-gas complexes in the Galaxy. Astron. Reports 39, 295-304 (1995).

Ross, D.J., Mennim, A., Heggie, D.C.: Escape from a tidally limited star cluster. Mon. Not. R. Astron. Soc. 284, 811-814 (1997). doi:10.1093/mnras/284.4.811 



\title{
Chapter 2 \\ Motion of Halo Stars in the Numerical Dynamical Models of Open Star Clusters
}

\begin{abstract}
In this chapter, we derive for the OSC periphery the stellar motion equations containing a small parameter $\mu$. $\mu$ values are given for six numerical dynamic models of OSC. For $\mu=0$ we give a general analytical solution of the equations of stellar motion. Applying the method of successive approximations we investigate the frequencies of stellar motion in the framework of the first order expansions (with respect to $\mu$ ) of the solutions of the stellar motion equations at the OSC periphery. Application of the obtained results is discussed.
\end{abstract}

\subsection{Introduction}

According to Danilov and Leskov (2005), in the numerical dynamic OSC models, which have different degrees of non-stationarity in a regular field, the trajectories of halo stars have small positive values of the maximum characteristic Lyapunov exponent $\lambda$ and not very complex Fourier spectra. According to Danilov and Leskov (2005), the Lyapunov times $t_{\lambda}=\lambda^{-1}$ for the stellar trajectories at the periphery of the OSC are 1-10 million years, which is comparable with the Lascar estimates (Laskar, 1989) for the orbits of planets in the Solar system. Three sinusoidal components with the largest amplitudes in the dependencies on the time of the star distance $r(t)$ from the cluster center account for 50-95\% of the total energy of Fourier spectrum for these dependencies. Amplitudes of the oscillations of the regular potential in the halo of non-stationary OSC models (Danilov and Dorogavtseva, 2003) are small and amount to less than $6 \%$ of the average regular potential value over its oscillation period. Such characteristics of stellar trajectories and gravitational potential in the OSC halo allow us to apply the methods of perturbation theory for an approximate description of stellar motion at the periphery of non-stationary OSC. According to Danilov and Leskov (2005), the stellar trajectory distributions in the OSC models by the periods of motion in the cluster have sharp resonant peaks at the periods commensurate with the period of regular field oscillations. Comparison of the periods of these "peaks" with the periods obtained as a result of applying the small parameter 
method to the study of stellar trajectories in such systems makes it possible to extract the main resonances in the halo stars' motion in the OSC models.

Periodic oscillations of the size, density, and regular field of the OSCs are largely ensured by the motion of the halo stars in the clusters. These oscillations lead to a number of complex processes observed in the numerical experiments on the dynamics of the OSC (energy transfer from the large-scale motions of stars to the small-scale motions, the oscillations in the entropy values of the system, etc. (Danilov, 2002b)). Therefore, it is important to understand the mechanisms for the formation of the trajectories of the OSC halo stars.

Objectives of Chapter 2: introduction of a small parameter in the equations of the OSC star motion; the study of frequencies of the star motion at the periphery of the OSC in the first order expansion of the solutions for the star motion equations by the small parameter; an extraction of the main resonances in the halo star motion in the OSC models.

\subsection{Description of OSC Models}

The paper of Danilov (2005) considers the open star cluster moving along a circular orbit in the plane of the Galaxy. It uses the equations of stellar motion (Chandrasekhar, 1942) in rotating coordinate system $\xi, \eta, \zeta$. Following to Chandrasekhar (1942), expansion of the Galactic regular potential in a series up to quadratic terms with respect to $\xi, \eta, \zeta$ coordinates is employed. Integrals of the motion of the cluster's center of mass are written in a usual way. In constructing of an initial approximation for the halo star trajectories, the following simplifications were used by Danilov (2005).

1. In order to estimate the dimensions of the critical surface of zero velocities in the cluster along the axes $\xi, \eta, \zeta$ the cluster was represented by a model in the form of a binary system containing a star with mass $m_{1}=1 m_{\odot}$ and point mass $m_{2}=M-m_{1}$, where $M$ is the mass of the cluster model $\left(M=500 m_{\odot}\right.$ is assumed here as well as by Danilov and Leskov (2005),). The integrals of the motion of the cluster mass center allow us to disconnect the systems of equations for the motion of bodies with masses $m_{1}$ and $m_{2}$ and to consider them independently. We can find three singular points from the system of equations of the motion of the star with mass $m_{1}: \xi=\eta=\zeta=0$ and $\xi= \pm\left|\xi_{t}\right|, \eta=\zeta=0$ (the last two points are points of saddle type and lie on the critical surface of zero velocities (King, 2002, p. 197)), where

$$
\left|\xi_{t}\right|=\left(-\frac{G m_{2}}{p^{2} \alpha_{1}}\right)^{1 / 3} \quad, \quad p=1+\frac{m_{1}}{m_{2}},
$$

as well as the Jacobi integral:

$$
\varepsilon=\frac{V^{2}+\alpha_{1} \xi^{2}+\alpha_{3} \zeta^{2}}{2}-\frac{G m_{2}}{p^{2} r}=\text { const }
$$


where $V$ and $r$ are moduli of vectors of the velocity and distance from the cluster mass center of a star with mass $m_{1}$;

$$
\alpha_{1}=\left(\frac{1}{R} \frac{\partial \Phi}{\partial R}-\frac{\partial^{2} \Phi}{\partial R^{2}}\right)_{0}<0 \quad ; \quad \alpha_{3}=-\left(\frac{\partial^{2} \Phi}{\partial Z^{2}}\right)_{0}>0 .
$$

Here, $R$ and $Z$ are cylindrical Galactocentric coordinates of the point. "0" indices indicate that the derivatives of the Galactic potential $\Phi$ are calculated at the point with the coordinates $R=R_{0}=8200 \mathrm{pc}$ and $Z=0$. The formula for $\xi= \pm\left|\xi_{t}\right|$, when $m_{1} / m_{2} \rightarrow 0$, passes into the formula (11.13) from the book of King (2002, p.198) for the tidal radius of the cluster in the gravitational field of the Galaxy. Substituting $V=0$ in (2.1), we find the equation of the zero velocities surface. Substituting $\xi=\left|\xi_{t}\right|, \eta=\zeta=0$ into this equation, we find the critical value of the Jacobi integral corresponding to the critical surface of zero velocities:

$$
\varepsilon_{t}=\frac{3 G m_{2}}{2 p^{2}\left|\xi_{t}\right|}
$$

The distances from the coordinates' origin to this surface along the axes $\eta$ and $\zeta$ are equal to

$$
\left|\eta_{t}\right|=\frac{2}{3}\left|\xi_{t}\right| \quad \text { and } \quad\left|\zeta_{t}\right|=\left|\xi_{t}\right| q^{1 / 3}\left[(\sqrt{1+q}+1)^{1 / 3}-(\sqrt{1+q}-1)^{1 / 3}\right] \simeq 0.5016,
$$

respectively. Here, $q=-\alpha_{1} / \alpha_{3}$. In order to estimate $\alpha_{1}$ and $\alpha_{3}$, we take the Galaxy potential according to the model of Kutuzov and Osipkov (1980). The relation $\left|\eta_{t}\right|=\frac{2}{3}\left|\xi_{t}\right|$ can also be found in the book of Spitzer (1987), where the dimensions of the critical surface of zero-velocity of the cluster are discussed, when both the Galaxy and the cluster are modeled in the form of point masses. In the numerical experiments of Terlevich (1987) and Danilov (1997a) the strong flattening of the OSC models in the $\zeta$ direction, and also the triaxiality of surfaces of equal density and equipotential cluster surfaces at its periphery (with the greatest elongation of the cluster along the axis $\xi$ and the smallest along the axis $\zeta$ ) was noted. The same results were obtained by Heggie and Ramamani (1995) during the construction of a stationary collisionless model with a tidal truncation of its density and potential at the critical value of the Jacobi integral. As an approximation, we determine the dimensions of the critical surface of zero velocities in the cluster along the axes $\xi$, $\eta, \zeta$ using the cluster model considered here.

2. The cluster model from the 1 st item (see above) and the equation (2.1) allow only an approximate description of the motion of stars at the periphery of the cluster, since a significant fraction of the stars in the OSC are located at the periphery of the clusters (Terlevich, 1987; Danilov, 1997a). Therefore, as an approximation, we take here the critical zero-velocity surface of the OSC model in the form of a surface of an ellipsoid with semiaxes $a=\left|\xi_{t}\right|, b=\left|\eta_{t}\right|, c=\left|\zeta_{t}\right| \simeq a / 2$, and propose to search the unperturbed potential of the OSC in the following form:

$$
U=U_{0}-\left(U_{0}+U_{1}\right) r^{2}+S, \quad r^{2}=\left(\frac{\xi}{a}\right)^{2}+\left(\frac{\eta}{b}\right)^{2}+\left(\frac{\zeta}{c}\right)^{2}, \quad S=\frac{\alpha_{1} \xi^{2}+\alpha_{3} \zeta^{2}}{2} .
$$


Here, $U_{0}$ is a potential in the center of the cluster at $r=0 ; U_{1}$ is the cluster potential on the critical surface of zero velocities at the points $\eta= \pm b, \xi=\zeta=0$. The unperturbed potential (2.2) corresponds to the initial approximation for the cluster potential, which is used later with the construction of the unperturbed trajectories of the halo cluster stars. The potential (2.2) is the potential on the interior point of some homogeneous ellipsoid with the density $\rho$ :

$$
\rho=-\frac{\Delta U}{4 \pi G}=\frac{2\left(U_{0}+U_{1}\right)\left(a^{-2}+b^{-2}+c^{-2}\right)-\alpha_{1}-\alpha_{3}}{4 \pi G} .
$$

The equation of the surface of equal potentials $U$ from (2.2) has the following form:

$$
\gamma^{2}=\left(\frac{\xi}{a_{u}}\right)^{2}+\left(\frac{\eta}{b_{u}}\right)^{2}+\left(\frac{\zeta}{c_{u}}\right)^{2}=\text { const },
$$

where $a_{u}=a / \sqrt{1-\alpha_{1} a^{2} / s} ; \quad b_{u}=b ; \quad c_{u}=c / \sqrt{1-\alpha_{3} c^{2} / s} ; \quad s=2\left(U_{0}+U_{1}\right)$. Here, $a_{u}<a, c_{u}>c$, as $\alpha_{1}<0, \alpha_{3}>0$. The surfaces given by the equation (2.4), have a form closer to spherical than the surfaces corresponding to $r^{2}=1$.

Taking into account the potential $U$ from (2.2), the equations of motion of the star in the field of cluster forces and in the linearized force field of the Galaxy (5.517)-(5.519) from the book of Chandrasekhar (1942) take the form

$$
\ddot{\xi}=-\beta_{1}^{2} \xi+2 \omega \dot{\eta}, \quad \ddot{\eta}=-\beta_{2}^{2} \eta-2 \omega \dot{\xi}, \quad \ddot{\zeta}=-\beta_{3}^{2} \zeta,
$$

where

$$
\dot{\xi}=\frac{d \xi}{d t} \quad ; \quad \ddot{\xi}=\frac{d \dot{\xi}}{d t} ;
$$

the values $\dot{\eta}, \dot{\zeta}, \ddot{\eta}, \ddot{\zeta}$ are defined similarly;

$$
\beta_{1}^{2}=2\left(U_{0}+U_{1}\right) / a^{2} \quad ; \quad \beta_{2}^{2}=\beta_{1}^{2} a^{2} / b^{2} \quad ; \quad \beta_{3}^{2}=\beta_{1}^{2} a^{2} / c^{2} ;
$$

$\omega$ is angular velocity of cluster motion relative to the center of the Galaxy; values $\beta_{i}^{2}>0$, as $\left(U_{0}+U_{1}\right)>0$ in the OSC models studied here, see below.

Using the adopted notation, we write the Jacobi integral for the system of equations (2.5) in the following form:

$$
\frac{V^{2}+\beta_{1}^{2} \xi^{2}+\beta_{2}^{2} \eta^{2}+\beta_{3}^{2} \zeta^{2}}{2}=\epsilon=\text { const }, \quad \epsilon \geq 0 .
$$

Substituting $V=0$ in (2.6), we find the equation of the surface of zero velocities for the homogeneous ellipsoid under consideration: $\left(U_{0}+U_{1}\right) r^{2}=\epsilon$. Setting here $r^{2}=1$, we find the critical value of the energy of star motion in the field of a homogeneous ellipsoid and the Galaxy: $\epsilon_{t}=\left(U_{0}+U_{1}\right)$. Thus, the surface of zero velocities of the star's motion in the joint field of the models of potentials of the cluster and the Galaxy considered here is indeed ellipsoidal, and, when $\epsilon=\epsilon_{t}$, this surface can be set by equation $r^{2}=1$. Therefore, we can use $M_{0}=4 \pi a b c \rho / 3$ as an estimate of the mass of the considered ellipsoid. In this case, cluster stars completely fill the entire volume under the critical surface of zero velocities, which is observed 
Table 2.1 Parameters of OSC models

\begin{tabular}{|c|c|c|c|c|c|c|}
\hline $\mathcal{N}$ & $U_{0}$ & $U_{1}$ & $\nu_{1}$ & $\nu_{2}$ & $\beta_{3}$ & $\mu$ \\
\hline 1 & 2 & 3 & 4 & 5 & 6 & 7 \\
\hline 1 & $0.43618 \pm 0.00684$ & $-0.26109 \pm 0.00496$ & 0.04461 & 0.10789 & 0.11329 & 0.03709 \\
\hline 2 & $0.45341 \pm 0.00854$ & $-0.27200 \pm 0.00619$ & 0.04568 & 0.10918 & 0.11532 & -0.00982 \\
\hline 3 & $0.48275 \pm 0.00952$ & $-0.29354 \pm 0.00690$ & 0.04696 & 0.11075 & 0.11777 & -0.06771 \\
\hline 4 & $0.49263 \pm 0.00997$ & $-0.30194 \pm 0.00723$ & 0.04721 & 0.11104 & 0.11823 & -0.07870 \\
\hline 5 & $0.49656 \pm 0.01066$ & $-0.30204 \pm 0.00773$ & 0.04783 & 0.11180 & 0.11941 & -0.10713 \\
\hline 6 & $0.48760 \pm 0.00857$ & $-0.29949 \pm 0.00621$ & 0.04678 & 0.11053 & 0.11743 & -0.05955 \\
\hline
\end{tabular}

in the numerical experiments of Terlevich (1987) and Danilov (1997a), and in the model of Heggie and Ramamani (1995).

The equation $\left(U_{0}+U_{1}\right) r^{2}=\epsilon$ for the case $\epsilon=\epsilon_{t}$ considering (2.2) can be reduced to the form $U(r=1)=-U_{1}+S$; here $U(r=1)$ matches the values $U(\xi, \eta, \zeta)$ on the surface $r^{2}=1$. Thus, on the critical surface of zero velocities for the potential $U$ a boundary condition of the same type as in the model of Heggie and Ramamani (1995) is satisfied.

Danilov (2005) determined the magnitudes $U_{0}$ and $U_{1}$ for six numerical models of the OSC (Danilov and Dorogavtseva, 2003) by the Marquardt method (Marquardt, 1963) approximating the cluster potential $U(\xi, \eta, \zeta)$, averaged over the oscillation period of a regular field for a series of points (at $r \in[0.5,1.2])$ on the cluster periphery by the equation (2.2). The method for specifying such points $(\xi, \eta, \zeta)$ was described in the work of Danilov (1997a). The results of calculations of the parameters of cluster models are given in the Table 2.1. In the first column of this table, the numbers of the OSC models considered by Danilov and Dorogavtseva (2003) are given (the initial parameters of these OSC models are described in Section 16.1). Values $U_{0}$ and $U_{1}$ in (pc/Myr) ${ }^{2}$ for these models are given in columns 2 and 3 of the Table 2.1.

According to the data of the Table 2.1 the mean values of the semi-axes $a_{u}, b_{u}, c_{u}$ can be obtained for the six models of Danilov and Dorogavtseva (2003). The ratios of these mean values $\left\langle a_{u}\right\rangle,\left\langle b_{u}\right\rangle,\left\langle c_{u}\right\rangle$ can be represented in the following form: $\left\langle a_{u}>:<b_{u}>:<c_{u}>\sim 1: 0.84: 0.98\right.$. Thus, in the OSC models of Danilov and Dorogavtseva (2003) the surfaces of the equal values of the potential $U$ are close to spheroidal, with the spheroid compression along the axis $\eta$ (along the direction of cluster motion in the Galaxy).

The third equation of the system (2.5) can be integrated independently of the first two ones. The general solution for the system (2.5) has the form

$$
\begin{aligned}
& \xi=C_{1} \cos \left(v_{1}\left(t-t_{1}\right)\right)+C_{2} \cos \left(v_{2}\left(t-t_{2}\right)\right), \\
& \eta=A C_{1} \sin \left(v_{1}\left(t-t_{1}\right)\right)+B C_{2} \sin \left(v_{2}\left(t-t_{2}\right)\right), \\
& \zeta=C_{3} \cos \left(\beta_{3}\left(t-t_{3}\right)\right)
\end{aligned}
$$

where 


$$
A=\frac{\beta_{1}^{2}-v_{1}^{2}}{2 \omega v_{1}} \quad ; \quad B=\frac{\beta_{1}^{2}-v_{2}^{2}}{2 \omega v_{2}} ;
$$

$v_{1}, v_{2}, \beta_{3}$ are natural frequencies of the system (2.5); $C_{i}, t_{i}$ are constant values $(i=$ $1,2,3)$. Constants $v_{1}, v_{2}$ are determined from the condition for the existence of non-zero solutions for the system of the first two equations (2.5) and are equal to

$$
v_{1,2}=\sqrt{\frac{P}{2}\left(1 \pm f^{2}\right)}, \quad P=\beta_{1}^{2}+\beta_{2}^{2}+4 \omega^{2}, \quad f^{2}=\sqrt{1-\frac{4 \beta_{1}^{2} \beta_{2}^{2}}{P^{2}}} .
$$

Thus, the solution of (2.7) for $\xi$ and $\eta$ coordinates is dual-frequency (with frequencies $v_{1}, v_{2}$ ), and for $\zeta$ coordinate the solution is single-frequency (with frequency $\beta_{3}$ ). In our models, $v_{1}<v_{2}<\beta_{3}$. Estimates of the values of these frequencies in the units of (1/Myr), corresponding to the estimated parameters $U_{0}$ and $U_{1}$ from the table, are given in columns $4-6$ of the Table 2.1.

Let us note that the values $U_{0}$ and $U_{1}$, obtained as a result of the approximation of the gravitational potential of the OSC models of Danilov and Dorogavtseva (2003) by the expression (2.2), depend on the accepted values of $\alpha_{1}$ and $\alpha_{3}$. Since $a$ and $b$ depend on $\alpha_{1}$, and $c$ depends on $\alpha_{1}$ and $\alpha_{3}$ (see the formulas for $\left.\left|\xi_{t}\right|,\left|\eta_{t}\right|,\left|\zeta_{t}\right|\right)$, the values of $\beta_{i}^{2}$ in (2.5) depend on the constants $\alpha_{1}$ and $\alpha_{3}$.

\subsection{Small Parameter in the Equations of Stellar Motion}

The equations of motion of a star in the joint force field of the cluster and the Galaxy can be written in a Hamiltonian form. Using the Lagrange function $L$ of the cluster written with taking into account the Lagrange function from the work of Chandrasekhar (1942) for a star moving in the joint force field of the cluster and the Galaxy, we find generalized momenta of the $i$-th cluster star:

$$
\begin{gathered}
p_{\xi_{i}}=\frac{\partial L}{\partial \dot{\xi}_{i}}=m_{i}\left(\dot{\xi}_{i}-\omega \eta_{i}\right), \quad p_{\eta_{i}}=\frac{\partial L}{\partial \dot{\eta}_{i}}=m_{i}\left(\dot{\eta}_{i}+\omega\left(R_{0}+\xi_{i}\right)\right), \\
p_{\zeta_{i}}=\frac{\partial L}{\partial \dot{\zeta}_{i}}=m_{i} \dot{\zeta}_{i},
\end{gathered}
$$

where $R_{0}$ is the distance of the center of the cluster mass from the center of the Galaxy. Unlike to Chandrasekhar (1942), here we used the system of signs which provides $\alpha_{1}<0, \alpha_{3}>0, \Phi>0, U>0$.

Using (2.8) and $L$ function of the cluster, we determine Hamiltonian of the cluster:

$$
\begin{gathered}
H=\frac{1}{2} \sum_{i=1}^{N} m_{i}\left(\Theta_{i}-\omega^{2}\left[\left(R_{0}+\xi_{i}\right)^{2}+\eta_{i}^{2}\right]\right)+W-\sum_{i=1}^{N} m_{i} \Phi_{i}, \\
\Theta_{i}=\left(\frac{p_{\xi_{i}}}{m_{i}}+\omega \eta_{i}\right)^{2}+\left(\frac{p_{\eta_{i}}}{m_{i}}-\omega\left(R_{0}+\xi_{i}\right)\right)^{2}+\left(\frac{p_{\zeta_{i}}}{m_{i}}\right)^{2}
\end{gathered}
$$


Here, $N$ and $W$ are the number of stars and the potential energy of the cluster, $\Phi_{i}$ is the Galactic potential at the point $\left(\xi_{i}, \eta_{i}, \zeta_{i}\right)$. The first sum in (2.9) multiplied by $\frac{1}{2}$ is the kinetic energy $T$ of the cluster. Then the unperturbed Hamiltonian of the ellipsoidal cluster can be written in the form

$$
H_{0}=T_{0}+W_{0}-\rho \int_{Q} \Phi d Q,
$$

where the integration of the potential $\Phi$ has performed by the volume $Q$ of the cluster ellipsoid; $T_{0}, W_{0}$ are kinetic and potential energies of the ellipsoidal cluster.

Now, let us estimate the value of the small parameter $\mu$. In order to do this, we define the value of $T_{0}$ from conditions of virial equilibrium of the cluster. When calculating $T_{0}$, we also use the expression obtained by Chandrasekhar (1942) for the integral $L_{\zeta}$ of the angular momentum of rotation of the cluster about the axis $\zeta$. This integral, according to Chandrasekhar (1942), exists for clusters with symmetry in the mass distribution, corresponding to (2.2). As in the OSC models of Danilov and Dorogavtseva (2003), we set $L_{\zeta}=0$ (in this case the cluster does not rotate relatively to the external galaxies). In order to calculate $\int_{Q} \Phi d Q$, we use the expression

$$
\Phi \simeq \Phi_{0}-\omega^{2} R_{0} \xi-\frac{\left(\alpha_{1}+\omega^{2}\right)}{2} \xi^{2}-\frac{\omega^{2}}{2} \eta^{2}-\frac{\alpha_{3}}{2} \zeta^{2} .
$$

Here, $\Phi_{0}$ is the potential $\Phi$ at the distance $R_{0}$ from the center of the Galaxy. Differentiation of this expression with respect to the coordinates $\xi, \eta, \zeta$ leads to the corresponding components of the force field of the Galaxy, obtained by Chandrasekhar (1942).

Let us note that the absence of rotational symmetry of this expression for potential $\Phi$ in cylindrical galactocentric coordinates just causes the absence of the $L_{\zeta}$ integral in the work of Chandrasekhar (1942) for the star cluster in the general case (that is, in the absence of the constraints on the symmetry of the cluster (Chandrasekhar, 1942)). If we use the exact equations of stellar motion and carry out calculation of $L_{\zeta}$ using the property of rotational symmetry potential $\Phi$ in cylindrical galactocentric coordinates, it is possible to obtain the value of the integral $L_{\zeta}$, that is persisting over time $t$, and the expression for which coincides with one obtained by Chandrasekhar (1942) in the presence of restrictions on the cluster symmetry.

In order to calculate

$$
W_{0}=-\frac{1}{2} \rho \int_{Q} U d Q
$$

we use the value $U$ from (2.2). Using the expressions obtained for $T_{0}, W_{0}, \int_{Q} \Phi d Q$, we find an expression for the constant $H_{0}$ :

$$
H_{0}=-M_{0}\left(\Phi_{0}+\frac{\omega^{2} R_{0}^{2}+U_{0}}{2}-\Xi\right),
$$




$$
\Xi=\frac{\left(3 \beta_{1}^{2}+4 \omega^{2}+\alpha_{1}\right) a^{2}+\left(3 \beta_{2}^{2}+4 \omega^{2}\right) b^{2}+\left(3 \beta_{3}^{2}+\alpha_{3}\right) c^{2}}{20} .
$$

Let us introduce a small constant parameter $\mu=\left(H-H_{0}\right) / H$. Then $H=H_{0}+\mu H$. Here the second term on the right-hand side can be regarded as a small perturbation of the Hamiltonian $H$. Taking into account (2.9)-(2.10) and the values $U_{0}, U_{1}$ from the Table 2.1 for six OSC models of Danilov and Dorogavtseva (2003) the values of $\mu$ are defined. The results of calculations for $\mu$ for the considered OSC models are given in the seventh column of the Table 2.1. Thus, for the OSC models of Danilov and Dorogavtseva (2003), the values $|\mu|$ are small in comparison with the unity $(|\mu| \sim 0.01-0.1)$, and the signs of $\mu$ can be different in different models.

Equations for the motion of the $i$-th star, corresponding to the record of the Hamiltonian $H$ in the form $H=H_{0}+\mu H$ and (2.9), have the following form:

$$
\begin{aligned}
\dot{p}_{\xi_{i}}=-\frac{\partial H}{\partial \xi_{i}} & =(1+\mu)\left(\omega p_{\eta_{i}}+m_{i} \frac{\partial \Phi}{\partial \xi_{i}}\right)+m_{i} \frac{\partial U}{\partial \xi_{i}}-\mu \frac{\partial W}{\partial \xi_{i}}, \\
\dot{p}_{\eta_{i}}=-\frac{\partial H}{\partial \eta_{i}} & =(1+\mu)\left(-\omega p_{\xi_{i}}+m_{i} \frac{\partial \Phi}{\partial \eta_{i}}\right)+m_{i} \frac{\partial U}{\partial \eta_{i}}-\mu \frac{\partial W}{\partial \eta_{i}}, \\
\dot{p}_{\zeta_{i}}=-\frac{\partial H}{\partial \zeta_{i}} & =(1+\mu)\left(m_{i} \frac{\partial \Phi}{\partial \zeta_{i}}\right)+m_{i} \frac{\partial U}{\partial \zeta_{i}}-\mu \frac{\partial W}{\partial \zeta_{i}} \\
\dot{\xi}_{i} & =\frac{\partial H}{\partial p_{\xi_{i}}}=(1+\mu)\left(\frac{p_{\xi_{i}}}{m_{i}}+\omega \eta_{i}\right), \\
\dot{\eta}_{i} & =\frac{\partial H}{\partial p_{\eta_{i}}}=(1+\mu)\left(\frac{p_{\eta_{i}}}{m_{i}}-\omega\left(R_{0}+\xi_{i}\right)\right), \\
\dot{\zeta}_{i} & =\frac{\partial H}{\partial p_{\zeta_{i}}}=(1+\mu)\left(\frac{p_{\zeta_{i}}}{m_{i}}\right) .
\end{aligned}
$$

Differentiating the equations (2.12) by time, proceeding to Lagrange equations and substituting in them the expressions for $\Phi, U$ and $W$, we find

$$
\begin{aligned}
& \ddot{\xi}_{i}=(1+\mu)\left(2 \omega \dot{\eta}_{i}-\left(\beta_{1}^{2}+\mu \alpha_{1}\right) \xi_{i}+\mu G \sum_{j \neq i} f_{i j}\left(\xi_{j}-\xi_{i}\right)\right), \\
& \ddot{\eta}_{i}=(1+\mu)\left(-2 \omega \dot{\xi}_{i}-\beta_{2}^{2} \eta_{i}+\mu G \sum_{j \neq i} f_{i j}\left(\eta_{j}-\eta_{i}\right)\right), \\
& \ddot{\zeta}_{i}=(1+\mu)\left(-\left(\beta_{3}^{2}+\mu \alpha_{3}\right) \zeta_{i}+\mu G \sum_{j \neq i} f_{i j}\left(\zeta_{j}-\zeta_{i}\right)\right)
\end{aligned}
$$

where

$$
\begin{gathered}
f_{i j}=m_{j} /\left(r_{i j}^{2}-e^{2}\right)^{3 / 2} ; \quad i, j=1, \ldots, N ; \\
r_{i j}^{2}=\left(\xi_{i}-\xi_{j}\right)^{2}+\left(\eta_{i}-\eta_{j}\right)^{2}+\left(\zeta_{i}-\zeta_{j}\right)^{2}
\end{gathered}
$$

$e^{2}=$ const is a small smoothing addition to $r_{i j}^{2}$ used by Danilov and Dorogavtseva (2003) in the study of numerical OSC models.

Equations (2.13) pass into equations (2.5) at $\mu=0$. Let us introduce the following notations: $\dot{\xi}=u, \dot{\eta}=v, \dot{\zeta}=w$. We write a solution of the system (2.13) in the following form: 


$$
\begin{aligned}
& \xi_{i}(t, \mu)=\xi_{i}(0,0)+\int_{0}^{t} u_{i}(t, 0) d t \\
& \eta_{i}(t, \mu)=\eta_{i}(0,0)+\int_{0}^{t} v_{i}(t, 0) d t, \\
& \zeta_{i}(t, \mu)=\zeta_{i}(0,0)+\int_{0}^{t} w_{i}(t, 0) d t, \\
& u_{i}(t, \mu)=u_{i}(0,0)+\int_{0}^{t} \dot{u}_{i}(t, 0) d t, \\
& v_{i}(t, \mu)=v_{i}(0,0)+\int_{0}^{t} \dot{v}_{i}(t, 0) d t, \\
& w_{i}(t, \mu)=w_{i}(0,0)+\int_{0}^{t} \dot{w}_{i}(t, 0) d t .
\end{aligned}
$$

Here, the second argument of the values $\xi, \eta, \zeta, u, v, w$ denotes the used value of $\mu$. In (2.13), assuming terms proportional to $\mu^{2}$ equal to zero, we find the solution of the system (2.13) in the first approximation by $\mu$ with the help of the Picard method. We use here the unperturbed solution (2.7) as a zero approximation for $\xi, \eta, \zeta, u, v, w$. In order to find the solution of (2.14), we decompose the function $f_{i j}$ in a series in powers of $\Delta_{i j}=r_{i j}^{2}-r_{i j}^{2}(0)$ limited to the terms $\sim \Delta_{i j}^{3}$ :

$$
f_{i j} \simeq m_{j}\left(s_{i j}^{-3}-\frac{3 \Delta_{i j}}{2 s_{i j}^{5}}+\frac{15 \Delta_{i j}^{2}}{8 s_{i j}^{7}}-\frac{35 \Delta_{i j}^{3}}{16 s_{i j}^{9}}+\ldots\right),
$$

where $s_{i j}^{2}=r_{i j}^{2}(0)+e^{2} ; r_{i j}(0)$ is the initial distance between the $i$-th star and the $j$-th one.

\subsection{Stellar Motion Frequencies in OSC Models}

The solution of the system (2.13) according to the scheme indicated in (2.14), was obtained by Danilov (2005) in the first approximation of $\mu$. Because of the large amount of calculations in the solution of (2.14), it is possible to use only the zero, first and second terms of the expansion of the function $f_{i j}$ in powers of $\Delta_{i j}$. The solution is very cumbersome, it is valid only at small intervals of time and therefore is not given here. However, we can briefly write the frequencies of the harmonic oscillations making up the solution in the following form:

1. For motions of stars in $\zeta$ and $w$ coordinates when using the zero and first terms of the expansion $f_{i j}$ in powers of $\Delta_{i j}$, we found the following frequencies (Danilov, 2005):

- $\pm \beta_{3}$

- $\pm 3 \beta_{3}$,

- $\pm \beta_{3} \pm v_{1} \pm v_{2}$,

- $\pm \beta_{3} \pm 2 v_{1}$,

- $\pm \beta_{3} \pm 2 v_{2}$

(a total of 20 frequencies). For the motion in $\xi, u$ and $\eta, v$ coordinates, we found the following frequencies:

- $\pm v_{1}$

- $\pm v_{2}$, 
- $\pm 3 v_{1}$,

- $\pm 3 v_{2}$,

- $\pm v_{1} \pm 2 \beta_{3}$,

- $\pm v_{2} \pm 2 \beta_{3}$,

- $\pm v_{1} \pm 2 v_{2}$

- $\pm v_{2} \pm 2 v_{1}$

(a total of 24 frequencies).

2. For stellar motions in $\zeta$ and $w$ coordinates, when using the zero, first, and second terms of the expansion $f_{i j}$ in powers of $\Delta_{i j}$, in addition to the ones mentioned in item 1 , the following frequencies were found:

- $\pm \beta_{3} \pm 2 v_{1} \pm 2 v_{2}$

- $\pm \beta_{3} \pm 3 v_{1} \pm v_{2}$,

- $\pm \beta_{3} \pm v_{1} \pm 3 v_{2}$,

- $\pm 3 \beta_{3} \pm 2 v_{1}$

- $\pm 3 \beta_{3} \pm 2 v_{2}$

- $\pm 5 \beta_{3}$,

- $\pm \beta_{3} \pm 4 v_{1}$,

- $\pm \beta_{3} \pm 4 v_{2}$,

- $\pm 3 \beta_{3} \pm v_{1} \pm v_{2}$

(a total of 50 frequencies). For the motion in $\xi, u$ and $\eta, v$ coordinates, when using the zero, first, and second terms of the expansion $f_{i j}$ in powers of $\Delta_{i j}$, in addition to the ones mentioned in item 1 , the following frequencies were found:

- $\pm 5 v_{1}$,

- $\pm 5 v_{2}$

- $\pm 4 v_{1} \pm v_{2}$

- $\pm v_{1} \pm 4 v_{2}$

- $\pm 2 \beta_{3} \pm 3 v_{1}$,

- $\pm 2 \beta_{3} \pm 3 v_{2}$,

- $\pm 2 v_{1} \pm 3 v_{2}$

- $\pm 3 v_{1} \pm 2 v_{2}$,

- $\pm 4 \beta_{3} \pm v_{1}$

- $\pm 4 \beta_{3} \pm v_{2}$

- $\pm 2 \beta_{3} \pm v_{1} \pm 2 v_{2}$,

- $\pm 2 \beta_{3} \pm 2 v_{1} \pm v_{2}$

(a total of 52 frequencies).

Thus, the frequencies obtained by Danilov (2005) are linear combinations of the three eigenfrequencies of the star motion in the joint field $\mathbf{F}$ of forces of the potential (2.2) and the Galactic potential. Danilov (2005) and Danilov and Leskov (2005) perform a comparison of these frequencies with frequencies obtained as a result of the Fourier analysis of the trajectories of halo stars (Danilov and Leskov, 2004, 2005) in the numerical model 1 of the OSC (Danilov and Dorogavtseva, 2003). It allows to distinguish several of the most sifnificant resonances in the motion of halo stars over $\zeta$ and $w$ coordinates at frequencies: 
- $2 \beta_{3}-v_{2} \simeq \frac{3}{5} \omega_{r}$,

- $3 \beta_{3}-2 v_{2} \simeq \frac{5}{8} \omega_{r}$,

- $\beta_{3}+3 v_{1}-v_{2} \simeq \frac{5}{7} \omega_{r}$,

- $\frac{4}{7} \omega_{r}$.

In the motion of halo stars along $\xi$, $u$ coordinates, the main resonances in the cluster model 1 (Danilov and Dorogavtseva, 2003) are at frequencies:

- $\beta_{3}-3 v_{1}+v_{2} \simeq \frac{2}{5} \omega_{r}$,

- $\frac{4}{9} \omega_{r}, v_{2} \simeq \frac{9}{17} \omega_{r}$,

- $2 v_{2}-3 v_{1} \simeq \frac{7}{17} \omega_{r}$,

- $\frac{3}{8} \omega_{r}$,

and in the motion of halo stars along $\eta, v$ coordinates, the main resonances are found at the following frequencies:

- $-\beta_{3}+2 v_{2} \simeq \frac{1}{2} \omega_{r}$,

- $2 \beta_{3}-3 v_{1} \simeq \frac{7}{15} \omega_{r}$,

- $\beta_{3}-3 v_{1}+v_{2} \simeq \frac{2}{5} \omega_{r}$,

- $3 \beta_{3}-2 v_{2} \simeq \frac{3}{5} \omega_{r}$,

where $\omega_{r}$ is the oscillation frequency of a regular cluster field. At these frequencies (or near them), 6-10 (sometimes up to 15) halo star trajectories in the model 1 are grouped (Danilov and Dorogavtseva, 2003). The frequencies noted here are in a rational relation to the oscillation frequency of the regular cluster field. The most probable reason for the formation of such groups of trajectories is the effect of "frequency pulling" or "frequency shift" , when oscillations are synchronizing; this effect was described in the literature for the self-oscillating systems with a small and large number of degrees of freedom (see Migulin et al. (1988, p.290) and Rabinovich and Trubetskov (2000, p.348)). As a result of such synchronization of oscillations, a set of frequencies, commensurate with the frequency of regular field oscillations, is formed in the system. At some of such frequencies, synchronous motion of stellar groups along trajectories is observed (motion with the same frequency). At the same time, a set of intervals of distances from the cluster center is formed in the system, the motion in which for halo stars becomes preferable in the presence of regular field oscillations.

\subsection{Conclusions}

In this part of the book, we have considered the potential model (2.2) which is approximating the gravitational potential at the periphery of numerical dynamic OSC models of Danilov and Dorogavtseva (2003) and admitting analytical solution for the equations of stellar motion in the joint force field $\mathbf{F}$ of potential (2.2) and the Galactic potential. For six OSC models of Danilov and Dorogavtseva (2003), we 
have listed parameters of the potential (2.2), the shape of its equipotential surfaces and the shape of the zero velocities surface of stellar motion in the field of forces $\mathbf{F}$.

2. We have written the equations of stellar motion at the OSC periphery, which contain a small parameter $\mu$. For six OSC models of Danilov and Dorogavtseva (2003) we list the values of $\mu$ obtained by Danilov (2005) considering the parameters of the potential (2.2) and the Galactic potential. We have presented a general analytical solution of the stellar motion equations for $\mu=0$. Stellar motion in the force field $\mathbf{F}$ is three-frequency (single-frequency motion along $\zeta$ coordinate, and two-frequency motion in each of the $\xi$ and $\eta$ coordinates). These frequencies are the eigenfrequencies of the stellar motion in the joint field of forces of the potential (2.2) and the Galactic potential.

3. We have given the frequencies of stellar motion in the first order expansions by $\mu$ of the solutions of the equations of stellar motion at the OSC periphery. These frequencies are linear combinations of the natural frequencies of stellar motion in the field of forces $\mathbf{F}$. The coefficients of such linear combinations are integer numbers. Several main resonances in the motion of halo stars in the cluster model 1 of Danilov and Dorogavtseva (2003) have been noted. The frequencies of these resonances are commensurate (or close to commensurability) with the oscillation frequency of a regular cluster field.

4. The small groups of halo star trajectories at these frequencies (or near them) are formed. It was noted for the numerical cluster model 1 in the work of Danilov and Leskov (2005). This fact indicates that a set of intervals of distances from the cluster center is formed in the system, the motion in which for halo stars becomes preferable in the presence of regular field oscillations.

\section{References}

Chandrasekhar, S.: Principles of Stellar Dynamics. Univ. Chicago Press, Chicago, Ill (1942). LCCN: 43-5608 (BKS1)

Danilov, V.M.: Numerical experiments simulating the dynamics of open clusters in the Galactic field. Astron. Reports 41, 163-173 (1997a).

Danilov, V.M.: Stellar Fluxes in Numerical Dynamical Models of Open Clusters. Astron. Reports 46, 887-899 (2002b). doi:10.1134/1.1522077

Danilov, V.M.: The Motion of Halo Stars in Dynamical Numerical Models of Open Clusters. Astron. Reports 49, 3604-610 (2005). doi:10.1134/1.2010649

Danilov, V.M., Dorogavtseva, L.V.: Estimates of Relaxation Times in Numerical Dynamical Models of Open Star Clusters. Astron. Reports 47, 483-491 (2003). doi:10.1134/1.1583775

Danilov, V.M., Leskov Ye.V.: Properties of stellar trajectiries in numerical dynamical models of open stellar clusters. In: Byrd, G.G., Kholshevnikov, K.V., Myllari, A.A., Nikiforov,I.I., Orlov, V.V. (eds) Order and chaos in stellar and planetary systems, ASP Conf. Ser. 316, 183-185 (2004) 
Danilov, V.M., Leskov, E.V.: Properties of Stellar Trajectories in Numerical Dynamical Models of Open Star Clusters. Astron. Reports 49, 190-200 (2005). doi: $10.1134 / 1.1882777$

Heggie, D.C., Ramamani, N.: Approximate self-consistent models for tidally trancated star clusters. Mon. Not. R. Astron. Soc. 272, 317-322 (1995). doi:10.1093/mnras/272.2.317

King, Ivan R.: An Introduction to Classical Stellar Dynamics. URSS, Moscow (2002) (In Russian).

Kutuzov, S.A., Osipkov, L.P.: A Generalized Model for the Three-Dimensional Gravitational Potential of Stellar Systems. Soviet Astron. 24, 17-22 (1980).

Laskar, J.: A numerical experiment on the chaotic behavior of the solar system. Nature 338, 237-238 (1989). doi:10.1038/338237a0

Marquardt, D.W.: An algoritm for least-squares estimation of nonlinear parameters. J. Soc. Indust. Appl. Math. 11, 431-441 (1963). doi:10.1137/0111030

Migulin V.V. et al.: Foundation of the oscillations theory, 2nd Ed. Nauka, Moscow (1988) (In Russian).

Rabinovich, M.I., Trubetskov, D.I.: Introduction to the theory of oscillations and waves. Sci.-Editorial Center "Regular and Chaotic Dynamics", Izhevsk (2000) (In Russian).

Spitzer, L.: Dynamical evolution of globular clusters. Princeton University Press, Princeton, NJ (1987). ISBN-13: 978-0691084602

Terlevich, E.: Evolution of n-body clusters. Mon. Not. R. Astron. Soc. 224, 193-225 (1987). doi:10.1093/mnras/224.1.193 



\title{
Chapter 3 \\ Properties of Stellar Trajectories in Numerical Dynamical Models of Open Stellar Clusters
}

\begin{abstract}
In this chapter, we consider stellar trajectories in the models of OSCs which are non-stationary in a regular field. Estimates of the maximum Lyapunov characteristic exponent $\lambda$ of the stellar trajectories in the OSC models are performed. The average $\lambda$ values in the considered OSC models are $\bar{\lambda} \simeq 1$ /Myr. Cluster cores are the regions of increased stochasticity; halos are the regions of ordered star motion. With the density of the cluster model increases, the value of $\bar{\lambda}$ grows, and the sizes of the high stochasticity region in the cluster core increase. An "adhesion" of the star trajectory in the phase space to the region with the given $\lambda$ value is noted. We perform the Fourier analysis of stellar trajectories in the considered OSC models and construct the distributions of the trajectories of the stars by the periods of their motions with the largest values of the power spectrum. In the distributions of stellar trajectories by the periods of the most significant oscillations, we detect the peaks with periods corresponding to (or close to) the periods commensurate with the oscillations periods of the regular field of the system. We discuss the features of the distributions of stellar trajectories by periods of the most significant oscillations and the reasons for the formation of these features in the considered OSC models.
\end{abstract}

\subsection{Introduction}

In the papers (Danilov, 2002a,b) we consider the models of open star clusters that are non-stationary in a regular field, close to gravitational instability and moving along circular orbits in the field of the Galaxy. Small-scale density oscillations due to stellar encounters in such systems are easily amplified to the level of large-scale ones. During the violent relaxation at $t<\tau_{v r}$, radial oscillations with practically constant amplitude and period are set (here, $t$ is time, $\tau_{v r}$ is violent relaxation time). In order to analyze stellar flux in OSC models, Danilov (2002b) and Danilov and Dorogavtseva (2003) carried out a statistical study of small segments of stellar trajectories, which made it possible to estimate relaxation times of stellar motion in the spaces of a number of parameters of stellar motion, as well as to detect the 
transfer of an energy from the large-scale stellar motions to the small-scale ones during the periodical changes in the regular field of the OSC model, etc.

The properties of the stellar trajectories in the OSC models of Danilov (2002b); Danilov and Dorogavtseva (2003) were first considered by Danilov and Leskov (2005). Danilov (2002b) has suggested that in the denser OSC models (with a lower degree of non-stationarity in a regular field) the number and influence of stochastic stellar trajectories increases, which probably leads to a decrease of the role and disappearance of barriers in the phase space (PS) of such systems. The estimates (Danilov, 2002a,b) show that the degree of stochasticity of stellar trajectories in cluster models of Danilov and Dorogavtseva (2003) does increase in denser models with a lower degree of non-stationarity.

In order to study the properties of stellar trajectories in the models of stellar systems, the following methods are usually applied:

- estimates of the Lyapunov characteristic exponents (Kandrup et al., 1997; Habib et al., 1997; Kandrup, 1998; Carpintero et al., 1999; Muzzio et al., 2001; Carpintero et al., 2003; Muzzio and Mosquera, 2004; Kandrup and Sideris, 2002; Caranicolas and Vozikis, 1999);

- Fourier analysis of stellar trajectories (Carpintero et al., 1999; Muzzio et al., 2001) and complexity indicators of trajectories based on the study of trajectory power spectra (two different definitions of the complexity of trajectories were given by Kandrup et al. (1997) and Kandrup and Sideris (2002));

- Fourier analysis of the deviation of close trajectories and the correlation analysis of time series corresponding to the dependency of the phase coordinates of stars on time (Vozikis et al., 2000);

- a geometric method using the Ricci curvature estimate (El-Zant, 1998);

- a method of the angular dynamic spectra (Efthymiopoulos et al., 1999; Voglis et al., 1999), and the parameters based on this method: parameters ROTOR and "spectral distance $D$ " of the spectra of two different initial vectors of deviations of perturbed trajectories from basic trajectory (Voglis et al., 1999);

- Poincaré section (Carpintero et al., 2003);

- mapping equations (Efthymiopoulos et al., 1999; Voglis et al., 1999; Lega and Froesle, 2001; Papaphilippou and Laskar, 1996);

- method of frequency maps (Lega and Froesle, 2001; Papaphilippou and Laskar, 1996);

- Fast Lyapunov Indicator (FLI) and the FLI card method (Lega and Froesle, 2001), etc.

As a rule, a combination of two (or more) different methods is used to study the properties of stellar trajectories. In the overwhelming majority of cases, these methods are used to study non-self-consistent, stationary and collisionless models of stellar systems and clusters. When the systems of $N$ gravitating point bodies are investigated (see, for example, El-Zant (1998)), most often the isolated clusters near virial equilibrium are considered.

Investigation of the properties of stellar trajectories in dynamic models of star clusters was performed by Carpintero et al. (1999),Muzzio et al. (2001),Carpintero 
et al. (2003),Muzzio and Mosquera (2004). These works consider stationary collisionless models of star clusters moving along a circular orbit around the center of the Galaxy. Carpintero et al. (1999) and Muzzio et al. (2001) noted an increase of the fraction of chaotic stellar trajectories in the outer parts of the globular cluster models, see fig. 6 from the paper of Carpintero et al. (1999) and fig. 3.1 from the paper of Muzzio et al. (2001) (stellar encounters were not taken into account when the fraction of chaotic trajectories were estimated by Carpintero et al. (1999) and Muzzio et al. (2001)). According to Carpintero et al. (1999), the proportion of chaotic trajectories at the cluster periphery increases up to $50-90 \%$. Muzzio et al. (2001) noted that the randomness on the cluster periphery arises as a result of mutual action on the star of three forces: the force of the attraction of the cluster, the centrifugal-centripetal force (which acts differentially), and the Coriolis force.

Let us note that consideration of the effect of stellar encounters in the OSC models made in our work leads to a significant increase in the degree of stochasticity and to the complication of the Fourier spectra of the trajectories of the cluster core stars in comparison with the estimates of Carpintero et al. (1999), Muzzio et al. (2001), Carpintero et al. (2003), and Muzzio and Mosquera (2004).

To reveal the role of synchronization of stellar motion in formation of the frequency spectrum of the oscillation motions of stars in the OSC models, the Fourier analysis of stellar trajectories is of considerable interest. It is also of interest to investigate the distributions of stellar trajectories in the clusters by the periods (obtained from the analysis of the PCS dependencies on time) with the largest values of the spectral density for the trajectory. An analysis of such distributions will make it possible to establish the most significant periodicity in stellar motions. A comparison of such distributions for the OSC models with the different degrees of non-stationarity will make it possible to study the effect of synchronization of radial stellar motions in clusters on the stellar motions with the frequencies incommensurate with the frequency of the radial oscillations of the OSC model.

According to Rabinovich and Trubetskov (2000, p.348), in the self-oscillating systems with a large number of freedom degrees, a synchronization of oscillations leads to a shift in frequency of oscillations and the formation in the system of a set of frequencies commensurate with the frequency of synchronous oscillations. If such a mechanism contributes to the evolution of the OSC models, its action will result in the extraction of the most significant frequencies (and periods) of stellar motion and the appearance of resonant peaks in the distributions of stellar trajectories by the periods (keeping in mind the resonances with radial oscillations of the cluster model). To study the features of synchronization of stellar motions in the models of non-stationary OSC, it is of interest to consider the consistency of the phases in the periodic components of stellar trajectories with the oscillation phases of the regular field of the cluster models.

The objective of this part of the work is to discuss the properties of stellar trajectories in the OSC models which are non-stationary in a regular field. 


\subsection{Basic Formulas and Notations}

Equations of stellar motion (5.517)-(5.519) from the book of Chandrasekhar (1942) can be written in the following form:

$$
\begin{aligned}
& \dot{\xi}_{i}=u_{i}, \quad \dot{\eta}_{i}=v_{i}, \quad \dot{\zeta}_{i}=w_{i} ; \\
& \dot{u}_{i}=2 \omega v_{i}-\alpha_{1} \xi_{i}+G \sum_{j=1, j \neq i}^{N}\left(\xi_{j}-\xi_{i}\right) / r_{i j}^{3}, \\
& \dot{v}_{i}=-2 \omega u_{i}+G \sum_{j=1, j \neq i}^{N}\left(\eta_{j}-\eta_{i}\right) / r_{i j}^{3}, \\
& \dot{w}_{i}=-\alpha_{3} \zeta_{i}+G \sum_{j=1, j \neq i}^{N}\left(\zeta_{j}-\zeta_{i}\right) / r_{i j}^{3}, \quad i=1, \ldots, N,
\end{aligned}
$$

where $N$ is the number of stars; $\xi_{i}, \eta_{i}, \zeta_{i}$ and $u_{i}, v_{i}, w_{i}$ are components of the radius vector $\mathbf{r}_{\mathbf{i}}$, and the velocity vector $\mathbf{v}_{\mathbf{i}}$ of the $i$-th star, respectively (the mass of a star in (3.1) is assumed to be equal to $1 M_{\odot}$, see above). Equations (3.1), linearized in the neighborhood of the $i$-th star trajectory, lead to the equations "in variations" for small perturbations modulo $\delta \xi_{i}, \delta \eta_{i}, \delta \zeta_{i}, \delta u_{i}, \delta v_{i}, \delta w_{i}$ :

$$
\begin{aligned}
& \delta \dot{\xi}_{i}=\delta u_{i}, \quad \delta \dot{\eta}_{i}=\delta v_{i}, \quad \delta \dot{\zeta}_{i}=\delta w_{i}, \\
& \delta \dot{u}_{i}=2 \omega \delta v_{i}-\alpha_{1} \delta \xi_{i}+G \sum_{j=1, j \neq i}^{N}\left[\delta \xi_{j}-\delta \xi_{i}-\left(\xi_{j}-\xi_{i}\right) a_{i j}\right] / r_{i j}^{3}, \\
& \delta \dot{v}_{i}=-2 \omega \delta u_{i}+G \sum_{j=1, j \neq i}^{N}\left[\delta \eta_{j}-\delta \eta_{i}-\left(\eta_{j}-\eta_{i}\right) a_{i j}\right] / r_{i j}^{3}, \\
& \delta \dot{w}_{i}=-\alpha_{3} \delta \zeta_{i}+G \sum_{j=1, j \neq i}^{N}\left[\delta \zeta_{j}-\delta \zeta_{i}-\left(\zeta_{j}-\zeta_{i}\right) a_{i j}\right] / r_{i j}^{3}, \\
& a_{i j}=3\left(\left(\mathbf{r}_{j}-\mathbf{r}_{i}\right) \delta\left(\mathbf{r}_{j}-\mathbf{r}_{i}\right)\right) / r_{i j}^{2}, \\
& r_{i j}^{2}=\left(\xi_{j}-\xi_{i}\right)^{2}+\left(\eta_{j}-\eta_{i}\right)^{2}+\left(\zeta_{j}-\zeta_{i}\right)^{2}+e^{2}, \\
& i=1, \ldots, N
\end{aligned}
$$

where $e=$ const is a small constant addition to the square of distance between the stars (that is a smoothing parameter (Danilov, 1997a)); $\alpha_{i}=$ const, $i=1,3, \alpha_{1}<0$, $\alpha_{3}>0 ; \omega=$ const, $\omega$ is the angular velocity of the OSC motion around the center of the Galaxy. The values $\alpha_{i}$ and $\omega$ are determined in this work with the help of the potential of the Galaxy of Kutuzov and Osipkov (1980).

In order to calculate stellar trajectories in the OSC models and the Maximum Lyapunov Characteristic Exponents (MLCE) $\lambda$, we numerically integrated the system of equations (3.1)-(3.2) in this work. To estimate the values of $\lambda$, we used the technique described by Lichtenberg and Lieberman (1983), see formula (5.3.10). As initial $\delta \xi_{i}, \delta \eta_{i}, \delta \zeta_{i}$, we choose the small magnitudes corresponding to $\delta r_{i} \leq 0.01 r_{i}$; 
Table 3.1 Parameters of OSC Models

\begin{tabular}{|c|c|c|}
\hline $\mathcal{N}$ & $\tau_{v r}$ & $\bar{\lambda}$ \\
\hline & $\mathrm{Myr}$ & $\mathrm{Myr}^{-1}$ \\
\hline 1 & 49.9 & $0.79 \pm 0.03$ \\
\hline 2 & 41.8 & $0.97 \pm 0.04$ \\
\hline 3 & 41.8 & $1.21 \pm 0.04$ \\
\hline 4 & 34.3 & $1.25 \pm 0.05$ \\
\hline 5 & 41.8 & $1.22 \pm 0.07$ \\
\hline 6 & 41.8 & $1.04 \pm 0.03$ \\
\hline
\end{tabular}

the initial $\delta u_{i}, \delta v_{i}, \delta w_{i}$ corresponded to $\delta \xi_{i}, \delta \eta_{i}, \delta \zeta_{i}$, and were obtained using the formula for the moduli of initial stellar velocities, see Section 16.1.

\subsection{The Lyapunov Times and Oscillation Periods of Stellar Trajectories}

Danilov and Leskov (2005) analyzed stellar trajectories in six OSC models, the parameters of which are listed in Table 3.1 and in Section 16.1, Table 16.1. Danilov (2002b), Danilov (2002a), Danilov and Dorogavtseva (2003), and Danilov and Leskov (2005), consider a cluster containing $N=500$ stars and moving in the Galactic plane along a circular orbit with a radius of $8200 \mathrm{pc}$ around the Galactic center. At the initial time $t=0$, the star cluster is modeled as a system of two gravitating balls simulating a halo and a core with coincident centers of mass. Six models of OSC are considered, the data of which are given in Section 16.1, Table 16.1, and in Table 3.1. The first column of Table 3.1 and the first column of Table 16.1 contain the numbers of OSC models. Danilov and Leskov (2005) performed the estimates of the MLCE $\lambda$ of the stellar trajectories in these OSC models and plotted the distributions $n\left(\lambda \tau_{v r}\right)$ of stellar trajectories by $\lambda \tau_{v r}$ values, see fig. 3.1. The values $\lambda$ obtained for stellar trajectories can be set in accordance with both the initial and final phase coordinates of the stars. Due to this, we also plot the diagrams of $\left(r(t), \lambda \tau_{v r}\right)$ and families of lines of equal values $\lambda \tau_{v r}=$ const in the space of $(r, v)$ for two time points $t / \tau_{v r}=0,2.6$.

Fig. 3.1 shows distributions $n\left(\lambda \tau_{v r}\right)$ in cluster models $1,3,5,6$. The distributions $n\left(\lambda \tau_{v r}\right)$ in clusters 2,4 (with the number of stars in the core $N_{1}=100$ ) have a form similar to the distribution $n\left(\lambda \tau_{v r}\right)$ in cluster model 1 . Therefore, distributions $n\left(\lambda \tau_{v r}\right)$ for models 2,4 are not shown here. A majority of the stellar trajectories in models 1, 2, 4 have small positive $\lambda \tau_{v r}\left(0<\lambda \tau_{v r} \leq 40-50\right)$. In this case, $0<\lambda<1 \mathrm{Myr}^{-1}$. These $\lambda$ values correspond to the stellar trajectories located at considerable distances from the cluster center. In cluster model 1 , these trajectories correspond to the Lyapunov time $t_{\lambda}=\lambda^{-1} \sim(1.2-12.5)$ Myr. Stellar trajectories 

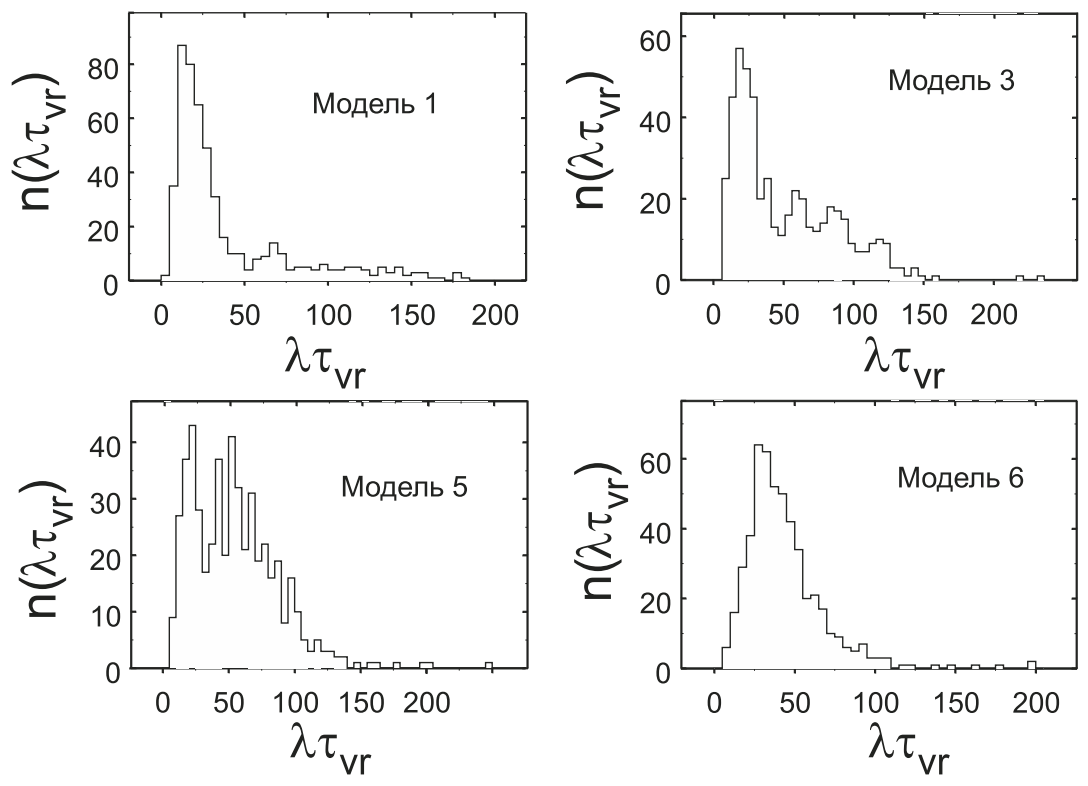

Fig. 3.1 Distributions of stellar trajectories by $\lambda \tau_{v r}$ in four OSC models

in the cluster core correspond to large $\lambda \sim\left(1 \mathrm{Myr}^{-1}<\lambda<(4-5) \mathrm{Myr}^{-1}\right)$, and, consequently, $t_{\lambda} \sim(0.2-1.0)$ Myr.

Using the distributions of the stellar trajectories with respect to $\lambda \tau_{v r}$, we obtained the mean MLCE values $\bar{\lambda}$ (and their errors) in the OSC models. $\bar{\lambda}$ increases with increasing density and decreasing degree of non-stationarity of the analyzed OSC models from $0.8\left(\mathrm{Myr}^{-1}\right)$ to $1.25\left(\mathrm{Myr}^{-1}\right)$, see Table 3.1. Initial values of $\tau_{v r}$ for OSC models (in Myr) are given in column 2 of Table 3.1, the mean $\bar{\lambda}$ of MLCE estimates $\lambda$ (in $\mathrm{Myr}^{-1}$ ) are given in column 3 of Table 3.1. Thus, the degree of stochasticity of stellar motions in OSC models decreases at large amplitudes of oscillations of a regular field (in strongly non-stationary systems).

The diagrams $\left(r(0), \lambda \tau_{v r}\right)$ for models 1,6 of the clusters are shown in fig. 3.2. The plots of $r(0)$ dependencies on $\lambda \tau_{v r}$ for models 2, 3, 4 have the form similar to this dependency for model 1 . The distributions of the points on diagrams $\left(r(0), \lambda \tau_{v r}\right)$ for models 5 and 6 also have similar appearance. Therefore, diagrams $\left(r(0), \lambda \tau_{v r}\right)$ for models $2-5$ are not shown here.

In fig. 3.2 for model 1, the regions occupied by the stars of the core and the halo are separated quite clearly by the line $\lambda \tau_{v r}=50$. With an increase in the number of stars in the core $N_{1}$, the size of the core increases (from $2.5 \mathrm{pc}$ in model 1 to $5.5 \mathrm{pc}$ in model 6), and the boundary value $\lambda \tau_{v r}$ separating trajectories of the core stars and the halo stars on the diagram $\left(r(0), \lambda \tau_{v r}\right)$, varies to a little degree: $\lambda \tau_{v r} \approx 40-50$.

In models $1-5$, dispersion of $\lambda$ (and $\lambda \tau_{v r}$ ) values of the core stars' trajectories is much larger than in the halo (fig. 3.1, 3.2). Probably, in the regions of phase transition 

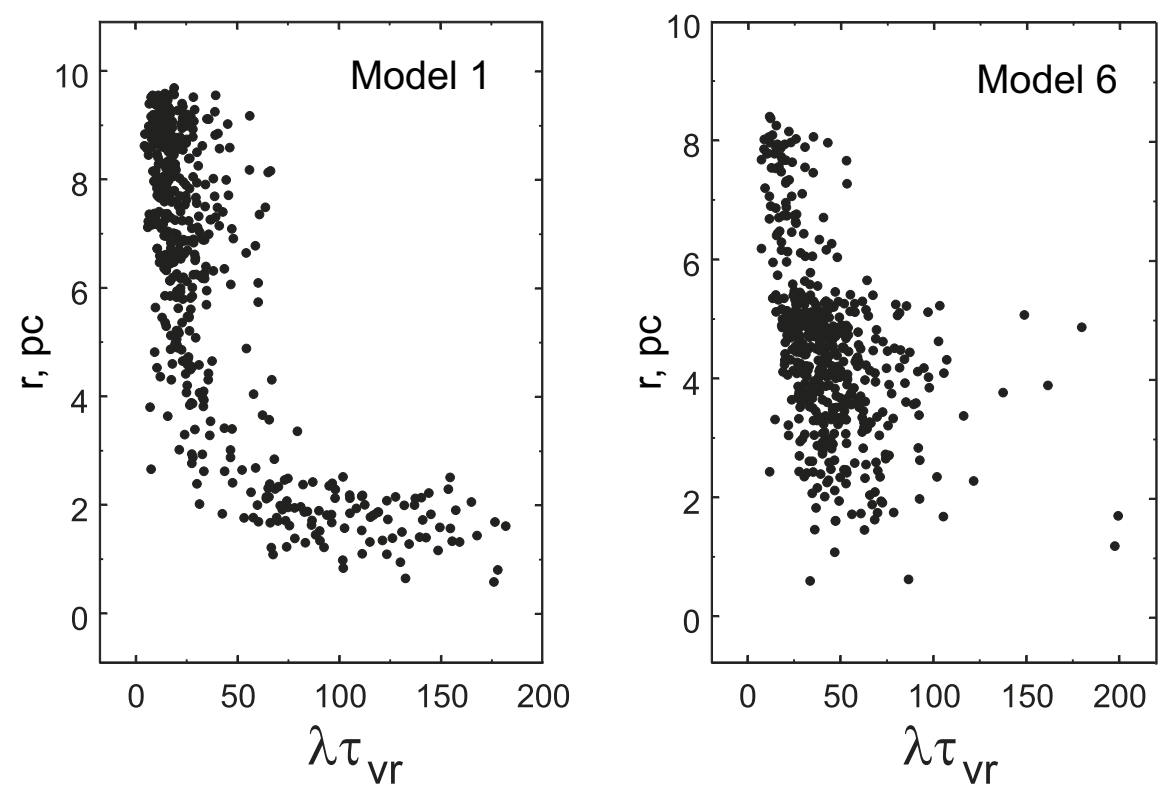

Fig. 3.2 Diagrams $\left(r(0), \lambda \tau_{v r}\right)$ for stellar trajectories in two OSC models

corresponding to the cluster core, the regions with high and low stochasticity of stellar trajectories largely coexist with each other, see fig. 3.2, 3.3.

In fig. 3.3, for cluster model 1 , the countour lines $\lambda \tau_{v r}=$ const are given in the space of $(r, v)$ for two time points $t$ ( $r$ is the distance from the cluster center (the center of mass) and $v$ is the absolute value of the velocity of the star). Numbers at the countour lines in fig. 3.3 show $\lambda \tau_{v r}$ value for a given countour line. $\lambda \tau_{v r} \in[4.2,181.8]$. White regions in $(r, v)$ plane are not occupied by stars at a corresponding point in time. Darker regions in $(r, v)$ plane correspond to large $\lambda \tau_{v r}$ values.

The countour lines of $\lambda \tau_{v r}=$ const have a similar form for other models of clusters considered here, therefore, the corresponding plots are not shown here. At the initial time, the region of significant stochasticity $(\lambda>\bar{\lambda})$ in the OSC models is located in the cores (at small distances $r$ from the cluster center) and in the region of small and intermediate $v$. During the evolution of OSC models, regions of significant stochasticity extend into the region of even smaller $r$ and $v$, see fig. 3.3. In the cores of OSC models, at the time of $t / \tau_{v r} \approx 2.7-3$ in the space of $(r, v)$, the extended regions of high stochasticity are penetrated by narrow regions with small stochasticity, and on the cluster periphery in regions of more ordered motion, we sometimes can find the small areas of an increased stochasticity. In models $1-6$, the regions of ordered and stochastic motion in the space $(r, v)$ are adjacent to each other.

Thus, stellar trajectories with large values $\lambda$ are located in the cores of OSC models, and trajectories with small $\lambda$ are located (generally) at the cluster periphery, 
323 Properties of Stellar Trajectories in Numerical Dynamical Models of Open Stellar Clusters
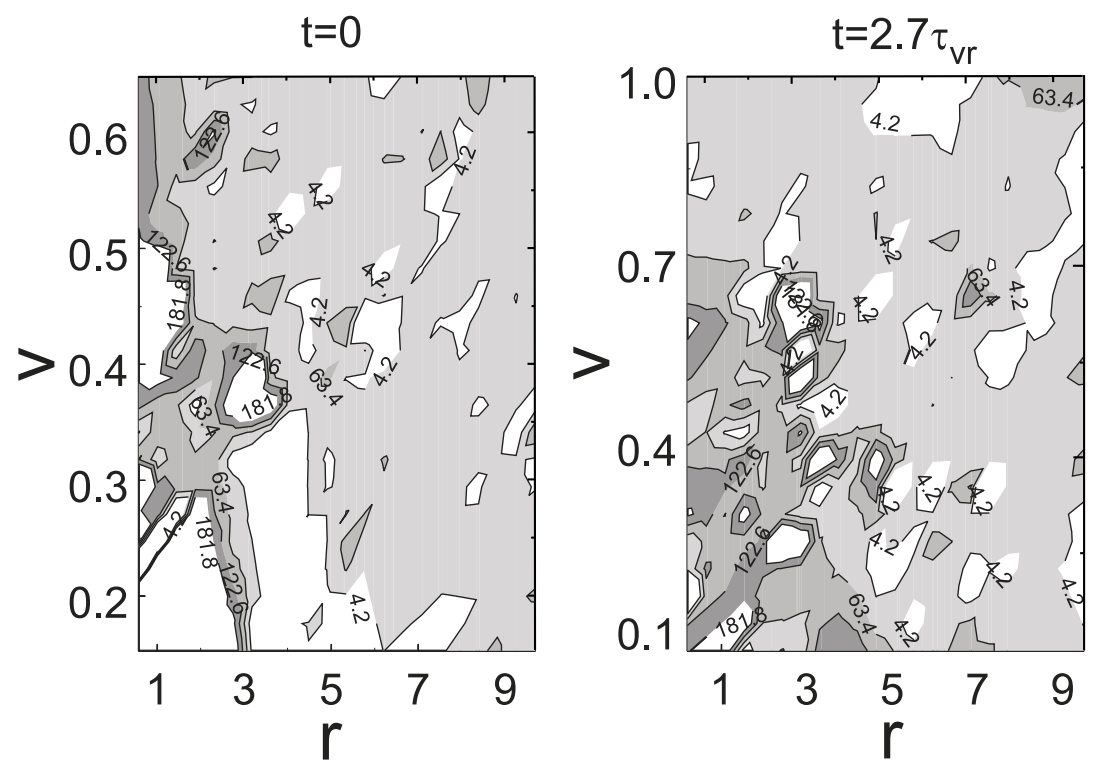

Fig. 3.3 Contour lines $\lambda=$ const in the space of $(r, v)$ for two time points $t$ in cluster model 1

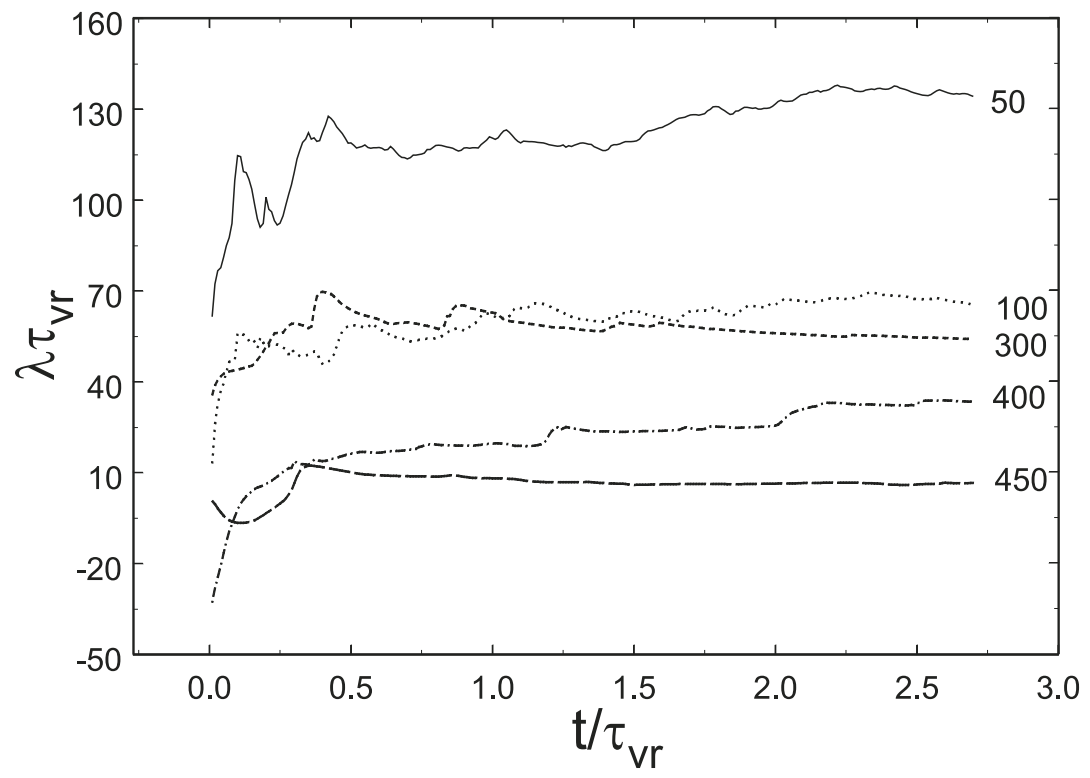

Fig. 3.4 Dependencies of the $\lambda \tau_{v r}$ estimates on time $t / \tau_{v r}$ for five stellar trajectories in cluster model 1. Numbers in the curves correspond to the number of the star in the cluster model. For these trajectories, a larger number of star corresponds, on average, to a larger distance of the trajectory from the cluster center 
see also fig. 3.2, 3.3. Consequently, in the non-stationary OSC, the cores of the clusters are the regions of increased stochasticity, and the halos form the regions of more regular stellar motions. In the denser OSC models, the dimensions of the increased stochasticity regions increase in the core.

The Lyapunov times (Danilov and Leskov, 2005) for "external" stellar trajectories at the clster periphery obtained by Danilov and Leskov (2005), are $\sim 42-83$ times less than $t_{\lambda}$ obtained by Carpintero et al. (1999) for external stellar trajectories in nonisolated collisionless models of globular clusters. Thus, the taking into account the effect of stellar encounters in estimating $t_{\lambda}$ values is of decisive importance, and leads to a significant decrease in $t_{\lambda}$ estimates in the models of star clusters.

When $\lambda$ is calculated with the method of Lichtenberg and Lieberman (1983) for each trajectory, the $\lambda$ estimate grows very rapidly over time, and reaches its highest value even at small $t$, after which the changes in $\lambda$ become insignificant, see fig. 3.4. Thus, there is an "adhesion" of stellar trajectory in the phase space (PS) to a region with the given $\lambda$.

For each trajectory Danilov and Leskov (2005) obtained amplitudes $A$, periods $P$, and phases $\Phi$ of the first three sinusoidal components (with the largest amplitudes) of the phase coordinates of stars (PCS) change over time. We used a technique for isolating the sinusoidal components (SC) of the stellar coordinates changes described in the papers of Carpintero and Aguilar (1998); Laskar (1988). We estimated the values of $q=E_{3} / E_{t}$, where $E_{3}$ and $E_{t}$ are the energies, which are obtained for the first three SCs and for the entire Fourier spectrum of the dependency $r=r(t)$ for each trajectory, respectively. Here and below, in the time dependencies of the considered values $r, \dot{r}, \theta, \xi, \eta, \zeta$, the trend, which is determined for these dependencies by the method of least squares in the time interval $\Delta t=3 \tau_{v r}$, is excluded. As a trend, we used a third-degree polynomial in time $t$. For estimates of the energies $E_{t}$ and $E_{3}$, we used the time representation of the trajectory and its first three SCs, as well as formulas (3.20) and (3.30) from the work of Max et al. (1981). According to our estimates, the first three SCs account for a significant part of the energies of the spectra considered (up to $95 \%$ ). In 75-92\% of the stellar trajectories in cluster models 1-6, the first three SCs collect more than $50 \%$ of the spectra energies of the dependencies $r=r(t)$ of the trajectories. Thus, the first three SCc of the dependencies $r=r(t)$ of trajectories represent stellar motion in our OSC models rather well and describe quite well the more ordered stellar motion in the halo. This result agrees with the data of Kandrup et al. (1997) that the complexity of the spectrum (the number of harmonics of the Fourier spectrum with a total energy of $95 \%$ of the spectrum energy) of stellar trajectories in collisionless systems decreases linearly with decreasing MLCE, see fig. 5, 8 from the paper of Kandrup et al. (1997).

$q$ values for the dependencies $\alpha=\alpha(t)$ in models $1-6$ are contained in the interval $q \in[0.66,0.93]$. Here, $\alpha$ means the cluster virial coefficient (see Section 16.1). Parameters of the first three SCs of the dependencies $\alpha=\alpha(t)$ in OSC models are given in Table 3.2.

Danilov and Leskov (2005) plotted the diagrams of $q$ dependencies on $\lambda \tau_{v r}$ for cluseter models 1-6. According to estimates of $q$ and $\lambda \tau_{v r}$ in models 2, 3, 5 with $R_{2} / R_{t}=0.8$, the fraction of trajectories with $q>0.5$ decreases from 0.81 
Table 3.2 Parameters $P_{i}, A_{i}, \Phi_{i}^{(\alpha)}$ of sinusoidal components of dependencies $\alpha=\alpha(t)$ in cluster models

\begin{tabular}{|c|c|c|c|c|c|c|c|c|c|}
\hline 1 & 2 & 3 & 4 & 5 & 6 & 7 & 8 & 9 & 10 \\
\hline $\mathcal{N}$ & $P_{1} / \tau_{v r}$ & $A_{1}$ & $\Phi_{1}^{(\alpha)}$ & $P_{2} / \tau_{v r}$ & $A_{2}$ & $\Phi_{2}^{(\alpha)}$ & $P_{3} / \tau_{v r}$ & $A_{3}$ & $\Phi_{3}^{(\alpha)}$ \\
\hline 1 & 0.62 & .1627 & $120.7^{\circ}$ & 0.52 & .0251 & $49.6^{\circ}$ & 1.12 & .0191 & $188.3^{\circ}$ \\
\hline 2 & 0.68 & .1338 & $93.4^{\circ}$ & 0.90 & .0253 & $215.8^{\circ}$ & 0.59 & .0188 & $65.9^{\circ}$ \\
\hline 3 & 0.64 & .0744 & $73.9^{\circ}$ & 0.84 & .0183 & $165.5^{\circ}$ & 1.21 & .0122 & $271.2^{\circ}$ \\
\hline 4 & 0.78 & .0964 & $103.0^{\circ}$ & 1.05 & .0117 & $194.2^{\circ}$ & 0.64 & .0103 & $31.4^{\circ}$ \\
\hline 5 & 0.67 & .0336 & $93.4^{\circ}$ & 1.15 & .0196 & $204.7^{\circ}$ & 0.37 & .0069 & $272.1^{\circ}$ \\
\hline 6 & 0.58 & .0444 & $102.9^{\circ}$ & 1.10 & .0441 & $131.3^{\circ}$ & 0.45 & .0099 & $275.3^{\circ}$ \\
\hline
\end{tabular}

to 0.76 as the number of stars in the core $N_{1}$ increases from 100 to $300 . q$ value for the dependencies $\alpha=\alpha(t)$ in models 2, 3, 5 also decreases from 0.93 to 0.66 . Apparently, this is due to the increase in the sizes of the region of high stochasticity in the core with increasing $N_{1}$ observed in models 2,3 , and 5.

In model 6 with $N_{1}=400$, the fraction of trajectories with $q>0.5$ reaches 0.92 . $q$ for the dependency $\alpha=\alpha(t)$ in model 6 reaches 0.91 . Judging by $q$ estimates for the stellar trajectories in cluster model 6 , this model has the most ordered stellar motion. We should note that in this model, during the periods of the greatest cluster compression to the plane $\zeta=0$, a toroidal structure is formed with an increased density of stars inside the torus with equatorial plane close to $\zeta=0$ (in the space $\xi, \eta, \zeta)$.

For a number of time points, Danilov and Leskov (2005) constructed distributions of the star number density $v(\rho, t)$ projected onto the plane $\zeta=0$ by distances $\rho$ from $\zeta$ axis, as well as radial distributions of the star number density $v(r, t)$ over distances $r$ from the cluster center. On the diagrams of dependencies $v=v(\rho, t)$, one can see the condensation waves propagating in the cluster core from $\zeta=0$ with a velocity comparable to an average velocity of peculiar stellar motions. The amplitude of the density $v(\rho, t)$ oscillations in the core of the 6th model is approximately 2 times greater than in the cores of cluster models 1-5. For this reason, the density of stars $v=v(\rho, t)$ in the intervals $\Delta \rho$, which are closer to $\zeta=0$ in the 6th model, at certain time points may be lower than in $\Delta \rho$ intervals, which are more distant from this axis. Similar density waves are observed for cluster models 1-6 also in the distributions $v(r, t)$ ( $r$ is the distance from the cluster center).

In models 1, 2, 4 with initial number of stars in the core $N_{1}=100$ and the value $R_{2} / R_{t}$ decreasing from 0.9 to 0.7 , the fraction of trajectories with $q>0.5$ increases from 0.75 to 0.85 . $q$ values for the dependencies $\alpha=\alpha(t)$ in models $1,2,4$ also increase from 0.89 to 0.97 . In our opinion, this is due to an increase in the number of trajectories of the halo stars associated with the OSC.

Danilov and Leskov (2005) plotted the sum of distributions of the trajectories by the periods $P$ of the first three SCs. These SCs were obtained from the study of dependencies $r=r(t), \dot{r}=\dot{r}(t), \theta=\theta(t)$, where $\dot{r}=\frac{d r}{d t} ; \theta$ is the angle between the radius vector of the star $\mathbf{r}$ at time $t$ and the positive direction of $\zeta$ axis. 
Such distributions allow us to see the frequencies and phases of the most significant oscillations in stellar motion. To plot these distributions, all trajectories were divided into two groups by $\lambda$ values: $\lambda<\bar{\lambda}$ and $\lambda>\bar{\lambda}$. $\bar{\lambda}$ values are given in Table 3.1. The results for the dependencies $\dot{r}=\dot{r}(t), \theta=\theta(t)$ are shown in fig. 3.5-3.6. The plots of the distributions of dependencies $r=r(t)$ and $\dot{r}=\dot{r}(t)$ by the periods $P$ differ little. Therefore, the distributions of the $r=r(t)$ dependencies by the periods $P$ are not given here.

These distributions of the first three SC (in the coordinates of the stars $r=r(t), \dot{r}=$ $\dot{r}(t), \theta=\theta(t))$ by the periods $P$ reveal the non-random and well-defined peaks at the points corresponding to (or close to) the periods commensurate with the period of oscillations of the regular field of the system $P_{r}$. When determining $P_{r}$, we used the frequency of the first SC (with the largest amplitude), extracted from the dependency $\alpha=\alpha(t)$ according to the procedure described by Carpintero and Aguilar (1998) and Laskar (1988). The SC parameters of the changes in the virial coefficient (and regular field) of OSC models over time are shown in Table 3.2.

Trajectories with small $P$ have the large values $\lambda$ and are located generally in the cluster core. Trajectories with $P \geq P_{r}$ have small $\lambda$ and are located generally at the cluster periphery. Contrary to the models $1-5$, in model 6 , the segregation of trajectories with different $\lambda$ values by the periods $P$ is absent, and in each interval $\Delta P$, two groups of trajectories with approximately the same ratio of ordered and chaotic trajectories are formed.

In cluster model 1 (which has the greatest degree of non-stationarity), a significant part of the stellar trajectories from the resonance peaks at $t \simeq 3 \tau_{v r}$ is located at large distances from the cluster center (at $r>R_{t}$ ). The small values of $\lambda$ correspond to these trajectories, see fig. 3.5-3.6. Apparently, oscillations of the regular field of the system play an important role in the dissipation of these stars from the cluster.

In the considered OSC models, with an increase of $N_{1}$, in more ordered trajectories with $\lambda<\bar{\lambda}$, the height (and population) of the resonance peaks of the distribution of trajectories by $P$ in the region $P<P_{r}$ is conserved, and in the region $P>P_{r}$, the height of these peaks decreases. With an increase of $N_{1}$, in our OSC models, the number of stars located deep beneath the tidal surface of the cluster in the field of the Galaxy (at $r<<R_{t}$ ) increases. For these stars, the energy of the escape from the cluster increases, and a longer time takes for transferring of the energy of the regular field oscillation to the star, and a larger number of regular field oscillations with period $P_{r}$ is required, in order to ensure the escape of these stars from the cluster and to provide the same population of resonance peaks, as for small $N_{1}$.

Distributions of the dependencies $\theta=\theta(t)$ for the main periods of the $P$ oscillations in the OSC models form two groups, the most noticeable of which are in the models 2, 4, 6; for the model 6 see fig. 3.6. In models 2 and 4, these are groups with periods $P<P_{r}$ and $P>P_{r}$, which basically correspond to cluster stars and stars that left the cluster to the large distances $r>R_{t}$ along the Galactic plane. Large $P$ values in the dependencies $\theta=\theta(t)$ correspond to large $r$, as well as to the stellar motions directed mainly along the plane $(\xi, \eta)$, which is parallel to the plane of the Galaxy. Ordered $(\lambda<\bar{\lambda})$ and chaotic $(\lambda>\bar{\lambda})$ trajectories in the first group with $P<P_{r}$ are 
363 Properties of Stellar Trajectories in Numerical Dynamical Models of Open Stellar Clusters
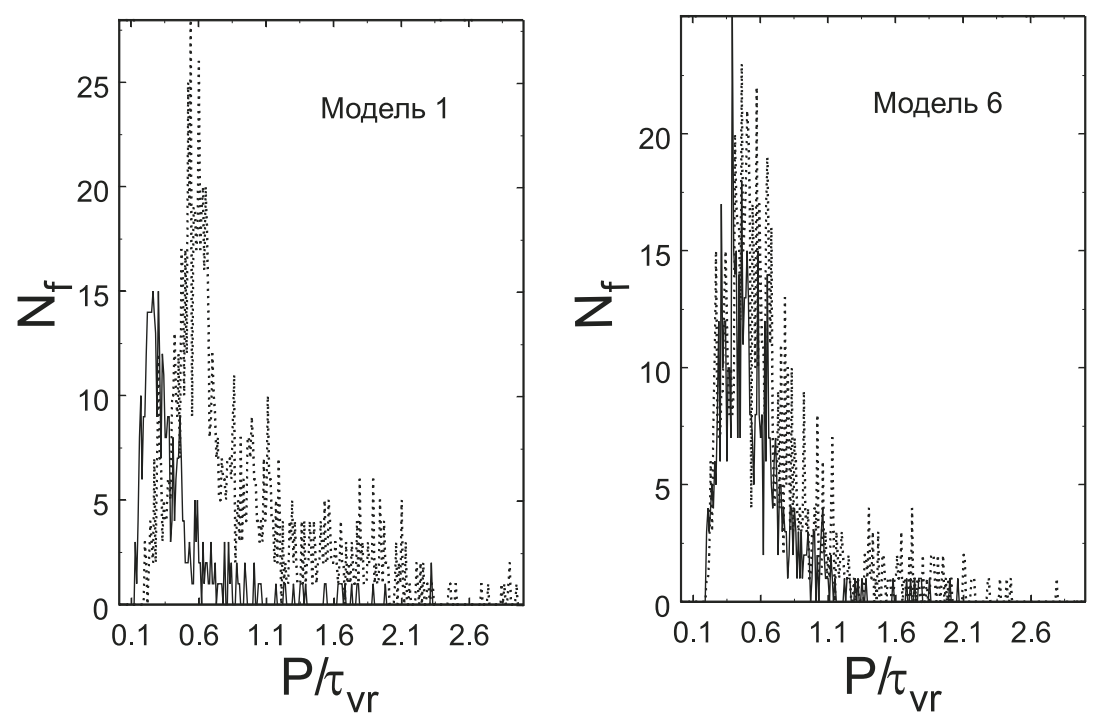

Fig. 3.5 The sum of distributions of the stellar trajectories by the periods $P$ for first three sinusoidal components of the dependencies $\dot{r}=\dot{r}(t)$ in two cluster models. Solid line denotes the distribution of the trajectories with $\lambda>\bar{\lambda}$. Dashed line shows distributions of the trajectories with $\lambda<\bar{\lambda}$
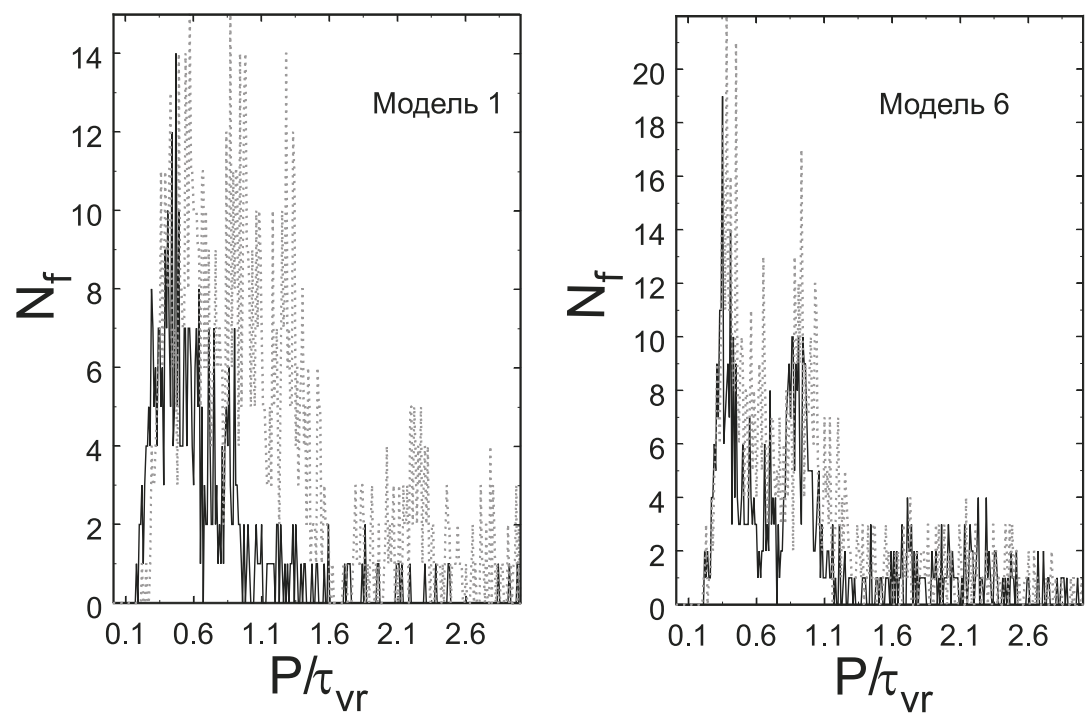

Fig. 3.6 The same as in fig. 3.5 for the dependencies $\theta=\theta(t)$ 
well mixed in the space of $P$ values. In models 2 and 4 , chaotic trajectories in the second group $\left(P>P_{r}\right)$ are encountered rarely.

In the core of model 6, there are two groups of stellar trajectories in the space of $P$, obtained in the study of the dependencies $\theta=\theta(t)$, see Fig. 3.6. When $t<3 \tau_{v r}$, the distance of these stars $r$ from the cluster center is less than $6 \mathrm{pc}$. For trajectories of the first group, $P$ is less than $0.62 \tau_{v r}$. For the trajectories of the second group, $P$ values are enclosed in an interval approximately equal to $P / \tau_{v r} \in[0.62,1.1]$. In the core of the 6th model, in groups 1 and 2 , there are both chaotic and more ordered trajectories, and in the second group the fraction of more ordered trajectories (with small $\lambda$ ) is greater than 0.5 , and larger than in the first group (with $P<0.62 \tau_{v r}$ ). The halos of the models are largely dominated by ordered motions. According to Danilov (2002b) and Danilov and Dorogavtseva (2003), in the 6th OSC model, there is a doubling of the periods of the large-scale oscillations of the star cluster: $P_{r} / \tau_{v r} \simeq 0.6$ and $P_{\xi, \eta} / \tau_{v r} \simeq 1.2$. The period $P_{\xi, \eta}$ corresponds to the oscillations of the outer cluster parts along the plane $(\xi, \eta)$ (that is, along the plane of the Galaxy). In the 6th model, a toroidal structure is periodically observed in the space $(\xi, \eta, \zeta)$, see above.

Danilov and Leskov (2005) plotted the sum of the distributions $N_{\Phi}$ of stellar trajectories by the phases $\Phi$ of the first three SC dependencies $r=r(t)$ for cluster models 1-6. The phase intervals $\Delta \Phi=2^{\circ}-18^{\circ}$ were used. Distribution of $N_{\Phi}$ obtained with the interval $\Delta \Phi=18^{\circ}$ for model 1 is shown by a dashed line in fig. 3.7. Solid line in fig. 3.7 shows the polynomial $\bar{N}_{\Phi}$ of the 6th power of the best mean-square approximation of the values $N_{\Phi}=N_{\Phi}(\Phi)$ for the dependencies $r=r(t)$.

We note that when the SC is selected from the $r=r(t)$ dependencies, initial values $\Phi=\Phi(0)$ are obtained according to the procedure of Carpintero and Aguilar (1998) and Laskar (1988). During the evolution of the cluster, phases $\Phi(t)$ of oscillations of the values $r=r(t)$ increase linearly with time: $\Phi(t)=\Phi(0)+\frac{2 \pi}{P} t$. In the distribution $N_{\Phi}$ of the dependencies $r=r(t)$ for the phases $\Phi(0)$ in fig. 3.7, we can see the predominance of the initial phases close to the initial oscillation phase of the regular cluster field (i.e., to the phase $\Phi_{1}^{(\alpha)}(0)$ of the first SC of the dependency $\alpha=\alpha(t)$, see Table 3.2). Such a consistency of the initial phases of radial stellar motions and cluster oscillations is more noticeable for models 1 and 2 with a greater degree of non-stationarity.

Vertical line $\Phi_{1}=120.7^{\circ}$ in fig. 3.7 marks the phase $\Phi_{1}^{(\alpha)}(0)$. It practically coincides with the phase of the maximum value of the polynomial $\bar{N}_{\Phi}$. Thus, only a small excess of the maximum value of $\bar{N}_{\Phi}$ over its minimum value (approximately 8-9 trajectories in the phase interval $\Delta \Phi=18^{\circ}$ ) indicates a tendency of the system to the in-phase radial star motions. The number of trajectories with phases $\Phi(0)$, differing by less than $90^{\circ}$ from $\Phi_{1}^{(\alpha)}(0)$, exceeds the number of remaining trajectories only by approximately $10 \%$. This is largely due to the method of specifying the initial velocities of the stars in our models (all initial directions of the vectors $\mathbf{v}$ are equally probable). In this case, the phases of radial stellar motions are distributed approximately uniformly. A significant contribution to the uniformity in the distri- 

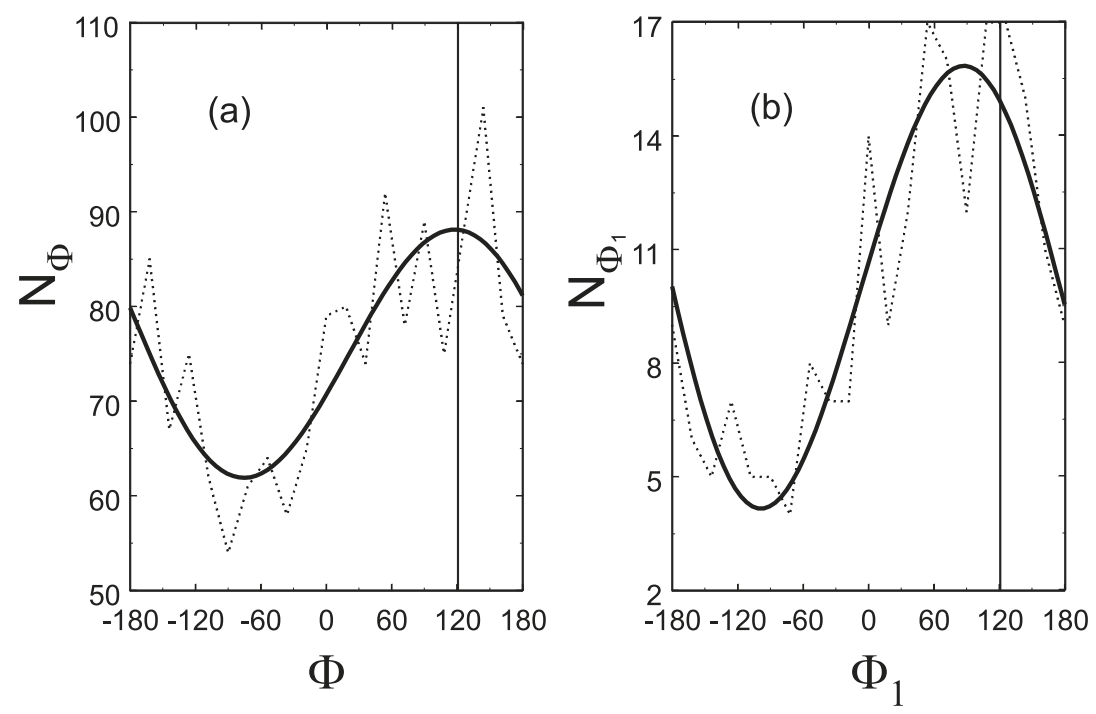

Fig. 3.7 The sum of distributions $N_{\Phi}$ of stellar trajectories for the stars of cluster model 1 by the initial phases $\Phi$ of the first three SC dependencies of $r=r(t)$ (a). Distribution $N_{\Phi_{1}}$ of the halo stars' trajectories in cluster model 1 by the initial phases $\Phi_{1}$ of the first SC dependencies of $r=r(t)$ (b)

bution of the current phases of dependencies $r=r(t)$ is made by the stochasticity of stellar trajectories (all $\lambda>0$ ) and the stochastic nature of stellar encounters.

For the halo stars, a consistency of the initial phases of radial motions increases noticeably. The trajectories of the halo stars in this work were selected among all trajectories in the clusters by the values $\lambda<\bar{\lambda}, q>0.5$ and the maximum distances $r_{\text {max }}$ of the star from the center not exceeding $20 \mathrm{pc}$ during the evolution of the cluster. In fig. 3.7,b the dotted line marks the distribution of the halo star trajectories $N_{\Phi_{1}}$ by the phases of the first SC in model 1. Solid line marks the sixth degree polynomial $\bar{N}_{\Phi_{1}}$ of the best mean-square approximation of the values $N_{\Phi_{1}}=N_{\Phi_{1}}\left(\Phi_{1}\right)$ for the dependencies $r=r(t)$. We used the phase interval $\Delta \Phi_{1}=18^{\circ}$. The excess of the maximum value of the polynomial $\bar{N}_{\Phi_{1}}$ over its minimal value in model 1 is approximately 12 trajectories in the phase interval $\Delta \Phi_{1}=18^{\circ}$. This is noticeably greater in percentage terms than for all the stellar trajectories of the cluster model, since only the trajectories of 203 stars in model 1 satisfy conditions $\lambda<\bar{\lambda}, q>0.5$ and $r_{\max }<20 \mathrm{pc}$. The number of halo star trajectories with phases $\Phi_{1}(0)$ differing by less than $90^{\circ}$ from $\Phi_{1}^{(\alpha)}(0)$, in this case exceeds the number of remaining halo star trajectories by $38 \%$ approximately.

Amplitudes of the radial stellar motions for a significant part of trajectories can increase at certain moments (and periods) of time due to a proximity of phases of the the first two SCs of dependencies $r=r(t)$ for these trajectories. Indeed, the number of dependencies $r=r(t)$ with the current phases $\Phi(t)$ of two SCs (of the 
first three ones), which differ by not more than $\Delta \Phi=18^{\circ}$, is on average $30 \%$ of the total trajectories number in model 1 , and at some time points reaches $50 \%$. With increasing of the considered interval $\Delta \Phi$, the fraction of the trajectories with close current phases of two (of the first three ones) SCs increases.
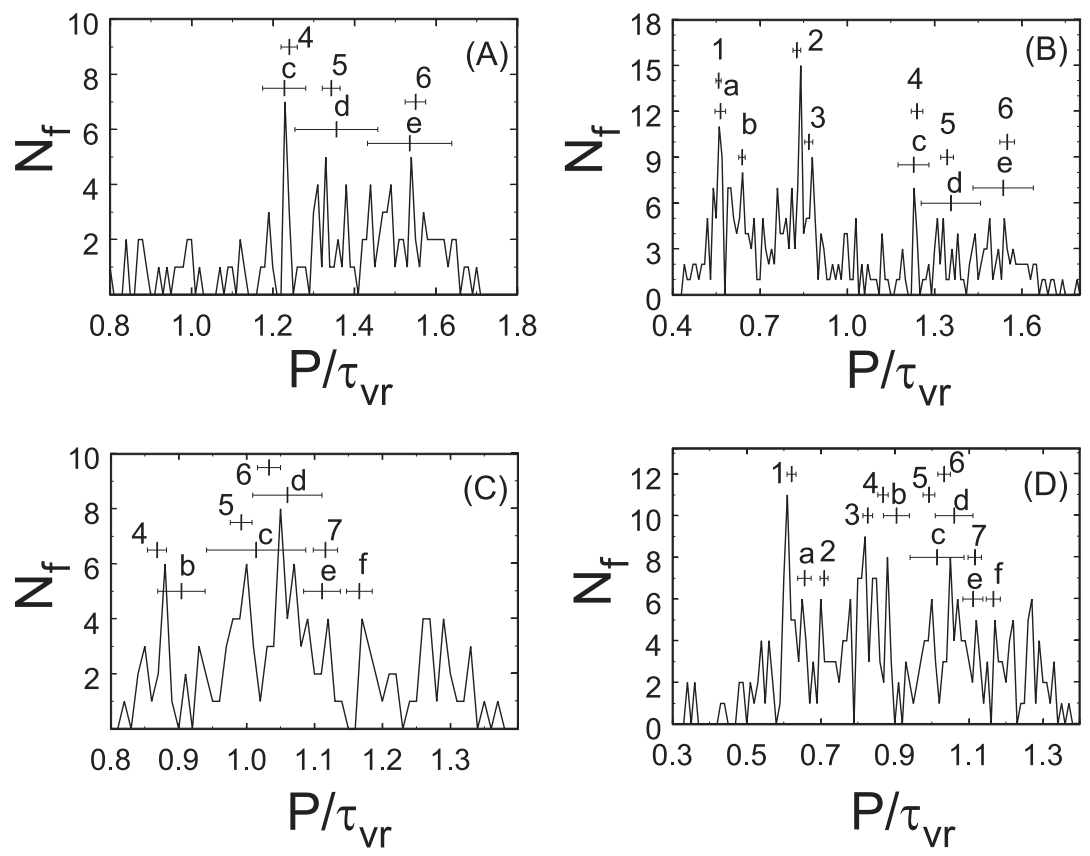

Fig. 3.8 Distributions $N_{f}$ of trajectories of the halo stars in the cluster model 1 by the periods $P$ : A, B are trajectory distributions for the first SC and for the first three SC dependencies $\eta=\eta(t)$, respectively; $\mathrm{C}, \mathrm{D}$ are the same distributions for dependencies $\zeta=\zeta(t)$

Danilov and Leskov (2005) presented distribution of trajectories of the halo stars in cluster model 1 by the periods $P$ of the first SC of dependencies $\xi=\xi(t), \eta=\eta(t)$, and $\zeta=\zeta(t)$. Similar distributions were also plotted for the periods of the first three SCs. In plotting these distributions, along with the criteria for sample of trajectories of halo stars described above, an additional constraint $E_{t r}<0.3\left(E_{t}+E_{t r}\right)$ was used, where $E_{t r}$ is the energy of the trend of a dependency $r=r(t)$. This restriction makes it possible to exclude from the consideration the trajectories of the halo stars, which traveled by the distances $r$ far beyond the tidal radius of the OSC during time $3 \tau_{v r}$, and then returned. In fig. 3.8, the digits indicate the numbers of the values of the periods $P$ for the highest peaks of distributions, and the lowercase letters indicate the labels of the theoretical estimates of these periods obtained by Danilov (2005) (here and below, $P$ values are expressed in the units of the violent relaxation time $\tau_{v r}$ ). 
The period of change of the regular cluster field $P_{r}=2 \pi / \omega_{r}=0.620 \pm 0.010$ ( $\omega_{r}$ is oscillation frequency of a regular field). For the dependencies $\eta=\eta(t)$, the following estimates are obtained by Danilov and Leskov (2005):

- $P_{a}\left(5 v_{1}\right)=0.564 \pm 0.018$,

- $P_{1}\left(\frac{9}{10} \omega_{r}\right)=0.568 \pm 0.009$,

- $P_{b}\left(2 v_{1}+v_{2}\right)=0.638 \pm 0.011$,

- $P_{2}\left(\frac{3}{4} \omega_{r}\right)=0.827 \pm 0.013$,

- $P_{3}\left(\frac{5}{7} \omega_{r}\right)=0.868 \pm 0.014$

- $P_{b}\left(\beta_{3}-2 v_{2}\right)=1.228 \pm 0.053$,

- $P_{4}\left(2 \omega_{r}\right)=1.240 \pm 0.020$

- $P_{5}\left(\frac{13}{6} \omega_{r}\right)=1.343 \pm 0.022$,

- $P_{c}\left(2 \beta_{3}-3 v_{1}\right)=1.356 \pm 0.102$,

- $P_{d}\left(-3 v_{1}+2 v_{2}\right)=1.536 \pm 0.104$,

- $P_{6}\left(\frac{5}{2} \omega_{r}\right)=1.550 \pm 0.025$,

where $v_{1}, v_{2}, \beta_{3}$ are natural frequencies of the halo star motion in the field of the homogeneous ellipsoidal cluster potential, which approximates the potential of the halo of model 1 of OSC averaged over the period $P_{r}$. The horizontal error bars (for the period) shown in fig. 3.8 correspond to errors in approximating of the potential of the cluster (averaged by the period $P_{r}$ ) by the potential of a homogeneous gravitating ellipsoid (Danilov, 2005) (when the letters are used) and the errors in determining $P_{r}$ (when the digits are used (Danilov and Leskov, 2005)).

For the dependencies $\zeta=\zeta(t)$ we find:

- $P_{1}\left(\omega_{r}\right)=0.620 \pm 0.010$,

- $P_{a}\left(-\beta_{3}+2 v_{2}+2 v_{1}\right)=0.656 \pm 0.018$,

- $P_{2}\left(\frac{7}{8} \omega_{r}\right)=0.709 \pm 0.011$,

- $P_{3}\left(\frac{3}{4} \omega_{r}\right)=0.827 \pm 0.013$,

- $P_{4}\left(\frac{5}{7} \omega_{r}\right)=0.868 \pm 0.014$,

- $P_{b}\left(3 v_{1}-v_{2}+\beta_{3}\right)=0.904 \pm 0.035$,

- $P_{5}\left(\frac{5}{8} \omega_{r}\right)=0.992 \pm 0.016$,

- $P_{c}\left(3 \beta_{3}-2 v_{2}\right)=1.014 \pm 0.073$,

- $P_{6}\left(\frac{3}{5} \omega_{r}\right)=1.033 \pm 0.017$,

- $P_{d}\left(-v_{2}+2 \beta_{3}\right)=1.060 \pm 0.051$,

- $P_{e}\left(\beta_{3}\right)=1.111 \pm 0.027$

- $P_{7}\left(\frac{5}{9} \omega_{r}\right)=1.116 \pm 0.018$

- $P_{f}\left(v_{2}\right)=1.166 \pm 0.019$.

For these periods (or near to them), from 6 to 15 trajectories of the halo stars are grouped in model 1. The periods indicated here are commensurate with $P_{r}$ (or are close to commensurability with $P_{r}$ ).

We note that the PCS for $t=0$ are the "random" values obtained with the help of continuous distributions in the phase space of the core and halo stars of the system. Star encounters in the cluster also have a random character. Therefore, one could expect that the frequencies and amplitudes of the stellar motion in the system will also be random and continuously distributed values. However, the resonance 
peaks are observed in the distribution of the trajectories by the periods $P$. The most probable reason for the formation of such trajectory groups is an effect of "frequency shift" when synchronizing the oscillations, described in the papers on self-oscillating systems with a large number of degrees of freedom (Rabinovich and Trubetskov, 2000, p. 348). As a result of such synchronization of oscillations, a set of frequencies commensurate with the frequency $\omega_{r}$ is formed in the system. At some of these frequencies, synchronous motion (with the same frequency) of small groups of stars along the trajectories is spontaneously established. Probably, in this case, a set of intervals of distances from the cluster center is formed in the system, the motion in which for the halo stars becomes preferable.

\subsection{Conclusions}

1. In this chapter, we have investigated trajectories of stellar motion in six OSC models which move along a circular orbit in the plane of the Galaxy, and are nonstationary in the field of the regular forces. We have performed estimates of the maximum Lyapunov characteristic exponents $\lambda$ of stellar trajectories in the OSC models. The mean values $\bar{\lambda}$ in OSC models increase with the increasing density and a decrease in the degree of non-stationarity of considered cluster models from $0.8(1 / \mathrm{Myr})$ to $1.25(1 / \mathrm{Myr})$. Thus, the degree of stochasticity of the stellar motions in OSC models decreases at large amplitudes of oscillations of a regular field (in strongly non-stationary systems).

2. Stellar trajectories with large $\lambda$ values are located in the cores of OSC models, and trajectories with small $\lambda$ - on the cluster periphery (generally). Thus, in a nonstationary OSC, the cluster cores form a region of increased stochasticity, and the halo form region of regular stellar motions. In the denser OSC models, dimensions of the stochasticity region in the core increase. During the evolution of OSC models, regions of significant stochasticity extend to the region of small $r$ and $v$. Regions of regular and stochastic motion in the space of $(r, v)$ are adjacent to each other.

3 . For each stellar trajectory, the estimate of $\lambda$ grows very rapidly with time, and reaches its maximum value at small values $t$, after which the changes of $\lambda$ become insignificant. Thus, there is an "adhesion" of the stellar trajectory in phase space to a region with a given $\lambda$.

4. We have performed the analysis of amplitudes, phases and periods of three sinusoidal components with the largest amplitudes in the stellar trajectories for six OSC models. To analyze the properties of trajectories, it is proposed to use the parameter $q$ (the ratio of the energy of these three SC, to the total energy of the Fourier spectrum of the trajectory). Joint use of the parameters $\lambda$ and $q$ allows to more accurately divide the ordered and stochastic trajectories. The parameter $q$ for the dependency $\alpha=\alpha(t)$ characterizes in general the complexity of the spectra of all trajectories in OSC model.

5. In models $1-5$, trajectories with low periods $P$ have large $\lambda$ values and are located generally in the cluster core; trajectories with low $\lambda$ have large $P$ and are 
located mainly on the cluster periphery. In the 6th model, segregation of trajectories with different $\lambda$ values over the periods $P$ is absent, which may be due to the formation of the toroidal structure in the stellar distribution of this model in the space $(\xi, \eta, \zeta)$.

6 . The distribution of the most significant oscillations in the stellar trajectories over periods of their motions in clusters has revealed nonrandom and well-defined peaks at periods $P$ commensurate (or close to commensurability) with oscillation period of the regular field in the cluster $P_{r}$. The periods obtained from the analysis of the halo stars trajectories corresponding to the peaks of these distributions agree with theoretical estimates of Danilov (2005).

7. A significant part of the stars from the resonant peaks in model 1 with the greatest degree of non-stationarity is located at large distances from the cluster center $\left(r>R_{t}\right)$ at the end of calculations (at $\left.t=3 \tau_{v r}\right)$. Perhaps, in the dissipation of these stars from the cluster, an important role the oscillations of the regular cluster field are played. For the cluster models with a greater degree of non-stationarity, we have also observed a greater consistency of the initial phases of the radial star motions along their trajectories and oscillations of the cluster.

\section{References}

Caranicolas, N.D., Vozikis, Ch.L.: Order and chaos in galactic maps. Astron. Astrophys. 349, 70-76 (1999).

Carpintero, D.D., Aguilar, L.A.: Orbit classification in arbitrary 2D and 3D potentials. Mon. Not. R. Astron. Soc. 298, 1-21 (1998). doi:10.1046/j.13658711.1998.01320.x

Carpintero, D.D., Muzzio, J.C., Wachlin, F.C.: Regular and chaotic motion in globular clusters. Celest. Mech. and Dyn. Astron. 73, 159-168 (1999). doi:10.1023/A:1008347013786

Carpintero, D.D., Muzzio, J.C., Vergne, M.M., Wachlin, F.C.: Chaotic Orbits in Galactic Satellites. Celest. Mech. and Dyn. Astron. 85, 247-267 (2003). doi:10.1023/A:1022509428034

Chandrasekhar, S.: Principles of Stellar Dynamics. Univ. Chicago Press, Chicago, Ill (1942). LCCN: 43-5608 (BKS1)

Danilov, V.M.: Numerical experiments simulating the dynamics of open clusters in the Galactic field. Astron. Reports 41, 163-173 (1997a).

Danilov, V.M.: Equilibrium After Violent Relaxation in Numerical Dynamical Models of Open Clusters. Astron. Reports 46, 443-450 (2002a). doi: $10.1134 / 1.1486176$

Danilov, V.M.: Stellar Fluxes in Numerical Dynamical Models of Open Clusters. Astron. Reports 46, 887-899 (2002b). doi:10.1134/1.1522077

Danilov, V.M.: The Motion of Halo Stars in Dynamical Numerical Models of Open Clusters. Astron. Reports 49, 604-610 (2005). doi:10.1134/1.2010649 
Danilov, V.M., Dorogavtseva, L.V.: Estimates of Relaxation Times in Numerical Dynamical Models of Open Star Clusters. Astron. Reports 47, 483-491 (2003). doi:10.1134/1.1583775

Danilov, V.M., Leskov, E.V.: Properties of Stellar Trajectories in Numerical Dynamical Models of Open Star Clusters. Astron. Reports 49, 190-200 (2005). doi: $10.1134 / 1.1882777$

Efthymiopoulos, C., Contopoulos, G., Voglis, N.: Cantori, islands and asymptotic curves in the stickness region. Celest. Mech. Dyn. Astron. 73, 221-230 (1999). doi:10.1023/A:1008359316512

El-Zant, A.A.: On the stability of motion of N-body systems: the effect of the variation of particle number, softening and rotation. Astron. Astrophys. 331, 782792 (1998).

Habib, S., Kandrup, H.E., Mahon, M.E.: Chaos and Noise in Galactic Potentials. Astrophys. J. 480, 155-166 (1997). doi:10.1086/303935

Kandrup, H.E.: Phase mixing in time-independent Hamiltonian systems. Mon. Not. R. Astron. Soc. 301, 960-974 (1998). doi:10.1046/j.1365-8711.1998.02063.x

Kandrup, H.E., Eckstein, B.L., Bradley, B.O.: Chaos, complexity, and short time Lyapunov exponents: two alternative characterisations of chaotic orbit segments. Astron. Astrophys. 320, 65-73 (1997).

Kandrup, H.E., Sideris, I.V.: Chaos in cuspy triaxial galaxies with a supermassive black hole: a simple toy model. Celest. Mech. and Dyn. Astron. 82, 61-81 (2002). doi:10.1023/A:1013859118402

Kutuzov, S.A., Osipkov, L.P.: A Generalized Model for the Three-Dimensional Gravitational Potential of Stellar Systems. Soviet Astron. 24, 17-22 (1980).

Laskar, J.: Secular evolution of the solar system over 10 million years. Astron. Astrophys. 198 341-362, (1988).

Lega, E., Froesle, C.: On the relationship between fast Lyapunov indicator and periodic orbits for symplectic mappings. Celest. Mech. and Dyn. Astron. 81, 129-147 (2001). doi:10.1023/A:1013323507265

Lichtenberg, A.J., Lieberman, M.A.: Regular and Chaotic Dynamics. SpringerVerlag, New York, NY (1983).

Max, J., et al.: Methodes et techniques de traitement du signal et applications aux mesures physiques. Tome 1. Principes generaux et methodes classiques. MASSON, Paris (1981).

Muzzio, J.C., Mosquera, M.E.: Spatial structure of regular and chaotic orbits in self-consistent models of galactic satellites. Celest. Mech. and Dyn. Astron. 88, 379-396 (2004). doi:10.1023/B:CELE.0000023411.87573.7a

Muzzio, J.C., Vergne, M.M., Wachlin, F.C., Carpintero, D.D.: Stellar Motions in Galactic Satellites. Celest. Mech. and Dyn.Astron. 81, 167-176 (2001). doi:10.1023/A:1013327608173

Papaphilippou, Y., Laskar, J.: Frequency map analysis and global dynamics in a galactic potential with two degrees of freedom. Astron. Astrophys. 307, 427-449 (1996). 
443 Properties of Stellar Trajectories in Numerical Dynamical Models of Open Stellar Clusters

Rabinovich, M.I., Trubetskov, D.I.: Introduction to the theory of oscillations and waves. Sci.-Editorial Center "Regular and Chaotic Dynamics", Izhevsk (2000) (In Russian).

Voglis, N., Contopoulos, G., Efthymiopoulos, C.: Detection of ordered and chaotic motion using the dynamical spectra. Celest. Mech. and Dyn. Astron. 73, 211-220 (1999). doi:10.1023/A:1008307332442

Vozikis, Ch.L., Varvoglis, M., Tsiganis, K.: The power spectrum of geodesic divergences as an early detector of chaotic motion. Astron. Astrophys. 359, 386-396 (2000). 


\title{
Chapter 4 \\ Dynamic Models of the Stellar Motion at the Periphery of Open Star Clusters
}

\begin{abstract}
In this chapter, we consider the model of homogeneous gravitating ellipsoidal star cluster moving along a circular orbit around the Galactic center. For this model, three independent isolating integrals of the stellar motion are written. An analysis is made of the features of cluster star motion, taking into account the influence of these three integrals of motion. On the periphery of the considered cluster model, the retrograde stellar motions predominate, and the velocity distribution of the stars is elongated along the direction of cluster motion. We construct a phase density function which depends on two integrals of motion and the distribution of the stellar velocities in the case of a three-integral phase density function. Applications of the results are discussed.
\end{abstract}

\subsection{Introduction}

Danilov (2005) constructed the model of a stationary homogeneous gravitating ellipsoid, the potential of which approximated the average potential at the periphery of the numerical dynamic OSC models (Danilov and Dorogavtseva, 2003), which consist of $N=500$ gravitating points. There are five integrals of the star motion in the field of a homogeneous stationary gravitating ellipsoid in the absence of rotation of the ellipsoid (Lynden-Bell, 1962). Rotation of the OSC, when it moves around the center of the Galaxy, significantly complicates the construction of the stellar motion integrals. In this case, we can easily find only the Jacobi integral and two independent integrals of the stellar motion in the plane of the Galaxy $\left(J_{1}\right)$ and perpendicularly to the Galactic plane $\left(J_{2}\right)$. An analytical solution for the trajectory of the stellar motion in the field of a homogeneous ellipsoid rotating in a circular orbit was obtained by Danilov (2005). It is of interest to construct a third independent integral $J_{3}$ of the stellar motion using this solution. Distribution of the stellar velocities in such ellipsoid can be obtained using the phase density function (PDF), which depends on independent single-valued integrals of motion. 
A satisfactory theoretical description of the stellar motion at the OSC periphery is currently lacking. The stationary collisionless cluster model (Heggie and Ramamani, 1995) depends only on one Jacobi integral of the stellar motion in the joint field of the cluster and the Galaxy. Distribution of the stellar velocities in such model is spherically symmetric. However, this velocity distribution is not formed in the numerical experiments on a modeling of the OSC dynamics. Known stationary models of isolated stellar clusters with the PDF $f(E, L)$ that depends on the integrals of energy $E$ and of the angular momentum $L$ (see, for example, King (2002, p. 189-191)) are inapplicable in this case, since the angular momentum of the stars in OSC is not conserved because of the influence of the Galaxy force field on the cluster. Numerical experiments show stellar motion at the OSC periphery, which is very different from that in the models with $f=f(E, L)$. According to Danilov (1997a), the distribution of the stellar velocities at the periphery of the numerical dynamic OSC models is elongated along the direction of the cluster motion in the Galaxy, and is also perpendicular to the Galactic plane. Nezhinskii (1990) considered the quasi-integral of Jacobi $h$ and the area quasi-integral $C$ (of the sixth order) for motion of the test body in the restricted circular three-body problem and analyzed the velocity field and the motion regions of the test body. Unfortunately, such a cluster model allows only an approximate description of the stellar motion in the Galactic plane at the cluster periphery, since a significant stellar mass in OSC is located on the clusters peripheries (Danilov and Dorogavtseva, 2003; Terlevich, 1987). In addition, Nezhinskii (1990) does not define the limits of the allowed $C$ values depending on the accepted $h$ value, and instead of considering the most typical situations, he discusses only two cases of trajectories with the $C$ values close to the extremely permissible one (in these cases, the signs of the angular momentum values and $C$ coincide, which is most often not realized in our cluster model).

It is of interest to analyze theoretically the stellar motions at the OSC periphery in the framework of the model of triaxial homogeneous ellipsoidal cluster; to construct a corresponding PDF that depends on two and on three independent single-valued integrals of motion. Such description will allow us to explain the features of stellar velocity distributions observed in numerical dynamic OSC models, making it possible to analyze observational data on stellar velocities in the vicinity of real OSC. The function of the phase density for the model of a homogeneous gravitating ellipsoid moving in the field of the Galaxy can be used to solve the following problems. First, such PDF can be used as an initial approximation in the theoretical elaborations while constructing more complex PDF in the stellar cluster models which consist of $N$ gravitating points. Secondly, the PDF of a homogeneous ellipsoidal cluster can be useful both for specifying the initial phase coordinates of stars in numerical dynamic OSC models, and for estimating the total masses of the observed OSC from data on the velocities and coordinates of stars in clusters.

The objectives of this chapter are as follows: discussion of the features of stellar motion at the OSC periphery; construction of the integrals of stellar motion in the model of Danilov (2005) of a homogeneous ellipsoidal cluster moving in a circular orbit around the center of the Galaxy; discussion of the phase density function and the velocity distribution of stars in this cluster model. 


\subsection{Equations and Integrals of Stellar Motion}

Following to Danilov (2005), we will consider the open stellar cluster moving along a circular orbit in the Galactic plane. The linearized equations of stellar motion in the rotating coordinate system $\xi, \eta, \zeta$ (Chandrasekhar, 1942, p. 222), according to Danilov (2005), can be written as

$$
\ddot{\xi}=-\beta_{1}^{2} \xi+2 \omega \dot{\eta}, \quad \ddot{\eta}=-\beta_{2}^{2} \eta-2 \omega \dot{\xi}, \quad \ddot{\zeta}=-\beta_{3}^{2} \zeta,
$$

where $\dot{\xi}=\frac{d \xi}{d t}, \ddot{\xi}=\frac{d \dot{\xi}}{d t}$, the values $\dot{\eta}, \dot{\zeta}, \ddot{\eta}, \ddot{\zeta}$ are defined similarly; values $\beta_{i}^{2}>0$ are defined by Danilov (2005) using the parameters of the ellipsoid potential of the cluster, see (4.2) and (4.6) in the paper of Danilov (2005); $\omega$ is angular velocity of cluster motion relative to the center of the Galaxy. According to Danilov (2005), the Jacobi integral for the system of equations (4.1) has the form

$$
\frac{V^{2}+\beta_{1}^{2} \xi^{2}+\beta_{2}^{2} \eta^{2}+\beta_{3}^{2} \zeta^{2}}{2}=\epsilon=\text { const }, \quad \epsilon \geq 0
$$

where $V^{2}=\dot{\xi}^{2}+\dot{\eta}^{2}+\dot{\zeta}^{2}$ is a square of the modulus of the stellar motion velocity. We note that the first two equations and the third equation of the system (4.1) can be integrated independently of each other and give two integrals $J_{1}$ and $J_{2}$ associated with the integral $\epsilon$ from (4.2):

$$
\dot{\xi}^{2}+\dot{\eta}^{2}+\beta_{1}^{2} \xi^{2}+\beta_{2}^{2} \eta^{2}=J_{1}=\text { const }, \quad \dot{\zeta}^{2}+\beta_{3}^{2} \zeta^{2}=J_{2}=\text { const } .
$$

It is easy to see that $J_{1}+J_{2}=2 \epsilon=$ const. A general solution of the system (4.1), obtained by Danilov (2005), can be written in the following form:

$$
\begin{aligned}
& \xi=C_{1} \cos \left(\phi_{1}\right)+C_{2} \cos \left(\phi_{2}\right), \\
& \eta=A C_{1} \sin \left(\phi_{1}\right)+B C_{2} \sin \left(\phi_{2}\right), \\
& \zeta=C_{3} \cos \left(\phi_{3}\right),
\end{aligned}
$$

where

$$
\begin{gathered}
\phi_{j}=v_{j}\left(t-t_{j}\right), \quad j=1,2, \\
\phi_{3}=\beta_{3}\left(t-t_{3}\right) ; \\
A=\frac{\beta_{1}^{2}-v_{1}^{2}}{2 \omega v_{1}} ; \\
B=\frac{\beta_{1}^{2}-v_{2}^{2}}{2 \omega v_{2}} ;
\end{gathered}
$$

$v_{1}, v_{2}, \beta_{3}$ are natural frequencies of the system (4.1). $C_{i}, t_{i}$ are constant values $(i=$ $1,2,3)$. Constants $v_{1}, v_{2}$ were defined by Danilov (2005) from a condition of the existence of the non-zero solutions for the system of the first two equations (4.1).

Following to Danilov (2006), we use the solution (4.4) in order to find new integrals of the system (4.1). We differentiate $\xi$ and $\eta$ from (4.4) by time $t$, and using 
the expressions for $\dot{\xi}, \dot{\eta}, \xi$, and $\eta$, we write the system of four linear equations for the values $\cos \left(\phi_{j}\right), \sin \left(\phi_{j}\right), \quad j=1,2$. Solving this system, we find

$$
\begin{array}{ll}
\cos \left(\phi_{1}\right)=\frac{\dot{\eta}-\xi \nu_{2} B}{s_{1} C_{1}}, & \sin \left(\phi_{1}\right)=-\frac{\dot{\xi} B+\eta v_{2}}{s_{2} C_{1}}, \\
\cos \left(\phi_{2}\right)=\frac{\xi \nu_{1} A-\dot{\eta}}{s_{1} C_{2}}, & \sin \left(\phi_{2}\right)=\frac{\eta v_{1}+\dot{\xi} A}{s_{2} C_{2}},
\end{array}
$$

where $s_{1}=v_{1} A-v_{2} B ; s_{2}=v_{1} B-v_{2} A$.

We use the first two equations of the system (4.1) to find the angular momentum $l_{\zeta}=\dot{\eta} \xi-\eta \dot{\xi}$ of the rotational stellar motion relatively to $\zeta$ axis:

$$
\left\{l_{\zeta}+\omega\left(\xi^{2}+\eta^{2}\right)\right\}_{0}^{t}=-\lambda \int_{0}^{t} \xi \eta d t,
$$

where $\{F(t)\}_{0}^{t}=F(t)-F(0), \lambda=\beta_{2}^{2}-\beta_{1}^{2}$.

Substituting $\xi$ and $\eta$ from (4.4) to the right-hand side of (4.6), we compute the integral of $\xi \eta$ for $t$. The expression obtained as a result of integration contains functions $\cos \left(\phi_{1} \pm \phi_{2}\right)$ and $\cos \left(2 \phi_{j}\right), \quad j=1,2$. Expressing them through $\cos \left(\phi_{j}\right)$ and $\sin \left(\phi_{j}\right)$, and expressing $\cos \left(\phi_{j}\right)$ and $\sin \left(\phi_{j}\right)$ through the phase coordinates of the star, according to (4.5) and (4.6), we find the third integral of the motion $J_{3}$ associated with $l_{\zeta}$ :

$$
\dot{\eta} \xi q_{1}-\dot{\xi} \eta q_{2}+\xi^{2} q_{3}+\eta^{2} q_{4}+\dot{\xi}^{2} q_{5}+\dot{\eta}^{2} q_{6}=J_{3}=\text { const },
$$

where $q_{1}=1+\lambda \Omega_{5} ; q_{2}=1-\lambda \Omega_{6} ; q_{3}=\omega+\lambda \Omega_{3} ; q_{4}=\omega+\lambda \Omega_{4} ; q_{5}=\lambda \Omega_{1}$; $q_{6}=\lambda \Omega_{2} ; q_{k}=$ const $; \quad k=1, \ldots, 6$.

Values $\Omega_{k},(k=1, \ldots, 6)$ can be written as follows:

$$
\begin{gathered}
\Omega_{1}=\frac{A B}{4 s_{2}^{2}}\left(\frac{B}{v_{1}}+\frac{A}{v_{2}}-Q_{1}+Q_{2}\right), \\
\Omega_{2}=-\frac{1}{4 s_{1}^{2}}\left(\frac{A}{v_{1}}+\frac{B}{v_{2}}-Q_{1}-Q_{2}\right), \\
\Omega_{3}=-\frac{A B}{4 s_{1}^{2}}\left(\frac{B v_{2}^{2}}{v_{1}}+\frac{A v_{1}^{2}}{v_{2}}-\left(Q_{1}+Q_{2}\right) v_{1} v_{2}\right), \\
\Omega_{4}=\frac{1}{4 s_{2}^{2}}\left(\frac{A v_{2}^{2}}{v_{1}}+\frac{B v_{1}^{2}}{v_{2}}+Q_{3}\right), \\
\Omega_{5}=\frac{A B}{2 s_{1}^{2}}\left(Q_{4}+Q_{5}\right), \\
\Omega_{6}=\frac{A B}{2 s_{2}^{2}}\left(Q_{4}+Q_{6}\right),
\end{gathered}
$$

where

$$
Q_{1}=\frac{2(A+B)}{v_{1}+v_{2}}
$$




$$
\begin{gathered}
Q_{2}=\frac{2(A-B)}{v_{1}-v_{2}}, \\
Q_{3}=v_{1} v_{2}\left(Q_{2}-Q_{1}\right), \\
Q_{4}=\frac{v_{2}}{v_{1}}+\frac{v_{1}}{v_{2}}, \\
Q_{5}=-\frac{v_{1} A+v_{2} B}{2 A B}\left(Q_{1}+Q_{2}\right), \\
Q_{6}=\frac{v_{1} B+v_{2} A}{2 A B}\left(Q_{2}-Q_{1}\right) .
\end{gathered}
$$

Performing the similar calculations of the integrals $J_{\eta}$ and $J_{\xi}$ of the system (4.1), related to the angular momenta $l_{\eta}=\dot{\xi} \zeta-\xi \dot{\zeta}$ and $l_{\xi}=\dot{\zeta} \eta-\zeta \dot{\eta}$ of the rotational stellar motion with respect to the axes $\eta$ and $\xi$, respectively, we find

$$
\begin{aligned}
& \dot{\xi} \zeta p_{1}-\xi \dot{\zeta \zeta} p_{2}-\eta \zeta p_{3}-\dot{\eta} \dot{\zeta} p_{4}=J_{\eta}=\text { const } \\
& \dot{\zeta} \eta r_{1}-\zeta \dot{\eta} r_{2}-\xi \zeta r_{3}-\dot{\xi} \dot{\zeta} r_{4}=J_{\xi}=\text { const }
\end{aligned}
$$

Expressions for the coefficients $p_{j}$ and $r_{j}$ for $j=1, \ldots, 4$ have a cumbersome form, and, for the sake of brevity, are not given here.

According to the data on the parameters $v_{1}, v_{2}, \beta_{i}(i=1, \ldots, 3)$ for homogeneous ellipsoids (Danilov, 2005), potentials of which approximate the gravitational potential at the periphery of the numerical dynamic OSC models 1-6 (Danilov and Dorogavtseva, 2003), we find: $q_{k}>0$ for $k=1, \ldots, 5, q_{6}<0 ; p_{j}=r_{j}=0$ for $j=1, \ldots, 4$. Thus, integrals $J_{\eta}$ and $J_{\xi}$ do not impose any restrictions on the stellar motion in the considered models of homogeneous ellipsoid, since any finite values of the phase coordinates of stars (PCS) are admissible for $p_{j}=r_{j}=0, j=1, \ldots, 4$.

Integrals $J_{1}, J_{2}$ and $J_{3}$ from (4.3) and (4.7) are represented by quadratic forms of PCS; they are independent and single-valued PCS functions, which can be used as arguments in the phase density function of the number of stars in the considered model of homogeneous ellipsoid. Equations for $J_{1}$ and $J_{3}$ in a general case define a circle and a hyperbola in the velocity space $(\dot{\xi}, \dot{\eta})$, respectively. Equation for $J_{2}$ in a general case defines an ellipse in the space $(\zeta, \dot{\zeta})$.

According to Danilov (2005) the generalized momenta of a star with mass $m$ moving in the homogeneous ellipsoid force field and in the linearized force field of the Galaxy, can be written as follows:

$$
P_{1}=m(\dot{\xi}-\omega \eta), \quad P_{2}=m\left(\dot{\eta}+\omega\left(R_{0}+\xi\right)\right), \quad P_{3}=m \dot{\zeta},
$$

where $R_{0}$ is distance of the center of the cluster masses from the center of the Galaxy. We consider equations (4.9) as a system for defining the values $\dot{\xi}, \dot{\eta}, \dot{\zeta}$. Solving this system and substituting values $\dot{\xi}, \dot{\eta}, \dot{\zeta}$ in (4.2), (4.3) and (4.7), we find hamiltonian $H$ for the motion of the star with mass $m$ in the joint field of the cluster and the Galaxy

$$
H=m \epsilon=\frac{m}{2}\left(J_{1}+J_{2}\right),
$$


as well as integrals $J_{i}$ in the independent variables: $\xi, \eta, \zeta$ and $P_{i},(i=1,2,3)$. We denote: $\xi=x_{1}, \eta=x_{2}, \zeta=x_{3}$. Calculating Poisson brackets

$$
\left\{H, J_{k}\right\}=\sum_{i=1}^{3}\left(\frac{\partial H}{\partial x_{i}} \frac{\partial J_{k}}{\partial P_{i}}-\frac{\partial J_{k}}{\partial x_{i}} \frac{\partial H}{\partial P_{i}}\right),
$$

we find:

$$
\left\{H, J_{i}\right\}=0, \quad\left\{J_{k}, J_{i}\right\}=0, \quad(i, k=1,2,3) .
$$

The relations (4.10) are satisfied for all six models of homogeneous ellipsoidal clusters considered by Danilov (2005). Thus, integrals $J_{1}, J_{2}, J_{3}$ (or, for example, $H, J_{2}, J_{3}$ ) are in involution to each other (the system (4.1) is completely integrable). Consequently, to find the remaining integrals of motion, we can use the Liouville theorem on the solution of dynamical problem with $n$ degrees of freedom, for which $n$ integrals of motion are known (Whittaker, 1937, p.354).

We write integrals $H, J_{2}, J_{3}$ of the system (4.1) in the form

$$
\frac{2}{m} H(\vec{x}, \dot{\vec{x}})=a_{1}, \quad J_{2}(\vec{x}, \dot{\vec{x}})=a_{2}, \quad J_{3}(\vec{x}, \dot{\vec{x}})=a_{3},
$$

where $\vec{x}=(\xi, \eta, \zeta) ; \dot{\vec{x}}=(\dot{\xi}, \dot{\eta}, \dot{\zeta}) ; a_{i}$ are arbitrary constants, $i=1,2,3$. We denote $\vec{a}=\left(a_{1}, a_{2}, a_{3}\right)$. To find new integrals of the system (4.1) it is necessary to solve the system of algebraic equations (4.11) relative to the values $\dot{x}_{i}$, express $\dot{x}_{i}$ through $P_{i}$ using (4.9), and to obtain the expressions for the generalized momenta in the form $P_{i}=P_{i}(\vec{x}, \vec{a}), i=1,2,3$. Calculating the value $P_{3}$ according to the second equation from (4.11), we easily find

$$
P_{3}= \pm m \sqrt{a_{2}-\beta_{3}^{2} \zeta^{2}} .
$$

The system of the first and third equations from (4.11) can be reduced to the form more convenient for solution:

$$
\begin{aligned}
& \dot{\xi}^{2}\left(q_{6}-q_{5}\right)+\xi^{2}\left(q_{6} \beta_{1}^{2}-q_{3}\right)+\eta^{2}\left(q_{6} \beta_{2}^{2}-q_{4}\right)-\dot{\eta} \xi q_{1}+\dot{\xi} \eta q_{2}=q_{6}\left(a_{1}-a_{2}\right)-a_{3}, \\
& \dot{\eta}^{2}\left(q_{5}-q_{6}\right)+\xi^{2}\left(q_{5} \beta_{1}^{2}-q_{3}\right)+\eta^{2}\left(q_{5} \beta_{2}^{2}-q_{4}\right)-\dot{\eta} \xi q_{1}+\dot{\xi} \eta q_{2}=q_{5}\left(a_{1}-a_{2}\right)-a_{3} .
\end{aligned}
$$

In this work, we obtain analytic solution of the system of equations (4.12) with respect to the values $\dot{\xi}, \dot{\eta}$ :

$$
\dot{\xi}_{l}=\dot{\xi}_{l}(\vec{x}, \vec{a}), \quad \dot{\eta}_{l}=\dot{\eta}_{l}(\vec{x}, \vec{a}), \quad l=1, \ldots, 4,
$$

that in a general case, gives the coordinates in the plane $(\dot{\xi}, \dot{\eta})$ of the four intersecton points of two branches of the hyperbola and the circle given by the equations (4.12). The solution (4.13) is very cumbersome, so its specific form is not given here. A complex form of the expressions (4.13) does not allow calculating the remaining integrals of the system (4.1) in analytical form according to the scheme indicated by 
Whittaker (1937, p. 354). However, the solution (4.13) was used by Danilov (2006) when studying the admitted region of $J_{1}$ and $J_{3}$ integrals.

\subsection{Stellar Motion in the Plane $(\xi, \eta)$}

Let $J_{2}=0$. In this case, according to (4.3), $J_{1}=2 \epsilon$. To study stellar motion on the periphery of a homogeneous ellipsoidal cluster, it is necessary to consider the values $\epsilon$ close to the critical one, $\epsilon=\epsilon_{t}$. The value $\epsilon_{t}$ was defined by Danilov (2005). With a given $\epsilon$, the boundary values of $J_{3}$ can be found using the solution (4.4) from the conditions: $C_{1} \neq 0, C_{2}=0$ and $C_{1}=0, C_{2} \neq 0$. To simplify the analysis of stellar motion, let us consider the trajectories beginning at the time point $t=0$ on the $\xi$ axis and satisfying the following conditions:

$$
\dot{\xi}_{0}=0, \quad \dot{\eta}_{0} \neq 0, \quad \xi_{0} \neq 0, \quad \eta_{0}=0, \quad t_{1}=t_{2}=0,
$$

here, $\xi_{0}=\xi(0)$; values $\dot{\xi}_{0}, \dot{\eta}_{0}, \eta_{0}$ are defined similarly.

According to (4.3) and (4.7), in this case we find

$$
J_{1}=\dot{\eta}_{0}^{2}+\beta_{1}^{2} \xi_{0}^{2}, \quad J_{3}=\dot{\eta}_{0} \xi_{0} q_{1}+\xi_{0}^{2} q_{3}+\dot{\eta}_{0}^{2} q_{6}
$$

Let us consider the case $C_{1} \neq 0, C_{2}=0$. In this case, the star motion in the plane $(\xi, \eta)$ is one-frequency (with the lowest frequency in this problem $v_{1} ; v_{1}<v_{2}<\beta_{3}$ (Danilov, 2005)). According to (4.4) and (4.14), we find

$$
\xi_{0}=C_{1}, \quad \dot{\eta}_{0}=A C_{1} v_{1}, \quad l_{\zeta}(0)=A C_{1}^{2} v_{1}>0
$$

(here, we used the values $A \simeq 0.48271>0, B \simeq-1.38109<0$, obtained for model 1 of a homogeneous ellipsoid by Danilov (2005); for the remaining five ellipsoid models, values $A$ and $B$ differ little from the values given here). As in the paper of Danilov (2005), the calculations were performed in the system of units: $1 m_{\odot}, 1 \mathrm{pc}$, 1 Myr. Using (4.4), we can obtain equation of the stellar orbit in the plane $(\xi, \eta)$ in the form

$$
\frac{\xi^{2}}{a_{p}^{2}}+\frac{\eta^{2}}{b_{p}^{2}}=1
$$

where $a_{p}^{2}=C_{1}^{2} ; b_{p}^{2}=A^{2} C_{1}^{2}$. Thus, $b_{p} / a_{p}=A<1$, see above, and the orbit is elliptical, elongated along $\xi$ axis. The star in such an orbit moves in a "prograde" direction. Substituting $\xi_{0}=C_{1}$ and $\dot{\eta}_{0}=A C_{1} v_{1}$ in (4.15), excluding $C_{1}^{2}$ from the expression for $J_{1}, J_{3}$, we find the maximum value

$$
J_{3, \text { max }}=J_{1}\left(\frac{q_{3}+A v_{1}\left(q_{1}+q_{6} A v_{1}\right)}{A^{2} v_{1}^{2}+\beta_{1}^{2}}\right)>0, \quad J_{3, \text { max }} \simeq 3.71651 \quad\left(\mathrm{pc}^{2} / \mathrm{Myr}\right) ;
$$

here we accepted $J_{1}=2 \epsilon_{t}$. 
Let us consider the case $C_{1}=0, C_{2} \neq 0$. In this case, the star motion in the plane $(\xi, \eta)$ is also one-frequency (with the frequency $\left.v_{2}\right)$. According to (4.4) and (4.14), we find: $\xi_{0}=C_{2}, \dot{\eta}_{0}=B C_{2} v_{2}, l_{\zeta}(0)=B C_{2}^{2} v_{2}<0$. The equation of the stellar orbit in the plane $(\xi, \eta)$ has the form

$$
\frac{\xi^{2}}{a_{r}^{2}}+\frac{\eta^{2}}{b_{r}^{2}}=1
$$

where $a_{r}^{2}=C_{2}^{2} ; b_{r}^{2}=B^{2} C_{2}^{2}$. Thus, $b_{r} / a_{r}=|B|>1$, the orbit is "retrograde", elliptical, elongated along the axis $\eta$. Substituting $\xi_{0}=C_{2}$ and $\dot{\eta}_{0}=B C_{2} v_{2}$ in (4.16), excluding $C_{2}^{2}$ from the expressions for $J_{1}, J_{3}$, we find the minimum value

$$
J_{3, \text { min }}=J_{1}\left(\frac{q_{3}+B v_{2}\left(q_{1}+q_{6} B v_{2}\right)}{B^{2} v_{2}^{2}+\beta_{1}^{2}}\right)<0, \quad J_{3, \text { min }} \simeq-1.48661 \quad\left(\mathrm{pc}^{2} / \mathrm{Myr}\right) .
$$

According to (4.4) angular momentum $l_{\zeta}$ is preserved when the star moves in these two orbits. Let us consider a general case: $C_{1} \neq 0, C_{2} \neq 0$. In this case, the angular momentum $l_{\zeta}$ in the stellar motion is not preserved. According to (4.5) and (4.14), we find

$$
C_{1}=\left(\dot{\eta}_{0}-\xi_{0} v_{2} B\right) / s_{1}, \quad C_{2}=\left(\xi_{0} v_{1} A-\dot{\eta}_{0}\right) / s_{1} .
$$

These formulas follow from the espressions (4.5) for $\cos \left(\phi_{i}\right)$ when $\phi_{i}=0, i=1,2$. When $\xi_{0}=0$, from the first equation (4.15) we find $\dot{\eta}_{0}^{2}=J_{1}, J_{3}=q_{6} J_{1}=J_{3}^{(0)}<0$. In this case, $l_{\zeta}(0)=\dot{\eta}_{0} \xi_{0}=0$. Let $a$ and $b$ be large and middle axes of the cluster ellipsoid and zero velocities ellipse (at $\epsilon=\epsilon_{t}$ ) in the plane $(\xi, \eta)$. At $\xi_{0}= \pm a$, the value $\dot{\eta}_{0}=0$ (and $\dot{\xi}_{0}=0$ ). In this case, also $l_{\zeta}(0)=0$, and corresponding $J_{1}$ and $J_{3}$ values, according to (4.15) and Danilov (2005), are

$$
J_{1}=\beta_{1}^{2} a^{2}=2 \epsilon_{t}, \quad J_{3}=a^{2} q_{3}=J_{3}^{(1)}>0 .
$$

At $\epsilon=\epsilon_{t}$, we find $J_{3}^{(0)} \simeq-0.82958\left(\mathrm{pc}^{2} / \mathrm{Myr}\right), J_{3}^{(1)} \simeq 3.05948\left(\mathrm{pc}^{2} / \mathrm{Myr}\right)$ and, consequently, we have the following inequalities: $J_{3, \min }<J_{3}^{(0)}<J_{3}^{(1)}<J_{3, \max }$. We express the value $\dot{\eta}_{0}$ in terms of $J_{1}$ from the first equation of the system (4.15) and substitute $\dot{\eta}_{0}$ in the second equation (4.15). After simple transformations, we obtain a biquadratic equation with respect to $\xi_{0}$, solving which, we find

$$
\xi_{0}= \pm \sqrt{\tau_{1} \pm \tau_{2}}, \quad \dot{\eta}_{0}= \pm e_{0},
$$

where

$$
\begin{gathered}
\tau_{1}=\left(2 e_{1} e_{2}+J_{1} q_{1}^{2}\right) /\left(2 e_{3}\right) ; \quad \tau_{2}=\sqrt{\tau_{1}^{2}-e_{1}^{2} / e_{3}} ; \\
e_{0}=\sqrt{J_{1}-\beta_{1}^{2} \xi_{0}^{2}} ; \quad e_{1}=J_{3}-q_{6} J_{1} ; \quad e_{2}=q_{3}-\beta_{1}^{2} q_{6} ; \quad e_{3}=e_{2}^{2}+\beta_{1}^{2} q_{1}^{2} .
\end{gathered}
$$

For the different values of $J_{1}$ and $J_{3}$, it is necessary to use the different quadruples of the eight roots $\left(\xi_{0} \dot{\eta}_{0}\right)$, given in (4.16). Let $J_{1}=2 \epsilon_{t}$. At $J_{3} \in\left[J_{3, \min }, J_{3}^{(0)}\right]$ 
substitution of roots in the equations (4.15) shows that the roots of the system (4.15) are the following values of $\left(\xi_{0}, \dot{\eta}_{0}\right)$ :

$$
\left(\xi_{0}=-\sqrt{\tau_{1} \pm \tau_{2}}, \dot{\eta}_{0}=e_{0}\right), \quad\left(\xi_{0}=\sqrt{\tau_{1} \pm \tau_{2}}, \dot{\eta}_{0}=-e_{0}\right) .
$$

At $J_{3} \in\left(J_{3}^{(0)}, J_{3}^{(1)}\right)$ we similarly find

$$
\left(\xi_{0}= \pm \sqrt{\tau_{1} \mp \tau_{2}}, \dot{\eta}_{0}=e_{0}\right), \quad\left(\xi_{0}= \pm \sqrt{\tau_{1} \pm \tau_{2}}, \dot{\eta}_{0}=-e_{0}\right) .
$$

In this group of roots, when calculating $\xi_{0}$, it is necessary to use either upper or lower signs. At $J_{3} \in\left[J_{3}^{(1)}, J_{3, \max }\right]$ we find

$$
\left(\xi_{0}=\sqrt{\tau_{1} \pm \tau_{2}}, \dot{\eta}_{0}=e_{0}\right), \quad\left(\xi_{0}=-\sqrt{\tau_{1} \pm \tau_{2}}, \dot{\eta}_{0}=-e_{0}\right) .
$$

At $J_{3}<J_{3, \min }$ and $J_{3}>J_{3, \max }$ the real roots of the equations (4.15) are absent.

Thus, the region of admissible $J_{3}$ values is the interval $\left[J_{3, \min }, J_{3, \max }\right]$. According to the solutions of the system of equations (4.15) for each value $J_{3}$ in the interval $J_{3} \in\left(J_{3, \min }, J_{3, \max }\right)$ there are two different values $l_{\zeta}(0)$ (about the two-valuedness of the velocity field of the test body, see also in the model of Nezhinskii (1990)). At $J_{3}=J_{3, \min }$, there is only one value $l_{\zeta}(0)=B C_{2}^{2} v_{2}<0$, see above. At $J_{3} \in$ $\left(J_{3, \min }, J_{3}^{(0)}\right)$ the both possible values $l_{\zeta}(0)<0$. At $J_{3}=J_{3}^{(0)}$ we have one value $l_{\zeta}(0)=0$ and one value $l_{\zeta}(0)<0$. At $J_{3} \in\left(J_{3}^{(0)}, J_{3}^{(1)}\right)$, we find one value $l_{\zeta}(0)>0$ and one value $l_{\zeta}(0)<0$. At $J_{3}=J_{3}^{(1)}$ we find one value $l_{\zeta}(0)=0$ and one value $l_{\zeta}(0)>0$. At $J_{3} \in\left(J_{3}^{(1)}, J_{3, \max }\right)$, the both possible values $l_{\zeta}(0)>0$. At $J_{3}=J_{3, \max }$, there is only one value $l_{\zeta}(0)=A C_{1}^{2} v_{1}>0$, see above.

In this work, we used the formulas (4.4) and initial conditions (4.14), (4.16) to calculate stellar trajectories in the cluster at $J_{1}=2 \epsilon_{t}$ for a number of values $J_{3} \in\left[J_{3, \min }, J_{3, \max }\right]$. Only in the intervals $J_{3} \in\left[J_{3, \min }, J_{3}^{(0)}\right], J_{3} \in\left[J_{3}^{(1)}, J_{3, \max }\right]$, for the values $J_{3}$ close to $J_{3, \min }$ and $J_{3, \max }$, signs of the values $l_{\zeta}(0), l_{\zeta}(t)$ and $J_{3}$ coincide. As the value $J_{3}$ deviates from $J_{3, \min }, J_{3, \max }$ in the indicated intervals of $J_{3}$ the oscillation amplitude of $l_{\zeta}(t)$ with respect to $l_{\zeta}(0)$ increases. Stellar trajectory is located in narrow elliptical annular zones in the planes $(\xi, \eta)$ and $(\dot{\xi}, \dot{\eta})$. In the plane $(\xi, \eta)$, these annular zones also contain the trajectories considered above

$$
\frac{\xi^{2}}{a_{p}^{2}}+\frac{\eta^{2}}{b_{p}^{2}}=1 \quad\left(J_{3} \simeq J_{3, \max }\right) \quad \text { and } \quad \frac{\xi^{2}}{a_{r}^{2}}+\frac{\eta^{2}}{b_{r}^{2}}=1 \quad\left(J_{3} \simeq J_{3, \min }\right) .
$$

The width of these annular zones increases with deviation $J_{3}$ from $J_{3, \min }$ and $J_{3, \max }$. Similar elliptical annular regions of possible motion of the test body are also noted in the model of Nezhinskii (1990). At $J_{3} \in\left(J_{3}^{(0)}, J_{3}^{(1)}\right)$, stellar trajectory in the plane $(\xi, \eta)$ largely fills the region inside the ellipse of zero velocities $\xi^{2} / a^{2}+\eta^{2} / b^{2}=1$. The shape of the region occupied by the trajectory does not depend on the sign of $l_{\zeta}(0)$. When the star moves, the value $l_{\zeta}(t)$ often changes a sign in the whole range of $l_{\zeta}(t)$ values admissible by the solution (4.4). At $J_{3} \simeq\left\langle J_{3}\right\rangle=\left(J_{3, \min }+J_{3, \max }\right) / 2$, 
the value $l_{\zeta}(t)$ equally often can be both positive and negative during the sufficiently large intervals of time $\Delta t$ of stellar motion. At $J_{3}>\left\langle J_{3}\right\rangle$, positive $l_{\zeta}(t)$ values are realized more often.

The formula (4.7) for the integral $J_{3}$ can be reduced to the form

$$
q_{5}\left(\dot{\xi}-\dot{\xi}_{h}\right)^{2}+q_{6}\left(\dot{\eta}-\dot{\eta}_{h}\right)^{2}=J_{3}+\xi^{2}\left(\frac{q_{1}^{2}}{4 q_{6}}-q_{3}\right)-\eta^{2}\left(\frac{q_{2}^{2}}{4 q_{5}}+q_{4}\right)=R,
$$

where

$$
\dot{\xi}_{h}=\left(\frac{q_{2}}{2 q_{5}}\right) \eta ; \quad \dot{\eta}_{h}=-\left(\frac{q_{1}}{2 q_{6}}\right) \xi
$$

are coordinates of the hyperbola center in the plane $(\dot{\xi}, \dot{\eta})$.

At the cluster periphery, the values $\left|\dot{\xi}_{h}\right|$ and $\left|\dot{\eta}_{h}\right|$ reach the highest values, and the hyperbola center (4.17) in the plane $(\dot{\xi}, \dot{\eta})$ is located at the greater distance from the origin $\dot{\xi}=\dot{\eta}=0$, than near the cluster center. Depending on the sign of $R$, the real axis of the hyperbola (4.17) in the velocity space can be located both on the axis $\dot{\xi}$ and on the axis $\dot{\eta}$. At $R=0$, we obtain the equations for the asymptotes of the hyperbola:

$$
\dot{\eta}-\dot{\eta}_{h}= \pm \sqrt{-\frac{q_{5}}{q_{6}}}\left(\dot{\xi}-\dot{\xi}_{h}\right) .
$$

The value

$$
\left(\frac{q_{1}^{2}}{4 q_{6}}-q_{3}\right)<0
$$

for models of homogeneous ellipsoidal clusters from the work of Danilov (2005). Therefore, the hyperbola branches degenerate in the line of asymptotes at $R=0$, $J_{3}>0$ for the points $(\xi, \eta)$, located on the ellipse:

$$
J_{3}=-\xi^{2}\left(\frac{q_{1}^{2}}{4 q_{6}}-q_{3}\right)+\eta^{2}\left(\frac{q_{2}^{2}}{4 q_{5}}+q_{4}\right) .
$$

When $R$ passes through $R=0$, the direction of the real and conjugate axes of the hyperbola changes on $90^{\circ}$.

Let $J_{1, \min }=\beta_{1}^{2} \xi^{2}+\beta_{2}^{2} \eta^{2}$ (see (4.3)), the point $(\xi, \eta)$ is located inside the region bounded by the ellipse of zero velocities, $J_{1}^{\prime} \in\left(J_{1, \text { min }}, 2 \epsilon_{t}\right], J_{3}^{\prime} \in\left(J_{3, \text { min }}, J_{3, \text { max }}\right]$. In the case $J_{1} \in\left[J_{1, \min }, J_{1}^{\prime}\right], J_{3} \in\left[J_{3, \min }, J_{3}^{\prime}\right]$ the region $S$ of admissible values $(\dot{\xi}, \dot{\eta})$ for a star located at the time $t$ in the point $(\xi, \eta)$, is determined by intersection of the circle with the radius

$$
v^{\prime}=\sqrt{J_{1}^{\prime}-\beta_{1}^{2} \xi^{2}-\beta_{2}^{2} \eta^{2}}
$$

with the center at the point $\dot{\xi}=\dot{\eta}=0$ and the region located between the branches of two hyperbolas (4.17), corresponding to the values $J_{3}=J_{3, \min }$ and $J_{3}=J_{3}^{\prime}$. When passing from one point $(\xi, \eta)$ to another, the shape and dimensions of the region $S$ in the plane $(\dot{\xi}, \dot{\eta})$ significantly change. There is also a change in the mutual arrangement of the circle corresponding to the given value $J_{1}$ from (4.3) and of the 
hyperbola branches (4.17) in the plane $(\xi, \eta)$. Analysis of these changes is related to the use of solutions (4.13), which makes it difficult to construct the PDF of the star number of a homogeneous ellipsoidal cluster in the analytical form. Therefore, we restrict ourselves here to consideration of only certain properties of this PDF.

Let us consider the following quadratic form of the phase coordinates of stars: $T=J_{1}+\sigma^{2} J_{3}=$ const $; \sigma^{2}=$ const $>0 . T$ value is conserved along the trajectory of a star. We can consider $T$ as an argument of the PDF. Substituting in $T$ the expressions for $J_{1}$ from (4.3) and $J_{3}$ from (4.7), we find

$$
T=\frac{\dot{\xi}^{2}}{a_{T}^{2}}+\frac{\dot{\eta}^{2}}{b_{T}^{2}}+\sigma^{2}\left(q_{1} \dot{\eta} \xi-q_{2} \eta \dot{\xi}\right)+\xi^{2} S_{1}+\eta^{2} S_{2},
$$

where

$$
a_{T}^{-2}=1+q_{5} \sigma^{2} ; \quad b_{T}^{-2}=1+q_{6} \sigma^{2} ; \quad S_{1}=\beta_{1}^{2}+\sigma^{2} q_{3} ; \quad S_{2}=\beta_{2}^{2}+\sigma^{2} q_{4} .
$$

At $\sigma^{2}<-1 / q_{6}$ the equation (4.18) in the plane $(\dot{\xi}, \dot{\eta})$ describes an ellipse with semiaxes $a_{T}$ and $b_{T}$. Since $q_{5}>0, q_{6}<0$, then $a_{T}<1, b_{T}>1$. Consequently, the velocity distribution of the stars at the point with coordinates $(\xi, \eta)$ is elongated along the axis $\dot{\eta}$ (along the direction of cluster motion in the Galaxy). The same result was obtained in the paper of Danilov (1997a) for the OSC models containing 500 stars and moving in the Galactic plane along a circular orbit around the center of the Galaxy. Let $\sigma_{\dot{\xi}}^{2}, \sigma_{\dot{\eta}}^{2}, \sigma_{\dot{\zeta}}^{2}$ be the dispersions of the residual velocities of stars along the axes $\xi, \eta, \zeta$, respectively. For the model 5 of Danilov (1997a) (OSC model with a halo having the largest sizes and number of stars; parameters of this model are closest to the parameters of model 1 of Danilov (2005)) from the ratio of the values $\sigma_{\dot{\xi}}^{2}$ and $\sigma_{\dot{\eta}}^{2}$ we find $\sigma^{2} \simeq 0.052\left(\mathrm{Myr}^{-1}\right)<-1 / q_{6} \simeq 0.422\left(\mathrm{Myr}^{-1}\right)$. Here is assumed $\sigma_{\dot{\eta}}^{2} / \sigma_{\dot{\xi}}^{2}=b_{T}^{2} / a_{T}^{2}=w$. Consequently, $\sigma^{2}=(w-1) /\left(q_{5}-q_{6} w\right)$.

In the paper of Danilov (2006), 132 trajectories of halo stars were selected for cluster model 1 (Danilov, 2005; Danilov and Dorogavtseva, 2003). According to Danilov and Leskov (2005), these trajectories correspond to small positive values of MLCE, which are smaller than the average MLCE value of stellar trajectories in this cluster model. These trajectories have the least complex Fourier spectra (for three sinusoidal components with the largest amplitudes in the dependencies of the stellar distance from the cluster center on the time, there is 41-95\% of the total energy of the Fourier spectrum of these dependencies (Danilov and Leskov, 2005)). The maximum distances of these stars from the center of the cluster during $1.5 \times 10^{8}$ years do not exceed (5-11) pc (the tidal radius $R_{t}$ of a stability of this cluster model, obtained according to King (2002, p. 198), is $R_{t} \simeq 10.44667$ pc). For the considered trajectories, the following mean values over the period of oscillations of a regular field are obtained: $\sigma_{\dot{\xi}}^{2} \simeq 0.07016 \pm 0.00315\left(\mathrm{pc}^{2} / \mathrm{Myr}^{2}\right), \sigma_{\dot{\eta}}^{2} \simeq 0.08861 \pm 0.00406$ $\left(\mathrm{pc}^{2} / \mathrm{Myr}^{2}\right), \sigma_{\dot{\zeta}}^{2} \simeq 0.14941 \pm 0.01457\left(\mathrm{pc}^{2} / \mathrm{Myr}^{2}\right)$. According to these estimates, we find $\sigma^{2} \simeq 0.03868\left(\mathrm{Myr}^{-1}\right)$, which is comparable with the estimate $\sigma^{2}$ for model 5 from the paper of Danilov (1997a). 
Assuming that $\xi$ and $\eta$ are constant values, we reduce the quadratic form (4.18) to the canonical form:

$$
T=\frac{\left(\dot{\xi}-\dot{\xi}_{c}\right)^{2}}{a_{T}^{2}}+\frac{\left(\dot{\eta}-\dot{\eta}_{c}\right)^{2}}{b_{T}^{2}}+\xi^{2}\left(S_{1}-q_{1}^{2} \sigma^{4} b_{T}^{2} / 4\right)+\eta^{2}\left(S_{2}-q_{2}^{2} \sigma^{4} a_{T}^{2} / 4\right),
$$

where

$$
\dot{\xi}_{c}=\left(q_{2} \sigma^{2} a_{T}^{2} / 2\right) \eta ; \quad \dot{\eta}_{c}=-\left(q_{1} \sigma^{2} b_{T}^{2} / 2\right) \xi
$$

are coordinates of the center of the stellar velocity distribution ellipse at the point $(\xi, \eta)$.

According to (4.19), we find $\dot{\xi}_{c} \simeq 0.19016$ (pc/Myr) and $\dot{\eta}_{c}=0$ in the point $\xi=0, \eta=b ; \dot{\eta}_{c} \simeq 0.17321(\mathrm{pc} / \mathrm{Myr})$ and $\dot{\xi}_{c}=0$ in the point $\xi=-a, \eta=0$ for model 1 of the homogeneous gravitating ellipsoid from the paper of Danilov (2005). Thus, on the periphery of a homogeneous ellipsoidal cluster in the plane $(\xi, \eta)$, the "retrograde" stellar motions predominate. This result also agrees with the data of the paper of Danilov (1997a), according to which the tidal cluster sizes for stars with "retrograde" motion are 1.5-2 times larger than ones for the stars with "prograde" motion. Greater stability of the "retrograde" stellar trajectories in unisolated clusters has also long been known (see, for example, Innanen (1979); Keenan and Innanen (1975)). The values $\dot{\xi}_{c}$ and $\dot{\eta}_{c}$ obtained here are $\sim 29$ and $\sim 32 \%$ of the velocity value $V_{t}=\sqrt{2 \epsilon_{t}}$, which is, according to (4.2), sufficient for the exit of the star from the center of the ellipsoidal cluster to the surface of zero velocities corresponding to $\epsilon=\epsilon_{t}$.

\subsection{Phase Density Function Depending on the Integrals $J_{1}$ and $J_{2}$}

Let the motion of a star be bounded in phase space by two hypersurfaces corresponding to some constant values of the integrals $J_{1}$ and $J_{2}$. Let us consider the following quadratic form of PCS: $T=J_{1}+\kappa^{2} J_{2}=$ const $>0, \kappa^{2}=$ const $>0$. As in the case (4.18), $T$ is unchanged along the trajectory of the motion of the star. Therefore, we can consider the expression for $T$ as the argument of the PDF. Substituting in $T$ the expressions for $J_{1}$ and $J_{2}$ from (4.3), we find

$$
T=\dot{\xi}^{2}+\dot{\eta}^{2}+\beta_{1}^{2} \xi^{2}+\beta_{2}^{2} \eta^{2}+\kappa^{2}\left(\dot{\zeta}^{2}+\beta_{3}^{2} \zeta^{2}\right)=K(\vec{x}, \dot{\vec{x}}),
$$

where vectors $\vec{x}, \dot{\vec{x}}$ are defined in the explanation of the formulas (4.11). In the space of velocities $(\dot{\xi}, \dot{\eta}, \dot{\zeta})$, the equation (4.20) specifies the surface of the spheroid elongated at $\kappa^{2}<1$ (or compressed at $\kappa^{2}>1$ ) along the axis $\dot{\zeta}$. The center of this spheroid coincides with the origin of coordinates $\dot{\xi}=\dot{\eta}=\dot{\zeta}=0$. Let us consider the zero-velocity surfaces given by equations

$$
J_{1}+J_{2}=2 \epsilon_{t}, \quad J_{1}+\kappa^{2} J_{2}=T
$$


at $\dot{\xi}=\dot{\eta}=\dot{\zeta}=0$. For brevity, we will call them as $\epsilon$ - and $T$-surfaces, and regions in the space $(\xi, \eta, \zeta)$, bounded by $\epsilon$ - and $T$-surfaces, as $\epsilon$ - and $T$-ellipsoids, respectively. Centers of these ellipsoids coincide with the origin of coordinates $\xi=\eta=\zeta=0$. $T$-ellipsoid in the case $\kappa^{2}<1\left(\kappa^{2}>1\right)$ is more elongated (compressed) along the axis $\zeta$, than $\epsilon$-ellipsoid. Excluding $J_{1}$ from the system (4.21) at $\dot{\xi}=\dot{\eta}=\dot{\zeta}=0$, expressing $T$ in terms of $\zeta$ and $\zeta$ in terms of $T$, we find

$$
T=2 \epsilon_{t}-\beta_{3}^{2} \zeta^{2}\left(1-\kappa^{2}\right), \quad \zeta= \pm \sqrt{\frac{2 \epsilon_{t}-T}{\beta_{3}^{2}\left(1-\kappa^{2}\right)}}=\zeta_{T} .
$$

According to (4.22), intersection of $\epsilon$ - and $T$-surfaces takes place on $\zeta$ coordinates equal to $\zeta_{T}$.

Let us first consider the case $\kappa^{2}<1$. At $\zeta_{T}= \pm c, \epsilon$ - and $T$-surfaces are tangent at two points: $\xi=\eta=0, \zeta= \pm c$, which corresponds to the value $T=T^{(1)}=2 \epsilon_{t} \kappa^{2}$ (since $\beta_{3}^{2}=2 \epsilon_{t} / c^{2}, c$ is the value of the semiminor axis of the cluster ellipsoid (Danilov, 2005)). When $\zeta_{T}=0$, according to (4.22), we find the value $T=T^{(2)}=$ $2 \epsilon_{t}$. In this case, $\epsilon$ - and $T$-surfaces are tangent along the line set by the equation $\xi^{2} / a^{2}+\eta^{2} / b^{2}=1$ in the plane $\zeta=0$. Thus, $0<T^{(1)}<T^{(2)}$.

Let us consider the case $\kappa^{2}>1$. At $\zeta_{T}=0$, we find $T=T^{(1)}=2 \epsilon_{t}$, since $T$ ellipsoid is more compressed along the $\zeta$ axis, than $\epsilon$-ellipsoid, and $\epsilon$ - and $T$-surfaces are tangent along the line, set by the equation $\xi^{2} / a^{2}+\eta^{2} / b^{2}=1$ in the plane $\zeta=0$. At $\zeta_{T}= \pm c$, we find $T=T^{(2)}=2 \epsilon_{t} \kappa^{2}$, and $\epsilon$ - and $T$-surfaces are tangent at two points: $\xi=\eta=0, \zeta= \pm c$. In this case also $0<T^{(1)}<T^{(2)}$, but $T^{(2)}$ at $\kappa^{2}>1$ is greater, than $T^{(2)}$ at $\kappa^{2}<1$. For any finite values of $\kappa^{2}>0$ the section lines of $\epsilon$ and $T$-surfaces by the plane $\zeta=$ const (for those values $\zeta$, at which such section is possible) are similar concentric ellipses, equations of which can easily be written in the coordinates $(\xi, \eta)$. At $T^{(1)}<T<T^{(2)}$, the motion of a star, which is bounded by hypersurfaces of the integrals $J_{1}$ and $J_{2}$, is possible in the intersection of $\epsilon$ - and $T$-ellipsoids.

We note that the values $\sigma^{2}$ from (4.18) and $\kappa^{2}$ from (4.20) are related to the conditions corresponding to the initial values of PCS in the numerical dynamic OSC models, assumed in the paper of Danilov (2005) as the initial ones for approximation by homogeneous gravitating ellipsoids. Depending on the initial conditions in the initial dynamic models of OSC, $\kappa^{2}$ can be either greater or less than unity. Let us define PDF, depending on the integrals $J_{1}$ and $J_{2}$, for the case $\kappa^{2}<1$. The formulas obtained for the PDF can easily be rearranged for $\kappa^{2}>1$ if necessary.

Let $\Gamma(T)$ be the volume of the phase space (PS) occupied by stars, the motion of which is described by the integral (4.20) with the value $T^{\prime} \in[0, T]$, and $\gamma(T)$ be the PS volume, occupied by the stars with $T^{\prime} \in[T, T+d T]$, corresponding to the interval $d T$ :

$$
\Gamma(T)=\int_{0}^{T} \gamma\left(T^{\prime}\right) d T^{\prime}, \quad \gamma(T)=\frac{d \Gamma(T)}{d T} .
$$

Functions $\Gamma(T)$ and $\gamma(T)$ are the integral and differential distributions with respect to $T$ of the PS volume, occupied by the stars from the corresponding intervals of $T$, 
see above. Taking into account (4.23), we write

$$
d N(T)=f(T) d T=\frac{f(T)}{\gamma(T)} d \Gamma(T) .
$$

Here, $d N(T)$ is the star number in the interval $d T, f(T)$ is stellar distribution in $T, \gamma(T)>0$, and $\Gamma(T)$ is monotonically increasing function of $T$. Assuming $T=T(\vec{x}, \dot{\vec{x}})$ in (4.24), we can define PDF in the form $F(\vec{x}, \dot{\vec{x}})=$ $f(T(\vec{x}, \dot{\vec{x}})) / \gamma(T(\vec{x}, \dot{\vec{x}}))$. Thus, in order to find PDF, it is necessary to find the functions $f(T)$ and $\gamma(T)$. Following to Danilov (2002a), we write the value $\Gamma(T)$ as an integral over the PS region where the condition $0 \leq K(\vec{x}, \dot{\vec{x}}) \leq T$ is satisfied:

$$
\Gamma(T)=\int_{0 \leq K(\vec{x}, \dot{\vec{x}}) \leq T} \cdots \int d^{3} x d^{3} \dot{x} .
$$

The third equation of the system (4.1) describes the motion of a star in $\zeta$-coordinate, and can be integrated independently of the first two equations. Therefore, at first, it is convenient to perform an integration in (4.25) by the variables $\dot{\zeta}$ and $\zeta$. Let $0<T<T^{(1)}$. To find the limits of integration with respect to $\dot{\zeta}$ and $\zeta$ in (4.25), we write the solution of equation (4.20) with respect to $\dot{\zeta}$ in the following form:

$$
\dot{\zeta}= \pm \beta_{3} \sqrt{\zeta_{\max }^{2}-\zeta^{2}}, \quad \zeta_{\max }^{2}=\frac{T-J_{1}}{\beta_{3}^{2} \kappa^{2}} .
$$

Assuming in (4.26) $\dot{\zeta}=0$, we find the maximum value $\zeta_{\max }^{2}$. At $\zeta \neq 0$ the equation (4.26) gives the maximum value $\dot{\zeta}^{2}=\dot{\zeta}_{\text {max }}^{2}$ for a given $T$. The value $\dot{\zeta}_{\text {max }}^{2}$ is the largest for all $T \geq J_{1}$. At $T=J_{1}$, according to (4.26), $\zeta_{\max }^{2}=0$ and $\dot{\zeta}_{\max }^{2}=0$. Let us introduce the following notations:

$$
\begin{gathered}
v^{2}=\dot{\xi}^{2}+\dot{\eta}^{2}, \quad \dot{\xi}=v \cos \varphi, \quad \dot{\eta}=v \sin \varphi, \\
r^{2}=\frac{\xi^{2}}{a^{2}}+\frac{\eta^{2}}{b^{2}}, \quad \xi=\operatorname{ar} \cos \psi, \quad \eta=b r \sin \psi,
\end{gathered}
$$

where $\varphi, \psi \in[0,2 \pi]$. Using the Jacobians of the transition from the coordinates $\dot{\xi}, \dot{\eta}$ and $\xi, \eta$ to the coordinates $v, \varphi$ and $r, \psi$, respectively, and taking into account the symmetries of $\epsilon$ - and $T$-ellipsoids considered above, we obtain

$$
d \dot{\xi} d \dot{\eta}=2 \pi v d v, \quad d \xi d \eta=2 \pi a b r d r .
$$

Taking into account accepted notations, we find from the equation $T=J_{1}$ the maximum value of $v^{2}$ for given values of $T$ and $r^{2}: v^{2}=T-2 \epsilon_{t} r^{2}=v_{\text {max }}^{2}$ (according to Danilov (2005) $\left.\beta_{1}^{2}=2 \epsilon_{t} / a^{2}, \beta_{2}^{2}=2 \epsilon_{t} / b^{2}\right)$. Setting $v_{\text {max }}^{2}=0$, we find the maximum value $r^{2}=T /\left(2 \epsilon_{t}\right)=r_{\text {max }}^{2}$. Passing to integration over $v$ and $r$ in (4.25), taking into account (4.26), we find: 


$$
\begin{gathered}
\Gamma(T)=16 \pi^{2} a b \int_{0}^{r_{\max }} r d r \int_{0}^{v_{\max }} v d v \int_{0}^{\zeta_{\max }} d \zeta \int_{0}^{\dot{\zeta}_{\max }} d \dot{\zeta}, \\
\Gamma(T)=\frac{\pi^{3} a b c T^{3}}{6 \kappa^{2}\left(2 \epsilon_{t}\right)^{3 / 2}},
\end{gathered}
$$

where $T \in\left[0, T^{(1)}\right] ; r_{\max }>0 ; v_{\max }>0 ; \zeta_{\max }>0 ; \dot{\zeta}_{\max }>0$; the value $\beta_{3}^{2}$ is expressed through $c^{2}$, see above. At $T \in\left(T^{(1)}, T^{(2)}\right]$ the expression for $\Gamma(T)$ can not be obtained analytically, but it is clear that in this range of values $T$, the value $\Gamma(T)$ increases less rapidly with increasing $T$, than in (4.27). At $T \rightarrow T^{(2)}$ and $T \in\left(T^{(1)}, T^{(2)}\right]$, the value $\zeta_{T}$ tends to zero, and only a part of the volume of $T$-ellipsoid is used in the integration (4.25). According to (4.23) and (4.27), we find

$$
\gamma(T)=\frac{d \Gamma(T)}{d T}=k^{2} T^{2}, \quad k^{2}=\frac{\pi^{3} a b c}{2 \kappa^{2}\left(2 \epsilon_{t}\right)^{3 / 2}}, \quad T \in\left[0, T^{(1)}\right] .
$$

Let us denote

$$
\Psi(T)=f(T) /\left(k^{2} T^{2}\right)=C\left(T^{(2)}-T\right),
$$

where $C=C(\xi, \eta, \zeta)$ is the normalization function for the $\operatorname{PDF}$, and $\Psi(T(\vec{x}, \dot{\vec{x}}))=$ $F(\vec{x}, \dot{\vec{x}})$. In this case, the distribution of stellar trajectories $f(T)$ by the value of the integral $T$ has the form:

$$
f(T)=C k^{2} T^{2}\left(T^{(2)}-T\right) \quad \text { at } \quad T \in\left[0, T^{(1)}\right]
$$

and

$$
f(T)=C \gamma(T)\left(T^{(2)}-T\right) \quad \text { at } \quad T \in\left[T^{(1)}, T^{(2)}\right] .
$$

The maximum value of the function $f(T)$ is reached at the point $T=2 T^{(2)} / 3=T_{m}$, if $T_{m} \leq T^{(1)}$. The value $T_{m}$ is defined by the equation:

$$
T_{m}=T^{(2)}-\gamma\left(T_{m}\right) /\left(\frac{d \gamma(T)}{d T}\right)_{T=T_{m}},
$$

if $T_{m} \in\left(T^{(1)}, T^{(2)}\right] ; f(T)=0$ at $T=0$ and $T=T^{(2)}$.

Let us define the normalization function $C=C(\xi, \eta, \zeta)$ for the function $\Psi(T)$. To do this, we compute the density $\rho$ at the point $(\xi, \eta, \zeta)$ of the cluster ellipsoid. We note that stars with the different values of the integrals $T^{\prime} \in\left[T, T^{(2)}\right]$ and $\epsilon^{\prime} \in\left[\epsilon, \epsilon_{t}\right]$ can arrive at the point $(\xi, \eta, \zeta)$. We denote

$$
v_{*}^{2}=g^{2}+\kappa^{2} \beta_{3}^{2} \zeta^{2}, \quad g^{2}=\beta_{1}^{2} \xi^{2}+\beta_{2}^{2} \eta^{2} .
$$

Then the value $T$ from (4.20) can be written in the form

$$
T=v^{2}+\kappa^{2} \dot{\zeta}^{2}+v_{*}^{2} .
$$

The motion of the cluster stars occurs under the surface of zero velocities within $\epsilon$-ellipsoid. Therefore, the maximum value of the integral $J_{1}+J_{2}$ for stars at the point 
$(\xi, \eta, \zeta)$ equals to

$$
2 \epsilon_{t}=v^{2}+g^{2}+\dot{\zeta}^{2}+\beta_{3}^{2} \zeta^{2}
$$

Consequently,

$$
v^{2}=2 \epsilon_{t}-\beta_{3}^{2} \zeta^{2}-g^{2}-\dot{\zeta}^{2} .
$$

At $\dot{\zeta}^{2}=0$, at the point $(\xi, \eta, \zeta) v^{2}$ reaches its maximum value, which is equal to

$$
v_{m}^{2}=2 \epsilon_{t}-\beta_{3}^{2} \zeta^{2}-g^{2} .
$$

We note that

$$
v_{m}^{2}+v_{*}^{2}=2 \epsilon_{t}-\beta_{3}^{2} \zeta^{2}\left(1-\kappa^{2}\right)=s^{2} .
$$

Taking into account the accepted notation for $s^{2}$ we find $v_{*}^{2}=s^{2}-v_{m}^{2}$. Substituting $v_{*}^{2}$ in the expression for $T$, and $v_{m}^{2}$ in the expression for $v^{2}$, we find

$$
T=v^{2}+\kappa^{2} \dot{\zeta}^{2}+s^{2}-v_{m}^{2}, \quad v^{2}=v_{m}^{2}-\dot{\zeta}^{2} .
$$

Consequently, the maximum value of $\dot{\zeta}^{2}$ at the point $(\xi, \eta, \zeta)$ equals to $\dot{\zeta}_{m}^{2}=v_{m}^{2}-v^{2}$. Integrating the function $\Psi(T)$ with respect to $v$ and $\dot{\zeta}$ and taking into account the accepted notation we find

$$
\rho=4 \pi C \int_{0}^{v_{m}} v d v \int_{0}^{\dot{\zeta}_{m}}\left(T^{(2)}-T\right) d \dot{\zeta}=4 \pi C \frac{v_{m}^{3}}{3}\left(e^{2}+\frac{3}{5}\left(1-\frac{\kappa^{2}}{3}\right) v_{m}^{2}\right),
$$

where

$$
\begin{gathered}
v_{m}>0 ; \quad \dot{\zeta}_{m}>0 ; \\
T^{(2)}-T=v_{m}^{2}-v^{2}+e^{2}-\kappa^{2} \dot{\zeta}^{2} ; \quad e^{2}=\beta_{3}^{2} \zeta^{2}\left(1-\kappa^{2}\right) .
\end{gathered}
$$

Since the expression for $\rho$ has been obtained by Danilov (2005), the equation (4.29) can be used to find the value $C$. Using the function $\Psi(T)$, we calculate the mean values for $v^{2}$ and $\dot{\zeta}^{2}$ at the point $(\xi, \eta, \zeta)$ :

$$
\left\langle v^{2}\right\rangle=\frac{1}{Q} \int_{0}^{v_{m}} v^{3} d v \int_{0}^{\dot{\zeta}_{m}} \Psi d \dot{\zeta}, \quad\left\langle\dot{\zeta}^{2}\right\rangle=\frac{1}{Q} \int_{0}^{v_{m}} v d v \int_{0}^{\dot{\zeta}_{m}} \dot{\zeta}^{2} \Psi d \dot{\zeta},
$$

where

$$
v_{m}>0 ; \quad \dot{\zeta}_{m}>0 ; \quad Q=\int_{0}^{v_{m}} v d v \int_{0}^{\dot{\zeta}_{m}} \Psi d \dot{\zeta} .
$$

Calculating the integrals from (4.30), we find

$$
\begin{aligned}
& \left\langle v^{2}\right\rangle=\frac{2}{5} v_{m}^{2}\left(\frac{e^{2}+\frac{3}{7}\left(1-\frac{1}{3} \kappa^{2}\right) v_{m}^{2}}{e^{2}+\frac{3}{5}\left(1-\frac{1}{3} \kappa^{2}\right) v_{m}^{2}}\right), \\
& \left\langle\dot{\zeta}^{2}\right\rangle=\frac{1}{5} v_{m}^{2}\left(\frac{e^{2}+\frac{5}{7}\left(1-\frac{3}{5} \kappa^{2}\right) v_{m}^{2}}{e^{2}+\frac{3}{5}\left(1-\frac{1}{3} \kappa^{2}\right) v_{m}^{2}}\right) .
\end{aligned}
$$



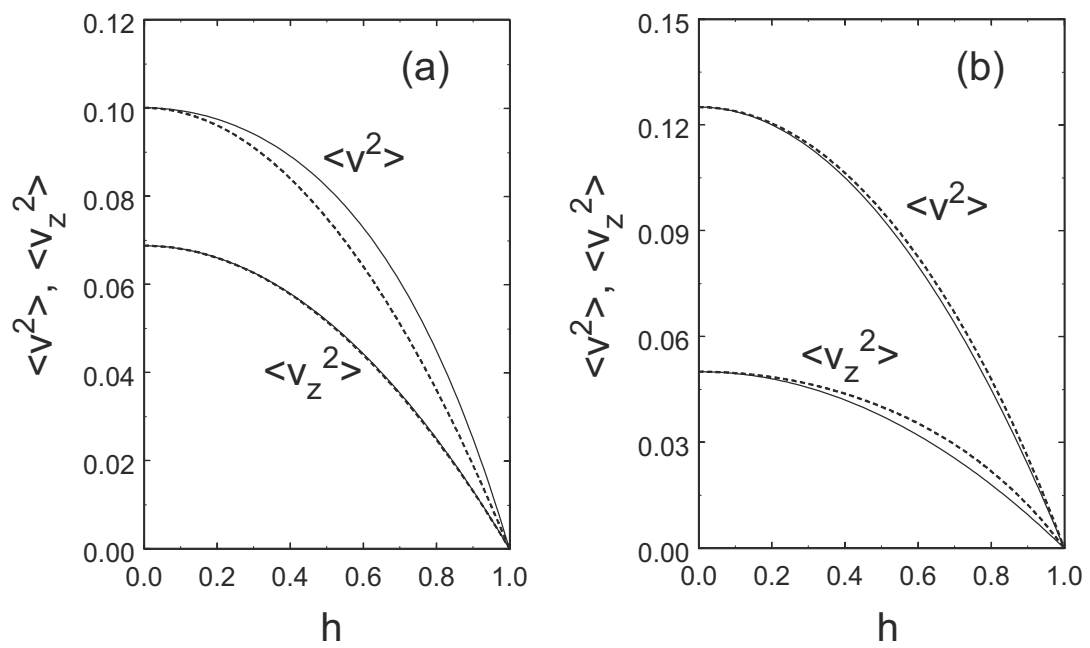

Fig. 4.1 Dependencies of $\left\langle v^{2}\right\rangle$ and $\left\langle v_{\zeta}^{2}\right\rangle=\left\langle\dot{\zeta}^{2}\right\rangle$ on $h$ for two cluster models with $\kappa^{2}=0.53873$ (a) and $\kappa^{2}=1.5$ (b). The solid lines correspond to $\xi=\eta=0$, the dashed lines indicate curves with $\zeta=0$

Since

$$
v_{m}^{2}=2 \epsilon_{t}\left(1-h^{2}\right), \quad h^{2}=\frac{\xi^{2}}{a^{2}}+\frac{\eta^{2}}{b^{2}}+\frac{\zeta^{2}}{c^{2}} \leq 1,
$$

see above, then $v_{m}^{2}=0$ at $h^{2}=1$ (on the $\epsilon$-surface) and $v_{m}^{2}=$ const at $h^{2}=$ const (on surfaces of ellipsoids similar to $\epsilon$-ellipsoid). Consequently, on the $\epsilon$-surface at $h^{2}=1$ the values $\left\langle v^{2}\right\rangle$ and $\left\langle\dot{\zeta}^{2}\right\rangle$ are equal to zero. In the center of the $\epsilon$-ellipsoid (at $h^{2}=0, \zeta=0$ ), according to (4.31), for the values $\left\langle v^{2}\right\rangle$ and $\left\langle\dot{\zeta}^{2}\right\rangle$, we find

$$
\left\langle v^{2}\right\rangle_{0}=\frac{4}{7} \epsilon_{t}, \quad\left\langle\dot{\zeta}^{2}\right\rangle_{0}=\frac{10}{21} \epsilon_{t}\left(\frac{1-\frac{3}{5} \kappa^{2}}{1-\frac{1}{3} \kappa^{2}}\right) .
$$

We note that

$$
\frac{2}{7} \epsilon_{t}<\left\langle\dot{\zeta}^{2}\right\rangle_{0} \leq \frac{10}{21} \epsilon_{t} \quad \text { at } \quad 0 \leq \kappa^{2}<1
$$

In the case $\zeta=$ const and $h^{2}=$ const $\left\langle 1\right.$, the values of $\left\langle v^{2}\right\rangle$ and $\left\langle\dot{\zeta}^{2}\right\rangle$ retain constant on the intersection line of the plane $\zeta=$ const and of the elliptical cylinder

$$
r^{2}=h^{2}-\frac{\zeta^{2}}{c^{2}}=\frac{\xi^{2}}{a^{2}}+\frac{\eta^{2}}{b^{2}}=\text { const } .
$$

With the increase of a distance from the cluster center along $\zeta$ axis and in the plane $(\xi, \eta)$, the values $\left\langle v^{2}\right\rangle$ and $\left\langle\dot{\zeta}^{2}\right\rangle$ decrease in comparison with the values indicated in (4.32), and reach zero on the $\epsilon$-surface at $h^{2}=1$, see fig. 4.1. 
Since $C \sim v_{m}^{-3}$, see (4.29), then the function $F(\vec{x}, \dot{\vec{x}}) \rightarrow \infty$ at $h^{2} \rightarrow 1$. The density of the matter $\rho$ inside the $\epsilon$-ellipsoid is constant, but the density in the velocity space tends to infinity as $h^{2} \rightarrow 1$, which is caused by the tendency to zero of the size of a range of admissible values of $\dot{\xi}, \dot{\eta}, \dot{\zeta}$ at $h^{2} \rightarrow 1$. Taking into account the equation (4.29), as well as the formulas for $v_{m}^{2}$ and $e^{2}$, we write an expression for the two-integral PDF of the cluster:

$$
F(\vec{x}, \dot{\vec{x}})=\frac{3 \rho}{4 \pi v_{m}^{3}}\left(\frac{e^{2}+v_{m}^{2}-v^{2}-\kappa^{2} \dot{\zeta}^{2}}{e^{2}+\frac{3}{5}\left(1-\frac{1}{3} \kappa^{2}\right) v_{m}^{2}}\right), \quad \kappa^{2}<1 .
$$

In the case $\kappa^{2}>1$ for the values $\rho,\left\langle v^{2}\right\rangle,\left\langle\dot{\zeta}^{2}\right\rangle$, and $F(\vec{x}, \dot{\vec{x}})$, we can use the expressions (4.29), (4.31), (4.33), in which it is necessary to use the value

$$
e^{2}=2 \epsilon_{t}\left(\kappa^{2}-1\right)\left(1-\zeta^{2} / c^{2}\right)
$$

as $e^{2}$. In this case, the formulas (4.32) have the following form:

$$
\left\langle v^{2}\right\rangle_{0}=\frac{4}{7} \epsilon_{t}\left(\frac{3 \kappa^{2}-2}{2 \kappa^{2}-1}\right), \quad\left\langle\dot{\zeta}^{2}\right\rangle_{0}=\frac{2}{7} \epsilon_{t} .
$$

We note that

$$
\left(\frac{3 \kappa^{2}-2}{2 \kappa^{2}-1}\right)>1 \quad \text { at } \quad \kappa^{2}>1 .
$$

Consequently, at $\kappa^{2}>1$, the value $\left\langle v^{2}\right\rangle_{0}$ is greater than at $\kappa^{2}<1$, and the value $\left\langle\dot{\zeta}^{2}\right\rangle_{0}$ at $\kappa^{2}>1$ is smaller than at $\kappa^{2}<1$.

\subsection{Distribution of the Stellar Velocities in the Case of Three-Integral PDF}

Let us consider the quadratic form for the PCS of the following type:

$$
T=J_{1}+\kappa^{2} J_{2}+\sigma^{2} J_{3} .
$$

$T$ is conserved along the stellar trajectory. Therefore, $T$ can be used as an argument in PDF. Taking into account the expressions for the integrals $J_{1}, J_{2}, J_{3}$, according to (4.19) and (4.20) we find

$$
\begin{aligned}
& T=\frac{\left(\dot{\xi}-\dot{\xi}_{c}\right)^{2}}{a_{T}^{2}}+\frac{\left(\dot{\eta}-\dot{\eta}_{c}\right)^{2}}{b_{T}^{2}}+\Omega, \\
& \Omega=\xi^{2}\left(S_{1}-q_{1}^{2} \sigma^{4} b_{T}^{2} / 4\right)+\eta^{2}\left(S_{2}-q_{2}^{2} \sigma^{4} a_{T}^{2} / 4\right)+\kappa^{2}\left(\dot{\zeta}^{2}+\beta_{3}^{2} \zeta^{2}\right) .
\end{aligned}
$$

According to (4.35) we write two relations for the value $\kappa^{2}$ : 


$$
\kappa^{2}=\left(\sigma_{\dot{\xi}}^{2} / \sigma_{\dot{\zeta}}^{2}\right) a_{T}^{-2} \quad \text { and } \quad \kappa^{2}=\left(\sigma_{\dot{\eta}}^{2} / \sigma_{\dot{\zeta}}^{2}\right) b_{T}^{-2} .
$$

Using values $\sigma_{\dot{\xi}}^{2}, \sigma_{\dot{\eta}}^{2}, \sigma_{\dot{\zeta}}^{2}$, obtained by Danilov (1997a) for the cluster model 5, as well as the value $\sigma^{2}$ for this model obtained above, we find $\kappa^{2} \simeq 0.387<1$ (both relations for estimation of $\kappa^{2}$ lead to the same value of $\kappa^{2}$ up to the first three digits after the decimal point). In the case of the cluster model 1 of Danilov (2005) and Danilov and Dorogavtseva (2003), both relations for $\kappa^{2}$ lead to the similar value of $\kappa^{2}=0.53873766$ to within the first eight digits after the decimal point. We note that in the cluster model 5 from Danilov (1997a) at the initial time $t=0$ a small excess of stars with the large initial values of $\zeta$ and $\dot{\zeta}$ is given in comparison with the cluster model 1 of Danilov and Dorogavtseva (2003). Therefore, the value $\sigma_{\dot{\zeta}}^{2}$ for the model 5 of Danilov (1997a) is greater, than for the model 1 of Danilov and Dorogavtseva (2003), and $\kappa^{2}$ for model 5 of Danilov (1997a) is smaller than for model 1 of Danilov and Dorogavtseva (2003).

Let us denote

$$
\theta^{2}=\frac{q_{3}+A v_{1}\left(q_{1}+q_{6} A v_{1}\right)}{A^{2} v_{1}^{2}+\beta_{1}^{2}}>0 .
$$

Then $J_{3, \max }=\theta^{2} J_{1}>0$, see explanations for the formula (4.15), where this expression for $J_{3, \max }$ was obtained at $J_{2}=0$. The same expression for $J_{3, \max }$ can be obtained also in the case $J_{2} \neq 0$, if the conditions $J_{1}=$ const, $J_{3}=$ const at $C_{1} \neq 0$, $C_{2}=0$ are taken into account. Substituting the values $J_{3}=J_{3, \max }$ in $T$, taking into account the limitation on the value $J_{1}+J_{2}$, we write

$$
T \leq v^{2} J_{1}+\kappa^{2} J_{2}, \quad J_{1}+J_{2} \leq 2 \epsilon_{t}, \quad \text { where } \quad v^{2}=1+\theta^{2} \sigma^{2} .
$$

These conditions allow us to define the maximum value $T_{\max }$ for the value of $T$ : $T_{\max }=2 \epsilon_{t} \kappa^{2}$ at $v^{2} \leq \kappa^{2}$ and $T_{\max }=2 \epsilon_{t} v^{2}$ at $v^{2}>\kappa^{2}$. For model 5 of Danilov (1997a) and model 1 of Danilov and Dorogavtseva (2003), according to Danilov (2006), $v^{2} \simeq 1.552$ and $v^{2} \simeq 1.410$, respectively.

Let us consider the function $\Psi(T)=C\left(T_{\max }-T\right)$. Substituting $T$ from (4.35), we can find the distribution of stellar velocities at the point $(\xi, \eta, \zeta)$ of a homogeneous ellipsoidal cluster accurate to the constant factor $C$. We consider the general distribution of stellar velocity in the ellipsoidal cluster. To do this, we calculate the integral of the function $\Psi(T)$ by the volume $Q$ of the cluster ellipsoid:

$$
\mathcal{F}(\dot{\xi}, \dot{\eta}, \dot{\zeta})=\langle C\rangle \int_{Q}\left(T_{\max }-T\right) d Q=\langle C\rangle Q\left(V_{0}^{2}-\frac{\dot{\xi}^{2}}{a_{T}^{2}}-\frac{\dot{\eta}^{2}}{b_{T}^{2}}-\kappa^{2} \dot{\zeta}^{2}\right),
$$

where $Q=\frac{4}{3} \pi a b c ;\langle C\rangle$ is the $C$ value at some inner point of the cluster ellipsoid (here, the value $\langle C\rangle$ was removed from the sign of the integral by $Q$ according to the mean-theorem);

$$
V_{0}^{2}=T_{\max }-\frac{1}{5}\left(S_{1} a^{2}+S_{2} b^{2}+\kappa^{2} \beta_{3}^{2} c^{2}\right) .
$$


Since both estimates of the value $\kappa^{2}$ received here correspond to $\kappa^{2}<1$, we choose the following value as $T_{\max }: T_{\max }=2 \epsilon_{t} v^{2} C_{v}$. Here, $C_{v}=$ const $>1$. The value $C_{v}$ is close to unity and is introduced as a free parameter for better matching of the velocity distribution of stars in the model of a homogeneous ellipsoid and in numerical dynamic cluster model 1 of Danilov (2005) and Danilov and Dorogavtseva (2003). To find the value $\langle C\rangle$, we integrate the function $\mathcal{F}(\dot{\xi}, \dot{\eta}, \dot{\zeta})$ by the volume $Q_{v}$ of the velocity ellipsoid

$$
V_{0}^{2}=\frac{\dot{\xi}^{2}}{a_{T}^{2}}+\frac{\dot{\eta}^{2}}{b_{T}^{2}}+\kappa^{2} \dot{\zeta}^{2}
$$

We obtain the number of stars $N$ in an ellipsoidal cluster (according to Danilov (2005) $N \simeq 500$ ):

$$
N=8 \pi a_{T} b_{T} V_{0}^{5}\langle C\rangle Q /(15 \kappa)
$$

This relation for $\langle C\rangle$ can be used as the initial one when approximating the velocity distribution in the numerical dynamic cluster model 1 of Danilov and Dorogavtseva (2003) by the function $\mathcal{F}(\dot{\xi}, \dot{\eta}, \dot{\zeta})$. In order to find the general distribution of stars in an ellipsoidal cluster with respect to $\dot{\xi}$, we calculate the integral

$$
F_{1}(\dot{\xi})=\int_{\Omega} \mathcal{F}(\dot{\xi}, \dot{\eta}, \dot{\zeta}) d \dot{\eta} d \dot{\zeta}
$$

over all values of $\dot{\eta}, \dot{\zeta}$ within the region $\Omega$, located inside the velocity ellipse

$$
V_{0}^{2}-\frac{\dot{\xi}^{2}}{a_{T}^{2}}=\frac{\dot{\eta}^{2}}{b_{T}^{2}}+\kappa^{2} \dot{\zeta}^{2} \leq V_{0}^{2}
$$

for a fixed value of $\dot{\xi}^{2}$. Performing the corresponding calculations of the functions $F_{2}(\dot{\eta}), F_{3}(\dot{\zeta})$ of distributions of stars by the values $\dot{\eta}$, $\dot{\zeta}$, we find

$$
\begin{aligned}
& F_{1}(\dot{\xi})=\langle C\rangle Q \frac{\pi b_{T}}{2 \kappa}\left(V_{0}^{2}-\frac{\dot{\xi}^{2}}{a_{T}^{2}}\right)^{2}, \\
& F_{2}(\dot{\eta})=\langle C\rangle Q \frac{\pi a_{T}}{2 \kappa}\left(V_{0}^{2}-\frac{\dot{\eta}^{2}}{b_{T}^{2}}\right)^{2}, \\
& F_{3}(\dot{\zeta})=\langle C\rangle Q \frac{\pi a_{T} b_{T}}{2}\left(V_{0}^{2}-\kappa^{2} \dot{\zeta}^{2}\right)^{2},
\end{aligned}
$$

where

$$
\dot{\xi}^{2} \leq V_{0}^{2} a_{T}^{2}, \quad \dot{\eta}^{2} \leq V_{0}^{2} b_{T}^{2}, \quad \dot{\zeta}^{2} \leq V_{0}^{2} / \kappa^{2} .
$$

Fig. 4.2 shows the distributions by the values $\xi, \eta, \zeta$ of the 132 halo stars from model 1 of Danilov and Dorogavtseva (2003) considered above, averaged over the period of oscillations of a regular field, as well as distributions $F_{1}(\dot{\xi}) \Delta \dot{\xi}, F_{2}(\dot{\eta}) \Delta \dot{\eta}, F_{3}(\dot{\zeta}) \Delta \dot{\zeta}$, obtained according to (4.37) at $C_{v}=1.2$. On the fig. 4.2, we used the notations $v_{\xi}=\dot{\xi}, v_{\eta}=\dot{\eta}, v_{\zeta}=\dot{\zeta}$. In constructing the theoretical distributions, we used the same values of the intervals $\Delta v_{\xi}, \Delta v_{\eta}, \Delta v_{\zeta}$, as for the corresponding numerical- 

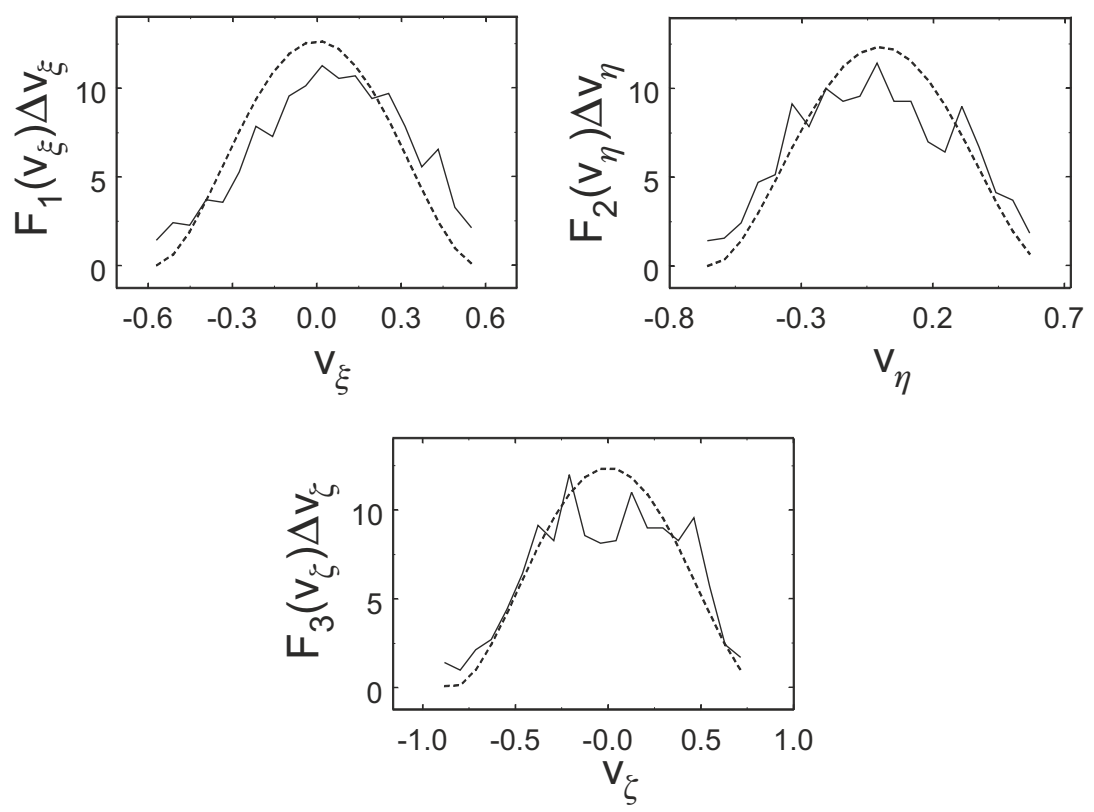

Fig. 4.2 The distributions of stars by the projections of the velocity vector for the cluster model 1 of Danilov and Dorogavtseva (2003). Solid lines correspond to the numerical experiment of Danilov and Dorogavtseva (2003); dashed lines show the curves for the ellipsoidal cluster model with $\kappa^{2}=0.53873$

experimental distributions. The obtained theoretical and numerical-experimental distributions of halo stars by the velocities of motion in the cluster model 1 of Danilov and Dorogavtseva (2003) are in good agreement with each other. However, a significant part of the halo stars $(\sim 2 / 3)$ from the considered sample has "energies" $\epsilon>\epsilon_{t}$. One of the reasons for this is the absence of a dense gravitating core in the considered model of the ellipsoidal cluster (the adding of a core at the center of this model will increase the $\epsilon_{t}$ and the work of the exit of a star from the cluster center to the cluster periphery). The second reason is the formation of a significant group of stars ( 70 stars) in the cluster model 1 of Danilov and Dorogavtseva (2003), located at the distances from one to two tidal radii $R_{t}$ of the cluster (ie, up to $\sim 20 \mathrm{pc}$ ) from its center (Danilov and Leskov, 2005). According to Danilov and Dorogavtseva (2003) and Danilov and Leskov (2005), these stars accompany the cluster during the all considered time interval $\left(1.5 \times 10^{8}\right.$ years $)$ and return to the cluster at distances from its center less than $R_{t}$ only briefly. These stars have the large "energy" $\epsilon$ and also contribute to the cluster potential averaged by the oscillation period of a regular field.

The results obtained here (a large number of the halo stars with "energies" $\epsilon>\epsilon_{t}$, and also $C_{v}>1$ ) indicate that for the cluster model 1 (Danilov and Dorogavtseva, 2003) the sizes of semi-axes $a, b, c$ and the mass of a homogeneous ellipsoid, the 
potential of which approximates the potential at the periphery of the cluster model 1 (Danilov, 2005), should be increased in comparison with those obtained by Danilov (2005) using the Jacobi integral.

The three-integral PDF is of great interest in the study of nonisolated homogeneous ellipsoidal OSC models. However, construction of a three-integral PDF requires the use of equations (4.12) and their solutions (4.13) in determining the range of admissible values of the integrals $J_{1}, J_{2}, J_{3}$ and the projections of the star velocity vector for each internal point of the cluster ellipsoid. Due to the cumbersome form of the obtained solutions (4.13), the construction of a three-integral PDF can only be performed numerically, which is beyond the scope of this study. Apparently, in order to construct a three-integral PDF, it is necessary to approximate the potential of the halo in the numerical dynamic models of OSC by the potential of a homogeneous ellipsoid, taking into account not only the corresponding Jacobi integral, but also the integral $J_{3}$, and also specifying the mean star motion with the velocity $\vec{v}_{c}=\left(\dot{\xi}_{c}, \dot{\eta}_{c}\right)$ at each point of the ellipsoid.

\subsection{Conclusions}

1. We have considered the model of a homogeneous gravitating ellipsoidal star cluster moving along a circular orbit around the Galactic center. For this model, we have written three independent isolating integrals of stellar motion. We have noted that these three integrals are in involution to each other. We have found the domain of the admissible values of the third integral $J_{3}$. Peculiarities of the cluster star motion have been considered taking into account the influence of three integrals of motion. On the periphery of the considered cluster model, the "retrograde" stellar motion predominates, and the distribution of stellar velocities is elongated along the direction of cluster motion. These conclusions are consistent with the results of numerical experiments on modeling OSC dynamics (Danilov, 1997a). The average velocities of "retrograde" stellar motion at the periphery of the homogeneous ellipsoidal cluster model reach $\sim 0.17-0.19$ (pc/Myr), which is approximately $30 \%$ of the escape velocity of the star from the center of the ellipsoidal cluster model to the zero-velocity surface corresponding to "energy" $\epsilon=\epsilon_{t}$.

2. We have made a comparison between the parameters of the distribution of the stellar motion velocity in a homogeneous ellipsoidal model and in two numerical dynamic models of OSC (Danilov and Dorogavtseva, 2003; Danilov, 1997a), consisting of $N$ gravitating points. Values $\sigma^{2}$ and $\kappa^{2}$ have been given, which ensure the proximity of the velocity distribution parameters in the model of a homogeneous gravitating ellipsoid and in two OSC models (Danilov and Dorogavtseva, 2003; Danilov, 1997a). In the range of values $T \in\left[0, T^{(1)}\right]$, we have presented the distribution of stellar trajectories by the value of the integral $T$ of the stellar motion in a two-integral homogeneous ellipsoidal cluster model; we have discussed the integral and differential distributions by $T$ of the phase space volume occupied by the stars from corresponding intervals of $T$ values. 
3. We have presented formulas for a phase density function that depends on two integrals of motion. The corresponding velocity distribution of the stars is spheroidal. Formulas for the mean squares of the residual velocities of stars in the two-integral model of an ellipsoidal cluster are presented and discussed. The average squares of residual stellar velocities in such model decrease with distance from the center of the cluster along $\zeta$ axis and in the plane $\zeta=0$ to zero at the cluster boundary.

4. In the case of the three-integral phase-density function, the local and general triaxial ellipsoidal distributions of stellar velocities in a homogeneous ellipsoidal cluster have been given. General distribution of stellar velocities agrees well with the distribution of halo stars' velocities, constructed from the results of calculations of corresponding numerical dynamic cluster model 1 of Danilov and Dorogavtseva (2003).

5. The distributions of stars by the velocities and the integral of motion $T$ considered in this chapter can be used for comparison with the corresponding distributions obtained in the numerical simulations of the OSC dynamics. The formulas considered above can also be used to analyze observational data on the radial velocities and the proper motions of stars in the vicinity of clusters; in constructing a PDF at the OSC periphery by the observational data; for estimating the masses of the observed OSCs by the data on the stellar velocities and coordinates; when specifying the initial conditions for constructing the numerical dynamic models of OSCs with given characteristics of the velocity distribution.

\section{References}

Chandrasekhar, S.: Principles of Stellar Dynamics. Univ. Chicago Press, Chicago, Ill (1942). LCCN: 43-5608 (BKS1)

Danilov, V.M.: Numerical experiments simulating the dynamics of open clusters in the Galactic field. Astron. Reports 41, 163-173 (1997a).

Danilov, V.M.: Equilibrium After Violent Relaxation in Numerical Dynamical Models of Open Clusters. Astron. Reports 46, 443-450 (2002a). doi: $10.1134 / 1.1486176$

Danilov, V.M.: The Motion of Halo Stars in Dynamical Numerical Models of Open Clusters. Astron. Reports 49, 3604-610 (2005). doi:10.1134/1.2010649

Danilov, V.M.: Dynamical models of stellar motions at the peripheries of open clusters. Astron. Reports 50, 346-357 (2006). doi:10.1134/S1063772906050027

Danilov, V.M., Dorogavtseva, L.V.: Estimates of Relaxation Times in Numerical Dynamical Models of Open Star Clusters. Astron. Reports 47, 483-491 (2003). doi:10.1134/1.1583775

Danilov, V.M., Leskov, E.V.: Properties of Stellar Trajectories in Numerical Dynamical Models of Open Star Clusters. Astron. Reports 49, 190-200 (2005). doi:10.1134/1.1882777 
Heggie, D.C., Ramamani, N.: Approximate self-consistent models for tidally truncated star clusters. Non. Not. R. Astron. Soc. 272, 317-322 (1995). doi:10.1093/mnras/272.2.317

Innanen, K.A.: The limiting radii of direct and retrograde satellite orbits, with applications to the solar system and to stellar systems. Astron. J. 84, 960-963 (1979). doi:10.1086/112499

Keenan, D.W., Innanen, K.A.: Numerical investigation of galactic tidal effects on spherical stellar systems. Astron. J. 80, 290-302 (1975). doi:10.1086/111744

King, Ivan R.: An Introduction to Classical Stellar Dynamics. URSS, Moscow (2002) (In Russian).

Lynden-Bell, D.: Stellar dynamics. Potentials with isolating integrals. Mon. Not. R. Astron. Soc. 124, 95-123 (1962). doi:10.1093/mnras/124.2.95

Nezhinskii, E.M.: Quasi-Integrals in the Restricted Circular Three-Body Problem. Soviet Astron. 34, 305-308 (1990).

Terlevich, E.: Evolution of n-body clusters. Mon. Not. R. Astron. Soc. 224, 193-225 (1987). doi:10.1093/mnras/224.1.193

Whittaker, E.T.: Treatise on the Analytical Dynamics of Particles and Rigid Bodies: With an Introduction to the Problem of Three Bodies. Cambridge University Press (1937). ISBN-13: 978-0521091329 


\title{
Chapter 5 \\ Investigation of the Stellar Trajectories in the Two-Point Model of Open Star Cluster
}

\begin{abstract}
In this chapter, we consider a two-point model of a non-isolated star cluster with a circular orbit in the Galactic plane. The equations of stellar motion are linearized in the neighborhood of a singular point on the critical zero-velocity surface (CZVS) and in the neighborhood of the point under CZVS on the trajectory with the "energy" of the star less than critical. We give the eigenvalues and eigenvectors of these equations. We note the instability of two singular points located on the CZVS and give a numerical solution for the separatrix line connecting these points with each other. For the trajectories located under the CZVS, the moduli of the eigenvalues of the linearized equations of motion increase with decreasing of the energy of the star and the maximum trajectory distance from the center of cluster masses. This leads to an increase in the numerical estimates of the maximum Lyapunov characteristic exponents (MLCE) of trajectories located closer to the mass center of the cluster. We consider the properties of stellar trajectories with the use of Poincare sections and MLCE. A number of periodic orbits is noted for different values of stellar "energy". The properties of trajectories in the neighborhood of these periodic orbits are discussed. Almost all stellar trajectories are stochastic; the degree of stochasticity increases with decreasing "energy" of the star. On Poincaré maps, regions with different degrees of stochasticity are marked.
\end{abstract}

\subsection{Introduction}

The study of stellar trajectories in numerical dynamic models of OSC was carried out in the paper of Danilov and Leskov (2005), in which the MLCE estimates $\Lambda$ of stellar trajectories were obtained and a Fourier analysis of these trajectories was carried out. Cluster cores are regions of increased stochasticity, and halos are the regions of much more ordered motions. The values of $\Lambda$ for the stellar trajectories in the cores are 200-250 times greater than in the halo. All the stellar trajectories considered by Danilov and Leskov (2005) are stochastic, and the corresponding $\Lambda$ values are positive. In the distributions of stellar trajectories by the periods of the 
most significant oscillations, we found the peaks with periods corresponding to (or close to) the periods commensurate with the oscillation period of a regular cluster field. Comparing the periods of these peaks with the periods obtained by Danilov (2005) as a result of applying the small parameter method to the study of stellar trajectories in such systems, we could highlight the main resonances in the motion of the halo stars in the OSC models. In the paper (Danilov, 2005), we write equations of stellar motion on the OSC periphery, containing the small parameter $\mu$. A general analytical solution of the star motion equations was obtained at $\mu=0$. As one of simplifications in the construction of initial approximation for the trajectories of halo stars (Danilov, 2005), the unperturbed potential of the OSC was proposed to be sought in the form of potential of a certain homogeneous ellipsoid (see (2) from Danilov (2005)). We note that a general solution of the stellar motion equations in the joint force field of the homogeneous ellipsoid and the Galaxy was also obtained much earlier (Bok, 1934; Mineur, 1939) (in this case, the solution of the equations of the stellar motion by Mineur (1939) and by Danilov (2005) was written in a more compact form than by Bok (1934)).

In the paper (Danilov, 2006) we considered the model of a homogeneous gravitating ellipsoidal star cluster moving along a circular orbit around the Galactic center. For this model, three independent isolating integrals of stellar motion were written. An analysis was made of the features of the cluster star motion, taking into account the influence of these three integrals of motion. At the periphery of the considered cluster model, the retrograde star motions prevail; stellar velocity distribution is elongated along the direction of cluster motion and perpendicular to the plane of the Galaxy (as in numerical dynamic models of OSC of Danilov and Dorogavtseva (2003)). We also note that Mineur (1939) obtained three adiabatic invariants $\gamma_{i}$ of the star motion in the joint force field of the homogeneous ellipsoid and the Galaxy by varying the constant parameters $u$ and $v$, which describe the system $(i=1,2,3$, see also formulas (28) of Mineur (1939)).

Invariants $\gamma_{1}$ and $\gamma_{2}$, corresponding to the stellar motion in the plane $(\xi, \eta)$ (Mineur, 1939), which coincides with the plane of the Galaxy, have much more complicated form in comparison with the integrals $J_{1}$ and $J_{3}$ of the stellar motion in this plane obtained in the paper (Danilov, 2006). Values $J_{1}$ and $J_{3}$ from the paper (Danilov, 2006) are related to the energy and angular momentum of the star in the plane $(\xi, \eta)$, respectively. The physical meaning of the invariants $\gamma_{1}$ and $\gamma_{2}$ remains unclear (their approximate constancy for a sufficiently slow change in the Hamiltonian of the system with time does not indicate a relationship between the values $\gamma_{1}$ and $\gamma_{2}$ with known physical characteristics of the motion). Apparently, values $\gamma_{1}$ and $\gamma_{2}$ can be represented as linear combinations of $J_{1}$ and $J_{3}$ from the paper (Danilov, 2006). The coefficients of these linear combinations are related to the system parameters, and are the constant values. The value $\gamma_{3}$ agrees with the value $J_{2}$ from (Danilov, 2006) with the accuracy of a multiplicative constant, and is therefore related to the energy of the stellar motion along the coordinate $\zeta$.

A two-point model of a non-isolated cluster considered by Danilov (2005) represents another extreme case of the mass density distribution at the OSC periphery in comparison with the mass density distribution in the model of a homogeneous 
ellipsoid. The study of the two-point cluster model in the linearized force field of the Galaxy has much in common with the study of equations for the circular bounded three-body problem. The equations of the body motion of the smallest mass in both problems have a similar form (the differences are only in the values of the constant factors for the corresponding terms of the equations). Therefore, it is of interest to discuss some of the results obtained recently in the study of the three-body problem.

Gidea and Masdemont (2007); Vela-Arevalo and Marsden (2004); Winter and Vieira Neto (2001); Paskowitz and Scheeres (2006), in the framework of the circular bounded planar and three-dimensional three-body problems, used the Poincaré sections in discussing a number of questions related to the motion of the body of an infinitesimal mass with the values of the Jacobi integral constant slightly above the critical value.

The Poincaré sections were used to analyze the manifolds of stable and unstable trajectories (Gidea and Masdemont, 2007) connected with the Lyapunov's orbits (periodic orbits near the libration point $L_{1}$ between the principal bodies), in the planar circular restricted three-body problem (CR3BP). The masses of two main (most massive) bodies were assumed by Gidea and Masdemont (2007) to be equal; Gidea and Masdemont (2007) consider the "energies" (the values of the Jacobi integral) slightly exceeding the critical value. The manifolds of stable and unstable trajectories are two-dimensional and degenerate into one-dimensional ones at the critical "energy". These two-dimensional manifolds are separatrices of the phase space. The branches of each of the manifolds of the motion of the body of an infinitesimally small mass in the plane $(x, y)$ form a tube of orbits (fig. 9, 14 from Gidea and Masdemont (2007)), asymptotically approaching to (or moving away) the corresponding Lyapunov orbit. They divide the phase space (PS) into two regions. The trajectories inside the tube correspond to the passage of orbits near the point $L_{1}$ from one main body to another. Trajectories outside the tube are reflected back to the region of their origin to make at least one complete revolution around the main body. The transverse (non-tangent) intersections of stable and unstable two-dimensional manifolds of trajectories on the Poincaré map make it possible to determine the homoclinic orbits for a body of an infinitesimal mass (see fig. 12, 13 from Gidea and Masdemont (2007), and determination of the homoclinic solution by Chandrasekhar (1942, p. 229)).

Vela-Arevalo and Marsden (2004) analyze the motions of the body with infinitesimal mass (comet) in the force field of the Sun - Jupiter system in the framework of a planar CR3BP applying the Poincaré section and the wavelet extractions of the instantaneous trajectory frequencies. The resonance ratios of the trajectory frequencies for the resonance islands on Poincaré maps are determined. The close neighborhood (mixture) of different frequencies in the "chaotic regions" of the PS indicates that the frequencies of the trajectories in these regions vary significantly and frequently with time $t$. Simultaneous application of two methods (Poincaré sections and wavelet analysis) when studying the trajectories in this problem allows: a) to trace the dynamics of chaotic trajectories (they can be temporarily captured near the resonant islands); b) to detect and investigate resonant transitions (i.e., the transitions of the trajectories from one PS region to another and, respectively, from one resonant is- 
land to another). Trajectories located always in the region of resonant islands have constant frequencies that satisfy the resonance relationship. Therefore, the change in the frequencies of the trajectories with time is used by Vela-Arevalo and Marsden (2004) as a criterion of the stochasticity of these trajectories.

Winter and Vieira Neto (2001), in the framework of a flat and a spatial CR3BP, applied the Poincaré sections in discussing the criterion and reasons for the departure of the spacecraft trajectory from the planet Uranus (in the force field of the Sun Uranus system). Poincaré sections in this problem allow them to localize in the phase space the position of periodic rtrograde orbits associated with the regions of stability of the spacecraft motion found earlier for estimating the dissipation time of spacecraft from the vicinity of the planet Uranus (see references in the paper of Winter and Vieira Neto (2001)). The stability of the plane retrograde trajectories of a spacecraft is caused by the existence of a family of periodic orbits and associated quasiperiodic trajectories that oscillate near one flat periodic orbit (the amplitude of these oscillations increases with the decreasing initial inclination $i$ of a quasiperiodic trajectory).

Paskowitz and Scheeres (2006) consider a dynamics of the temporarily trapped trajectories in the planar and spatial CR3BP with reference to the spacecraft orbits near Europe (in the system Jupiter - Europe). They discuss the methods for the spacecraft transition from a safe trajectory (not leading to a spacecraft impact on the surface of Europe) to a long-term, more stable trajectory. To analyze the spacecraft trajectories captured by the force field of Europe, they apply the pericentric Poincare section, obtained with the help of the following conditions at the pericenter of the trajectory: $\dot{r}=0, \ddot{r}>0$, where $r$ is the spacecraft distance from the mass center of Europe (see fig. 1 from the paper of Paskowitz and Scheeres (2006)), $\dot{r}=\frac{d r}{d t}$, $\ddot{r}$ is defined similarly. In this case, the Poincare map is obtained by mapping the pericentric passages of the spacecraft onto the plane $(x, y)$, which coincides with the plane of motions of the main bodies (Jupiter and Europe). This approach allows: a) to easily identify on the Poincaré map the safety zones for the spacecraft preventing its collisions with the surface of Europe; b) to investigate the spacecraft motion under the open zero-velocity surface (ZVS) for energies slightly exceeding the critical value (see fig. 2 from the paper of Paskowitz and Scheeres (2006)). Fig. 4 from the paper of Paskowitz and Scheeres (2006) shows the pericentric Poincaré maps for a series of values of the Jacobi integral. According to this figure, the trapped trajectories lead to dissipation from the vicinity of Europe after passing through the dissipation region at the first pericentric passage. Overlapping of the regions of capture and dissipation on the pericentric Poincare map yields the path (mechanism), following which the trajectory captured by Europe leads further to a dissipation.

The results obtained by Gidea and Masdemont (2007); Vela-Arevalo and Marsden (2004); Winter and Vieira Neto (2001); Paskowitz and Scheeres (2006) can be applied at a qualitative level in the discussion of: a) a dynamics of the stellar motion in the OSC in the neighborhood of singular points, as well as motion in the vicinity of some planar periodic orbits in the cluster, considered in Section 5.2; b) a dissipation of stars from the cluster; c) formation in the OSC vicinity of a stellar grouping with the "energies" greater than critical one, which accompany the cluster for a 
sufficiently long time interval (Danilov and Leskov, 2005; Danilov, 2005, 2006), and then gradually leave it to the long distances. Since Gidea and Masdemont (2007); Vela-Arevalo and Marsden (2004); Winter and Vieira Neto (2001); Paskowitz and Scheeres (2006) and we consider the different systems (the force field of the Galaxy in the vicinity of the cluster is not the point mass field; when describing the motions of the body of the smallest mass, Gidea and Masdemont (2007); Vela-Arevalo and Marsden (2004); Winter and Vieira Neto (2001); Paskowitz and Scheeres (2006) and we use the different approximations), it is of interest to analyze the stellar motions in the joint force field of the OSC and the Galaxy.

It is also of interest to apply the Poincare sections to study the properties of stellar trajectories in the two-point model of a nonisolated cluster at "energies" less than the critical value. The use of Poincaré sections in this problem allows us to present data on a very large number of trajectories in a concentrated form; to determine the parameters and localize the position of stable and unstable periodic orbits in the PS (with greater accuracy for the stable orbits); to estimate the sizes of the regions of regular and stochastic motion in this model (see, for example, the paper of Winter and Vieira Neto (2001)). When discussing the properties of stellar trajectories in a two-point OSC model, it is very useful to use estimates of MLCE for these trajectories.

The objective of this chapter is to study the properties of stellar trajectories in the two-point OSC model at the "energies" below the critical value. The simplicity of such model allows us to advance further in the study of the properties of stellar trajectories in the OSC.

\subsection{Two-Point Model of Non-Isolated Star Cluster}

Let us consider the cluster model in the form of a system of two point masses, one of which $\left(m_{2}\right)$ is approximately equal to the mass of the cluster, and the other one $\left(m_{1}\right)$ is equal to the mass of the Sun. In the presence of an external field of the Galaxy, such a model describes approximately the stellar motion at the cluster periphery with a dense massive core and an extended low-density halo with the small mass. Such a model has already been considered by Danilov (2005), in which the dimensions of the critical ZVS (CZVS) in the cluster along the coordinate axes were determined using an unisolated two-point cluster model. Danilov (2005) adopted the form of the CZVS of a homogeneous ellipsoidal OSC model as a surface of an ellipsoid with the semiaxes equal to the dimensions of the CZVS in a two-point cluster model.

Let the mass of the cluster be equal to $M=m_{1}+m_{2}$, where the star mass $m_{1}=m_{\odot}$;

$m_{2}=M-m_{1}$. Following to Danilov and Leskov (2005); Danilov (2005), we take $M=500 m_{\odot}$ and consider a cluster moving along a circular orbit of radius $R=R_{0}$ in the Galactic plane.

According to Chandrasekhar (1942), the equations of motion of the star with mass $m_{1}$ in the cluster force field and in the linearized force field of the Galaxy can be written in the following form: 


$$
\begin{aligned}
\ddot{\xi}_{1}-2 \omega \dot{\eta}_{1}+\alpha_{1} \xi_{1} & =-g\left(\xi_{1}-\xi_{2}\right), \\
\ddot{\eta}_{1}+2 \omega \dot{\xi}_{1} & =-g\left(\eta_{1}-\eta_{2}\right), \\
\ddot{\zeta}_{1}+\alpha_{3} \zeta_{1} & =-g\left(\zeta_{1}-\zeta_{2}\right),
\end{aligned}
$$

where $\dot{\xi}_{1}=\frac{d \xi_{1}}{d t}, \ddot{\xi}_{1}=\frac{d \dot{\xi}_{1}}{d t}$, values $\dot{\eta}_{1}, \dot{\zeta}_{1}, \ddot{\eta}_{1}, \ddot{\zeta}_{1}$ are defined similarly; $\omega$ is the constant angular velocity of the cluster motion around the center of the Galaxy; $g=G m_{2} / r_{1,2}^{3}$, $G$ is the gravitational constant; $r_{1,2}$ is the distance between two gravitating masses $m_{1}$ and $m_{2}$. According to Chandrasekhar (1942), the system of equations (5.1) is written in the coordinate system $(\xi, \eta, \zeta)$ rotating at angular velocity $\omega$; the origin of coordinates coincides with the mass center of the cluster, axis $\xi$ is directed from the center of the Galaxy, axis $\eta$ - in the direction of cluster motion in the Galactic plane, axis $\zeta$ is perpendicular to the Galactic plane.

$$
\alpha_{1}=\left(\frac{1}{R} \frac{\partial \Phi}{\partial R}-\frac{\partial^{2} \Phi}{\partial R^{2}}\right)_{0}<0, \quad \alpha_{3}=-\left(\frac{\partial^{2} \Phi}{\partial Z^{2}}\right)_{0}>0 .
$$

Here, $R$ and $Z$ are cylindrical Galactocentric coordinates of a point. Indices "0" indicate that derivatives of the Galaxy potential $\Phi$ are calculated at the point with coordinates $R=R_{0}=8200 \mathrm{pc}$ and $Z=0$.

Following toDanilov and Chernova (2008), we use the integrals of motion of the center of mass for the system of bodies $m_{1}$ and $m_{2}$ in order to unlock the equations of motion of these bodies. As a result, for a star with mass $m_{1}$ we find

$$
\begin{aligned}
\ddot{\xi}_{1}-2 \omega \dot{\eta}_{1}+\left(\alpha_{1}+\beta\right) \xi_{1} & =0, \\
\ddot{\eta}_{1}+2 \omega \dot{\xi}_{1}+\beta \eta_{1} & =0, \\
\ddot{\zeta}_{1}+\left(\alpha_{3}+\beta\right) \zeta_{1} & =0,
\end{aligned}
$$

where $\beta=\gamma / r_{1}^{3} ; \gamma=G m_{2} / \mu^{2} ; \mu=1+m_{1} / m_{2} ; r_{1,2}=\mu r_{1} ; r_{1}=\sqrt{\xi_{1}^{2}+\eta_{1}^{2}+\zeta_{1}^{2}}$ is a distance of a star with mass $m_{1}$ from the mass center of a cluster. The system (5.2) is a closed system of nonlinear differential equations. Further calculations in our paper relate to the motion of a star with a mass of $m_{1}$. Therefore, for the sake of brevity, we will omit the index " 1 " for all the values characterizing the motion and parameters of a star with the mass $m_{1}$.

From the conditions $\dot{\xi}=\dot{\eta}=\dot{\zeta}=\ddot{\xi}=\ddot{\eta}=\ddot{\zeta}=0$, we find three singular points (the rest, or equilibrium points) for the system (5.2): 1) $\xi= \pm\left|\xi_{t}\right|, \eta=\zeta=0$, where $\left.\left|\xi_{t}\right|=\left(-\gamma / \alpha_{1}\right)^{1 / 3}=R_{t} ; 2\right) \xi=\eta=\zeta=0$. The value $r=R_{t}$ closely agrees with the value of the tidal radius of the spherical cluster model in the field of the Galaxy (King, 2002, p. 198), since $\mu^{2} \simeq 1$. The point $\xi=\eta=\zeta=0$ coincides with the mass center of the cluster.

The Jacobi integral of the system (5.2) can be written in the following form:

$$
\frac{\dot{\xi}^{2}+\dot{\eta}^{2}+\dot{\zeta}^{2}+\alpha_{1} \xi^{2}+\alpha_{3} \zeta^{2}}{2}-\frac{\gamma}{r}=\varepsilon=\text { const }
$$

Substituting $\dot{\xi}=\dot{\eta}=\dot{\zeta}=0$ in (5.3), we obtain the ZVS equation for the star with "energy" $\varepsilon$ : 


$$
\frac{\alpha_{1} \xi^{2}+\alpha_{3} \zeta^{2}}{2}-\frac{\gamma}{r}=\varepsilon
$$

We substitute the coordinates of one of the singular points in (5.4): $\xi= \pm\left|\xi_{t}\right|$, $\eta=\zeta=0$. Taking into account that $\alpha_{1}=-\gamma /\left|\xi_{t}\right|^{3}$, we find the corresponding value the critical "energy" $\varepsilon: \varepsilon_{t}=-3 \gamma /\left(2\left|\xi_{t}\right|\right)$. Substituting $\varepsilon=\varepsilon_{t}$ in (5.4), we can obtain the CZVS equation for the given OSC model. Substituting $\xi=\zeta=0$ in the equation, we find $\varepsilon_{t}=-\gamma /\left|\eta_{t}\right|$, where $\left|\eta_{t}\right|$ is the distance from the origin to the CZVS along the axis $\eta$. Comparing it with $\left|\xi_{t}\right|$, we find $\left|\eta_{t}\right|=2\left|\xi_{t}\right| / 3$. The same relation between $\left|\eta_{t}\right|$ and $\left|\xi_{t}\right|$ is given by Spitzer (1987), when he discusses the dimensions of the cluster CZVS in the case when both the Galaxy and the cluster are modeled by the point masses. Let $\left|\zeta_{t}\right|$ be distance from the origin to the CZVS along the $\zeta$ axis in our cluster model. Substituting $\xi=\eta=0, \varepsilon=\varepsilon_{t}$ and $S=\left|\zeta_{t}\right| /\left|\xi_{t}\right|$ in (5.4), we obtain the equation $S^{3}+3 q S-2 q=0$, the real root of which, according to Cardano's formulas (Korn and Korn, 1961), can be written in the following form:

$$
\left|\zeta_{t}\right|=\left|\xi_{t}\right| q^{1 / 3}\left[(\sqrt{1+q}+1)^{1 / 3}-(\sqrt{1+q}-1)^{1 / 3}\right] \simeq 0.5016\left|\xi_{t}\right|
$$

where $q=-\alpha_{1} / \alpha_{3}$, and in order to determine the values $\left|\zeta_{t}\right|, \alpha_{1}$ and $\alpha_{3}$, we applied the Galaxy potential model of Kutuzov and Osipkov (1980).

The coordinates of three singular points considered above are particular solutions of the equations (5.2). It is important to perform stability analysis of these points (see, for example, Markeev (1978)) when studying stellar motion in the vicinity of these points.

In order to discuss the properties of stellar motion in the neighborhood of solutions of the system (5.2) (of periodic orbits, nonclosed trajectories, singular points), we linearize the system (5.2) in the neighborhood of some solution of this system. We denote $\dot{\xi}=u, \dot{\eta}=v, \dot{\zeta}=w$. Let $\delta \xi, \delta \eta, \delta \zeta, \delta u, \delta v, \delta w$ be variations of the functions $\xi(t), \eta(t), \zeta(t), u(t), v(t), w(t)$, which have small absolute values. Following to Nemytsky and Stepanov (1947, p. 184), we write the variational equations for the system (5.2):

$$
\begin{gathered}
\delta \dot{\xi}=\delta u, \delta \dot{\eta}=\delta v, \delta \dot{\zeta}=\delta w, \\
\delta \dot{u}=2 \omega \delta v-\left(\alpha_{1}+\beta\right) \delta \xi+\xi \delta s, \\
\delta \dot{v}=-2 \omega \delta u-\beta \delta \eta+\eta \delta s, \\
\delta \dot{w}=-\left(\alpha_{3}+\beta\right) \delta \zeta+\zeta \delta s,
\end{gathered}
$$

where $\delta s=3 \beta(\xi \delta \xi+\eta \delta \eta+\zeta \delta \zeta) / r^{2}$. Values $\beta, r, \xi, \eta, \zeta$ here are determined by the solution of system (5.2), in the vicinity of which the system (5.5) defines the variations of phase coordinates of stars.

Let us consider the equations (5.5) in the neighborhood of a singular point: $\xi=-\left|\xi_{t}\right|, \eta=\zeta=0$. In this case, the values of $\beta$ and $r$ are constant: $\beta=\gamma /\left|\xi_{t}\right|^{3}$, $r=\left|\xi_{t}\right| ; \delta s=-3 \beta \delta \xi /\left|\xi_{t}\right|$, and the equations (5.5) are simplified:

$$
\begin{gathered}
\delta \dot{\xi}=\delta u, \delta \dot{\eta}=\delta v, \delta \dot{\zeta}=\delta w, \\
\delta \dot{u}=2 \omega \delta v+3 \beta \delta \xi, \\
\delta \dot{v}=-2 \omega \delta u-\beta \delta \eta, \\
\delta \dot{w}=-\left(\alpha_{3}+\beta\right) \delta \zeta .
\end{gathered}
$$


The characteristic equation of system (5.6) has the form

$$
\left[\lambda^{4}+2 \lambda^{2}\left(2 \omega^{2}-\beta\right)-3 \beta^{2}\right]\left(\lambda^{2}+\alpha_{3}+\beta\right)=0 .
$$

The roots of the equation (5.7) can be written in the following form:

$$
\begin{gathered}
\lambda_{1,2}= \pm \sqrt{\nu_{1}}, \text { where } v_{1}=q_{1}+q_{2}>0, \\
\lambda_{3,4}= \pm i \sqrt{-\nu_{2}}, \text { where } v_{2}=q_{1}-q_{2}<0, \\
\lambda_{5,6}= \pm i \sqrt{\alpha_{3}+\beta},
\end{gathered}
$$

where $q_{1}=\beta-2 \omega^{2} ; q_{2}=2 \sqrt{\omega^{4}-\omega^{2} \beta+\beta^{2}}$. The estimates of these values and the signs of $v_{1}$ and $v_{2}$ are obtained for the parameters of the cluster and its orbit assumed here. Thus, the characteristic equation (5.7) has one pair of real roots $\left(\lambda_{1,2}\right)$ and two pairs of imaginary roots $\left(\lambda_{3,4}, \lambda_{5,6}\right)$. One of the real roots is positive. Therefore, according to Lyapunov's Theorem 1 (Markeev, 1978, p. 25), the singular point with coordinates $\xi=-\left|\xi_{t}\right|, \eta=\zeta=0$ is unstable. The same conclusion can easily be obtained for the singular point $\xi=\left|\xi_{t}\right|, \eta=\zeta=0$. The instability of these points (solutions) arises when the star moves in the plane $(\xi, \eta)$. The equations (5.5) and (5.6) describe the motion of a star with the "energy" close to $\varepsilon_{t}$ (including the case $\varepsilon>\varepsilon_{t}$; in this case, the periodic motions of the star around the singular points $\xi= \pm\left|\xi_{t}\right|, \eta=\zeta=0$ are possible).

Let us consider in more detail the motion of a star in the plane $(\xi, \eta)$ in the neighborhood of the point $\xi=-\left|\xi_{t}\right|, \eta=\zeta=0$. With such a motion, $\zeta=w=\delta \zeta=$ $\delta w=0$; the roots of the characteristic equation are $\lambda_{1,2}$ and $\lambda_{3,4}$, see (5.8), and the singular point itself is a "saddle-center" point (the same type also has the point $L_{1}$ in the planar CR3BP (Gidea and Masdemont, 2007), see also (Markeev, 1978, p.26)).

In this case, the general solution of the system (5.6) can be written in the following form:

$$
\begin{aligned}
& \delta \xi=C_{1} \exp \left(\lambda_{1} t\right)+C_{2} \exp \left(-\lambda_{1} t\right)+C_{3} \cos (n t)+C_{4} \sin (n t), \\
& \delta \eta=A\left(C_{1} \exp \left(\lambda_{1} t\right)-C_{2} \exp \left(-\lambda_{1} t\right)\right)-B\left(C_{3} \sin (n t)-C_{4} \cos (n t)\right),
\end{aligned}
$$

where $C_{i}$ are the integration constants $(i=1, \ldots, 4), n=\sqrt{-v_{2}}, A=\left(\lambda_{1}^{2}-\right.$ $3 \beta) /\left(2 \omega \lambda_{1}\right), B=\left(n^{2}+3 \beta\right) /(2 \omega n)$. Values $\delta u$ and $\delta v$ can be obtained by time $t$ differentiation of $\delta \xi$ and $\delta \eta$ from (5.9). Within the framework of our cluster model, we find $\lambda_{1}^{-1} \simeq 1.61775 \times 10^{7}$ years, $T=2 \pi / n \simeq 1.13422 \times 10^{8}$ years.

At $\varepsilon>\varepsilon_{t}, C_{1}=C_{2}=0$, and small amplitudes $C_{3}$ and $C_{4}$, the periodic orbits of the linearized system in the neighborhood of the singular point are elliptic, elongated along the axis $\eta$ (semiaxis of the orbit along the $\eta$ axis is $\sim 2.87117$ times larger than along the $\xi$ axis). Near the singular point, it is possible to construct the periodic Liapunov orbits for the initial (non-linearized) system (5.2). The method of constructing of such orbits is given by Gidea and Masdemont (2007). The frequencies of the Lyapunov orbits for small amplitudes $C_{3}$ and $C_{4}$ are close to $n$, see an explanation to formulas (3.5) from the paper of Gidea and Masdemont (2007). 


\subsection{Calculation of Separatrix}

According to (5.9), at $C_{1}>0, C_{2}=C_{3}=C_{4}=0$ and $\xi>-\left|\xi_{t}\right|$ in the vicinity of the singular point with coordinates $\xi=-\left|\xi_{t}\right|, \eta=\zeta=0$, we obtain an unstable solution. A separatrix "emerging" from a singular point corresponds to this solution. The solution (5.9) can be used to construct the trajectory segments close to the separatrix of the original system (5.2). At $t=0$, according to (5.9), we find

$$
\delta \xi=C_{1}, \quad \delta \eta=A C_{1}<0, \quad \delta u=C_{1} \lambda_{1}>0, \quad \delta v=A C_{1} \lambda_{1}<0 .
$$

Let $C_{1}=0.1 \mathrm{pc}$. Within the framework of our cluster model, $\left|\xi_{t}\right| \simeq 10.44467 \mathrm{pc}$, and $C_{1}<<\left|\xi_{t}\right|$. We write the initial conditions for the stellar trajectory given by the equations (5.2), in the form

$$
\begin{gathered}
\xi(0)=-\left|\xi_{t}\right|+C_{1}, \eta(0)=A C_{1}, u(0)=C_{1} \lambda_{1} \\
v(0)=A C_{1} \lambda_{1}, \zeta(0)=w(0)=0
\end{gathered}
$$

Fig. 5.1 shows the segments of two trajectories located between two singular points $\xi= \pm\left|\xi_{t}\right|, \eta=\zeta=0$, obtained by numerical integration of the equations (5.2) with initial conditions (5.10). Numerical integration was carried out applying the 4th order Runge-Kutta method until the third intersection of the trajectory with $\eta$ axis. A further path of the trajectory was obtained by applying the properties of a symmetry of the equations (5.2) and their solutions to the change of the signs of $t$ and the phase coordinates of the star (see, for example, formulas (2.6) and (2.7) from Gidea and Masdemont (2007)). The same way of constructing the homoclinic orbits was used in the paper of Gidea and Masdemont (2007). The direct numerical integration of the equations (5.2) to the second singular point $\xi=\left|\xi_{t}\right|, \eta=\zeta=0$ leads to formation of a "homoclinic tangle" (see the term and fig. 2.57 in Contopoulos (2002, p. 146)) near the second singular point due to the instability of the considered trajectory. However, a solution of the type (5.9) in the neighborhood of the second singular point can be obtained analytically, and then numerically, by specifying the initial conditions near this point and by reverse integration of the equations (5.2). As a result, we can obtain a separatrix "entering" to the second singular point.

The trajectory segments shown in fig. 5.1 are close to the corresponding separatrix segments. The larger the value $C_{1}$ in (5.10), the less accurate are the linearized equations (5.6). At $C_{1}=0.1 \mathrm{pc}$, according to (5.10) and (5.3), we find $\varepsilon=1.000000265 \varepsilon_{t}$; in this case, the maximum relative error in the "energy" $|\Delta \varepsilon / \varepsilon|$ is $\sim 0.74 \times 10^{-9}$ when calculating the trajectory. Therefore, the numerically obtained trajectories, strictly speaking, do not pass through singular points, but very close to them. The trajectories corresponding to (5.10), at $C_{1}=0.05 \mathrm{pc}$ and $C_{1}=0.1 \mathrm{pc}$ differ very little from each other (the greatest distance between them does not exceed $\sim 0.00028$ pc), and practically coincide on the plots similar to fig. 5.1 . At $C_{1}=0.05 \mathrm{pc}$, we find $\varepsilon=1.000000033 \varepsilon_{t}$; the maximum value $|\Delta \varepsilon / \varepsilon|$, when calculating the trajectory, is $\sim 0.79 \times 10^{-9}$. The closer the trajectory of a star approaches a singular point, 

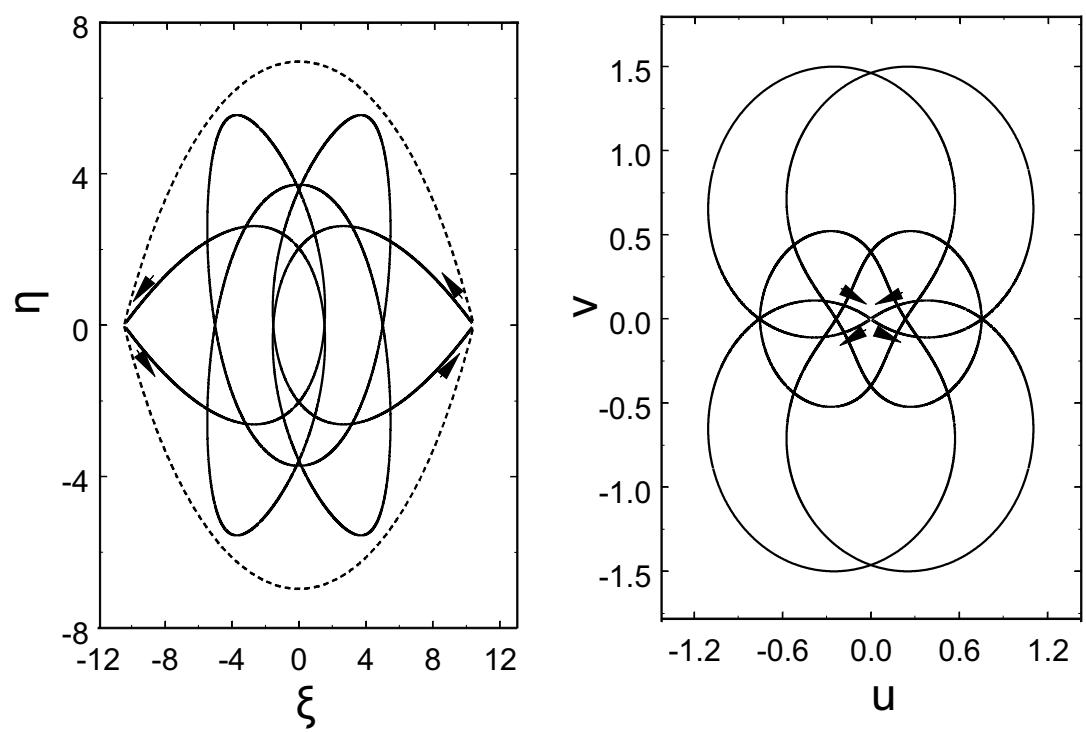

Fig. 5.1 Separatrix branches near two singular points of the cluster model. Dashed line shows zerovelocity line in the plane $(\xi, \eta)$. Arrows indicate the direction of motion along the trajectories

the greater amount of computation is required and, consequently, the accuracy of numerical integration decreases.

According to fig. 5.1, when moving along the separatrix from one singular point $\left(\xi=-\left|\xi_{t}\right|, \eta=\zeta=0\right)$ to another $\left(\xi=\left|\xi_{t}\right|, \eta=\zeta=0\right)$, the star in the cluster makes 2.5 turns around its center of mass. Moreover, it can pass fairly close $(r \sim 2 \mathrm{pc})$ to the center of mass of the cluster. In the real OSC, such distances from the center can be well achieved on the boundary of the cluster core. A star with an energy of $\varepsilon>\varepsilon_{t}$, dissipating from the cluster, moves near such a trajectory.

\subsection{Canonical Form of Equations in Variations}

Following to Nemytsky and Stepanov (1947, p. 94), as well as Gantmakher (1966) and Gidea and Masdemont (2007), we transform the system (5.6) to a simpler form. Let us consider the star motion in the plane $(\xi, \eta)$. In this case, $\delta \zeta=\delta w=0$. The system of equations (5.6) can be written in the following form:

$$
\dot{\mathbf{x}}=C \mathbf{x},
$$




$$
\text { where } \mathbf{x}=\left(\begin{array}{l}
\delta \xi \\
\delta \eta \\
\delta u \\
\delta v
\end{array}\right), \quad C=\left(\begin{array}{cccc}
0 & 0 & 1 & 0 \\
0 & 0 & 0 & 1 \\
3 \beta & 0 & 0 & 2 \omega \\
0 & -\beta & -2 \omega & 0
\end{array}\right) .
$$

According to Gantmakher (1966, p. 93), we write the adjoint matrix $D$ for the matrix $C$ in the following form:

$$
D=\left(\begin{array}{cccc}
\lambda\left(\lambda^{2}+4 \omega^{2}+\beta\right) & -2 \beta \omega & \lambda^{2}+\beta & 2 \lambda \omega \\
-6 \beta \omega & \lambda\left(\lambda^{2}+4 \omega^{2}-3 \beta\right) & -2 \lambda \omega & \lambda^{2}-3 \beta \\
3 \beta\left(\lambda^{2}+\beta\right) & -2 \beta \lambda \omega & \lambda\left(\lambda^{2}+\beta\right) & 2 \lambda^{2} \omega \\
-6 \beta \lambda \omega & -\beta\left(\lambda^{2}-3 \beta\right) & -2 \lambda^{2} \omega & \lambda\left(\lambda^{2}-3 \beta\right)
\end{array}\right) .
$$

Non-zero columns of the matrix $D$, corresponding to the value $\lambda=\lambda_{i}$, are eigenvectors of the matrix $C$ (Gantmakher, 1966). However, for an every eigenvalues $\lambda_{i}$ of the matrix $C$ all columns of the matrix $D$ turn out to be non-zero. Therefore, when writing $i$-th eigenvector of the matrix $C$, we used the $i$-th column of the matrix $D$, obtained for $\lambda=\lambda_{i}$. Let us compose the matrix $U$ of such columns:

$$
U=\left(\begin{array}{cccc}
2 \lambda_{1} \Omega_{1} & -2 \beta \omega & -2 \Omega_{2} & -2 \lambda_{3} \omega \\
-6 \beta \omega & -2 \lambda_{1} \Omega_{2} & -2 \lambda_{3} \omega & -2 \Omega_{1} \\
-6 \beta \Omega_{3} & 2 \beta \lambda_{1} \omega & -2 \lambda_{3} \Omega_{2} & -2 \omega \Omega_{4} \\
-6 \beta \lambda_{1} \omega & 2 \beta \Omega_{5} & 2 \omega \Omega_{4} & 2 \lambda_{3} \Omega_{1}
\end{array}\right),
$$

where $\Omega_{1}=\omega^{2}+\beta+f, \Omega_{2}=\omega^{2}-\beta+f, \Omega_{3}=\omega^{2}-\beta-f, \Omega_{4}=2 \omega^{2}-\beta+2 f$, $\Omega_{5}=\omega^{2}+\beta-f, f=q_{2} / 2, \lambda_{1}=\sqrt{\nu_{1}}, \lambda_{3}=i n$, see above.

The eigenvectors are determined with accuracy to a constant (non-zero) factor (Korn and Korn, 1961, p. 382). We multiply all the eigenvectors found by $k=1$ $(\mathrm{Myr})^{3}$. In this case, the dimensions of the corresponding coordinates of the vectors $\mathbf{x}$ and $\mathbf{x}^{\prime}$, connected with each other by relation $\mathbf{x}=U \mathbf{x}^{\prime}$, coincide, and the determinant of the matrix $U$ is equal to

$|U|=768 \beta^{2} \omega^{2}\left[\beta^{3}-2\left(\omega^{6}+f^{3}\right)+3 \beta \omega^{2}\left(\omega^{2}-\beta\right)\right] \simeq-0.21874 \times 10^{-13}(\mathrm{Myr})^{2} \neq 0$.

Therefore, the matrix $U^{-1}$, reverse to the matrix $U$, exists. Transformation $\mathbf{x}=U \mathbf{x}^{\prime}$ introduces four eigenvectors of the matrix $C$ as a coordinate basis. We compute $U^{-1}$ (multiplication of the columns of the matrix $U$ by $k$ leads to multiplication of the matrix $U^{-1}$ by $k^{-1}$ ). Substituting $\mathbf{x}=U \mathbf{x}^{\prime}$ to (5.11), multiplying the resulting equation by $U^{-1}$ from the left, we find $\dot{\mathbf{x}}^{\prime}=U^{-1} C U \mathbf{x}^{\prime}=L \mathbf{x}^{\prime}$, or $\dot{\mathbf{x}}^{\prime}=L^{\prime} \mathbf{x}^{\prime}$, where

$$
L=\left(\begin{array}{cccc}
\lambda_{1} & 0 & 0 & 0 \\
0 & -\lambda_{1} & 0 & 0 \\
0 & 0 & \text { in } & 0 \\
0 & 0 & 0 & -i n
\end{array}\right), \quad L^{\prime}=\left(\begin{array}{cccc}
\lambda_{1} & 0 & 0 & 0 \\
0 & -\lambda_{1} & 0 & 0 \\
0 & 0 & 0 & n \\
0 & 0 & -n & 0
\end{array}\right)
$$

The method of constructing the matrix $L^{\prime}$ in the presence of complex conjugate roots of the characteristic equation $|C-\lambda E|=0$, is described by Nemytsky and Stepanov (1947, p. 96). Here, $E$ is the identity matrix. In order to the matrix $L$ of 
the considered system of equations to be real, it is replaced by an equivalent matrix $L^{\prime}$, having the same eigenvalues as the matrix $L$.

Equations $\dot{\mathbf{x}}^{\prime}=L^{\prime} \mathbf{x}^{\prime}$ can be applied both for their integration over $t$, and, together with $\mathbf{x}=U \mathbf{x}^{\prime}$, when setting the initial conditions for the systems (5.2) and (5.6) in the case $\zeta=w=\delta \zeta=\delta w=0$. The solution of equations of the type $\dot{\mathbf{x}}^{\prime}=L^{\prime} \mathbf{x}^{\prime}$ is written and analyzed by Gidea and Masdemont (2007) for CR3BP.

Thus, in the phase space, there are four directions along which the matrix $C$ performs the "stretching" with different coefficients $\lambda_{j}, j=1, \ldots, 4$ (Gantmakher, 1966 , p. 85). One of them is the direction of unstable motion (corresponding to $\left.\lambda=\lambda_{1}>0\right)$, the second is the direction of stable motion $\left(\lambda=\lambda_{2}<0\right)$, as well as "two-dimensional direction" of the motion (Gidea and Masdemont, 2007) with respect to the center at the point $\xi=-\left|\xi_{t}\right|, \eta=0$. The coordinate transformation $\mathbf{x}=U \mathbf{x}^{\prime}\left(\right.$ or $\left.\mathbf{x}^{\prime}=U^{-1} \mathbf{x}\right)$ makes it possible to establish these directions. For the sake of brevity, the matrix $U^{-1}$ is not given here (due to the cumbersome form of its elements).

Let us consider equations (5.5) for the neighborhood of the point located under CZVS. Let this point be located on some trajectory with energy $\varepsilon \leq \varepsilon_{t}$. Let us consider the motion of a star in the plane $(\xi, \eta)$. In this case, $\zeta=w=\delta \zeta=\delta w=0$, and the matrix $F$ of the system (5.5) takes the form

$$
F=\left(\begin{array}{cccc}
0 & 0 & 1 & 0 \\
0 & 0 & 0 & 1 \\
a & b & 0 & 2 \omega \\
b & c & -2 \omega & 0
\end{array}\right)
$$

where

$$
\left\{\begin{array}{l}
a=-\alpha_{1}-\beta\left(1-3 \xi^{2} / r^{2}\right), \\
b=3 \beta \xi \eta / r^{2} \\
c=-\beta\left(1-3 \eta^{2} / r^{2}\right)
\end{array}\right.
$$

$\xi, \eta$ are coordinates of the considered point; $r^{2}=\xi^{2}+\eta^{2}$.

The roots of the characteristic equation $|F-\lambda E|=0$ are

$$
\lambda_{1,2}= \pm \sqrt{Q+K}, \lambda_{3,4}= \pm \sqrt{Q-K},
$$

where $Q=\left(a+c-4 \omega^{2}\right) / 2 ; K=\sqrt{Q^{2}-a c+b^{2}}$. Calculations similar to those performed in (5.12), lead to the matrix $V$ of transformation $\mathbf{x}=V \mathbf{x}^{\prime}$; the columns of this matrix are eigenvectors of the matrix $F$ :

$$
V=\left(\begin{array}{cccc}
\lambda_{1} a-K_{1} & K_{2} & K_{6} & K_{3} \\
K_{5} & -\lambda_{1} c+K_{1} & K_{3} & \lambda_{3}^{2}-a \\
\lambda_{1}^{2} a+K_{4} & -\lambda_{1} K_{2} & \lambda_{3} K_{6} & -\lambda_{3} K_{3} \\
\lambda_{1} K_{5} & \lambda_{1}^{2} c+K_{4} & \lambda_{3} K_{3} & \lambda_{3}\left(a-\lambda_{3}^{2}\right)
\end{array}\right),
$$

where $\quad K_{1}=\lambda_{1} \lambda_{3}^{2}-2 \omega b ; \quad K_{2}=-\lambda_{1} b+2 \omega c ; \quad K_{3}=-2 \lambda_{3} \omega+b ; \quad K_{4}=$ $b\left(2 \lambda_{1} \omega+b\right)-a c ; \quad K_{5}=\lambda_{1} b-2 \omega a ; \quad K_{6}=\lambda_{3}^{2}-c$. 
According to (5.14), for all points under the CZVS, we find: 1) the determinant of the matrix $V$ is not equal to zero (its modulus in this region increases with the decreasing $r$ in comparison with the modulus of the determinant of the matrix $U) ; 2)$ values $\lambda_{1,2}$ are real $\left(\lambda_{1}=-\lambda_{2}>0\right)$, and values $\lambda_{3,4}= \pm i p$ are imaginary $\left(\lambda_{3}=i p=-\lambda_{4}, p>0\right)$, as in the case (5.8). Therefore, the variational equations for the motion in the neighborhood of the considered point can be written in the form

$$
\dot{\mathbf{x}}^{\prime}=V^{-1} F V \mathbf{x}^{\prime}=L_{1} \mathbf{x}^{\prime},
$$

or $\dot{\mathbf{x}}^{\prime}=L_{1}^{\prime} \mathbf{x}^{\prime}$, where $L_{1}$ and $L_{1}^{\prime}$ are matrices of the same type as $L$ and $L^{\prime}$ in (5.13). $\lambda_{1}$ and $p$ increase with decreasing $r$ (due to an increase of $\beta$ in the formulas for $a$, $b, c$, see matrix $F$ ). In the considered range of $r$, the values of $\lambda_{1}$ and $p$ change in several times. For example, if the point under consideration is displaced along $\xi$ axis (at $\eta=0$ ) from $\xi=-\left|\xi_{t}\right| \simeq-10.44467 \mathrm{pc}$ to $\xi=-3 \mathrm{pc}, \lambda_{1}$ increases in $\sim 6.60708$ times, and $p$ increases in $\sim 5.24892$ times.

General solution of the system $\dot{\mathbf{x}}^{\prime}=V^{-1} F V \mathbf{x}^{\prime}$ can be written as a linear combination of four particular solutions (including into the fundamental system of solutions) in which the coefficients of the linear combination are constants. In the expression for $\mathbf{x}=V \mathbf{x}^{\prime}$, at sufficiently large $t$, the term $\sim \exp \left(\lambda_{1} t\right)$ begins to dominate, and, at numerical estimates of MLCE, gives the $\lambda_{1}$ value for the given trajectory (Loskutov and Mikhailov, 1990, p. 132-133). Thus, the MLCE values increase for the trajectories located at smaller distances from the mass center of the cluster. Numerical estimates of the MLCE for the trajectories considered by Danilov and Leskov (2005), as well as in Section 5.5 below, have the same character.

According to (5.15) and $\mathbf{x}=V \mathbf{x}^{\prime}$, four directions of motion can be defined for each point of the stellar trajectory with energy $\varepsilon \leq \varepsilon_{t}$; the characteristics of these directions are discussed above in the explanation to (5.13). The directions of stable and unstable motion with respect to the initial trajectory can be used in the construction of families of stable and unstable trajectories in a given model of a star cluster, as in the paper of Gidea and Masdemont (2007). We can use as the initial trajectory either the separatrix considered in Section 5.3 or any of the periodic trajectories discussed in Section 5.5 below.

\subsection{Construction of Poincaré Sections}

Following to Danilov and Chernova (2008), we determine the maximum distance $\xi_{\varepsilon}$ of a star from the origin along the axis $\xi$ at the star "energy" $\varepsilon=l \varepsilon_{t}$, where $l>1$. Let $\xi_{\varepsilon}>0, \eta=\zeta=0$. Using (5.4), denoting $S_{\varepsilon}=\xi_{\varepsilon} /\left|\xi_{t}\right|$, we obtain the equation for $S_{\varepsilon}$ :

$$
S_{\varepsilon}^{3}-3 l S_{\varepsilon}+2=0 .
$$

The smallest positive root $S_{\varepsilon} \in[0,1]$ of this equation, according to Korn and Korn (1961), equals to

$$
S_{\varepsilon}=\sqrt{l}(\cos (\varphi / 3)-\sqrt{3} \sin (\varphi / 3)),
$$


where $\varphi=\arctan \sqrt{l^{3}-1}$. At $l=1$, we find $\varphi=0, S_{\varepsilon}=1$, that is, $\xi_{\varepsilon}=\left|\xi_{t}\right|$.

Let $\zeta=w=0, \varepsilon / \varepsilon_{t}>1$. We consider two initial conditions for the system of equations (5.2):

$$
\begin{gathered}
\text { 1) } \eta(0)=v(0)=u(0)=0, \quad \xi(0)=-\xi_{\varepsilon} ; \\
\text { 2) } \eta(0)=u(0)=0, \xi(0)=-\xi_{\varepsilon}+C^{\prime}, C^{\prime} \in\left(0,\left|\xi_{t}\right|\right), v(0) \neq 0 .
\end{gathered}
$$

We find the value of $v(0)$ from the Jacobi integral (5.3):

$$
v(0)=-\sqrt{2(\varepsilon+\gamma /|\xi(0)|)-\alpha_{1} \xi^{2}(0)}<0 .
$$

Here, we use the sign " - " in front of the square root, and consider the trajectory for which the vector of the initial angular momentum of the star relative to the mass center of the cluster is parallel to the angular momentum vector of the Galaxy. In this case, the trajectory of the star passes far enough from the center of mass of the cluster, and the numerical integration of equations (5.2) is simplified.

Let the "energy" of the star motion $\varepsilon=\varepsilon(\xi, \eta, u, v)=$ const. We consider the cross-section of the stellar trajectories by $\eta$ axis. In this case, $\xi=0$. Let $u>0$ (the trajectory intersects the $\eta$ axis when the star moves from the region $\xi<0$ to the region $\xi>0$ ). The values of the phase coordinates $\eta$ and $v$ at $\xi=0$ set a point on the Poincaré map, see fig. 2, as well as Contopoulos (2002, p. 94).

In the case $\xi=u=0$, we find from the Jacobi integral (5.3)

$$
v_{1,2}= \pm \sqrt{2(\gamma /|\eta|+\varepsilon)}
$$

We note that $v_{1}=v_{2}=0$ at $\eta=\eta_{0}$, where $\left|\eta_{0}\right|=-\gamma / \varepsilon=2\left|\xi_{t}\right| /(3 l)<\left|\eta_{t}\right|$, since $l>1$, see notation for $\varepsilon_{t}$ and $\left|\eta_{t}\right|$ in explanations for the formula (5.3). The equations (5.16) correspond to two curves in the plane $(\eta, v)$, symmetric with respect to the axis $\eta$ and mutually symmetric with respect to the $v$ axis. At $\xi=0, u>0$, from Jacobi integral we find

$$
|v|=\sqrt{2(\gamma /|\eta|+\varepsilon)-u^{2}}<\left|v_{1,2}\right| .
$$

The values $v$ corresponding to the values $|v|$ on the curves (5.16) are reached for large values $|\eta|$. Thus, the envelopes $v_{1,2}=v_{1,2}(\eta)$ of the region of the possible values of $(\eta, v)$ for the given "energy" $\varepsilon$ (or "tangents", see Gidea and Masdemont (2007)) in the plane $(\eta, v)$ are located at greater distances from the axis $v$, than for any $u \neq 0$. The envelopes considered here intersect the $\eta$ axis at the points with coordinates $v=0, \eta= \pm\left|\eta_{0}\right|$. In the plane $(\xi, \eta)$ the points with coordinates $\xi=0, \eta= \pm\left|\eta_{0}\right|$ are located on the ZVS for a star with the "energy" $\varepsilon$.

If $\xi=0$ and $u>0$, then with the change of the sign of $\xi$ at a given step of integration $\Delta t$ we have $\xi(t) \xi(t+\Delta t)<0$ (this condition is used to detect intersection of the trajectory with $\eta$ axis). Let $t_{k}=t+s_{k} \Delta t$, where $t_{k}$ is the moment of the $\xi$ sign change. Let $\Delta t>0,0<s_{k}<1$, and $\xi\left(t_{k}\right)=0$. Assuming the dependency $\xi=\xi(t)$ near $t=t_{k}$ as linear, we find the value $s_{k}=-\xi(t) /[\xi(t+\Delta t)-\xi(t)]>0$. From the data on $\eta(t), v(t)$ and $\eta(t+\Delta t), v(t+\Delta t)$ we find $\eta\left(t_{k}\right)=\eta(t)+s_{k}[\eta(t+\Delta t)-\eta(t)]$, 
Table 5.1 Orbit parameters

\begin{tabular}{|c|c|c|c|c|}
\hline $\mathcal{N}$ & $\varepsilon / \varepsilon_{t}$ & $C^{\prime}, \mathrm{pc}$ & $T$, milliom years & $I$ \\
\hline 1 & 1.003 & 1.48275279 & 577.0999 & 9 \\
\hline 2 & 1.01 & 0.05662418 & 323.2832 & 5 \\
\hline 3 & 1.02 & 0.04520202 & 292.3900 & 5 \\
\hline 4 & 1.05 & 0.50570390 & 306.6827 & 6 \\
\hline 5 & 1.07 & 0.38881780 & 282.3036 & 6 \\
\hline 6 & 1.0927 & 0 & 233.0558 & 6 \\
\hline 7 & 1.5199885 & 0 & 221.1408 & 13 \\
\hline
\end{tabular}

$v\left(t_{k}\right)=v(t)+s_{k}[v(t+\Delta t)-v(t)]$. In this case, the functions $\eta(t), v(t)$ near $t=t_{k}$ are also considered as linear. The values $\eta\left(t_{k}\right), v\left(t_{k}\right)$ for the constant "energy" $\varepsilon$ were used later in constructing the Poincaré map in the plane $(\eta, v)$. We considered seven $\varepsilon$ values from the interval $\varepsilon / \varepsilon_{t} \in[1.003,1.52)$. For each of these values of $\varepsilon$, a periodic orbit (or trajectory very close to periodic) has been found. Data on these orbits are given in Table 5.1, where the values $\mathcal{N}, T, I$ denote the serial number, the period and the number of the orbit turns (the revolutions relatively to the mass center of the cluster during the time $T$ ), respectively. The seventh orbit was discovered and investigated by T.S. Chachina. The parameters of this orbit were further refined by V.M. Danilov.

Danilov and Chernova (2008) established instability of orbits 5-7 from the Table 5.1. In order to analyze the stability of motions in the vicinity of a periodic orbit, we used the system of equations (5.5) in the case $\zeta=w=\delta \zeta=\delta w=0$, as well as multipliers of the monodromy matrix (Yakubovich and Starzhinsky (1972, p. 82) and Demidovich (1967, p. 183)) for this case of the system (5.5). Numerical integration of the equations (5.2) and (5.5) was carried out by Danilov and Chernova (2008) with the use of the 4 th order Runge-Kutta method. The average relative error in the "energy" $|\Delta \varepsilon / \varepsilon|$ in calculating of the periodic orbits is $\sim 10^{-13}$ (the maximum value is $\sim 10^{-11}$, the minimum is $\sim 10^{-15}$ ). In order to check the accuracy of the integrating of the system of equations in variations, we used the integral of this system, obtained according to Nemytsky and Stepanov (1947, p. 185), in the following form:

$$
\left.u \delta u+v \delta v\left(\alpha_{1}+\gamma / r^{3}\right)\right) \xi \delta \xi+\left(\gamma / r^{3}\right) \eta \delta \eta=h=\text { const } .
$$

In the joint integration of the equations (5.2) and (5.5), the value $h$ is usually preserved with relative accuracy by $2-3$ orders of magnitude lower than $\varepsilon$ at identical intervals of integration time.

When calculating the Poincaré maps, Danilov and Chernova (2008) for each value of $\varepsilon_{i} / \varepsilon_{t}$ performed the calculation of the trajectories with initial condition 2 during the time interval $10 T_{i}$, where $T_{i}$ are the periods indicated in Table 5.1 for the energy $\varepsilon_{i}$ at $i=1, \ldots, 7$. The mean relative error in the "energy" for the considered choices of the trajectories is $|\Delta \varepsilon / \varepsilon| \sim 10^{-10}$ (maximum $\sim 10^{-7}$, minimal $\sim 10^{-13}$ ). When 
the value $C^{\prime} \in\left(0, \xi_{\varepsilon}\right)$ changes with the step $\Delta C^{\prime}=0.001 \xi_{\varepsilon}>0$, the region corresponding to the initial condition 2 is selected on the Poincare map in the plane $(\eta, v)$, see fig. 5.2. We obtained the estimates of the MLCE $\Lambda$ for all trajectories. In order to estimate $\Lambda$, we applied the procedure described by Lichtenberg and Lieberman (1983), see formula (5.3.10) there. We used the small values, which were obtained as the difference of the initial phase coordinates of two trajectories satisfying the initial condition 2 with close $C^{\prime}$ values differing by $\delta C^{\prime}=0.0001 \Delta C^{\prime}$, as initial values of $\delta \xi, \delta \eta, \delta u, \delta v$ in the equations (5.5).

In order to find the periodic orbits at $C^{\prime} \neq 0$ and the "energy" $\varepsilon=\varepsilon_{i}$ Danilov and Chernova (2008) calculated the distance $d$ between two points on Poincaré map, which were obtained for each value of $C^{\prime}$ on the first turn of the trajectory during the time intervals $t \in\left[0, T_{i}\right]$ and $t \in\left(5 T_{i}, 6 T_{i}\right]$, see Table 5.1. If the trajectory is close to periodic, $d$ is small, what leads to appearance of a local minimum on the dependency $d=d\left(C^{\prime}\right)$. The small changes of $C^{\prime}$ within the interval $\left[C^{\prime}-\Delta C^{\prime}, C^{\prime}+\Delta C^{\prime}\right]$ allow us to determine the exact values of $C^{\prime}$ and period $T$ for the detected closed orbit.

\subsection{Discussion of The Properties of Stellar Trajectories}

For all trajectories with initial conditions 2 at the "energies" $\varepsilon$ indicated in Table 5.1, $\Lambda$ values found by Danilov and Chernova (2008) are positive. Consequently, all these trajectories are stochastic. All trajectories for each $\varepsilon$ were divided into groups according to $\Lambda$. The equal intervals $\Delta \Lambda=\left(\Lambda_{\max }-\Lambda_{\min }\right) / n_{g}$ were considered, which contained each of the groups of trajectories, where $\Lambda_{\max }$ is the maximum value, and $\Lambda_{\min }$ is the minimum value of $\Lambda$ for a given "energy" $\varepsilon$, and $n_{g}=3-5$ is the number of trajectory groups.

Fig. 5.2 shows the fragments of the Poincare map from the paper of Danilov and Chernova (2008) for the case $\varepsilon=1.02 \varepsilon_{t}, n_{g}=3$. The dashed line in fig. 5.2a and $5.2 \mathrm{~b}$ indicates the envelope of the region of admissible values $(\eta, v)$, obtained according to (5.16). In the fig 5.2 (and in fig. 5.3), darker points correspond to the trajectories with larger $\Lambda$. To increase the contrast of the image, the points in fig. $5.2 \mathrm{c}$ and fig. $5.2 \mathrm{~d}$ are shown darker than the points for the same trajectories in fig. 5.2a and fig. 5.2b. According to fig. 5.2a, trajectories with large values of $\Lambda$ are located near the axis $v$ (at $\eta \simeq 0$ ), as well as at some distance from the envelope line, indicating that the motions are stochastic in these regions. In the plane $(\xi, \eta)$ trajectories with the largest $\Lambda$ are located near the point $\eta=0, \xi=0$ (here, an important role is played by the sufficiently close encounters of bodies with masses $m_{1}$ and $m_{2}$, which leads to an increase in the role of the nonlinearity of the equations of motion and to an increased stochasticity of the trajectories). A small number of points for such trajectories are scattered over the accessible region $(\eta, v)$. The size of the PS region available for such trajectories may be large enough, but the area occupied by such trajectories on the Poincaré map is small. Trajectories with small values of $\Lambda$ are located near the envelope line in the region $(\eta, v)$. At $\eta \simeq \eta_{0}$ in this region, the star moves slowly and is at the system periphery most of the time. Therefore, near the 
point $(\eta, v)=\left(\eta_{0}, 0\right)$, the close trajectories diverge slowly, which leads to small $\Lambda$ for these trajectories. Apparently, motions along $\eta$ axis at $\xi=0$ near the envelope line (5.16), have a "more regular" character at large $v$. Trajectories with mean $\Lambda$ values occupy symmetric regions with respect to the line $v=0$.

(a)

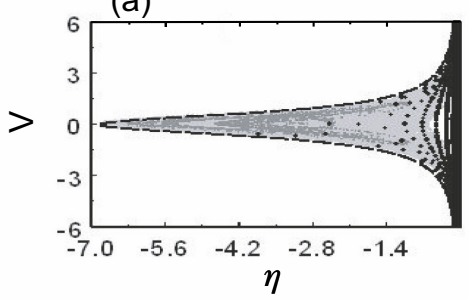

(c)

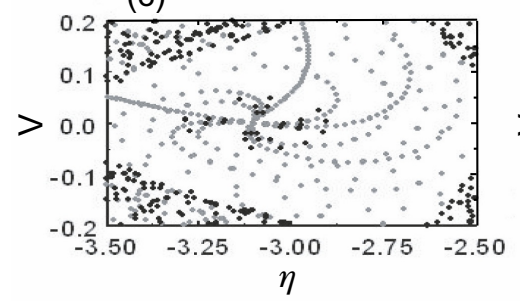

(b)

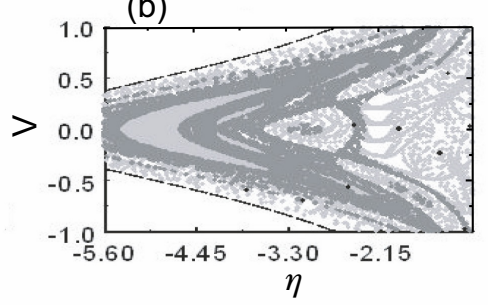

(d)

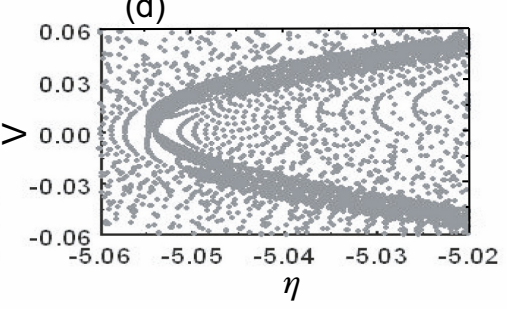

Fig. 5.2 Fragments (a)-(d) of Poincaré map at the "energy" of a star $\varepsilon=1.02 \varepsilon_{t}$

Fig. 5.2b shows in higher resolution (in comparison with Fig. 5.2a) the region $(\eta, v)$ at the system periphery, where the difference in the location of trajectories with large and small $\Lambda$ is more noticeable. The region with the average values of $\Lambda$ is clearly visible. In fig. 5.2b, the areas of avoidance of trajectories in the region accessible to the stellar motion in the plane $(\eta, v)$, where there are no points at all, are clearly visible. This is due to the use of not very large time intervals of integration $\Delta t=10 T_{i}$, see Table 5.1. If we integrate the equations of motion further along $t$ (at $\left.t>10 T_{i}\right)$, then all regions that are not occupied by points on the plane $(\eta, v)$ between the curve (5.16) and axis $v(\eta=0)$ will be filled. Trajectories with small values of $\Lambda$ are located in almost all regions of PS accessible to motion, although there are regions of avoidance for such trajectories in the plane $(\eta, v)$ (see above). The regions $(\eta, v)$ with different stochasticity are closely intermixed in the PS (on the Poincaré map and in the PS, there is a mutual penetration of regions with different degrees of stochasticity).

With increasing spatial resolution, a large number of structural elements are observed in the $(\eta, v)$ plane: star-shaped structures of the first type, formed by the intersection at one point of several lines set by chains of points with different $C^{\prime}$ values (fig. 5.2c), as well as structures of the second type - the ribbon-like regions with a width of the "ribbon", decreasing to zero at a certain point (fig. 5.2d), etc. 
Table 5.2 Values $\eta, v$ and $C^{\prime}$ for four points in fig. 5.2c

\begin{tabular}{|c|c|c|c|}
\hline $\mathcal{N}$ & $v, \mathrm{pc} /$ million years & $\eta, \mathrm{pc}$ & $C^{\prime}, \mathrm{pc}$ \\
\hline 1 & 0 & -3.1025 & 0.045202021 \\
\hline 2 & 0.015 & -3.075 & 0.063282830 \\
\hline 3 & 0.022 & -3.0625 & 0.0723232343 \\
\hline 4 & 0.03 & -3.05 & 0.081363639 \\
\hline
\end{tabular}

Table 5.2 gives approximate coordinates of four points in fig. 5.2c, taken along one of the "rays" of the starlike structure, starting from its "center" (point $\mathcal{N}=1$ ). Columns 1, 2, 3, 4 of the Table 5.2 give the numbers of points $\mathcal{N}$, values of $\eta$, $v$, and corresponding value of $C^{\prime}$. Small changes of $C^{\prime}$ near the value $C^{\prime}$ corresponding to a point with $\mathcal{N}=1$ in Table 5.2, allow us to detect a five-turn closed orbit with a period of $T=292.2434746 \mathrm{Myr}$ and $C^{\prime}=0.045615881 \mathrm{pc}$, one of the points of which on the plane $(\eta, v)$ is located near the "center" of considered structure of the 1 st type. Analysis of the monodromy matrix multipliers for this orbit shows that it is stable. On the plane $(\eta, v)$, this orbit is represented by five points (for six deviations in distance $r$ from the center of the system, the star in the plane $(\xi, \eta)$ passes the interval $\Delta \varphi=10 \pi$ through azimuth angle $\varphi$ ). If we plot these five points in fig. $5.2 \mathrm{~b}$, these points will be surrounded by the invariant "curves" which are ellipses of different degrees of elongation corresponding to the $C^{\prime}$ value given in Table 5.2. The values MLCE for the periodic orbits found by Danilov and Chernova (2008) were obtained from the data on the periods of these orbits and on the multipliers of the monodromy matrices corresponding to these orbits (see formula (2.22) from Yakubovich and Starzhinsky (1972, p.87) and Demidovich (1967, p.189))).

In the middle of the horseshoe-like region with small values of $\Lambda$ in fig. $5.2 \mathrm{~b}$ there is a point with coordinates $\eta \simeq-5.05384 \mathrm{pc}, v=0$, which corresponds to a one-turn unstable elliptical orbit with the period $T=53.85359851 \mathrm{Myr}$ and $C^{\prime}=4.604446465 \mathrm{pc}$, with large semiaxes $\sim 4.6 \mathrm{pc}$ (in $\xi$-coordinate) and $\sim 5.1 \mathrm{pc}$ (in $\eta$-coordinate). The value of MLCE for this trajectory equals $\Lambda=0.001875267$ $(\mathrm{Myr})^{-1}$, which corresponds to Lyapunov time $t_{\Lambda} \simeq 5.3 \times 10^{8}$ years. The value of $t_{\Lambda}$ for this orbit is $\sim 2.7$ times greater than an average OSC lifetime $\tau \simeq 2 \times 10^{8}$ years (Wielen, 1971). Then, this orbit can be considered as close to stable or practically stable at time intervals comparable to $\tau$. In fig. 5.2d, a point corresponds to this orbit at which the extended ribbon-like region (with small $\Lambda$ ) contracts to zero width; the same structure (of the second type) is also observed on the Poincare map for $\varepsilon=1.01 \varepsilon_{t}$. The points of the invariant "lines", corresponding to the same $C^{\prime}$, with an increasing time $t$, are moved away from the point $(\eta, v)=(-5.05384 \mathrm{pc}, 0)$, which is formed by the single-turn periodic orbit considered above.

Thus, structures of the 1st and 2nd types, which are most clearly seen in fig. 5.2, are associated with stable and practically stable periodic orbits.

On the Poincaré maps with $\varepsilon / \varepsilon_{t}=1.01,1.05,1.07$ the same structural elements are observed as in the case of "energy" $\varepsilon / \varepsilon_{t}=1.02$. On the Poincaré maps with 
$\varepsilon / \varepsilon_{t}=1.0927,1.5199885$ at a sufficiently large resolution, structures of the first type are also observed, which, in these cases, differ to some extent from those considered at $\varepsilon / \varepsilon_{t}=1.02$. The difference is that the "rays" of these structures are located within small vertical angles (with a vertex at the center of the "asterisk" - at the intersection of all lines, ("rays") of the structure), these angles are less than in the case shown in fig. 5.2c. At $\varepsilon / \varepsilon_{t}=1.0927,1.5199885$, points of invariant curves with a fixed value $C^{\prime}$, just like on the map with $\varepsilon / \varepsilon_{t}=1.02$, traverse the vertex of an "asterisk" along arcs that, with increasing $t$, can form ellipse-shaped curves surrounding the vertex of the "asterisk".
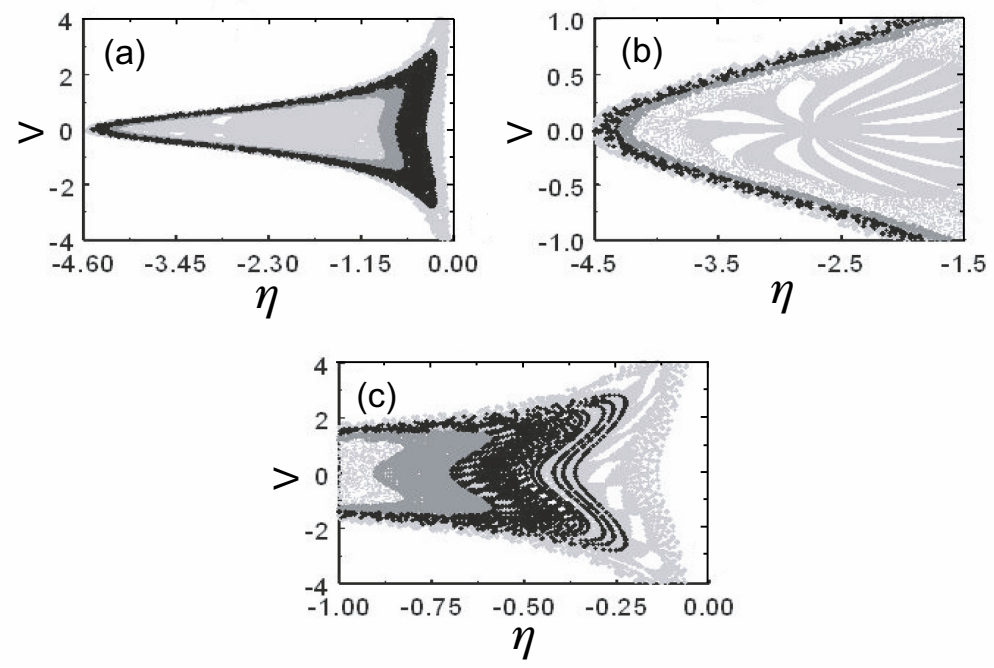

Fig. 5.3 Fragments (a, b, c) of Poincaré map at the "energy" of star $\varepsilon=1.5199885 \varepsilon_{t}$

Fig. 5.3 shows the fragments of the Poincare map from the paper of Danilov and Chernova (2008) with the "energy" $\varepsilon / \varepsilon_{t}=1.5199885$. Here, as in fig. 5.2, the boundaries between regions occupied by trajectories with different values of $\Lambda$ at $n_{g}=5$ are clearly visible. The size of the points for the second group of trajectories (with $\Lambda \in\left(\Lambda_{\min }+\Delta \Lambda, \Lambda_{\min }+2 \Delta \Lambda\right]$ ) has been reduced in comparison with the size of points for the trajectories of the remaining groups. In fig. 5.3, identical points of the darkest shade correspond to the 4th and 5th groups of trajectories with the largest values of $\Lambda$. Points with small values of $\Lambda$ are present practically in all structural elements of fig. 5.3, which, as in the case of fig. 5.2, indicates the mutual penetration of regions with different degrees of stochasticity in the PS. The points with the maximum $\Lambda$ are in the "annular triangular" region, symmetric with respect to the line $v=0$, see fig. 5.3a. Inside this "annular" region, one can see an area of smaller values of $\Lambda$, near the center of which, there is the largest (for this map) 
Table 5.3 Values $C^{\prime}$ at $d\left(C^{\prime}\right) \simeq 0$

\begin{tabular}{|c|c|c|c|c|}
\hline$\varepsilon / \varepsilon_{t}$ & \multicolumn{4}{|c|}{$C^{\prime}, \mathrm{pc}$} \\
\hline \multirow{3}{*}{1.02} & 0.04520202 & 0.1808081 & 1.419343 & 2.395707 \\
\cline { 2 - 5 } & 3.435354 & 4.212828 & 4.601566 & 4.990303 \\
\cline { 2 - 5 } & 5.668333 & 5.695455 & 6.527172 & 6.563334 \\
\cline { 2 - 5 } & 7.223283 & 7.81995 & & \\
\hline 1.5199885 & 0.009609614 & 2.147749 & 4.540543 & 4.646249 \\
\cline { 2 - 5 } & 4.713516 & & & \\
\hline
\end{tabular}

structure of the 1 st type, see fig. 5.3b. We note that this map shows more structures of the 1 st type than the map at $\varepsilon / \varepsilon_{t}=1.0927$, see fig. 5.3c. One of them, with a vertex near the point $\eta=-2.695 \mathrm{pc}, v=0$, see fig.5.3b, corresponds to a one-turn periodic nearly circular orbit in the plane $(\xi, \eta)$ with radius $r_{*} \simeq 2.7 \mathrm{pc}$. The values of $C^{\prime}=2.149548799 \mathrm{pc}$ and period $T=19.97273 \mathrm{Myr}$ correspond to this orbit. The value MLCE for this orbit is $\Lambda \simeq 0.39272 \times 10^{-4}(\mathrm{Myr})^{-1}$, it corresponds to the Lyapunov time $t_{\Lambda} \simeq 2.5 \times 10^{10}$ years, which exceeds the age of our Galaxy. Therefore, this orbit can be considered as practically stable. Another 13 structures of the 1st type have centers on the plane $(\eta, v)$, corresponding to the points of the 13 -wobble unstable periodic orbit. It corresponds to the value $C^{\prime}=4.7135821 \mathrm{pc}$, period $T=222.637 \mathrm{Myr}, \Lambda \simeq 0.04(\mathrm{Myr})^{-1}, t_{\Lambda} \simeq 25$ million years. The centers of the eight structures of the first type associated with a given orbit are easily seen in fig. $5.3 \mathrm{c}$ in the range of values $\eta \in[-0.35 \mathrm{pc},-0.15 \mathrm{pc}]$.

In comparison with fig. 5.2, at "energy" $\varepsilon=1.003 \varepsilon_{t}$, the whole region accessible to motion is more evenly and chaotically filled in the plane $(\eta, v)$. The smaller the "energy" $\varepsilon<0$, the more structures of the first types appear on the Poincaré maps, and the average stochasticity of the trajectories increases (the mean $\Lambda$ at $\varepsilon=1.003 \varepsilon_{t}$ is $\bar{\Lambda} \simeq(0.1775 \pm 0.1366)(\mathrm{Myr})^{-1}$, and at $\varepsilon=1.5199885 \varepsilon_{t}$, we find $\left.\bar{\Lambda} \simeq(0.3346 \pm 0.1053)(\mathrm{Myr})^{-1}\right)$. With a decreasing of $\varepsilon$, the maximum possible number of turns in periodic orbits in the plane $(\xi, \eta)$ increases; the radial elongation of these turns and the degree of instability of multi-turn periodic orbits increase. The dependency of $\bar{\Lambda}$ on $\varepsilon$ in the interval $\varepsilon / \varepsilon_{t} \in[1.003,1.5199885]$ is consistent with the data on the increase of the moduli of eigenvalues of the matrix $F$ with decreasing $r$, obtained in Section 5.4.

Applying the functions $d=d\left(C^{\prime}\right)$, see above, in the cases $\varepsilon=1.02 \varepsilon_{t}$ and $\varepsilon=1.5199885 \varepsilon_{t}$, we have found 14 and 5 periodic orbits (or trajectories very close to periodic), see Table 5.3. For these orbits, $d\left(C^{\prime}\right) \simeq 0$. Varying $C^{\prime}$ in the neighborhood of each value $C^{\prime}$, we can refine $C^{\prime}$ and determine the periods $T$ of the found orbit.

In this part of the work, we have considered only "prograde" trajectories, satisfying the initial conditions 1 and 2 in Section 5.5. The consideration of "retrograde" trajectories leads to less stochastic motions and the formation of a large number of stable periodic orbits. Also, it leads to closer and more frequent encounters of bodies 
with masses $m_{1}$ and $m_{2}$, which significantly complicates calculation of trajectories. In this case, the two-point cluster model describes to a lesser extent the situation in OSC (due to the absence of the point cores in the real OSC). Therefore, "retrograde" trajectories of stellar motion in this model were not considered. At the same time, this cluster model provides an increase in the stochasticity of motions with the decreasing of "energy" $\varepsilon$ and the largest distance of the stellar trajectory from the center of mass, which agrees with the conclusion about increased stochasticity of the cluster cores in the OSC models (Danilov and Leskov, 2005).

\subsection{Conclusions}

1. We have provided the formulas for the coordinates of three singular points of the system of equations of stellar motion in a two-point model of the OSC with a circular orbit in the plane of the Galaxy. We have found eigenvalues and eigenvectors of the equations in variations written both for the neighborhood of the singular point $\xi=-\left|\xi_{t}\right|, \eta=\zeta=0$, and for neighborhood of the point located under CZVS on a trajectory with "energy" $\varepsilon \leq \varepsilon_{t}$.

2. The singular points with coordinates $\xi= \pm\left|\xi_{t}\right|, \eta=\zeta=0$ are points of the "saddle-center" type; motion in the neighborhood of these points is unstable (as in the corresponding case of CR3BP). We have written general solution of the system of equations in variations in the neighborhood of singular point $\xi=-\left|\xi_{t}\right|, \eta=\zeta=0$. Using this solution, a separatrix is found numerically, which connecting the singular points $\xi= \pm\left|\xi_{t}\right|, \eta=\zeta=0$.

3. At $\varepsilon \leq \varepsilon_{t}$, for the trajectories under CZVS, the moduli of the eigenvalues of the system of equations in variations increase with decreasing the maximum distance of the trajectory from the mass center of the cluster. It leads to an increase in the numerical estimates of the MLCE of the trajectories with a decreasing $\varepsilon$ in Section 5.6.

4. In the coordinate system associated with the eigenvectors of the considered systems of the variational equations, the canonical variational equations are written that can be used both for their numerical integration and for a setting of the initial conditions for the constructing of the stable (and unstable) trajectories relatively to the original trajectory in the PS.

5. On the Poincaré maps, a large number of structural elements of two types are observed: structures of the first type, formed by the intersection at one point of several lines given by chains of points with different values of $C^{\prime}$, and also structures of the second type - the ribbon-like regions, with the "ribbon" width decreasing to zero at a certain point.

6 . At $C^{\prime} \neq 0$, all the found values of the MLCE of the trajectories are positive, and the trajectories themselves are stochastic. Zones with different stochasticity are very closely intermixed in the phase space. The smaller $\varepsilon$, the more structures of the first type appear on the Poincaré maps, and a stochasticity of the trajectories increases. 
7. A number of periodic orbits have been found in the considered model of the OSC. The structures of the first and second types that are most noticeable on the Poincaré map at $\varepsilon / \varepsilon_{t}=1.02$, are associated with stable and practically stable periodic orbits. A large number of structures of the first type on the Poincare map for $\varepsilon / \varepsilon_{t}=1.5199885$ is due to the existence of multiturn unstable periodic orbits. With a decreasing of the $\varepsilon$ for such orbits, the MLCE value and the maximum possible number of turns in the plane $(\xi, \eta)$ increase; the radial extension of these turns also increases.

\section{References}

Bok, B.J.: The Stability of Moving Clusters. Harvard Observ. Circ. 384, 1-41 (1934). Chandrasekhar, S.: Principles of Stellar Dynamics. Univ. Chicago Press, Chicago, Ill (1942). LCCN: 43-5608 (BKS1)

Contopoulos, G.J.: Order and chaos in dynamical astronomy. Springer-Verlag, Berlin, Heidelberg, New York (2002). ISBN: 978-3642077708

Danilov, V.M.: The Motion of Halo Stars in Dynamical Numerical Models of Open Clusters. Astron. Reports 49, 3604-610 (2005). doi:10.1134/1.2010649

Danilov, V.M.: Dynamical models of stellar motions at the peripheries of open clusters. Astron. Reports 50, 346-357 (2006). doi:10.1134/S1063772906050027

Danilov, V.M., Chernova, O.A.: Analysis of stellar trajectories in an open cluster model. Astron. Reports 52, 27-39 (2008). doi:10.1134/S1063772908010046

Danilov, V.M., Dorogavtseva, L.V.: Estimates of Relaxation Times in Numerical Dynamical Models of Open Star Clusters. Astron. Reports 47, 483-491 (2003). doi: $10.1134 / 1.1583775$

Danilov, V.M., Leskov, E.V.: Properties of Stellar Trajectories in Numerical Dynamical Models of Open Star Clusters. Astron. Reports 49, 190-200 (2005). doi:10.1134/1.1882777

Demidovich, B.P.: Lectures on the mathematical theory of stability. Nauka, Moscow (1967) (In Russian).

Gantmakher F.R.: Theory of matrices. Nauka, Moscow (1966) (In Russian).

Gidea, M., Masdemont, J.J.: Geometry of Homoclinic Connections in a Planar Circular Restricted Three-Body Problem. International Journal of Bifurcation and Chaos 17, 1151-1169 (2007). doi:10.1142/S0218127407017744

King, Ivan R.: An Introduction to Classical Stellar Dynamics. URSS, Moscow (2002) (In Russian).

Korn, G.A., Korn, T.M: Mathematical Handbook for Scientists and Engineers. McGraw-Hill, New York, Toronto, London (1961).

Kutuzov, S.A., Osipkov, L.P.: A Generalized Model for the Three-Dimensional Gravitational Potential of Stellar Systems. Soviet Astron. 24, 17-22 (1980).

Lichtenberg, A.J., Lieberman, M.A.: Regular and Chaotic Dynamics. SpringerVerlag, New York, NY (1983). 
Loskutov, A.Yu., Mikhailov, A.S.: An introduction to sinergetics. Nauka, Moscow (1990) (In Russian).

Markeev, A.P.: The libration points in celestial mechanics and space dynamics. Nauka, Moscow (1978) (In Russian).

Mineur, H.: Équilibre des nuages galactiques et des amas ouverts dans la Voie Lactée. Évolution des amas. Ann. d'Astropys. 2, 1-244 (1939).

Nemytsky, V.V., Stepanov, V.V.: A qualitative theory of differential equations. OGIZ, Moscow, Leningrad (1947) (In Russian).

Paskowitz, M.E, Scheeres, D.J.: Robust Capture and Transfer Trajectories for Planetary Satellite Orbiters. Journal of Guidance, Control, and Dynamics 29, 342-363 (2006). doi:10.2514/1.13761

Spitzer, L.: Dynamical evolution of globular clusters. Princeton University Press, Princeton, NJ (1987). ISBN-13: 978-0691084602

Vela-Arevalo, L.V., Marsden, J.E.: Time frequency analysis of the restricted threebody problem: transport and resonance transitions. Classical Quantum Gravity 21, 351-375 (2004). doi:10.1088/0264-9381/21/3/022

Wielen, R.: On the Lifetimes of Galactic Clusters. Astrophys. Space Sci. 13, 300-308 (1971). doi:10.1007/BF00649160

Winter, O.C., Vieira Neto, E.: Time analysis for temporary gravitational capture. Stable orbits. Astron. Astrophys. 377, 1119-1127 (2001). doi:10.1051/00046361:20011165

Yakubovich, V.A., Starzhinsky, V.M.: Linear differential equations with periodical coefficients and its applications. Nauka, Moscow (1972) (In Russian). 



\title{
Chapter 6 Time Scales of Dynamic Evolution Mechanisms of Open Star Clusters
}

\begin{abstract}
In this chapter, we consider the data on the time dependencies of the dispersions of the star motion velocities in three mutually perpendicular directions in the halo and the cluster core for a number of the non-stationary OSC models. In the course of the dynamic evolution of the OSC models, the values of the velocity dispersions undergo oscillations that do not damp for 5-10 intervals of violent relaxation time $\tau_{v r}$. We discuss the estimates of the synchronization time $t_{s}$ of the rotation of the examined OSC models with their motion around the center of the Galaxy. Depending on the parameters of the OSC models, the synchronization times are $t_{s} \simeq(5-27) \tau_{v r}$. The mechanisms of a synchronization of the OSC models are discussed. We note a prominent role of the tidal friction in the decay of such systems in the field of the Galaxy. We consider the estimates of the formation time for the spherical distribution of stellar velocities in the cluster models $t_{\sigma} \simeq(6-25) \tau_{v r}$. We discuss the effect of instability in the motion of the cluster stars on the formation of the spherical distribution of stellar velocities in the considered OSC models. We note a tendency toward weakening of the dependency of a coarse-grained phase density of the cluster on the effect of the small initial perturbations of the phase coordinates of stars in the cores of the cluster models for time instants which are $\sim 5$ times more distant than the time of a violent relaxation.
\end{abstract}

\subsection{Introduction}

Long and Weinberg (1993) and Weinberg (1993) investigated the tidal action of the Galaxy on the shape of its satellites (dwarf galaxies and globular clusters). They showed, at the base of numerical experiments with initially oblate satellites of the Galaxy, that the synchronization of satellite rotation with its orbital motion occurs in a time approximately equal to five revolutions of the satellite around the center of the Galaxy in the tidal field, the value of which is below a certain critical value. In stronger tidal fields, in which the satellite-cluster loses more than half of its stars, 
synchronization does not occur, and after the tidal truncation the cluster takes a spheroidal shape.

Mechanisms of tidal interactions in binary stars (BS) were considered in the papers of Lecar et al. (1976); Hut (1981); Mardling and Aarseth (2001) (see also references in these papers). If the distances between two stars and their mutual orientation change with time slowly enough, then the tides are called equilibrium tide (or static tide (Surdin, 2002, p. 10)), otherwise the tides are called dynamic. Dynamic tides occur at resonances between the natural frequencies of stellar oscillations and the frequency of tide. Equilibrium tides exist in the case of rotation of the BS component synchronous with its orbital motion. In the presence of damping of the tidal oscillations, we observe the lags (or leads) of the tidal humps (protrusions on the surface) of a star relative to a straight line connecting the centers of mass of two stars (Hut, 1981; Mardling and Aarseth, 2001). The equilibrium tide model (Hut, 1981) with weak tidal friction describes the evolution of the angular moments of stars and their orbits with the not very large eccentricities. Mardling and Aarseth (2001) considered a chaotic behavior of the orbits of the BS components for large eccentricity of orbits and very close encounters of the BS components.

It is of interest to analyze a tidal friction in the OSC models in the case when the rotation of the cluster is not synchronized with its motion along the orbit. Dissipation of the energy of a tidal hump in such OSC models can occur both in the interaction of single cluster stars with the field of the tidal hump (eventually, with the stars forming the tidal hump) and at resonances between the oscillation frequencies of the OSC and the frequency of the tide. Due to these mechanisms, during the time $t_{s}$, the cluster rotation synchronizes with its orbital motion, and the cluster itself "heats up" and disrupts, partially or completely.

According to observations in the infrared, $\mathrm{X}$-ray and radio wavelengths, the OSC are formed in the cores of giant molecular clouds (GMC) (Allen et al., 2007). In this case, the velocities of the gas in the cloud are inherited by the emerging stars, which makes it possible to get some idea about the characteristics of the initial stellar velocity field in the OSC. Chernin and Efremov (1995) and Shchekinov and Zinchenko (2004) discuss the various mechanisms for the formation of the GMCs and the torque in these clouds. Chernin and Efremov (1995) presents the estimates of the angular momenta for the most rapidly rotating and the "ordinary" GMCs (see Table 1 in the paper of Chernin and Efremov (1995)), and also gives the examples of three clouds, two of which rotate in "retrograde" direction, and one rotates in "prograde" direction (in these cases, the angular momentum vectors of the cloud and the Galaxy are antiparallel and parallel, respectively). Shchekinov and Zinchenko (2004) have shown that the rotational momentun of clouds can be oriented not necessarily along the direction of the Galaxy rotation.

According to Phillips (1999), the observed orientations of the angular rotation velocities of 156 molecular clouds (MC) form a distribution with two maxima near the directions to the northern and southern poles of the Galaxy. At the same time, the dispersion of this distribution (Phillips, 1999) near the maxima is sufficiently large; orientations of the $\mathrm{MC}$ rotation angular momenta can be random, and an appreciable part of the MCs has an orientation of rotation along the Galactic plane. Goodman 
et al. (1993) and Pirogov et al. (2003) had studied the rotation in the dense and massive MC cores by the data of radio observations. Goodman et al. (1993) showed that in $29 \mathrm{MCs}$ (out of 43 ones), the gas motions are consistent with the model of homogeneous rotation of the cores; an orientation of the gradient of the gas velocities projections onto celestial sphere is approximately conserved in the whole range of the considered densities (they used the radio observations of the molecules $\mathrm{NH}_{3}$, $C^{18} O, C S$ ). Goodman et al. (1993) found the relation between the specific angular momentum $j$ and the size $R$ (a diameter) of the cloud: $j \sim R^{3 / 2}$; they did not note the correlation between the directions of the axis of rotation and the elongation of the cloud. Pirogov et al. (2003), according to the radio observations of the molecule $\mathrm{N}_{2} \mathrm{H}^{+}$in $35 \mathrm{MCs}$, determined the sizes of the MC cores of $(0.3-2.1) \mathrm{pc}$ and their virial masses of $(30-3000) M_{\odot}$. The typical ratio of the rotational energy of MC to its gravitational energy, according to Goodman et al. (1993), is $\beta \sim 0.02$, and, according to Pirogov et al. (2003), $\beta \sim 4 \times 10^{-4}-7.1 \times 10^{-2}$ (that is, a rotation does not keep the MC in an equilibrium). Pirogov et al. (2003) noted the correlation between the directions of the axis of rotation and the elongation of the cloud. Data on the angular momenta of the MC cores can also be found in Section 5.3.1 of the review of Ballesteros-Paredes (2007).

Danilov and Dorogavtseva (2003) considered the dynamic evolution of the OSC models moving along the circular orbits in the plane of the Galaxy and not rotating relatively to the outer galaxies at the initial point of time $t=0$. They obtained the estimates of the local time of a violent relaxation $t_{r}$ from the data on the instability of the phase density function (PDF) of clusters relatively the small initial perturbations of the phase coordinates of stars; the estimates of the initial time of a violent relaxation of the OSC models $\tau_{v r}$, as well as the estimates of the relaxation time of these models from the data on star fluxes in the spaces of a number of the stellar motion parameters: $\varepsilon, l_{\zeta}, \varepsilon_{\zeta}, r$ and $v$ (energy, angular momentum of the star with respect to $\zeta$ axis and the energy of the stellar motion perpendicular to the Galactic plane per unit of the star mass, the module of the radius vector and the module of stellar velocity, respectively). According to Danilov and Dorogavtseva (2003), the time of the "collisional" relaxation $\tau_{s t}$ for such systems (due to the stellar encounters) is $1.8-2.6$ times larger than $\tau_{v r}$. It means that the interactions of stars with the timevarying regular field dominate in the relaxation of the considered OSC models with the number of stars $N=500$. Estimates of the relaxation time of the OSC models in the spaces of values $\varepsilon, l_{\zeta}, \varepsilon_{\zeta}, r$ and $v$ satisfy the relations $\tau_{\varepsilon}>\tau_{l_{\zeta}}>\tau_{\varepsilon_{\zeta}}>\tau_{v}$ and $\tau_{r}>\tau_{v}$.

Danilov and Dorogavtseva (2003) discussed the possible reasons for the formation of such inequalities. During the evolution of the cluster models, the relaxation times in all the considered spaces increase, which indicates a decrease in the rate of evolution of the OSC in the spaces of the values $\varepsilon, l_{\zeta}, \varepsilon_{\zeta}, r, v$ (Danilov and Dorogavtseva, 2003). In the course of a violent relaxation, stars with energies $\varepsilon$ occupy all regions accessible to them in the OSC, first in the space of $v$, and then in the space of $r$ (in models of Danilov and Dorogavtseva (2003) $\tau_{r} \simeq(1.3-2.6) \tau_{v}$ ). A connection between the growth rates of the PDF perturbations during the evolution of the OSC models with the differences in the relaxation rates of the models in the $v$ and $r$ spaces 
is noted. In the OSC models of Danilov and Dorogavtseva (2003) and Danilov (2002b), the balance of star fluxes in the spaces $\varepsilon, l_{\zeta}, \varepsilon_{\zeta}, r$ is noted; the equilibrium distributions of stars are present in the spaces $\varepsilon, l_{\zeta}, \varepsilon_{\zeta}$. The equilibrium PDF, which corresponds to the balance of the star fluxes in the space $\varepsilon$, determines the structure of the OSC and the models of such clusters both during the violent relaxation (in the time intervals $t \in\left[0, \tau_{v r}\right]$ ) and during the subsequent, slowly damping density oscillations.

It is of interest to estimate the time intervals, during which the oscillations of the dispersion of the velocities decay, and the parameters of the velocity distribution formed at this time point, and to analyze the evolution of the PDF perturbations due to the instability of the stellar trajectories to small changes in the initial PCS in the considered OSC models. Comparison of the decay time of the oscillations of the velocity dispersions with the relaxation times of the OSC models will allow us to find the mechanisms that delay the "virialization" of such systems. The dynamics of the PDF perturbations will make it possible to investigate the effect of the initial (and current) PDF perturbations on the conclusions about the parameters of the considered systems. In order to obtain these estimates, it is necessary to calculate the cluster models at the time intervals $t$, which are longer than ones considered by Danilov and Dorogavtseva (2003).

Calculations of the cluster models in the work of Danilov and Dorogavtseva (2003) were performed by integrating the equations of the stellar motion using the difference schemes of the 8th and 9th order of accuracy in the time interval $t \in\left[0, t_{0}\right]$, where $t_{0} / \tau_{v r} \simeq 2.7-3.5 . t_{0}$ is the time interval of the dynamic evolution of the OSC model, during which the statistical criterion for the accuracy of calculations is fulfilled (Danilov, 1997b), see also Section 16.3. In this case, the accuracy of calculation of the cluster's PDF can be considered sufficient for the conclusions about the statistical properties of the PDF. To increase $t_{0}$, a further increase is necessary in the order of accuracy of the difference schemes used in the calculations.

The objective of this chapter is to discuss the features of the dynamic evolution of the OSC models that are non-stationary in the regular field and have different initial rotation speeds; to estimate the synchronization time of the cluster rotation with its motion around the Galactic center. Also, the goal of this part of the present work is to increase the order of accuracy of the integration method used to calculate the stellar trajectories in the OSC models.

\subsection{Description of OSC Models}

Following to Danilov and Dorogavtseva (2003); Danilov (2002b); Danilov and Dorogavtseva (2008), we consider a cluster containing $N=500$ stars and moving in the Galactic plane in a circular orbit with a radius of $8200 \mathrm{pc}$ around the Galactic center with an angular velocity $\omega=$ const. At the initial time $t=0$, the star cluster is modeled as a system of two gravitating balls simulating a halo and a core with the coincident centers of mass. 12 OSC models are considered, the data of which are 
given in Table 6.1. The 1st column of Table 6.1 and Table 6.2 contains the number of the OSC model. Models 1-6 at $t=0$ do not rotate with respect to outer galaxies and coincide with models 1-6 of Danilov and Dorogavtseva (2003); the parameters of these models are listed also in Table 16.1 of Section 16.1. Models 1a-6a at $t=0$ rotate synchronously with their orbital motion. Rotation around $\zeta$ axis of $I$ a models at $t=0$ is solid-state with angular velocity $\omega$ relative to $I$ models $(I=1-6)$. Initial parameters $R_{1} / R_{2}$ and $N_{1} / N_{2}$ (see the 2 nd and 3rd columns of Table 6.1) in all considered OSC models satisfy the relation $R_{1} / R_{2} \simeq 0.39 \times\left(N_{1} / N_{2}\right)^{0.35}$ obtained from observations (Danilov and Seleznev, 1994), where $R_{1}$ and $R_{2}$ are the radii of the core and the halo of the cluster, $N_{1}$ and $N_{2}$ are the numbers of the core stars and the halo stars of the clusters (the initial value of $N_{1}$ is given in the 4th column of the Table 6.1).

Table 6.1 Parameters of the OSC Models

\begin{tabular}{|c|c|c|c|c|c|}
\hline $\mathcal{N}$ & $R_{1} / R_{2}$ & $N_{1} / N_{2}$ & $N_{1}$ & $R_{2} / R_{t}$ & $\tau_{v r}$ \\
\hline 1 and 1a & 0.24 & 0.25 & 100 & 0.9 & 49.9 \\
\hline 2 and 2a & 0.24 & 0.25 & 100 & 0.8 & 41.8 \\
\hline 3 and 3a & 0.34 & 0.67 & 200 & 0.8 & 41.8 \\
\hline 4 and 4a & 0.24 & 0.25 & 100 & 0.7 & 34.3 \\
\hline 5 and 5a & 0.45 & 1.50 & 300 & 0.8 & 41.8 \\
\hline 6 and 6a & 0.63 & 4.00 & 400 & 0.8 & 41.8 \\
\hline
\end{tabular}

Let us consider the motion of the cluster stars in the rotating coordinate system $(\xi, \eta, \zeta)$, associated with the mass center of the cluster. The axes $\xi, \eta$ and $\zeta$ are directed from the mass center of the cluster to the Galactic anticenter, along the direction of cluster motion in the Galactic plane and perpendicular to the plane of the Galaxy, respectively. In order to calculate the OSC models, we applied the equations of stellar motion (5.517)-(5.519) from the paper of Chandrasekhar (1942) written in the coordinate system $(\xi, \eta, \zeta)$. The potential of the Galaxy was taken in the form proposed by Kutuzov and Osipkov (1980). The method for specifying the initial positions and the velocities of the stars in the OSC models is described by Danilov and Dorogavtseva (2003). The initial coordinates of the stars in the models 1a-6a and in the corresponding models 1-6 coincide, and the initial velocities of the stars in the models $1 \mathrm{a}-6 \mathrm{a}$ are specified so that the cluster does not rotate in the coordinate system $(\xi, \eta, \zeta)$. We use the following system of units: pc, $\mathrm{Myr}, M_{\odot}$. The smoothing of the force functions in the right-hand parts of the equations of the stellar motion was used (the technique and the smoothing parameter are described by Danilov (1997a)).

Column 5 of Table 6.1 lists the initial values $R_{2} / R_{t}$ for the cluster models. $R_{t}$ is the tidal radius of the cluster stability in the field of the Galaxy, obtained according to King (1962). Column 6 of Table 6.1 lists the estimates of the initial time of a 
Table 6.2 Estimates of Synchronization Time

\begin{tabular}{|c|c|c|c|}
\hline $\mathcal{N}$ & $t_{s, c} / \tau_{v r}$ & $t_{s, h} / \tau_{v r}$ & $t_{s} / \tau_{v r}$ \\
\hline 1 & $6.2 \pm 0.4$ & $8.9 \pm 0.3$ & $7.5 \pm 0.2$ \\
\hline 2 & $8.0 \pm 1.0$ & $9.3 \pm 0.5$ & $8.7 \pm 0.4$ \\
\hline 3 & $5.7:$ & $11.6 \pm 0.7$ & $12.6 \pm 1.1$ \\
\hline 4 & $8.7 \pm 1.1$ & $12.7 \pm 1.1$ & $10.3 \pm 0.7$ \\
\hline 5 & $8.3:$ & $14.1:$ & $12.0:$ \\
\hline 6 & $9.9:$ & $26.6 \pm 4.0$ & $17.8::$ \\
\hline $\mathcal{N}$ & $t_{s, c} / \tau_{v r}$ & $t_{s, h} / \tau_{v r}$ & $t_{s} / \tau_{v r}$ \\
\hline $1 \mathrm{a}$ & $3.4 \pm 0.1$ & $6.2 \pm 0.2$ & $4.6 \pm 0.1$ \\
\hline $2 \mathrm{a}$ & $5.2 \pm 0.3$ & $10.4 \pm 0.8$ & $7.4 \pm 0.3$ \\
\hline $3 \mathrm{a}$ & $4.7 \pm 0.5$ & $12.2 \pm 2.0$ & $7.4 \pm 0.5$ \\
\hline $4 \mathrm{a}$ & $6.4 \pm 0.8$ & $14.2:$ & $12.0 \pm 1.9$ \\
\hline $5 \mathrm{a}$ & $6.6:$ & $8.5 \pm 1.2$ & $7.6::$ \\
\hline $6 \mathrm{a}$ & - & - & - \\
\hline
\end{tabular}

violent relaxation $\tau_{v r}$ (in Myr), obtained by Danilov and Dorogavtseva (2003) for the models $1-6$ and used here as the corresponding time scale for the models $1 \mathrm{a}-6 \mathrm{a}$.

In Table 6.2, columns 2-4 contain the estimates of a synchronization time of the cluster (and its subsystems) rotation with the orbital motion of the cluster for models 1-6 and 1a-6a, respecfively, obtained by Danilov and Dorogavtseva (2008): $t_{s, c}-$ for the core, $t_{s, h}$ - for the halo of the cluster, $t_{s}$ for the cluster as a whole, obtained in the units of $\tau_{v r}$.

\subsection{Basic Calculation Techniques}

In the work of Danilov and Dorogavtseva (2008), an integration of the equations of stellar motion is carried out by the 10th order Runge-Kutta method (Hairer et al., 1987, p.203) applying the Richardson extrapolation, which provides the 11th accuracy order for approximation of the desired solution (Hairer et al., 1987, p. 176). The calculations were made with an accuracy of 15-16 decimal digits. The maximum relative error in calculating of the "energy" $E$ of the cluster (see (5.522) from Chandrasekhar (1942)) reached in the time interval $t_{0}$ in the considered models, is modulo $\sim(1-4) \times 10^{-13}$. The statistical criterion for the accuracy of the PDF computation (Danilov, 1997a) was fulfilled during the time intervals $t_{0} / \tau_{v r}$ in the range of $3.0-3.9$ in the center to $3.6-5.1$ at the periphery of the considered cluster models. The use of methods of the 10th and 11th accuracy orders makes it possible to increase the values $t_{0} / \tau_{v r}$ in comparison with those obtained by Danilov and Dorogavtseva (2003), see above.

In order to estimate the values $t_{s, c}, t_{s, h}$ and $t_{s}$, given in the Table 6.2, we applied the time dependencies of the mean values of the velocities $\left\langle v_{\varphi}\right\rangle$ for the motion of 
the stars of the cluster core, the cluster halo, and the cluster as a whole around the axis $\zeta$ in the plane $(\xi, \eta)$. These dependencies $\left\langle v_{\varphi}\right\rangle=\left\langle v_{\varphi}(t)\right\rangle$ were approximated by the linear dependencies at the specially chosen sections of the curves close to the moment of intersection of the $t$ axis by the curve $\left\langle v_{\varphi}\right\rangle=\left\langle v_{\varphi}(t)\right\rangle$. The time, at which the straight line approximating the dependence $\left\langle v_{\varphi}(t)\right\rangle$ intersects the $t$ axis, was assumed as the corresponding synchronization time. According to Table 6.2, the estimates of $t_{s, c}$ and $t_{s, h}$, even for the halos of the OSC models, are several times higher than $t_{0} / \tau_{v r}$ for these cluster models. We note that, in comparison with the PDF, the values $\left\langle v_{\varphi}\right\rangle$ depend to a lesser extent on the initial and current PDF perturbations, since they contain averaging in the space of coordinates and velocities of the stars. In order to analyze the influence of errors of integration of the equations of a stellar motion on the values $\left\langle v_{\varphi}\right\rangle$ (and on the estimates of $t_{s, c}, t_{s, h}, t_{s}$ ), we perform the statistical comparison of the time dependencies of the values $\left\langle v_{\varphi}\right\rangle_{10}$ and $\left\langle v_{\varphi}\right\rangle_{11}$ obtained with the use of the numerical methods of 10th and 11th accuracy orders, respectively, for integrating the equations of a stellar motion. As a measure of correlation between $\left\langle v_{\varphi}\right\rangle_{10}$ and $\left\langle v_{\varphi}\right\rangle_{11}$, we used the correlation coefficient $k_{1,2}$ of the time dependencies $\left\langle v_{\varphi}(t)\right\rangle_{10}$ and $\left\langle v_{\varphi}(t)\right\rangle_{11}$. The radius of the cluster core $r=R_{c}$ at the time $t$ was determined using the radial profiles of the spatial density of the number of stars as a position of the outer boundary (by $r$ ) of the region of the maximum density gradient modulus.

We assumed the cluster halo radius to be equal to the tidal radius $r=R_{t}$ when estimating the values of $t_{s, h}$ and $t_{s}$. The synchronization times $t_{s, c}, t_{s, h}$ and $t_{s}$ were determined from the data on the $\left\langle v_{\varphi}\right\rangle$ obtained with the use of the PCS with distances $r$ from the center of the cluster satisfying to the following inequalities, respectively: $r \leq R_{c}, R_{c}<r \leq R_{t}$ and $r \leq R_{t}$. Integration of the equations of motion of stars was carried out to the time values of $t=2 t_{0}$, where $t_{0}$ was the largest value $t_{0}$, obtained for the periphery of the cluster model. The maximum relative error of calculating the "energy" $E$ at the time point $t=2 t_{0}$ in the considered models, was $\sim(1-4) \times 10^{-13}$ (as in the case of $t=t_{0}$ ). Consequently, the greatest values of the error of $E$ are attained at small values of $t<t_{0}$ in the early stages of the dynamic evolution of the cluster models. At $k_{1,2} \in[0.65,0.95]$, the time interval $t^{*}$ (during which a significant correlation between the dependencies $\left\langle v_{\varphi}(t)\right\rangle_{10}$ and $\left\langle v_{\varphi}(t)\right\rangle_{11}$ takes place for the considered OSC models) equals to $t^{*} / \tau_{v r} \simeq 5.8-10.1$ for the core, $t^{*} / \tau_{v r} \simeq 6.9-10.1$ for the halo, and $t^{*} / \tau_{v r} \simeq 7.1-10.1$ for the entire cluster (here, the largest value of $t^{*}$ reaches $t^{*}=2 t_{0} \simeq 10.1 \tau_{v r}$ ).

In order to analyze the time evolution of the velocity distribution of stars in the core and the halo of clusters over time intervals equal to $2 t_{0}$, we have calculated the time dependencies of $\sigma_{v_{\xi}}^{2}, \sigma_{v_{\eta}}^{2}$ and $\sigma_{v_{\zeta}}^{2}$ for the dispersions of the stellar motion velocities along the axes $\xi, \eta$ and $\zeta$, respectively. Comparison of the time dependencies of the velocity dispersions obtained using the 10th and 11th accuracy methods leads to the following time intervals $t^{*}$, during which a significant correlation between these dependencies takes place. When studying the cores of the OSC models from the data on the values $\sigma_{v_{\zeta}}^{2}$, we find $t^{*} / \tau_{v r} \simeq 7.1-10.1$ at $k_{1,2} \in[0.82,0.97]$, and, for dependencies $\sigma_{v_{\xi}}^{2}(t)$, we find $t^{*} / \tau_{v r} \simeq 5.7-10.1$ at $k_{1,2} \in[0.66,0.85]$. In the case of the halo of the OSC models: $t^{*} / \tau_{v r} \simeq 7.1-10.1$ at $k_{1,2} \in[0.84,0.93]$ 
and $t^{*} / \tau_{v r} \simeq 6.1-10.1$ at $k_{1,2} \in[0.68,0.85]$ for dependencies $\sigma_{v_{\zeta}}^{2}(t)$ and $\sigma_{v_{\xi}}^{2}(t)$, respectively. Thus, at $t \leq t^{*}$ the values $\left\langle v_{\varphi}\right\rangle, \sigma_{v_{\zeta}}^{2}$ and $\sigma_{v_{\varepsilon}}^{2}$ obtained within the framework of the 11th accuracy order can be used properly for the analysis of the dynamics of the considered OSC models. Let $t_{\text {min }}^{*}$ be the smallest value $t^{*}$ for each of the variables $\left\langle v_{\varphi}\right\rangle, \sigma_{v_{\zeta}}^{2}$ and $\sigma_{v_{\xi}}^{2}$. Then the largest values of $k_{1,2}$ and $t_{\min }^{*}$ are attained for the dependencies $\sigma_{v_{\zeta}}^{2}=\sigma_{v_{\zeta}}^{2}(t)$. Consequently, the dependencies $\sigma_{v_{\zeta}}^{2}=\sigma_{v_{\zeta}}^{2}(t)$ for the considered OSC models are obtained most accurately.

In the Table 6.2, for models 3, 4a, 5, 5a, 6, the symbol ":" indicates uncertain estimates of $t_{s, c}$ and $t_{s, h}$, for which the value $\left\langle v_{\varphi}\right\rangle=0$ was achieved many times over a sufficiently large time interval or near the time $t=t_{s, c}$ or $t=t_{s, h}$, there was a noticeable difference in $\left\langle v_{\varphi}\right\rangle_{10}$ and $\left\langle v_{\varphi}\right\rangle_{11}$, as well as their corresponding synchronization time estimates. The symbol "::" in the Table 6.2 for models 5 a and 6 indicates even more uncertain estimates of the synchronization time of the cluster model $t_{s}$ obtained by the formula $t_{s}=\left(t_{s, c}+t_{s, h}\right) / 2$. In this case, the estimate of $t_{s}$ from the data on $\left\langle v_{\varphi}(t)\right\rangle_{11}$ is either impossible, or contains the extrapolation in $t$ over a large time interval.

The procedure for calculating of the PDF perturbations $\psi_{j}(t)$, which are the mean relative differences of the PDF of two OSC model versions with small initial differences of PCS, is described in detail by Danilov and Dorogavtseva (2003). All 500 stars in one of the compared cluster model versions were divided into groups of 50 stars in order of increasing $r$ (Danilov and Dorogavtseva, 2003). According to the data on the velocities of stars from the intervals of distances $r$, corresponding to these groups, according to formula (6) from the paper of Danilov and Dorogavtseva (2003), we obtain the values $\psi_{j}(t)(j=1, \ldots, 10)$. Functions $\psi_{j}(t)$, obtained for different values of $j$, allow us to evaluate the instability of the PDF of the OSC models to small initial PCS perturbations at different distances from the cluster center.

Changes with time $t$ of the radii of the concentric spheres with the center in the mass center of the cluster and "enclosing" (bounding relatively to the distance from the cluster center) a fixed number of cluster stars, allow us to evaluate the influence of the tidal field of the Galaxy on the groups of stars located at different distances from the cluster center. The method of the similar calculations of the radii of spheres in the star cluster models was considered by Spitzer and Thuan (1972); Henon (1973).

\subsection{Changes in the Parameters of Stellar Velocity Distribution with Time. Instability of Phase Density to Small Initial Perturbations}

Synchronization of rotation of the considered OSC models with their orbital motion in the Galaxy is accompanied by a "heating" and a partial destruction of the clusters. After the beginning of evolution in models 1-6 during the time intervals of $\Delta t \simeq$ $(3-4) \tau_{v r}$, the values $\left\langle v_{\varphi}\right\rangle<0$ and change (decrease) only slightly with time due to an escape from the cluster of a part of the stars with "prograde" trajectories (with 
positive angular momentum of motion $l_{\zeta}$ with respect to the $\zeta$ axis). A significant role also is played by the transitions of stars with $l_{\zeta}>0$ from the core to the halo. As a result of such transitions, the values of $\left\langle v_{\varphi}\right\rangle$, obtained for the cluster core, decrease with increasing of $t$ (at $t \leq(3-4) \tau_{v r}$ ); for models 1 and 4 , see fig. 6.1.
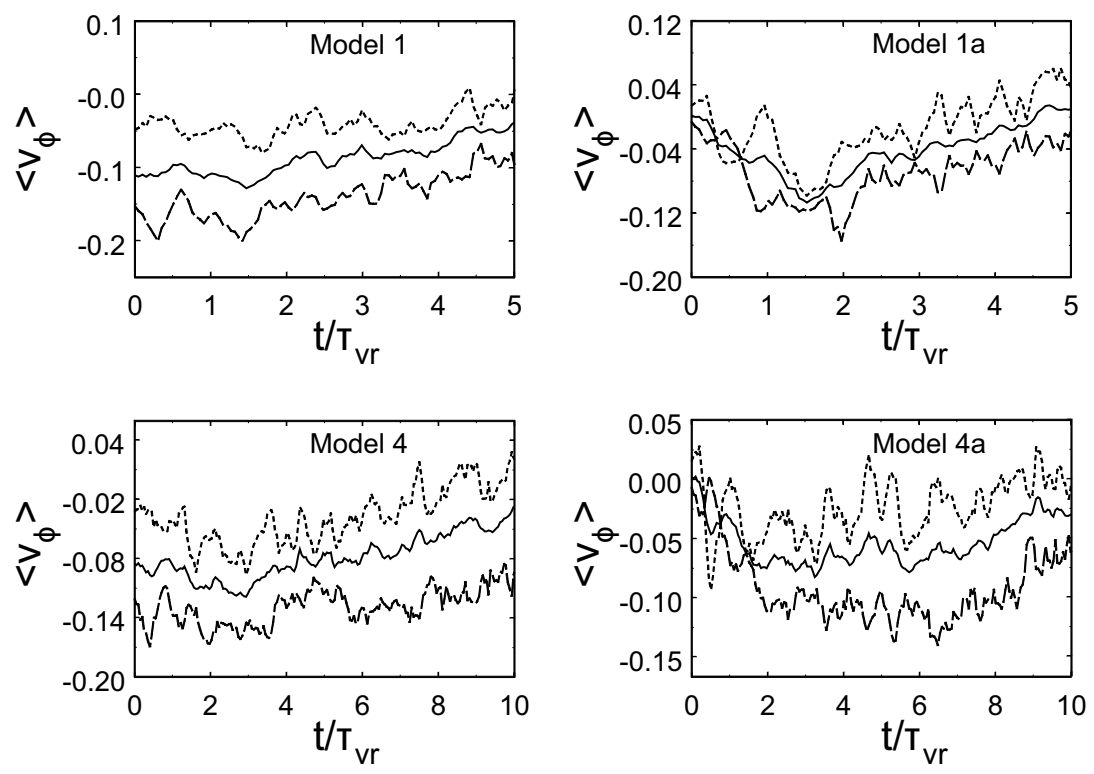

Fig. 6.1 The time dependencies of $\left\langle v_{\varphi}\right\rangle$ in models 1, 1a, 4, 4a. Solid line, short-dash and longdash lines, mark the curves obtained from the data on stars with $r \leq R_{t}, r<R_{c}, R_{c}<r \leq R_{t}$ respectively

In models 1a-6a during $\Delta t \simeq(3-4) \tau_{v r}$, the loss of stars with $l_{\zeta}>0$ from the cluster is more significant. As a result, stars with $l_{\zeta}<0$ begin to dominate in the estimates of $\left\langle v_{\varphi}\right\rangle$, and the cluster "spins" in the "retrograde" direction (clockwise in the $(\xi, \eta)$ plane). By the moment $t \simeq(3-4) \tau_{v r}$, the values $\left\langle v_{\varphi}\right\rangle$ in these models also become negative, see fig. 6.1. Then the considered cluster models due to a tidal friction and the subsequent relaxation of the energy of the tidal perturbations begin to "spin" in "prograde" direction, which leads to an increase of $\left\langle v_{\varphi}\right\rangle$ with time, and subsequent synchronization of the intrinsic and orbital rotations of clusters. Short-period changes in the values of $\left\langle v_{\varphi}\right\rangle$ with time in the core and the halo occur in antiphase (the acceleration of the rotation of the core in the coordinate system $(\xi, \eta, \zeta)$ is accompanied by the halo deceleration and vice versa). With an increase of $t$ (at $t \simeq 10.1 \tau_{v r}$ ) the values $\left\langle v_{\varphi}\right\rangle$ for the core and the halo approach each other. It indicates an increase in the angular rotation rate of the core in comparison with angular velocity of rotation of the halo. However, these velocities are small, and when the OSC models are synchronized, they tend to zero. The values of $t_{s}$ increase with the increasing number of stars $N_{1}$ in the core (see data on models 2, 3, 5, 6 with 
the same value $R_{2} / R_{t}=0.8$ in Tables 6.1 and 6.2). It is caused by a lesser influence of tidal forces on the denser (and more massive) core of the cluster in comparison with their effect on the extended and rarefied halo of the cluster. For the same reason, larger estimates of $t_{s}$ were obtained for denser cluster models (see data on models 1 and 4, 1a and 4a in Tables 6.1 and 6.2).

The estimate of the rotation synchronization time for the cluster-satellite of the Galaxy obtained by Weinberg $(1993)\left(\sim 1.1 \times 10^{9}\right.$ years for the radius of the cluster orbit considered in the present work) is quite comparable with our largest estimates of $t_{s} \simeq 5.3 \times 10^{8}$ years and $t_{s} \simeq 7.4 \times 10^{8}$ years for models 3 and 6 , respectively. The differences in the values of $t_{s}$ of (1.5-2 times) in the paper of Weinberg (1993) and in the present work may well be due to the difference in the parameters of clusters and models of the force field of the Galaxy assumed by Weinberg (1993) and in the present work.

It is convenient to study the destructive effect of the tidal field of the Galaxy on the cluster applying the time dependencies of the average distance of stars $\langle r\rangle$ from the cluster center for stars with $r \leq R_{t}$, see fig. 6.2. Almost in all the analyzed OSC
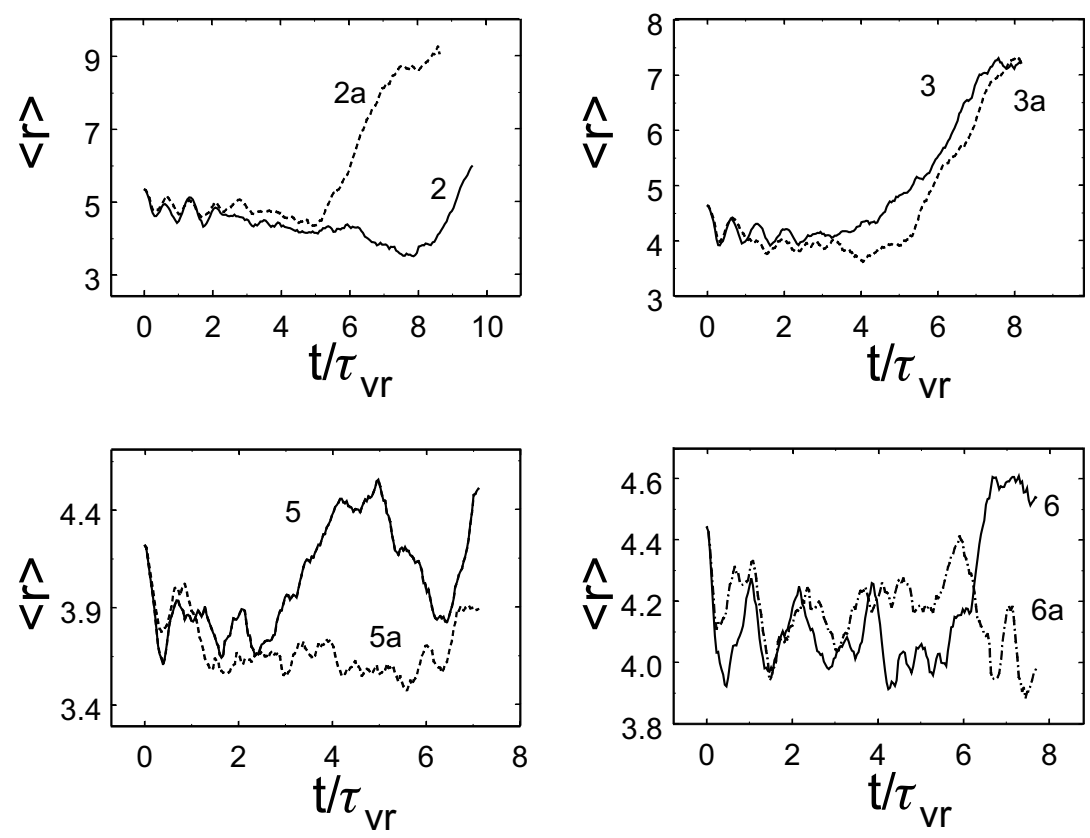

Fig. 6.2 Time dependencies of $\langle r\rangle$ in cluster models 2, 2a, 3, 3a, 5, 5a, 6, 6a. The curves are marked by the numbers corresponding to the numbers of specified models

models (except model 6a), the value of $\langle r\rangle$ after the onset of evolution first decreases with increasing $t$. It indicates an increase in the density of the cores of the OSC models (due to the dissipation of stars and the corresponding relaxation processes). 
Then, starting from the moment $t_{\langle r\rangle}$, the value of $\langle r\rangle$ begins to increase rapidly with increasing $t$ (due to tidal "heating" of the cluster). The tendency of the considered OSC models to synchronize their rotation with the orbital one (see fig. 6.1) is an indication of the role of the tidal forces in this expansion. In addition, the rate of a tidal "heating" of the cluster is largely determined by the cluster size. The equation for the rate of change in the rotational energy of the cluster model due to the action of tidal perturbations in the framework of a simple model of the equilibrium tide can be written in a form analogous to equation (A30) from the paper of Hut (1981) for the BS components. In this case, according to Hut (1981), the rate of the energy transfer of rotation to the cluster is proportional to the value of $\sim\langle\omega\rangle R^{8} / M$, where $\langle\omega\rangle$ is a mean angular velocity of the cluster rotation with respect to $\zeta$ axis in the coordinate system $(\xi, \eta, \zeta), R$ and $M$ are the radius and mass of the cluster model, respectively. Therefore, the increase of the size of the cluster within the model of Hut (1981) at $\langle\omega\rangle \neq 0$ makes a decisive contribution to the change in the cluster energy.

In model 6a, the time $t_{\langle r\rangle}$ (when a decay begins) can not be determined, since after a compression and small expansion, this model again begins to compress. According to Table 6.3, the values of $t_{\langle r\rangle}$ in the considered models are enclosed in the interval of $t$ from $t_{\langle r\rangle}^{(1 a)} \simeq(1.1 \pm 0.1) \tau_{v r}$ (in model 1a) to $t_{\langle r\rangle}^{(2)} \simeq(7.7 \pm 0.4) \tau_{v r}$ (in model 2), which roughly corresponds to the $t$ values from (54.9 \pm 5.0$)$ Myr to (322.2 \pm 16.7$) \mathrm{Myr}$. The values $t_{\langle r\rangle}$ (and their errors) are determined using polynomials of the second degree approximating the dependency $\langle r(t)\rangle$ in a certain vicinity of the time point at which the cluster begins to extend. The errors of $t_{\langle r\rangle}$ are due to the errors in the coefficients of the polynomials approximating the dependency $\langle r(t)\rangle$; the errors in $\langle r\rangle$ and $t_{\langle r\rangle}$ are related to each other by the $\left.\langle r(t)\rangle\right)$ relationship. For the subsystem of stars with $r \leq R_{t}$ by the time $t=t_{\langle r\rangle}$ in models $1-6$, the angular rotational velocities $\langle\omega\rangle$ relatively to the $\zeta$ axis in the coordinate system $(\xi, \eta, \zeta)$ are approximately equal to each other and on average are $\langle\omega\rangle=\left\langle v_{\varphi}\right\rangle /\langle r\rangle \simeq-0.0198 \pm 0.0022(\mathrm{Myr})^{-1}$, which is by absolute value $\sim(1.4289 \pm 0.1588)$ times less than the angular velocity $\omega$ of the motion of the considered cluster models around the center of the Galaxy. At the time $t=t_{\langle r\rangle}$ in models 1-6, as a result of the action of tidal friction and the relaxation of tidal perturbations, approximately the same changes occur in the value of $\langle\omega\rangle$ towards the direction of synchronization with the frequency of the orbital motion of the OSC model in comparison with the initial value $\langle\omega(0)\rangle=-\omega$. In models $1 \mathrm{a}-5 \mathrm{a}$ by the moment $t=t_{\langle r\rangle}$, the values $\langle\omega\rangle$ are in the range from $\sim(-0.021 \pm 0.001)(\mathrm{Myr})^{-1}$ (in the model 1a) to $\sim(-0.008 \pm 0.001)\left(\mathrm{Myr}^{-1}\right.$ (in the model 5a). The averages values of $\langle\omega\rangle$ on the interval $t \in\left[0, t_{\langle r\rangle}\right]$ are modulo $\sim(2-5)$ times smaller, than in models $1-6$ by the moment $t=t_{\langle r\rangle}$. In the models 1-6 by the time $t_{\langle r\rangle}$ the larger angular rotation speeds $\langle\omega\rangle$ are formed in "retrograde" direction (in comparison to models $1 \mathrm{a}-5 \mathrm{a}$ ). Also, there are large deviations from the direction toward the Galactic center of straight line of tidal hump (which connects the mass centers of the humps in the plane $(\xi, \eta)$, see fig. 1 from the paper of Hut (1981)). This deviation indicates a somewhat larger role of tidal effects in models $1-6$.

With an increase of the density and a decrease of $\langle r\rangle$ in the considered OSC models, the role of the tidal forces in an evolution decreases, which leads to an 
increase in $t_{\langle r\rangle}$. For example, for models 1 and 2 , we find $t_{\langle r\rangle}^{(2)} \simeq(2.3 \pm 0.3) t_{\langle r\rangle}^{(1)}$ (the mean radii of these models before a beginning of an expansion satisfy the relation $\langle r\rangle_{1} \simeq(1.3 \pm 0.1)\langle r\rangle_{2}$, and the values $\langle\omega\rangle$ are practically the same). However, when comparing models 1 and $1 \mathrm{a}$, we find $t_{\langle r\rangle}^{(1)} \simeq(2.5 \pm 0.4) t_{\langle r\rangle}^{(1 a)}$ at $\langle r\rangle_{1 a} \simeq(1.0 \pm 0.1)\langle r\rangle_{1}$, $\langle\omega\rangle_{1} \simeq(1.0 \pm 0.1)\langle\omega\rangle_{1 a}$ before the expansion of the models begins. In model 1a, at the time $t_{\langle r\rangle}$, the loss of stars with angular momenta $l_{\zeta}>0$ is greater than in the model 1 (due to the instability of such trajectories and a bigger number of stars with $l_{\zeta}>0$ at $t=0$ in model 1a). On average, in time interval $t \in\left[0, t_{\langle r\rangle}\right]$, the value $\langle\omega\rangle_{1} \simeq 2\langle\omega\rangle_{1 a}$. Loss of stars in the cluster model 1a leads to a noticeable weakening of the force field and acceleration of the decay of model 1a in comparison with model 1. When comparing models $1 \mathrm{a}$ and $2 \mathrm{a}$, we find $t_{\langle r\rangle}^{(2 a)} \simeq(3.6 \pm 0.4) t_{\langle r\rangle}^{(1 a)}$ at $\langle r\rangle_{1 a} \simeq$ $(1.1 \pm 0.1)\langle r\rangle_{2 a},\langle\omega\rangle_{1 a} \simeq(1.9 \pm 0.2)\langle\omega\rangle_{2 a}$ before the expansion of the models begins. In model $1 \mathrm{a}$, the role of tidal forces increases in comparison with model $2 \mathrm{a}$ due to the increase in the angular velocity $\langle\omega\rangle$. It leads to a greater deviation of the tidal hump line from the direction to the Galactic center. Comparing models 2 and $2 \mathrm{a}$, we find $t_{\langle r\rangle}^{(2)} \simeq(1.6 \pm 0.1) t_{\langle r\rangle}^{(2 a)}$ at $\langle r\rangle_{2 a} \simeq(1.2 \pm 0.1)\langle r\rangle_{2},\langle\omega\rangle_{2} \simeq(1.8 \pm 0.2)\langle\omega\rangle_{2 a}$. The difference in the proportions $t_{\langle r\rangle}^{(1)} \simeq(2.5 \pm 0.4) t_{\langle r\rangle}^{(1 a)}$ and $t_{\langle r\rangle}^{(2)} \simeq(1.6 \pm 0.1) t_{\langle r\rangle}^{(2 a)}$ is due to the greater density of models 2 and $2 \mathrm{a}$ in comparison to models 1 and $1 \mathrm{a}$, which reduces the role of tidal forces in the dynamics of denser OSC models. Comparison of the parameters $t_{\langle r\rangle},\langle r\rangle,\langle\omega\rangle$ of the rest of the OSC models indicates the effect of the considered above factors in the dynamic evolution of OSC: density of the model, degree of concentration of stars toward the center of the model, the preferred loss of stars with $l_{\zeta}>0$, and a deviation from zero of the mean angular velocity $\langle\omega\rangle$ in the coordinate system $(\xi, \eta, \zeta)$.

For all the OSC models considered in sections 6.2-6.4, Danilov and Dorogavtseva (2008) constructed the time dependencies of the radii $r_{n}$ of the concentric spheres with the center in the mass center of the cluster, which limit a fixed number of stars $n$ by distances $r \leq r_{n}$ (Spitzer and Thuan, 1972; Henon, 1973). The dependencies $r_{n}=r_{n}(t)$ have a similar character for all models. At small $n \sim 5-50$, the values $r_{n}$ depend weakly on time. At large $n \sim 250-450$, on average with respect to the oscillation period of the regular field $P_{r}$, after a small and short decrease, the $r_{n}$ values increase with time experiencing small oscillations with the period $P_{r}$, see, for example, fig. 6.3.

Table 6.3 contains the data on the number $n$ of stars satisfying the condition $r_{n} \leq R_{t}$ by the time $t=t_{0}$, obtained by Danilov and Dorogavtseva (2008) with the use of the dependencies $r_{n}=r_{n}(t)$ for the considered OSC models (the errors of the values $n$ in the Table 6.3 are \pm 5 ). According to Table 6.3, models 5, 5a, 6 and 6a are the most steady to a decay in the field of Galactic forces. By the time $t=t_{0}$, they retain $82-92 \%$ of the initial number of stars. We note that models 5 and 6 are the closest ones to a virial equilibrium among models 1-6 considered by Danilov and Dorogavtseva (2003). Interestingly, in model 6, during the periods of the greatest compression of the cluster to the plane $\zeta=0$, a toroidal structure is formed; this structure has an increased density of the number of stars inside the torus (in the space $\xi, \eta, \zeta$ ), and an equatorial plane close to $\zeta=0$ (Danilov and Leskov, 2005). 

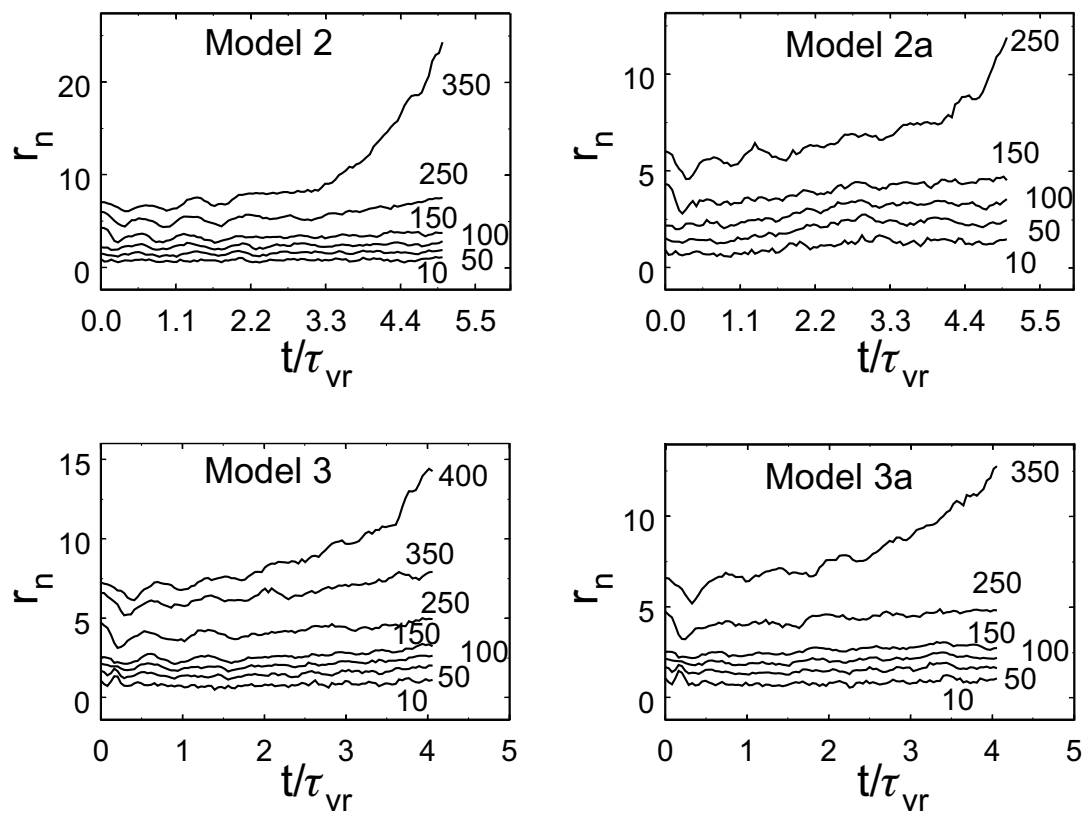

Fig. 6.3 Time dependencies of $r_{n}$ in cluster models 2, 2a, 3, 3a. Numbers indicate the curves corresponding to the specified number of stars $n$ with $r \leq r_{n}$

Perhaps the results noted here indicate the stability of toroidal (or close to toroidal) systems moving along circular orbits in the plane of the Galaxy. The least dense cluster models 1a and 1 are the most unstable ones to decay among the considered models. By the time $t=t_{0}$, they conserve only $30-32 \%$ of the initial number of stars. According to Danilov and Dorogavtseva (2003), model 1 is the most distant one from a virial equilibrium among models 1-6.

For all the considered OSC models, for stars of the core $\left(r \leq R_{c}\right)$ and the halo $\left(R_{c}<r \leq R_{t}\right)$ of clusters, on the time interval $t \in\left[0,2 t_{0}\right]$, we constructed the time dependencies of the values $\sigma_{v_{\xi}}^{2}, \sigma_{v_{\eta}}^{2}$ and $\sigma_{v_{\zeta}}^{2}$ which are the stellar velocity dispersions along the axes $\xi, \eta$ and $\zeta$, respectively (for models 2 and 2a, see fig. 6.4).

The values $\sigma_{v_{\zeta}}^{2}$ demonstrate the largest magnitudes and the largest oscillation amplitudes over time. Therefore, the largest contribution to the non-stationarity of the cluster models is made by their oscillations along the $\zeta$-coordinate. The amplitude of the oscillations of the values $\sigma_{v_{\zeta}}^{2}$ at $t \leq t_{\langle r\rangle}$ changes over time like the amplitude of oscillations of the virial coefficient $\alpha=2 E / W$, where $E$ and $W$ are total and potential energy of the stellar subsystem with $r \leq R_{t}$ in the cluster model. The amplitudes of the oscillations of the values $\sigma_{v_{\zeta}}^{2}$ and $\alpha$ decrease with time until the time point corresponding to either the instant of the establishing of the "equilibrium" oscillations with almost constant amplitude (for example, in model 6), or the time point after which the amplitudes of $\sigma_{v_{\zeta}}^{2}$ and $\alpha$ oscillations increase again. At $t \rightarrow 2 t_{0}$, 

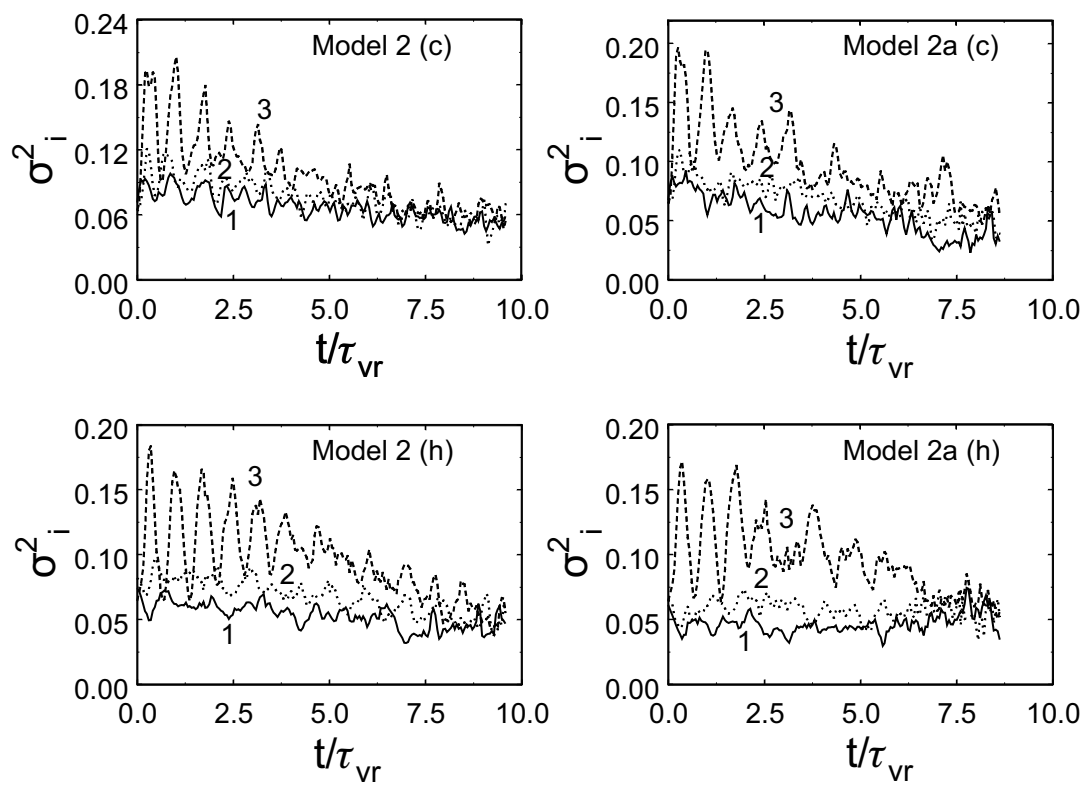

Fig. 6.4 Time dependencies of the dispersion of stellar velocities $\sigma_{v_{\xi}}^{2}=\sigma_{1}^{2}, \sigma_{v_{\eta}}^{2}=\sigma_{2}^{2}$ and $\sigma_{v_{\zeta}}^{2}=\sigma_{3}^{2}$ in cluster models 2 and 2a. Numbers 1, 2, 3 indicate curves corresponding to the values $\sigma_{1}^{2}, \sigma_{2}^{2}$, and $\sigma_{3}^{2}$. Letters $c$ and $h$ indicate the dependencies obtained for the core and halo of the model, respectively

the virial equilibrium in the OSC models is not achieved. Oscillations of the OSC models along $\zeta$ axis do not completely damp and support models in the non-stationary state on the time interval $t \in\left[0,2 t_{0}\right]$. We note that the relaxation time of models 1-6 in the space of specific energies $\varepsilon_{\zeta}$ of the stellar motion along $\zeta$-coordinate, according to the data of Danilov and Dorogavtseva (2003) on stellar fluxes in the space of $\varepsilon, l_{\zeta}, \varepsilon_{\zeta}, r$, is a smallest one. On average, for models $1-6$ of Danilov and Dorogavtseva (2003), the following proportions take place: $\tau_{\varepsilon} \simeq(4.2 \pm 0.3) \tau_{\varepsilon_{\zeta}}$, $\tau_{l_{\zeta}} \simeq(2.9 \pm 0.2) \tau_{\varepsilon_{\zeta}}, \tau_{r} \simeq(1.4 \pm 0.1) \tau_{\varepsilon_{\zeta}}$. This indicates the efficiency of the energy transfer to the cluster from the large-scale motions along the $\zeta$-coordinate. The transfer of the energy of a stellar motion along the $\zeta$ axis to a stellar motion along $\xi$ - and $\eta$-coordinates leads to expansion of the cluster along the plane $(\xi, \eta)$ and to a partial loss of stars from the cluster during the motion of stars along this plane. The main reasons for a maintaining the non-stationarity and oscillations of the considered cluster models along the $\zeta$ axis on the time interval $t \in\left[0,2 t_{0}\right]$ are:

- the large initial degree of deviation of the cluster from an equilibrium in the stellar motions along $\zeta$ axis (at $t=0$, the models are spherically symmetric);

- the constant loss of stars from the cluster (which becomes more significant after the beginning of cluster expansion at $\left.t \geq t_{\langle r\rangle}\right)$; 
- the proximity of the OSC models to gravitational instability noted earlier by Danilov and Dorogavtseva (2003) and Danilov (2002b).

In the latter case, the small and small-scale density oscillations in the cluster are easily amplified to the level of the large-scale ones, which is visible on the plots of dependencies of $\alpha$ and $\sigma_{v_{\zeta}}^{2}$ on time $t$.

As a rule, in the course of calculations, the relations $\sigma_{v_{\zeta}}^{2}>\sigma_{v_{\eta}}^{2}>\sigma_{v_{\xi}}^{2}$ are fulfilled, see, for example, fig. 6.4. As a result of the action of the relaxation mechanisms (violent relaxation, stellar encounters) in the models of the OSC, the values of $\sigma_{v_{\zeta}}^{2}$ (averaged by the oscillation period) decrease with time until the moment $t=t_{\sigma}$, when the dispersions $\sigma_{v_{\xi}}^{2}, \sigma_{v_{\eta}}^{2}$ and $\sigma_{v_{\zeta}}^{2}$ approximately equalize with each other. It does not happen in all models in the considered time intervals. At least, on the time intervals $t \in\left[0,2 t_{0}\right]$, in the halo of models $4,5 \mathrm{a}$ and $6 \mathrm{a}$, the values of $\sigma_{v_{\zeta}}^{2}$ are only slightly approaching to $\sigma_{v_{\xi}}^{2}, \sigma_{v_{\eta}}^{2}$, which indicates the elongation of the velocity ellipsoid along the $\zeta$ axis in these clusters at $t \rightarrow 2 t_{0}$. In the halo of models $4,5 \mathrm{a}$ and 6a, the values $\sigma_{v_{\zeta}} / \sigma_{v_{\xi}}$ oscillate near the values of $1.283 \pm 0.039,1.261 \pm 0.054$ and $1.262 \pm 0.048$, respectively, and the values $\sigma_{v_{\zeta}} / \sigma_{v_{\eta}}$ - near the values of 1.137 $\pm 0.041,1.179 \pm 0.040$ and $1.149 \pm 0.044$, respectively.

In this work, the estimate of $t_{\sigma}$ for the cores and the halos of the OSC models was obtained according to the data on dependencies of $\sigma_{v \xi}^{2}$ and $\sigma_{v \zeta}^{2}$ on time $t$. These dependencies were approximated by linear functions of $t$ at specially chosen time intervals contained in the intervals $t \in\left[0, t^{*}\right]$, during which a significant correlation takes place between the dependencies on time of the velocity dispersions obtained from the integration of the stellar motion equations of the 10th and 11th accuracy order. Solution of the system of linear regression dependencies $\sigma_{v_{\xi}}^{2}(t)$ and $\sigma_{v_{\zeta}}^{2}(t)$, obtained from the 11th-accuracy-order calculations, determines the value $t=t_{\sigma}$. The errors in the coefficients of regressions $\sigma_{v_{\xi}}^{2}(t)$ and $\sigma_{v_{\zeta}}^{2}(t)$ set an error of the estimate of $t_{\sigma}$. The value $t_{\sigma}$ can be considered as an approximate estimate of the transition time of the system to almost spherical distribution of stellar velocities. The estimates of $t_{\sigma}$ for the core $\left(t_{\sigma, c}\right)$ and halo $\left(t_{\sigma, h}\right)$ of the considered OSC models in the units of a violent relaxation time $\tau_{v r}$ are given in the Table 6.3. According to the Table 6.3, the time $t_{\sigma}$ is 6.1-24.9 times higher than the value $\tau_{v r}$, which indicates a very slow spherization of the velocity distribution in the considered models.

Comparison of the values of $t_{\sigma}$ in models 1, 2, 4 (as well as in models $1 \mathrm{a}, 2 \mathrm{a}$, 4a) shows that $t_{\sigma, c}$ and $t_{\sigma, h}$ grow with the increasing density of the cluster model. In these models, on average, $t_{\sigma, c}<t_{\sigma, h}$, although in statistical sense the difference between $t_{\sigma, c}$ and $t_{\sigma, h}$ is small. Comparison of the values $t_{\sigma}$ in models 2, 3, 5, 6 (as well as in models 2a, 3a, 5a, 6a) shows that $t_{\sigma, c}$ and $t_{\sigma, h}$ grow with increasing number of stars $N_{1}$ in the cluster core. Thus, in models 1 and 1a with the lowest initial density and the lowest number of stars in the core, the estimates of $t_{\sigma}$ are the smallest ones. According to Danilov and Dorogavtseva (2003), the estimates of the relaxation time $\tau_{\varepsilon}$ in the cluster models $1-6$ decrease with the increasing cluster density and number of stars in the model core. Consequently, the process of spherization of the velocity distribution is mainly not due to the action of relaxation processes, but to the proximity of the OSC models to a gravitational instability and the development of 
Table 6.3 Estimates of the values $t_{\langle r\rangle}, n, t_{\sigma, c}, t_{\sigma, h}$

\begin{tabular}{|c|c|c|c|c|}
\hline $\mathcal{N}$ & $t_{\langle r\rangle} / \tau_{v r}$ & $n$ & $t_{\sigma, c} / \tau_{v r}$ & $t_{\sigma, h} / \tau_{v r}$ \\
\hline 1 & $2.8 \pm 0.3$ & 160 & $7.6 \pm 1.0$ & $8.9 \pm 1.1$ \\
\hline 2 & $7.7 \pm 0.4$ & 265 & $8.3 \pm 1.1$ & $10.2 \pm 0.9$ \\
\hline 3 & $2.9 \pm 0.1$ & 375 & $11.8 \pm 1.8$ & $9.0 \pm 1.1$ \\
\hline 4 & $4.2 \pm 0.4$ & 380 & $17.8 \pm 2.9$ & $>20.0$ \\
\hline 5 & $1.9 \pm 0.2$ & 410 & $14.4 \pm 0.8$ & $13.7 \pm 0.6$ \\
\hline 6 & $5.2 \pm 0.4$ & 460 & $21.5 \pm 1.1$ & $24.9 \pm 1.3$ \\
\hline $\mathcal{N}$ & $t_{\langle r\rangle} / \tau_{v r}$ & $n$ & $t_{\sigma, c} / \tau_{v r}$ & $t_{\sigma, h} / \tau_{v r}$ \\
\hline $1 \mathrm{a}$ & $1.1 \pm 0.1$ & 150 & $9.9 \pm 2.2$ & $6.1 \pm 0.6$ \\
\hline 2a & $4.7 \pm 0.3$ & 235 & $9.1 \pm 2.2$ & $8.7 \pm 0.6$ \\
\hline $3 \mathrm{a}$ & $4.0 \pm 0.2$ & 320 & $14.0 \pm 4.0$ & $9.7 \pm 1.2$ \\
\hline $4 \mathrm{a}$ & $5.8 \pm 0.4$ & 315 & $14.6 \pm 2.4$ & $16.0 \pm 1.7$ \\
\hline $5 \mathrm{a}$ & $5.4 \pm 0.5$ & 420 & $21.7 \pm 1.1$ & $13.5 \pm 0.5$ \\
\hline $6 \mathrm{a}$ & - & 415 & $19.4 \pm 0.9$ & $22.8 \pm 1.1$ \\
\hline
\end{tabular}

some types of instabilities in the motion of stars in the cluster models. For example, according to Ogorodnikov (1958, p. 398), in the stellar subsystem participating in a development of the dynamic instability, a rotation ("quasi-precession") of the ellipsoid of velocities of these stars in the plane $\left(v_{\xi}, v_{\eta}\right)$ leads to the fact that general distribution of stellar velocities of the system in this plane becomes circular.

A similar effect is also observed in our models, see, for example, the decrease with time of the differences between the values $\sigma_{v_{\eta}}^{2}$ and $\sigma_{v_{\xi}}^{2}$ in fig. 6.4. Usually for the considered OSC models, a relation $t_{s} \leq t_{\sigma}$ is fulfilled, see Tables 6.2 and 6.3. Perhaps the non-synchronous rotation of OSC relatively its orbital motion prevents the formation of a spherical distribution of stellar velocities in the cluster models. According to Tables 6.1-6.3, the values $t_{s}$ and $t_{\sigma}$ are several times larger than an average OSC lifetime $\sim 2 \times 10^{8}$ years (Wielen, 1971). This is an argument in favor of the fact that the process of synchronization of the rotational and orbital OSC motions, as well as the process of formation of the spherical distribution of stellar velocities in most of the observed OSC have not yet finished.

Let us note that the mean values $\sigma_{v_{\xi}}^{2}, \sigma_{v_{\eta}}^{2}$, and $\sigma_{v_{\zeta}}^{2}$ (by the oscillation period) mostly decrease over time, which is caused by tidal "heating" of the cluster and its expansion. Such changes with time of the mean $\sigma_{v_{\xi}}^{2}, \sigma_{v_{\eta}}^{2}$, and $\sigma_{v_{\zeta}}^{2}$ are more visible in models 1-4 and 1a-4a with a higher degree of non-stationarity in a regular field. The considered tides in the models in our work are dynamic. Therefore, the role of resonances between the frequency of the tide and oscillation frequencies of models 1-4 and 1a-4a can be significant in the energy dissipation of the tidal humps of these models. For the degree of non-stationarity of the models $1-4$, see Table 1 from the paper of Danilov and Dorogavtseva (2003). For the analysis of resonances between the periods of stellar motion and the period of oscillations of a regular field $P_{r}$ in models 1-6 see Danilov and Leskov (2005). In models 5, 6, and 5a, 6a, the stellar encounters may play a more important role in the dissipation of the energy 

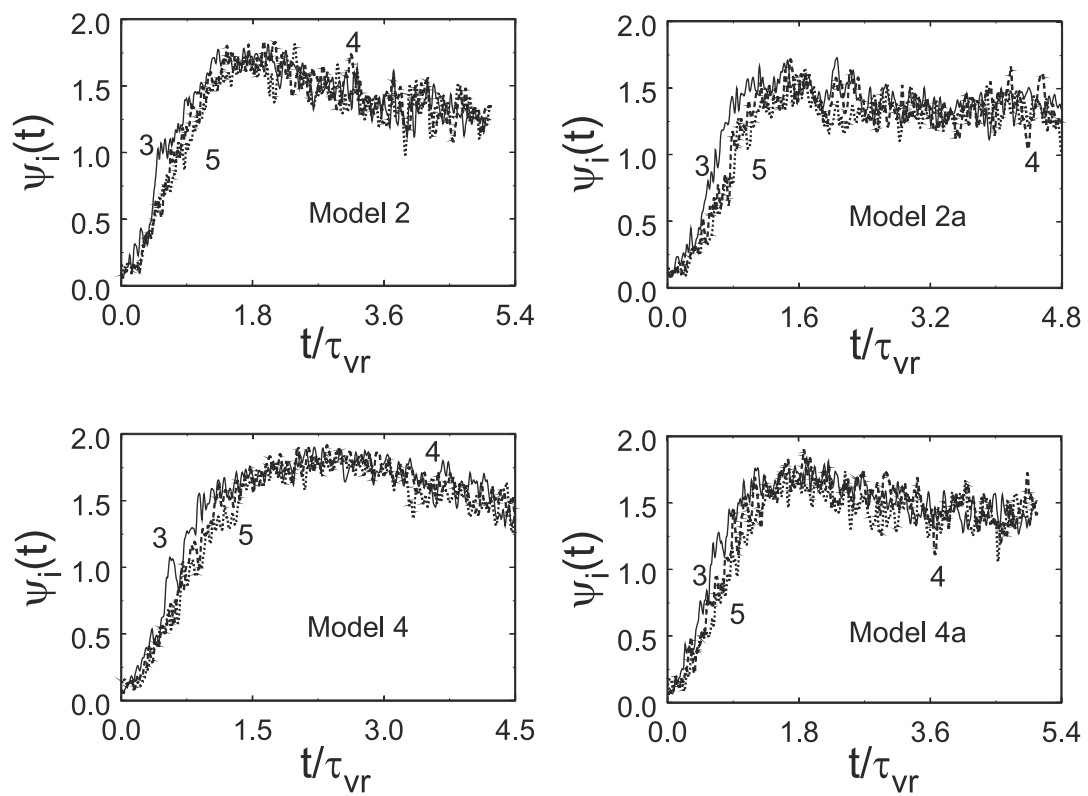

Fig. 6.5 The time dependencies of $\psi_{i}(t)$ in the cluster models 2, 2a, 4 and 4a. Digits 3, 4 and 5 indicate the curves corresponding to $i=3,4$, and 5

of tidal perturbations. As a result of the action of these mechanisms in time $t_{s}$, the rotation of the cluster is synchronized with its orbital motion, and the cluster itself "heats up" and partially disrupts.

Let us note that in the halo of models $2 \mathrm{a}, 3$ and $3 \mathrm{a}$, the mean values $\sigma_{v_{\xi}}^{2}, \sigma_{v_{\eta}}^{2}$ (averaged over the oscillation period) in the interval $t \in\left[t_{0}, 2 t_{0}\right]$ grow insignificantly with increasing $t$, which indicates the possibility of transferring of the part of the kinetic energy of the stellar motions along $\zeta$ axis to the stellar motions in the plane $(\xi, \eta)$ at time intervals $\Delta t \sim t_{0}$.

For all the considered cluster models, we have constructed the time dependencies of $\psi_{i}$ at the time interval $t \in\left[0, t_{0}\right]$. Analysis of the dependencies $\psi_{i}=\psi_{i}(t)$ confirms the conclusion of Danilov and Dorogavtseva (2003) about the decrease in the values of $\psi_{i}$ with time in the cores of the OSC models (with the larger number of models and for longer periods of time). The value $t_{0}$ grows with the increase of $i$ (in the regions of the cluster that are further from its center). Therefore, a decrease in the values $\psi_{i}$ (over time $t$ ) can be traced to the longest extent in time at the periphery of the cores of the cluster models, where the density of the number of stars and the frequency of stellar encounters are still large enough (according to Danilov and Dorogavtseva (2003)) that lead to a decrease in $\psi_{i}$ over time $t$.

According to fig. 6.5, in the cluster models $2 \mathrm{a}$ and $4 \mathrm{a}$, which do not rotate at $t=0$ relatively $\zeta$ axis in the coordinate system $(\xi, \eta, \zeta)$, the values $\psi_{i}$ only slightly decrease with time after reaching the highest values. This is due to greater non-stationarity 
and greater loss of stars in models $2 \mathrm{a}$ and $4 \mathrm{a}$ in comparison with models 2 and 4 (see fig. 6.5 and Table 6.3). The tendency to a weakening of the dependency of the cluster PDF on the influence of the small initial PCS perturbations with increasing $t$ at the considered time intervals is more noticeable in the cluster models $1-6$, that do not rotate relatively the external galaxies at $t=0$, contain a larger number of stars with angular momenta $l_{\zeta}<0$, and are more resistant to decay in the field of the Galaxy in comparison with models $1 \mathrm{a}-6 \mathrm{a}$.

\subsection{Conclusions}

1. We have considered the results of calculations of the dynamic evolution of 12 models of non-stationary OSC on time intervals $\Delta t=2 t_{0} \simeq(7.2-10.1) \tau_{v r}$. In the course of the dynamic evolution of the OSC models, dispersions of the stellar velocities in three mutually perpendicular directions undergo oscillations that do not damp for more than $(5-10) \tau_{v r}$. The main reasons for maintaining non-stationarity and oscillations of the considered cluster models along $\zeta$ axis are large initial deviation of the cluster from equilibrium in stellar motions along $\zeta$ axis, the constant loss of stars from the cluster, and the proximity of the OSC models to a gravitational instability mentioned earlier by Danilov and Dorogavtseva (2003); Danilov (2002b).

2 . We have given estimates of the synchronization time $t_{s}$ of the rotation of the OSC models with their motion around the center of the Galaxy. Depending on the parameters of the OSC models, the synchronization times are $t_{s} \simeq(5-27) \tau_{v r}$. For the cores of the considered OSC models, $t_{s}$ values are 1.4-2.7 times less than for the halo. The tides in the considered OSC models are dynamic. Therefore, an important role in dissipation of the energy of tidal perturbations in such OSC models should be played by resonances between the oscillation frequencies of the OSC and the frequency of the tide. In models 5, 5a, 6, 6a, which are closer to a virial equilibrium, the stellar encounters can play an appreciable role in the energy dissipation of the tidal perturbations. As a result of these mechanisms, during the time $t_{s}$, the cluster rotation is synchronized with its orbital motion, and the cluster itself "heats up" and partially disrupts. During the time $t_{0} \simeq(3.6-5.1) \tau_{v r}$, in the OSC models at distances $r \leq R_{t}$ from the cluster center, between 30 and $92 \%$ of the cluster stars are conserved.

3. We have performed the estimates of $t_{\sigma}$, the time of the formation of a spherical distribution in the OSC models. The values $t_{\sigma} 6.1-24.9$ times exceed the value $\tau_{v r}$, which indicates a very slow spherization of the velocity distribution in the considered models. $t_{\sigma}$ increase with the increase of the density of the model and the number of stars in the core of the cluster model. We have noticed the effect of instability in the cluster star motion on the process of spherization of the stellar velocity distribution in the considered OSC models. Usually, for these cluster models the relation $t_{s} \leq t_{\sigma}$ is fulfilled. Perhaps, the non-synchronous rotation of the OSC relatively to its orbital motion prevents the formation of a spherical distribution of stellar velocities in the cluster models. The values $t_{s}$ and $t_{\sigma}$ are several times higher than the mean OSC 
lifetime, which indicates that the process of synchronization of the rotational and orbital OSC motions, as well as the formation of the spherical distribution of stellar velocities in most of the observed OSC have not yet finished.

4. We have confirmed the conclusion of Danilov and Dorogavtseva (2003) (for a larger number of cluster models and at larger periods of time) about the presence in the cores of the OSC models of a tendency to decrease $\psi_{i}$ and to weaken the dependency of a coarse-grained PDF on the influence of the small initial PCS perturbations for the time points that are $\sim 5$ times further than the violent relaxation time.

5. We have noted that it is possible to monitor statistically the calculation accuracy of the characteristics of the OSC models $\left\langle v_{\varphi}\right\rangle$ and $\sigma_{i}^{2}, i=1,2,3$, averaged in the space of PCS, as well as the accuracy of $t_{s}, t_{\sigma}$ obtained from the data on $\left\langle v_{\varphi}\right\rangle$ and $\sigma_{i}^{2}$ at $t \in\left[t_{0}, t^{*}\right], t^{*}>t_{0}$. Comparison of the dependencies $\left\langle v_{\varphi}(t)\right\rangle$ (or $\sigma_{i}^{2}(t)$ ), obtained using the 10th and 11th accuracy orders at the time intervals $t \in\left[0, t^{*}\right]$, reveals a significant correlation between them $\left(k_{1,2} \in[0.65,0.98]\right)$. In the considered models, $t^{*} / \tau_{v r} \simeq(5.8-10.1)$ for the core, and $t^{*} / \tau_{v r} \simeq(6.1-10.1)$ for the halo of the cluster.

\section{References}

Allen, L., Megeath, S.T., Gutermuth, R., et al.: Structure and evolution of young stellar clusters. In: Reipurth, B., Jewitt, D., Keil, K. (eds) Protostars and Planets V. Univ. of Arizona Press, Tucson (2007), 361-376.

Ballesteros-Paredes, J., Klessen, R.S., Mac Low, M.-M., et al.: Molecular cloud turbulence and star formation. In: Reipurth, B., Jewitt, D., Keil, K. (eds) Protostars and Planets V. Univ. of Arizona Press, Tucson (2007), 63-80.

Chandrasekhar, S.: Principles of Stellar Dynamics. Univ. Chicago Press, Chicago, Ill (1942). LCCN: 43-5608 (BKS1)

Chernin, A.D., Efremov, Yu.N.: Rotation of giant molecular clouds and hydrodynamic effects in the Galactic disc. Mon. Not. R. Astron. Soc. 275, 209-216 (1995). doi:10.1093/mnras/275.2.209

Danilov, V.M.: Numerical experiments simulating the dynamics of open clusters in the Galactic field. Astron. Reports 41, 163-173 (1997a).

Danilov, V.M.: Statistical analysis of dynamical open-cluster models with small differences in their initial stellar phase coordinates. Astron. Letters, 23, 322-326 (1997b).

Danilov, V.M.: Stellar Fluxes in Numerical Dynamical Models of Open Clusters. Astron. Reports 46, 887-899 (2002b). doi:10.1134/1.1522077

Danilov, V.M., Dorogavtseva, L.V.: Estimates of Relaxation Times in Numerical Dynamical Models of Open Star Clusters. Astron. Reports 47, 483-491 (2003). doi:10.1134/1.1583775 
Danilov, V.M., Dorogavtseva, L.V.: Timescales for mechanisms for the dynamical evolution of open star clusters. Astron. Reports 52, 467-478 (2008). doi:10.1134/S1063772908060048

Danilov, V.M., Leskov, E.V.: Properties of Stellar Trajectories in Numerical Dynamical Models of Open Star Clusters. Astron. Reports 49, 190-200 (2005). doi:10.1134/1.1882777

Danilov, V.M., Seleznev, A.F.: The catalogue of structural and dynamical characteristics of $103 \mathrm{OCl}$ and the first results of its investigations. Astron. Astrophys. Trans. 6, 85-156 (1994). doi:10.1080/10556799408232061

Goodman, A.A., Benson, P.J., Fuller, G.A. et al.: Dense cores in dark clouds. VIII Velocity gradients. Astrophys. J. 406, 528-547 (1993). doi:10.1086/172465

Hairer, E., Norsett, S.P., Wanner, G.: Solving Ordinary Differential Equations I. Nonstiff Problems. Springer-Verlag, Berlin, Heidelberg (1987). doi:10.1007/9783-662-12607-3

Henon, M.: Collisional Dynamics in Spherical Stellar Systems. In: Contopoulos, G., Henon, M., Lynden-Bell, D. (eds) Dynamical Structure and Evolution of Stellar Systems., Observatoire de Geneve, 1290 Sauverny, Switzerland (1973), 183-260.

Hut P.: Tidal evolution in close binary systems. Astron. Astrophys. 99, 126-140 (1981).

King, I.R.: The structure of star clusters. I. An empirical density law. Astron. J. 67, 471-485 (1962). doi:10.1086/108756

Kutuzov, S.A., Osipkov, L.P.: A Generalized Model for the Three-Dimensional Gravitational Potential of Stellar Systems. Soviet Astron. 24, 17-22 (1980).

Lecar, M., Wheeler, J.C., McKee, C.F.: Tidal circularization of the binary X-ray sources Hercules X-1 and Centaurus X-3. Astrophys. J. 205, 556-562 (1976). doi: $10.1086 / 154311$

Long, K., Weinberg, M.D.: Tidal Influence on Satellite Shapes. In: American Astronomical Society, 183rd AAS Meeting, id.108.14; Bulletin of the American Astronomical Society, 25, 1453 (1993).

Mardling, R.A., Aarseth, S.J.: Tidal interactions in star cluster simulations. Mon. Not. R. Astron.Soc. 321, 398-420 (2001). doi:10.1046/j.1365-8711.2001.03974.x

Ogorodnikov, K.F.: Dynamics of stellar systems. GIFML, Moscow (1958) (In Russian).

Phillips, J.P.: Rotation in molecular clouds. Astron. Astrophys. Suppl. Ser. 134, 241-254 (1999). doi:10.1051/aas:1999137

Pirogov, L., Zinchenko, I., Caselli, P., et al.: N2H+(1-0) survey of massive molecular cloud cores. Astron. Astrophys. 405, 639-654 (2003). doi:10.1051/00046361:20030659

Shchekinov, Yu.A., Zinchenko, I.I.: The Origin of Giant Molecular Clouds. Astron. Reports 48, 629-637 (2004). doi:10.1134/1.1787065

Spitzer, L., Thuan, T.X.: Random Gravitational Encounters and the Evolution of Spherical Systems. IV. Isolated Systems of Identical Stars. Astrophys. J. 175, 31-61 (1972). doi:10.1086/151537

Surdin, V.G.: The fifth force. MCNMO, Moscow (2002) (In Russian). 
Weinberg, M.D.: How Has Dynamical Evolution Changed the Galactic Globular Clusters? In: Smith, G.H., Brodie, J.P. (eds) The globular cluster-galaxy connection. ASP Conf. Ser., 48, 689-700 (1993).

Wielen, R.: On the Lifetimes of Galactic Clusters. Astrophys. Space Sci. 13, 300-308 (1971). doi:10.1007/BF00649160 



\title{
Chapter 7
}

\section{Analysis of Density Oscillations in Models of Open Star Clusters}

\begin{abstract}
In the numerical dynamic OSC models of Danilov and Dorogavtseva (2003); Danilov (1999); Danilov and Dorogavtseva (2008), the large-scale oscillfations of a density and of a regular field are spontaneously and rapidly developing. We studied these oscillations and, on a basis of that, established and investigated the instability of solutions for phase density function (PDF) to small initial perturbations of the phase coordinates of stars (PCS) (Danilov and Dorogavtseva, 2003; Danilov, 1999). Danilov and Dorogavtseva (2008) used the possibility of the gravitational instability development in the considered OSC models to explain some of the results obtained numerically (oscillations of the values of the virial coefficient and dispersion of the cluster stars' velocities that do not decay during large time intervals; decrease of the time of a spherization of the stellar velocity distribution in the least dense OSC models with the greatest degree of non-stationarity in a regular field). We note that, at present, the theoretical description of the gravitational instability in such complex, inhomogeneous in a density and non-isolated objects as OSC is absent.
\end{abstract}

\subsection{Introduction}

In the papers Danilov $(2005,2006)$ we considered the theoretical models of a stellar motion on the periphery of OSC. Danilov (2005) used the model of the potential of a nonisolated homogeneous gravitating ellipsoid in order to approximate the average (by the oscillation period) regular field of a potential at the periphery of the numerical dynamic OSC models. We could completely integrate the equations of a stellar motion in the joint field of such an ellipsoid and the Galaxy, written according to Chandrasekhar (1942), and also obtained three integrals of motion. An analysis of these integrals made it possible to construct a two-integral PDF and a three-integral general stellar velocity distribution in the considered model of a homogeneous ellipsoid. 
An amplitudes of the regular potential oscillations near the center and at the periphery of the numerical models of a non-stationary OSC (Danilov and Dorogavtseva, 2003), according to Danilov (2005), are at least $6 \%$ of the regular potential averaged over its oscillation period $P_{r}$. The mean value of the potential (averaged over $P_{r}$ ) near the centers of the OSC models (Danilov and Dorogavtseva, 2003) is 2.6-3.3 times greater than at the periphery (near the critical surface of zero velocities of stellar motion (Danilov, 2005)). Therefore, the amplitudes of the potential oscillations in the cores of the cluster models are the greatest in absolute value. The stellar motions in the cores (Danilov and Dorogavtseva, 2003) of the OSC models have the greatest degree of stochasticity (Danilov and Leskov, 2005). Properties of the stellar trajectories and the cluster potential noted here complicate the analysis of the stellar motions and the perturbations of the PDF in the OSC cores. Theoretical description of the phase density oscillations in the cores of a non-stationary non-isolated OSC is currently possible only, if one actively apply the results of a numerical modeling of the dynamics for such clusters.

The points of a great interest are: 1) the gross-dynamic analysis of the density oscillation frequencies in the non-isolated ellipsoidal OSC models (the results of such study can be applied to simplify the analysis of the phase-density oscillations in such systems); 2) the use of the model of a potential of a non-isolated homogeneous gravitating ellipsoid to construct an equilibrium three-integral PDF and describe the dynamics of the OSC central regions; 3 ) application of the Boltzmann equation for the analysis of the phase density oscillations in the OSC cores.

The objective of this chapter is to discuss the oscillations of a density and a phase density in the non-isolated ellipsoidal OSC models.

\subsection{Oscillations of a Homogeneous Triaxial Gravitating Ellipsoid}

Following to Danilov (2005), we consider the cluster moving in the Galactic plane along the circular orbit around the Galactic center with an angular velocity $\omega=$ const. The equations of a stellar motion are written in the rotating coordinate system $\xi, \eta, \zeta$ (Chandrasekhar, 1942). Following to Chandrasekhar (1942), we expanded the regular potential of the Galaxy in a series up to quadratic terms in the coordinates $\xi, \eta, \zeta$. According to Danilov (2005) and Chandrasekhar (1942), the equations of a stellar motion in the joint force field of the Galaxy and the cluster have the following form:

$$
\ddot{\xi}=-\beta_{1}^{2} \xi+2 \omega \dot{\eta}, \quad \ddot{\eta}=-\beta_{2}^{2} \eta-2 \omega \dot{\xi}, \quad \ddot{\zeta}=-\beta_{3}^{2} \zeta,
$$

where $\dot{\xi}=\frac{d \xi}{d t}, \ddot{\xi}=\frac{d \dot{\xi}}{d t}$, the values $\dot{\eta}, \dot{\zeta}, \ddot{\eta}, \ddot{\zeta}$ are defined similarly;

$$
\beta_{l}^{2}=2\left(U_{0}+U_{1}\right) / a_{l}^{2}, \quad l=1,2,3,
$$

$a_{1}=a, a_{2}=b, a_{3}=c$ 


$$
-\beta_{1}^{2} \xi=\frac{\partial U}{\partial \xi}-\alpha_{1} \xi, \quad-\beta_{2}^{2} \eta=\frac{\partial U}{\partial \eta}, \quad-\beta_{3}^{2} \zeta=\frac{\partial U}{\partial \zeta}-\alpha_{3} \zeta
$$

$\alpha_{1}$ and $\alpha_{3}$ are constants characterizing the force field of the Galaxy in the vicinity of the circular cluster orbits (their numerical values are defined by Danilov (2005) applying the model of a Galactic potential of Kutuzov and Osipkov (1980)).

In equations (7.1), a potential $U$ of the cluster is used in the form

$$
U=U_{0}-\left(U_{0}+U_{1}\right) k^{2}+S, \quad k^{2}=\left(\frac{\xi}{a}\right)^{2}+\left(\frac{\eta}{b}\right)^{2}+\left(\frac{\zeta}{c}\right)^{2}, \quad S=\frac{\alpha_{1} \xi^{2}+\alpha_{3} \zeta^{2}}{2} .
$$

Constant values $U_{0}$ and $U_{1}$ were obtained by Danilov (2005) for six OSC models by an approximating of the cluster potential at its periphery by the potential of some homogeneous ellipsoid (for the sake of brevity, we will henceforth call it $H$-ellipsoid); potential of this ellipsoid is set by the formula (7.2); $a, b, c$ are the semiaxes of $H$-ellipsoid, dimensions of which coincide with the dimensions of a critical surface of zero velocities of the cluster (Danilov, 2005).

We use a gross-dynamic description of the evolution of a gravitating ellipsoid in order to study its oscillations near the corresponding to $H$-ellipsoid state of a virial equilibrium (Danilov, 2005). In this case, the integral characteristics of a gravitating ellipsoid as a whole are usually considered (Kuzmin, 1965).

Danilov (2008) obtained the equations for the total kinetic energies $T_{l}$ of the cluster star motions along the axes $\xi, \eta, \zeta$ in the force field of the Galaxy and $H$ ellipsoid applying the equations (7.1). The same equations are applied to obtain the angular momentum $L_{3}$ of the $H$-ellipsoid rotation around $\zeta$ axis,

$$
\dot{T}_{l}=-\frac{\beta_{l}^{2}}{2} \dot{I}_{l}, \quad \dot{L}_{3}=-\omega\left(\dot{I}_{1}+\dot{I}_{2}\right),
$$

as well as the equations for the diagonal elements of the inertia tensor of $H$-ellipsoid $I_{l}, l=1,2,3$. The equation for $L_{3}$ is easily integrated:

$$
L_{3}=-\omega\left(I_{1}+I_{2}\right)+L_{3}(0), \text { where } L_{3}(0)=\text { const } .
$$

Let $L_{3}(0)=0$. According to Danilov (2006), a general three-integral velocity distribution of stars in the model of $H$-ellipsoid is triaxial and ellipsoidal. Taking into account an expression for $L_{3}$, as well as the symmetry of the star distribution in the model of the $H$-ellipsoid in the space of coordinates and velocities, we reduce the equations for $I_{l}$ values to the following form:

$$
\begin{aligned}
& \ddot{I}_{1}=4 T_{1}-2 \beta_{1}^{2} I_{1}-2 \omega^{2}\left(I_{1}+I_{2}\right) ; \\
& \ddot{I}_{2}=4 T_{2}-2 \beta_{2}^{2} I_{2}-2 \omega^{2}\left(I_{1}+I_{2}\right) ; \\
& \ddot{I}_{3}=4 T_{3}-2 \beta_{3}^{2} I_{3} .
\end{aligned}
$$

The first three equations from (7.3) together with the equations (7.4) form a closed system for the values $T_{l}, I_{l}, l=1,2,3$. We apply these equations in order to estimate the oscillation frequencies of gravitating ellipsoid near the equilibrium state 
Table 7.1 Parameters of homogeneous ellipsoids

\begin{tabular}{|c|c|c|}
\hline $\mathcal{N}$ & $k_{h}$ & $U_{0} / U_{0}^{\prime}$ \\
\hline 1 & 0.37988 & 0.99617 \\
\hline 2 & 0.38026 & 0.98740 \\
\hline 3 & 0.37836 & 0.99429 \\
\hline 4 & 0.38416 & 1.00431 \\
\hline 5 & 0.38275 & 0.98633 \\
\hline 6 & 0.38453 & 1.01201 \\
\hline
\end{tabular}

corresponding to $H$-ellipsoid (for the sake of brevity, we shall refer to these oscillations as $H$-ellipsoid oscillations). The $\beta_{l}^{2}$ values depend nonlinearly on $I_{l}$. The potential $U_{0}^{\prime}$ in the center of an isolated homogeneous ellipsoid with density $\rho$ and semiaxes $a, b, c$ is related to the potential energy $W^{\prime}$ of this ellipsoid as follows: $W^{\prime}=-\frac{2}{5} M U_{0}^{\prime}$, where $M$ is the ellipsoid mass (Subbotin, 1949, p. 40-41). For six numerical dynamic non-isolated OSC models considered by Danilov (2005), the values $k_{h}=-W_{0} /\left(M U_{0}\right)$ for $H$-ellipsoid and $U_{0} / U_{0}^{\prime}$ have been defined by Danilov (2008) and are listed in Table 7.1. Here, $W_{0}$ and $U_{0}$ are the potential energy and the potential at the center of $H$-ellipsoid.

According to Table 7.1, $k_{h}$ in the considered $H$-ellipsoid models is close to 0.4 , and the value of $U_{0} / U_{0}^{\prime}$ differs little from unity. Differences in the potentials $U_{0}$ and $U_{0}^{\prime}$ are 1.5-4.7 times smaller than the approximation errors for the $U_{0}$ value (Danilov, 2005). Therefore, for an approximate evaluation of the variations $\delta \beta_{l}^{2}$ of the $\beta_{l}^{2}$ values, we will further apply the relations $U_{0} \simeq U_{0}^{\prime}$ and $W_{0}=-k_{h} U_{0} M$.

Following to Danilov (2005), for the value $-W_{0}$ we write

$$
k_{h} U_{0} M=\frac{M}{2}\left\{U_{0}-0.1\left[6\left(U_{0}+U_{1}\right)-\alpha_{1} a^{2}-\alpha_{3} c^{2}\right]\right\} .
$$

From this relation, we find

$$
U_{0}+U_{1}=\left[h U_{0}+0.5\left(\alpha_{1} a^{2}+\alpha_{3} c^{2}\right)\right] / 3,
$$

where $h=5\left(1-2 k_{h}\right)$. Let us to use $U_{0}^{\prime}$ as $U_{0}$ in the expression for $U_{0}+U_{1}$. Varying $U_{0}+U_{1}$, we find

$$
\delta\left(U_{0}+U_{1}\right)=-\frac{5 G h}{8} \sum_{l=1}^{3} A_{l} \delta I_{l}+\frac{5}{6 M}\left(\alpha_{1} \delta I_{1}+\alpha_{3} \delta I_{3}\right)
$$

where

$$
\begin{gathered}
A_{l}=\int_{0}^{\infty} \frac{d s}{\left(a_{l}^{2}+s\right) \Delta(s)} ; \\
\Delta(s)=\sqrt{\left(a_{1}^{2}+s\right)\left(a_{2}^{2}+s\right)\left(a_{3}^{2}+s\right)}
\end{gathered}
$$


(Danilov, 2006); $a_{l}^{2}=5 I_{l} / M ; l=1,2,3 ; G$ is the gravitational constant.

$$
\delta \beta_{l}^{2}=\left[2 \delta\left(U_{0}+U_{1}\right)-\beta_{l}^{2} \delta a_{l}^{2}\right] / a_{l}^{2} .
$$

The formula (7.5) allows to define the values $\delta \beta_{l}^{2}$ according to the data on $H$-ellipsoid parameters. Let $I_{l}=I_{0, l}+\delta I_{l}, T_{l}=T_{0, l}+\delta T_{l}$, where $I_{0, l}, T_{0, l}$ are constant values; $l=1,2,3$. Let $\left|\delta I_{l}\right|<<I_{0, l},\left|\delta T_{l}\right|<<T_{0, l}$. Varying first three equations from (7.3), equations (7.4), considering the expressions obtained for $\delta \beta_{l}^{2}$, we write linearized equations for the values $\delta I_{l}, \delta T_{l}$ :

$$
\begin{gathered}
\delta \ddot{I}_{1}=4 \delta T_{1}-\delta Q, \quad \delta \ddot{I}_{2}=4 \delta T_{2}-\delta Q ; \\
\delta \ddot{I}_{3}=4 \delta T_{3}+2 \tau_{1} \delta I_{1}+2 \tau_{2} \delta I_{2}-2 Q_{3} \delta I_{3}, \quad \delta \dot{T}_{l}=-\frac{\beta_{l}^{2}}{2} \delta \dot{I}_{l},
\end{gathered}
$$

where

$$
\begin{gathered}
\delta Q=2\left(Q_{1} \delta I_{1}+Q_{2} \delta I_{2}+Q_{3} \delta I_{3}\right) ; \\
Q_{1}=\omega^{2}-\tau_{1}<0 ; \quad Q_{2}=\omega^{2}-\tau_{2}<0 ; \quad Q_{3}=-\frac{G h M A_{3}}{4}+\alpha_{3} / 3>0 ; \quad l=1,2,3 ; \\
\tau_{1}=\frac{G h M A_{1}}{4}-\alpha_{1} / 3>0 ; \quad \tau_{2}=\frac{G h M A_{2}}{4}>0 .
\end{gathered}
$$

The signs of the values $Q_{i}$ and $\tau_{j}$ are given here considering the data on the $\mathrm{H}$ ellipsoid parameters in the cluster model 1 of Danilov (2005).

Characteristic equation of the system (7.6) has the form

$$
\mu^{3}\left(\mu^{6}+2 L \mu^{4}+4 N \mu^{2}+8 P\right)=0,
$$

where $\mu$ is an eigenvalue of the matrix of system (7.6);

$$
\begin{gathered}
L=\sum_{i=1}^{3}\left(\beta_{i}^{2}+Q_{i}\right) ; \\
N=Q_{1}\left(\beta_{2}^{2}+\beta_{3}^{2}\right)+Q_{2}\left(\beta_{1}^{2}+\beta_{3}^{2}\right)+Q_{3}\left(2 \omega^{2}+\beta_{1}^{2}+\beta_{2}^{2}\right)+\beta_{1}^{2} \beta_{2}^{2}+\beta_{3}^{2}\left(\beta_{1}^{2}+\beta_{2}^{2}\right) ; \\
P=\beta_{1}^{2} \beta_{2}^{2} \beta_{3}^{2}+Q_{1} \beta_{2}^{2} \beta_{3}^{2}+Q_{2} \beta_{1}^{2} \beta_{3}^{2}+Q_{3}\left[\beta_{1}^{2} \beta_{2}^{2}+\omega^{2}\left(\beta_{1}^{2}+\beta_{2}^{2}\right)\right] .
\end{gathered}
$$

The first three roots of the equation (7.7) equal to zero $\left(\mu_{1,2,3}=0\right)$. We denote $\lambda=\mu^{2}$. Equating the expression in the parentheses in (7.7) to zero, we obtain a bicubic equation for $\lambda$ :

$$
\lambda^{3}+2 L \lambda^{2}+4 N \lambda+8 P=0 .
$$

With a help of a standard substitution $\lambda=\chi-2 L / 3$ (Korn and Korn, 1968, p. 44) this equation can be reduced to a simpler (incomplete) form:

$$
\chi^{3}+p \chi+q=0
$$

where

$$
p=-(2 L)^{3} / 3+4 N ; \quad q=2(2 L / 3)^{3}-8 L N / 3+8 P .
$$


In the case of $H$-ellipsoid of the OSC model 1 (Danilov, 2005), we find

$$
(p / 3)^{3}+(q / 2)^{2}<0, \quad p<0, \quad q>0 .
$$

According to Korn and Korn (1968), there are three different real roots of the equation $\chi^{3}+p \chi+q=0$

$$
\begin{gathered}
\chi_{1}=2 \sqrt{-p / 3} \cos (\alpha / 3), \quad \chi_{2,3}=-2 \sqrt{-p / 3} \cos (\alpha / 3 \pm \pi / 3), \\
\cos (\alpha)=-q /\left(2 \sqrt{(-p / 3)^{3}}\right) .
\end{gathered}
$$

The obtained values $\chi_{l}$ in our case correspond to $\lambda_{l}<0, l=1,2,3$. Therefore, the roots $\mu_{4}, \ldots, \mu_{9}$ of the equation (7.7) are imaginary, and can be written in the form $\mu_{4,5}= \pm i \omega_{1}, \mu_{6,7}= \pm i \omega_{2}, \mu_{8,9}= \pm i \omega_{3}$, where $i=\sqrt{-1}$. In the case of $H$-ellipsoid of the cluster model 1 (Danilov, 2005), the periods $P_{l}$ corresponding to the frequencies $\omega_{l}$ are: $P_{1} \simeq 38.08723 \mathrm{Myr}, P_{2} \simeq 52.86999 \mathrm{Myr}, P_{3} \simeq 84.46301 \mathrm{Myr}$. The $P_{3}$ value is in a good agreement with the largest periods of the halo stars motion in the cluster model 1 (Danilov, 2005) (see fig. 8a,b in the paper of Danilov and Leskov (2005)).

Thus, oscillations of the considered triaxial homogeneous ellipsoid are stable, which agrees with the conclusion of Ossipkov (2001) on the stability of oscillations of biaxial gravitating ellipsoid in the joint force field of this ellipsoid and the Galaxy.

Let us consider a simpler case of $H$-ellipsoid oscillations under the condition that the relations between the ellipsoid semiaxes preserve constant. In this case, the equations (7.3) and (7.4) lead to the following equations for the moment of inertia $I=\sum_{l=1}^{3} I_{l}$ of the ellipsoid and of total kinetic energy $T=\sum_{l=1}^{3} T_{l}$ of star motion in this ellipsoid:

$$
\ddot{I}=4 T-2\left(\beta^{2}+2 \omega^{2} \gamma^{2}\right) I, \quad \dot{T}=-\frac{\beta^{2}}{2} \dot{I},
$$

where

$$
\beta^{2}=\sum_{l=1}^{3} \beta_{l}^{2} / q_{l} ; \quad q_{l}=\sum_{j=1}^{3} a_{j}^{2} / a_{l}^{2} ; \quad I_{l}=I / q_{l} ; \quad \gamma^{2}=q_{1}^{-1}+q_{2}^{-1} .
$$

Let $I=I_{0}+\delta I, T=T_{0}+\delta T,|\delta I|<<I_{0},|\delta T|<<T_{0} ; I_{0}$ and $T_{0}$ are constant values. After a linearization of equations (7.8) we find

$$
\ddot{\delta} I=4 \delta T-2 K \delta I, \quad \dot{\delta} T=-\frac{\beta^{2}}{2} \dot{\delta} I,
$$

where

$$
\begin{gathered}
K=\beta^{2}+2 \omega^{2} \gamma^{2}+F / 4 ; \\
F=\left(-3 G h M A_{1}-4 \beta_{1}^{2}+4 \alpha_{1}\right) / q_{1}-\left(3 G h M A_{2}+4 \beta_{2}^{2}\right) / q_{2}+\left(-3 G h M A_{3}-4 \beta_{3}^{2}+4 \alpha_{3}\right) / q_{3} .
\end{gathered}
$$

The characteristic equation of the system (7.9) has the form

$$
\mu^{3}+2 \mu\left(K+\beta^{2}\right)=0 .
$$


The roots of this equation are

$$
\mu_{1}=0, \mu_{2,3}= \pm \sqrt{-2\left(K+\beta^{2}\right)}= \pm i \omega_{h},
$$

where $\omega_{h}^{2}=2\left(K+\beta^{2}\right)>0 ; \omega_{h}$ is the oscillation frequency of $H$-ellipsoid at the constant values of $q_{j} ; j=1,2,3$. In the case of $H$-ellipsoid of the OSC model 1 (Danilov, 2005), the frequency $\omega_{h}$ of the ellipsoid homologous oscillations corresponds to the oscillation period of $P_{h} \simeq 68.33937 \mathrm{Myr}$. The value $P_{h}$ is slightly different (1.16873 times) from the mean value of $P_{0}=\frac{1}{3} \sum_{l=1}^{3} P_{l} \simeq 58.47341 \mathrm{Myr}$, where $P_{l}$ are the periods obtained using the equation (7.7). As in the case of (7.7), the considered oscillations of $H$-ellipsoid are stable.

\subsection{Nonhomological Oscillations of $\boldsymbol{H}$-Ellipsoid}

Following to Danilov (2008), we consider non-homological oscillations of the $\mathrm{H}$ ellipsoid. In this case, the distributions of a density along the distance from the center in the ellipsoid at different time points can not be considered as similar. Suppose that the perturbation $\delta \rho$ of ellipsoid density $\rho$ does not lead to a change of a symmetry in the matter distribution in the ellipsoid; $\rho=\rho_{0}+\delta \rho,|\delta \rho|<<\rho_{0}, \rho_{0}=$ const. Let

$$
\delta \rho=\sum_{n=0}^{\infty} b_{n} k^{2 n}
$$

where the value of $k^{2}$ was defined in (7.2). The condition of a constancy of the ellipsoid mass leads to the following restriction in $b_{n}$ :

$$
\sum_{n=0}^{\infty} \frac{b_{n}}{2 n+3}=0 .
$$

We carried out the calculation of the integrals, corresponding to (7.12), over the ellipsoid volume in the coordinate system $k, \theta$, $\phi$, where $k \in[0,1] ; \theta \in[0, \pi]$; $\phi \in[0,2 \pi]$. These coordinates are connected with $\xi, \eta, \zeta$ by the following relations: $\xi=a k \sin (\theta) \cos (\phi), \eta=b k \sin (\theta) \sin (\phi), \zeta=c k \cos (\theta)$. The volume elements in the considered coordinate systems are connected by a relation

$$
d V=d \xi d \eta d \zeta=a b c k^{2} d k \sin (\theta) d \theta d \phi
$$

where $V$ is the volume of the $H$-ellipsoid in the coordinate system $\xi, \eta, \zeta$. The condition (7.12) means that $\int_{V} \delta \rho d V=0$ (in this case, the value of $\delta \rho$ averaged by volume $V$ equals to zero, i.e. $\langle\delta \rho\rangle=0$ ). Since the mass of the $H$-ellipsoid $M=V\langle\rho\rangle=V \rho_{0}=$ const, then $\delta M=\rho_{0} \delta V=0$. Consequently, $\delta V=0$ and $V=$ const . Let the largest $n$ be $n_{m}=1$. Then, from the a condition $M=$ const in (7.12), we find $b_{1}(t)=-5 b_{0}(t) / 3$. 
Following Danilov (2005) and Duboshin (1975, p. 149), we write the equations of a stellar motion in the joint field of inhomogeneous ellipsoid and the Galaxy:

$$
\begin{aligned}
& \ddot{\xi}=-\beta_{1}^{2} \xi+2 \omega \dot{\eta}-\lambda_{0} b_{0} \xi \sigma_{1}, \\
& \ddot{\eta}=-\beta_{2}^{2} \eta-2 \omega \dot{\xi}-\lambda_{0} b_{0} \eta \sigma_{2}, \\
& \ddot{\zeta}=-\beta_{3}^{2} \zeta-\lambda_{0} b_{0} \zeta \sigma_{3},
\end{aligned}
$$

where

$$
\begin{gathered}
\lambda_{0}=2 \pi G a b c, \quad \sigma_{l}=A_{l}-\frac{5}{3} \sum_{j=1}^{3} x_{j}^{2} A_{l j} ; \\
x_{1}=\xi ; \quad x_{2}=\eta ; \quad x_{3}=\zeta ; \quad A_{l j}=\int_{0}^{\infty} \frac{d s}{\left(a_{l}^{2}+s\right)\left(a_{j}^{2}+s\right) \Delta(s)} .
\end{gathered}
$$

Let $q_{l}=$ const $, l=1,2,3$. Calculations similar to those performed in (7.8) lead to the following equations for the moment of inertia $I$ and the kinetic energy $T$ :

$$
\begin{gathered}
\ddot{I}=4 T-2\left(\beta^{2}+2 \omega^{2} \gamma^{2}+\lambda_{0} b_{0} g^{2}\right) I+\frac{50}{21} \lambda_{0} b_{0} s_{1}^{2} B I^{2} /\left(C^{2} M\right), \\
\dot{T}=-\frac{\beta^{2}+\lambda_{0} b_{0} g^{2}}{2} \dot{I},
\end{gathered}
$$

where

$$
\begin{aligned}
& g^{2}=\sum_{l=1}^{3} A_{l} / q_{l} ; \quad C=1-4 b_{0} /\left(21 \rho_{0}\right) ; \quad B=1-8 b_{0} /\left(27 \rho_{0}\right) \\
& s_{1}^{2}=3 \sum_{l=1}^{3} A_{l l} / q_{l}^{2}+2\left(A_{12} /\left(q_{1} q_{2}\right)+A_{13} /\left(q_{1} q_{3}\right)+A_{23} /\left(q_{2} q_{3}\right)\right) .
\end{aligned}
$$

Here, the moment of inertia of the inhomogeneous ellipsoid is written in the form $I=I_{0}+\delta I=I_{0} C$, where $I_{0}=$ const corresponds to $I$ at $b_{0}=0$ and $\delta \rho=0$. Therefore, $\dot{I}=\dot{\delta} I$ and the $I$ oscillation frequency $\omega_{n h}$ equals to the $b_{0}(t)$ oscillation frequency.

We linearize the equations (7.14) by setting $|\delta I|<<I_{0}$, considering $I_{0}=I / C$ in the first equation (7.14) and expressing $b_{0}$ and $B$ through $I$ and $I_{0}$ :

$$
\ddot{\delta} I=4 \delta T-2 K_{1} \delta I, \quad \dot{\delta} T=-\frac{\beta^{2}}{2} \dot{\delta} I
$$

where

$$
K_{1}=\beta^{2}+2 \omega^{2} \gamma^{2}+\lambda_{0} \rho_{0}\left(25 s_{1}^{2} I_{0} / M-21 g^{2}\right) / 4 .
$$

The characteristic equation of the system (7.15) has the form

$$
\mu^{3}+2 \mu\left(K_{1}+\beta^{2}\right)=0 .
$$

The roots of this equation are 


$$
\mu_{1}=0, \quad \mu_{2,3}= \pm \sqrt{-2\left(K_{1}+\beta^{2}\right)}= \pm i \omega_{n h},
$$

where

$$
\omega_{n h}^{2}=2\left(K_{1}+\beta^{2}\right)>0 \text {. }
$$

In the case of the $H$-ellipsoid of the cluster model 1 (Danilov, 2005), the period of the non-homological oscillations corresponding to the frequency $\omega_{n h}$ equals $P_{n h} \simeq 59.24792$ Myr. Therefore, $P_{n h}<P_{h}\left(P_{n h} \simeq P_{h} / 1.15345\right)$, and $\omega_{n h}>\omega_{h}$. Thus, the non-homological oscillations of the $H$-ellipsoid considered here are also stable. The value $\omega_{n h}=0$ is a critical one for the stability of such oscillations. Therefore, the oscillations of the type (7.11) have a larger "reserve" of stability than the homological oscillations considered in section 7.2.

\subsection{Three-Integral Equilibrium Function of Phase Density}

In order to describe the stellar motions in the cluster core, we can apply the threeintegral phase density function (PDF) in the form considered by Danilov (2006):

$$
\Psi=\Psi(\mathcal{T}), \quad \text { where } \quad \mathcal{T}=J_{1}+\kappa^{2} J_{2}+\sigma^{2} J_{3} ;
$$

where $J_{l}$ are the integrals of the motion $(l=1,2,3) ; \kappa^{2}$ and $\sigma^{2}$ are the constant values. To calculate the function $\Psi=\Psi(\mathcal{T})$, we need to approximate the potential in the center (and near the center) of the numerical dynamic cluster model (averaged over the oscillation period of a regular field) by the potential of a dense homogeneous ellipsoid (for brevity, we will call it $C$-ellipsoid). Such approximation can be performed using the technique described by Danilov (2005) in constructing of the model of the $H$-ellipsoid. In this case, the sizes of semi-axes of the $C$ - and $H$-ellipsoids coincide.

According to Danilov $(2006,2008)$ the function $\Psi(\mathcal{T})$ can be written in the form

$$
\Psi(\mathcal{T})=Q\left(\mathcal{T}_{\max }-\mathcal{T}\right), \quad \mathcal{T}=\frac{\left(\dot{\xi}-\dot{\xi}_{0}\right)^{2}}{a_{T}^{2}}+\frac{\left(\dot{\eta}-\dot{\eta}_{0}\right)^{2}}{b_{T}^{2}}+\kappa^{2} \dot{\zeta}^{2}+\psi(\xi, \eta, \zeta),
$$

where $\mathcal{T}_{\text {max }}, a_{T}^{2}$ and $b_{T}^{2}$ are constant values; $\dot{\xi}_{0}$ and $\dot{\eta}_{0}$ are linear functions of $\eta$ and $\xi$ respecrively (formulas for the values $a_{T}^{2}, b_{T}^{2}, \dot{\xi}_{0}$, and $\dot{\eta}_{0}$ are given in the explanations for the formulas (18), (19) in the paper of Danilov (2006));

$$
\psi(\xi, \eta, \zeta)=a_{Q}^{-2} \xi^{2}+b_{Q}^{-2} \eta^{2}+c_{Q}^{-2} \zeta^{2} .
$$

Formulas for the constant coefficients $a_{Q}^{2}, b_{Q}^{2}, c_{Q}^{2}$ can easily be obtained by using formula (35) from the paper of Danilov (2006).

As a result of an approximating of the potential $U$ (averaged by the oscillation period of the regular field) near the center (at $k \in[0,0.1]$ ) of the cluster model 1 (Danilov, 2005) by the formula (7.2), the following constants were obtained by 
Danilov (2008): $U_{0} \simeq 0.65059 \pm 0.032908(\mathrm{pc} / \mathrm{Myr})^{2}, U_{1} \simeq-0.21736 \pm 0.023245$ $(\mathrm{pc} / \mathrm{Myr})^{2}$. The eigenfrequencies of the stellar motion in the joint field of $C$-ellipsoid and the Galaxy, according to formulas (7) from the paper of Danilov (2005), are: $v_{1} \simeq$ $0.07891(\mathrm{Myr})^{-1}, v_{2} \simeq 0.15093(\mathrm{Myr})^{-1}, \beta_{3} \simeq 0.17821(\mathrm{Myr})^{-1}$; it is, respectively, $\sim 1.76884,1.39888,1.57304$ times larger than the values $v_{1}, v_{2}, \beta_{3}$ obtained for the stellar motion at the periphery of the $H$-ellipsoid of the cluster model 1 by Danilov (2005); $\rho_{0} \simeq 0.90429 M_{\odot} / \mathrm{pc}^{3}$ and $M \simeq 1439.48916 M_{\odot}$ are the density and the mass of $C$-ellipsoid, respectively.

In order to estimate $\sigma^{2}$ and $\kappa^{2}$, Danilov (2008) considers a sample of 368 stellar trajectories in the cluster model 1 (Danilov and Dorogavtseva, 2003; Danilov, 2005). According to Danilov and Leskov (2005), these trajectories correspond to the large positive values of MLCE which exceed the mean value of MLCE of stellar trajectories in this cluster model. According to the estimates made by Danilov and Leskov (2005), these trajectories have the most complex Fourier spectra. During the oscillation period $P_{r}$ of the regular field of the cluster model 1 (Danilov, 2005), the stars moving along the considered trajectories pass one time (or more times) near the cluster center $(k \leq 0.1)$ and contribute to the formation of the stellar velocity distribution in this area (averaged by the period $P_{r}$ ). We obtain for these trajectories the mean values (over the period $P_{r}$ ) of the dispersions of the residual stellar velocities along the axes $\xi, \eta, \zeta$, respectively: $\sigma_{\dot{\xi}}^{2} \simeq 0.09336 \pm 0.00707(\mathrm{pc} / \mathrm{Myr})^{2}, \sigma_{\dot{\eta}}^{2} \simeq 0.12404 \pm 0.00968$ $(\mathrm{pc} / \mathrm{Myr})^{2}, \sigma_{\dot{\zeta}}^{2} \simeq 0.15378 \pm 0.03106(\mathrm{pc} / \mathrm{Myr})^{2}$, which, according to formulas from the paper of Danilov (2006) for $\sigma^{2}$ and $\kappa^{2}$, leads to the following values: $\sigma^{2} \simeq$ $0.06127(\mathrm{Myr})^{-1}$ and $\kappa^{2} \simeq 0.71484$. Since $\kappa^{2}<1<v^{2}$, then $\mathcal{T}_{\text {max }}=2 \epsilon_{t} v^{2}$ (see the explanation to formula (36) from Danilov (2006)), where $v^{2}$ is defined by Danilov (2006), and $\epsilon_{t}=U_{0}+U_{1}$ is the critical value of the stellar motion energy in the joint field of the homogeneous ellipsoid and the Galaxy.

We define the function $Q=Q(\xi, \eta, \zeta)$. We use the fact that the value $\mathcal{T}_{\text {max }}-\mathcal{T}$ is the integral of motion. We introduce the variables $u$ and $\varphi$, so that $\dot{\xi}-\dot{\xi}_{0}=$ $a_{T} u \cos (\varphi), \dot{\eta}-\dot{\eta}_{0}=b_{T} u \sin (\varphi)$, where $u \geq 0$ and $\varphi \in[0,2 \pi]$. Substituting $u$ and $\varphi$ in the formula for $\mathcal{T}$, we find

$$
\mathcal{T}=u^{2}+\kappa^{2} \dot{\zeta}^{2}+\psi(\xi, \eta, \zeta)
$$

In case $\mathcal{T}=0$ and $\dot{\zeta}^{2}=0$, the maximum value of $u^{2}$ for the star at the point $(\xi, \eta, \zeta)$ is $u^{2}=-\psi$. If $\mathcal{T}=\mathcal{T}_{\text {max }} \neq 0$ and $\dot{\zeta}^{2}=0$, then the maximum value of $u^{2}$ is $\mathcal{T}_{\text {max }}-\psi$. Consequently, $u^{2} \in\left[-\psi, \mathcal{T}_{\text {max }}-\psi\right]$. In the considered $C$-ellipsoid model, $\psi \geq 0$. Therefore, $u^{2} \in\left[0, \mathcal{T}_{\text {max }}-\psi\right]$, and the maximum value of $u^{2}$ for the star at point $(\xi, \eta, \zeta)$ is $u_{m}^{2}=\mathcal{T}_{\text {max }}-\psi$. According to (7.18), $u_{m}^{2}=u^{2}+\kappa^{2} \dot{\zeta}^{2}$. For a given value $u^{2} \neq 0$, the maximum value of $\dot{\zeta}^{2}=\dot{\zeta}_{m}^{2}$ for the star at point $(\xi, \eta, \zeta)$ is $\dot{\zeta}_{m}^{2}=\left(u_{m}^{2}-u^{2}\right) / \kappa^{2}$. We calculate the density $\rho$ at point $(\xi, \eta, \zeta)$. In order to do that, we integrate the $\operatorname{PDF} \Psi(\mathcal{T})$ over all possible values of stellar velocities. While integrating the PDF, we pass to the variables $u(), \varphi, \dot{\zeta}$, take into account that $d \dot{\xi} d \dot{\eta}=a_{T} b_{T} u d u d \varphi$ and find 


$$
\rho=4 \pi Q a_{T} b_{T} \int_{0}^{u_{m}} u d u \int_{0}^{\dot{\zeta}_{m}}\left(u_{m}^{2}-u^{2}-\kappa^{2} \dot{\zeta}^{2}\right) d \dot{\zeta}=\frac{8 \pi a_{T} b_{T} Q u_{m}^{5}}{15 \kappa} .
$$

Since the value $\rho=\rho_{0}$, see above, then, according to (7.19) and (7.17), we find $Q$ and $\Psi(\mathcal{T})=f_{0}(\xi, \eta, \zeta, u, \dot{\zeta})$ :

$$
\begin{gathered}
Q=\frac{15 \rho \kappa}{8 \pi a_{T} b_{T}\left(\mathcal{T}_{\max }-\psi\right)^{5 / 2}}, \\
f_{0}(\xi, \eta, \zeta, u, \dot{\zeta})=\frac{15 \rho \kappa\left(\mathcal{T}_{\max }-\psi-u^{2}-\kappa^{2} \dot{\zeta}^{2}\right)}{8 \pi a_{T} b_{T}\left(\mathcal{T}_{\max }-\psi\right)^{5 / 2}} .
\end{gathered}
$$

Comparing the values $Q=Q(\xi, \eta, \zeta)$ and $Q_{0}=Q(0,0,0)$, we find $Q / Q_{0}=$ $1 /\left(1-\psi / \mathcal{T}_{\text {max }}\right)^{5 / 2}$. The value of $Q \rightarrow \infty$ at $\psi / \mathcal{T}_{\text {max }} \rightarrow 1$. The value $Q=\infty$ is achieved on the surface of the ellipsoid with the semiaxes: $a_{Q} \simeq 1.02963 a$, $b_{Q} \simeq 1.18682 b, c_{Q} \simeq 1.35977 c$ (see the formula for $\psi$ in the explanation for (7.17)). Thus, the semiaxis values of $C$-ellipsoid can be increased in comparison with the values $a, b, c$. This conclusion agrees with the analogous conclusion obtained by Danilov (2006) at the qualitative level for the $H$-ellipsoid during the discussion of the general three-integral velocity distribution of stars in the model of this ellipsoid. The shape of the surface of such an ellipsoid differs slightly from the shape of the envelope surface tangent to the trajectories in the model of nonisolated homogeneous ellipsoid. The shape of the surface tangent to the stellar trajectories must be determined by the values of all three integrals of motion $J_{l}$, and the dimensions of the zero-velocity surface of the cluster (and the formula (7.2) for the potential $U$ ) are obtained by Danilov (2005) using only the Jacobi integral. As a result, in the paper of Danilov (2006), the three-integral PDF at the periphery of a non-isolated homogeneous ellipsoid has not been constructed.

The PDF (7.20) in Danilov (2008) was used to describe the regions with approximately constant density in the cores of the numerical dynamic OSC models (Danilov and Dorogavtseva, 2003; Danilov, 2005). These regions correspond to the central regions of the $C$-ellipsoids of the models of Danilov and Dorogavtseva (2003); Danilov (2005), where $k \leq(0.1-0.2)$. We note, that for the $C$-ellipsoid of model 1 of Danilov (2005) at $k \leq 0.3$ the value of $Q$ varies little with the distance from the center and can be approximately considered to be constant and equal to $Q_{0}$, since as $\xi$ increases from 0 to $0.3 a$ at $\eta=\zeta=0$, the value of $Q / Q_{0}$ changes from 1 to $Q / Q_{0} \simeq 1.11728$, and as $\zeta$ increases from 0 to $0.3 c$ at $\xi=\eta=0$, the value of $Q / Q_{0}$ changes from 1 to $Q / Q_{0} \simeq 1.06436$.

\subsection{The Boltzmann Equation}

Following to Danilov (2008), we consider the evolution of non-isolated gravitating ellipsoid at sufficiently short intervals of time $\Delta t$, which are small in comparison with the time of collisional relaxation of the core of the cluster model 1 of Danilov 
(2005). In this case, we can neglect the effects of collisional relaxation in describing the oscillations of the ellipsoid. We write the Boltzmann equation for the PDF $f$ of an inhomogeneous gravitating ellipsoid with a potential $\Omega$ moving along a circular orbit in the Galactic plane in a rotating coordinate system $\xi, \eta, \zeta$ in the following form:

$$
\frac{\partial f}{\partial t}+\left(\mathbf{v} \frac{\partial f}{\partial \mathbf{r}}\right)+\left(\frac{\partial \Omega}{\partial \xi}+2 \omega \dot{\eta}-\alpha_{1} \xi\right) \frac{\partial f}{\partial \dot{\xi}}+\left(\frac{\partial \Omega}{\partial \eta}-2 \omega \dot{\xi}\right) \frac{\partial f}{\partial \dot{\eta}}+\left(\frac{\partial \Omega}{\partial \zeta}-\alpha_{3} \zeta\right) \frac{\partial f}{\partial \dot{\zeta}}=0,
$$

where $\mathbf{r}=(\xi, \eta, \zeta) ; \mathbf{v}=(\dot{\xi}, \dot{\eta}, \dot{\zeta})$. Let $f=f_{0}+\delta f, \Omega=U+\delta \Omega$, where $|\delta f|<<f_{0}$; $|\delta \Omega|<<U$. Taking into account the adopted restrictions on functions $\delta f$ and $\delta \Omega$, we linearize equation (7.21):

$$
\frac{\partial \delta f}{\partial t}+\left(\mathbf{v} \frac{\partial \delta f}{\partial \mathbf{r}}\right)+\left(-\beta_{1}^{2} \xi+2 \omega \dot{\eta}\right) \frac{\partial \delta f}{\partial \dot{\xi}}-\left(\beta_{2}^{2} \eta+2 \omega \dot{\xi}\right) \frac{\partial \delta f}{\partial \dot{\eta}}+\left(-\beta_{3}^{2} \zeta\right) \frac{\partial \delta f}{\partial \dot{\zeta}}=-\left(\frac{\partial \delta \Omega}{\partial \mathbf{r}} \frac{\partial f_{0}}{\partial \mathbf{v}}\right)
$$

According to Frank-Kamenetsky (1968, p. 249), equation (7.22) can be written in the following form:

$$
\frac{d \delta f}{d t}=-\left(\frac{\partial \delta \Omega}{\partial \mathbf{r}} \frac{\partial f_{0}}{\partial \mathbf{v}}\right)=-\Lambda,
$$

where $\frac{d}{d t}$ is a derivative over the unperturbed stellar trajectory in the joint force field of the Galaxy and $C$-ellipsoid. Assuming $n_{\max }=1$, as in (7.13), we write

$$
\frac{\partial \delta \Omega}{\partial \mathbf{r}}=-\lambda_{0} b_{0} \mathbf{e}, \quad \text { where } \quad \mathbf{e}=\left(\xi \sigma_{1}, \eta \sigma_{\mathbf{2}}, \zeta \sigma_{\mathbf{3}}\right)
$$

Function $f_{0}$ is defined in Section 7.4. Therefore, function $\Lambda$ in the right-hand side of equation (7.23) is known. Equation (7.23) is convenient to solve by the method of integration over the unperturbed trajectory (Frank-Kamenetsky, 1968). Integrating (7.23) over $t$, we find

$$
\delta f(\mathbf{r}, \mathbf{v}, t)=-\int_{-\infty}^{t} \Lambda\left(\mathbf{r}\left(t^{\prime}\right), \mathbf{v}\left(t^{\prime}\right)\right) d t^{\prime},
$$

where $\mathbf{r}\left(t^{\prime}\right), \mathbf{v}\left(t^{\prime}\right)$ are radius vector and velocity vector of a star moving along unperturbed trajectory at the time point $t^{\prime}$, provided that the star at time $t$ is at the point with the phase coordinates $\mathbf{r}(t), \mathbf{v}(t)$. Let $\Lambda \rightarrow 0$ at $t^{\prime} \rightarrow-\infty$, which is necessary for convergence of the integral at the right-hand side of (7.24) (FrankKamenetsky, 1968). To study the natural oscillations of an inhomogeneous ellipsoid, we write $\delta f=f_{1}(\mathbf{r}, \mathbf{v}) \exp (-i \lambda t)$. According to (7.23), in this case we find $\Lambda=$ $\Lambda_{1}(\mathbf{r}, \mathbf{v}) \exp (-i \lambda t)$. Assuming $t=0$ in (7.24), we find

$$
f_{1}(\mathbf{r}, \mathbf{v})=-\int_{-\infty}^{0} \Lambda_{1}\left(\mathbf{r}\left(t^{\prime}\right), \mathbf{v}\left(t^{\prime}\right)\right) \exp \left(-i \lambda t^{\prime}\right) d t^{\prime} .
$$


At $\lambda=\lambda_{1}+i \lambda_{2}$ and $\lambda_{2}>0$, the integral in (7.25) converges. At $\lambda_{2} \leq 0$, function $f_{1}(\mathbf{r}, \mathbf{v})$ must be considered as an analytic continuation of function (7.25) from the domain $\lambda_{2}>0$ (Krall and Trivelpiece, 1973, p. 317).

Integrating function $f_{1}(\mathbf{r}, \mathbf{v})$ over stellar velocities (at the region $E_{\mathbf{v}}$ ), we find the density perturbation (an amplitude of the density oscillation) $\rho_{1}$ at time $t=0$ :

$$
\rho_{1}(\mathbf{r})=\int_{E_{\mathbf{v}}} f_{1}(\mathbf{r}, \mathbf{v}) d^{3} v=b_{0}\left(1-\frac{5}{3} k^{2}\right)
$$

(here $b_{0}=b_{0}(0)$ and the condition $M=$ const is applied). Considering (7.20), we write

$$
\frac{\partial f_{0}}{\partial \dot{\xi}}=-2\left(\dot{\xi}-\dot{\xi}_{0}\right) Q / a_{T}^{2}, \quad \frac{\partial f_{0}}{\partial \dot{\eta}}=-2\left(\dot{\eta}-\dot{\eta}_{0}\right) Q / b_{T}^{2}, \quad \frac{\partial f_{0}}{\partial \dot{\zeta}}=-2 \kappa^{2} \dot{\zeta} Q .
$$

We substitute $\frac{\partial \delta \Omega}{\partial \mathbf{r}}$ and $\frac{\partial f_{0}}{\partial \mathbf{v}}$ in (7.25). Integrating this equation over stellar velocities, reducing both sides of the equation by $b_{0}\left(b_{0} \neq 0\right)$, we write

$$
1-\frac{5}{3} k^{2}=-2 \lambda_{0} \int_{E_{\mathbf{v}}} d^{3} v \int_{-\infty}^{0} d t^{\prime} \mathcal{F}\left(\mathbf{r}^{\prime}, \mathbf{v}^{\prime}\right) Q^{\prime} \exp \left(-i \lambda t^{\prime}\right),
$$

where the primes of the values under the integral sign over $t^{\prime}$ mean that these values are considered at the time point $t^{\prime}$;

$$
\mathcal{F}(\mathbf{r}, \mathbf{v})=e_{1}\left(\dot{\xi}-\dot{\xi}_{0}\right) / a_{T}^{2}+e_{2}\left(\dot{\eta}-\dot{\eta}_{0}\right) / b_{T}^{2}+e_{3} \kappa^{2} \dot{\zeta} ;
$$

$e_{j}$ are coordinates of the vector $\mathbf{e}, j=1,2,3$.

At $k=0$ (and, consequently, at $\xi=\eta=\zeta=0$ ), the roots $\lambda_{l}$ of equation (7.26) describe the density oscillations at the center of the ellipsoid model. The expression on the right side of (7.26) contains the stellar coordinates only at the time point $t^{\prime}=0$ (since the integrand vanishes here at $t^{\prime}=-\infty$ ). Equation (7.26) at different points $(\xi, \eta, \zeta)$ at $k \neq 0$ (at different distances from the cluster center) must have different coefficients and roots $\lambda_{l}$. In order to study the oscillations of the cluster core, we average the equation (7.26) by volume $V_{c}$ of the central part of $C$-ellipsoid (in the range of values $k \leq 0.1$ ), which approximately corresponds to the core of the cluster model 1 of Danilov and Dorogavtseva (2003). To do this, it suffices to integrate equation (7.26) by volume $V_{c}$. An integral of the function on the left-hand side of (7.26) has the form

$$
\int_{V_{c}}\left(1-\frac{5}{3} k^{2}\right) d^{3} r=4 \pi a b c 0.1^{3}\left(1-0.1^{2}\right) / 3=(0.99 / 1000) V,
$$

where $V=\frac{4}{3} \pi a b c$ is the volume of an ellipsoid. Therefore, the equation giving an approximate description of the density oscillations in the core of model 1 of Danilov (2005) can be written in the following form: 


$$
1=-\frac{2000 \lambda_{0}}{0.99 V} \int_{V_{c}} d^{3} r \int_{E_{\mathbf{v}}} d^{3} v \int_{-\infty}^{0} d t^{\prime} \mathcal{F}\left(\mathbf{r}^{\prime}, \mathbf{v}^{\prime}\right) Q^{\prime} \exp \left(-i \lambda t^{\prime}\right)
$$

Calculation of integrals on the right-hand side of equations (7.26) and (7.27) is a complex task and can not be performed analytically. To simplify the calculations, we consider a particular case of equation (7.26) at the point $\xi=\eta=\zeta=0$ at $b_{1}=0$ (the homological oscillations of ellipsoid). In this case, $\sigma_{j}=A_{j}, j=1,2,3$. Let $Q \simeq Q_{0}$, which is valid for points near the center of the ellipsoid, see section 7.4. Taking into account the accepted restrictions, equation (7.26) takes the following form:

$$
1=-2 \lambda_{0} Q_{0} \int_{E_{\mathrm{v}}} d^{3} v \int_{-\infty}^{0} d t^{\prime}\left\{\frac{A_{1}}{a_{T}^{2}} \xi\left(\dot{\xi}-\dot{\xi}_{0}\right)+\frac{A_{2}}{b_{T}^{2}} \eta\left(\dot{\eta}-\dot{\eta}_{0}\right)+A_{3} \kappa^{2} \zeta \dot{\zeta}\right\}^{\prime} \exp \left(-i \lambda t^{\prime}\right) .
$$

According to (4) from the paper of Danilov (2006), a general solution of equations of motion for the unperturbed stellar trajectory is written in the following form:

$$
\begin{gathered}
\xi=C_{1} \frac{\exp \left(i \phi_{1}\right)+\exp \left(-i \phi_{1}\right)}{2}+C_{2} \frac{\exp \left(i \phi_{2}\right)+\exp \left(-i \phi_{2}\right)}{2 i}, \\
\eta=C_{1} A \frac{\exp \left(i \phi_{1}\right)-\exp \left(-i \phi_{1}\right)}{2 i}+C_{2} B \frac{\exp \left(i \phi_{2}\right)-\exp \left(-i \phi_{2}\right)}{2 i}, \\
\zeta=C_{3} \frac{\exp \left(i \phi_{3}\right)+\exp \left(-i \phi_{3}\right)}{2},
\end{gathered}
$$

where $C_{l}$ are the constants of an integration $(l=1,2,3), \phi_{\varsigma}=v_{\varsigma} t+\phi_{0, \varsigma}, \varsigma=$ 1,$2 ; \phi_{3}=\beta_{3} t+\phi_{0,3}, \phi_{0, j}$ are initial phases $(j=1,2,3)$. Constant values $A$ and $B$ are defined by Danilov (2006). Following to Danilov (2006), we write $\dot{\xi}_{0}=q_{x} \eta$, $\dot{\eta}_{0}=q_{y} \xi$, where $q_{x}=q_{2} \sigma^{2} a_{T}^{2} / 2, q_{y}=-q_{1} \sigma^{2} b_{T}^{2} / 2$, and the constant values $q_{1}$ and $q_{2}$ are defined in Danilov (2006). After substitution (7.29) in $\dot{\xi}_{0}, \dot{\eta}_{0}$ and in (7.28), we reduce the expression in curly brackets in (7.28) to the following form:

$$
\{. .\}=\sum_{l=1}^{10} g_{l} \exp \left(i\left(\mathbf{m}_{l}, \mathbf{u}\right)\right)
$$

where $\left(\mathbf{m}_{l}, \mathbf{u}\right)$ is a scalar product of vectors $\mathbf{m}_{l}$ and $\mathbf{u}, \mathbf{u}=\left(\phi_{1}, \phi_{2}, \phi_{3}\right), \mathbf{m}_{1}=(1,1,0)$, $\mathbf{m}_{2}=(-1,-1,0), \mathbf{m}_{3}=(1,-1,0), \mathbf{m}_{4}=(-1,1,0), \mathbf{m}_{5}=(2,0,0), \mathbf{m}_{6}=(-2,0,0)$, $\mathbf{m}_{7}=(0,2,0), \mathbf{m}_{8}=(0,-2,0), \mathbf{m}_{9}=(0,0,2), \mathbf{m}_{10}=(0,0,-2) ; g_{l}$ are the constant imaginary values $(1=1, \ldots, 10)$. Calculating the integral over $t^{\prime}$ in equation (7.28), we find

$$
\int_{-\infty}^{0} \sum_{l=1}^{10} g_{l} \exp \left(i\left(\left(\mathbf{m}_{l}, \mathbf{u}\right)-\lambda\right) t^{\prime}\right) d t^{\prime}=-i \sum_{l=1}^{10} g_{l} \exp \left(i\left(\mathbf{m}_{l}, \mathbf{u}_{0}\right)\right) /\left(\left(\mathbf{m}_{l}, \mathbf{w}\right)-\lambda\right),
$$

where $\mathbf{u}_{0}=\mathbf{u}(t=0), \mathbf{w}=\left(v_{1}, v_{2}, \beta_{3}\right)$. Passing in the formula for

$$
\exp \left(i\left(\mathbf{m}_{l}, \mathbf{u}_{0}\right)\right)=\cos \left(\left(\mathbf{m}_{l}, \mathbf{u}_{0}\right)\right)+i \sin \left(\left(\mathbf{m}_{l}, \mathbf{u}_{0}\right)\right)
$$


to the functons $\cos \left(\mathbf{u}_{0}\right), \sin \left(\mathbf{u}_{0}\right)$, using for $\cos \left(\mathbf{u}_{0}\right), \sin \left(\mathbf{u}_{0}\right)$ the expressions (5) from the paper of Danilov (2006) at $t=0$ and $\cos \left(\phi_{0,3}\right)=\zeta(0) / C_{3}, \sin \left(\phi_{0,3}\right)=$ $-\dot{\zeta}(0) /\left(C_{3} \beta_{3}\right)$, see (4) from the paper of Danilov (2006), for the integral

$$
\int_{-\infty}^{0} \sum_{l=1}^{10} g_{l} \exp \left(i\left(\left(\mathbf{m}_{l}, \mathbf{u}\right)-\lambda\right) t^{\prime}\right) d t^{\prime}
$$

we obtain the expression in the form of the second degree polynomial with respect to PCS at $t=0$ (here, $\sin \left(\mathbf{u}_{0}\right)$ gets the values $\sin \left(\phi_{0,1}\right), \sin \left(\phi_{0,2}\right) ; \sin \left(\phi_{0,3}\right) ; \cos \left(\mathbf{u}_{0}\right)$ is defined similarly).

Carrying out the integrations of the obtained polynomial with respect to stellar velocities in (7.28) at $t=0$, applying, as in (7.19), variables $u, \varphi, \dot{\zeta}(0)$, separating the real part of the integration result, using it in equation (7.28), and substituting $\xi(0)=\eta(0)=\zeta(0)=0$ into the final equation, we obtain an algebraic equation of the fifth degree with respect to $\chi=\lambda^{2}$ :

$$
\sum_{j=0}^{5} p_{j} \chi^{j}=0
$$

where the constant values $p_{j}$ can be presented as

$$
p_{j}=H_{0, j}+\sum_{k=1}^{3} H_{k, j} C_{k}^{2},
$$

where $H_{k, j}$ are the constant values, and $k=0, \ldots, 3 ; j=0, \ldots, 5$. A general form of formulas for the coefficients $p_{i}$ is not given here due to very cumbersome form of these formulas.

\subsection{Instability of Phase Density Oscillations in the Cores of OSC Models. Estimates of Parameters and Consequences of Such Oscillations}

The constants $C_{j}(j=1,2,3)$ are directly proportional to the amplitudes of the unperturbed stellar motion in $\xi, \eta, \zeta$-coordinates and have the same order of magnitude as these amplitudes, see (7.29). The values $C_{j}^{2}$ are directly proportional to the energy $\epsilon$ of a stellar motion. The larger the values $C_{j}^{2}$ considered, the greater the stellar energy and the distance from the center of ellipsoid reached by the star as it moves (hereinafter, for brevity, we denote $C_{1,2,3}=C_{j}(j=1,2,3)$ ).

Let us consider two cases: $C_{1,2,3}=0$ and $C_{1,2,3}=1 \mathrm{pc}$. The first case corresponds to the position of an unperturbed star in the cluster center, and the second case for the cluster model 1 of Danilov (2005) approximately provides the maximum removal of this star to the boundary of the cluster core $(|\xi| / a \simeq 0.1,|\zeta| / c \simeq 0.2)$ as this 
Table 7.2 The values of the coefficients $p_{k}$ and roots $\chi_{k}=\lambda_{k}^{2}$

\begin{tabular}{|c|c|c|}
\hline & $C_{1,2,3}=0$ & $C_{1,2,3}=1 \mathrm{pc}$ \\
\hline$p_{0}(\mathrm{Myr})^{-10}$ & $0.4180460326 \times 10^{-7}$ & $0.4245915893 \times 10^{-7}$ \\
\hline$p_{1}(\mathrm{Myr})^{-8}$ & $-0.1176728365 \times 10^{-4}$ & $-0.1195862196 \times 10^{-4}$ \\
\hline$p_{2}(\mathrm{Myr})^{-6}$ & $0.8285419864 \times 10^{-3}$ & $0.8423261216 \times 10^{-3}$ \\
\hline$p_{3}(\mathrm{Myr})^{-4}$ & $-0.2340173146 \times 10^{-1}$ & $-0.2364231243 \times 10^{-1}$ \\
\hline$p_{4}(\mathrm{Myr})^{-2}$ & 0.2654871362 & 0.2663156562 \\
\hline$p_{5}$ & -1 & -1 \\
\hline$\lambda_{1}^{2}(\mathrm{Myr})^{-2}$ & $0.5183374833 \times 10^{-2}$ & $0.5183417921 \times 10^{-2}$ \\
\hline$\lambda_{2,3}^{2}(\mathrm{Myr})^{-2}$ & $0.2759885969 \times 10^{-1}$ & $0.2791520605 \times 10^{-1}$ \\
& $\pm i 0.717579758 \times 10^{-2}$ & $\pm i 0.626130831 \times 10^{-2}$ \\
\hline$\lambda_{4}^{2}(\mathrm{Myr})^{-2}$ & $0.7807410158 \times 10^{-1}$ & $0.7965084085 \times 10^{-1}$ \\
\hline$\lambda_{5}^{2}(\mathrm{Myr})^{-2}$ & 0.1270319404 & 0.1256509853 \\
\hline
\end{tabular}

star moves. The values of the coefficients $p_{k}$ and roots $\chi_{k}=\lambda_{k}^{2}(k=1, \ldots, 5)$ of equation (7.30) at $C_{1,2,3}=0$ and $C_{1,2,3}=1 \mathrm{pc}$ are given in Table 7.2. According to Table 7.2 in the considered cases, the values $\lambda_{2,3}^{2}$ are complex, which indicates an instability of the phase density oscillations at the cluster center of the model 1 of Danilov (2005) (since $\sqrt{\lambda_{2,3}^{2}}= \pm\left(\gamma_{1} \pm i \gamma_{2}\right)$, where $\gamma_{1}=0.1675042251(\mathrm{Myr})^{-1}$, $\gamma_{2}=0.2141975098 \times 10^{-1}(\mathrm{Myr})^{-1}$ at $C_{1,2,3}=0$ and $\gamma_{1}=0.1681130474(\mathrm{Myr})^{-1}$, $\gamma_{2}=0.1862231519 \times 10^{-1}(\mathrm{Myr})^{-1}$ at $\left.C_{1,2,3}=1 \mathrm{pc}\right)$. This instability is the cause of a development of the large-scale density oscillations in the numerical dynamic OSC models (Danilov and Dorogavtseva, 2003). The value $\gamma_{2}$ is the growth increment of the phase density perturbation, and the value $t_{\lambda}=1 / \gamma_{2}$ is a characteristic time of a perturbation growth. When $C_{1,2,3}=0$ the value $t_{\lambda}=t_{\lambda, 0} \simeq 46.68588 \mathrm{Myr}$, and at $C_{1,2,3}=1 \mathrm{pc}$, we find $t_{\lambda}=t_{\lambda, 1} \simeq 53.69902 \mathrm{Myr} ; t_{\lambda, 1}>t_{\lambda, 0}$.

Instability of the solutions for the PDF of the numerical dynamic OSC models to small initial perturbations is known, and was studied in detail while calculating the relative perturbations $\Psi_{l}(t)$ of the PDF in the papers of Danilov and Dorogavtseva (2003) and Danilov (1999) $(l=1, \ldots, 10$; larger values of $l$ correspond to greater distances from the cluster center). The time $t_{\Psi}$ of the PDF instability development near the center of the cluster model 1 of Danilov and Dorogavtseva (2003) can be estimated as a value reverse to the rate of an increase of the relative PDF perturbations: $t_{\Psi} \simeq(49.0 \pm 2.4) \mathrm{Myr}$, which completely agrees with the estimates of the values of $t_{\lambda}$ considered here (an estimate of the $t_{\Psi}$ value is obtained from the data of the paper of Danilov and Dorogavtseva (2003) for small values $\Psi_{1}(t) \leq 0.3$, at $t / \tau_{v r} \in[0,0.22]$, since in equations (7.22)-(7.28) the small perturbations are considered in the linear approximation $|\delta f| / f_{0}$ ). According to the results of section 7.4 , a consideration of the non-homology of the density oscillations should lead to an increase of the estimates $t_{\lambda}$ as a result of a partial suppression of the instability considered here. Since $t_{\lambda, 1}>t_{\lambda, 0}$, then stars with higher energies reduce the rate of 
the instability development both near the center of the ellipsoid model considered, and in the OSC cores.

Halo stars passing near the system center with the large velocities and energies can also contribute to the suppression of the instability considered, which should lead to an increase in the estimates of $t_{\lambda}$. The same effect of stars with high energies and velocities of motion on instability also occurs in the case of gravitational instability of the infinite homogeneous gravitating systems (King, 2002, p.103). The reason for development of the instability considered in our work are small stellar velocities, which is due to the low density of OSCs and their models (Danilov and Dorogavtseva, 2003).

We note that the exponential instability of stellar trajectories in the cores of the OSC models also contributes to the increase rate of $\Psi_{1}(t)$ with time. Therefore, only part of the $t_{\Psi}^{-1}$ value is due to the gravitational instability in the cluster. However, contribution of the exponential instability of stellar trajectories to the estimate $t_{\Psi}$ at $\Psi_{1}(t) \leq 0.3$ and $t / \tau_{v r} \in[0,0.22]$ is small. According to Danilov and Dorogavtseva (2003), the radius $r_{1}$ of a sphere containing 50 stars closest to the cluster center, from the data on the velocities of which the function $\Psi_{1}(t)$ is obtained, equals to $r_{1} \simeq$ (1.62-1.72) pc for the cluster model 1. Dimensions of the exponential divergence of stellar trajectories in the time intervals $\Delta t \leq 0.22 \tau_{v r}$ usually do not exceed the dimensions of a sphere with the radius $r_{1}$. Therefore, the exponential instability of the stellar trajectories does not have a sufficient time to change significantly the values of the coarse-grained PDF used in the estimates of the function $\Psi_{1}(t)$. However, the contribution of the stochastic trajectories to $\Psi_{1}(t)$ at large $\Psi_{1}(t)$ and $t$ becomes significant.

At $t / \tau_{v r} \geq 0.5-0.6, \Psi_{1}(t) \simeq$ const, and in the OSC models of Danilov and Dorogavtseva (2003), the large-scale oscillations of a density and a regular field occur. One of the reasons for this persistence of the values of $\Psi_{l}(t)$ is discussed in detail by Danilov (1999). It is related to the fact that in the compared versions $\mathbf{a}$ and b of the OSC models (with small differences in initial PCS), used in the calculation of $\Psi_{1}(t)$, by the time point $t / \tau_{v r} \simeq 0.5-0.6$ under conditions of the exponential instability of trajectories, the stars already occupy, with a density unequal to zero, all the regions of phase space available for motion, and the PDF in the versions a and $\mathbf{b}$ of the OSC models begin vary with time slowly and approximately in the same way. The second important reason for approximate constancy of the values of function $\Psi_{1}(t)$ at $t / \tau_{v r} \geq 0.5-0.6$ is the balance of the rates of a development and a damping of the PDF perturbations; this balance is achieved in the system as a result of combined effect of the gravitational instability and a violent relaxation (see below).

The rate of a growth of the relative PDF perturbations, according to Danilov and Dorogavtseva (2003); Danilov (1999), decreases with distance from the cluster center. Therefore, the estimates of $t_{\Psi}$ and $t_{\lambda}$ must increase for points $(\xi, \eta, \zeta)$, located at greater distances from the system center.

Let us note that $\delta f \sim \exp \left(\mp i\left(\gamma_{1} \pm i \gamma_{2}\right) t\right)$ at $\chi=\chi_{2,3}$. Therefore, a development of the instability in the system leads rather quickly to the dominance of the oscillation amplitudes with frequency $\gamma_{1}$. In the case $C_{1,2,3}=1 \mathrm{pc}$ the period $P_{\gamma}=2 \pi / \gamma_{1} \simeq$ 
37.37476 Myr, which completely agrees with the estimate of oscillation period of a regular field $P_{r} \simeq 31.0 \mathrm{Myr}$ for the cluster model 1, obtained from the Fourier analysis of the time dependency of this model's virial coefficient (see Table 2 in the paper of Danilov and Leskov (2005)). The taking into account of the non-homology of the density oscillations in the gravitating ellipsoid model, according to section 7.3 , should lead to an increase in the oscillation frequency $\gamma_{1}$ and a decrease of $P_{\gamma}$. In addition, halo stars passing near the system center and having high velocities and energies can also contribute to a decrease of $P_{\gamma}$ (since $P_{\gamma}\left(C_{1,2,3}=1 \mathrm{pc}\right.$ ) $<P_{\gamma}\left(C_{1,2,3}=0\right)$, where $P_{\gamma}\left(C_{1,2,3}=0\right)$ is $P_{\gamma}$ obtained at $C_{1,2,3}=0$; the value of $P_{\gamma}\left(C_{1,2,3}=1 \mathrm{pc}\right.$ is defined similarly $)$.

Thus, the oscillations of the values of the virial coefficients and the moments of inertia of the OSC models (Danilov and Dorogavtseva, 2003) with practically constant period and amplitude are the result of the gravitational instability development in these models.

According to Table 2 from the paper of Danilov and Dorogavtseva (2003), the estimates of violent relaxation time $t_{r, c}^{(1)}$ of the cluster core obtained from the data on functions $\Psi_{1}(t)$, are $t_{r, c}^{(1)} \simeq 0.5 \tau_{v r}$ for the cluster models $1-5$, and $t_{r, c}^{(1)} \simeq(0.6$ $0.7) \tau_{v r}$ for the cluster model 6 (the values $t_{r, c}^{(1)}$ agree well with the initial relaxation time $\tau_{v}$ of the cluster model, obtained by Danilov and Dorogavtseva (2003) from the data on stellar fluxes in the space of the moduli of stellar velocity; for the calculation of function $\Psi_{1}(t)$, we also used the data on stellar velocities). Initial ratios of the radii of the core and halo, as well as of the numbers of stars in the core and halo, are the same in the cluster models 1, 2, 4 (Danilov and Dorogavtseva, 2003), and the density of these models increases from the first to the fourth model (see Table 1 in the paper of Danilov and Dorogavtseva (2003)). Estimates of $\varpi=t \Psi / t_{r, c}^{(1)}$ from the data on functions $\Psi_{1}(t)$ in the range of values $\Psi_{1}(t) \leq 0.3$ for models 1,2 , 4 , are $\varpi \simeq 1.96 \pm 0.10,2.50 \pm 0.08,2.72 \pm 0.30$, respectively. Thus, the time of the gravitational instability development near the cluster centers expressed in the units of a violent relaxation time of the cores at $t / \tau_{v r} \in[0,0.22]$ increases with the increasing of the cluster density. This leads to a decrease in the rate and degree of the gravitational instability development near cluster centers with an increasing of the cluster density. For larger values $\Psi_{1}(t)$ and at the highest rate of increase of the PDF relative perturbations (at $t / \tau_{v r} \in[0.22,0.5]$ ), the values $\varpi$ for models $1,2,4$ decrease in $\vartheta$ times, where $\vartheta \sim 3.8-4.6$, which leads to the development of the density and regular-field oscillations in these cluster models. Probably, at $t / \tau_{v r} \geq 0.5$, in the cores of models $1,2,4$ the rates of growth and damping of the phase density perturbations are equalized, which leads to the establishment of the oscillations of the virial coefficient values with almost constant amplitudes and periods observable in these models.

According to Table 1 from the paper of Danilov and Dorogavtseva (2003), in the cluster models $2,3,5,6$, the number of stars in the core and the size of the core increase from model 2 to model 6 with the identical initial radii of the halo. $\varpi$ values for models $2,3,5,6$ are $\varpi \simeq 2.50 \pm 0.08,1.70 \pm 0.07,1.82 \pm 0.14,1.31 \pm 0.06$, respectively. Consequently, with the increasing size and mass of the cores of the 
cluster models at $t / \tau_{v r} \in[0,0.22]$, the rates of a development and a damping of the phase density perturbations in the cluster cores approach each other. For the larger values of $\Psi_{1}(t)$ and the fastest rate of increase of the relative PDF perturbations (at $t / \tau_{v r} \in[0.22,0.5]$ ), the values $\varpi$ decrease in $\vartheta$ times, where $\vartheta \sim 4.6 \pm 0.4$, $2.5 \pm 0.1,3.7 \pm 0.3,1.7 \pm 0.1$ for models $2,3,5,6$, respectively. It leads to the development of the density and regular field oscillations in these cluster models. We note that $\vartheta$ values in the considered models are only partially due to the action of the gravitational instability caused by the influence of the exponential instability of the stellar trajectories on the $\Psi_{1}(t)$ curves at $t / \tau_{v r}$ close to 0.5 . Therefore, the estimates of $\vartheta$ given here can only be considered as upper (overstated) estimates of $\vartheta$ when discussing the effect of the gravitational instability development in the cores of the OSC models at $t / \tau_{v r} \in[0.22,0.5]$.

Apparently, the approximate equality of the rates of a development and damping of the phase density perturbations as a result of the combined action of a gravitational instability and a violent relaxation in the cores of the OSC models (and in star cluster models of Danilov and Dorogavtseva (2003) as a whole) leads to the establishment of the large-scale density and regular-field oscillations, which continue in these models for fairly long periods of time.

According to Danilov and Dorogavtseva (2008), the cluster models with smaller density and larger amplitude of the regular field oscillations get destroyed (with an expanding in the Galaxy field) in shorter time than the models of the denser clusters. The reasons for the accelerated decay of the OSCs with a lower density in the Galactic field is the tidal "heating" of such clusters by the Galactic force field (Danilov and Dorogavtseva, 2008), as well as a dynamic instability (Ogorodnikov, 1958, p.389) and the development of the gravitational instability in such systems. According to Danilov (1982), the large-scale oscillations of the cluster density in the Galactic field can lead to an impulsive and significant loss of mass of the cluster (see fig. 2 and Table 2 from the paper of Danilov (1982)). In this case, the stars with higher velocities and energies leave the cluster earlier than other stars. It leads to a compression and a subsequent stabilization of the remaining gravitationally related part of the cluster (the loss of the high-energy stars by cluster can be one of the reasons for a decrease of the amplitude of oscillations of a density and regular cluster field).

Considering (7.25), as well as the constraints adopted to simplify computations in (7.28), the expression for $f_{1}(\mathbf{r}, \mathbf{v})$ at the point $\xi=\eta=\zeta=0$ at $C_{1,2,3}=1 \mathrm{pc}$ can be written in the following form:

$$
f_{1}(0, \mathbf{v})=d_{1} \dot{\xi}^{2}+d_{2} \dot{\eta}^{2}+d_{3} \dot{\xi} \dot{\eta}+d_{4}
$$

where constant values $d_{l}$ are:

$$
\begin{gathered}
d_{1}=0.2808910230+i 0.7086888676 \times 10^{-1}, \\
d_{2}=0.4708932329-i 0.7443246439 \times 10^{-1}, \\
d_{3}=0.2883002901+i 0.4348075779,
\end{gathered}
$$




$$
d_{4}=-0.7266762684 \times 10^{-2}+i 0.5009804792 \times 10^{-2} ;
$$

the dimensions of $d_{1,2,3}$ values equal to $M_{\odot}(\mathrm{Myr})^{5} / \mathrm{pc}^{8}$, the dimension of $d_{4}$ value equals to $M_{\odot}(\mathrm{Myr})^{3} / \mathrm{pc}^{6}$. The general formulas for $d_{l}$ values is not given here due to a very cumbersome form of these formulas. Assuming the value $f_{1}(0, \mathbf{v})$ in (7.31) equal to a small positive constant, isolating the real part of $f_{1}(0, \mathbf{v})$, we obtain the equation of an ellipse in the space of the values $(\dot{\xi}, \dot{\eta})$ with the center at point $\dot{\xi}=\dot{\eta}=0$, with a ratio of the large semiaxis $a_{v}$ to the small one $b_{v}$, which equals $a_{v} / b_{v} \simeq 1.64279$. The main axis of this ellipse is inclined to the axis $\dot{\xi}$ at the angle $\varphi \simeq-0.49405 \mathrm{rad}$ (see formulas for $a_{v}, b_{v}$ and $\varphi$ in Korn and Korn (1968, p. 66)). Thus, a distribution of the perturbations of the stellar velocities in the center of the considered cluster model is biaxial, having an elliptic symmetry and elongated along the main axis of the ellipse (7.31). At $C_{1,2,3}=0$ and $t=0$, we similarly find $a_{v} / b_{v} \simeq 1.58968$ and $\varphi \simeq-0.49084$ rad. Consequently, a distribution of the perturbations of the stellar motion velocities with the higher energies has a larger elongation along the main axis of the velocity ellipse. Using the real part of $\delta f=f_{1}(0, \mathbf{v}) \times \exp \left(-i\left( \pm \gamma_{1}+i \gamma_{2}\right) t\right)$ at $t=2 \mathrm{Myr}$ and $C_{1,2,3}=1 \mathrm{pc}$, we find $a_{v} / b_{v} \simeq 2.04887, \varphi \simeq-0.63226 \mathrm{rad}$ (regardless of the sign facing the value $\gamma_{1}$ ). Thus, regardless of the sign of $\gamma_{1}$ value, the ellipse of the velocity perturbation distribution rotates with an angular velocity $\gamma_{1}$ in the direction opposite to the cluster motion in the Galaxy. In this case, the velocity perturbation ellipse extends along its main axis. A similar result was described in the book of Ogorodnikov (1958) when discussing a dynamic instability of the Local system and studying the evolution of the velocity perturbation distribution of stars leaving the system (see the description of the quasi-tide and quasi-precession of the ellipsoid of velocities of stars of the Local system on p. 397 in the book of Ogorodnikov (1958)). However, in the book of Ogorodnikov (1958), the angular velocity of a rotation of the ellipse of the stellar velocity perturbations is $\sim 2.97106$ times less than in the case of stars with energies sufficient only to reach the boundary of the cluster core. According to Ogorodnikov (1958), a combined influence of the quasi-tide and quasi-precession on the joint distribution of the perturbed and undisturbed stellar velocities leads to the formation of circular symmetry of the general stellar velocity distribution in the vicinity of the Local system. Such an equalization of the stellar velocity dispersions along $\xi$ and $\eta$ axis in the course of an evolution was also noted in the numerical dynamic OSC models (Danilov and Dorogavtseva, 2008).

\subsection{Conclusions}

1. In this chapter, we have discussed the estimates of the frequencies of the homological and non-homological density oscillations in the unisolated ellipsoidal models of open star clusters. The considered oscillations of the inertia momentum and of the diagonal elements of the inertia tensor are stable. The frequencies of the non-homological oscillations are greater than the frequencies in the case of the ho- 
mologous oscillations of ellipsoids. The non-homological oscillations have a larger "margin" of stability than the homological oscillations.

2. For the central regions of the numerical dynamic OSC models, we have written a Boltzmann equation for the phase density function $f$. We have constructed equilibrium three-integral phase density function $f_{0}$, which is employed to derive the linearized equation for small perturbation of the function $f$. Applying the method of integration over an unperturbed trajectory, we have written down the equations for the small perturbation of the function $f$ and the frequencies of the natural oscillations of an inhomogeneous ellipsoid. We have noted the instability of the phase density natural oscillations in the center of a homogeneous ellipsoid ( $C$-ellipsoid), parameters of which (the dispersion of stellar velocities along the coordinate axes; the density and potential near the center of ellipsoid) are close to the mean (with respect to the regular field oscillations) values of these parameters in the cores of the numerical dynamic model 1 (Danilov and Dorogavtseva, 2003) of the non-stationary cluster.

3. Development of the instability of the phase density oscillations at the center of the model of a $C$-ellipsoid of the cluster is caused by complex frequencies $\lambda=$ $\pm\left(\gamma_{1} \pm i \gamma_{2}\right)$ of the phase density natural oscillations and leads to the dominance of the oscillations with the frequency $\gamma_{1}$, corresponding to the increment of growth $\gamma_{2}$ of this instability. The estimates of $\gamma_{1}$ agree quite well with the estimates of an oscillation frequency of the virial coefficient and the regular field (Danilov and Leskov, 2005) in model 1 of Danilov and Dorogavtseva (2003). Estimates of the time of the phase density instability development at the center of the $C$-ellipsoid model agree quite well with time $t_{\Psi}$ for the development of the PDF instability to small initial perturbations near the center of the numerical dynamic cluster model 1 (Danilov and Dorogavtseva, 2003). In the process of the instability development, the ellipse of the stellar velocity perturbations distribution rotates with an angular velocity $\gamma_{1}$ in the direction opposite to the direction of the cluster motion in the Galaxy. In this case, the velocity perturbation ellipse extends along its main axis. The reason for the development of the instability of the phase-density oscillations is low stellar velocity, which is due to a low density of the OSCs and their models (Danilov and Dorogavtseva, 2003).

4. We have performed the analysis of the development and damping of the phase density oscillations in the cores of the numerical dynamic OSC models (Danilov and Dorogavtseva, 2003). Probably, the approximate equality in the rates of a development and damping of the phase density perturbations as a result of the combined effect of the gravitational instability and a violent relaxation in the cores of the OSC models (and in OSC models as a whole (Danilov and Dorogavtseva, 2003)) leads to the large-scale density and regular-field oscillations continuing in these models for sufficiently large time intervals. 


\section{References}

Chandrasekhar, S.: Principles of Stellar Dynamics. Univ. Chicago Press, Chicago, Ill (1942). LCCN: 43-5608 (BKS1)

Danilov, V.M.: The Early Dynamical Evolution of Open Clusters - Part Two. Soviet Astron. 26, 490-499 (1982).

Danilov, V.M.: Some properties of the phase-space density function in numerical dynamical models of open star clusters. Astron. Reports 43, 74-79 (1999).

Danilov, V.M.: The Motion of Halo Stars in Dynamical Numerical Models of Open Clusters. Astron. Reports 49, 3604-610 (2005). doi:10.1134/1.2010649

Danilov, V.M.: Dynamical models of stellar motions at the peripheries of open clusters. Astron. Reports 50, 346-357 (2006). doi:10.1134/S1063772906050027

Danilov, V.M.: Analysis of density fluctuations in models of open clusters. Astron. Reports 52, 888-899 (2008). doi:10.1134/S1063772908110036

Danilov, V.M., Dorogavtseva, L.V.: Estimates of Relaxation Times in Numerical Dynamical Models of Open Star Clusters. Astron. Reports 47, 483-491 (2003). doi: $10.1134 / 1.1583775$

Danilov, V.M., Dorogavtseva, L.V.: Timescales for mechanisms for the dynamical evolution of open star clusters. Astron. Reports 52, 467-478 (2008). doi:10.1134/S1063772908060048

Danilov, V.M., Leskov, E.V.: Properties of Stellar Trajectories in Numerical Dynamical Models of Open Star Clusters. Astron. Reports 49, 190-200 (2005). doi:10.1134/1.1882777

Duboshin, G.N.: Celestial Mechanics. The main problems and methods. Nauka, Moscow (1975) (In Russian).

Frank-Kamenetsky, D.A.: Lectures on the plasma physics. Atomizdat, Moscow (1968) (In Russian).

King, Ivan R.: An Introduction to Classical Stellar Dynamics. URSS, Moscow (2002) (In Russian).

Korn, G.A., Korn, T.M: Mathematical Handbook for Scientists and Engineers. McGraw-Hill, New York, San Francisco, Toronto, London, Sydney (1968).

Krall, N.A., Trivelpiece, A.W.: Principles Of Plasma Physics. McGraw-Hill, New York (1973).

Kutuzov, S.A., Osipkov, L.P.: A Generalized Model for the Three-Dimensional Gravitational Potential of Stellar Systems. Soviet Astron. 24, 17-22 (1980).

Kuzmin, G.G.: On the modern state and some next targets of a stellar dynamics. In: Kinematics and dynamics of stellar systems and physics of an interstellar matter. Proc. of Astrophys. Inst. of Acad. Sci. of Kaz.SSR, Alma-Ata (1965) (In Russian).

Ogorodnikov, K.F.: Dynamics of stellar systems. GIFML, Moscow (1958) (In Russian).

Ossipkov, L.P.: Gross-Dynamics of Open Clusters in the Galaxy. In: Deiters, S., Fuchs, D., Just, A., Spurzem, R., Wielen R. (eds) Dynamics of Star Clusters and Milky Way. ASP Conf. Ser. 228, 341 (2001).

Subbotin, M.F.: Course of celestial mechanics. Vol.3. Nauka, Leningrad, Moscow (1949) (In Russian). 


\title{
Chapter 8 \\ On Phase Density Oscillations at the Centers of Six Open Star Clusters
}

\begin{abstract}
In this chapter, we consider the instability of the natural oscillations of a phase density in the centers of six open star clusters. We determine the boundaries of the instability region of such oscillations in the space of the mean mass density of the cluster core. For the central parts of these clusters and for several numerical dynamic models of the open star clusters, we obtain estimates of a number of the dynamic parameters (potentials; dispersions of the stellar velocities; the mass density; periods of the phase density oscillations; instability development time of such oscillations, etc.). We derive an equation for estimating the dynamic mass of a cluster, taking into account the influence of the external field of the Galaxy on the cluster and of a non-stationary nature of the cluster. The influence of these effects leads to a decrease of the dynamic mass of the cluster in comparison with the virial mass for an isolated cluster. Astrophysical applications of the results are discussed.
\end{abstract}

\subsection{Introduction}

In the numerical dynamic OSC models (Danilov and Dorogavtseva, 2003; Danilov, 1999; Danilov and Dorogavtseva, 2008), the large-scale oscillations of a density and of regular field are spontaneously and rapidly developed. The possibility of an instability development in the OSC models is used by Danilov and Dorogavtseva (2008) to explain the variations in the values of the virial coefficient and dispersion of the cluster star velocities that do not decay during the large time intervals, as well as to explain small times of the spherization of the distribution of stellar velocities in the least dense OSC models with the greatest degree of a non-stationarity in a regular field. The theoretical description of an instability of the phase density oscillations in the central parts of the numerical dynamic OSC models was carried out by Danilov (2008) using the Boltzmann equation. It is of interest to use the formulas obtained by Danilov (2008) (equation (31) and the coefficients $p_{j}$ of this equation) to study the observed OSC. We note that an appropriate processing of the observational data on a spatial structure of the OSCs makes it possible to use the formulas and results 
of Danilov (2008) without a preliminary construction of the numerical dynamic models of these OSCs. At the same time, a detailed discussion of the characteristics of the numerical dynamic OSC models (Danilov and Dorogavtseva, 2008) and their comparison with the observational data on the open star clusters makes it easier to analyze the structural and dynamic parameters of the observed OSCs.

The following points are of particular interest: 1 ) to use the formulas obtained by Danilov (2008) for analyzing the instability of the phase density oscillations in the cores of the observed OSCs; 2 ) to determine the boundaries of this instability and discuss the parameters of the density distributions in the OSCs, which are resistant to such oscillations; 3 ) to estimate the dynamic characteristics of the analyzed OSCs; 4) to compare the dynamic characteristics of the considered OSCs and the OSC models (Danilov and Dorogavtseva, 2008).

The objectives of this chapter are: 1) to study the density and phase density oscillations in the central parts of six observed OSCs; 2) to estimate the structural and dynamic characteristics of the considered star clusters.

\subsection{Structural Parameters of Six OSC}

Following to Danilov (2010), let us consider well-studied clusters of different ages: NGC 188, NGC 1912, NGC 6705, NGC 6819, NGC 7654, IC 1848. Danilov and Seleznev (1994) determined the structural and dynamic parameters of these clusters most accurately. We selected the clusters with the number of stars $N_{c}>300$ from the catalogue of Danilov and Seleznev (1994). Such sampling allows us to reduce the role of stellar encounters in the dynamic evolution of the clusters (the coefficients $p_{j}$ of the equation (31) from Danilov (2008) were obtained in the collisionless approximation). According to the estimates of Henon (1964) and Danilov (1980) in the clusters with $N_{c}>250$ the collisional relaxation time $\tau_{s t}$ due to the single stars encounters is longer than the time of violent relaxation $\tau_{v r}$ due to the interaction of stars with a regular force field of a non-stationary cluster. To calculate the distributions of the apparent density of the number of stars in the selected clusters, Danilov (2010) used the data on the numbers of the stars $N(r)$ obtained by star counts performed by Danilov and Seleznev (1994). Here, $N(r)$ is the number of stars within a circle of radius $r$ in the projection onto the tangent plane. Comparison of the numbers $N(r)$ in the cluster region and in several nearby non-overlapping regions of the star field allows us to estimate the errors of the apparent density values. Calculation of the apparent density of the number of stars $F(r)$ on the interval $r \in\left[r^{\prime}, r^{\prime}+\Delta r\right)$ by Danilov (2010) was carried out using two methods. One of them is described in the book of Kholopov $(1981$, p. 307), where $F(r)$ is defined as the ratio of the number of stars within the annular zone (in the projection onto the tangent plane) to the area of this zone, and $\Delta r$ is the width of the annular zone. Unlike to Kholopov (1981), $F(r)$ here was assigned to the middle of the interval $r \in\left[r^{\prime}, r^{\prime}+\Delta r\right)$, and the "interval error" (Danilov, 2010) was eliminated by introducing the correction in $F(r)$ using the technique described by Vasilevsky $(1985$, p. 24). $F(r)$ at the boundary of the 
annular zone was calculated from the formula

$$
F(r)=\frac{1}{2 \pi r} \frac{d N(r)}{d r} .
$$

Danilov (2010) smoothed the functions $F(r)$ and $N(r)$ before performing a numerical differentiation with the use of the symmetric nonrecursive five-point digital filters, see, for example, in Hamming (1977, p. 54-55).

The parameters $k, r_{c}, r_{t}$ of the King's distribution of the apparent density $F(r)$ (see formula (8.38) in Kholopov (1981)) for the considered clusters are given in the catalogue of Danilov and Seleznev (1994) (here, $k$ is the value associated with the apparent density in the center of the cluster, $r_{c}$ is the radius of cluster core, $r_{t}$ is the cluster radius). However, this distribution only approximately reproduces an apparent density in the OSC. Usually, in the OSC the number of stars

$$
N_{c, F}=2 \pi \int_{0}^{r_{t}} r^{\prime} F\left(r^{\prime}\right) d r^{\prime}
$$

is noticeably less than the number of stars $N_{c}$, obtained in star counts of Danilov and Seleznev (1994). For example, in the old clusters NGC 188 and NGC 6819, the value $N_{c, F}$ obtained from the King distribution is, respectively, $\sim 1.35$ and $\sim 1.52$ times smaller than the values of $N_{c}$, obtained by Danilov and Seleznev (1994) for these clusters. In the young OSCs this ratio can reach $\sim 2.0-2.5$. The halos of the OSCs are usually much more populated by stars than it follows from the King's distribution. In this connection, the following modified King's distribution was used to describe the OSCs by Danilov (2010):

$$
F(r)=k_{m}\left(\frac{1}{\left(1+\left(r / r_{c, m}\right)^{2}\right)^{1 / \gamma}}-\frac{1}{\left(1+\left(r_{t} / r_{c, m}\right)^{2}\right)^{1 / \gamma}}\right)^{\gamma} .
$$

Such a distribution is a particular case of the apparent density distribution proposed by Veltmann (1965) and written down by Kholopov (1981) in the form of formula (8.40). The expression (8.1) becomes a usual King distribution at $\gamma=2, k_{m}=k$, $r_{c, m}=r_{c}$. We note that at $\gamma<0$, the formula (8.1) loses its meaning and ceases to describe the distribution of the apparent density in the stellar clusters (since in this case it is necessary to raise a negative number to the power of $\gamma$ ). In the case $0<\gamma<2$, the formula (8.1) allows us to match $N_{c}$ values obtained in star counts and $N_{c, F}$ values.

The distributions of the apparent density $F(r)$ obtained by Danilov (2010) for six open clusters were approximated by distributions of the form (8.1) applying the Marquardt method (Marquardt, 1963). In this case, the observed radius $r_{t}$ of the cluster was not varied, but was taken equal to that obtained by Danilov and Seleznev (1994) (see Table A.2.1 there; in all estimates, scale of distances to the clusters was used corresponding to P.N. Kholopov's ZAMS). After a finding of the distribution parameters $k_{m}, r_{c, m}, \gamma$, their mean values were refined using the condition $N_{c}=N_{c, F}$. For this, $\gamma$ was varied near the average $\gamma$ found within the error 
Table 8.1 Structural Parameters of Six OSC

\begin{tabular}{|c|c|c|c|c|c|c|c|}
\hline № & OSC & $k_{m}$ & $r_{c, m}$ & $\gamma$ & $\rho(0)$ & $\frac{\sigma_{N_{c, F}}}{N_{c, F}}(\%)$ & $q_{e}$ \\
\hline 1 & 2 & 3 & 4 & 5 & 6 & 7 & 8 \\
\hline 1 & NGC 188 & $\begin{array}{c}17.69 \\
\pm 1.18 \\
( \pm 1.18)\end{array}$ & $\begin{array}{c}1.69 \\
\pm 0.14 \\
( \pm 0.14)\end{array}$ & $\begin{array}{c}1.44 \\
\pm 1.06 \\
( \pm 0.32)\end{array}$ & \begin{tabular}{|c|}
7.2 \\
\pm 1.1
\end{tabular} & $\begin{array}{c}9.5 \\
(3.5)\end{array}$ & 1.00 \\
\hline 2 & NGC 1912 & $\begin{array}{c}22.61 \\
\pm 0.62 \\
( \pm 0.18)\end{array}$ & $\begin{array}{c}1.47 \\
\pm 0.05 \\
( \pm 0.01)\end{array}$ & $\begin{array}{c}0.16 \\
\pm 0.24 \\
( \pm 0.15)\end{array}$ & \begin{tabular}{|l|}
12.3 \\
\pm 0.8
\end{tabular} & $\begin{array}{c}1.0 \\
(0.4)\end{array}$ & 0.29 \\
\hline 3 & NGC 6705 & $\begin{array}{c}56.13 \\
\pm 2.38 \\
( \pm 0.31)\end{array}$ & $\begin{array}{c}1.11 \\
\pm 0.06 \\
( \pm 0.01)\end{array}$ & $\begin{array}{c}0.15 \\
\pm 0.27 \\
( \pm 0.14)\end{array}$ & \begin{tabular}{|l|}
71.2 \\
\pm 4.8
\end{tabular} & $\begin{array}{c}1.5 \\
(0.3)\end{array}$ & 0.13 \\
\hline 4 & NGC 6819 & $\begin{array}{c}35.89 \\
\pm 1.11 \\
( \pm 0.16)\end{array}$ & $\begin{array}{c}1.40 \\
\pm 0.05 \\
\pm 0.01)\end{array}$ & $\begin{array}{c}0.15 \\
\pm 0.87 \\
( \pm 0.14)\end{array}$ & \begin{tabular}{|l|}
23.9 \\
\pm 1.2
\end{tabular} & $\begin{array}{c}1.7 \\
(0.3)\end{array}$ & 0.14 \\
\hline 5 & NGC 7654 & \begin{tabular}{|c|}
26.60 \\
\pm 3.88 \\
$( \pm 0.39)$
\end{tabular} & $\begin{array}{c}1.31 \\
\pm 0.20 \\
( \pm 0.20)\end{array}$ & \begin{tabular}{|c|}
0.98 \\
\pm 0.57 \\
$( \pm 0.59)$
\end{tabular} & \begin{tabular}{|l|}
37.7 \\
\pm 8.0
\end{tabular} & $\begin{array}{c}6.5 \\
(6.6)\end{array}$ & 1.00 \\
\hline 6 & IC 1848 & $\begin{array}{c}11.88 \\
\pm 1.02 \\
( \pm 0.05)\end{array}$ & $\begin{array}{c}1.63 \\
\pm 0.24 \\
( \pm 0.01)\end{array}$ & $\begin{array}{c}0.15 \\
\pm 1.57 \\
( \pm 0.14)\end{array}$ & \begin{tabular}{|l|}
17.4 \\
\pm 2.6
\end{tabular} & $\begin{array}{c}4.6 \\
(0.3)\end{array}$ & 0.05 \\
\hline
\end{tabular}

(the standard deviation) of this value. Generally, this was enough to match the values $N_{c, F}$ and $N_{c}$ from the catalog of Danilov and Seleznev (1994) with the difference $\Delta N=N_{c, F}-N_{c}$ not exceeding \pm 0.01 . In those cases, when the changes in $\gamma$ were insufficient for such matching of $N_{c}$ and $N_{c, F}$, a similar refinement of the average values of $k_{m}, r_{c, m}$ was carried out. Since the changes in the parameters $k_{m}, r_{c, m}$, $\gamma$ with this refinement have a non-random character, the errors of these parameters are equal to those obtained for the initial approximation of the apparent-density distributions by the function (8.1).

The results of calculations of Danilov (2010) are given in the columns 3-5 of the Table 8.1. The first and second columns of Table 8.1 show the number (in an order) and the name of the cluster, respectively.

According to the Table 8.1, the errors $\sigma_{\gamma}$ in the determination of the values $\gamma=\bar{\gamma} \pm \sigma_{\gamma}$ in the considered clusters are the largest. We note that the use of conditions $N_{c}=N_{c, F}$ and $\gamma>0$ imposes restrictions on the errors $\sigma_{\gamma}, \sigma_{k_{m}}$, $\sigma_{r_{c, m}}$ of the values $\gamma, k_{m}, r_{c, m}$. The range of admissible values of these parameters can be determined as follows. Following to Danilov (2010), we variate the relation $N_{c}=N_{c, F}$ by setting $\delta N_{c}=0$ and using the function $F(r)$ from (8.1). We obtain

$$
\delta N_{c}=I_{1} \delta k_{m}+I_{2} \delta r_{c, m}+I_{3} \delta \gamma=0 \quad \text { or } \quad(\mathbf{I}, \delta \mathbf{n})=0,
$$

where 


$$
\begin{aligned}
& I_{1}=\int_{0}^{r_{t}} r^{\prime} \frac{\partial F\left(r^{\prime}\right)}{\partial k_{m}} d r^{\prime}, \quad I_{2}=\int_{0}^{r_{t}} r^{\prime} \frac{\partial F\left(r^{\prime}\right)}{\partial r_{c, m}} d r^{\prime}, \quad I_{3}=\int_{0}^{r_{t}} r^{\prime} \frac{\partial F\left(r^{\prime}\right)}{\partial \gamma} d r^{\prime}, \\
& \mathbf{I}=\left(I_{1}, I_{2}, I_{3}\right), \quad \delta \mathbf{n}=\mathbf{n}-\langle\mathbf{n}\rangle, \quad \mathbf{n}=\left(k_{m}, r_{c, m}, \gamma\right), \quad\langle\mathbf{n}\rangle=\left(\bar{k}_{m}, \bar{r}_{c, m}, \bar{\gamma}\right) .
\end{aligned}
$$

The equation (8.2) is the euqation of the plane in the space of parameters $\left(k_{m}, r_{c, m}, \gamma\right)$ (on the plane defined by this equation, the value of $N_{c}=$ const). Solving the equation (8.2) together with the equations of planes $k_{m}=\bar{k}_{m} \pm \sigma_{k_{m}}$ and $r_{c, m}=\bar{r}_{c, m} \pm \sigma_{r_{c, m}}$, we find the following four values of $\gamma$ :

$$
\gamma^{(1,2,3,4)}=\bar{\gamma}-\frac{\left( \pm \sigma_{k_{m}}\right) I_{1}+\left( \pm \sigma_{r_{c, m}}\right) I_{2}}{I_{3}}
$$

The mean values of $\gamma^{(i)}$ coincide with $\bar{\gamma}$, and the largest deviations of $\gamma^{(i)}$ from $\bar{\gamma}$ make it possible to judge the size of the range of admissible values for $\gamma$. In most of the cases considered, the conditions $\gamma^{(i)}>0$ can not be achieved without decreasing the sizes of the range of admissible values in the spaces $k_{m}$ and $r_{c, m}$ in $q_{e}$ times. The admissible deviations of $k_{m}, r_{c, m}$, gamma from their mean values, corresponding to the conditions $N_{c}=N_{c, F}$ and $\gamma>0$, for the considered clusters are given in columns 3-5 of Table 8.1 in brackets. $q_{e}$ values are listed in column 8 of Table 8.1. In column 7 of this table, the relative errors of $N_{c, F}$ are listed, caused by the errors of $k_{m}, r_{c, m}, \gamma$, as well as by the admissible deviations of these values from the mean (in parentheses).
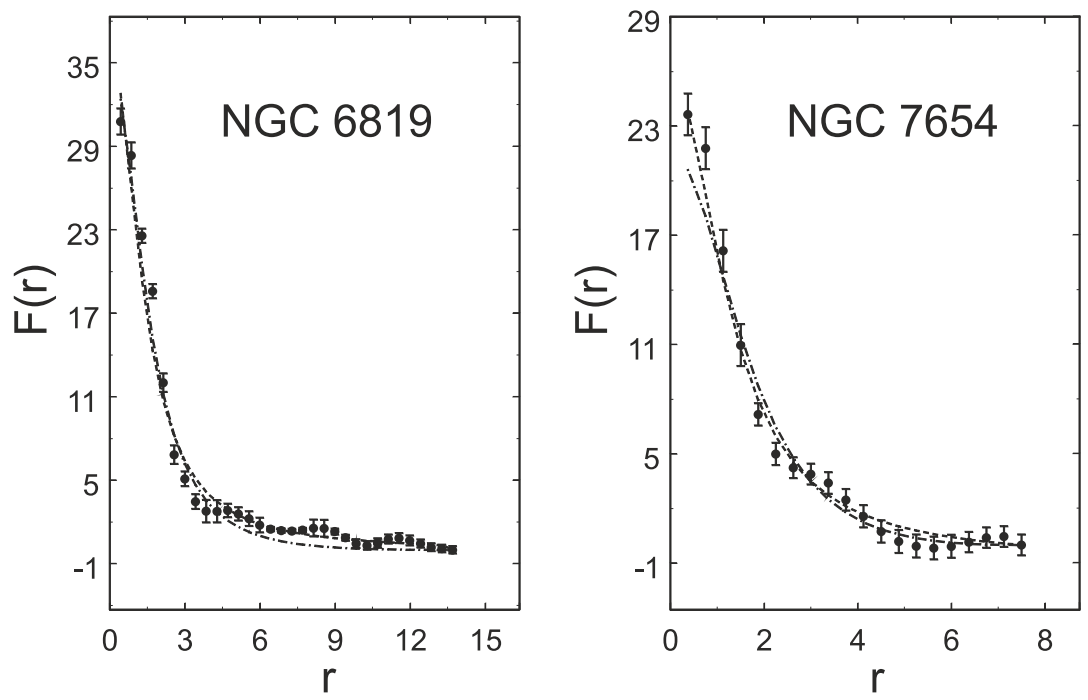

Fig. 8.1 The distribution of an apparent density of the number of stars by the distance $r$ from the centers of clusters NGC 6819, NGC 7654. Dashed lines correspond to the modified King distribution; the dash-and-dot lines indicate the curves for the usual King distribution 
For all the OSCs considered, the relative errors (and the permissible deviations from $N_{c}$ ) of the values $N_{c, F}$ are small. In addition, in all cases (with the exception of NGC 188), the values of $\gamma$ significantly differ from 2 . Therefore, the apparent density distributions in the considered clusters differ noticeably from the usual King distribution (8.38) from Kholopov (1981). The function $F(r)$ from (8.1) and the value $k_{m}=\bar{k}_{m}, r_{c, m}=\bar{r}_{c, m}, \gamma=\bar{\gamma}$ allow us to approximate better the distribution of the apparent density near the center and at the periphery of the considered OSC than the usual King distribution (8.38) from Kholopov (1981). For example, fig. 8.1 shows the apparent density distributions in NGC 6819 and NGC 7654 (shown as points with error bars), as well as functions (8.1) (dashed line) with parameters $k_{m}=\bar{k}_{m}, r_{c, m}=\bar{r}_{c, m}, \gamma=\bar{\gamma}$ from Table 8.1 and the distribution (8.38) from Kholopov (1981) (the dash-and-dot line) with parameters $k=45.16 \pm 0.92 \mathrm{pc}^{-2}$; $r_{c}=1.70 \pm 0.07 \mathrm{pc}$ for NGC 6819 and $k=43.26 \pm 1.41 \mathrm{pc}^{-2} ; r_{c}=1.84 \pm 0.14 \mathrm{pc}$ for NGC 7654 (these values are obtained by an approximating of the distributions of an apparent density in the clusters by the function (8.38) from Kholopov (1981)); a small difference between these values and those indicated by Danilov and Seleznev (1994) is due to the fact that parameters $k$ and $r_{c}$ are obtained by Danilov and Seleznev (1994) by an approximating of the observational data on numbers of stars $N(r)$ by the corresponding function (3) from Danilov and Seleznev (1994)).

For a transition from the apparent density distribution of the number of stars $F\left(r^{\prime}\right)$ to the spatial density distribution $f(r)$ we used (Danilov, 2010) the assumption of the spherical symmetry of the distribution of stars (and mass) in the cluster, as well as a solution of the Abel integral equation (see (8.5) in Kholopov (1981)) for the function $f(r)$ written in the form (8.7), (8.8) from Kholopov (1981). Performing the calculations given by (8.7) and (8.8) from Kholopov (1981), taking into account the distribution (8.1), we find

$$
f(r)=\frac{2 k_{m} I}{\pi r_{c, m}^{2}}, \quad I=\int_{0}^{z_{m}} \frac{d z}{u^{2}\left[1-\left(u / u_{0}\right)^{1 / \gamma}\right]^{1-\gamma}},
$$

where

$$
z_{m}=\sqrt{r_{t}^{2}-r^{2}}, \quad u=1+\left(r^{2}+z^{2}\right) / r_{c, m}^{2}, \quad u_{0}=1+r_{t}^{2} / r_{c, m}^{2} .
$$

At $\gamma<1$, an integral $I$ becomes improper, and the integrand in $I$ becomes unbounded. In this case, for $z \rightarrow z_{m}$, the value $u \rightarrow u_{0}$, which complicates the numerical integration in (8.3). If $r \rightarrow r_{t}$ and $\gamma<0.5$, the contribution to the integral $I$ of the regions of $z$ close to $z_{m}$ becomes significant. In such cases, it is convenient to use the following semi-analytic estimate of the integral $I$. We introduce a small value $\varepsilon>0$. Then we divide the region of integration over $z$ in (8.3) into two regions: $z \in\left[0,(1-\varepsilon) z_{m}\right]$ and $z \in\left[(1-\varepsilon) z_{m}, z_{m}\right]$.

In the first interval the integration is easily performed numerically (we denote this part of the integral as $I_{1-\varepsilon}$ ). In the second interval we note that the values of the function $u$ vary very little near the value $u_{0}$ (at $z \rightarrow z_{m}$, the value of $u \rightarrow u_{0}$ ). Therefore, the value $u^{2}$ (the factor in front of the square bracket in the denominator 
of integrand) can be replaced in some approximation by $u_{0}^{2}$. Expanding the function $g(z)=1-\left(u / u_{0}\right)^{1 / \gamma}$ in a Taylor series in powers of $z-z_{m}$ and limiting to the first term of the expansion, we find

$$
g(z) \simeq-\frac{2 z_{m}\left(z-z_{m}\right)}{\gamma\left(r_{c, m}^{2}+r_{t}^{2}\right)} .
$$

Substituting the function $g(z)$ in the integral $I$ at the interval $z \in\left[(1-\varepsilon) z_{m}, z_{m}\right]$, we find

$$
I_{\varepsilon}=\int_{(1-\varepsilon) z_{m}}^{z_{m}} \frac{d z}{u_{0}^{2} g(z)^{1-\gamma}}=\frac{1}{u_{0}^{2} z_{m}^{1-2 \gamma}}\left(\frac{\varepsilon}{\gamma}\right)^{\gamma}\left(\frac{r_{c, m}^{2}+r_{t}^{2}}{2}\right)^{1-\gamma} .
$$

Let us denote $I_{s}=I_{1-\varepsilon}+I_{\varepsilon} \simeq I$. Choosing the value $\varepsilon$ to be sufficiently small, it is easy to obtain a good estimate for the integral $I$. For example, in the case of cluster NGC 6819 at $\varepsilon=10^{-5}$, the relative error $\epsilon=\left|\left(I_{S}-I\right) / I\right|$ is $(0.17-0.69) \times 10^{-8}$ at $r=0-3 \mathrm{pc}$. If $r \rightarrow r_{t}$, the value $\epsilon$ increases by $1-2$ orders of magnitude. To check the accuracy of the obtained values of $f(r)$, we also used the condition

$$
N_{c}=4 \pi \int_{0}^{r_{t}} r^{2} f(r) d r .
$$

We performed the calculations of the spatial density distributions of the number of stars $f(r)$ in the clusters with $\gamma \leq 0.155$ taking into account the analytical estimate (8.4) at $\varepsilon=10^{-5}, 10^{-6}$ (Danilov, 2010). The values of $\epsilon$ in these cases are $\sim 10^{-8}-10^{-7}$ at $r=0-3 \mathrm{pc}$. Maximum errors in the values of $N_{c}$ were achieved for clusters NGC 6819, NGC 6705, IC 1848: 2.7, 2.8, $5.1 \%$, respectively. To reduce the relative errors of the $N_{c}$ values to $(1-2) \%$, the values $\varepsilon=\varepsilon(r)$ decreasing with increasing of $r$ were used (Danilov, 2010). For clusters NGC 188 and NGC 7654, it became possible to obtain the functions $f(r)$ by the Simpson method over the entire range of values $r \in\left[0, r_{t}\right]$ with the relative error $\sim 10^{-8}-10^{-7}$, which corresponds to relative errors in $N_{c}$ equal to 0.026 and $0.005 \%$, respectively.

In column 6 of Table 8.1, the values of the spatial mass density $\rho(0)=m f(0)$ in the centers of the considered clusters are listed; here, $m$ is an average mass of the star in the cluster, obtained by Danilov and Seleznev (1994). According to Table 8.1, among the considered clusters, the largest density $\rho(0)$ is reached in NGC 6705, and the smallest one in NGC 188. The errors in $\rho(0)$ are due to the errors in the values $k_{m}, r_{c, m}, \gamma$, and have been obtained by varying the expression (8.3) in $k_{m}, r_{c, m}, \gamma$ for $r=0$. The greatest contribution to the error of $\rho(0)$ for cluster NGC 188 is made by the error of $\gamma$, while for the remaining clusters - by the error in $r_{c, m}$. The most complicated for an integrating with respect to $z$ for $\gamma<1$ is the integrand in the expression for $\frac{\partial \rho(0)}{\partial \gamma}$. In this case, the integral over $z$ becomes improper, and the integrand is unbounded (at $z \rightarrow z_{m}$, its values tend to $-\infty$ ): 


$$
\begin{gathered}
\frac{\partial \rho(0)}{\partial \gamma}=\frac{2 m k_{m}}{\pi r_{c, m}^{2} u_{0}^{2} \gamma} J, \\
J=\int_{0}^{z m} \frac{[\gamma g \ln (g)-(1-\gamma)(1-g) \ln (1-g)] d z}{g^{2-\gamma}(1-g)^{2 \gamma}},
\end{gathered}
$$

where $g=1-\left(u / u_{0}\right)^{1 / \gamma}$, see above. To calculate the integral in(8.5), as in the case (8.3), we divide the region of integration over $z$ into two: $z \in\left[0,(1-\varepsilon) z_{m}\right]$ and $z \in\left[(1-\varepsilon) z_{m}, z_{m}\right]$. In the first interval over $z$, the integration is easily carried out numerically (we denote this part of integral $J$ as $J_{1-\varepsilon}$ ). In the second interval, we use the change of variables $z=r_{c, m} \sqrt{u_{0}(1-g)^{\gamma}-1}$ for the transition to integration over $g$. At $z=z_{m}$, we find $g=0$, and at $z=(1-\varepsilon) z_{m}$ we find

$$
\left.g=g_{\varepsilon}=1-\left(1+(1-\varepsilon)^{2} z_{m}^{2} / r_{c, m}^{2}\right) / u_{0}\right)^{1 / \gamma} .
$$

Swapping the limits of integration, for the part of integral $J$ corresponding to the interval $z \in\left[(1-\varepsilon) z_{m}, z_{m}\right]$ we obtain

$$
J_{\varepsilon}=\frac{r_{c, m} u_{0} \gamma}{2} \int_{0}^{g_{\varepsilon}} \frac{[\gamma g \ln (g)-(1-\gamma)(1-g) \ln (1-g)] d g}{g^{2-\gamma}(1-g)^{1+\gamma} \sqrt{u_{0}(1-g)^{\gamma}-1}} .
$$

Expanding the function $\ln (1-g)$ in Taylor series in powers of $g$ and restricting by the first term of the expansion, we find $\ln (1-g) \simeq-g$. In the interval of values $g \in\left[0, g_{\varepsilon}\right]$, for sufficiently small $\varepsilon>0$, such an approximation for the function $\ln (1-g)$ is quite acceptable. We substitute $\ln (1-g) \simeq-g$ in (8.6). We note that the values of the function $1-g$ at $g \in\left[0, g_{\varepsilon}\right]$ vary little near 1 . Therefore, the value $1-g$ in (8.6) can be replaced in some approximation by 1 . After these transformations, we find

$$
J_{\mathcal{\varepsilon}} \simeq \frac{r_{c, m} u_{0} \gamma}{2 \sqrt{u_{0}-1}} \int_{0}^{g_{\varepsilon}} \frac{[\gamma g \ln (g)+(1-\gamma) g] d g}{g^{2-\gamma}}=\frac{r_{c, m} u_{0} \gamma}{2 \sqrt{u_{0}-1}} g_{\varepsilon}^{\gamma}\left(\ln \left(g_{\varepsilon}\right)-1\right) .
$$

The first term in the integrand in (8.7) can be easily integrated by parts, and the remainder of this function is integrated as a power function. In calculating the integrals $J_{\varepsilon}$ in the paper Danilov (2010) we used the values $\varepsilon=10^{-7}$, which allows to get the estimates $J_{\varepsilon}$ with a relative error $\epsilon \sim 10^{-8}-10^{-7}$. With the same accuracy, it is not difficult to obtain the estimates of the value $J=J_{1-\varepsilon}+J_{\varepsilon}$.

The relative errors of the $\rho(0)$ quantities for the considered here OSC are within the interval $\sigma_{\rho(0)} / \rho(0) \sim 0.05-0.21$. We note that here we do not take into account the relative errors in the values $m$, which, according to Danilov and Seleznev (1994), are assumed to be on average equal to $\sigma_{m} / m \simeq 0.25$. Considering the errors $\sigma_{m} / m$, the values $\sigma_{\rho(0)} / \rho(0)$ will be enclosed in the interval $\sim 0.30-0.46$. 


\subsection{Structural and Dynamic Parameters of Six OSC Models}

For further analysis of the structural and dynamic characteristics of the OSC considered in this part of the work, it is necessary to clarify certain parameters of the numerical dynamic OSC models 1 - 6 (Danilov and Dorogavtseva, 2003, 2008). In these papers, the cluster models consisting of 500 stars, moving along circular orbit of radius $R_{G}=8200 \mathrm{pc}$ in the plane of the Galaxy around its center, are considered. In the papers Danilov and Dorogavtseva (2003, 2008), the cluster models 1-6 are numbered in descending order depending on the degree of model's non-stationarity in a regular field, see Table 1 from Danilov and Dorogavtseva (2003). The initial parameters of the OSC models are listed in Table 1 from Danilov and Dorogavtseva (2003, 2008). The gravitational potential in the core and the periphery of the OSC models was averaged over the period of the oscillations $P_{r}$ of a regular field (Danilov, $2008,2005,2006)$ on the interval of the relative time values $t / \tau_{v r} \in[1.3, \vartheta]$, where $\vartheta=1.9-2.0 ; \tau_{v r}$ is the initial time of a violent relaxation obtained according to the formula $\tau_{v r} \simeq 2.6 \bar{t}_{c r}$ (Aarseth, 1974); $\bar{t}_{c r}$ is the average initial time of the cluster' intersection by a star (the crossing time). At time $t / \tau_{v r}=1.3$, the equilibrium regime of the density and regular field oscillations is already established in models 1-6.

In columns 1 and 2 of Table 8.2 for the considered OSC models, we indicate the model number (according to the numbering of these models by Danilov and Dorogavtseva $(2003,2008))$ and the value of the potential

$$
U(0)=\sum_{i=1}^{N_{t}} G m_{i} / r_{i}
$$

in the center of the cluster model (averaged over the period $P_{r}$ ); here, $G$ is the gravitational constant, $r_{i}$ is the distance of the $i$-th star from the mass center of the cluster, $N_{t}$ is the number of stars in the cluster model with $r_{i}<R_{t}, R_{t}$ is tidal radius of the cluster (King, 2002, p. 198), $m_{i}$ is the mass of the $i$-th star (for the OSC models 1-6 (Danilov and Dorogavtseva, 2003, 2008), the star masses were assumed to be equal to one mass of the Sun $m_{i}=m_{\odot}$; in the papers of Danilov and Dorogavtseva $(2003,2008)$ and in this study, we used the following system of units: $1 m_{\odot}, 1 \mathrm{pc}$, 1 Myr). Column 3 of Table 8.2 contains the value of the mean mass density of the cluster core $\rho_{c}$ averaged over the period $P_{r}$ (obtained by $N_{s}$ stars closest to the center of mass of the cluster model and located within the cluster core; the radius of the cluster core corresponds to the distance from the center, in which the spatial density attains the values characteristic for the cluster halo; the moduli of the spatial density gradients in the halo are minimal). The value $N_{s}$ is given in the 4 th column of the Table 8.2. Column 5 of Table 8.2 gives the value of the mean mass density at the center of the cluster $\rho(0)$ averaged over the period $P_{r}$ (obtained by five cluster stars closest to the center). Column 6 of Table 8.2 gives the mass density $\rho_{e}$ of a homogeneous ellipsoid with semiaxes $a, b, c$ which approximately correspond to the distance of zero-velocity surface of the numerical cluster model from its center along the coordinate axes $\xi, \eta, \zeta$ (Danilov and Dorogavtseva, 2003; Danilov, 2008). 
Table 8.2 Structural and dynamic parameters of six OSC models

\begin{tabular}{|c|c|c|c|c|c|c|c|c|}
\hline № & $U(0)$ & $\rho_{c}$ & $N_{s}$ & $\rho(0)$ & $\rho_{e}$ & $\varepsilon_{t}$ & $\sigma_{v}^{2}$ & $\sigma_{v i r}^{2}$ \\
\hline 1 & 2 & 3 & 4 & 5 & 6 & 7 & 8 & 9 \\
\hline 1 & 0.62 & 0.93 & 125 & 11.4 & 0.90 & 0.43 & 0.27 & 0.20 \\
& \pm 0.03 & \pm 0.16 & & \pm 4.1 & \pm 0.09 & \pm 0.04 & \pm 0.03 & \pm 0.01 \\
\hline 2 & 0.70 & 0.93 & 150 & 16.5 & 0.88 & 0.42 & 0.28 & 0.23 \\
& \pm 0.03 & \pm 0.11 & & \pm 10.6 & \pm 0.09 & \pm 0.04 & \pm 0.02 & \pm 0.01 \\
\hline 3 & 0.75 & 0.95 & 240 & 7.1 & 0.96 & 0.46 & 0.30 & 0.25 \\
& \pm 0.02 & \pm 0.07 & & \pm 3.0 & \pm 0.096 & \pm 0.04 & \pm 0.02 & \pm 0.01 \\
\hline 4 & 0.84 & 1.03 & 180 & 12.4 & 0.99 & 0.47 & 0.33 & 0.26 \\
& \pm 0.05 & \pm 0.07 & & \pm 6.9 & \pm 0.10 & \pm 0.04 & \pm 0.02 & \pm 0.01 \\
\hline 5 & 0.79 & 1.08 & 300 & 4.9 & 1.08 & 0.51 & 0.331 & 0.284 \\
& \pm 0.02 & \pm 0.06 & & \pm 1.8 & \pm 0.11 & \pm 0.05 & \pm 0.006 & \pm 0.004 \\
\hline 6 & 0.69 & 0.84 & 300 & 2.2 & 0.87 & 0.42 & 0.35 & 0.27 \\
& \pm 0.03 & \pm 0.13 & & \pm 0.8 & \pm 0.09 & \pm 0.04 & \pm 0.02 & \pm 0.01 \\
\hline
\end{tabular}

Potential of this ellipsoid $U_{e}$ near the center of mass of each cluster model is given by the equation (2) from Danilov (2008):

$$
U_{e}=U_{0}-\left(U_{0}+U_{1}\right) r_{e}^{2}+S, \quad r_{e}^{2}=\left(\frac{\xi}{a}\right)^{2}+\left(\frac{\eta}{b}\right)^{2}+\left(\frac{\zeta}{c}\right)^{2}, \quad S=\frac{\alpha_{1} \xi^{2}+\alpha_{3} \zeta^{2}}{2}
$$

where $\alpha_{1}$ and $\alpha_{3}$ are the constants characterizing a Galactic force field in the neighborhood of the cluster circular orbit (their numerical values are defined in Danilov and Dorogavtseva $(2003,2008)$ using the Galaxy potential model of Kutuzov and Osipkov (1980); $\alpha_{1}<0$ and $\alpha_{3}>0$ ). The constants $U_{0}$ and $U_{1}$ for model 1 are obtained by Danilov (2008). These constants for models 2-6 are obtained in the present work by means of approximation (by the method of Marquardt (1963)) of the potential of a numerical cluster model near its center, averaged over the period $P_{r}$, by a potential of the form (8.8). In the 7th column of Table 8.2, the values of $\varepsilon_{t}=U_{0}+U_{1}$ is given. According to Danilov (2005), the values $\rho_{e}$ and $\varepsilon_{t}$ are related by the ratio

$$
\rho_{e}=\frac{2 \varepsilon_{t}\left(a^{-2}+b^{-2}+c^{-2}\right)-\alpha_{1}-\alpha_{3}}{4 \pi G},
$$

obtained by Danilov (2005) using the Poisson equation for a homogeneous ellipsoid with the potential (8.8).

In a derivation of relation (8.9), Danilov (2005) used equations (5.517)-(5.519) from Chandrasekhar (1942), describing stellar motion in the joint force field of the Galaxy and a homogeneous gravitating ellipsoid with potential 8.9). According to Danilov (2010), the equations (5.517)-(5.519) from Chandrasekhar (1942), written for the motion of a star in the force field of the Galaxy and the open star cluster, yield the following (virial) relation for the mean square of a residual velocity of the motion of a star in the cluster: 


$$
\sigma_{v i r}^{2}=\frac{1}{M_{c}}\left[-W+\sum_{i=1}^{N_{t}} m_{i}\left(\alpha_{1} \xi_{i}^{2}+\alpha_{3} \zeta_{i}^{2}\right)\right],
$$

where $M_{c}=m N_{t}$ and $W$ are the mass and the potential cluster energy. In (8.10), the angular momentum of a rotation of the cluster relatively to $\zeta$ axis in the rotating coordinate system $(\xi, \eta, \zeta)$ (Chandrasekhar, 1942) is assumed to be zero,

$$
W=-\frac{G}{2} \sum_{i=1}^{N_{t}} \sum_{j=1}^{N_{t}} \frac{m_{i} m_{j}}{r_{i j}}, \quad(i \neq j),
$$

$r_{i, j}$ is the distance between the $i$-th and $j$-th cluster stars (Chandrasekhar, 1942). According to Bagin (1969), for the isolated spherical stellar systems in the case of local virial equilibrium at a distance of $r$ from the center of the system, the mean square of the residual stellar velocities is $\overline{v^{2}(r)}=U(r) / 2$, where $U(r)$ is the system potential at a distance $r$ from its center. We note that for a star cluster moving along a planar circular orbit in the field of forces of the Galaxy, the relation

$$
\overline{v^{2}(\mathbf{r})}=\frac{U(\mathbf{r})}{2}+\alpha_{1} \xi^{2}+\alpha_{3} \zeta^{2}
$$

after multiplying both parts of (8.11) by the mass element $d M_{c}=\rho(\mathbf{r}) d V$ and integrating over an entire mass of the cluster, allow us to obtain the relation (8.10); here, $\rho(\mathbf{r})$ and $d V$ are the mass density at the point $\mathbf{r}=(\xi, \eta, \zeta)$ and the element of the cluster volume respectively. At $\mathbf{r} \rightarrow \mathbf{0}=(0,0,0)$, the relation (8.11) passes into $\overline{v^{2}(0)}=U(0) / 2$. Relation (8.11) is the condition for local virial equilibrium of the star cluster at the point $\mathbf{r}$.

Column 8 of Table 8.2 gives the value of the average (with respect to $P_{r}$ period) dispersion of the stellar velocities $\sigma_{v}^{2}$ in the cluster model obtained by $N_{v}$ stars closest to the center of mass of the cluster model. For $\sigma_{v}^{2}$ estimates, we used the value $N_{v}=300$. With an increase of $N_{v}$ (at $N_{v} \leq 300$ ) in the considered models, $\sigma_{v}^{2}$ increases (or remains approximately constant) within errors of the values of $\sigma_{v}^{2}$ specified in column 8 , and then decreases (at $N_{v}>300$ ) within the limits of the indicated errors. Such variation of $\sigma_{v}^{2}$ with an increase of $N_{v}$ is associated with the tidal "heating" of the cluster and a subsequent dissipation (rather slow) of the tidal perturbations in the cluster.

Column 9 of Table 8.2 gives $\sigma_{v i r}^{2}$ value from (8.10) averaged over the period $P_{r}$. In all cases, except model 6 , the value of $\sigma_{v}^{2}$ is approximately equal to the half-sum of $\sigma_{v i r}^{2}$ and $\overline{v^{2}(0)}$ also averaged over the period $P_{r}$. For model 6 on average over $P_{r}$, we can write $\sigma_{v}^{2} \simeq \overline{v^{2}(0)}$ (within the errors of $\sigma_{v}^{2}$ and $\overline{v^{2}(0)}$ averaged over $P_{r}$ ). Since the OSCs with the same "halo-core" parameters of the structure, like those of model 6 are quite rare (Danilov and Seleznev, 1994; Danilov and Dorogavtseva, 2008), then the most common for the OSCs is the following relation

$$
\sigma_{v}^{2} \simeq \frac{\left(\sigma_{v i r}^{2}+\overline{v^{2}(0)}\right)}{2}
$$


From the Table 8.2, it is clear that for the considered OSC models, the values $\rho_{e}$ and $\rho_{c}$, taking into account the errors of these values, do not differ statistically $\left(\rho_{e} \simeq \rho_{c}\right)$. We note that the values $U_{0}$ and $U(0)$ also agree well with each other, but in all OSC models the average value of $U_{0}$ is somewhat less than the average value of $U(0)$ (usually the difference between the average values of $U_{0}$ and $U(0)$ remains within the sum of the errors of these values).

Relations (8.12), $\rho_{e} \simeq \rho_{c}$ and $U_{0} \lesssim U(0)$ between the parameters of the models allow us to estimate the dynamic characteristics of the observed OSCs and analyze the dynamic state of such star clusters considering the data on the structure, size and mass of the OSC.

\subsection{Dynamic Parameters of Six OSC and Two OSC Models}

The spatial densities of the number of stars $f(r)$ in the considered OSC for $\sim 200$ values of $r$ from the interval $r \in\left(0, r_{t}\right)$ with a given step $\Delta r$ were obtained in this work using formulas (8.3), (8.4). Then, using the random number generator, the spatial positions of $6 N_{c}$ stars in the spherical coordinate system $(r, \theta, \varphi)$ were generated for each star cluster. The function $f(r)$ was used to calculate the distribution density

$$
p_{a}(r)=4 \pi \int_{0}^{r} f\left(r^{\prime}\right) r^{\prime 2} d r^{\prime} / N_{c}
$$

for the probabilities of a star to fall into the interval $r \in\left(0, r_{t}\right) ; p_{a}(0)=0, p_{a}\left(r_{t}\right)=1$. A discrete random value $r$ with a given density $p_{a}(r)$ was distributed in the interval $r \in\left(0, r_{t}\right)$ according to the procedure described by $\operatorname{Sobol}(1985, \mathrm{p} .26)$. The values of $\theta$ and $\varphi$ were distributed in the intervals $\theta \in(0, \pi)$ and $\varphi \in(0,2 \pi)$ with the densities $p_{b}(\theta)=\frac{1}{2} \sin (\theta)$ and $p_{c}(\varphi)=\frac{1}{2 \pi}$, respectively (densities $p_{b}(\theta)$ and $p_{c}(\varphi)$ ensure the uniform stellar distribution by the angles $\theta$ and $\varphi$ for each fixed value of $r$ ). As a result, we obtain a sets of values $\left(\left(r_{i}, \theta_{i}, \varphi_{i}\right), i=1, \ldots, 6 N_{c}\right.$. Each of the six sets of coordinates of $N_{c}$ stars simulates a given cluster of stars. When estimating the dynamic parameters of the OSC, the mean values of these parameters and standard deviations from the mean over the six sets of coordinates of $N_{c}$ stars were calculated.

The results of the estimations of the dynamic parameters of the six analyzed OSCs and two OSC models are given in Table 8.3. Models 1 and 6 of Danilov and Dorogavtseva (2003) also characterize the structure and the dynamic state of a number of the observed OSCs with corresponding parameters of the "halo-core" structure (Danilov and Seleznev, 1994). Moreover, models 1 and 6 have the largest and the least degree of non-stationarity in a regular field, which makes it easier to analyze the results obtained for the six OSCs considered in our study.

In columns 1 and 2 of Table 8.3, the number and a name of the object are listed. Columns 3 and 4 of Table 8.3 give the estimates of the time of a violent $\left(\tau_{v r}\right)$ and a collisional $\left(\tau_{s t}\right)$ relaxation (in Myr). The $\tau_{v r}$ is obtained according to the formula $\tau_{v r} \simeq 2.6 \bar{t}_{c r}$ (Aarseth, 1974). $\bar{t}_{c r}=2 \bar{R} / \sigma_{v}$, where $\bar{R}$ is the mean cluster 
Table 8.3 Dynamic parameters of six OSC and two OSC models

\begin{tabular}{|c|c|c|c|c|c|c|c|c|c|c|}
\hline № & $\begin{array}{c}\text { Name } \\
\text { of the object }\end{array}$ & $\tau_{v r}$ & $\tau_{s t}^{1}$ & $U(0)$ & $\sigma_{\text {vir }}$ & $\sqrt{\overline{v^{2}(0)}}$ & $\varepsilon_{t}$ & $\rho_{e}$ & $P_{\gamma}(1)$ & $t_{\lambda}(1)$ \\
\hline 1 & 2 & 3 & 4 & 5 & 6 & 7 & 8 & 9 & 10 & 11 \\
\hline 1 & NGC 188 & 38.7 & 31.2 & 0.85 & 0.56 & 0.65 & 0.47 & 0.68 & 43.9 & 72.6 \\
& & \pm 4.0 & \pm 3.9 & \pm 0.03 & \pm 0.08 & \pm 0.10 & \pm 0.03 & \pm 0.05 & \pm 1.5 & \pm 3.6 \\
\hline 2 & NGC 1912 & 17.8 & 19.5 & 1.91 & 0.80 & 0.98 & 1.53 & 1.57 & 29.0 & 49.9 \\
& & \pm 0.7 & \pm 2.4 & \pm 0.07 & \pm 0.07 & \pm 0.10 & \pm 0.09 & \pm 0.10 & \pm 1.0 & \pm 0.7 \\
\hline 3 & NGC 6705 & 9.3 & 12.0 & 4.22 & 1.15 & 1.45 & 3.81 & 4.10 & 17.9 & 33.2 \\
& & \pm 1.0 & \pm 1.5 & \pm 0.03 & \pm 0.06 & \pm 0.04 & \pm 0.26 & \pm 0.29 & \pm 0.7 & \pm 0.3 \\
\hline 4 & NGC 6819 & 22.3 & 36.0 & 2.33 & 0.88 & 1.08 & 1.97 & 1.90 & 26.5 & 42.7 \\
& & \pm 2.3 & \pm 4.5 & \pm 0.06 & \pm 0.06 & \pm 0.08 & \pm 0.07 & \pm 0.07 & \pm 0.5 & \pm 0.4 \\
\hline 5 & NGC 7654 & 14.1 & 12.4 & 2.69 & 0.93 & 1.16 & 2.34 & 2.34 & 23.5 & 45.2 \\
& & \pm 1.2 & \pm 1.5 & \pm 0.14 & \pm 0.09 & \pm 0.11 & \pm 0.18 & \pm 0.18 & \pm 1.0 & \pm 0.3 \\
\hline 6 & IC 1848 & 24.6 & 21.4 & 2.02 & 0.81 & 1.01 & 1.64 & 1.31 & 31.9 & 51.4 \\
& & \pm 1.6 & \pm 2.7 & \pm 0.07 & \pm 0.07 & \pm 0.10 & \pm 0.08 & \pm 0.07 & \pm 0.8 & \pm 0.7 \\
\hline 7 & Model 1 & 46.8 & 121.9 & & 0.45 & 0.56 & & & 37.4 & 53.7 \\
& & \pm 2.8 & \pm 12.2 & & \pm 0.10 & \pm 0.12 & & & \pm 1.8 & \pm 2.9 \\
\hline 8 & Model 6 & 38.2 & 72.6 & & 0.52 & 0.59 & & & 38.1 & 56.3 \\
& & \pm 2.0 & \pm 7.2 & & \pm 0.10 & \pm 0.11 & & & \pm 1.8 & \pm 3.3 \\
\hline
\end{tabular}

\begin{tabular}{|c|c|c|c|c|c|c|c|}
\hline № & $\begin{array}{c}\text { Name } \\
\text { of the object }\end{array}$ & $\frac{\gamma_{1}(1)}{\omega}$ & $\rho_{\max , 0}$ & $\rho_{\min , 0}$ & $\rho_{\max , 1}$ & $\rho_{\min , 1}$ & $\rho_{e} / \rho_{t}$ \\
\hline 1 & 2 & 12 & 13 & 14 & 15 & 16 & 17 \\
\hline 1 & NGC 188 & 5.7 & 683.37 & 0.33 & 4.28 & 0.35 & 8.1 \\
\pm 0.2 & \pm 0.05 & \pm 0.05 & \pm 0.05 & \pm 0.05 & \pm 0.6 \\
\hline 2 & NGC 1912 & 8.9 & 632.53 & 0.30 & 5.36 & 0.31 & 20.0 \\
& & \pm 0.3 & \pm 0.20 & \pm 0.10 & \pm 0.10 & \pm 0.09 & \pm 1.3 \\
\hline 3 & NGC 6705 & 9.9 & 1348.96 & 0.65 & 11.14 & 0.68 & 26.2 \\
& & \pm 0.4 & \pm 0.30 & \pm 0.29 & \pm 0.29 & \pm 0.29 & \pm 1.8 \\
\hline 4 & NGC 6819 & 8.0 & 950.26 & 0.46 & 8.31 & 0.47 & 16.8 \\
& & \pm 0.1 & \pm 0.10 & \pm 0.07 & \pm 0.07 & \pm 0.07 & \pm 0.6 \\
\hline 5 & NGC 7654 & 10.6 & 691.09 & 0.33 & 61.80 & 0.34 & 27.6 \\
& & \pm 0.4 & \pm 0.18 & \pm 0.18 & \pm 0.18 & \pm 0.18 & \pm 2.1 \\
\hline 6 & IC 1848 & 8.4 & 538.95 & 0.27 & 5.95 & 0.29 & 17.8 \\
& & \pm 0.2 & \pm 0.14 & \pm 0.07 & \pm 0.07 & \pm 0.07 & \pm 0.9 \\
\hline 7 & Model 1 & 5.9 & 865.05 & 0.37 & 36.71 & 0.40 & 8.6 \\
& & \pm 0.3 & \pm 0.14 & \pm 0.09 & \pm 0.10 & \pm 0.09 & \pm 0.9 \\
\hline 8 & Model 6 & 5.8 & 864.97 & 0.38 & 4.44 & 0.40 & 8.3 \\
& & \pm 0.3 & \pm 0.18 & \pm 0.09 & \pm 0.09 & \pm 0.09 & \pm 0.9 \\
\hline
\end{tabular}

Note - 1 - Danilov and Seleznev (1994); Danilov and Dorogavtseva (2003). 
radius. For six OSCs, the values $\sigma_{v}$ were obtained according to (8.12), and for two OSC models, the values $\sigma_{v}$ were used in accordance with Table 8.2. The values of $\tau_{s t}$ for the OSCs are given according to Danilov and Seleznev (1994), and for the OSC models - according to Danilov and Dorogavtseva (2003). The errors of $\tau_{v r}$ and $\tau_{s t}$ are due to the the errors of $\sigma_{v}$ and parameters of OSCs (Danilov and Seleznev, 1994). Columns 5-9 of Table 8.3 give the values $U(0), \sigma_{v i r}, \sqrt{\overline{v^{2}(0)}}$, $\varepsilon_{t}=U_{0}+U_{1}$ and $\rho_{e}$ (averaged for six sets of coordinates of $N_{c}$ stars of the OSCs) obtained with the use of formulas for $U(0)$, as well as (8.10) and (8.11). The method of an approximation of the potential $U(\mathbf{r})$ of the cluster model by the potential $U_{e}$ of a homogeneous ellipsoid (8.8) was described by Danilov (2008) and Danilov (2005). The values $U(0), \varepsilon_{t}, \rho_{e}$ for models 1 and 6 are given in Table 8.2, therefore in the Table 8.3 they are not listed. The values $\sigma_{v i r}$ and $\sqrt{\overline{v^{2}(0)}}$ for these models are obtained using the data from Table 8.2.

According to Table 8.3, the values of $\tau_{v r}$ are noticeably smaller than $\tau_{s t}$ only in NGC 6819 and in models 1 and 6 (in this case, a violent relaxation acts more efficiently in the system). In the other clusters, the values $\tau_{v r}$ and $\tau_{s t}$ are not statistically different (in these clusters, the effects of the violent and the collisional relaxations are comparable).

The values $\sigma_{v i r}$ for the considered objects are in the range $0.45-1.15 \mathrm{pc} / \mathrm{Myr}$, and the values of $\sqrt{\overline{v^{2}(0)}}$ are in the interval $0.55-1.45 \mathrm{pc} / \mathrm{Myr}$ (see Table 8.3). Among the OSCs from Table 8.3, the largest values of $U(0), \sigma_{v i r}, \sqrt{\overline{v^{2}(0)}}$ are achieved in NGC 6705, and the smallest ones in NGC 188. For clusters NGC 6705 and NGC 188 , we find $\sigma_{v} \simeq 1.31 \pm 0.04 \mathrm{pc} / \mathrm{Myr}$ and $\sigma_{v} \simeq 0.61 \pm 0.06 \mathrm{pc} / \mathrm{Myr}$, respectively. According to the data on the mean-square-root radial residual stellar velocities in the clusters NGC 6705 (Mathieu, 1985) and NGC 188 (Geller et al., 2008), assuming a spherical symmetry in the velocity distribution of the cluster stars, we find $\sigma_{v} \simeq \sqrt{3} \times(1.21 \pm 0.35) \simeq 2.10 \pm 0.61 \mathrm{~km} / \mathrm{s}$ and $\sigma_{v} \simeq \sqrt{3} \times(0.41 \pm 0.04) \simeq 0.71 \pm 0.07$ $\mathrm{km} / \mathrm{s}$, respectively $(1 \mathrm{~km} / \mathrm{s} \simeq 1 \mathrm{pc} / \mathrm{Myr})$. According to de Grijs et al. (2008), the $\sigma_{v}$ value, obtained with a taking into account of the data on the proper motions and the radial velocities of stars in NGC 6705 is $\sigma_{v} \simeq 2.0 \pm 0.8 \mathrm{~km} / \mathrm{s}$. The estimates of $\sigma_{v}$ given here for NGC 6705 are either completely consistent, or do not differ statistically. Estimates of $\sigma_{v}$ for NGC 188 obtained from the data on the radial velocities of stars and with taking into account of the relation (8.12) do not differ statistically. We note that the dynamic masses of NGC 6705 and NGC 188 obtained by Geller et al. (2008); de Grijs et al. (2008) for the case of an isolated virialized cluster are $(5717 \pm 4956) m_{\odot}$ and $(2300 \pm 460) m_{\odot}$, respectively. In our estimates of $\sigma_{v}$, we used the masses $M_{c}=(2250 \pm 660) m_{\odot}$ and $M_{c}=(690 \pm 180) m_{\odot}$ of NGC 6705 and NGC 188, respectively, obtained by Danilov and Seleznev (1994) with the help of star counts and the estimates of an average mass of a cluster star.

Thus, the taking into account the effect of the external field of the Galaxy and of a non-stationary nature of the OSCs on the estimates of dynamic cluster masses, makes it possible to reduce noticeably these estimates of cluster masses and to bring together the observational and theoretical estimates of $\sigma_{v}$. The difference in the 
values of $M_{c}$ (in 2.5-3.3 times) between the $M_{c}$ values obtained for NGC 6705 and NGC 188 by Danilov and Seleznev (1994) and by Geller et al. (2008) and de Grijs et al. (2008) can be partially explained by a difference in the data on the cluster sizes used in these studies. The equation for estimating the dynamic mass of the OSC is easily obtained as follows. Following to Danilov (2010), substituting (8.10) and (8.11) at $\mathbf{r}=\mathbf{0}$ into (8.12), assuming $N_{t}=N_{c}$, we obtain

$$
\sigma_{v}^{2} \simeq \frac{1}{2}\left\{\frac{1}{M_{c}}\left[-W+\sum_{i=1}^{N_{c}} m_{i}\left(\alpha_{1} \xi_{i}^{2}+\alpha_{3} \zeta_{i}^{2}\right)\right]+\frac{U(0)}{2}\right\} .
$$

In this case, the relation (8.10) can be considered as Lagrange-Jacobi equation (averaged over the period $P_{r}$ of the oscillations of a regular cluster field), with the value of $\frac{d^{2} I_{c}}{d t^{2}}$ (averaged over the period $P_{r}$ ) equal to zero, where $I_{c}$ is the momentum of inertia of the cluster. The values $W$ and $U(0)$ in (8.13) depend on the mass $M_{c}$, the size of the cluster, and the mass distribution in the cluster.

Danilov (2008) used the three-integral phase density function (PDF) in the form $\Psi=\Psi(\mathcal{T})$, in order to describe stellar motions in the cluster core, where $\mathcal{T}$ is a linear combination of three integrals of stellar motion in the joint field of forces of the Galaxy and a homogeneous ellipsoid with potential $U_{e}$ from (8.8); the coefficients of this linear combination are $1, \kappa^{2}$, and $\sigma^{2}$. The value $\mathcal{T}$ is also an integral of a motion. The expression for the function $\Psi(\mathcal{T})$ is given in the form of formula (21) by Danilov (2008). We estimate the values of $\kappa^{2}$ and $\sigma^{2}$ for OSCs from Table 8.3. Let $\sigma_{\dot{\xi}}^{2}, \sigma_{\dot{\eta}}^{2}$ and $\sigma_{\dot{\xi}}^{2}$ be the dispersions of the stellar velocities in the OSC cores along axes $\xi, \eta$, and $\zeta$, respectively. Let the distribution of the stellar velocities in the cores of the considered OSCs be spherically symmetric. Then, $w=\sigma_{\dot{\eta}}^{2} / \sigma_{\dot{\xi}}^{2}=b_{T}^{2} / a_{T}^{2}=1$, and $\sigma^{2}=0$, since $\sigma^{2} \sim(w-1)$, see formulas (18) and their explanations in Danilov (2006); the values $b_{T}^{2}$ and $a_{T}^{2}$ are constant and are defined in Danilov (2006). In our case, $b_{T}^{2}=a_{T}^{2}=1$. According to (35) from Danilov (2006), there are two relations for $\kappa^{2}: \kappa^{2}=\left(\sigma_{\dot{\xi}}^{2} / \sigma_{\dot{\zeta}}^{2}\right) a_{T}^{-2}$ and $\kappa^{2}=\left(\sigma_{\dot{\eta}}^{2} / \sigma_{\dot{\zeta}}^{2}\right) b_{T}^{-2}$. In the case of a spherical symmetry of the stellar velocity distribution, we find $\kappa^{2}=1$ and $\mathcal{T}_{\text {max }}=2 \varepsilon_{t}$, see the explanation to formula (36) from Danilov (2006); $\mathcal{T}_{\text {max }}=$ const is the largest value of $\mathcal{T} ; \mathcal{T}_{\text {max }}$ is included in the expression (21) for the function $\Psi(\mathcal{T})$ from Danilov (2008).

The values $\kappa^{2}$ and $\sigma^{2}$ for OSC model 1 are given by Danilov (2008). Taking into account the values of $\sigma_{\dot{\xi}}^{2} \simeq 0.112 \pm 0.016, \sigma_{\dot{\eta}}^{2} \simeq 0.143 \pm 0.016$ and $\sigma_{\grave{\zeta}}^{2} \simeq 0.166 \pm 0.027$ (averaged over the regular field oscillation period $P_{r}$ ) for the core stars of model 6 , we find $\sigma^{2} \simeq 0.052 \pm 0.039 \mathrm{Myr}^{-1}, \kappa^{2} \simeq 0.776 \pm 0.182$ (we used data on the velocities of 100 stars closest to the center of the cluster model 6). As in the paper of Danilov (2008), the average values of $\kappa^{2}$ and $\sigma^{2}$ should be used for estimating the parameters of the equilibrium PDF (the errors of these values are due to the non-stationarity of the OSC models $1-6$ and the oscillations of the values of $\sigma_{\dot{\xi}}^{2}, \sigma_{\dot{\eta}}^{2}$, and $\sigma_{\dot{\zeta}}^{2}$ during the period $P_{r}$ ). Since $\kappa^{2}<1<v^{2}$ (see the explanation to formula (36) from Danilov (2006)), then $\mathcal{T}_{\max }=2 \varepsilon_{t} v^{2}$, where the value $v^{2}$ was defined by 
Danilov (2006). The obtained values $\sigma^{2}, \kappa^{2}$ and the relations for $\mathcal{T}_{\text {max }}$, as well as the values $\rho_{e}$ and $\varepsilon_{t}$ from Tables 8.2 and 8.3 were used further in order to analyze the stability of the phase-density oscillations in the examined OSCs and OSC models.

When studying the natural oscillations of a non-isolated homogeneous gravitating ellipsoid, whose potential and density are equal to the corresponding parameters of the core of the numerical dynamic cluster model, the following form of the PDF $f_{*}$ perturbations was considered by Danilov (2008): $\delta f_{*}(\mathbf{r}, \mathbf{v}, t)=f_{1}(\mathbf{r}, \mathbf{v}) \exp (-i \lambda t)$. The $f_{1}(\mathbf{r}, \mathbf{v})$ is the initial amplitude of the phase density oscillations, and $\lambda$ is determined from the fifth degree algebraic equation with respect to $\chi=\lambda^{2}$ :

$$
\sum_{j=0}^{5} p_{j} \chi^{j}=0 .
$$

The constants $p_{j}$ can be represented in the form

$$
p_{j}=H_{0, j}+\sum_{k=1}^{3} H_{k, j} C_{k}^{2},
$$

where $H_{0, j}, H_{k, j}, C_{k}$ are constant values and $k=1, \ldots, 3 ; j=0, \ldots, 5$; the $C_{k}$ values are directly proportional to the amplitudes of unperturbed stellar motion in $\xi-, \eta-, \zeta$-coordinates, and have the same order of magnitude as these amplitudes. Here, $\mathbf{r}, \mathbf{v}$ are phase coordinates of the star. Equation (8.14) was obtained by Danilov (2008) using the Boltzmann equation for the PDF of gravitating ellipsoid.

Danilov (2010) obtained the solutions of the equation (8.14) for six OSCs and two OSC models considered here for a number of the $\varepsilon_{t}$ values (including the $\varepsilon_{t}$ values indicated in Tables 8.3 and 8.2) at $C_{k}=0$ and $C_{k}=1 \mathrm{pc}(k=1,2,3)$. The case $C_{k}=0$ corresponds to the position of an unperturbed star in the cluster center, and the case $C_{k}=1 \mathrm{pc}$ approximately ensures the maximum distance of this star from the center by the distance $\sim 1 \mathrm{pc}$ to the boundary of the cluster core as this star moves. Both at $C_{k}=0$ and at $C_{k}=1 \mathrm{pc}$ for all the considered objects at the $\varepsilon_{t}$ values indicated in Tables 8.2 and 8.3, the phase density oscillations at the centers of these systems are unstable (among the solutions of the equation (8.14), there is a pair of complex conjugate roots). The corresponding solutions for $\delta f_{*}$ have the form $\delta f_{*}=f_{1}(\mathbf{r}, \mathbf{v}) \exp \left( \pm i\left(\gamma_{1} \pm i \gamma_{2}\right) t\right)$. Therefore, the development of an instability in the system leads rather quickly to the dominance of the amplitudes of the oscillations with the frequency $\gamma_{1}$. In the case $C_{k}=1 \mathrm{pc}$, the period $P_{\gamma}(1)=2 \pi / \gamma_{1}(1)$ and the instability development time $t_{\lambda}(1)=1 / \gamma_{2}(1)$ in Myr are given in columns 10 and 11 of Table 8.3. $\gamma(1) / \omega$ are given in column 12 of Table 8.3; here, $\omega$ is the angular velocity of the OSC (or the OSC model) motion around the Galactic center ( $\omega$ is determined with an assumption of the plane circular orbits of the OSCs using the Galaxy potential model of Kutuzov and Osipkov (1980) and the data of Danilov and Seleznev (1994) on the distances of the OSCs $R_{G}$ to the center of the Galaxy).

Let us determine the boundaries of the instability region in the considered objects (the OSCs and the OSC models). Increasing (or decreasing) the value of $\varepsilon_{t}$ while 
preserving the number of stars and the mass of the object, according to (8.9), we obtain the ellipsoids with a greater (or lesser) density $\rho_{e}$ which is approximately equal to the mean core density of the object $\rho_{c}$ (see above). Calculating the roots of the equation (8.14) for the new values $\varepsilon_{t}$ and $\rho_{e}$ (and with the previous values Of all other parameters included in the equations for the coefficients of the equation (8.14)) we can establish the boundary of the instability region of the object. In the columns 13 and 14 of Table 8.3 for the case $C_{k}=0$, we indicate the maximum and minimum values of $\rho_{e}\left(\rho_{\max , 0}\right.$ and $\left.\rho_{\min , 0}\right)$, at which the phase density oscillations in the center of masses of the object become stable. In columns 15 and 16 of Table 8.3, we give the same by a sense values of $\rho_{e}\left(\rho_{\max , 1}\right.$ and $\left.\rho_{\min , 1}\right)$ for the case $C_{k}=1$. Densities in columns 9, 13-16 of Table 8.3 are given in $m_{\odot} / \mathrm{pc}^{3}$. The values of the densities in columns 13-16 of Table 8.3 were obtained for the mean values of $\varepsilon_{t}$ from column 8 of Table 8.3 corresponding to each object. Errors of the values in columns $13-16$ of Table 8.3 are caused both by the errors of $\varepsilon_{t}$, and by the errors in the finding of the boundary of the unstable oscillations region at an average value of $\varepsilon_{t}$ from column 8 of Table 8.3. In column 17 of Table 8.3, we give the values $\rho_{e} / \rho_{t}$, where $\rho_{e}$ is the value of the ellipsoid density taken from column 9 of Table 8.3, and $\rho_{t}$ is the tidal density of the cluster $\rho_{t}=3 M_{c} /\left(4 \pi R_{t}^{3}\right)=-3 \alpha_{1} /(4 \pi G), \alpha_{1}<0$ (see above, and also at the p. 201 of the book of King (2002)).

\subsection{Analysis of Dependencies Between the Parameters of Unstable Oscillations of Phase Density in the Centers of the Considered OSCs and Cluster Models}

For the considered objects, the ratios $\rho_{\max , 0} / \rho_{e}$ from Table 8.3 are in the range from $\sim 332.3$ (for NGC 6705) to $\sim 1004.0$ (for NGC 188); the ratio $\rho_{e} / \rho_{\min , 0}$ are in the interval from $\sim 2.1$ (for NGC 188) to $\sim 7.1$ (for NGC 7654); the ratios $\rho_{\max , 1} / \rho_{e}$ are contained in the interval from $\sim 2.7$ (for NGC 6705) to $\sim 40.6$ (for model 1); ratio $\rho_{e} / \rho_{\min , 1}$ is in the interval from $\sim 2.0$ (for NGC 188) to $\sim 6.8$ (for NGC 7654). Thus, the considered objects are deep inside the region of instability of the regarded oscillations, and have a large "instability reserve" relatively to to the core density $\rho_{c} \simeq \rho_{e}$ (and energy $\left.\varepsilon_{t}\right)$.

We note that the condition $M_{c}=$ const with an increase of $\varepsilon_{t}$ and $\rho_{e}$ leads to a decrease in the size (and mass) of the core. In this case, for the stars capable to reach the boundary of the core during their motion, the values $C_{k} \neq 0$ should tend to zero, and the boundary of the region of the unstable oscillations of the phase density should approach to the boundary obtained for the case $C_{k}=0$. The "halocore" structure of the clusters with the stable oscillations of the phase density should resemble more the structure of globular clusters. However, at such large densities of clusters' cores, we can no longer use the collisionless approximation used by Danilov (2008) when deriving the equation (8.14). It's worth mentioning that such high densities are not observed in the OSCs (except the cases of protoclusters in the cores of giant molecular clouds (see Surdin (2001, p.148) and Elmegreen (2008)). 
Thus, the oscillations in the OSC cores considered above are unstable up to the largest possible values of the average cluster density (in the OSCs, the collisionless approximation is quite applicable for the time intervals shorter than the collisional relaxation time).

Taking into account the influence of the less bright stars with stellar magnitudes $B>16^{m}$ exceeding the limiting magnitude of the stars considered by Danilov and Seleznev (1994) can not significantly change the position of the OSC within the region of the unstable oscillations of a phase density. Indeed, the taking into account such stars can lead to an increase in the mass and the central density of the cluster only in 2-3 times (see above the estimates of the virial masses and the masses $M_{c}$ obtained by Danilov and Seleznev (1994) using star counts for clusters NGC 6705 and NGC 188). Usually, the total masses of OSCs do not differ significantly from the dynamic masses (de Grijs et al., 2008; Miller and Scalo, 1979). The total masses of the clusters NGC 1912, NGC 6705, NGC 7654, IC 1848 with ages $t<10^{8}$ years (Danilov and Seleznev, 1994), obtained by Danilov (2010) using the initial mass function of Miller and Scalo (1979) for the stars with the masses $m \geq 0.1 m_{\odot}$, taking into account the data on minimum and maximum masses used by Danilov and Seleznev (1994) for an estimating of $M_{c}$, are respectively $\sim 2.1,2.6,2.7,2.6$ times higher than the estimates of $M_{c}$ in the catalog ofDanilov and Seleznev (1994). The average mass density of the cluster equal to $\rho_{t}$, does not change, see above. In addition, the stars of small masses (and luminosities) are usually located at the cluster periphery. Therefore, consideration of such stars can lead only to an insignificant increase in $\rho(0)$ and, consequently, does not change our conclusions about the instability of the phase density oscillations in the cores of the considered OSCs.

The condition $M_{c}=$ const, as $\varepsilon_{t}$ and $\rho_{e}$ decrease, leads to an increase of the size (and mass) of the core, as well as to a decrease in $\rho_{c}$ and $\rho(0)$. We note that the OSC models 5 and 6 with the most extended massive cores and the smallest $\rho(0)$ values (see Table 8.2) have the least degree of non-stationarity (Danilov and Dorogavtseva, 2003). These models decay more slowly than models $1-4$, and the time of a transition of models 5, 6 to the spherical velocity distribution is the greatest among models 1-6, see Table 8.3 (Danilov and Dorogavtseva, 2008). According to Danilov and Dorogavtseva (2008), this is due to a decrease in the role of the gravitational and dynamic instabilities in the dynamics of such systems. In model 6, during the periods of greatest compression to the Galactic plane $\zeta=0$, a toroidal structure with an increased density of number of stars inside the torus (in the space $(\xi, \eta, \zeta)$ ) is formed with the equatorial plane close to $\zeta=0$ (Danilov and Leskov, 2005). According to Danilov and Dorogavtseva (2008), the results noted here for models 5 and 6 can be considered as arguments in a favor of the stability of the toroidal (or close to toroidal) systems moving along the circular orbits in the Galactic plane.

Among 103 OSCs from the sample of Danilov and Seleznev (1994), one can find only five clusters with the parameters of the "halo-core" structure $R_{1} / R_{2} \geq 0.6$, $N_{1} / N_{2}>2.5$, close to the structural parameters of models 5 and 6 (see Table A.3 from Danilov and Seleznev (1994); here $R_{1}, N_{1}$ are the radius of the core and the number of stars in the cluster core, and $R_{2}, N_{2}$ are the halo radius and the number of stars in the halo of the cluster). If we add stars of the intermediate density zone in 
the cluster (Danilov and Seleznev, 1994) to the stars of the cluster core, and assume the radius of the intermediate zone to be the radius of the core, we get 8 clusters with parameters $R_{1} / R_{2} \geq 0.6, N_{1} / N_{2}>2.5$ in the sample of Danilov and Seleznev (1994). The clusters chosen using these two methods of estimating the structural parameters of the OSCs do not coincide. Therefore, the total number of selected clusters with the extended massive cores is 13 (among the 103 OSCs of the sample of Danilov and Seleznev (1994)). According to Table A.2.2 (column 7) of Danilov and Seleznev (1994), these 13 OSCs have the least degrees of a non-stationarity (the amplitudes $\delta \alpha=\langle\delta \alpha\rangle_{t h}$ of the virial coefficients $\alpha$ are $\delta \alpha=0.10 \pm 0.02$ and $\delta \alpha=0.08 \pm 0.03$ for the first and the second way of evaluating the structural parameters, respectively; in the first case, we used the data on $\delta \alpha$ for 4 clusters, and in the second case for 7 clusters, since the data on $\delta \alpha$ for two of 13 selected clusters are absent in the catalog of Danilov and Seleznev (1994); here, $\alpha=2 E / W, E$ is the total energy of the cluster).

A small number of clusters, in the centers of which the phase density oscillations can be stable, most likely does not reflect the real prevalence of such clusters. Danilov and Seleznev (1994) considered the clusters with a quite high probability of existence (Danilov et al., 1985). Danilov and Seleznev (1994) and Danilov et al. (1985) noted the influence of large-scale fluctuations in a field-star density in the vicinity of clusters on the possibility of determining the size of clusters. According to Danilov et al. (1985), the distribution of field stars in the vicinity of the OSCs does not follow the Poisson's law, and angular dimensions of such large-scale field-star density fluctuations are comparable with the angular dimensions of the considered OSCs. If at least some of the over-densities of this type are objects that are resistant to the oscillations of the phase density, then the number of such objects in the Galaxy can be quite large.

In fig. 8.2a and $8.2 \mathrm{~b}$ in the coordinates of $\left(\ln \left(\rho / \rho_{e}\right), \ln \left(\rho_{e} / \rho_{t}\right)\right.$ and $\left(\ln \left(\rho / \rho_{e}\right)\right.$, $\left.\rho(0) / \rho_{e}\right)$, respectively, the dots indicate the positions of the objects considered in our study inside the region of the unstable oscillations of a phase density at the centers of these objects. Here, $\rho$ is the density of an ellipsoid with the potential $U_{e}$ obtained by varying the energy $\varepsilon_{t}$. Solid lines in fig. 8.2 indicate the upper and lower boundaries of the region of the unstable phase density oscillations at $C_{i}=0$, and the dashed lines indicate the boundary of this region at $C_{i}=1 \mathrm{pc}$. Here and in all the other figures, the points corresponding to OSCs NGC 188, NGC 6819, IC 1848, NGC 1912, NGC 6705, NGC 7654 are labeled with letters $a, b, c, d, e, f$. Numbers 1 and 6 indicate the points corresponding to models 1 and 6 . The lower boundaries of the instability region (the solid line and the dashed line at $C_{i}=0$ and $C_{i}=1$ $\mathrm{pc}$, respectively) in these coordinates almost coincide. The upper boundary of this region (as well as the lower one) should be located between corresponding solid and dashed lines (closer to the solid line). According to fig. 8.2a with an increase of the average mass density of the cluster core $\left(\rho_{c} \simeq \rho_{e}\right)$ approaches the upper boundary of the instability region considered here (and moves away from the lower boundary). Consequently, for clusters with dense cores and large values of the star concentration to the center $\left(r_{t} / r_{c}\right)$, the degree of non-stationarity should decrease at increasing of $\rho_{c} / \rho_{t}$. 

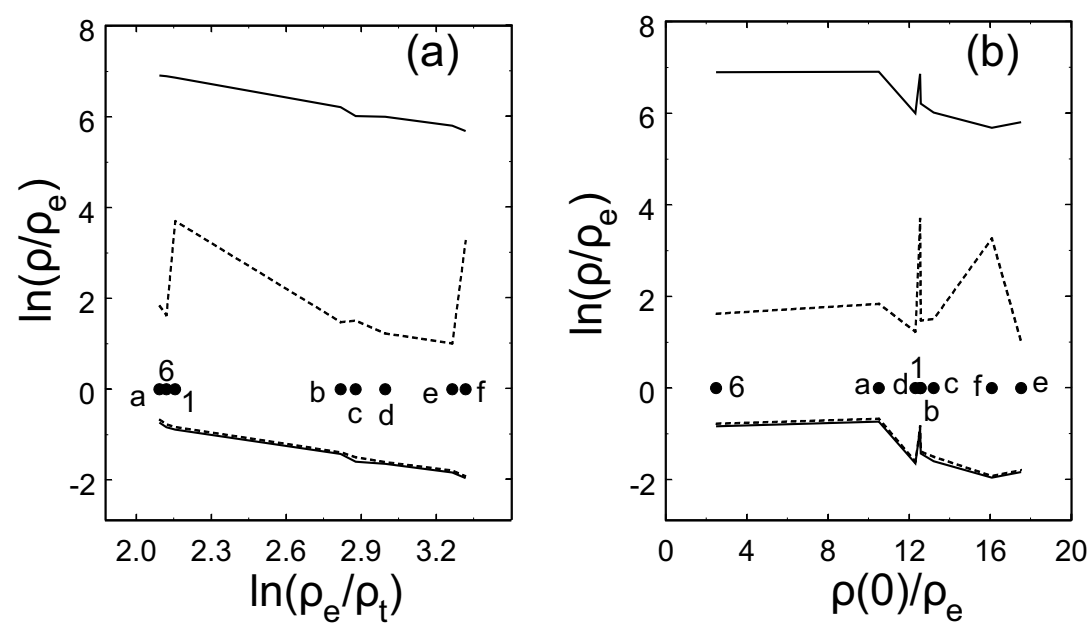

Fig. 8.2 The boundaries of the region of the unstable phase density oscillations in the centers of the considered OSCs and OSC models. Positions of these eight objects inside the instability region

Indeed, according to Table. A.2.2 from Danilov and Seleznev (1994), the values $\delta \alpha=\langle\delta \alpha\rangle_{t h}$ for clusters NGC 1912, NGC 6705, NGC 7654 (denoted here by the letters $d, e, f$ ) are minimal: $\delta \alpha \simeq 0.037,0.028,0.044$, respectively. For cluster NGC 188 (letter $a$ in fig. 8.2), $\delta \alpha$ values is not defined in Danilov and Seleznev (1994), but should be as high as possible, since for this cluster $r_{t}>R_{t}$ (probably, this cluster is observed at the stage of an impulsive loss of a significant part of the halo stars in the field of the Galaxy). The degree of non-stationarity of model 1 is also maximal (see, for example, Table 1 from Danilov and Leskov (2005)). According to Table A.2.2 from Danilov and Seleznev (1994), for cluster NGC 6819 (letter $b$ in fig. 8.2) the value of $\delta \alpha \simeq 0.062$. In fig. 8.2a models 1,6 and NGC 188 are located side by side, although model 6 has an extended massive core and a minimal degree of non-stationarity (see Table 1 from Danilov and Leskov (2005)). For the clusters with the extended massive cores, an important role is played by the proximity of their position to the upper dashed boundary of instability region in fig. 8.2a.

In fig. 8.2b the positions of models 1 and 6 along the axis $\rho(0) / \rho_{e}$ differ significantly. If we consider only clusters and model 1 , then, according to fig. $8.2 \mathrm{~b}$, with the increase in the mass density at the center of the object (and of the $\rho(0) / \rho_{e}$ value), positions of the considered objects approach to the upper boundary of an instability region, which also indicates a decrease in the degree of a non-stationarity of such systems.

On the example of NGC 6705 in fig. 8.3a and 8.3b, for the values of $C_{k}=0$ and $C_{k}=1$, respectively, we show the dependencies of the values $t_{\lambda}(1)$ (solid line) and $P_{\gamma}(1)$ (dashed line) on density $\rho$ for the $\rho$ values corresponding to the unstable oscillations of a phase density. The vertical arrows indicate the "observed" value of $\rho_{e}\left(\rho_{e} \simeq \rho_{c}\right)$, see 9 th column of Table 8.3. When $\rho$ changes from the "observed" 

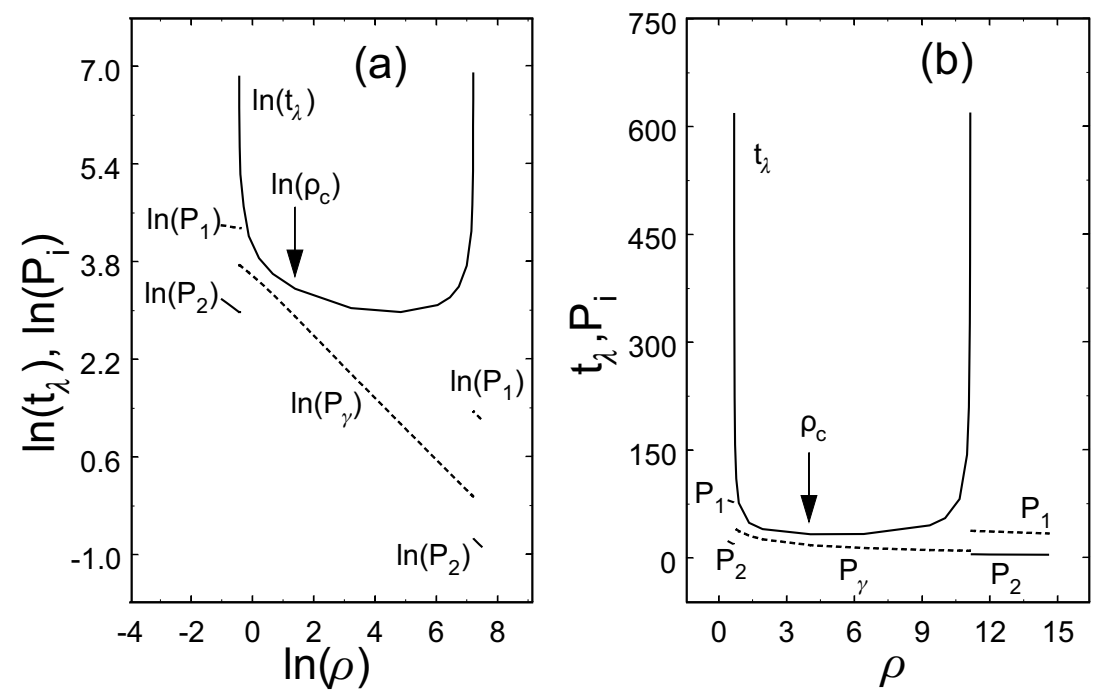

Fig. 8.3 The dependencies of $t_{\lambda}, P_{\gamma}, P_{1}$ and $P_{2}$ on the density $\rho$ for cluster NGC 6705

values $\rho_{e}$ to the values $\rho$ on the boundary of an instability region, the value $t_{\lambda}(1)$ increases indefinitely (in this case, the rate of an instability development in the phase density oscillations decreases to zero). The period of dominant (natural) oscillations $P_{\gamma}(1)$ decreases with an increase of $\rho$. Outside the region of instability, according to (8.14), five periods of the stable phase density oscillations are obtained. Among them, the maximum $\left(P_{1}\right)$ and the minimum $\left(P_{2}\right)$ periods are indicated in fig. 8.3 by dashed and solid lines, respectively. According to fig. 8.3, the values of $P_{1}$ and $P_{2}$ decrease with an increase of $\rho$.

Positions of the objects in fig. 8.4 form the dependencies of $P_{\gamma}(1)$ and $\tau_{v r}$ on $t_{\lambda}(1)$ for the considered objects. According to fig. 8.4, the values of $P_{\gamma}(1)$ and $\tau_{v r}$ decrease with a decrease of $t_{\lambda}(1)$ and $\tau_{v r} \sim P_{\gamma}(1)$. The same dependency of $\tau_{v r}$ on the oscillation period $P_{r}$ of the regular field of a stellar system was obtained by Lynden-Bell (1967) (see formula (10) from Lynden-Bell (1967)) with a description of the violent relaxation of the system. The smallest values of $t_{\lambda}(1), P_{\gamma}(1)$ and $\tau_{v r}$ are obtained for cluster NGC 6705. Therefore, in the central region of this cluster, the speed of an action of the collective processes (a violent relaxation, the oscillations in a density and a phase density, as well as an instability of these oscillations) should be the greatest, which is due to the largest values of $\rho(0), \rho_{e}$ and $\rho(0) / \rho_{e}$, see Tables 8.1, 8.3 and fig. 8.2b. However, among the clusters considered in our study, the degree of non-stationarity in NGC 6705 is the smallest one (Danilov and Seleznev, 1994), which is most likely due to the greatest closeness of the position of this cluster to the upper dashed boundary of the instability region in fig. 8.2. Apparently, in NGC 6705 (and in model 6), the role of high-energy stars in the suppression of the phase-density oscillations is the greatest, see fig. 8.2a, and also Danilov (2008). 

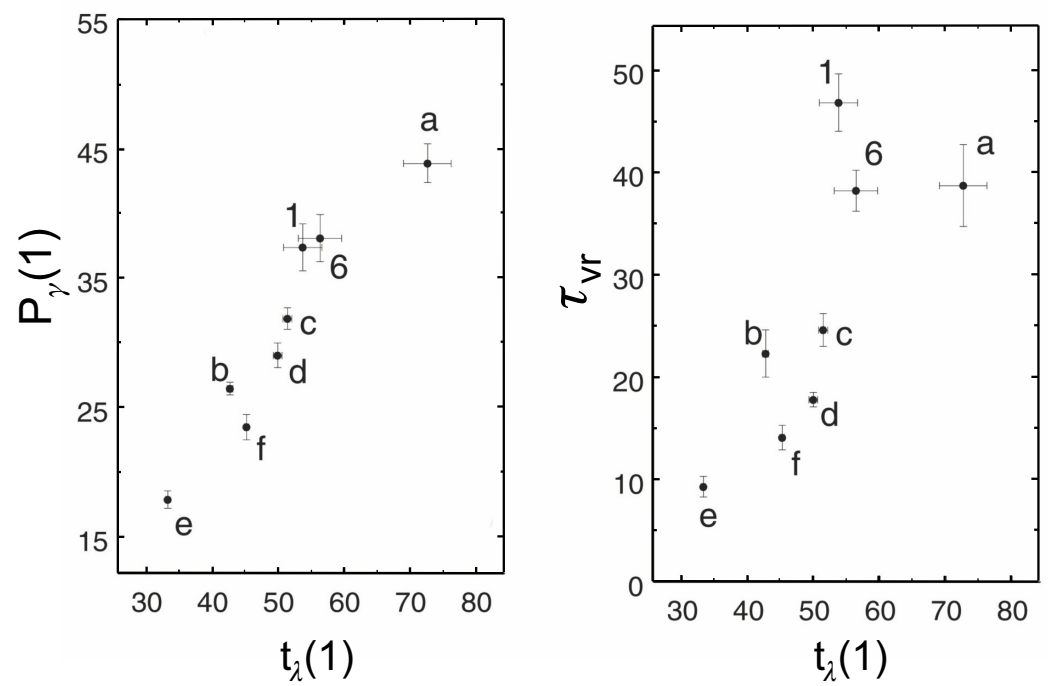

Fig. 8.4 Positions of the considered OSCs and OSC models in the coordinate systems $\left(t_{\lambda}(1), P_{\gamma}(1)\right)$ and $\left(t_{\lambda}(1), \tau_{v r}\right)$
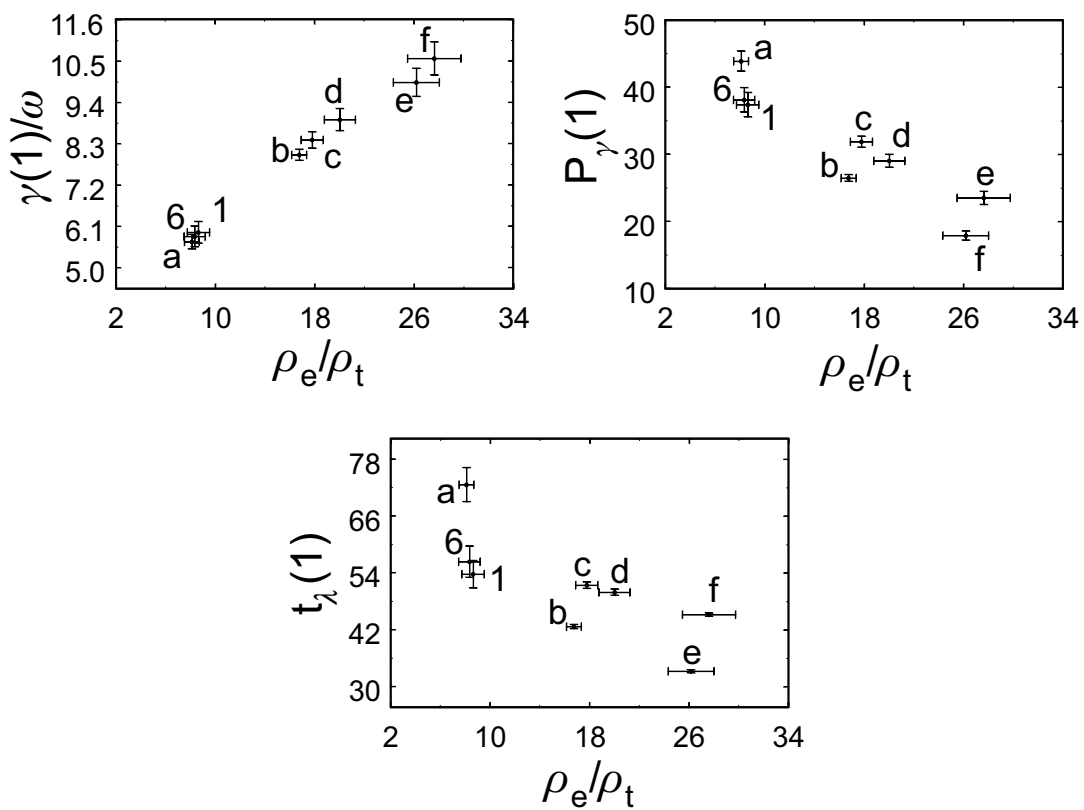

Fig. 8.5 Positions of the considered OSCs and OSC models in the coordinate systems $\left(\rho_{e} / \rho_{t}, \gamma_{1}(1) / \omega\right),\left(\rho_{e} / \rho_{t}, P_{\gamma}(1)\right)$ and $\left(\rho_{e} / \rho_{t}, t_{\lambda}(1)\right)$ 
The positions of the objects in fig. 8.5 form the dependencies of $\gamma_{1}(1) / \omega, P_{\gamma}(1)$, $t_{\lambda}(1)$ on $\rho_{e} / \rho_{t}$. The value of $\gamma_{1}(1) / \omega$ increases, and the values of $P_{\gamma}(1)$ and $t_{\lambda}(1)$ decrease with an increase of $\rho_{e} / \rho_{t}$. Such dependencies are quite typical for nonstationary stellar systems. According to Lynden-Bell (1967), in such systems, the relation $P_{r} \sim 1 / \sqrt{\langle\rho\rangle}$ is fulfilled (see formula (9) from Lynden-Bell (1967)), where $\langle\rho\rangle$ is the density of the system averaged over the period $P_{r}$. Thus, as in the case of fig. 8.4, the speed of action of the considered above collective processes in the OSCs and the OSC models increases with an increase of $\rho_{e} / \rho_{t}$ and an increase of the density of the cores of the considered objects.

According to Table 8.3, the values $\gamma_{1}(1) / \omega$ within the indicated limits of the errors can be quite represented as integers or rational numbers. Therefore, the resonances between the frequencies of the natural density oscillations of the considered objects and the frequency of the tide can play an important role in the dissipation of the energy of tidal perturbations of the OSCs and the OSC models by the force field of the Galaxy (see also Danilov and Dorogavtseva (2008)).

\subsection{Conclusions}

1. In this part of the study, we have considered the estimates of the parameters of the modified King distribution of the apparent density of star number for six OSCs. As an approximation to the construction of the spatial distributions $f(r)$ of the density of star number in these OSC, we have used the assumption on a spherical symmetry of the star distribution in the clusters. We have performed the analytic estimates (8.4) and (8.7) of improper integrals $I_{\varepsilon}$ and $J_{\varepsilon}$ arising in a calculation of the function $f(r)$ and its errors at $r=0$.

2. In order to determine the structural and dynamic parameters of OSCs (see Table 8.3), the Monte Carlo method have been applied for setting the spatial positions of the stars corresponding to the distribution $f(r)$ of stars in these OSCs.

3. Structural and dynamic parameters for six OSC models have been listed (see Table 8.2). We have presented relations $\rho_{e} \simeq \rho_{c}$ and (8.12) between parameters of the models, which allow us to analyze the dynamic state and to estimate the dynamic characteristics of OSCs. An equation (8.13) have been derived which makes it possible to estimate the dynamic masses of OSCs taking into account the influence on the cluster of the Galactic force field and the non-stationarity of the OSC. It has been shown that taking these effects into account allows to significantly reduce the estimates of the dynamic masses of OSCs in comparison with the estimates obtained in the framework of models of the isolated virialized clusters.

4. We have discussed the natural oscillations of a phase density in the centers of the examined OSCs and two OSC models. Instability of such oscillations in these objects is noted. We have considered the boundaries of the instability region of such oscillations in the space of the mean mass density of the cluster core (see Table 8.3). Possible candidates for the role of objects, central parts of which are resistant to the phase density oscillations, are OSCs with a small central density and the massive 
extended cores, as well as some objects (over-densities) with the dimensions and the densities characteristic of the large-scale field-star density fluctuations in the vicinity of the observed OSCs.

5. We have noted the dependencies between the parameters of the central regions of six OSCs and two OSC models. Such dependencies are characteristic for nonstationary stellar systems in the stage of a violent relaxation. The role of the highenergy stars in a suppressing the the phase density oscillations in the central regions of NGC 6705 and model 6 is noted, as well as the possible role of resonances between the frequency of the phase density oscillations and the frequency of a tide in the dissipation of an energy of the tidal perturbations of the OSCs and the OSC models in the force field of the Galaxy.

\section{References}

Aarseth, S.J.: Dynamical evolution of simulated star clusters. I - Isolated models. Astron. Astrophys. 35, 237-250 (1974).

Bagin, V.M.: A Model for Globular Clusters with a Nonuniform Star Population. Astronomicheskii Zhurnal, 46, 1201-1206 (1969) (In Russian).

Chandrasekhar, S.: Principles of Stellar Dynamics. Univ. Chicago Press, Chicago, Ill (1942). LCCN: 43-5608 (BKS1)

Danilov, V.M.: On the dynamics of open star clusters. In: Stellar aggregates, Ural State University, Sverdlovsk (1980) (In Russian).

Danilov, V.M.: Some properties of the phase-space density function in numerical dynamical models of open star clusters. Astron. Reports 43, 74-79 (1999).

Danilov, V.M.: The Motion of Halo Stars in Dynamical Numerical Models of Open Clusters. Astron. Reports 49, 3604-610 (2005). doi:10.1134/1.2010649

Danilov, V.M.: Dynamical models of stellar motions at the peripheries of open clusters. Astron. Reports 50, 346-357 (2006). doi:10.1134/S1063772906050027

Danilov, V.M.: Analysis of density fluctuations in models of open clusters. Astron. Reports 52, 888-899 (2008). doi:10.1134/S1063772908110036

Danilov, V.M.: Phase-Density Fluctuations at the Centers of Six Open Clusters. Astron. Reports 54, 514-527 (2010). doi:10.1134/S1063772910060053

Danilov, V.M., Dorogavtseva, L.V.: Estimates of Relaxation Times in Numerical Dynamical Models of Open Star Clusters. Astron. Reports 47, 483-491 (2003). doi:10.1134/1.1583775

Danilov, V.M., Dorogavtseva, L.V.: Timescales for mechanisms for the dynamical evolution of open star clusters. Astron. Reports 52, 467-478 (2008). doi:10.1134/S1063772908060048

Danilov, V.M., Leskov, E.V.: Properties of Stellar Trajectories in Numerical Dynamical Models of Open Star Clusters. Astron. Reports 49, 190-200 (2005). doi:10.1134/1.1882777

Danilov, V.M., Matkin, N.V., Pylskaya, O.P.: Statistical Boundaries for Open Clusters. Soviet Astron. 29, 621-625 (1985). 
Danilov, V.M., Seleznev, A.F.: The catalogue of structural and dynamical characteristics of $103 \mathrm{OCl}$ and the first results of its investigations. Astron. Astrophys. Trans. 6, 85-156 (1994). doi:10.1080/10556799408232061

Elmegreen, B.G.: Variations in Stellar Clustering with Environment: Dispersed Star Formation and the Origin of Faint Fuzzies. Astrophys. J. 672, 1006-1012, (2008). doi:10.1086/523791

Geller, A.M., Mathieu, R.D., Harris, H.C. et al.: WIYN Open Cluster Study. XXXII. Stellar Radial Velocities in the Old Open Cluster NGC 188. Astron. J. 135, 22642278 (2008). doi:10.1088/0004-6256/135/6/2264

de Grijs, R., Goodvin, S.P., Kouwenhoven, M.B.N., et al.: Open cluster stability and the effects of binary stars. Astron. Astrophys. 492, 685-693 (2008). doi:10.1051/0004-6361:200810251

Hamming, R.W.: Digital Filters. Prentice-Hall, Englewood Cliffs, NJ (1977).

Henon, M.: Lévolution initiale dún amas sphérique. Annales d' Astrophys. 27, 83-91 (1964).

Kholopov, P.N.: Star clusters. Nauka, Moscow (1981) (In Russian).

King, Ivan R.: An Introduction to Classical Stellar Dynamics. URSS, Moscow (2002) (In Russian).

Kutuzov, S.A., Osipkov, L.P.: A Generalized Model for the Three-Dimensional Gravitational Potential of Stellar Systems. Soviet Astron. 24, 17-22 (1980).

Lynden-Bell, D.: Statistical mechanics of violent relaxation in stellar systems. Mon. Not. R. Astron. Soc. 136, 101-121 (1967). doi:10.1093/mnras/136.1.101

Marquardt, D.W.: An algoritm for least-squares estimation of nonlinear parameters. J. Soc. Indust. Appl. Math. 11, 431-441 (1963). doi:10.1137/0111030

Mathieu, R.D.: The structure and internal kinematics of open clusters. In: Goodman, J., Hut, P. (eds) Dynamics of star clusters, IAU Symp. 113, Reidel, Dordrecht (1985), 427-446.

Miller, G.E., Scalo, J.M.: The initial mass function and stellar birthrate in the solar neighborhood. Astrophys. J. Suppl. Ser. 41, 513-547 (1979). doi:10.1086/190629

Sobol, I.M.: Monte-Carlo method. Nauka, Moscow (1985) (In Russian).

Surdin, V.G.: Birth of stars. URSS, Moscow (2001) (In Russian).

Vasilevsky, A.E.: Methods of the stellar statistics. Ural State University, Sverdlovsk (1985) (In Russian).

Veltmann, U.-I.: On Phase Density of Spherical Stellar Systems. Publications de L'Observatoire Astronomique de l'Universite de Tartu, 35, 5-26 (1965). 



\title{
Chapter 9 On the Dynamics of Open Star Clusters
}

\begin{abstract}
In this chapter, we consider a stellar kinematics in the models of open star clusters. The central regions of these models are quite "cold". This leads to a gravitational instability in the cores of clusters and their models. In the cluster models, a temporary virialisation is noted, when a significant part of cluster oscillations energy temporarily passes into a kinetic energy of the peculiar stellar motions. The duration of this stage can reach $\sim 10^{8}$ years. The reasons for the temporary virilization of clusters are discussed. An instability of the natural oscillations of a phase density in the centers of six clusters and six cluster models is investigated. The motion of clusters and their models to a state of a stable equilibrium is possible both with decreasing and increasing density of the cluster core. The structure of the instability regions of the phase density fluctuations in the centers of six clusters is studied. The resonance curves are constructed for the amplitudes of the steady-state fluctuations of a phase density at the center of NGC 6705. An analysis of the structure of the instability regions in clusters indicates the significant rates of an oscillation energy loss in clusters under the action of the relaxation effects. With an increase of the cluster's distance from the center of the Galaxy, the instability increments and the widths of the instability regions decrease.
\end{abstract}

\subsection{Introduction}

Heggie and Ramamani (1995) used the Jacobi integral in order to generalize the King models (King, 1966) for the case of a non-isolated star cluster moving in a circular orbit around the center of the Galaxy. The distribution of the velocities of the cluster stars in this case is spherically symmetric, and the cluster is tidally truncated and compressed to the Galactic plane. The models of King (1966) and Heggie and Ramamani (1995) are commonly used in discussing the structure of globular clusters. In the numerical dynamic OSC models of Danilov and Dorogavtseva (2008), a slow evolution is observed toward a formation of the spherical distribution of the stellar velocities. However, OSC models of Danilov and Dorogavtseva (2008) do not reach 
this state and, as a rule, they are destroyed before a stationary state and the velocity and density distributions noted in the model of Heggie and Ramamani (1995) (or close to them) are formed.

In the OSC models of Danilov and Dorogavtseva (2008), the large-scale oscillations of a density and a regular field develop spontaneously and rapidly. A development of an instability in the OSC models is used by Danilov and Dorogavtseva (2008) and Danilov (2008) to explain the oscillations of the virial coefficient values and the velocity dispersion of cluster stars that do not decay during the large time intervals. Danilov (2008) performed a theoretical description of the phase density oscillations in the central parts of the numerical dynamic OSC models. Danilov (2010) used the formulas obtained by Danilov (2008) for the study of the phase density oscillations at the centers of six observed OSCs and two OSC models (models 1 and 6 of Danilov and Dorogavtseva (2008) were considered). Danilov (2010) showed that the PD oscillations are unstable at the centers of all considered objects (i.e. OSCs and their models).

Thus, the equilibrium state and the equilibrium phase density function found for the objects considered by Danilov (2008) and Danilov (2010) are unstable. Dynamic evolution of the OSCs and their models must take place in the form of the oscillations relative to this equilibrium state, and in the direction of a formation of a stable equilibrium. Otherwise, it should be an evolution toward the equilibrium states with a lower degree of instability, if in the considered period of time the OSC does not expand and does not experience a large-scale loss of stars caused by heating of the cluster by the tidal field of the Galaxy. Fig. 2 from the paper of Danilov (2010) shows the position of the considered objects inside the region of the unstable PD oscillations (in the coordinates related to the core density, the tidal density, and the central cluster density). The further investigation of the PD oscillations in the centers of the OSCs (with a much smaller step in the core density than in the paper of Danilov (2010)) revealed an extra regions of an instability of the PD oscillations in addition to ones revealed by Danilov (2010). It is of interest to refine the position of the boundaries of an unstable oscillation region for the clusters considered by Danilov (2010), to find these boundaries for all six models of Danilov and Dorogavtseva (2008), as well as to determine the positions of these models in the diagrams (the same as fig. 2 from Danilov (2010)) for two noticeably different time points. This will allow us to judge of the direction of the dynamic evolution of the OSCs and their models in the diagrams of fig. 2 of Danilov (2010), as well as to judge of the mechanisms that affect the development of the PD oscillation instability in the OSCs. An important role in the development of the gravitational instability considered by Danilov (2008) and Danilov (2010) in the central parts of the OSCs is played by the heating of the outer parts of the clusters by the tidal field of the Galaxy, which is revealed when studying the stellar kinematics in the OSC models.

The objectives of this chapter are: 1) to analyze the dispersion of the stellar velocities in the OSC models of Danilov and Dorogavtseva (2008) and to discuss the causes of the PD oscillation instability development in the central regions of the OSCs; 2) to search the new areas of an instability of the phase density oscillations in the cores of some OSCs and the OSC models of Danilov and Dorogavtseva (2008); 
3) to determine the boundaries of this instability in the OSC models of Danilov and Dorogavtseva (2008) for two noticeably different time points and to discuss the mechanisms and the directions of the dynamic evolution of the OSCs.

\subsection{Stellar Velocity Dispersion in the OSC Models}

Danilov (2011) obtained the estimates of a stellar velocity dispersion $\sigma_{v}^{2}$ in the numerical dynamic OSC models according to the data of Danilov and Dorogavtseva (2008) on the phase coordinates of stars in the cluster models for several time points $t$ (see the description of the OSC models in Section 16.1).

Table 9.1 Velocity Dispersion in the OSC Models

\begin{tabular}{|c|c|c|c|c|c|c|c|}
\hline \multirow{2}{*}{ № } & $t_{1} / \tau_{v r}$ & \multicolumn{7}{|c|}{$\sigma_{v}^{2}$} \\
\cline { 3 - 8 } & & $n=50$ & $n=100$ & $n=200$ & $n=300$ & $n=400$ & $n=500$ \\
\hline 1 & 2 & 3 & 4 & 5 & 6 & 7 & 8 \\
\hline 1 & 0.025 & 0.169 & 0.154 & 0.190 & 0.200 & 0.204 & 0.203 \\
& & \pm 0.018 & \pm 0.011 & \pm 0.007 & \pm 0.008 & \pm 0.007 & \pm 0.005 \\
\hline 2 & 0.025 & 0.190 & 0.172 & 0.210 & 0.220 & 0.221 & 0.218 \\
& & \pm 0.020 & \pm 0.013 & \pm 0.007 & \pm 0.007 & \pm 0.007 & \pm 0.004 \\
\hline 3 & 0.021 & 0.258 & 0.248 & 0.224 & 0.246 & 0.252 & 0.246 \\
& & \pm 0.017 & \pm 0.013 & \pm 0.004 & \pm 0.003 & \pm 0.004 & \pm 0.017 \\
\hline 4 & 0.025 & 0.216 & 0.197 & 0.238 & 0.246 & 0.246 & 0.241 \\
& & \pm 0.023 & \pm 0.015 & \pm 0.008 & \pm 0.007 & \pm 0.006 & \pm 0.003 \\
\hline 5 & 0.018 & 0.291 & 0.281 & 0.269 & 0.255 & 0.264 & 0.265 \\
& & \pm 0.014 & \pm 0.011 & \pm 0.007 & \pm 0.003 & \pm 0.002 & \pm 0.001 \\
\hline 6 & 0.019 & 0.263 & 0.263 & 0.260 & 0.256 & 0.248 & 0.249 \\
& & \pm 0.010 & \pm 0.010 & \pm 0.005 & \pm 0.005 & \pm 0.003 & \pm 0.002 \\
\hline
\end{tabular}

In Tables 9.1-9.3 for three time points $t_{i}(i=1,2,3)$, we give the estimates of $\sigma_{v}^{2}$ (in $\mathrm{pc}^{2} / \mathrm{Myr}^{2}$ ) obtained by Danilov (2011) by to $n$ stars closest to the center of a cluster model. In the case $i=1, \sigma_{v}^{2}$ in Tables 9.1-9.3 is equal to the mean $\sigma_{v}^{2}$ relative to two time points $t=0$ and $t=100 h$, where $h$ is the initial step of an integrating the equations of a stellar motion in time. In the case $i=2,3, \sigma_{v}^{2}$ is equal to the mean $\sigma_{v}^{2}$ relative to the period $P_{r}$ of the regular field oscillations of the cluster model. In every cases $i=2,3$ for each cluster model, the time period $P_{r}$ is determined by the time dependencies of the virial coefficient $\alpha=2 E / W$, where $E$ and $W$ are total and potential energies of the cluster model, respectively. The first and second columns of Tables 9.1-9.3 show the number of the cluster model and the time point $t_{i}$ (in the units of the time $\tau_{v r}$ ) for which the values of $\sigma_{v}^{2}$ are indicated in the following columns of Tables 9.1-9.3 at different $n$ (here, $\tau_{v r}$ is the initial time of the violent 
Table 9.2 Velocity Dispersion in the OSC Models

\begin{tabular}{|c|c|c|c|c|c|c|}
\hline \multirow{2}{*}{ } & $t_{2} / \tau_{v r}$ & \multicolumn{5}{|c|}{$\sigma_{v}^{2}$} \\
\cline { 3 - 7 } & & $n=50$ & $n=100$ & $n=200$ & $n=300$ & $n=400$ \\
\hline 1 & 2 & 3 & 4 & 5 & 6 & 7 \\
\hline 1 & 1.60 & 0.224 & 0.235 & 0.258 & 0.267 & 0.268 \\
& & \pm 0.022 & \pm 0.017 & \pm 0.020 & \pm 0.025 & \pm 0.031 \\
\hline 2 & 1.60 & 0.234 & 0.257 & 0.284 & 0.285 & 0.279 \\
& & \pm 0.042 & \pm 0.023 & \pm 0.022 & \pm 0.021 & \pm 0.026 \\
\hline 3 & 1.65 & 0.325 & 0.315 & 0.294 & 0.299 & 0.290 \\
& & \pm 0.036 & \pm 0.024 & \pm 0.016 & \pm 0.018 & \pm 0.019 \\
\hline 4 & 1.65 & 0.288 & 0.298 & 0.336 & 0.332 & 0.316 \\
& & \pm 0.039 & \pm 0.026 & \pm 0.023 & \pm 0.023 & \pm 0.022 \\
\hline 5 & 1.60 & 0.389 & 0.378 & 0.353 & 0.331 & 0.319 \\
& & \pm 0.027 & \pm 0.020 & \pm 0.010 & \pm 0.006 & \pm 0.006 \\
\hline 6 & 1.60 & 0.453 & 0.421 & 0.375 & 0.348 & 0.320 \\
& & \pm 0.041 & \pm 0.035 & \pm 0.028 & \pm 0.024 & \pm 0.019 \\
\hline
\end{tabular}

Table 9.3 Velocity Dispersion in the OSC Models

\begin{tabular}{|c|c|c|c|c|c|c|}
\hline \multirow{2}{*}{ o } & $t_{3} / \tau_{v r}$ & \multicolumn{5}{|c|}{$\sigma_{v}^{2}$} \\
\cline { 3 - 7 } & & $n=50$ & $n=100$ & $n=200$ & $n=300$ & $n=400$ \\
\hline 1 & 2 & 3 & 4 & 5 & 6 & 7 \\
\hline 1 & 2.677 & 0.212 & 0.220 & 0.241 & 0.245 & 0.281 \\
& & \pm 0.027 & \pm 0.024 & \pm 0.018 & \pm 0.014 & \pm 0.019 \\
\hline 2 & 2.738 & 0.254 & 0.280 & 0.284 & 0.284 & 0.273 \\
& & \pm 0.028 & \pm 0.024 & \pm 0.016 & \pm 0.017 & \pm 0.019 \\
\hline 3 & 2.821 & 0.332 & 0.324 & 0.308 & 0.306 & 0.294 \\
& & \pm 0.035 & \pm 0.027 & \pm 0.016 & \pm 0.010 & \pm 0.009 \\
\hline 4 & 2.484 & 0.295 & 0.314 & 0.338 & 0.333 & 0.313 \\
& & \pm 0.042 & \pm 0.028 & \pm 0.016 & \pm 0.014 & \pm 0.014 \\
\hline 5 & 2.915 & 0.374 & 0.374 & 0.356 & 0.334 & 0.317 \\
& & \pm 0.032 & \pm 0.024 & \pm 0.016 & \pm 0.010 & \pm 0.007 \\
\hline 6 & 3.592 & 0.362 & 0.354 & 0.336 & 0.312 & 0.291 \\
& & \pm 0.045 & \pm 0.027 & \pm 0.016 & \pm 0.010 & \pm 0.010 \\
\hline
\end{tabular}

relaxation obtained according to the formula $\tau_{v r} \simeq 2.6 \bar{t}_{c r}$ (Aarseth, 1974); $\bar{t}_{c r}$ is the mean initial time of intersection of the cluster by a star (a crossing time)).

Danilov and Dorogavtseva (2008) performed the calculations of the cluster models by an integration of the equations of a stellar motion using the difference schemes of the 10th and 11 th accuracy orders in the time interval $t \in\left[0, t_{0}\right], t_{0} / \tau_{v r} \simeq 3.9-5.1$, where $t_{0}$ is the time interval for the dynamic evolution of the OSC model, during which a statistical criterion for the accuracy of calculations is performed (Danilov, 1997b) (see Section 16.1, 16.3). By the time $t / \tau_{v r}=1.3$, in models $1-6$ of Danilov 
and Dorogavtseva (2008), an equilibrium regime of the density and a regular field oscillations is established. The values of $t_{i}(i=2,3)$ are assumed to be equal here: $t_{2}=1.3 \tau_{v r}+0.5 P_{r}\left(t_{2}\right)$ and $t_{3}=t_{0}-0.5 P_{r}\left(t_{3}\right)$ (the values $P_{r}$ in the models of Danilov and Dorogavtseva (2008) change slowly with time, therefore the corresponding time points are indicated in parentheses for $P_{r}$ ). From Tables 9.2, 9.3, it is clear that the value of $t_{3}-t_{2}$ in models of Danilov and Dorogavtseva (2008) is more than $1 \tau_{v r}$, and in model $6 t_{3}-t_{2} \simeq 2 \tau_{v r}$. Thus, the considered time interval $t_{3}-t_{2}$ is quite sufficient for a registering the possible changes with time in the stellar motions and in the dynamic state of the OSC models of Danilov and Dorogavtseva (2008).

Fig. 9.1 shows the dependencies $\sigma_{v}^{2}=\sigma_{v}^{2}(n)$ for OSC models 1-6 of Danilov and Dorogavtseva (2008). Figs. 9.1a-c correspond to the dependencies $\sigma_{v}^{2}=\sigma_{v}^{2}(n)$ for the time points $t_{1}, t_{2}$ and $t_{3}$, respectively. Numbers near the curves $\sigma_{v}^{2}=\sigma_{v}^{2}(n)$ in figs. 9.1 a-c correspond to the numbers of the OSC models. Comparison of $\sigma_{v}^{2}$ in fig. 9.1a and in figs. 9.1b,c reveals that during the evolution $\sigma_{v}^{2}$ increases in all models, which is caused by both compression of the cluster models at $t \lesssim 0.5 P_{r}$ and "heating" of clusters by a tidal field of the Galaxy at $t \sim t_{i}(i=2,3)$. According to Danilov and Seleznev (1994), the OSCs with the massive extended cores (i.e. with the "halo-core" structure parameters as models 5 and 6 have) are quite rare. Basically, the sample of 103 OSC (Danilov and Seleznev, 1994) includes the clusters for which the structural parameters are close to the parameters of models 1-4.

The rate of a dynamic evolution of the considered OSC models is related to the slope and the distance from the $n$ axis of the curves $\sigma_{v}^{2}=\sigma_{v}^{2}(n)$ in fig. 9.1. The lower curves in fig. 9.1 correspond to the fastest evolving model 1 of Danilov and Dorogavtseva (2008). In time $t_{0}$, model 1 passes through the most of its evolution towards a formation of a stable equilibrium state (see below). According to (Danilov, 2011), the time for the formation of the spherical velocity distribution of stars $t_{\sigma}$ in model 1 is $t_{\sigma} \simeq(7.6-8.9) \tau_{v r}$, and in model $6-t_{\sigma} \simeq(21.5-24.9) \tau_{v r}$, see Table 3 in Danilov and Dorogavtseva (2008). The synchronization time $t_{s}$ of the cluster rotation with its motion around the center of the Galaxy in model 1 is $t_{s}=(7.5 \pm 0.2) \tau_{v r}$, and in model $6 t_{s}=(26.6 \pm 4.0) \tau_{v r}$, see Table 2 in Danilov and Dorogavtseva (2008). The dependencies $\sigma_{v}^{2}=\sigma_{v}^{2}(n)$ at $t_{i}(i=2,3)$ show a slight increase or constancy of $\sigma_{v}^{2}$ with increasing $n$ for models $1-4$, and a slight decrease of $\sigma_{v}^{2}$ with increasing $n$ for models 5, 6, see fig. 9.1.

We note that the matter densities in the OSCs and in the central parts of OSCs are sufficiently small, which leads to small values of the potential and the dispersion of the stellar velocities near cluster centers (Danilov, 2010). According to Danilov (2010), the OSC halo are usually much more populated by stars than follows from the King distribution (eq. (14) from King (1962)). Tidal "heating" of the OSCs and their models is more significant at the cluster periphery. The contribution of the halo stars to $\sigma_{v}^{2}(n)$ increases rapidly with an increase of $n$. Therefore, the $\sigma_{v}^{2}(n)$ values for models 1, 2, 4 increase with increasing $n$. As a result, the central regions of OSC models 1, 2, 4 are more "cold" than the entire cluster, which leads to the formation of gravitational instability of the OSCs and their models noted in Danilov (2008) and Danilov (2010). In the core of model $3, \sigma_{v}^{2}$ is only slightly higher than $\sigma_{v}^{2}$ in the 

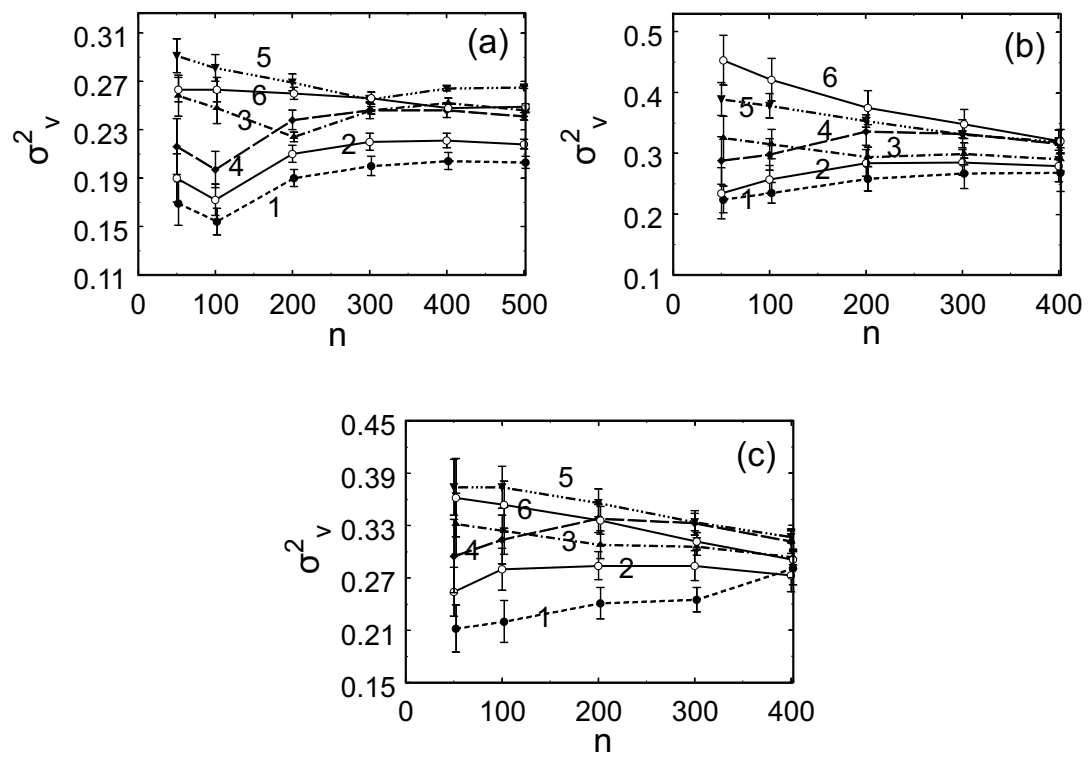

Fig. 9.1 Dependencies of the stellar velocity dispersion in the OSC models on $n$

whole cluster, which also ensures the formation of a gravitational instability at the center of this cluster model, see below.

Thus, the small densities and a tidal "heating" of the OSCs provide the formation of "cold" cores of these clusters which therefore are gravitationally unstable.

Models 5, 6 evolve slowly (in comparison with models 1-4), and during time $t_{0}$ they undergo only a small part of their evolution in the direction of formation of the stable equilibrium state. Therefore, the form of the curves $\sigma_{v}^{2}=\sigma_{v}^{2}(n)$ for these models varies little at different $t_{i}$. Dependencies $\sigma_{v}^{2}=\sigma_{v}^{2}(n)$ for models 5 and 6 change places of each other by the distance from $n$ axis passing from $t=t_{2}$ to $t=t_{3}$ (at equal $n$, model 5 at $t=t_{3}$ has greater $\sigma_{v}^{2}(n)$ than model 6 , and at $t=t_{2}$, model 5 has smaller $\sigma_{v}^{2}(n)$ than model 6). We note that at $t / \tau_{v r} \in[2.3,4.6]$, model 5 shows a temporary decrease in the amplitude of oscillations of the virial coefficient $\alpha$ (temporal virialisation), which is observed on the plots of $\alpha=\alpha(t)$ dependencies obtained by Danilov and Dorogavtseva (2008). In this case, most of the energy of the radial oscillations of the cluster temporarily passes into the energy of the peculiar stellar motions, and the cluster temporarily expands (see fig. 2 from Danilov and Dorogavtseva (2008) for model 5). At $t>4.6 \tau_{v r}$, the $\alpha$ oscillation amplitude in model 5 again increases significantly. The same temporal virialisation (to different degrees and at different time intervals) is observed in other OSC models, with the exception of model 1. The value of $t_{\langle r\rangle}$ from Table 3 in Danilov and Dorogavtseva (2008) (the time point in which the average distance $\langle r\rangle$ of the star from the cluster center begins to increase) approximately coincides with the beginning of the temporal virialisation stage in models 2-6. 
In model 2, a decrease in the amplitude of the cluster oscillations (and the $\alpha$ values) near $t=t_{3}$ (at $t / \tau_{v r} \in[2.40,3.14]$ ) also leads to an increase in $\sigma_{v}^{2}(n)$, which is noticeable in comparison with $\sigma_{v}^{2}(n)$ for model 1 (see fig. 9.1b and 9.1c; the mutual arrangement of the $\sigma_{v}^{2}=\sigma_{v}^{2}(n)$ curves for models 1 and 2). However, at $t / \tau_{v r}>3.14$, the large-scale oscillations in this cluster model continue, the core continues to contract, which should lead to an increase in $\sigma_{v}^{2}(n)$ at $n \simeq 100-150$.

The reason for a temporal virialization of the analyzed OSC models is the "heating" of the cluster core by the energy flow from the periphery toward the cluster center caused by the large-scale cluster oscillations at the previous stage of evolution. Such energy flow in cluster model 1 was detected and investigated by Danilov (2002b). At the stage of sa temporal virialization, the gravitational instability in the cluster cores is largely suppressed (due to a temporarily increased dispersion of the stellar velocities). Further "cooling" of the OSC core due to the expansion of the core and the entire cluster again leads to the development of a gravitational instability near the center, and to an increase in the amplitude of cluster oscillations. In model 1 , the stage of temporal virialization is not observed, since the mechanism of a heating and an expansion of the cluster core considered here rapidly leads to the loss of a significant part of the halo stars in this model, and to a relative stabilization of the model's core near the stable equilibrium state (see the position of model 1 at $t=t_{3}$ in fig. 9.2). This state is probably the final stage in the evolution of the OSCs and their models.

We note that the external field of the Galaxy not only "heats up" the outer parts of the cluster, but also largely prevents the entry of the high-energy stars into the cluster center (due to the non-radiality of the cluster star orbits in the presence of the Galaxy field). Therefore, the transfer of the energy of the regular field oscillations toward the cluster center (Danilov, 2002b) plays an important role in the "heating" of the cluster core. The energy transfer into the cluster core also is partially ensured by the diffusion of stars in the velocity space during the stellar encounters. In our OSC models, such mechanism is less efficient, since estimates of the collision relaxation time $\tau_{s t}$ of the models (due to stellar encounters) exceed $\tau_{v r}$ in 1.9-2.6 times (see, for example, Table 3 from Danilov (2010) for models 1,6). We also notice that the slope of the curves $\sigma_{v}^{2}=\sigma_{v}^{2}(n)$ in fig. 9.1 at small $n \simeq 50-200$ in models 1, 2, 4 indicates the presence of an external source of the cluster "heating".

Thus, for the OSCs, a temporal virialization is possible, during which a significant part of the cluster oscillation energy temporarily transforms into a kinetic energy of the peculiar stellar motions. Among the considered OSC models, the greatest duration $\Delta t$ of this stage is achieved in model 5 ( $\Delta t \simeq 2.3 \tau_{v r}$, which corresponds to $\Delta t \simeq 9.8 \times 10^{7}$ years).

Observational data on the radial dependencies of the stellar velocity dispersions $\sigma=\sigma(r)$ in the OSCs are very few. The errors in the stellar velocity dispersion are either too large for reliable conclusions about dependencies $\sigma=\sigma(r)$, or not given in the papers on this topic. We shall mention only the following two papers. According to the data on the proper motions of stars in cluster NGC 2669, Sagar and Bhatt (1989) have shown that the stellar velocity dispersions depend weakly on the distance $r$ from the cluster center at $r \geq 45^{\prime}$ (arcminutes), and increase at $r \geq 45^{\prime}$ 
(see Table 3 from Sagar and Bhatt (1989)). Geller et al. (2008), from the data on the radial stellar velocities in cluster NGC 188 , have shown that the stellar velocity dispersions do not change within errors with an increasing distance from the cluster center after a considering the influence of the binary stars in the cluster core, that are not resolved into the single stars in the observations. Such observed dependencies $\sigma=\sigma(r)$ quite can lead to dependencies $\sigma_{v}^{2}=\sigma_{v}^{2}(n)$ shown in fig. 9.1.

\subsection{Instability Parameters of OSC and their Models}

Danilov (2008) and Danilov (2010) used a three-integral PDF in the form $\Psi=\Psi(\mathcal{T})$ to describe the motion of stars in the cluster core, where $\mathcal{T}=J_{1}+\kappa^{2} J_{2}+s J_{3}, J_{l}$ are the integrals of motion $\left((l=1,2,3) ; \kappa^{2}\right.$ and $s$ are the constant values. $\kappa^{2}$ and $s$ are related to the ratios of the mean stellar velocity dispersions along the coordinate axes (averaged over the period $P_{r}$ of the regular field oscillations). The formulas for $\kappa^{2}$ and $s$ are given in the explanations to formulas (35) and (18) from Danilov (2006). The $s$ value in the Danilov (2008), Danilov (2010), and Danilov (2006) papers is denoted as $\sigma^{2}$, since in these papers at $t=t_{2}$ models 1,6 were considered, for which $s>0$. In models 3 and 5 at $t=t_{2}$ and in models 4 and 5 at $t=t_{3}$, the mean values of the coefficient $s<0$, so a denotation $s$ is used here instead of $\sigma^{2}$, which does not affect the possibility of an estimating the instability parameters of the PDF oscillations at the cluster center.

Results of an estimating of the dynamic parameters of the OSC models of Danilov and Dorogavtseva (2008) for two time points $t=t_{2}, t_{3}$ are given in Table 9.4. In the 1 st column of Table 9.4, the number of the cluster model is indicated. In columns 2 , 3 and 9,10 of Table 9.4, for the time points $t=t_{2}$ and $t_{3}$, respectively, the values of the coefficients $\kappa^{2}$ and $s$ are given in the case of a stellar motion in the joint force field of the Galaxy and a homogeneous ellipsoid with the potential $U_{e}$ ( $C$-ellipsoid), see formula (8) from Danilov (2010).

In the papers of Danilov (2008) and Danilov (2010) and in this work, the mean potential (averaged over the period $P_{r}$ ) near the center of the numerical dynamic cluster model was approximated by the $U_{e}$ potential. The eigenvalues of $\lambda$ in the problem of the PDF value oscillations near the center of $C$-ellipsoid were obtained by solving equation (31) from Danilov (2008). The complex conjugate roots of this equation $\lambda=\gamma_{1} \pm i \gamma_{2}$ allow to estimate the period $P_{\gamma}=2 \pi / \gamma_{1}$ of the oscillations with a dominating amplitude and the time $t_{\lambda}=1 / \gamma_{2}$ of the instability development of the PDF oscillations near the center of the cluster model. In columns 4, 5 and 11, 12 of Table 9.4 at $t=t_{2}$ and $t=t_{3}$, respectively, the values of $P_{\gamma}(1)$ and $t_{\lambda}(1)$ in Myr are given. The unity in the parentheses at $P_{\gamma}$ and $t_{\lambda}$ means that the considered stars have energies $\varepsilon$, which are sufficient to remove a star by $1 \mathrm{pc}$ from the cluster center (till the cluster core boundary) during its motion within the cluster. In this case, $C_{k}=1 \mathrm{pc}, k=(1,2,3)$, see formulas (30) from Danilov (2008); the $C_{k}$ values are directly proportional to the amplitudes of an unperturbed stellar motion with respect to the spatial coordinates, and have the same order of magnitude as these 
amplitudes. In columns $6-8$ of Table 9.4 for $t=t_{3}$, the following values are given: $\rho(0)$ (the mean density near the center of the cluster model averaged over $P_{r}$ and obtained from the 5-6 stars closest to the cluster center); $\rho_{e}$ (the density of the $C$-ellipsoid approximately equal to the mean density of the cluster model averaged over $P_{r}$, see Danilov (2010)); $\varepsilon_{t}$ (the energy per unit of the star mass, which is necessary for the star to exit from the center to the surface of $C$-ellipsoid). $\rho(0)$, $\rho_{e}$ are given in the units of $m_{\odot} / \mathrm{pc}^{3}$, and $\varepsilon_{t}$ is given in the units of $(\mathrm{pc} / \mathrm{Myr})^{2}$. The values of $\rho(0), \rho_{e}$ and $\varepsilon_{t}$ for models $1-6$ at the time point $t=t_{2}$ are given in Table 2 from Danilov (2010). The values of $\rho_{e}, \varepsilon_{t}, \kappa^{2}, s, C_{k}, k=(1,2,3)$, as well as data on the mass, the tidal sizes of the cluster model, and the parameters of the force field of the Galaxy $\alpha_{1}$ and $\alpha_{3}$ (Chandrasekhar, 1942), taken in accordance with the model of a Galactic potential of Kutuzov and Osipkov (1980), were used in this work to find the coefficients of equation (31) from Danilov (2008) and to calculate the values of $P_{\gamma}$ and $t_{\lambda}$.

When calculating the boundaries of the unstable PD oscillation region in the space of $\rho$ (the mass density of the cluster core) in the OSC models and in the clusters considered in Danilov (2010), for the cases $C_{k}=0,1 \mathrm{pc}, k=1,2,3$, we used the technique described in Danilov (2010) (the variation of the $\varepsilon_{t}$ and $\rho_{e}$ values at the constant cluster mass $M_{c}=$ const). In comparison with Danilov (2010), the range $E_{t}$ of the considered values of $\varepsilon_{t} \in E_{t}$ was extended. The $E_{t}$ range was investigated in steps of $\Delta \varepsilon$ equal to $\Delta \varepsilon=\left(10^{-4}-10^{-6}\right) \times \varepsilon_{t}$ (Danilov (2010) used the step $\Delta \varepsilon=(1-10) \times \varepsilon_{t}$, and when the stable oscillations were found, the position of the boundary of the instability region was refined by a "bisection method"). The results of the $\rho$ calculations are shown in fig. 9.2. In fig. 9.2, $\rho_{e}$ values for models $1-6$ at the time $t=t_{3}$ are taken from Table 2 of Danilov (2011), see also in Table 9.4; in the case $t=t_{2}-$ the values $\rho_{e}$ are taken from Table 3 of Danilov (2010). For six OSC considered in Danilov (2010), the $\rho_{e}$ values are also taken from Table 3 of Danilov (2010). As in Danilov (2010), the tidal density $\rho_{t}$ of the OSCs and their models was calculated using a formula $\rho_{t}=-3 \alpha_{1} /(4 \pi G)$, where $G$ is gravitational constant, and $\alpha_{1}<0$ (see above, and also Geller et al. (2008); Danilov (2006)). The numerical values of $\rho$ at the boundaries of the instability regions for the considered objects in the form of tables are not given here due to a cumbersome form of such tables. The errors $\sigma_{\rho}$ of $\rho$ obtained for the OSCs and their models at the boundaries of the instability regions do not exceed the errors of $\rho$ indicated in columns 13-16 of Table 3 from Danilov (2010), and in the case of regions II and II', see fig. 9.2, the $\sigma_{\rho}$ values are $\sim(0.64-7.10) \%$ of the width $\Delta \rho$ of these regions relative to $\rho$ (here, $\Delta \rho$ is obtained at the mean $\varepsilon_{t}$ for the given model of the object).

In fig. 9.2 and 9.3 in coordinates $\left(\ln \left(\rho_{e} / \rho_{t}\right), \ln \left(\rho / \rho_{e}\right)\right)$ and $\left(\rho(0) / \rho_{e}, \ln \left(\rho / \rho_{e}\right)\right)$, the dots indicate the positions of the objects considered in our work (the OSCs and the OSC models) within the region of the unstable PD oscillations at the centers of these objects. Letters a, b, c, d, e, f mark the points corresponding to the OSCs NGC 188, NGC 6819, IC 1848, NGC 1912, NGC 6705, NGC 7654 (as in Danilov (2010)). Numbers 1-6 mark the points corresponding to the OSC models $1-6$ at $t=t_{2}$, and numbers $1^{\prime}-6^{\prime}$ mark the points corresponding to these models at $t=t_{3}$. Solid and dashed lines in figs. 9.2, 9.3 indicate the boundaries of the unstable PD oscillations 


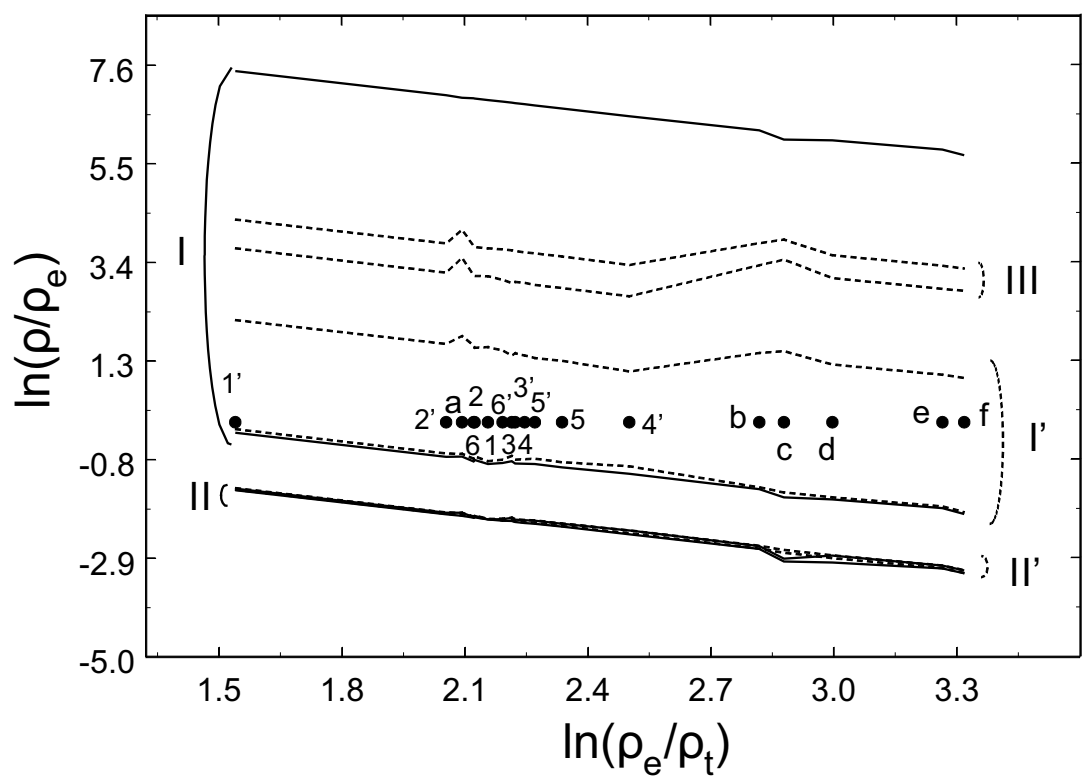

Fig. 9.2 The boundaries of the regions of the unstable phase density oscillations at the centers of six OSCs and considered OSC models; positions of these objects within the instability region

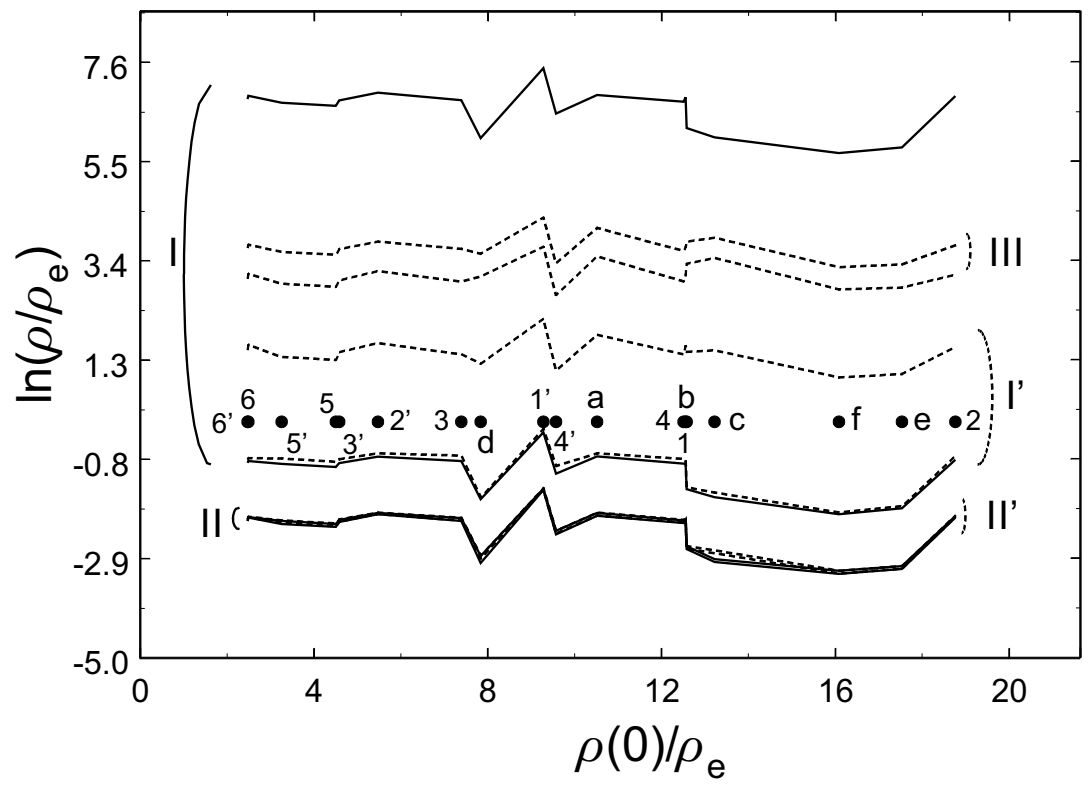

Fig. 9.3 The same as in fig. 9.2 in coordinates $\left(\rho(0) / \rho_{e}, \ln \left(\rho / \rho_{e}\right)\right)$ 
at $C_{k}=0$ and $C_{k}=1 \mathrm{pc}$, respectively. Solid (and dashed) vertical arcs with numbers I, II (and I', II', III) in figs. 9.2, 9.3 indicate the regions of an instability of these oscillations at $C_{k}=0$ (and $C_{k}=1 \mathrm{pc}$ ). All considered objects in figs. 9.2, 9.3 are located within regions $\mathrm{I}$ and $\mathrm{I}^{\prime}$. In addition to the unstable PD oscillation regions indicated in Danilov (2010), the present study reveals the very narrow regions II and $\mathrm{II}^{\prime}$ (at the density values of $\rho=\rho_{I I, I I^{\prime}} \sim(0.114-0.127) m_{\odot} / \mathrm{pc}^{3}$ for models $1-6\left(R_{G}=8200 p c\right.$, see above $)$ and $\rho_{I I, I I^{\prime}} \sim(0.181-0.192) m_{\odot} / \mathrm{pc}^{3}$ in the case of NGC $6705, R_{G}=6820 \mathrm{pc}, \rho_{I I, H I^{\prime}} \sim(0.068-0.086) m_{\odot} / \mathrm{pc}^{3}$ in the case of IC $1848, R_{G}=9520 \mathrm{pc} ; R_{G}$ values are assumed here according to Danilov and Seleznev (1994), Table A.2.1; the $\rho_{I I, I I^{\prime}}$ values are usually $\sim(9-21) \%$ larger than $\rho_{t}$ ). An upper bound of the values of $\rho=\rho_{I^{\prime}}$ in the OSC models is reached at $\rho_{I I^{\prime}} \sim(3.8-4.5) m_{\odot} / \mathrm{pc}^{3}$. The lower bounds of the $\rho_{I^{\prime}}$ values in six OSCs and in OSC models 1, 6 at $t=t_{2}$ are listed in Table 3 of Danilov (2010), column 15. At $C_{k}=1 \mathrm{pc}$, this study also reveals the region III of the unstable PD oscillations (the density values $\rho=\rho_{I I I} \sim(18.6-37.3) m_{\odot} / \mathrm{pc}^{3}$ in the OSC models), see figs. 9.2, 9.3.

According to fig. 9.2, model 1 in time $\Delta t=t_{3}-t_{2}$ evolves toward the reducing of the core density. The point in fig. 9.2 corresponding to model 1 herewith moves to the left almost till the boundary of the unstable oscillation region I' (and region I). Thus, the movement of the OSC toward the state of a stable equilibrium is possible with a decrease of the density of the cluster core (probably, this is the most frequent version of the OSC evolution). Models 2 and 5 at $t \in\left[t_{2}, t_{3}\right]$ also evolve toward a stable equilibrium state with the decreasing core density (see fig. 9.2).

The most noticeable movement to the right in fig. 9.2 is detected for model 4 (the densest cluster model with the largest initial concentration of stars toward the center, the same as in models 1, 2, see Table 1 from Danilov and Dorogavtseva (2008), column 2). In this case, model 4 begins to approach the upper boundary of region $\mathrm{I}^{\prime}$ and, therefore, evolves toward the state of a stable equilibrium. Models 3 and 6 also show a slow evolution with the increasing core density towards the upper boundary of region $\mathrm{I}^{\prime}$ in fig. 9.2. Models 5 and 6 show an opposing motion in fig. 9.2. In this case, model 5 approaches a steady equilibrium, and model 6 moves away from it, which is associated with an increase of the velocity dispersion $\sigma_{v}^{2}(n)$ in model 5 and a decrease of $\sigma_{v}^{2}(n)$ in model 6 at $t \in\left[t_{2}, t_{3}\right]$, see above.

Thus, if the effects of core "heating" (caused by the oscillations of a regular field and by a tidal field of the Galaxy) dominate in the cluster, then the cluster in fig. 9.2 moves to the left (in the same direction the evolution of the OSC is accelerated if we take into account the influence on the evolution of the encounters between the single stars (SS) and the dynamically active close binary stars (CBS) (Danilov, 1977, 1978)). If the effects of the encounters between SS dominate in the cluster, then a compression and an increase in the core density take place. Such cluster moves to the right in fig. 9.2. At $\rho_{e} / \rho_{t} \gtrsim 17.8$ (see cluster IC 1848, point "c" in fig. 9.2), an evolution with the core compression gradually leads the cluster to the state of a stable equilibrium. During the dynamic evolution of the cluster, the roles of the mechanisms operating in the cluster can change (for example, a temporal virialization of the cluster or a replacement of the dominant type of the encounters 
between SS by the encounters between SS and CBS as a result of an accumulation of the total binding energy in CBS (Danilov, 1977)). In this case, after a prolonged stage of the cluster compression, a prolonged cluster expansion may occur, see fig. 1, 2 from Danilov (1977). The critical cluster density value $\rho_{m}$, at which the cluster compression is replaced with an expansion, is obtained by Danilov (1977) within the framework of the gross-dynamic description of the OSC (in the form of the $\rho_{m}$ dependency on the number and the parameters of the CBSs, see formula (17) and fig. 3 from Danilov (1977)).

We note that changes $\Delta x$ in the position of the OSC models 2, 3, 5, 6 along the axis $x=\ln \left(\rho_{e} / \rho_{t}\right)$ in time $\Delta t=t_{3}-t_{2}$ in fig. 9.2 are within the limits of the errors $\sigma_{x}$. In model $4, \Delta x$ is $\sim 2.5$ times larger than $\sigma_{x}$, and in model $1, \Delta x$ is $\sim 6.0$ times larger than $\sigma_{x}$. The errors $\sigma_{x}$ are defined by the errors $\sigma_{\rho_{e}}$ of $\rho_{e}$, and are given in Table 2 from Danilov (2010) for $t=t_{2}$ and in Table 9.3 of this work for $t=t_{3}$. Usually, the $\sigma_{\rho_{e}}$ values are $\sim 10 \%$ of the $\rho_{e}$ values. They are determined by the errors in defining the $\varepsilon_{t}$ (see formula (9) from Danilov (2010)), and are an upper bound of errors $\sigma_{\rho_{e}}$, since in addition to the random deviations of the gravitational potential $U$ of the model from the mean value (averaged over the $P_{r}$ period), the $U$ values also characterize the amplitude of the $U$ oscillations near the center of the cluster model.

Table 9.4 Dynamic parameters of six OSC models

\begin{tabular}{|c|c|c|c|c|c|c|c|c|c|c|c|}
\hline \multirow[b]{2}{*}{ № } & \multicolumn{4}{|c|}{$t=t_{2}$} & \multicolumn{7}{|c|}{$t=t_{3}$} \\
\hline & $\kappa^{2}$ & $s$ & $\overline{P_{\gamma}(1)}$ & $t_{\lambda}(1)$ & $\rho(0)$ & $\rho_{e}$ & $\varepsilon_{t}$ & $\kappa^{2}$ & $S$ & $P_{\gamma}(1)$ & $t_{\lambda}(1)$ \\
\hline 1 & 2 & 3 & 4 & 5 & 6 & 7 & 8 & 9 & 10 & 11 & 12 \\
\hline \multirow[t]{2}{*}{1} & 0.71 & 0.061 & 37.4 & 53.7 & 4.53 & 0.49 & 0.25 & 0.75 & 0.037 & 49.2 & 128.4 \\
\hline & \pm 0.16 & \pm 0.024 & \pm 1.8 & \pm 2.9 & \pm 1.94 & \pm 0.05 & \pm 0.02 & \pm 0.21 & \pm 0.040 & \pm 2.0 & $(100.1-244.1)$ \\
\hline \multirow[t]{2}{*}{2} & 0.75 & 0.034 & 38.2 & 60.5 & 4.57 & 0.82 & 0.40 & 0.77 & 0.039 & 39.4 & \begin{tabular}{|l|}
61.9 \\
\end{tabular} \\
\hline & \pm 0.19 & \pm 0.044 & \pm 1.8 & \pm 3.7 & \pm 1.52 & \pm 0.08 & \pm 0.04 & \pm 0.19 & \pm 0.034 & \pm 1.9 & \pm 4.4 \\
\hline \multirow[t]{2}{*}{3} & 0.90 & -0.006 & 37.0 & 67.1 & 4.43 & 0.97 & 0.46 & 0.85 & 0.023 & 36.6 & 59.4 \\
\hline & \pm 0.21 & \pm 0.037 & \pm 1.8 & \pm 4.0 & \pm 2.00 & \pm 0.10 & \pm 0.04 & \pm 0.19 & \pm 0.043 & \pm 1.8 & \pm 3.2 \\
\hline \multirow[t]{2}{*}{4} & 0.81 & 0.012 & 36.2 & 61.4 & 12.22 & 1.28 & 0.59 & 0.82 & -0.026 & 32.8 & 64.8 \\
\hline & \pm 0.20 & \pm 0.047 & \pm 1.9 & \pm 3.5 & \pm 7.62 & \pm 0.13 & \pm 0.06 & \pm 0.21 & \pm 0.055 & \pm 1.3 & \pm 2.5 \\
\hline \multirow[t]{2}{*}{5} & 0.86 & -0.0006 & 34.8 & 61.7 & 3.30 & 1.01 & 0.48 & 0.85 & -0.003 & 35.9 & 64.4 \\
\hline & \pm 0.13 & \pm 0.0330 & \pm 1.7 & \pm 2.8 & \pm 1.68 & \pm 0.10 & \pm 0.04 & \pm 0.14 & \pm 0.037 & \pm 1.8 & \pm 3.5 \\
\hline \multirow[t]{2}{*}{6} & \begin{tabular}{|l|}
0.78 \\
\end{tabular} & 0.052 & 38.1 & 56.3 & 2.32 & 0.94 & 0.45 & 0.84 & 0.034 & 37.0 & 57.8 \\
\hline & \pm 0.18 & \pm 0.039 & \pm 1.8 & \pm 3.3 & \pm 1.48 & \pm 0.10 & \pm 0.04 & \pm 0.16 & \pm 0.034 & \pm 1.8 & \pm 3.2 \\
\hline
\end{tabular}

According to fig. 9.3, cluster models $1-6$ at $t \in\left[t_{2}, t_{3}\right]$ evolve with decreasing $\rho(0) / \rho_{e}$. The most significant decrease in $y=\rho(0) / \rho_{e}$ is observed in model 2 . Apparently, the clusters marked by letters in fig. 9.3 evolve in the same direction. With an increase of $t$, the central densities in the OSCs gradually decrease in comparison with the mean cluster core density. We note that the errors $\sigma_{y}$ of $y$ are $\sim 14.2 \%$ of the $y$ values. The changes $\Delta y$ in the position of models 1-5 along the axis $\rho(0) / \rho_{e}$ 
in time $\Delta t$ in fig. 9.3 go beyond $2 \sigma_{y}$ (the value of $\Delta y<\sigma_{y}$ only in the case of model 6 , but for model $2 \Delta y \simeq 10 \sigma_{y}$ ).

\subsection{Structure of Regions of the PD Oscillation Instability in the Centers of Six OSCs}

Fig. 9.4 shows (according to Danilov (2011)) the dependencies of the PD oscillation parameters in the center of NGC 6705 on the density $\rho$ and $P_{\gamma}^{-1}$ for $C_{k}=0$ (fig. 9.4a and 9.4c) and $C_{k}=1 \mathrm{pc}$ (fig. 9.4b and 9.4d) according to Danilov (2011). Solid (and dashed) lines in fig. 9.4a, 9.4b indicate dependencies of $\ln \left(t_{\lambda}\right)$ (and $\ln \left(P_{\gamma}\right)$ ) on $\ln (\rho)$ within the instability regions. In comparison with fig. 3 from Danilov (2010), three new instability regions are added here. Moreover, fig. $3 \mathrm{~b}$ from Danilov (2010) contains typos (the ordinate axis name in fig. 3b from Danilov (2010) must be $t_{\lambda}, P_{i}$ instead of $\ln \left(t_{\lambda}\right), \ln \left(P_{i}\right)$; the values changing along the abscissa axis are $\rho$, not $(\rho)$ ). Outside the instability regions, according to (31) from Danilov (2008), five periods of the stable phase density oscillations are obtained. Of these, the maximum $\left(P_{1}\right)$ and minimum $\left(P_{2}\right)$ periods are indicated in fig. $9.4 \mathrm{a}, \mathrm{b}$ by dashed and solid lines, respectively. The vertical arrows in fig. 9.4 mark the "observed" values of $\rho_{e}$ and $P_{\gamma}^{-1}$ (see Table 3 from Danilov (2010), column 9 for $\rho_{e}$ and column 10 for $P_{\gamma}$ at $C_{k}=1$ pc). When $\rho$ is changing from $\rho_{e}$ to the $\rho$ value on the boundaries of instability regions, $t_{\lambda}$ in fig. 9.4a,b increases indefinitely (in this case, the growth rate of the PD oscillation amplitude decreases to zero). According to fig. 9.4a,b, the dominant oscillation period $P_{\gamma}$ and the periods $P_{1}, P_{2}$ decrease with increasing density $\rho$. Fig. 9.4c, d, shows the resonance curves (Strelkov, 2005) for the PD oscillation amplitude in the cluster center within the instability zones.

Resonance curves were obtained by Danilov (2011) under the assumption that the amplitude of the steady-state PD oscillations at the cluster center is directly proportional to the instability growth rate (an increment) $\gamma_{2}$ of such oscillations. Values of the coefficients $A_{i}$ were chosen so that it would be convenient to compare the resonance curves obtained for different instability regions. The index values $i$ in fig. 9.4c,d mark their corresponding resonance curves. At $C_{k}=0$, the values $i=1,2$ correspond to regions II, I in fig. 9.2 and 9.3 , and at $C_{k}=1 \mathrm{pc}$, the values $i=1,2,3$ correspond to regions $\mathrm{II}^{\prime}, \mathrm{I}^{\prime}$, III, see above. At $C_{k}=0$, the values $A_{i}$ (in Myr) are assumed to be: $A_{1}=50, A_{2}=1$, and at $C_{k}=1 \mathrm{pc}$, they are assumed to be: $A_{1}=100$, $A_{2}=3, A_{3}=1$. We note that for other clusters and cluster models considered in this work, the dependencies of $\ln \left(t_{\gamma}\right)$ and $P_{\gamma}$ on $\ln (\rho)$, as well as the resonance curves have a form similar to that shown in fig.9.4 for cluster NGC 6705. The amplitudes (and energies) of the established PD oscillations in the cluster center (and the cluster as a whole) decrease when passing from the large frequencies $\gamma_{1}=2 \pi P_{\gamma}^{-1}$ to smaller ones. When passing from $C_{k}=1 \mathrm{pc}$ to $C_{k}=0$ (to the lower motion energy of the stars in the cluster core), the number of the regions of the unstable oscillations decreases, but the width of the regions I and I' of the unstable PD oscillations, containing clusters and their models, increases. When the core is "heated", the number of the 

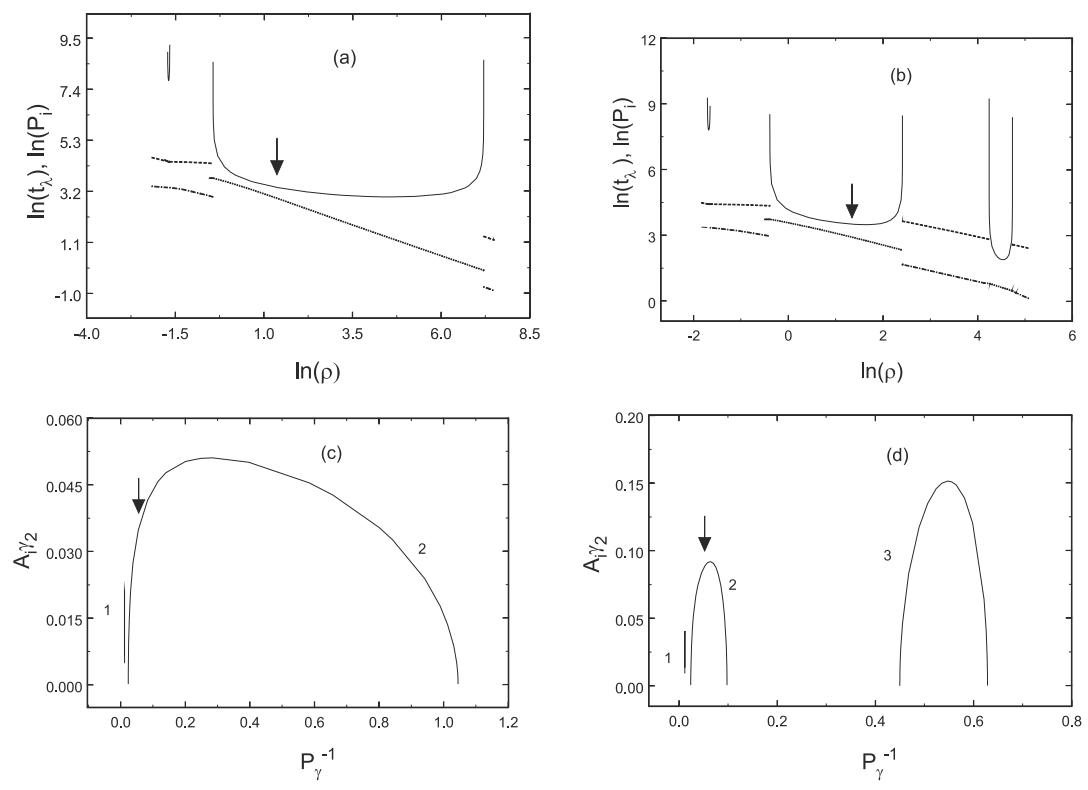

Fig. 9.4 The dependencies of the PD oscillation parameters in the center of cluster NGC 6705 on the density $\rho$ and the $P_{\gamma}^{-1}$ value

complex conjugate roots $\lambda$ of equation (31) from Danilov (2008) increases, which should lead to an increase in the number of the instability regions in figs. 9.2-9.4 and to a decrease in their width in the spaces of $\ln (\rho)$ and $\gamma_{1}$. In this case, we can expect the OSCs and their models to fall into the region of stable PD oscillations, which contains a larger number of the narrower instability zones.

The OSCs and their models considered here are not in the maxima of the resonance curves of regions I and $\mathrm{I}^{\prime}$, although they are located near these maxima. In order to describe the instability regions, it is convenient to introduce the following value: $Q=\gamma_{1_{0}} / \Delta \gamma_{1}=P_{\gamma_{0}}^{-1} / \Delta P_{\gamma}^{-1}$, where $\gamma_{1_{0}}$ and $P_{\gamma_{0}}$ correspond to the frequency and the PD oscillation period at the maximum of a resonance curve, and $\Delta \gamma_{1}$ and $\Delta P_{\gamma}^{-1}$ correspond to the width of this instability region at $\gamma_{2}=0$. A similar parameter is introduced when describing the quality factor $Q$ of the simplest oscillation mechanical systems (Strelkov, 2005). However, Strelkov (2005) determines $\Delta \gamma_{1}$ for $Q$ at the level of the values $\gamma_{2}=\frac{1}{2} \gamma_{2_{0}}$, where $\gamma_{2_{0}}$ corresponds to the maximum of the resonance curve. According to Strelkov (2005), $Q$ characterizes the relative energy loss of a mechanical oscillation system due to the energy dissipation caused by friction on an interval of time equal to one oscillation period. In our OSC models, the energy of the system oscillations passes into the energy of the peculiar stellar motions under the action of the relaxation mechanisms, and remains largely within the system. In linear mechanical systems (Strelkov, 2005, p.53), the source of oscil- 
lations is the external driving force, and in our OSC models, the oscillation source (the gravitational instability) is inside the system.

Different ways of an estimating of $\Delta \gamma$ by Strelkov (2005) and by Danilov (2011) for OSCs do not lead to a significant discrepancy in $Q$, which is explained by the shape of the resonance curves (see fig. 9.4c,d). In the case of NGC 6705 at $C_{k}=1$ pc, the use of the values $\gamma_{2}=\frac{1}{2} \gamma_{2_{0}}$ leads to an increase of $Q$ by $12.4,12.6,18.2 \%$ in the regions $\mathrm{II}^{\prime}, \mathrm{I}^{\prime}, \mathrm{III}$, respectively, in comparison with the $Q$ values obtained at $\gamma_{2}=0$ (in the case $C_{k}=0$, an increase of $Q$ at $\gamma_{2}=\frac{1}{2} \gamma_{2_{0}}$ is 11.8 and $24.0 \%$ in regions II and I, respectively, in comparison with $Q$ obtained at $\gamma_{2}=0$ ).

Table 9.5 Parameters of the instability regions of the PD oscillations in the centers of six OSCs

\begin{tabular}{|c|c|c|c|c|c|}
\hline \multirow[t]{2}{*}{ OSC } & \multicolumn{5}{|c|}{$C_{k}=0$} \\
\hline & Region & $\bar{Q}$ & $\gamma_{2_{0}}$ & $\overline{P_{\gamma_{0}}}$ & $\gamma_{1_{0}} / \omega$ \\
\hline 1 & 2 & 3 & 4 & 5 & 6 \\
\hline \multirow[t]{4}{*}{ NGC 188} & I & 0.27614 & 0.03636 & 4.9811 & 50.146 \\
\hline & & \pm 0.00002 & $\pm 10^{-9}$ & \pm 0.0004 & \pm 0.004 \\
\hline & II & 74.7 & 0.00031 & 117.236 & 2.13059 \\
\hline & & \pm 0.7 & $\pm 0.8 \times 10^{-10}$ & \pm 0.001 & \pm 0.00002 \\
\hline \multirow[t]{4}{*}{ NGC 1912} & $\bar{I}$ & 0.276 & 0.03498 & 5.17745 & 50.14634 \\
\hline & & \pm 0.001 & $0.6 \times \pm 10^{-10}$ & $\pm 0.4 \times 10^{-6}$ & $\pm 0.5 \times 10^{-5}$ \\
\hline & $\overline{\mathrm{II}}$ & 77.4 & 0.00030 & \begin{tabular}{|l|}
121.862 \\
\end{tabular} & 2.13053 \\
\hline & & \pm 7.4 & $\pm 0.4 \times 10^{-7}$ & \pm 0.001 & \pm 0.00002 \\
\hline \multirow[t]{4}{*}{ NGC 6705} & $\bar{I}$ & 0.27617 & 0.05109 & 3.5454 & 50.141 \\
\hline & & \pm 0.00003 & $0.6 \pm 10^{-10}$ & \pm 0.0002 & \pm 0.003 \\
\hline & II & 79.0 & 0.00042 & 83.430 & 2.13076 \\
\hline & & \pm 2.1 & $\pm 0.8 \times 10^{-8}$ & \pm 0.002 & \pm 0.00005 \\
\hline \multirow[t]{4}{*}{ NGC 6819} & I & 0.27615 & 0.04288 & 4.22401 & 50.14368 \\
\hline & & $\pm 0.5 \times 10^{-6}$ & $\pm 10^{-10}$ & $\pm 0.3 \times 10^{-6}$ & $\pm 0.4 \times 10^{-5}$ \\
\hline & II & 77.7 & 0.00036 & 99.4103 & 2.13064 \\
\hline & & \pm 1.4 & $\pm 0.4 \times 10^{-9}$ & \pm 0.0005 & \pm 0.00001 \\
\hline \multirow[t]{4}{*}{ NGC 7654} & I & 0.27614 & 0.03657 & 4.9533 & 50.146 \\
\hline & & $\pm 0.9 \times 10^{-6}$ & $\pm 0.5 \times 10^{-10}$ & $\pm 0.3 \times 10^{-6}$ & $\pm 0.3 \times 10^{-5}$ \\
\hline & II & \begin{tabular}{|l|}
76.3 \\
\end{tabular} & 0.00031 & \begin{tabular}{|l|}
116.580 \\
\end{tabular} & 2.13061 \\
\hline & & \pm 2.2 & $\pm 0.7 \times 10^{-9}$ & \pm 0.001 & \pm 0.00002 \\
\hline \multirow[t]{4}{*}{ IC 1848} & I & 0.27630 & 0.03229 & 5.60964 & 47.74339 \\
\hline & & $\pm 0.6 \times 10^{-6}$ & $\pm 0.4 \times 10^{-10}$ & $\pm 0.5 \times 10^{-6}$ & $\pm 0.4 \times 10^{-5}$ \\
\hline & II & 86.8 & 0.00024 & 131.915 & 2.03027 \\
\hline & & \pm 5.8 & $\pm 0.9 \times 10^{-10}$ & \pm 0.003 & \pm 0.00005 \\
\hline
\end{tabular}

Tables 9.5 and 9.6 give the parameters of the instability regions of the PD oscillations in the centers of six OSCs. Columns 1 and 2 of Tables 9.5 and 9.6 indicate cluster names and numbers of the unstable oscillation regions (coinciding with their numbers in figs. 9.2,9.3). The $Q$ values obtained at $\gamma_{2}=0$, as well as $\gamma_{2_{0}}\left(\right.$ in $\left.\mathrm{Myr}^{-1}\right)$, $P_{\gamma_{0}}$ (in Myr) and $\gamma_{1_{0}} / \omega$ are given in columns 3-6 of Tables 9.5 and 9.6 at $C_{k}=0$ and $C_{k}=1 \mathrm{pc}$, respectively. Here, $\omega$ is the frequency of the cluster rotation around 
Table 9.6 Parameters of instability regions of PD oscillations in the centers of six OSC

\begin{tabular}{|c|c|c|c|c|c|}
\hline \multirow[t]{2}{*}{ OSC } & \multicolumn{5}{|c|}{$C_{k}=1 \mathrm{pc}$} \\
\hline & Region & $Q$ & $\gamma_{2_{0}}$ & $P_{\gamma_{0}}$ & $\gamma_{1_{0}} / \omega$ \\
\hline 1 & 2 & 3 & 4 & 5 & 6 \\
\hline \multirow[t]{6}{*}{ NGC 188} & $\mathrm{I}^{\prime}$ & 0.917 & 0.0194 & 25.1 & 10.0 \\
\hline & & \pm 0.054 & \pm 0.0011 & \pm 1.5 & \pm 0.6 \\
\hline & $\mathrm{II}^{\prime}$ & 2.6 & 0.10790 & 3.1 & 80.6 \\
\hline & & \pm 0.2 & \pm 0.00001 & \pm 0.2 & \pm 5.2 \\
\hline & III & 96.7 & 0.00029 & 117.221 & \begin{tabular}{|l|}
2.13086 \\
\end{tabular} \\
\hline & & \pm 11.4 & $\pm 0.3 \times 10^{-5}$ & \pm 0.005 & \pm 0.00009 \\
\hline \multirow[t]{6}{*}{ NGC 1912} & $\mathrm{I}^{\prime}$ & 0.856 & 0.0212 & \begin{tabular}{|l|}
22.7 \\
\end{tabular} & \begin{tabular}{|l|}
11.4 \\
\end{tabular} \\
\hline & & \pm 0.048 & \pm 0.0009 & \pm 1.3 & \pm 0.7 \\
\hline & $\overline{\mathrm{II}^{\prime}}$ & 3.11 & 0.10379 & 2.6 & 99.9 \\
\hline & & \pm 0.25 & $\pm 0.4 \times 10^{-5}$ & \pm 0.2 & \pm 7.7 \\
\hline & III & 80.3 & 0.00029 & 121.846 & 2.13081 \\
\hline & & \pm 1.8 & $\pm 0.2 \times 10^{-5}$ & \pm 0.003 & \pm 0.00005 \\
\hline \multirow[t]{6}{*}{ NGC 6705} & $\mathrm{I}^{\prime}$ & 0.862 & 0.306 & 15.7 & 11.3 \\
\hline & & \pm 0.049 & \pm 0.001 & \pm 0.9 & \pm 0.7 \\
\hline & $\overline{\Pi^{\prime}}$ & 3.07 & 0.15158 & 1.82 & 97.7 \\
\hline & & \pm 0.25 & \pm 0.000006 & \pm 0.15 & \pm 8.1 \\
\hline & III & 82.6 & 0.00041 & 83.420 & 2.13102 \\
\hline & & \pm 2.3 & $\pm 0.3 \times 10^{-5}$ & \pm 0.003 & \pm 0.00008 \\
\hline \multirow[t]{6}{*}{ NGC 6819} & $\mathrm{I}^{\prime}$ & 0.851 & 0.026 & 18.2 & 11.6 \\
\hline & & \pm 0.047 & \pm 0.001 & \pm 1.0 & \pm 0.6 \\
\hline & $\overline{I I}^{\prime}$ & 3.2 & 0.12722 & 2.1 & 100.9 \\
\hline & & \pm 0.3 & \pm 0.000004 & \pm 0.2 & \pm 9.6 \\
\hline & III & 80.2 & 0.00035 & 99.399 & 2.13088 \\
\hline & & \pm 1.5 & $\pm 0.2 \times 10^{-5}$ & \pm 0.001 & \pm 0.00002 \\
\hline \multirow[t]{6}{*}{ NGC 7654} & $\mathrm{I}^{\prime}$ & 0.852 & 0.022 & 21.46 & 11.6 \\
\hline & & \pm 0.047 & \pm 0.001 & \pm 1.19 & \pm 0.6 \\
\hline & $\overline{\mathrm{II}^{\prime}}$ & 3.2 & 0.10848 & 2.5 & 99.4 \\
\hline & & \pm 0.3 & \pm 0.000004 & \pm 0.2 & \pm 8.0 \\
\hline & III & 80.0 & 0.00030 & 116.568 & 2.13082 \\
\hline & & \pm 2.5 & $\pm 0.2 \times 10^{-5}$ & \pm 0.003 & \pm 0.00005 \\
\hline \multirow[t]{6}{*}{ IC 1848} & $\mathrm{I}^{\prime}$ & 0.829 & 0.0218 & 21.6 & 12.4 \\
\hline & & \pm 0.045 & \pm 0.0008 & \pm 1.2 & \pm 0.7 \\
\hline & $\mathrm{II}^{\prime}$ & 3.5 & 0.10061 & 2.4 & 111.6 \\
\hline & & \pm 0.3 & \pm 0.000002 & \pm 0.2 & \pm 9.3 \\
\hline & III & $\overline{77.0}$ & 0.00028 & 125.696 & \begin{tabular}{|l|}
2.13072 \\
\end{tabular} \\
\hline & & \pm 1.5 & $\pm 0.2 \times 10^{-5}$ & \pm 0.002 & \pm 0.00003 \\
\hline
\end{tabular}


the Galaxy center obtained by Danilov (2010) assuming the planar circular orbit of the cluster. Errors $\sigma_{Q}, \sigma_{\gamma_{2_{0}}}, \sigma_{P_{\gamma_{0}}}, \sigma_{\gamma_{1_{0}} / \omega}$ of $Q, \gamma_{2_{0}}, P_{\gamma_{0}}, \gamma_{1_{0}} / \omega$ given in Tables 9.5 and 9.6 are caused by the errors in evaluation of the cluster mass $M_{c}$ (related to the errors in the number of cluster stars $N_{c}$ and in the mean mass of a cluster star, see Danilov and Seleznev (1994)), as well as by the errors in a defining the position of the maxima of the resonance curves and the boundaries of the instability regions (related to the step value $\Delta \varepsilon$, used with a studying the region $E_{t}$, see above). Probably, small values of $\sigma_{Q}, \sigma_{\gamma_{2_{0}}}, \sigma_{P_{\gamma_{0}}}, \sigma_{\gamma_{1_{0}} / \omega}$ in Table 9.3 are due to a weak dependency of the parameters $Q, \gamma_{2_{0}}, P_{\gamma_{0}}, \gamma_{1_{0}} / \omega$ on the sources of errors mentioned above.

According to Tables 9.5 and 9.6, for all six clusters, the $Q$ values for region I approximately equal $Q_{I} \simeq 0.276$, and for region $\mathrm{I}^{\prime}$, the $Q$ values are $Q_{I^{\prime}} \simeq$ $0.829-0.917$. With a decrease of the density $\rho$ of the cluster core in region II' $^{\prime}$, the $Q$ values reach $Q_{I I^{\prime}} \simeq 2.6-3.5$, and in region II, the $Q$ values are $Q_{I I} \simeq 74.7-86.8$. With a decreasing density $\rho$ in region III, the $Q$ values are $Q_{I I I} \simeq 77.0-96.7$.

All the considered clusters are located in regions I, $\mathrm{I}^{\prime}$ with small $Q$ values, which indicates a low $\mathrm{Q}$ factor (goodness) of such systems. In this case, the rate of the energy loss of the system's oscillations (see, for example, Strelkov $(2005$, p.312,175)) due to the relaxation mechanisms must be large, and for a maintaining of the oscillations, the sufficiently large growth rates $\gamma_{2}$ of the instability increase are needed. Indeed, the relaxation times $\tau_{\varepsilon}$ in models 1-6, obtained by Danilov and Dorogavtseva (2003) from data on the star fluxes in the space of an energy $\varepsilon$, are large enough, and the relation $\tau_{\varepsilon}>t_{\lambda}(1)$ is fulfilled (see Table 9.4 of this work and Table 2 from Danilov and Dorogavtseva (2003); the values of $\tau_{v r}$ can be found, for example, in Table 1 from Danilov and Dorogavtseva (2008)). According to the estimates of Danilov and Dorogavtseva (2003) and of this work for models $1-6$, we find $\tau_{\varepsilon} / t_{\lambda}(1) \sim 2.0-4.8$ at $t=t_{2}$ and $\tau_{\varepsilon} / t_{\lambda}(1) \sim 2.0-3.2$ at $t=t_{3}$. Consequently, the values of $\gamma_{2}$ for models $1-6$ are sufficiently large in comparison with the velocity $\gamma_{\varepsilon}$ of the system relaxation in the space of $\varepsilon\left(\gamma_{\varepsilon} \sim 1 / \tau_{\varepsilon}\right)$.

According to Table 2 from Danilov and Dorogavtseva (2003) and 9.4 for models $1-6$, the following relations are satisfied: $t_{\lambda}(1)>\tau_{v r}, t_{\lambda}(1)>\tau_{v}$. In the case of the OSC models 1 and $2, t_{\lambda}(1) \lesssim \tau_{r}$, and for models $3-6$, the relation $t_{\lambda}(1)>\tau_{r}$ is fulfilled. Here, $\tau_{v}$ and $\tau_{r}$ are the relaxation times of models $1-6$ obtained by Danilov and Dorogavtseva (2003) from the data on the stellar fluxes in the spaces of $v$ (the stellar velocity moduli) and distances $r$ of the star from the mass center of the cluster, respectively. $\tau_{v r}, \tau_{v}, \tau_{r}$ determine the time of the system's transition to a virial equilibrium in the absence of the conditions for a gravitational instability in it. Relations between $t_{\lambda}(1)$ and $\tau_{v r}, \tau_{v}, \tau_{r}$ indicate a fairly rapid loss of the oscillation energy due to the relaxation processes in the OSCs and their models.

Thus, the estimates of $Q_{I}$ and $Q_{I^{\prime}}$ considered here correspond to systems with low $\mathrm{Q}$ factor. Since $Q_{I^{\prime}}>Q_{I}$, the $\mathrm{Q}$ factor for a subsystem of stars of a core with the higher energies increases, and the relative losses of an oscillation energy under the action of relaxation mechanisms decrease (the faster the core star moves, the weaker it interacts with an alternating force field of the system).

According to Tables 9.5 and 9.6, for very narrow regions II, II' at small $\rho$, we find $Q_{I I} \gg Q_{I I^{\prime}}$. In this case, $C_{k}=1 \mathrm{pc}$ (and the star energy $\varepsilon$ ) used in the calculations 
may be insufficient to describe the dynamics of a stellar subsystem reaching the boundary of the cluster core. Probably, for such stars and such systems, it is necessary to use $C_{k}$, which are comparable with the average cluster radius. In this case, we can expect the characteristic values of $Q<Q_{I I^{\prime}}\left(\right.$ or $\left.Q \ll Q_{I I^{\prime}}\right)$. In region III, in the systems with a high density of cores $\rho$, the values of $C_{k}=1 \mathrm{pc}$ used in calculations can be overestimated (with an increase of $\rho$ the cluster mass remains constant, and the core dimensions decrease, see Danilov (2010)). Unfortunately, in a case of $C_{k}=0$, for large values of $\rho$, an instability region could not be detected. Probably, the region of the unstable oscillations in this case is formed for $C_{k}=(0.1-0.5) \mathrm{pc}$.

We note that the resonance curves in fig. 9.4c, d characterize not only star cluster NGC 6705, but also the force field of the Galaxy. Fig. 9.5 shows the dependencies of $\gamma_{2_{0}}$ on the distance $R_{G}$ of the clusters from the center of the Galaxy, obtained from data on the clusters considered here. The values of $R_{G}$ (in kpc) are taken here according to Danilov and Seleznev (1994). The curves in fig. 9.5 are labeled with the numbers of instability regions for which these curves are constructed. It is easy to see that the values of $\gamma_{2_{0}}$ decrease with increasing distance $R_{G}$. Thus, the presence of an external field of the Galaxy is an essential factor in the development of an instability in the centers of the OSCs.
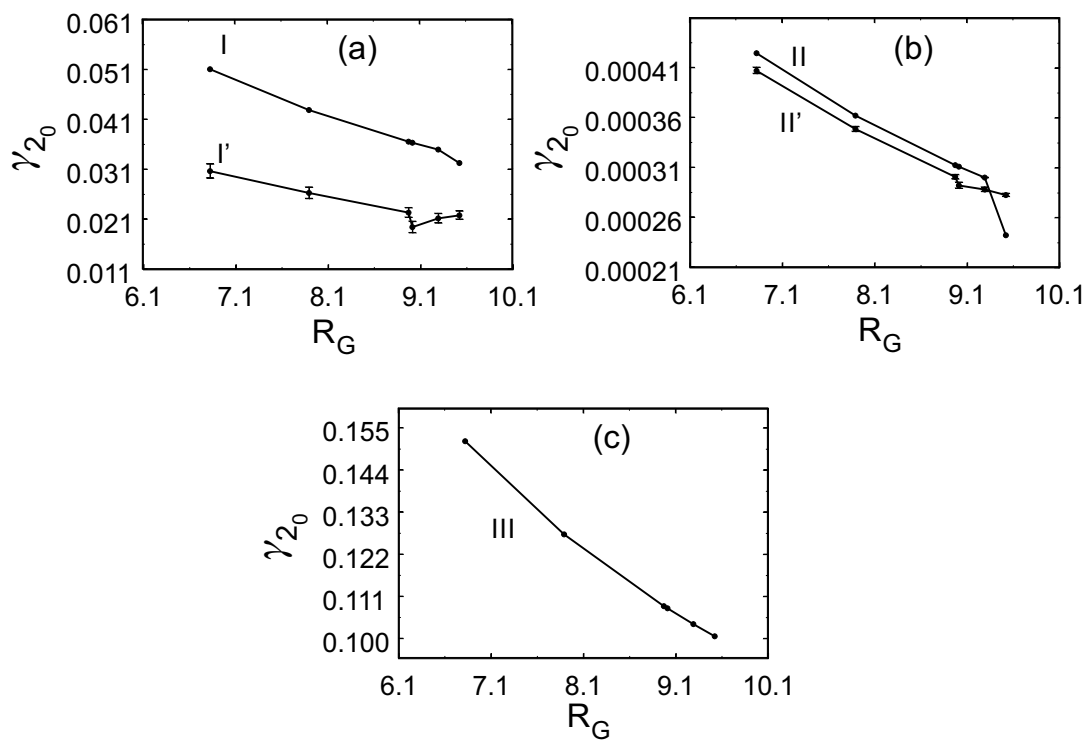

Fig. 9.5 Dependencies of $\gamma_{2_{0}}$ on $R_{G}$

As $R_{G}$ increases, the periods $P_{\gamma_{0}}$ increase on average, and the oscillation frequencies $\gamma_{1_{0}}$ corresponding to these periods decrease on average. Figs. 9.6a,b show the dependencies of $P_{i}^{-1}-P_{j}^{-1}$ on $R_{G}$. The numbers of the corresponding instability regions of the PD oscillations are used as $i$ indices of $P_{i}=P_{\gamma_{0}}$. According to fig. 

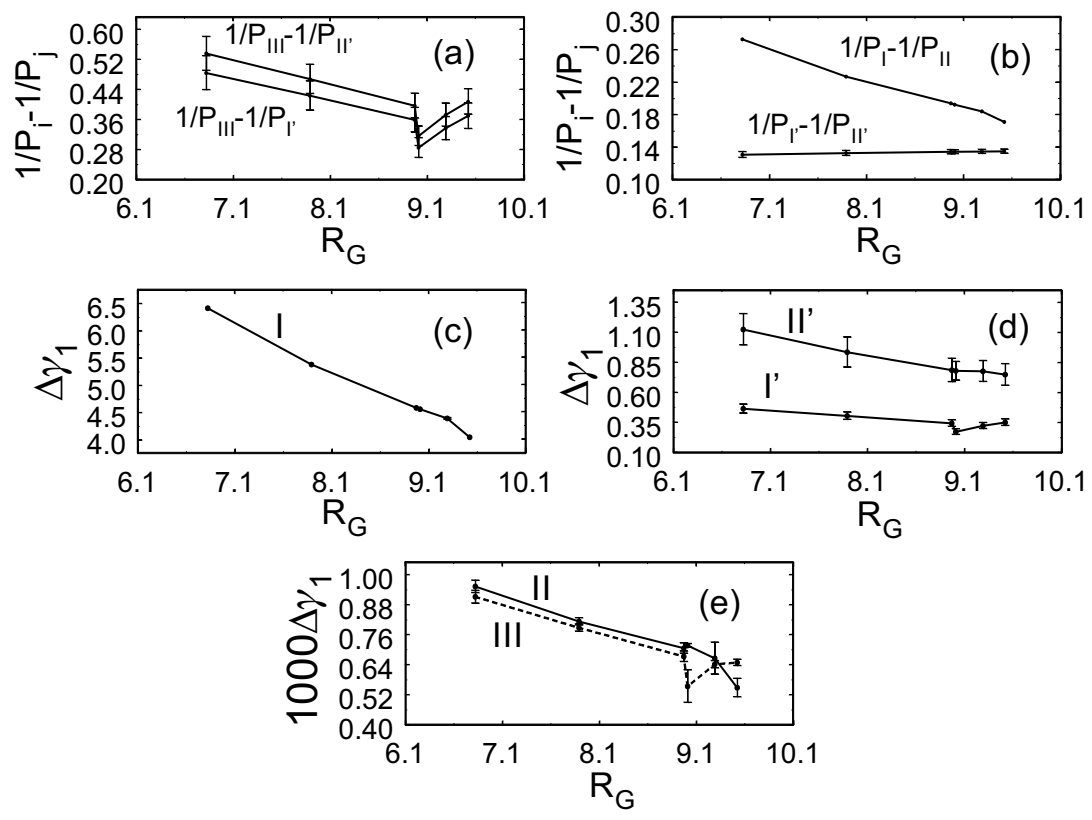

Fig. 9.6 Dependencies of $P_{i}^{-1}-P_{j}^{-1}$ and $\Delta \gamma_{1}$ on $R_{G}$

9.6, $P_{i}^{-1}-P_{j}^{-1}$ and $\Delta \gamma_{1}=2 \pi /\left(Q P_{\gamma_{0}}\right)$ decrease on average with increasing $R_{G}$ in all cases, except for the dependency of $P_{I^{\prime}}^{-1}-P_{I I^{\prime}}^{-1}$ on $R_{G}$ (see fig. 9.6b). In this case, the values $P_{I^{\prime}}^{-1}-P_{I I^{\prime}}^{-1}$ are observed to be constant or weakly growing as $R_{G}$ increases. In figs. 9.6c,d,e, the dependencies of the width of the instability region $\Delta \gamma_{1}$ on $R_{G}$ are marked by the numbers of these regions. Thus, as the distance from the center of the Galaxy increases, the maxima of the resonance curves and the instability regions on the plots shown in fig. 9.4 approach each other in frequency. If we consider the plots in fig. 9.4c,d as the spectra of a certain signal (Strelkov, 2005, p.154) formed with the participation of the Galaxy force field in the cluster core (by creating the conditions for the formation of a PD oscillation instability in the cluster core), then the amplitude of such a signal decreases, and the width of the spectrum decreases with an increasing distance of the cluster from the center of the Galaxy.

The frequencies $\gamma_{1_{0}}$ of the resonance curves maxima in regions II and III (for $C_{k}=0$ and $C_{k}=1 \mathrm{pc}$, respectively) practically coincide each other (see Table 9.5 and 9.6) for every considered clusters. Therefore, when constructing the resonance curves on the basis of the numerical experiments on the integration of the equations of a stellar motion in the OSC, these curves (spectra) for subsystems of stars with the different energies will be superimposed on each other, which will complicate the interpretation of such curves.

According to Tables 9.5 and 9.6 , the values $\gamma_{1_{0}} / \omega$ within the limits of the indicated errors can be completely represented as integers or rational numbers. Therefore, the 
resonances between the frequency $\omega$ and the frequencies of the PD oscillations in the centers of the considered OSCs for the stellar subsystems with the different energies $\varepsilon$ can play an important role in the formation of the instability regions.

\subsection{Conclusions}

1. We have performed an analysis of the stellar velocity dispersions in the OSC models. The central regions of these models are rather "cold" (or more "cold" than the cluster as a whole), which leads to a gravitational instability in the cores of the OSCs and their models noted by Danilov (2008) and Danilov (2010). An external field of the Galaxy not only "heats up" the outer parts of the cluster, but also largely prevents an entry of the high-energy stars to the cluster center (due to a non-radiality of the cluster stars' orbits in the presence of the Galactic field).

2. In the OSC models, a temporary virialization have been noted, when a significant part of a cluster oscillation energy temporarily transfers into the kinetic energy of the peculiar stellar motions. The duration of this stage can reach $\sim 10^{8}$ years. The main cause of a temporary OSC virialization is a "heating" of the cluster core by the energy flow from the periphery to the center of the cluster (Danilov, 2002b) caused by the large-scale cluster oscillations at the previous stage of evolution. During the stage of a temporal virialization, a gravitational instability in the cluster cores is largely suppressed. A further "cooling" of the OSC core caused by the expansion of the core and the cluster as a whole again leads to the development of a gravitational instability near the center and to an increase of the amplitude of the cluster oscillations.

3. We have considered the natural oscillations of the phase density in the centers of six OSCs and six OSC models. Instability of such oscillations in these objects is shown. In addition to the areas of the unstable PD oscillations indicated by Danilov (2010), several new regions have been noted (see above). We have revealed the possibility of the motion of the OSCs and their models towards the state of a stable equilibrium, both with the decreasing and increasing density of the cluster core. If the effects of the core "heating" by the regular field oscillations and by the tidal Galactic field dominate in the cluster, then the cluster evolves to the state of a stable equilibrium with a decrease in the core density. If the encounters of single stars dominates in the cluster, then the cluster evolves to the state of a stable equilibrium with the increasing core density. In the considered time intervals $\Delta t \simeq(1-2) \tau_{v r}$, on average (relative to the period of regular field oscillations), the central densities in the OSC models decrease in comparison with an average cluster core density.

4. We have considered the structure of the PD oscillation instability regions in the centers of six OSCs. We have performed the resonance curves obtained under the assumption that the amplitude of the steady-state PD oscillations at the cluster center is directly proportional to the growth rate (an increment) of the instability $\gamma_{2}$ of such oscillations. In order to describe the instability regions in the OSCs, we introduce a $Q$ coefficient, similar to $\mathrm{Q}$ factor in the simplest mechanical systems. 
In regions I and I', for the considered OSCs, $Q$ equals $Q \simeq 0.276-0.917$, which indicates the significant rates of loss of the OSC oscillation energy under the action of relaxation processes. With an increase of the cluster distance from the center of the Galaxy, the largest increment $\gamma_{2_{0}}$ and the width $\Delta \gamma_{1}$ of the instability region decrease on average for all regions of the unstable oscillations. An important role in the formation of the instability regions can be played by the resonances between an

$\omega$ frequency of the orbital cluster motion in the Galaxy and the frequencies of the natural PD oscillations at the centers of the analyzed OSCs.

\section{References}

Aarseth, S.J.: Dynamical evolution of simulated star clusters. I - Isolated models. Astron. Astrophys. 35, 237-250 (1974).

Chandrasekhar, S.: Principles of Stellar Dynamics. Univ. Chicago Press, Chicago, Ill (1942). LCCN: 43-5608 (BKS1)

Danilov, V.M.: Late stages in the evolution of open star clusters. Astrophysics, 13, 406-412 (1977). doi:10.1007/BF01006618

Danilov, V.M.: Dynamics of close binaries and single stars in open clusters. Astrophysics, 14, 19-30 (1978). doi:10.1007/BF01005359

Danilov, V.M.: Statistical analysis of dynamical open-cluster models with small differences in their initial stellar phase coordinates. Astron. Letters, 23, 322-326 (1997b).

Danilov, V.M.: Stellar Fluxes in Numerical Dynamical Models of Open Clusters. Astron. Reports 46, 887-899 (2002b). doi:10.1134/1.1522077

Danilov, V.M.: Dynamical models of stellar motions at the peripheries of open clusters. Astron. Reports 50, 346-357 (2006). doi:10.1134/S1063772906050027

Danilov, V.M.: Analysis of density fluctuations in models of open clusters. Astron. Reports 52, 888-899 (2008). doi:10.1134/S1063772908110036

Danilov, V.M.: Phase-Density Fluctuations at the Centers of Six Open Clusters. Astron. Reports 54, 514-527 (2010). doi:10.1134/S1063772910060053

Danilov, V.M.: On the dynamics of open clusters, Astron. Reports 55, 473-486 (2011). doi:10.1134/S1063772911060035

Danilov, V.M., Dorogavtseva, L.V.: Estimates of Relaxation Times in Numerical Dynamical Models of Open Star Clusters. Astron. Reports 47, 483-491 (2003). doi:10.1134/1.1583775

Danilov, V.M., Dorogavtseva, L.V.: Timescales for mechanisms for the dynamical evolution of open star clusters. Astron. Reports 52, 467-478 (2008). doi:10.1134/S1063772908060048

Danilov, V.M., Seleznev, A.F.: The catalogue of structural and dynamical characteristics of $103 \mathrm{OCl}$ and the first results of its investigations. Astron. Astrophys. Trans. 6, 85-156 (1994). doi:10.1080/10556799408232061 
Geller, A.M., Mathieu, R.D., Harris, H.C. et al.: WIYN Open Cluster Study. XXXII. Stellar Radial Velocities in the Old Open Cluster NGC 188. Astron. J. 135, $2264-$ 2278 (2008). doi:10.1088/0004-6256/135/6/2264

Heggie, D.C., Ramamani, N.: Approximate self-consistent models for tidally trancated star clusters. Non. Not. R. Astron. Soc. 272, 317-322 (1995). doi:10.1093/mnras/272.2.317

King, I.R.: The structure of star clusters. I. An empirical density law. Astron. J. 67, 471-485 (1962). doi:10.1086/108756

King, I.R.: The structure of star clusters. III. Some simple dynamical model. Astron.J. 71, 64-75 (1966). doi:10.1086/109857

Kutuzov, S.A., Osipkov, L.P.: A Generalized Model for the Three-Dimensional Gravitational Potential of Stellar Systems. Soviet Astron. 24, 17-22 (1980).

Sagar, R., Bhatt, H.C.: Internal kinematics of open star clusters. Mon. Not. R. Astron. Soc. 236, 865-874 (1989). doi:10.1093/mnras/236.4.865

Strelkov, S.P.: An introduction to oscillation theory. Lan', St.Petersburg (2005) (In Russian). 


\title{
Chapter 10 \\ Parameters of Nonstationarity of Open Star Clusters
}

\begin{abstract}
In this chapter, we consider the estimates of a number of dynamic parameters of 103 open star clusters (contrast of densities in the cluster core; stellar velocity dispersion obtained with an allowance for the effect of the external field of the Galaxy and the cluster non-stationarity on the cluster; the oscillation periods of the cluster and the cluster core, etc.). The analytic solutions of the equations of a gross dynamics are given for the simple cluster models. These solutions are used to estimate a number of values characterizing the degree of the cluster non-stationarity (an oscillation amplitude of the virial cluster coefficient, an oscillation amplitude of the cluster core radius, etc.). The astrophysical applications of the obtained results are discussed.
\end{abstract}

\subsection{Introduction}

At the present time, a number of features of the OSC structure is known, which indicate the non-stationary nature of these clusters (an irregular shape of the cores that does not correspond to the equilibrium state of the OSCs, a presence of the stepped structures in the radial density profiles (Barkhatova (1956); Seleznev (1988), Kholopov (1981, p.328)), etc.). Formation of the stepped structures in the radial density profiles of the non-stationary OSC models is noted by Danilov (1984); Danilov and Ryazanov (1985) using two different methods of studying the dynamic evolution of the OSCs. Estimates of the degree of the OSC non-stationarity from the data on the structural parameters of the OSCs had been fulfilled by Danilov and Seleznev (1994). In the OSC models of Danilov and Dorogavtseva (2008), the large-scale oscillations of the density and a regular field develop spontaneously and rapidly. Estimates of the gravitational instability parameters of the OSCs and their models were performed by Danilov (2008) and Danilov (2010). Development of an instability in the OSC models is used by Danilov and Dorogavtseva (2008) and Danilov (2008) to explain the oscillations in the values of the virial coefficient and the dispersions of the cluster stars' velocities that do not decay during the large time 
intervals. A theoretical description of the phase density oscillations in the central parts of the numerical dynamic OSC models was performed by Danilov (2008). In the paper of Danilov (2010), the formulas obtained by Danilov (2008) are used to study the phase density (PD) oscillations in the centers of six observed OSC and the OSC models 1, 6 of Danilov and Dorogavtseva (2008). Danilov (2010) has shown that the PD oscillations are unstable at the the centers of all considered objects (the OSCs and their models). According to Danilov (2011), an important kinematic sign of the OSC non-stationarity is an increase of the dispersion of the stellar velocities with a distance from the cluster center. However, the clusters, in which stellar velocities decrease slightly with distance from the cluster center, are also non-stationary, but with a lesser degree of a non-stationarity and with a slower rate of a dynamic evolution (Danilov, 2011) (the degree of the cluster instability is characterized here by the amplitude $\delta \alpha$ of the virial coefficient oscillations $\alpha=2 E / W$, where $E=T+W ; T$ is a kinetic energy of the stellar motions in the cluster, $W$ is a potential cluster energy).

We denote by $v=\rho(0) / \rho_{c}$ the ratio of the density of star number $\rho(0)$ at the cluster center to the average density of star number $\rho_{c}$ of the cluster core (hereinafter, for brevity, $v$ will be called as a density contrast in the cluster core). According to Danilov (2011), the small $v$ values in the cluster cores are formed due to the dynamic evolution of the numerical OSC models. The small $v$ values indicate the effect of an instability and a non-stationarity on the radial profile of the OSC density. An increase in the dispersion of $v$ with the increasing amplitude $\delta \alpha$ of oscillations of the values of the virial coefficient $\alpha$ in the sample of the OSCs is also a sign of the clusters' non-stationarity.

We denote, as in the paper of Danilov and Seleznev (1994), $\xi=R_{1} / R_{2}, \mu=$ $N_{1} / N_{2}$, where $R_{1}$ and $R_{2}$ are the radii of the core and the halo, respectively (the halo radius equals the cluster radius); $N_{1}$ and $N_{2}$ are the numbers of stars in the core and in the halo, respectively. Standard deviations $\sigma_{R_{1}}$ of $R_{1}$ from the mean $R_{1}$ (averaged over the oscillation period of a regular cluster field) can be used as estimates of $\delta R_{1}$ being the oscillation amplitude of the $R_{1}$ value. In the OSC models of Danilov and Dorogavtseva (2008), the values of $\sigma_{R_{1}}$ vary from $\sigma_{R_{1}} \simeq(0.25 \pm 0.01) R_{1}$ in model 1 with the greatest degree of non-stationarity to $\sigma_{R_{1}} \simeq(0.17 \pm 0.01) R_{1}$ in model 6 with the least degree of non-stationarity. Oscillations of the OSC cores with such amplitudes can quite affect the width $\Delta \xi(\mu)=\xi_{\max }(\mu)-\xi_{\min }(\mu)$ of the band occupied by the clusters in the diagram $(\xi, \mu)$, see Danilov and Seleznev (1994), fig. 21 (or fig. $10.3 \mathrm{c}$ of the present work), and $\Delta \xi(\mu)$ can also determine the degree of the OSC non-stationarity with a given $\mu$. Here, $\xi_{\max }(\mu)$ and $\xi_{\min }(\mu)$ are the maximum and minimum values of $\xi$ at a given $\mu$.

Estimates of $\delta \alpha=\delta \alpha_{t h}$ of Danilov and Seleznev (1994) were based on the assumption that the oscillations of the regular field of the OSC are caused by the action of the stellar encounters and the thermal fluctuations of the OSC density. The formula for $\delta \alpha_{t h}$ in Danilov and Seleznev (1994) was obtained considering the data of the numerical experiments on the dynamic evolution of the OSC. However, $\delta \alpha_{t h}$ in these numerical experiments was determined by the stars with the distances $r \leqslant R_{t}$ from the cluster center, where $R_{t}$ is the tidal cluster radius obtained according to King (1962). We note that several clusters from the sample of Danilov and Seleznev 
(1994) have radii $R_{2}>R_{t}$. For them, the very large values of $\delta \alpha_{t h}$ were obtained. Apparently, in such cases, it is better to determine $\delta \alpha_{t h}$ values only for a system of stars with $r \leqslant R_{t}$. It is also of interest to redefine the degree of non-stationarity $\delta \alpha$ for the OSCs of a sample of Danilov and Seleznev (1994) applying other methods (for example, within a gross dynamic description) and considering the results of Danilov (2008) and Danilov (2010) on the development of a gravitational instability in the OSC cores.

It is of interest to estimate the following values: periods $P_{1}$ and $P_{2}$ of the $R_{1}$ and $R_{2}$ oscillations for the OSCs of a sample of Danilov and Seleznev (1994); the violent relaxation times $\tau_{v r}$ of these clusters; the amplitudes $\delta R_{1}$ of the oscillations of the radii of the cores; equilibrium values $\alpha_{0}$ of a virial coefficient (with respect to which the $\alpha$ oscillations with the $\delta \alpha$ amplitude occur). The above parameters characterize in more detail the non-stationarity and the oscillations of the force field in the OSCs in comparison with the data of Danilov and Seleznev (1994) only on $\delta \alpha_{t h}$. The values of $\delta R_{1}, \delta R_{1} / R_{1}, \alpha_{0}, \delta \alpha / \alpha_{0}$ have not been previously determined for the OSCs. The $\tau_{v r}$ values for the OSCs also have not been determined by Danilov and Seleznev (1994).

During the study of the numerical dynamic OSC models 1-6 of Danilov and Dorogavtseva (2008) in Danilov (2010), formula (13) was obtained to estimate the dispersion $\sigma_{v}^{2}$ of the stellar velocities in the OSCs, considering the influence of a non-stationarity and of the external force field of the Galaxy on the cluster. Since the $\sigma_{v}^{2}$ values are often used for the virial estimates of the OSC masses without taking into account the effect of the Galactic force field on the cluster (see, for example, Geller et al. (2008); de Grijs et al. (2008)), it is of interest to find how different are the estimates of $\sigma_{v}^{2}$ (and of the dynamic masses of the OSCs) obtained with and without considering the influence of the cluster non-stationarity and of the Galactic force field on the OSC. For these purposes, it is convenient to use formula (13) from Danilov (2010) and the data on the OSCs from the sample of Danilov and Seleznev (1994).

The objectives of this chapter are: 1$)$ to consider a number of parameters $(v, \delta \alpha$, $\tau_{v r}, P_{R_{1,2}}, \delta R_{1} / R_{1}, \alpha_{0}, \delta \alpha / \alpha_{0}$, etc.) characterizing the non-stationarity of the OSCs from Danilov and Seleznev (1994); 2) to discuss the values of these parameters and the OSC distributions in the spaces of the indicated parameters in order to reveal new signs of the clusters' non-stationarity according to the data on the OSC structure; 3 ) to analyze the influence of the OSC non-stationarity and of the external force field of the Galaxy on the estimates of the dynamic masses of the OSCs; 4) to discuss the solutions of the gross dynamics equations of the non-isolated OSC models; to apply these solutions to the analysis of the OSC non-stationarity.

\subsection{Contrast of Densities in the OSC Cores}

In order to estimate the $v$ values, we use the King distribution $f\left(r, k, r_{c}, R_{2}\right)$ for the spatial density of the number of stars at the distance $r$ from the cluster center, see 


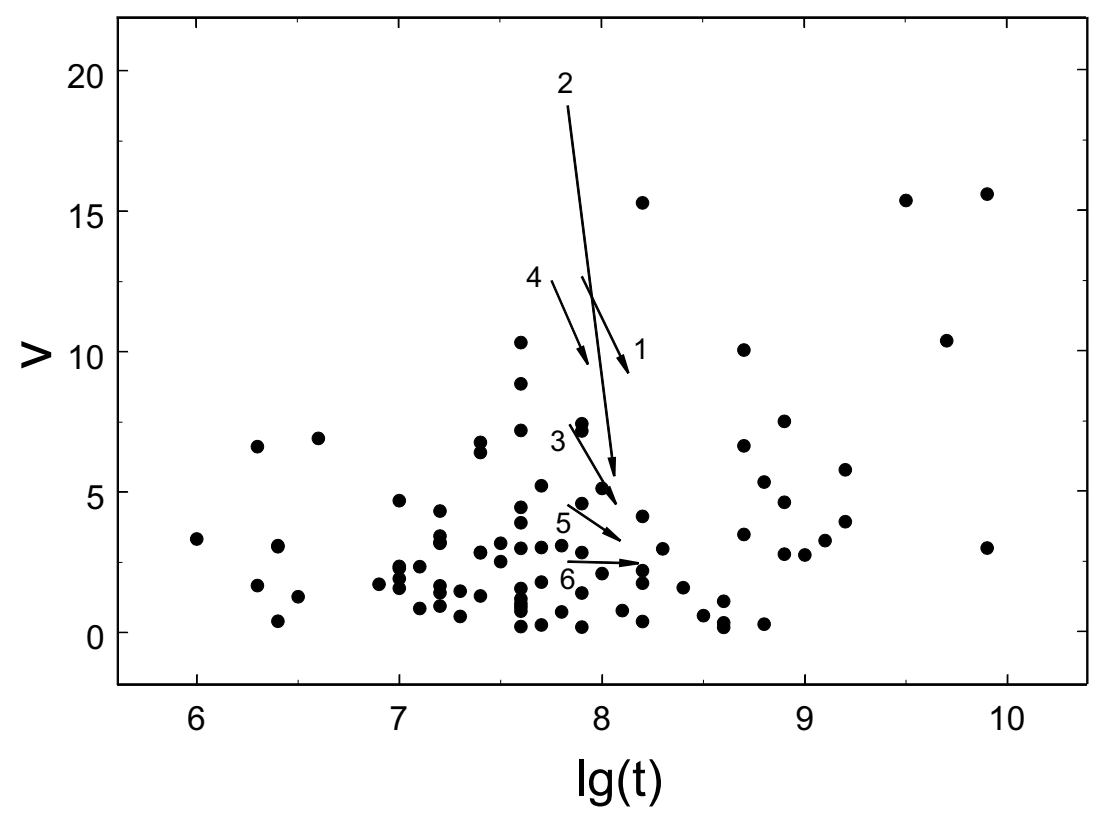

Fig. 10.1 Positions of the OSCs and the OSC models 1-6 of Danilov and Dorogavtseva (2008) in coordinates $(v, \lg (t))$

King (1962), formula (27) (or Kholopov (1981), formula (8.41)). Here, $k, r_{c}, R_{2}$ are the King distribution parameters of an apparent density of the cluster star number. $\rho(0)=f\left(0, k, r_{c}, R_{2}\right)$. Since the cluster star number $N_{c}=N_{1}+N_{2}$, and $N_{1}=\mu N_{2}$, $R_{1}=\xi R_{2}, \rho_{c}=3 N_{1} /\left(4 \pi R_{1}^{3}\right)$, then

$$
\rho_{c}=3 \mu N_{c} /\left(4 \pi(1+\mu) \xi^{3} R_{2}^{3}\right) .
$$

Consequently,

$$
v=4 \pi(1+\mu) \xi^{3} R_{2}^{3} f\left(0, k, r_{c}, R_{2}\right) /\left(3 \mu N_{c}\right) .
$$

Formula (10.1) was used by Danilov and Putkov (2012a) to obtain the estimates of $v$ for 89 OSCs from Danilov and Seleznev (1994). When calculating $v$, the values of $\xi, \mu, N_{c}, R_{2}$ were used, as well as the parameters $k, r_{c}$ of a King distribution all from Danilov and Seleznev (1994). The results of the $v$ calculation by Danilov and Putkov (2012a) for the OSCs and six cluster models of Danilov (2011) are shown in fig. 10.1 depending on the decimal logarithm of the cluster age $t$ ( $t$ values are taken here according to Danilov and Seleznev (1994)). The $v$ values for the cluster models were determined by Danilov (2011) for two time points $t_{1}$ and $t_{2}$, therefore in fig. 10.1 , they are shown as arrows, the beginning and end of which correspond to the time points $t_{1}$ and $t_{2}$, respectively; direction of the arrow indicates the direction from $t_{1}$ to $t_{2}\left(t_{1}<t_{2}\right)$. The numbers near the arrows in fig. 10.1 indicate the numbers of the cluster models of Danilov and Dorogavtseva (2008). 
Fig. 10.1 does not show the positions of three clusters NGC 2169, NGC 2194, NGC 7235 from the sample of Danilov and Seleznev (1994) with the largest values $v \gtrsim 30$. The values $v$ for these OSCs are more than $3 \sigma_{v}$ different from the mean $v$, which is $\bar{v}=7.19 \pm 0.80\left(\sigma_{v} \simeq 7.55\right.$ is a standard deviation of $v$ from $\left.\bar{v}\right)$. Without considering these three clusters, the mean $v$ for OSCs from the sample of Danilov and Seleznev (1994) is $\bar{v}=3.62 \pm 0.36\left(\sigma_{v} \simeq 3.34\right)$. These estimates of $\bar{v}$ and $\sigma_{v}$ were obtained under the assumption of an equal accuracy of $v$ in the considered OSCs. To estimate the $v$ errors, we used the data on $\frac{\partial v}{\partial \mathbf{x}}$, where $\mathbf{x}=\left(R_{1}, N_{1}, k, r_{c}, R_{2}\right)$ is the vector of the parameters entering into the expression for $v$. We used the data on the errors of $N_{c}, R_{2}, k, r_{c}$ given by Danilov and Seleznev (1994). $\xi$ and $\mu$ errors were assumed equal to 0.05 and $0.37 \mu^{0.65}$, respectively (Danilov and Seleznev, 1989). As a result, the large enough errors $\sigma(v)$ were obtained for the $v$ values. The mean $\sigma(v) / v$ over the sample of 86 OSCs are $\overline{\sigma(v) / v}=1.00 \pm 0.05$ (three clusters mentioned above were not taken into account here). If we neglect the estimates with $\sigma(v) / v>1$, then for the remaining 52 OSCs we find $\bar{v}=3.92 \pm 0.51, \sigma_{v} \simeq 3.64$, $\overline{\sigma(v) / v}=0.71 \pm 0.02$. We note that the values of $\bar{v}$ obtained here are not statistically different. $\sigma(v)$ and $v$ correlate fairly closely $(v \sim \sigma(v)$, since the OSCs with a small number of stars in Danilov and Seleznev (1994) appear to have smaller errors of $R_{2}$ and $N_{c}$ than the clusters with the medium and large values of the indicated parameters). Therefore, the calculation of the weighted mean values of $v$ is not given. The total contribution of the $N_{1}$ and $R_{1}$ errors to $\sigma(v)$ on average is $\sim 56 \%$ of $\sigma(v)$.

Thus, $\bar{v}$ values are small enough and are comparable to the $v$ values for the OSC models 5, 6 of Danilov and Dorogavtseva (2008). Small $v$ for most of the considered OSCs can be caused by the following reasons: 1) OSCs are formed with small $v$, and then the $v$ values are kept small due to the gravitational instability of the clusters' cores and a non-stationarity of the OSCs;2) at $t<10^{6}$ years, OSCs are formed both with large and small $v$, and then, under the action of the regular-field oscillations, the radial density profiles of the OSCs evolve towards the profiles with the small $v$ (as in the cluster models 1-5 of Danilov and Dorogavtseva (2008)). If, then, at $t>10^{9}$ years (fig. 10.1) the $v$ values in some clusters begin to increase with increasing $t$, then in the dynamic evolution of such clusters, the role of stellar encounters increases, and the radial density profiles in such OSCs begin to resemble the density profiles of the globular clusters.

Fig. 10.2 for the same OSCs, as in fig. 10.1, shows the $v$ depending on $\delta \alpha$, which characterizes the degree of a cluster non-stationarity in the gross-dynamic description of the cluster (see below). According to fig. 10.2, the maximum $v$ values and a dispersion of $v$ at a given $\delta \alpha$ grow with increasing $\delta \alpha$. These dependencies can be due to the following reasons: 1) the OSC oscillations at a given $\delta \alpha$ have a non-homological character (in this case, the density distribution by a distance from the cluster center at different times can not be considered as similar; we observe the clusters with the different phases of the radial oscillations); 2) during a dynamic evolution of the OSCs, their positions in fig. 10.2 are gradually shifted towards the small $\delta \alpha$ (as in the OSC models of Danilov (2011)). In this case, the cluster approaches the state of a stable equilibrium. 
We note that taking into account the influence of the less bright stars with the stellar magnitudes $m_{B}>16^{m}$ exceeding the limiting magnitude of stars considered in Danilov and Seleznev (1994) can not significantly change $v$ for the OSCs considered here, since usually the stars of the small masses (and luminosities) are located at the cluster periphery.

\subsection{Homologous Oscillations of the OSC Models}

Following to Chandrasekhar (1942), we consider the cluster moving in the Galactic plane along a circular orbit at the distance $R_{G}$ from the Galactic center with an angular velocity $\omega=$ const. We write the equations of a stellar motion in the rotating coordinate system $(x, y, z)$ and use the expansion of the Galactic regular potential in a series up to quadratic terms with respect to $x, y, z$ coordinates. The $x, y, z$ axes used in our work coincide with $\xi, \eta, \zeta$ axes from Chandrasekhar (1942, fig.24, p.222). We make use of the gross-dynamic (GD) description of the OSC evolution in order to estimate $\delta \alpha$ of the clusters and to study the OSC oscillations near the equilibrium state. Within the framework of a GD description, the integral characteristics of the cluster as a whole are usually considered (Kuzmin, 1965). Taking into account the equations of a stellar motion in the field of the forces of the Galaxy and the cluster, it is easy to obtain the equations for the momentum of the cluster inertia $I$, the kinetic energy $T$ of the cluster star motion, and the angular momentum $L_{z}$ of the cluster rotation around the $z$ axis which is perpendicular to the Galactic plane and passing through the cluster's center of mass (see, for example, formulas (3)-(5) from Danilov (2008)). For a cluster with a spherically symmetric mass distribution, we write

$$
\ddot{I}=4 T+4 \omega L_{z}-\frac{2 q^{2}}{3} I, \quad \dot{T}=-\frac{q^{2}}{6} \dot{I}, \quad \dot{L}_{z}=-\frac{2 \omega}{3} \dot{I},
$$

where $\dot{I}=\frac{d I}{d t}$; the values $\dot{T}, \dot{L}_{z}$ are defined similarly; $\ddot{I}=\frac{d \dot{I}}{d t} ; q^{2}=\alpha_{1}+\alpha_{3}+3 \beta$; $\alpha_{1}, \alpha_{3}$ are the constants characterizing a Galactic force field in the vicinity of the cluster's circular orbit (Chandrasekhar, 1942) (their numerical values are defined in our work using the Galactic potential model of Kutuzov and Osipkov (1980)). $\beta$ characterizes a force field of the cluster, $\frac{\partial U}{\partial r}=-\beta r, U(r)$ is the gravitational potential of the cluster, $r$ is the distance from the cluster's center of mass.

Following to Danilov and Putkov (2012a), we consider the OSC model in the form of a homogeneous gravitating sphere with the mass $M_{c}$ and radius $R_{2}$. In this case, $\beta=G M_{c} / R_{2}^{3}$. The third equation in (10.2) is easily integrated by the time $t$ : $L_{z}=-2 \omega I / 3+L_{z}(0), L_{z}(0)=$ const. Let $L_{z}(0)=0$ (in this case, the cluster does not rotate with respect to external galaxies). We substitute $L_{z}$ to (10.2). We linearize the resulting system by setting $I=I_{0}+\delta I, T=T_{0}+\delta T,|\delta I| \ll I_{0},|\delta T| \ll T_{0}$, where $I_{0}$ and $T_{0}$ are the equilibrium values of $I$ and $T$. As a result of these transformations, we find 


$$
\ddot{\delta} I=4 \delta T-K \delta I, \quad \dot{\delta} T=-\frac{q_{0}^{2}}{6} \dot{\delta} I,
$$

where

$$
K=2\left(4 \omega^{2}+q_{0}^{2}-9 p^{2} /\left(2 I_{0}^{3 / 2}\right)\right) / 3,
$$

$q_{0}^{2}$ is an equilibrium value of $q^{2}, p^{2}=G M_{c}\left(0.6 M_{c}\right)^{3 / 2}$; in this case, $W=-p^{2} / \sqrt{I}$ (a similar approach to the study of the oscillations of the spheroidal stellar systems' models was applied by Ossipkov (2001); see the conditions for the quasi-homology oscillations of a stellar system, as well as references in Ossipkov (2001)).

Characteristic equation of the system (10.3) and its roots has the form

$$
\lambda^{3}+\lambda\left(\frac{2}{3} q_{0}^{2}+K\right)=0, \quad \lambda_{1}=0, \quad \lambda_{2,3}= \pm i \omega_{h}
$$

where $i=\sqrt{-1} ; \omega_{h}=\sqrt{2 q_{0}^{2} / 3+K}$. The positive $\omega_{h}^{2}$ values indicate the stability of the considered oscillations, $\omega_{h}$ is the frequency of such oscillations.

Integrating the second equation of system (10.3), we find

$$
\delta T=-\frac{q_{0}^{2}}{6} \delta I+\delta T(0),
$$

where $\delta T(0)=$ const (it is the $\delta T$ value in the time point $t=0$ ). Let us consider the case $\lambda_{2,3}= \pm i \omega_{h}$. According to (10.3)-(10.4), the values of $\delta T, \delta I$ can be represented by the linear combinations of the functions $\sin \left(\omega_{h} t\right), \cos \left(\omega_{h} t\right)$. Let $\delta T(0)=0$. Then $\delta I(0)=0$ and $\delta I \sim \sin \left(\omega_{h} t\right)$. Varying $\alpha$, considering $\delta T=-\frac{q_{0}^{2}}{6} \delta I$ and $\delta W=p^{2} \delta I /\left(2 I^{3 / 2}\right)$ we find

$$
\delta \alpha=-\left[1-q_{0}^{2} I_{0}^{3 / 2} /\left(3 p^{2}\right)-E_{0} / W_{0}\right] \delta I / I_{0} .
$$

Here, indices " 0 " indicate the equilibrium values. In order to find the equilibrium values $E_{0}, W_{0}$, we integrate the second equation of system (10.2). We obtain the integral of the cluster energy:

$$
E_{c}=T+\left(\alpha_{1}+\alpha_{3}\right) I / 6-p^{2} / \sqrt{I}=E+\left(\alpha_{1}+\alpha_{3}\right) I / 6=\text { const } .
$$

Substituting integrals $E_{c}$ and $L_{z}$ (see above) in the first equation of system (10.2), we obtain a Lagrange-Jacobi equation in the following form:

$$
\ddot{I}=4 E_{c}+2 p^{2} / \sqrt{I}-4\left(\alpha_{1}+\alpha_{3}+2 \omega^{2}\right) I / 3 .
$$

Assuming $\ddot{I}=0$, we find from this equation the value $E_{c}$. We use this value to calculate $E=E_{0}$ with the help of the energy integral. An equilibrium value

$$
\alpha_{0}=2 E_{0} / W_{0}=1-\left(\alpha_{1}+\alpha_{3}+4 \omega^{2}\right) I_{0}^{3 / 2} /\left(3 p^{2}\right)<1 .
$$


An inequality $\alpha_{0}<1$ is consistent with the data of the numerical experiments (Danilov and Dorogavtseva, 2008). For the models of the isolated clusters, $\alpha_{0}=1$, since $\alpha_{1}=\alpha_{3}=\omega=0$.

Let us estimate the kinetic energy of the oscillations of our cluster model $T_{f}$. According to Danilov (1984), the solution of the continuity equation for the nonstationary spherically-symmetric homogeneous star clusters leads to a linear dependency of the radial velocity $v_{r}$ of the star flow at the distance $r$ from the cluster center: $v_{r}=\frac{r \dot{R}_{2}}{R_{2}}$. For a homogeneous ball with the mass density $\bar{\rho}$, we find

$$
T_{f}=2 \pi \bar{\rho} \int_{0}^{R_{2}} r^{2} v_{r}^{2} d r=2 \pi \bar{\rho} R_{2}^{3} \dot{R}_{2}^{2} / 5
$$

Considering $\bar{\rho}=3 M_{c} /\left(4 \pi R_{2}^{3}\right)$, we find $T_{f}=0.3 M_{c} \dot{R}_{2}^{2}$.

Let $\delta T_{m}, \delta I_{m}$ be the amplitudes of the $\delta T, \delta I$ values (the maximum moduli of $\delta T$ and $\delta I$ ). Then $\delta T_{m}=\frac{q_{0}^{2}}{6} \delta I_{m}$. For our cluster model, we find $\dot{I}=1.2 M_{c} R_{2} \dot{R}_{2}$. In the case of the small oscillation amplitudes of the cluster radius, the maximum modulus of $I$ equal $\delta I_{m} \omega_{h}$. Consequently, the maximum value

$$
{\dot{R_{2}}}^{2}=\delta I_{m}^{2} \omega_{h}^{2} /\left(1.2 M_{c} R_{2}\right)^{2} .
$$

Therefore, the maximum value $T_{f}$ is

$$
\delta T_{m}=b \omega_{h}^{2} \delta I_{m}^{2}, \quad \text { where } \quad b=0.3 M_{c} /\left(1.2 M_{c} R_{2}\right)^{2} .
$$

Considering that $\delta I_{m}=6 \frac{\delta T_{m}}{q_{0}^{2}}$, see above, we find

$$
\delta T_{m}=q_{0}^{4} /\left(36 b \omega_{h}^{2}\right) .
$$

Let $M_{c}=500 M_{\odot}, R_{G}=8200 \mathrm{pc}$. When the value $R_{2}$ is changed from $1 \mathrm{pc}$ to $10 \mathrm{pc}<R_{t} \simeq 10.468 \mathrm{pc}$ in the OSC model in the form of a homogeneous sphere, $\delta \alpha$ decreases from 1.994 to $0.973, \alpha_{0}$ decreases from 0.999 to $-0.357, \delta I_{m} / I_{0}$ decreases from 3.986 to $1.375, \delta T_{m} / T_{0}$ decreases from 3.984 to 1.101 , the period of the cluster oscillations $P_{R_{2}}$ increases from 4.163 to $56.338 \mathrm{Myr}$. In order to estimate the influence of the cluster mass distribution on $\delta \alpha, \delta I_{m} / I_{0}, \delta T_{m} / T_{0}$ etc., we have done the calculations, similar to the above ones, for the cluster models with a density $\rho(r, t)=\rho_{0}(t) / r^{2}$. In this case, the continuity equation for the cluster model also leads to a solution $v_{r}=\frac{r \dot{R}_{2}}{R_{2}}$, see above. At $M_{c}=500 M_{\odot}, R_{G}=8200 \mathrm{pc}$, at the change in $R_{2}$ from 1 to $10 \mathrm{pc}$ in the model with $\rho(r, t)=\rho_{0}(t) / r^{2}$, the value $\delta \alpha$ decreases from 1.997 to $0.895, \alpha_{0}$ decreases from to 0.9995 to $0.548, \delta I_{m} / I_{0}$ decreases from 3.995 to $2.077, \delta T_{m} / T_{0}$ decreases from 3.995 to 1.852 , the period of the cluster oscillations $P_{R_{2}}$ increases from 2.407 to $48.228 \mathrm{Myr}$. Thus, in the considered GD-models of the OSCs, the degree of a non-stationarity is very high (10-20 times exceeding the corresponding estimates for the numerical dynamic 
OSC models of Danilov and Dorogavtseva (2008)). This result depends a little on the accepted density distribution for the cluster model.

The periods $P_{r}$ of the radial oscillations of the considered GD-models of the OSCs with parameters $M_{c}=500 M_{\odot}, R_{2}=(0.7-0.9) R_{t}, R_{G}=8200 \mathrm{pc}$ decrease from $P_{r}=53.2 \pm 2.4 \mathrm{Myr}$ to $P_{r}=42.6 \pm 1.8 \mathrm{Myr}$ with a transition from the cluster models with a homogeneous density to the models with the density $\rho \sim 1 / r^{2}$. For the numerical dynamic OSC models (Danilov and Dorogavtseva, 2008) with the same initial parameters $M_{c}, R_{2}$ at $R_{G}=8200 \mathrm{pc}$, the $P_{r}$ values are $P_{r}=27.6 \pm 0.9 \mathrm{Myr}$. Let us specify the periods of the homologous oscillations for the GD-models of the OSCs. To do so, we apply the King distribution $f=f\left(r, k, r_{c}, R_{2}\right)$ for the spatial density of the number of stars in the cluster. Under a condition of the homologous oscillations of the cluster, $s=R_{2} / r_{c}=$ const. We write the function $f$ in the following form:

$$
f=\gamma\left(k, R_{2}, s\right) g\left(r, R_{2}, s\right),
$$

where

$$
\begin{gathered}
\gamma=k s /\left(\pi R_{2}\left(1+s^{2}\right)^{1.5}\right) ; \quad g=\arccos (z) / z^{3}-\sqrt{1-z^{2}} / z^{2} ; \\
z=\sqrt{\left(1+\left(r s / R_{2}\right)^{2}\right) /\left(1+s^{2}\right)},
\end{gathered}
$$

see King (1962). Using the data on the values $k, r_{c}, R_{2}$ for the OSCs from the catalogue of Danilov and Seleznev (1994) and function $f$, we find the numbers of stars in the clusters:

$$
N_{k}=4 \pi \int_{0}^{R_{2}} r^{2} f d r=4 \pi \gamma \int_{0}^{R_{2}} r^{2} g d r .
$$

Comparison of $N_{k}$ with the number of stars $N_{c}$ in the cluster obtained by Danilov and Seleznev (1994) with the star counts, shows that $N_{k} / N_{c}$ on average over the OSC sample of Danilov and Seleznev (1994) equals $\overline{N_{k} / N_{c}}=0.62 \pm 0.02$. A standard deviation of $N_{k} / N_{c}$ from $\overline{N_{k} / N_{c}}$ is $\sigma_{N_{k} / N_{c}} \simeq 0.19 . N_{k} / N_{c} \in[0.17,1.08]$. Thus, the distribution $f=f\left(r, k, r_{c}, R_{2}\right)$ gives an understated number of stars in the OSC (when using the function $f$, the stars located at the OSC periphery are not fully considered, see also Danilov (2010)). To take into account $\delta N=N_{c}-N_{k}>0$ of stars when estimating the dynamic parameters of the OSCs, let us consider the following modified King distribution model:

$$
f^{*}=f\left(r, k, r_{c}, R_{2}\right)+\delta f, \quad \text { where } \delta f=3 \delta N /\left(4 \pi R_{2}^{3}\right) .
$$

In this case, the density of the number of stars in the cluster center increases only slightly as compared with $f\left(0, k, r_{c}, R_{2}\right)$, since usually $\delta f / f\left(0, k, r_{c}, R_{2}\right) \ll 1$, but at the cluster periphery, the contribution of $\delta f$ to $f^{*}$ (and to $M_{c}$ ) becomes significant. Distribution $f^{*}$ is corresponded to the apparent density distribution of star number $F^{*}=F\left(r^{\prime}\right)+\delta F\left(r^{\prime}\right)$, where $F\left(r^{\prime}\right)$ is an apparent density of the number of stars by King (1962);

$$
\delta F\left(r^{\prime}\right)=2 R_{2} \sqrt{1-\left(r^{\prime} / R_{2}\right)^{2}} \delta f ;
$$


$r^{\prime}$ is the distance from the cluster center in the tangent plane, see, for example, equation (8.5) on p. 299 in the book of Kholopov (1981).

Using the function $f^{*}$, we write the expression for the momentum of inertia and the potential energy of the cluster in the following form:

$$
\begin{gathered}
I=4 \pi \bar{m} \gamma \int_{0}^{R_{2}} g r^{4} d r+0.6 \bar{m} \delta N R_{2}^{2}, \\
W=-4 \pi \bar{m} G\left[\gamma \int_{0}^{R_{2}} M(r) g r d r+\frac{3 \delta N}{4 \pi R_{2}^{3}} \int_{0}^{R_{2}} M(r) r d r\right],
\end{gathered}
$$

where $\bar{m}=$ const is the mean star mass in the cluster;

$$
M(r)=4 \pi \bar{m} \gamma \int_{0}^{r} g\left(r^{\prime}, R_{2}, s\right) r^{\prime 2} d r^{\prime}+\bar{m} \delta N r^{3} / R_{2}^{3}
$$

is the mass of a cluster stars with the distances from its center $r^{\prime} \leq r$. For a spherically symmetric cluster model with the distribution of a star number $f^{*}$, we can write:

$$
W=-c^{2} G M_{c}^{2.5} / \sqrt{I}, \quad \text { where } c^{2}=c^{2}\left(k, s, N_{c}, N_{k}\right)=\text { const }
$$

(a constancy of $c^{2}$ is revealed when calculating the values of $-W \sqrt{I}$ at a fixed $M_{c}$ ). After simple transformations made in Danilov and Putkov (2012a), the system of equations (10.2) for this model can be written in the following form:

$$
\begin{gathered}
\ddot{I}=4 T-\frac{2}{3}\left(\alpha_{1}+\alpha_{3}+4 \omega^{2}\right) I+2 W, \\
\dot{T}=-\frac{\alpha_{1}+\alpha_{3}}{6} \dot{I}-G \sum_{j} \frac{m_{j} M\left(r_{j}\right)}{r_{j}^{2}} \dot{r}_{j} .
\end{gathered}
$$

The first equation of the system (10.5) is obtained after a substitution of the integral $L_{z}$ of the third equation of system (10.2) in the first equation (see the explanation for equations (10.2)). Summation over $j$ in the second equation of system (10.5) is carried out for all cluster stars, $m_{j}=$ const and $r_{j}$ are a mass and a distance of the $j$-th star from the mass center of a cluster, respectively. To calculate the sum by $j$ in the second equation of (10.5), we consider the velocity of a radial flux of stars $v_{r}$ in this cluster model. Integrating the continuity equation corresponding to our OSC model

$$
\frac{\partial f^{*}}{\partial t}+\frac{1}{r^{2}} \frac{\partial}{\partial r}\left(r^{2} v_{r} f^{*}\right)=0
$$

by $r$, we find

$$
v_{r}=-\frac{\dot{\gamma} \int_{0}^{r} g r^{\prime 2} d r^{\prime}+\gamma \dot{R}_{2} \int_{0}^{r} \frac{\partial g}{\partial R_{2}} r^{\prime 2} d r^{\prime}+\dot{\delta f} \int_{0}^{r} r^{\prime 2} d r^{\prime}}{r^{2}(f+\delta f)} .
$$


Let $N_{k}=$ const . In this case,

$$
\dot{N}_{k}=4 \pi\left(\dot{\gamma} \int_{0}^{R_{2}} g r^{2} d r+\gamma \dot{R}_{2} \int_{0}^{R_{2}} \frac{\partial g}{\partial R_{2}} r^{2} d r\right)=0 .
$$

Consequently,

$$
\dot{\gamma}=-\gamma \dot{R}_{2} \int_{0}^{R_{2}} \frac{\partial g}{\partial R_{2}} r^{2} d r / \int_{0}^{R_{2}} g r^{2} d r .
$$

We substitute $\dot{\delta f}=-9 \delta N \dot{R}_{2} /\left(4 \pi R_{2}^{4}\right)$ and the expression for $\dot{\gamma}$ in the formula for $v_{r}$ and find

$$
\frac{v_{r}}{\dot{R}_{2}}=\frac{\mathcal{S}+\frac{r^{3} \delta f}{R_{2}}}{r^{2}(f+\delta f)},
$$

where

$$
\mathcal{S}=\gamma\left[\int_{0}^{R_{2}} \frac{\partial g}{\partial R_{2}} r^{2} d r \int_{0}^{r} g r^{\prime 2} d r^{\prime} / \int_{0}^{R_{2}} g r^{2} d r-\int_{0}^{r} \frac{\partial g}{\partial R_{2}} r^{\prime 2} d r^{\prime}\right] .
$$

A numerical integration of the integrands in the formula for $\mathcal{S}$ and calculation of $v_{r} / \dot{R}_{2}$ leads to the linear dependency on $r$ of $v_{r}=r \dot{R}_{2} / R_{2}$. Here and below, in the considered integrals by $r^{\prime}, g\left(r, R_{2}, s\right)$ is assumed equal to $g\left(r^{\prime}, R_{2}, s\right)$.

The mass of the stars with the distances from the cluster center $r^{\prime} \leq r$ at a time point $t$ for our cluster model is

$$
M(r)=4 \pi \bar{m} \int_{0}^{r} f^{*} r^{\prime 2} d r^{\prime}=4 \pi \bar{m} \gamma \int_{0}^{r} g r^{\prime 2} d r^{\prime}+\bar{m} \delta N r^{3} / R_{2}^{3} .
$$

Here, it is assumed $f^{*}(r)=f^{*}\left(r^{\prime}\right)$ (for a brevity, we omit the values of the parameters of the $f^{*}(r)$ function). During the oscillations of cluster models, while $r$ varies proportionally to the change of $R_{2}, M(r)$ remains constant. Indeed,

$\dot{M}(r)=4 \pi \bar{m}\left[\dot{\gamma} \int_{0}^{r} g r^{\prime 2} d r^{\prime}+\gamma \dot{R}_{2} \int_{0}^{r} \frac{\partial g}{\partial R_{2}} r^{\prime 2} d r^{\prime}+\gamma g r^{2} \dot{r}\right]+\frac{\bar{m} \delta N 3 r^{2}}{R_{2}^{3}}\left(\dot{r}-\frac{r \dot{R}_{2}}{R_{2}}\right)$.

We substitute in $\dot{M}(r)$ the expression for $\dot{\gamma}$. Considering that $v_{r} / \dot{R}_{2}=r / R_{2}=\dot{r} / \dot{R}_{2}$, we find $\dot{M}(r)=0$. Consequently, under the condition of the homologous oscillations of the cluster model, $M(r)=$ const. Differentiating the potential energy of the cluster model $W=-G \sum_{j} m_{j} M\left(r_{j}\right) / r_{j}$ by $t$, we find $\dot{W}=G \sum_{j} m_{j} M\left(r_{j}\right) \dot{r}_{j} / r_{j}^{2}$. In this case, the second equation of system (10.5) can be written in the form

$$
\dot{T}=-\frac{\alpha_{1}+\alpha_{3}}{6} \dot{I}-\dot{W} .
$$

Integrating this equation by $t$, we find the energy integral of the cluster model

$$
E=T+W+\frac{\alpha_{1}+\alpha_{3}}{6} I=\text { const }
$$


which agrees with the energy integral (5.522) from Chandrasekhar (1942). Varying the first equation of system (10.5), considering $\delta E=0, \delta W=c^{2} G M_{c}^{2.5} \delta I /\left(2 I_{0}^{1.5}\right)$ and taking into account only the linear terms in $\delta I$, we obtain the equation $\ddot{\delta} I+\omega_{h}^{2} \delta I=$ 0 , where $\omega_{h}=\sqrt{4\left(\alpha_{1}+\alpha_{3}+2 \omega^{2}\right) / 3+c^{2} G M_{c}^{2.5} / I_{0}^{1.5}}$ is the frequency of the small $I$ oscillations for the considered cluster model. The period of such oscillations is $P_{2}=2 \pi / \omega_{h}$.

Danilov and Putkov (2012a) performed the estimates of $P_{2}$ for OSCs from the sample of Danilov and Seleznev (1994). The mean $P_{2}$ has been obtained equal to $\overline{P_{2}}=31.8 \pm 1.7 \mathrm{Myr}$, a standard deviation of $P_{2}$ from $\overline{P_{2}}$ has been obtained equal to $\sigma_{P_{2}} \simeq 16.1 \mathrm{Myr}, P_{2} \in[6.0,70.7] \mathrm{Myr}$. For seven clusters Danilov and Seleznev (1994) gives the radii $R_{2}>R_{t}$. In these cases, the $P_{2}$ values were recalculated considering only stars with the distances from the cluster center $r \leq R_{t}$. As a result of this refinement, the following values of the parameters of the OSC distribution by $P_{2}$ were obtained: $\overline{P_{2}}=31.4 \pm 1.6 \mathrm{Myr}, \sigma_{P_{2}} \simeq 15.3 \mathrm{Myr}, P_{2} \in[6.0,67.0] \mathrm{Myr}$. The estimates of $\overline{P_{2}}$ and $\sigma_{P_{2}}$ are obtained here under the assumption of an equal accuracy of $P_{2}$ in the considered OSCs. On average for our sample of clusters, we find $\overline{\sigma\left(P_{2}\right) / P_{2}}=0.17 \pm 0.01$, where $\sigma\left(P_{2}\right)$ is the error of $P_{2} . P_{2}$ and $\sigma\left(P_{2}\right)$ are weakly correlated with each other (a correlation coefficient $k_{1,2} \simeq 0.23$ ). The weighted mean value of $P_{2}$ is $\left(P_{2}\right)_{p}=23.3 \pm 0.3 \mathrm{Myr}$, and a weighted standard deviation of $P_{2}$ from $\left(P_{2}\right)_{p}$ is $\sigma_{\left(P_{2}\right)_{p}} \simeq 18.8 \mathrm{Myr}$. According to Danilov and Seleznev (1994), the errors of $R_{2}$ and $N_{c}$ increase with increasing $R_{2}$ and $N_{c}$. The $P_{2}$ values for the considered star clusters on average decrease with decreasing $M_{c}$. Therefore, $\left(P_{2}\right)_{p}<\overline{P_{2}}$.

Following the procedure of Lynden-Bell (1967), assuming $\left|\frac{\partial U}{\partial t}\right| \simeq U / P_{2}$, in the framework of a GD description of a dynamics of the isolated star cluster models with density $\rho \sim 1 / r^{2}$, it is easy to obtain an expression for the time of a violent relaxation $\tau_{v r} \simeq 0.75 P_{2}=0.75 \sqrt{\pi /(G \bar{\rho})}$ (here, $U=U(r, t)$ is a gravitational potential of the system, $\bar{\rho}$ is a mean mass density in the system). The estimate of $\tau_{v r} \simeq 0.75 P_{2}$ is in a good agreement with the estimate of

$$
\tau_{v r} \simeq 2.6 \overline{t_{c r}} \simeq 0.72 \sqrt{\pi /(G \bar{\rho})},
$$

obtained by Aarseth (1974) in the framework of the numerical experiments on a modeling of the OSC dynamics (here, $\overline{t_{c r}}$ is the mean crossing time). $\tau_{v r}$ is comparable in magnitude with $P_{2}$; it characterizes the time in which the cluster transits from a non-stationary state in the regular field to a stationary and equilibrium state (Lynden-Bell, 1967) in the absence of the conditions for a gravitational instability; $\tau_{v r}^{-1}$ characterizes the rate of the cluster oscillations' energy loss during a violent relaxation (Danilov, 2011). 


\subsection{Oscillations in the Cores of OSC and OSC Models}

The reason for overestimation of $\delta \alpha, \delta I_{m} / I_{0}, \delta T_{m} / T_{0}$ in the GD models is a nonhomology of the oscillations in the real OSCs and a difference in the velocity of the star flux in the OSCs from $v_{r}=\frac{r \dot{R}_{2}}{R_{2}}$. Indeed, in the OSC models of Danilov and Dorogavtseva (2008) with increasing $r$ outside the cluster core, $v_{r}$ decreases, and the tangential flow velocity increases (see formula (18) and its explanation in Danilov (2006), as well as fig. 1 from Danilov and Dorogavtseva (2008) for the OSC models $1,4)$. The instability of the phase density oscillations develops to a greater extent near the center and in the cluster core (Danilov and Dorogavtseva, 2008; Danilov, 2008), which is approximately spherical during an evolution. The effect of the density and force field oscillations in the cluster core on a halo star's motion decreases with increasing $r$ outside the cluster core. According to Danilov (2005), the amplitudes of the oscillations of the regular potential in the halo of the non-stationary OSC models (Danilov and Dorogavtseva, 2003) are small, and amount to less than $6 \%$ of the mean regular potential averaged by the oscillation period.

In order to obtain the more accurate estimates of $\delta \alpha, \delta I_{m} / I_{0}, \delta T_{m} / T_{0}$ in the framework of a GD description, we consider a cluster model consisting of two homogeneous concentric spheres simulating the core and the halo of a cluster (Danilov, 1984) moving along a circular orbit in the Galactic plane. The use of the spherical halo models instead of ellipsoidal ones is dictated by the following considerations. The density oscillations near the cluster center depend a little on the symmetry of the density distribution at the cluster periphery (the difference in the attraction force between the spherical and ellipsoidal halos of equal masses at small distances from the cluster center is small). Non-stationary nature of the cluster is mainly determined by the oscillations of the cluster core. Therefore, our estimates of the non-stationarity parameters of the OSCs are consistent with the results of the determination of these values from the calculation data of Danilov and Dorogavtseva (2008), see below. The spherical models allow us to obtain the analytical formulas for the non-stationarity parameters, which can be used for the mass estimates of new (previously undetected) characteristics of the OSCs.

Let us consider the oscillations with the finite amplitudes of the cluster core under the condition that the mass and the halo cluster radius are constant. In this case, the oscillations of the entire cluster are non-homologous, and a lower estimate of the cluster's non-stationarity degree can be obtained.

Let $M_{j}, R_{j}, I_{j}, W_{j}$, and $T_{j}$ be mass, radius, inertia momentum, potential and kinetic energy of the $j$-th subsystem of cluster stars. Indices $j$ correspond to the core $(j=1)$ and halo $(j=2)$ of the cluster. The potential $U(r)$ of the cluster at $r \leq R_{1}$, $R_{1}<R_{2}$, is

$$
U(r)=1.5 G\left(M_{1} / R_{1}+M_{2} / R_{2}\right)-0.5 G\left(M_{1} / R_{1}^{3}+M_{2} / R_{2}^{3}\right) r^{2},
$$

see formula (27) from Danilov (1988). According to Danilov and Putkov (2012a), the GD equations for the cluster core can be written as follows: 


$$
\ddot{I}_{1}=4 T_{1}+4 \omega L_{1, z}-\frac{2 q_{1}^{2}}{3} I_{1}, \quad \dot{T}_{1}=-\frac{q_{1}^{2}}{6} \dot{I}_{1}, \quad \dot{L}_{1, z}=-\frac{2 \omega}{3} \dot{I}_{1},
$$

where $q_{1}^{2}=\alpha_{1}+\alpha_{3}+3 \beta_{1} ; \beta_{1}=G\left(M_{1} / R_{1}^{3}+M_{2} / R_{2}^{3}\right) ; L_{1, z}$ is the angular momentum of a rotation of the cluster core around the $z$ axis. Let us introduce the following notation:

$$
p_{0}^{2}=1.5 G M_{1} M_{2} / R_{2}, \quad p_{1}^{2}=\left(0.6 M_{1}\right)^{1.5} G M_{1}, \quad p_{2}^{2}=0.5 G M_{2} / R_{2}^{3} .
$$

The inertia momentum of the cluster core is $I_{1}=0.6 M_{1} R_{1}^{2}$. From the last relation, we find $R_{1}=\sqrt{I_{1} /\left(0.6 M_{1}\right)}$. A potential energy of the cluster core is $W_{1}=W_{1,1}+W_{1,2}$, where $W_{1,1}$ is the energy of interaction between the core stars, and $W_{1,2}$ is the energy of interaction between the core stars and the halo stars (Danilov, 1984). Following the notations adopted, according to Danilov (1984), we find

$$
W_{1,2}=-p_{0}^{2}+p_{2}^{2} I_{1}, \quad W_{1,1}=-p_{1}^{2} / \sqrt{I_{1}}, \quad q_{1}^{2}=\alpha_{1}+\alpha_{3}+3\left(p_{1}^{2} / I_{1}^{1.5}+2 p_{2}^{2}\right) .
$$

We substitute $q_{1}^{2}$ to the second equation of system (10.6) and integrate it by $t$. We obtain the energy integral of the core:

$$
E_{1}=T_{1}-p_{1}^{2} / \sqrt{I_{1}}+\left(\alpha_{1}+\alpha_{3}+6 p_{2}^{2}\right) I_{1} / 6=\text { const } .
$$

From the third equation of system (10.6), we find

$$
L_{1, z}=-2 \omega I_{1} / 3+L_{1, z}(0) \text {. }
$$

Assuming $L_{1, z}=0$, we substitute $L_{1, z}$ and $E_{1}$ in the first equation of system (10.6). After the simple transformations, we find

$$
\ddot{I}_{1}=4 E_{1}+2 p_{1}^{2} / \sqrt{I_{1}}-2 a I_{1},
$$

where $a=2\left(\alpha_{1}+\alpha_{3}+6 p_{2}^{2}+2 \omega^{2}\right) / 3$. Assuming $\ddot{I}_{1}=0$ in (10.7), we find $E_{1}=$ $0.5\left(a I_{1,0}-p_{1}^{2} / \sqrt{I_{1,0}}\right)$, where $I_{1,0}$ is an equilibrium value of $I_{1}$. We multiply (10.7) by $\dot{I}_{1}$ and integrate the resulting equation by $t$ :

$$
\dot{I}_{1}^{2}=2 f\left(I_{1}\right) ; \quad f\left(I_{1}\right)=-a I_{1}^{2}+4 E_{1} I_{1}+4 p_{1}^{2} \sqrt{I_{1}}+C, \quad C=\text { const } .
$$

Assuming $f\left(I_{1}\right)=0$ in (10.8), we find

$$
C=a I_{1, m}^{2}-4 E_{1} I_{1, m}-4 p_{1}^{2} \sqrt{I_{1, m}},
$$

where $I_{1, m}$ is the minimum $\left(I_{1, \min }\right)$ or the maximum $\left(I_{1, \max }\right)$ value of $I_{1}$ at a given $E_{1}$ value. The considered oscillations of the size of a cluster core occur under condition $\mu=M_{1} / M_{2}=$ const . In deriving the formulas (10.8), we applied the same method as in Ferronsky et al. (1978) for solving the Lagrange-Jacobi equation for the spherical isolated systems.

Let us consider two possible situations in the dynamics of the observed OSCs. 
1. If the cluster radius $R_{1}$ obtained from the observations determines $I_{1,0}$ value, and the maximum (or the minimum) value of $\xi=\xi(\mu)$ determines one of the values $I_{1, m}$, then the equation $f\left(I_{1}\right)=0$ is an algebraic equation of the fourth degree with respect to $\tau=\sqrt{I_{1}}$ and allows us to find the second $I_{1, m}$ value. The solution of equation (10.8) can be written in the following form:

$$
\pm \int_{I_{1}(0)}^{I_{1}} d I_{1} / \sqrt{2 f\left(I_{1}\right)}=t
$$

where $I_{1}(0)$ is the initial value of $I_{1}$. The period $P_{1}$ of a core oscillation in this cluster model is

$$
P_{1}=2 \int_{I_{1, \min }}^{I_{1, \max }} \frac{d I_{1}}{\sqrt{2 f\left(I_{1}\right)}}
$$

By substitution $\tau^{2}=I_{1}$, integral (10.9) can be reduced to

$$
P_{1}=2 \sqrt{2} \int_{\tau_{1}}^{\tau_{4}} \tau d \tau / \sqrt{f\left(\tau^{2}\right)}
$$

where $f\left(\tau^{2}\right)=-a\left(\tau-\tau_{1}\right)\left(\tau-\tau_{2}\right)\left(\tau-\tau_{3}\right)\left(\tau-\tau_{4}\right)$ is the fourth-degree polynomial in $\tau ; \tau_{k}$ are the roots of equation $f\left(\tau^{2}\right)=0, k=1, \ldots, 4$.

In the considered versions of clusters, $a>0, \tau_{1}=\sqrt{I_{1, \min }}$ and $\tau_{4}=\sqrt{I_{1, \max }}$ are real roots, $0<\tau_{1}<\tau_{4} ; \tau_{2}$ and $\tau_{3}$ are the complex conjugate roots. Therefore, the integral for $P_{1}$ is improper, and the integrand in it is unbounded in the neighborhoods of the points $\tau=\tau_{1}, \tau_{4}$. If $\tau_{2,3}=\theta \pm i \phi$, then $\left(\tau-\tau_{2}\right)\left(\tau-\tau_{3}\right)=(\tau-\theta)^{2}+\phi^{2}$ (here, $\theta=$ const,$\phi=$ const (Korn and Korn, 1968). The integrals of the form of $P_{1}$ are elliptical, and can be reduced to the normal elliptic ones (Korn and Korn, 1968), but we apply the following method of calculating such integrals. We divide the domain of integration over $\tau$ into two equal parts: $\tau \in\left[\tau_{1}, \tau_{s}\right]$ and $\tau \in\left[\tau_{s}, \tau_{4}\right]$, where $\tau_{s}=\left(\tau_{1}+\tau_{4}\right) / 2$. Next, we use the substitutions $v^{2}=\tau-\tau_{1}$ and $v^{2}=\tau_{4}-\tau$ in the integrals over $\tau$ for the first and second range of the $\tau$ values, respectively. These substitutions lead to integrals over $v$ from the bounded functions. Such integrals are easily computed using the numerical methods.

2. If the radius $R_{1}$ of the cluster core obtained from the observations determines $I_{1, m}=I_{1, \min }$ (or $I_{1, m}=I_{1, \max }$ ), and the maximum (or the minimum) value of $\xi=\xi(\mu)$ determines the second value of $I_{1, m}$, then from the equation for $C$ (see explanations for (10.8)), we can determine

$$
E_{1}=a\left(I_{1, \text { max }}+I_{1, \text { min }}\right) / 4-p_{1}^{2} /\left(\sqrt{I_{1, \max }}+\sqrt{I_{1, \min }}\right)
$$

at $I_{1, \max } \neq I_{1, \min }$. Substituting $E_{1}$ in the expression for $E_{1}$, obtained from (10.7) at $\ddot{I}_{1}=0$, we can determine $I_{1,0}$ from the cubic equation for $\tau_{0}=\sqrt{I_{1,0}}$ :

$$
\tau_{0}^{3}-2 E_{1} \tau_{0} / a-p_{1}^{2} / a=0
$$


In the considered cluster versions, this equation has two complex conjugate roots and one real root $\tau_{0}>0$, which determines the $I_{1,0}$ values. The $I_{1, m}$ values allow us to find $C$, to determine function $f\left(I_{1}\right)$ and the $P_{1}$ value according to (10.8),(10.9).

Thus, for different positions of the point representing the OSC in the plane $(\xi, \mu)$, we can obtain the values of $\delta I_{1}=\left|I_{1, m}-I_{1,0}\right|, \delta I_{1} / I_{1,0}$ and $\delta R_{1} / R_{1,0}=$ $\left|R_{1, m}-R_{1,0}\right| / R_{1,0}$, characterizing the degree of a cluster non-stationarity (here, $R_{1, m}$ and $R_{1,0}$ are determined by the corresponding $I_{1}$ values, see above). In order to obtain the equilibrium values of the kinetic energies $T_{j, 0}$, as well as a potential energy $W_{0}$ and a virial coefficient $\alpha_{0}$ of the cluster, corresponding to the conditions $\ddot{I}_{j}=0, j=1,2$, it is convenient to use equations (14) for $\ddot{I}_{j}$ and formulas (31), (32), (35) from Danilov (1984), written for the cluster model considered in our work. Considering conditions $\ddot{I}_{j}=0$ and the notations adopted here, according to Danilov (1984) and Danilov and Putkov (2012a), we write

$$
\begin{gathered}
T_{1,0}=\omega_{0} I_{1,0}+\frac{1}{2}\left(\frac{p_{1}^{2}}{\sqrt{I_{1,0}}}+\frac{2 p_{0}^{2} I_{1,0} M_{2}}{5 I_{2,0} M_{1}}\right), \\
T_{2,0}=\omega_{0} I_{2,0}+\frac{1}{2}\left[\frac{p_{3}^{2}}{\sqrt{I_{2,0}}}+p_{0}^{2}\left(1-\frac{3 I_{1,0} M_{2}}{5 I_{2,0} M_{1}}\right)\right],
\end{gathered}
$$

where $\omega_{0}=\left(\alpha_{1}+\alpha_{3}+4 \omega^{2}\right) / 6, p_{3}^{2}=G M_{2}\left(0.6 M_{2}\right)^{1.5}$. The value of $T_{1,0}-\omega_{0} I_{1,0}$ (see the first equation from (10.10)) can also be written directly from the first and the third equations of system (10.6) in a different form (using constants $p_{1}^{2}, p_{2}^{2}$ ). These two forms of an expression for $T_{1,0}$ give the same $T_{1,0}$ values. According to Danilov (1984), a potential energy of an interaction of the cluster stars is $W=\frac{1}{2} \sum_{j=1}^{2} W_{j}=$ $W_{1,1}+W_{2,2}+W_{1,2}$, where $W_{2,2}$ is the energy of the interaction of the halo stars with each other. Considering our notation, we find

$$
W=-p_{1}^{2} / \sqrt{I_{1}}-p_{3}^{2} / \sqrt{I_{2}}-p_{0}^{2}\left(1-I_{1} M_{2} /\left(5 I_{2} M_{1}\right)\right) .
$$

In the case $I_{j}=I_{j, 0}$, we find the equilibrium values of a potential energy $W_{0}$ and a virial coefficient $\alpha_{0}=2\left(T_{1,0}+T_{2,0}+W_{0}\right) / W_{0}$ for our cluster model. We note that $\dot{T}_{2}=0$, and $T_{2}=$ const, since $\dot{T}_{2} \sim \dot{I}_{2}=0$, and the equation for $\dot{T}_{2}$ has the form similar to the second equation of system (10.6) for $\dot{T}_{1}$. Moreover, $W_{2}=W_{2,2}+W_{1,2}$, and for the isolated cluster model in this case $\dot{T}_{2}=\dot{E}_{2}-\dot{W}_{2}=0$, see (33) from Danilov (1984); $E_{2}$ is determined in Danilov (1984) as the total energy of the subsystem of the halo stars $(j=2)$.In the considered cluster model, consisting of two homogeneous concentric spheres simulating the core and the halo, $\alpha_{0}<1$ (as in the case of the GD models of a cluster with a homogeneous density and a cluster with the density $\rho \sim 1 / r^{2}$ ). The difference of $\alpha_{0}$ from unity characterizes the degree of an influence of the external Galactic field on the cluster. Minimum and maximum values of $\alpha=\alpha_{m}$ can be obtained by substituting $I_{1, m}$ to formula for $T_{1}$ (see above, the energy integral of a core $\left.E_{1}\right)$ and in formula $\alpha=2\left(T_{1}+T_{2,0}+W\right) / W$. The values of $\delta \alpha=\left|\alpha_{m}-\alpha_{0}\right|$ and $\delta \alpha / \alpha_{0}=\left|\alpha_{m}-\alpha_{0}\right| / \alpha_{0}$ characterize the degree of non-stationarity of the considered cluster model. 


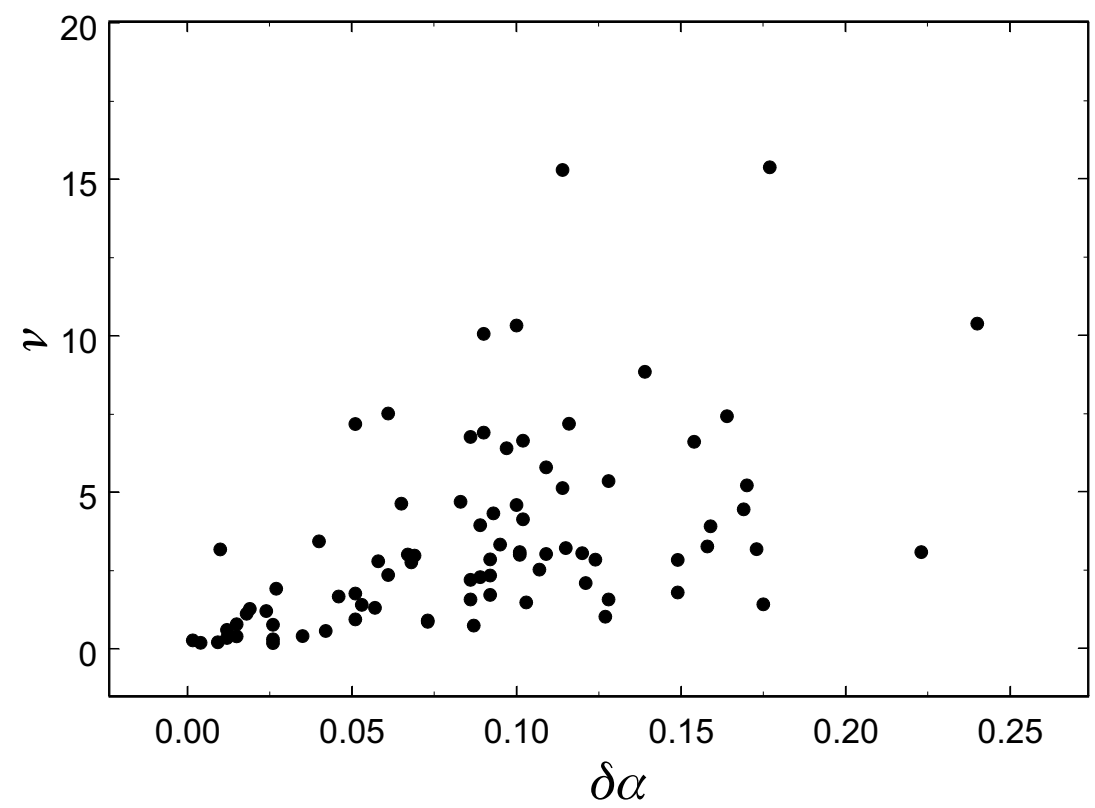

Fig. 10.2 Positions of OSC in coordinates $(\nu, \delta \alpha)$
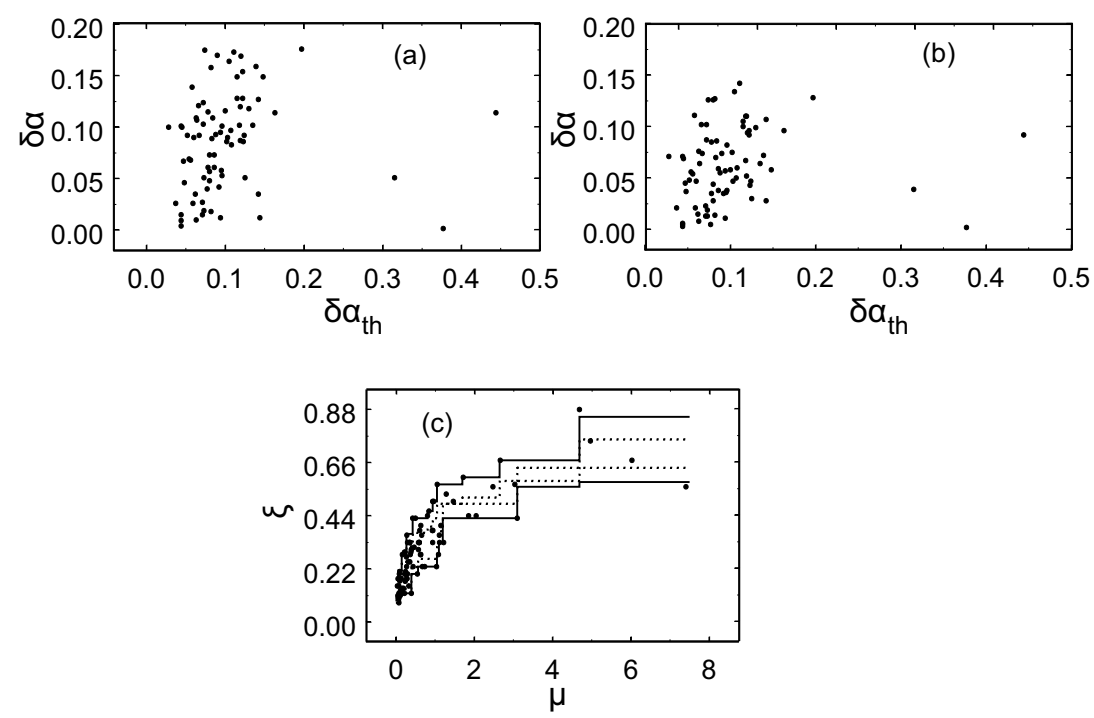

Fig. 10.3 Comparison of $\delta \alpha$ and $\delta \alpha_{t h}$ (a, b) for two methods of a conducting of the lines $\xi(\mu)_{\max , \min }$ in the diagram $(\xi, \mu)$ indicated in (c) 
Figs. 10.3a,b show the results of calculations of $\delta \alpha$ for OSCs from the catalogue of Danilov and Seleznev (1994) using the formulas from Section 10.3 of this book. $\delta \alpha$ in fig. 10.3 are given in comparison with $\delta \alpha_{t h}$, obtained by Danilov and Seleznev (1994). In those cases when the several estimates of $\delta \alpha$ were possible (for the different phases of the oscillations of the observed clusters), for such a cluster in fig.10.3 the average estimate of $\delta \alpha$ is used. Fig. 10.3c shows two versions of the upper and lower "enveloping" dependencies of $\xi=\xi(\mu)$ for the considered OSCs in the plane $(\xi, \mu)$. The solid lines indicate lines $\xi=\xi(\mu)$ approximately corresponding to the maximum and minimum $\xi$ values for a given $\mu$ value at $\mu<4$.69. For four OSCs with $\mu \geq 4.69$, due to the small number of clusters, the $\xi(\mu)_{\max , \min }$ values were assumed as $\xi(\mu)_{\text {max }, \min }=\overline{\xi(\mu)} \pm \sigma_{\xi}$, where $\overline{\xi(\mu)}$ is the mean $\xi$ for these four clusters, and $\sigma_{\xi}$ is the standard deviation of $\xi$ from $\overline{\xi(\mu)}$. Fig. 10.3a gives $\delta \alpha$ obtained using this version of the "envelopes". We note that the $\xi$ values from Danilov and Seleznev (1994) are known with the errors $\sigma(\xi)$ of $\sim 0.14 \xi$ (since the relative errors of a determination of the radii of the core and the halo in Danilov and Seleznev (1994) are $\sim 10 \%$ ). The errors $\sigma(\xi)$ of $\xi$ must lead to an increase of the width $\Delta \xi(\mu)$ of the band occupied by the clusters in the plane $(\xi, \mu)$. To estimate the effect of the changes in the width of $\Delta \xi(\mu)$ on the $\delta \alpha$ values in our work, we plot the dependencies $\xi(\mu)_{\text {max } \text { min }}^{\prime}=0.86 \xi(\mu)_{\max }, 1.14 \xi(\mu)_{\min }$, shown in fig. $10.3 \mathrm{c}$ by the dashed lines. Fig.10.3b gives $\delta \alpha$ values obtained using the dependencies $\xi(\mu)_{\text {max }, \text { min }}^{\prime}$.

The values $\delta \alpha$, used in figs.10.3a,b, show a significant correlation (with a probability $P>0.999$ ) with $\delta \alpha_{t h}$. A sample correlation coefficient $k_{1,2}$ and statistics of $t^{\prime}$ with a Student's t-distribution, in the case of the sample $\delta \alpha$ corresponding to fig. 10.3a, are obtained equal to $k_{1,2}=0.67$ and $t^{\prime}=6.81$ at critical $t^{\prime} \simeq 3.49$, and in the case of the sample $\delta \alpha$, corresponding to fig. $10.3 \mathrm{~b}, k_{1,2}=0.39$ and $t^{\prime}=3.56$ at critical value $t^{\prime} \simeq 3.45$ (Korn and Korn, 1968) (the values of $k_{1,2}$ and $t^{\prime}$ are obtained here without consideration of the clusters with $\delta \alpha_{t h}>0.3$, see fig. 10.3a,b). We note that the $P_{1}$ and $P_{2}$ values of the oscillation periods of the core and a homologous cluster mentioned here and in the previous sections of this book are also significantly correlating with each other (with a probability $P>0.999$ ). In this case, $k_{1,2} \simeq 0.83$, $t^{\prime} \simeq 13.88$, a critical value $t^{\prime} \simeq 3.43$.

Let $\mathbf{X}=\left(\delta \alpha, \delta R_{1} / R_{1,0}, \delta \alpha / \alpha_{0}, \alpha_{0}, P_{1}\right)$. The average (over the OSC sample) values of coordinates $X_{i}(i=1, \ldots, 5)$ of the vector $\mathbf{X}$, as well as standard deviations $\sigma_{X_{i}}$ of $X_{i}$ from the mean $\overline{X_{i}}$ values in the cases corresponding to fig. 10.3a,b are given in Table $10.1\left(\overline{P_{1}}\right.$ and $\sigma_{P_{1}}$ in Table 10.1 are given in $\operatorname{Myr}, \overline{X_{i}}$ values at $i=1, \ldots, 4$ are dimensionless). The values $\sigma_{X_{i}}$ in the case corresponding to fig. $10.3 \mathrm{~b}$, differ a little from $\sigma_{X_{i}}$ given for the case corresponding to fig. 10.3a, and, therefore, they are not indicated in Table 10.1. Thus, the above mentioned decrease in $\Delta \xi(\mu)$ practically does not affect the $\overline{\alpha_{0}}$ and $\overline{P_{1}}$ values, and it decreases the $\overline{\delta \alpha}, \frac{\delta\left(\mu / R_{1} / R_{1,0}\right.}{\delta \alpha / \alpha_{0}}$ values approximately in $\sim 1.5$ times. For the OSCs with $\delta \alpha_{t h}<0.3$ considered here, $\overline{\delta \alpha_{t h}}=0.10 \pm 0.01$ (see also Danilov and Seleznev (1994)), which is in complete agreement with the estimate of $\overline{\delta \alpha}$ in the case of fig. 10.3a.

We note that in the case of the homologous oscillations of the OSC models with a density $\rho(r, t) \sim \rho_{0}(t) / r^{2}$, such value of $\overline{\delta \alpha_{t h}}$ corresponds to the relative amplitude of the oscillations of the cluster radius $\delta R_{2} / R_{2,0} \simeq 0.111 \pm 0.002$. Here, 
Table 10.1 Parameters of the non-stationarity of OSC

\begin{tabular}{|c|c|c|}
\hline \multicolumn{3}{|c|}{$\xi(\mu)_{\min , \max } \quad$ (fig. 10.3, a) } \\
\hline $\begin{array}{r}\overline{\overline{\delta \alpha}}=0.09 \\
\pm 0.01\end{array}$ & $\sigma_{\delta \alpha} \simeq 0.05$ & $\begin{aligned} \overline{\overline{\sigma(\delta \alpha) / \delta \alpha}}=0.53 \\
\\
\pm 0.04\end{aligned}$ \\
\hline $\begin{aligned} \overline{\frac{\delta R_{1}}{R_{1,0}}} & =0.34 \\
& \pm 0.01\end{aligned}$ & $\sigma_{\delta R_{1} / R_{1,0}} \simeq 0.11$ & $\begin{aligned} \frac{\sigma\left(\delta R_{1} / R_{1,0}\right)}{\delta R_{1} / R_{1,0}} & =0.34 \\
& \pm 0.03\end{aligned}$ \\
\hline $\begin{aligned} \overline{\delta \alpha / \alpha_{0}} & =0.14 \\
& \pm 0.02\end{aligned}$ & $\sigma_{\delta \alpha / \alpha_{0}} \simeq 0.17$ & $\begin{aligned} \frac{\overline{\sigma\left(\delta \alpha / \alpha_{0}\right)}}{\delta \alpha / \alpha_{0}} & =0.53 \\
& \pm 0.04\end{aligned}$ \\
\hline $\begin{aligned} \overline{\overline{\alpha_{0}}} & =0.77 \\
& \pm 0.03\end{aligned}$ & $\sigma_{\alpha_{0}} \simeq 0.28$ & $\begin{aligned} \overline{\sigma\left(\alpha_{0}\right) / \alpha_{0}} & =0.07 \\
& \pm 0.04\end{aligned}$ \\
\hline $\begin{aligned} \overline{P_{1}}= & 16.8 \\
& \pm 1.3\end{aligned}$ & $\sigma_{P_{1}} \simeq 12.6$ & $\begin{aligned} \overline{\sigma\left(P_{1}\right) / P_{1}} & =0.180 \\
& \pm 0.005\end{aligned}$ \\
\hline
\end{tabular}

\begin{tabular}{|c|}
\hline$\xi(\mu)_{\min , \max }^{\prime}$ (fig. 10.3, b) \\
\hline$\overline{\delta \alpha}=0.062 \pm 0.004$ \\
\hline$\overline{\delta R_{1} / R_{1,0}}=0.25 \pm 0.01$ \\
\hline$\overline{\delta \alpha / \alpha_{0}}=0.08 \pm 0.01$ \\
\hline$\overline{\alpha_{0}}=0.76 \pm 0.03$ \\
\hline$\overline{P_{1}}=16.7 \pm 1.3$ \\
\hline
\end{tabular}

we used the values $\overline{R_{G}}=8806 \pm 94 \mathrm{pc}, \overline{R_{2} / R_{t}}=0.576 \pm 0.032$ averaged over the OSC sample of Danilov and Seleznev (1994) as well as the expressions for $\delta \alpha, \alpha_{0}$ obtained for this cluster model in the same way as was done for the homogeneous cluster model, see above. We also used a relation $\delta W / W_{0}=-\delta R_{2} / R_{2,0}$; in this case, $\delta R_{2} /\left(R_{2,0} \delta \alpha\right)$ does not depend on $M_{c}$. Thus, on average for the OSC sample, $\delta R_{1} / R_{1,0}$ may be approximately 2.3-3.1 times larger than $\delta R_{2} / R_{2,0}$ obtained for the homologous oscillations of the cluster. Such difference of $\overline{\delta R_{1} / R_{1,0}}$ from $\delta R_{2} / R_{2,0}$, as well as the difference of $\overline{P_{1}}$ from $\overline{P_{2}} \simeq 1.9 \overline{P_{1}}$ indicates the non-homology of the OSC oscillations. Non-homologous oscillations of the OSC models of Danilov and Dorogavtseva (2008) are another argument against the homology of the OSC oscillations.

The value $\overline{\delta R_{1} / R_{1,0}}$ in the case corresponding to fig. $10.3 \mathrm{~b}$, quite agrees with the estimates of $\sigma_{R_{1}} / R_{1,0}$ for the cluster model 1 with the greatest degree of nonstationarity (Danilov and Dorogavtseva, 2008), see above. The $\overline{\delta \alpha / \alpha_{0}}$ value in the case corresponding to fig. 10.3a, is not statistically different from $\overline{\delta \alpha / \alpha_{0}}=0.21 \pm 0.07$ obtained for six OSC models of Danilov and Dorogavtseva (2003), for which the standard deviation $\delta \alpha / \alpha_{0}$ from $\overline{\delta \alpha / \alpha_{0}}$ is $\sigma_{\delta \alpha / \alpha_{0}} \simeq 0.18$.

Thus, the oscillations of the OSC cores with such amplitudes quite can determine the width $\Delta \xi(\mu)$ of the band occupied by the clusters in the diagram $(\xi, \mu)$, see fig. 21 from Danilov and Seleznev (1994), and the $\Delta \xi(\mu)$ value quite can determine the non-stationarity degree of the OSCs with a given $\mu$. We note that at $\mu \gtrsim 2.5$, 
the method for calculating the non-stationarity parameters $\delta \alpha, \delta R_{1} / R_{1,0}, \delta \alpha / \alpha_{0}$ applied here can give overestimated results for these values. According to Danilov and Dorogavtseva (2008), during the periods of the greatest compression to the plane of the Galaxy in the clusters with $\mu>2.5$, the toroidal structures can form with an increased density of the number of stars inside the torus, and with the equatorial plane coinciding with the plane of the Galaxy (see, for example, the cluster model 6 of Danilov and Dorogavtseva (2008), for which the initial value of $\mu=4$ ). This leads to an increase in the non-homology of the oscillations and to a decrease in the degree of non-stationarity of such cluster models (Danilov and Dorogavtseva, 2008; Danilov, 2008). Probably, in such cases, in order to estimate the degree of the cluster non-stationarity, it is necessary to carry on the numerical experiments on the simulation of the OSC dynamics (as in Danilov and Dorogavtseva $(2008,2003)$ ). The indicated effect only weakly affects the mean values of the OSC non-stationarity parameters obtained here, since the clusters with $\mu>2.5$ in our sample are only seven.

The estimates of the $\overline{X_{i}}(i=1, \ldots, 5)$ values are obtained here assuming that the values $X_{i}$ have an equal accuracy for different OSCs. Let $\sigma\left(X_{i}\right), \sigma\left(R_{2}\right), \sigma\left(R_{t}\right)$ be the errors of $X_{i}, R_{2}, R_{t}$ for the considered OSCs. The values $\sigma\left(X_{i}\right) / X_{i}$ averaged over the sample of the clusters with $R_{2}+\sigma\left(R_{2}\right)<R_{t}-\sigma\left(R_{t}\right)$ are given in the 3rd column of Table 10.2. All $X_{i}$ values, except for $\delta R_{1} / R_{1,0}$, correlate fairly well with $\sigma\left(X_{i}\right)$. The weighted mean value estimate of $\delta R_{1} / R_{1,0}$ for the considered sample of clusters was obtained equal to $\left(\delta R_{1} / R_{1,0}\right)_{p}=0.33 \pm 0.01$, which is not statistically different from $\overline{\delta R_{1} / R_{1,0}}$, see above. The weighted mean square deviation of $\delta R_{1} / R_{1,0}$ from $\left(\delta R_{1} / R_{1,0}\right)_{p}$ is obtained equal to $\sigma_{\left(\delta R_{1} / R_{1,0}\right)_{p}} \simeq 0.09$, which also agrees with the estimate of $\sigma_{\delta R_{1} / R_{1,0}}$.

Let $\mathbf{y}=\left(M_{c}, R_{2}, \xi, \mu\right)$. The coordinates $y_{j}(j=1, \ldots, 4)$ of a vector $\mathbf{y}$ allow to determine a vector $\mathbf{X}$. When estimating the errors $\sigma\left(X_{i}\right)$ for each OSC, the $y_{j}$ values were assumed to be distributed according to the normal law with a probability density

$$
w(\mathbf{y}) \sim \exp \left(-\sum_{j=1}^{4} t_{j}^{2} / 2\right),
$$

where $t_{j}=\left(y_{j}-\left\langle y_{j}\right\rangle\right) / \sigma\left(y_{j}\right),\left\langle y_{j}\right\rangle$ and $\sigma\left(y_{j}\right)$ are the parameters of a distribution $w(\mathbf{y})$. In this case, the equation of the ellipsoid of the "one-sigma" errors of $\sigma\left(y_{j}\right)$ in the $t_{j}$ coordinates has the form

$$
\sum_{j=1}^{4} t_{j}^{2} / 2=1
$$

(an equation of a sphere with the radius $\sqrt{2}$ in the 4-dimensional space $\left(t_{1}, \ldots, t_{4}\right)$ ). Assuming by the order an every value of $t_{j}$ equal to zero in this equation, we obtain four equations of spheres in 3-dimensional spaces. Passing in these equations to the spherical coordinates and varying the angular variables in steps of $\pi / 4$, we specify 96 points on the surface of the ellipsoid of the "one-sigma" errors $\sigma\left(y_{j}\right)$. The indicated 
points and the center of the "one-sigma" error ellipsoid are used to calculate $96 X_{i}$ values and the $\left\langle X_{i}\right\rangle$ values. The $\sigma\left(X_{i}\right)$ values were defined as standard deviations of $X_{i}$ from $\left\langle X_{i}\right\rangle$.

We note that the errors $\sigma(\bar{m})$ of the mean mass of a cluster star do not affect the estimates of $\sigma(\delta \alpha), \sigma\left(\delta R_{1} / R_{1,0}\right), \sigma\left(\delta \alpha / \alpha_{0}\right)$. The contribution of the $\sigma(\bar{m})$ values to $\sigma\left(P_{1}\right)$ and $\sigma\left(\alpha_{0}\right)$ on average for the sample of the considered OSCs is $\sim 25$ and $\sim 33 \%$, respectively.

How can the taking into account the less bright stars with stellar magnitudes $m_{B}>$ $16^{m}$, which exceed the limiting magnitude of the stars considered by Danilov and Seleznev (1994), to affect the estimates of the OSC dynamic parameters? Estimates of the OSC parameters performed in our work using the catalogue of Danilov and Seleznev (1994) show that $\delta \alpha, \alpha_{0}$ (and $\bar{\rho}=3 M_{c} /\left(4 \pi R_{2}^{3}\right)$ ) on average (relatively to $M_{c}$ in several selected $M_{c}$ intervals) decrease, while $P_{1}$ and $P_{2}$ on average grow with an increase of $M_{c}$. Although, at $M_{c}>350 M_{\odot}$, the indicated changes in the mean values do not exceed the standard deviations of these values from the corresponding mean values. The $\delta R_{1} / R_{1,0}$ and $\delta \alpha / \alpha_{0}$ values on average (relatively to $M_{c}$, see above) are practically independent of $M_{c}$. Therefore, at $m_{B}>16^{m}$, it is likely that the values of $\delta \alpha$ and $\alpha_{0}$ obtained in our work can be considered as overestimated. The values of $P_{1}$ and $P_{2}$ can be considered as the lower estimates of these values for the considered OSCs. Estimates of $\delta R_{1} / R_{1,0}$ and $\delta \alpha / \alpha_{0}$ do not seem to depend on the limiting magnitude $m_{B}$ of stars at $m_{B}>16^{m}$.

\subsection{Dynamic Dispersions of Stellar Velocities in OSC}

In order to estimate the dispersions of the stellar velocities $\sigma_{v}^{2}$ in the OSCs according to the data on the structural-dynamic parameters of the clusters, we use equation (13) from Danilov (2010). Following to Danilov and Putkov (2012a), for the model of a spherically symmetric cluster, we write this equation as follows:

$$
\sigma_{v}^{2} \simeq \frac{1}{2}\left\{\frac{1}{M_{c}}\left[-W+\frac{1}{3}\left(\alpha_{1}+\alpha_{3}\right) I\right]+\frac{U(0)}{2}\right\},
$$

where $U(0)$ is the potential in the cluster center. According to Danilov (2010), this equation takes into account the influence of the force field of the Galaxy and the non-stationary nature of the cluster on $\sigma_{v}^{2}$. For the cluster model consisting of two homogeneous concentric spheres imitating the core and the halo of the cluster, we find $U(0)=1.5 G\left(M_{1} / R_{1}+M_{2} / R_{2}\right)$, see $U=U(r)$, as well as the formula for $W$ in the explanations for equations (10.6) and (10.10).

Following to Danilov and Putkov (2012a), we write the equation (13) from Danilov (2010) for a cluster model with a homogeneous spherical core and an ellipsoidal halo with coincident centers of mass as follows:

$$
\sigma_{v}^{2} \simeq \frac{1}{2}\left\{\frac{1}{M_{c}}\left[-W+\frac{\left(\alpha_{1}+\alpha_{3}\right) M_{1} R_{1}^{2}+\left(\alpha_{1} a^{2}+\alpha_{3} c^{2}\right) M_{2}}{5}\right]+\frac{U(0)}{2}\right\},
$$


Table 10.2 Values $\bar{\lambda}$ and $\overline{\lambda^{2}}$ of OSC

\begin{tabular}{|c|c|c|c|}
\hline Halo model & $a=b=c$ & $a>b>c$ & $b>a>c$ \\
\hline $\bar{\lambda}$ & $1.17 \pm 0.01$ & $1.15 \pm 0.01$ & $1.16 \pm 0.01$ \\
\hline$\lambda_{\min }$ & $1.07 \pm 0.01$ & $1.07 \pm 0.01$ & $1.07 \pm 0.01$ \\
\hline$\lambda_{\max }$ & $1.36 \pm 0.15$ & $1.29 \pm 0.15$ & $1.36 \pm 0.15$ \\
\hline$\overline{\lambda^{2}}$ & $1.38 \pm 0.02$ & $1.32 \pm 0.01$ & $1.35 \pm 0.02$ \\
\hline$\lambda_{\min }^{2}$ & $1.14 \pm 0.02$ & $1.14 \pm 0.02$ & $1.14 \pm 0.02$ \\
\hline$\lambda_{\max }^{2}$ & $1.9 \pm 0.5$ & $1.7 \pm 0.5$ & $1.9 \pm 0.5$ \\
\hline
\end{tabular}

where

$$
\begin{gathered}
U(0)=G\left(1.5 M_{1} / R_{1}+0.75 M_{2} J_{0}\right), \\
J_{0}=\int_{0}^{\infty} d s / \Delta(s) \\
\Delta(s)=\sqrt{\left(a^{2}+s\right)\left(b^{2}+s\right)\left(c^{2}+s\right)}
\end{gathered}
$$

$a, b, c$ are the semiaxes of the ellipsoid of the halo directed along the coordinate axes $x, y, z$, respectively;

$$
W=-G\left(0.6 M_{1}^{2} / R_{1}+0.3 M_{2}^{2} J_{0}+0.75 M_{1} M_{2}\left(J_{0}-0.4 R_{1}^{2} /(a b c)\right)\right) .
$$

Let us consider the case of equal densities (and volumes) of a spherical and ellipsoidal cluster halo $a b c=R_{2}^{3}$ and $c / a=0.5, b / a=2 / 3, a=R_{2} \sqrt[3]{3}$ (such semi-axis ratios are adopted taking into account the results on the dimensions of the critical surface of zero-velocity in the two-point non-isolated OSC model (Danilov and Chernova, 2008)).

In the case of an isolated virialized cluster, $\sigma_{v}^{2}$ equals $\sigma_{v}^{\prime 2}=-W / M_{c}$ (Chandrasekhar, 1942). Using the data on the $\xi, \mu, N_{c}, M_{c}, R_{c}=R_{2}, R_{G}$ values for the OSCs from Danilov and Seleznev (1994), we find the $\sigma_{v}, \sigma_{v}^{\prime}$ and $\lambda=\sigma_{v} / \sigma_{v}^{\prime}$ values. The results of calculations for a cluster with an ellipsoidal halo in the case $a>b>c$ are shown in fig. 10.4. The points and triangles in fig. 10.4 indicate the values of $\sigma_{v}$ (in $\mathrm{pc} / \mathrm{Myr} \simeq \mathrm{km} / \mathrm{s}$ ) and $\lambda$ (in dimensionless units), respectively, depending on the cluster mass $M_{c}$. According to fig. 10.4, the $\sigma_{v}$ values grow with an increasing mass $M_{c}$; the growth rate of $\sigma_{v}$ with an increasing $M_{c}$ decreases at large $M_{c}$. The largest values of $\sigma_{v}$ are noted for clusters NGC 6705 and NGC 6755 $\left(\sigma_{v}^{(N G C 6705)}>\sigma_{v}^{(N G C 6755)}\right)$. According to fig. 10.4 , the $\lambda$ values vary slightly as $M_{c}$ increases. The mean values of $\lambda$ and $\lambda^{2}$ for a spherical halo $\left(a=b=c=R_{2}\right)$ and for an ellipsoidal halo $(a>b>c),\left(b>a>c\right.$, at $\left.b=R_{2} \sqrt[3]{3}, a=2 b / 3, c=0.5 b\right)$ are given in Table 10.2. The latter case corresponds to a halo ellipsoid elongated in the direction of the cluster motion. In the case of a spherical halo $\left(a=b=c=R_{2}\right)$, the equations (10.11) and (10.12) give the same values of $\sigma_{v}$ and $\lambda$, but the use of 


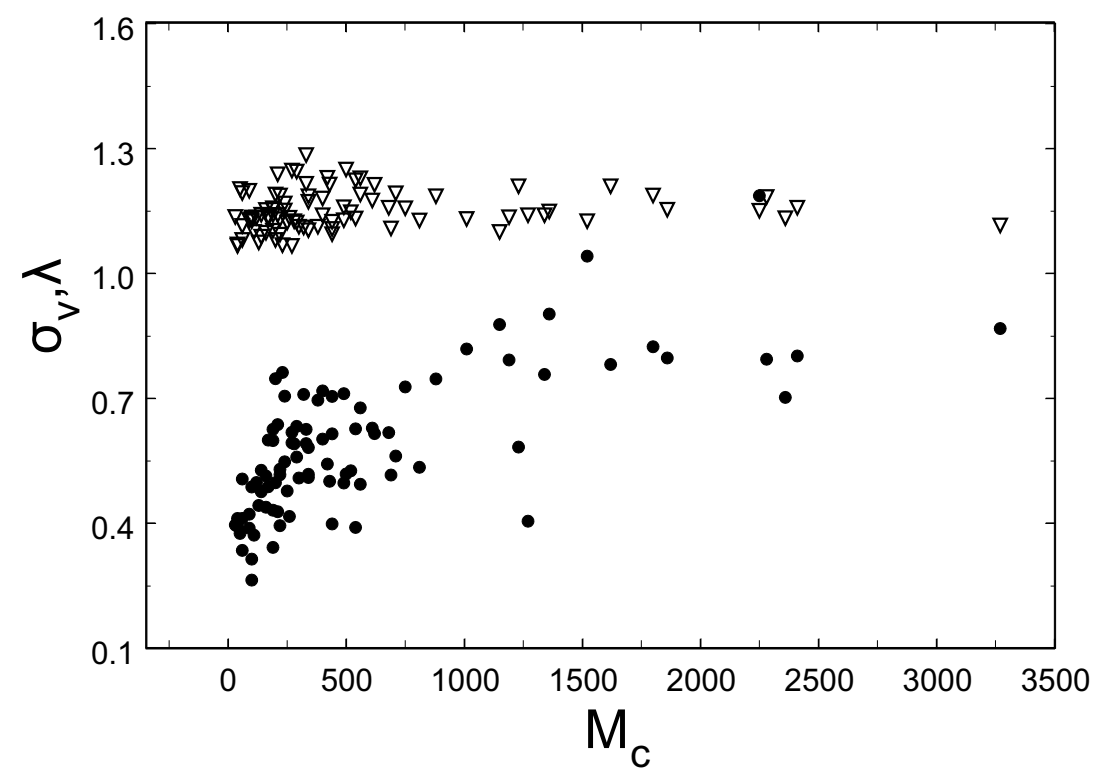

Fig. 10.4 The dependencies of $\sigma_{v}(\bullet)$ and $\lambda(\nabla)$ on the cluster mass $M_{C}$

the equation (10.11) is preferable, since it does not require the calculation of the improper integral $J_{0} ; \lambda_{\min }$ and $\lambda_{\max }$ correspond to the smallest and largest $\lambda$ values in the sample. In all three cases, the $\overline{\lambda^{2}}$ values in Table 10.2 differ a little from each other $\left(\overline{\lambda^{2}} \simeq 1.3-1.4\right)$. We can note a some decrease of $\overline{\lambda^{2}}$ in the case of a halo ellipsoid with $a>b>c$ as compared to $\overline{\lambda^{2}}$ for a spherical halo. $\lambda_{\text {max }}^{2} \simeq 1.7-1.9$.

Within the framework of a spherical halo model, a standard deviation of $\lambda^{2}$ from $\overline{\lambda^{2}}$ equals $\sigma_{\lambda^{2}} \simeq 0.16 . \overline{\lambda^{2}}$ is obtained under the assumption that $\lambda^{2}$ values for the considered OSCs are equally accurate. If we take into account an unequal accuracy of $\lambda^{2}$ for the considered OSCs, we find on the average $\overline{\sigma\left(\lambda^{2}\right) / \lambda^{2}}=0.10 \pm 0.01$, where $\sigma\left(\lambda^{2}\right)$ is an error of $\lambda^{2}$. The values of $\sigma\left(\lambda^{2}\right)$ and $\lambda^{2}$ correlate rather closely $\left(\lambda^{2} \sim \sigma\left(\lambda^{2}\right)\right)$. Therefore, we did not calculate the average weighted values of $\lambda^{2}$. The contribution of the error of the average star mass $\bar{m}$ to $\sigma\left(\lambda^{2}\right)$ is, on average, $\sim 16 \%$ of $\sigma\left(\lambda^{2}\right)$.

Thus, the impact of the Galactic field on the cluster and the non-stationarity of the cluster increase $\sigma_{v}^{2}$ on average in $\sim \overline{\lambda^{2}}$ times. If we use $\sigma_{v}^{2}$ obtained from the observational data on the stellar velocities in order to estimate the virial mass $M_{c}^{\prime}$ of an isolated cluster, then $M_{c}^{\prime} \simeq M_{c} \overline{\lambda^{2}}$. Consequently, the $M_{c}^{\prime}$ values for such estimates are overrated in comparison with $M_{c}$ on average in $\sim \overline{\lambda^{2}}$ times (the largest overestimation of $M_{c}^{\prime}$ relative to $M_{c}$ reach $\lambda_{\text {max }}^{2}$ times, see Table 10.2). Unfortunately, the source of inaccuracy in the estimates of the dynamic OSC masses indicated here does not lead to the most significant errors in $M_{c}$. The 2.5-3.3 times difference in the $M_{c}$ estimates obtained for NGC 6705, NGC 188 in Danilov and Seleznev (1994) 
and Geller et al. (2008); de Grijs et al. (2008) noted in the Danilov (2010) may be partially due to the difference in the data on the size of clusters used in these papers.

\subsection{Conclusions}

In this part of the book, we have considered the estimates of a number of parameters characterizing the non-stationarity of the OSCs and the various manifestations of the non-stationarity in these clusters. We have shown that it is necessary to take into account the non-stationarity of OSCs when estimating the dynamic masses of these clusters.

1. The average value of the density contrast in the OSC cores from Danilov and Seleznev (1994) in the work of Danilov and Putkov (2012a) was obtained to be $\bar{v}=3.62 \pm 0.36$. Such $v$ values are comparable to the smallest estimates of $v$ for the cores of the OSC models of Danilov and Dorogavtseva (2008). The most probable causes of small $v$ in the OSCs are: 1) formation of the OSCs with small $v$ values; 2) an effect of a gravitational instability of the OSC cores and the non-stationarity of the OSCs on the density distribution in the cores of such clusters.

2. We have considered a gross-dynamic description of the homologous oscillations of the OSC models. We wrote the expressions for $\delta \alpha$ and $\alpha_{0}$ in the models of a homogeneous sphere and a sphere with the density $\rho \sim 1 / r^{2}$. We have presented the estimates of the kinetic energy $T_{f}$ of such oscillations in these OSC models. The $T_{f}$ values in these models are very large and, in comparison with the data of numerical experiments of Danilov and Dorogavtseva (2008), lead to an overestimation in 10-20 times of $\delta \alpha$ and other parameters characterizing the degree of the cluster non-stationarity. The reasons for overestimation of these parameters are: 1) a non-homology of the OSC oscillations; 2) a difference of the velocity $v_{r}$ of the radial flux of stars in the OSCs from the relation $v_{r} \sim r$. It is noted that the use of King's distribution for the spatial density of the number of stars $f=f\left(r, k, r_{c}, R_{2}\right)$ leads to an underestimated number of stars in the OSCs on average $0.62 \pm 0.02$ times in comparison with the number of stars obtained in Danilov and Seleznev (1994) by star counts (when one uses the $f$ function, the stars located at the periphery of the OSC are not completely taken into account). We have considered a grossdynamic description of the homologous oscillations of a combined cluster model consisting of a homogeneous sphere and a sphere with the spatial density distribution $f\left(r, k, r_{c}, R_{2}\right)$. Within the framework of this model, the dependency $v_{r} \sim r$ is shown; estimates of the periods $P_{2}$ of the oscillations of the OSCs from Danilov and Seleznev (1994) are given. The average value of $P_{2}$ is $\overline{P_{2}}=31.8 \pm 1.7 \mathrm{Myr}$.

3 . We have considered a gross-dynamic description of the non-homogeneous oscillations of the OSC models consisting of two homogeneous concentric spheres imitating a core and a halo of the cluster. Subject to the constancy of the mass and the halo cluster radius, we have examined the lower bounds of $\delta \alpha$ and $\delta R_{1} / R_{1,0}$ for the OSCs in the framework of such cluster model. The mean $\delta \alpha$ and $\delta R_{1} / R_{1,0}$ for the OSCs from Danilov and Seleznev (1994) are: $\bar{\alpha}=0.09 \pm 0.01$ and $\overline{\delta R_{1} / R_{1,0}}=$ 
$0.34 \pm 0.01$. Solution of the gross-dynamic equations for this OSC model is reduced to quadratures. We have obtained the estimates of $P_{1}$ periods of the OSC core oscillations. Mean $P_{1}$ for the OSCs from Danilov and Seleznev (1994) is $\overline{P_{1}}=$ $16.8 \pm 1.3 \mathrm{Myr} . \overline{\delta R_{1} / R_{1,0}}$ values are $2.3-3.1$ times larger than the relative amplitude of the oscillations of the cluster's halo radius $\delta R_{2} / R_{2,0}$ obtained for the homological oscillations of the cluster model with density $\rho \sim 1 / r^{2}$ and mean $M_{c}, R_{G}, R_{2} / R_{t}$ for the OSCs from Danilov and Seleznev (1994). According to Danilov and Putkov (2012a), the $\delta \alpha$ values show a significant (with probability $P>0.999$ ) correlation with $\delta \alpha_{t h}$ values obtained in another way by Danilov and Seleznev (1994); the mean $\delta \alpha$ values from Danilov and Putkov (2012a) and $\delta \alpha_{t h}$ from Danilov and Seleznev (1994) are quite consistent with each other. Consequently, the oscillations of the OSC cores with the relative amplitudes $\overline{\delta R_{1} / R_{1,0}}$, see above, can quite determine the width $\Delta \xi(\mu)$ of the band occupied by clusters in the diagram $(\xi, \mu)$, and $\Delta \xi(\mu)$ value can also determine the degree of non-stationarity of the OSC with a given $\mu$.

4. We have given the estimates of the dispersion of stellar velocities $\sigma_{v}^{2}$ in the clusters obtained by Danilov and Putkov (2012a) from the data on the structuraldynamic parameters of the OSCs from Danilov and Seleznev (1994). These estimates take into account the influence of the force field of the Galaxy and the non-stationarity of the cluster on $\sigma_{v}^{2}$ value. The effect of these factors increases $\sigma_{v}^{2}$ in OSCs on average in $\overline{\lambda^{2}}=1.3-1.4$ times as compared to $\sigma_{v}^{2}$ estimates for the isolated virialized clusters. According to estimates of the dispersion of the stellar velocities $\sigma_{v}^{2}$ in the clusters performed by Danilov and Putkov (2012a), the largest $\lambda^{2}$ value reaches $\lambda_{\text {max }}^{2} \simeq 1.7-1.9$. Consequently, the virial masses of OSCs obtained from the observational data on the stellar velocities without taking into account the effect

of the external field of the Galaxy on the cluster, are on average $\overline{\lambda^{2}}$ times overvalued estimates of the dynamic masses of the OSCs.

\section{References}

Aarseth, S.J.: Dynamical evolution of simulated star clusters. I - Isolated models. Astron. Astrophys. 35, 237-250 (1974).

Barkhatova, K.A.: An open star cluster NGC 7086. Astronomicheskii Zhurnal, 34, 556-562 (1956) (In Russian).

Chandrasekhar, S.: Principles of Stellar Dynamics. Univ. Chicago Press, Chicago, Ill (1942). LCCN: 43-5608 (BKS1)

Danilov, V.M.: Dynamically isolated groups of stars in non-stationary star clusters. In: Barkahatova, K.A. (ed.) Metody astron.-geod. issled. Ural State Univ. Publ., Sverdlovsk (1984), 94-117 (In Russian).

Danilov, V.M.: Dynamics of Core / Halo Structures in Spherical Stellar Clusters. Sov. Astron. 32, 374-379 (1988).

Danilov, V.M.: The Motion of Halo Stars in Dynamical Numerical Models of Open Clusters. Astron. Reports 49, 3604-610 (2005). doi:10.1134/1.2010649 
Danilov, V.M.: Dynamical models of stellar motions at the peripheries of open clusters. Astron. Reports 50, 346-357 (2006). doi:10.1134/S1063772906050027

Danilov, V.M.: Analysis of density fluctuations in models of open clusters. Astron. Reports 52, 888-899 (2008). doi: $10.1134 /$ S1063772908110036

Danilov, V.M.: Phase-Density Fluctuations at the Centers of Six Open Clusters. Astron. Reports 54, 514-527 (2010). doi:10.1134/S1063772910060053

Danilov, V.M.: On the dynamics of open clusters, Astron. Reports 55, 473-486 (2011). doi:10.1134/S1063772911060035

Danilov, V.M., Chernova, O.A.: Analysis of stellar trajectories in an open cluster model. Astron. Reports 52, 27-39 (2008). doi:10.1134/S1063772908010046

Danilov, V.M., Dorogavtseva, L.V.: Estimates of Relaxation Times in Numerical Dynamical Models of Open Star Clusters. Astron. Reports 47, 483-491 (2003). doi: $10.1134 / 1.1583775$

Danilov, V.M., Dorogavtseva, L.V.: Timescales for mechanisms for the dynamical evolution of open star clusters. Astron. Reports 52, 467-478 (2008). doi:10.1134/S1063772908060048

Danilov, V.M., Putkov, S.I.: Non-stationarity parameters of open clusters. Astron. Reports 56, 609-622 (2012a). doi:10.1134/S106377291208001X

Danilov, V.M., Ryazanov, A.P.: On modelling spherical instationary collisionless stellar systems. In: Barkahatova, K.A. (ed.) Astronomical-geodetical investigations. Ural State Univ. Publ., Sverdlovsk (1985), 19-47 (In Russian).

Danilov, V.M., Seleznev, A.F.: Estimates of the parameters of cores and halos of open star clusters. In: Vasilevsky, A.E. (ed.) Astronomical-geodetical investigations. Ural State Univ. Publ., Sverdlovsk (1989), 26-32 (In Russian).

Danilov, V.M., Seleznev, A.F.: The catalogue of structural and dynamical characteristics of $103 \mathrm{OCl}$ and the first results of its investigations. Astron. Astrophys. Trans. 6, 85-156 (1994). doi:10.1080/10556799408232061

Ferronskii, V.I., Denisik, S.A., Ferronskii, S.V.: The solution of Jacobi's virial equation for celestial bodies. Cel. Mech., 18, 113-140 (1978). doi:10.1007/BF01228711

Geller, A.M., Mathieu, R.D., Harris, H.C. et al.: WIYN Open Cluster Study. XXXII. Stellar Radial Velocities in the Old Open Cluster NGC 188. Astron. J. 135, 22642278 (2008). doi:10.1088/0004-6256/135/6/2264

de Grijs, R., Goodvin, S.P., Kouwenhoven, M.B.N., et al.: Open cluster stability and the effects of binary stars. Astron. Astrophys. 492, 685-693 (2008). doi:10.1051/0004-6361:200810251

Kholopov, P.N.: Star clusters. Nauka, Moscow (1981) (In Russian).

King, I.R.: The structure of star clusters. I. An empirical density law. Astron. J. 67, 471-485 (1962). doi:10.1086/108756

Korn, G.A., Korn, T.M: Mathematical Handbook for Scientists and Engineers. McGraw-Hill, New York, San Francisco, Toronto, London, Sydney (1968).

Kutuzov, S.A., Osipkov, L.P.: A Generalized Model for the Three-Dimensional Gravitational Potential of Stellar Systems. Soviet Astron. 24, 17-22 (1980). 
Kuzmin, G.G.: On the modern state and some next targets of a stellar dynamics. In: Kinematics and dynamics of stellar systems and physics of an interstellar matter. Proc. of Astrophys. Inst. of Acad. Sci. of Kaz.SSR, Alma-Ata (1965) (In Russian). Lynden-Bell, D.: Statistical mechanics of violent relaxation in stellar systems. Mon. Not. R. Astron. Soc. 136, 101-121 (1967). doi:10.1093/mnras/136.1.101

Ossipkov, L.P.: Gross-Dynamics of Open Clusters in the Galaxy. In: Deiters, S., Fuchs, D., Just, A., Spurzem, R., Wielen R. (eds) Dynamics of Star Clusters and Milky Way. ASP Conf. Ser. 228, 341 (2001).

Seleznev, A.F.: The Determination of the Star Number Surface Density in Star Clusters. Astronomicheskii Tsirkulyar. No. 1531/SEPT, 9-10 (1988). (In Russian). 



\title{
Chapter 11 \\ On the Dynamics of Correlations in Models of Open Star Clusters
}

\begin{abstract}
In this chapter, we consider two-time and two-point (two-particle) correlations for a number of parameters of stellar motion, as well as for the density and the phase density of the models of the open star clusters in the vicinity of these stars. We consider estimates of the time and the radius of a correlation of the OSC models in the spaces of indicated parameters. We discuss distributions of two-point correlations over distances between stars in the spaces of the coordinates and the velocities of stars. We determine the parameters of a density, a potential, and the phase density waves in the models of star clusters. The analysis of the fine structure of the two-point correlations' condensations in the space of the mutual distances between stars indicates the formation of the polarization clouds near a number of such distances. We consider the distributions of the correlations of the phase density values. The dynamics of these distributions is discussed. We note a growth of the correlations with time for $50 \%$ of the considered star cluster models. The fluxes of the correlations of the phase density values are discussed. The dominant flux of correlations from the region of strong correlations to the region of weak correlations is detected. Such a flux leads to the appearance of a kinetic energy flux towards the cluster center. The estimates of the rate of heating of the cluster models' cores by this flux is discussed. We note the signs of a weak turbulence in the motions of the stars located in the cluster core of the model with the greatest degree of non-stationarity in a regular field.
\end{abstract}

\subsection{Introduction}

The encounters of stars in the OSCs result in the formation and change with time of both the stellar velocity distributions and the various correlations in the positions of stars in the phase space. Due to a long-range nature of the Newtonian forces, the interactions of stars are continuously ongoing, and have a non-Markovian character (Prigogine and Severne, 1966; Prigogine, 1962). In this case, the rate of change of the velocity distribution function at the time point $t$ depends on the values of this 
function at the previous time points $t-\tau$, where $\tau \in[0, t]$ is the value associated with a nonzero duration of the stellar encounters. Also we refer the reader to the non-Markovian evolution equation (2.1) from Prigogine and Severne (1966) for the velocity distribution function of stars in a homogeneous and infinite gravitating system (gravitational plasma); the derivation of equation (2.1) can be found in the book of Prigogine (1962), formula (11.9). As a result of the interactions, the different states of the correlations (binary, triple, etc.) pass into each other. A flux of the correlations arises, it is an irreversible process (Prigogine and Stengers, 1994, p.174,175). Two types of a correlations' propagation in the system are shown in the book of Prigogine (1962), see figs. 80, 81 there. In the first case, the propagation of a correlation is accompanied by its scattering by other particles. In the second case, this correlation is "born" from the correlation of a lower order.

In the theoretical papers, when studying the spatially inhomogeneous stellar systems, the differential equations for the single-particle distribution functions of stars and the two-particle correlation functions are also used. When writing such equations, the different simplifying assumptions are usually applied (Severne and Haggerty, 1976; Gilbert, 1968, 1970). However, until 2012, the calculations of the correlation functions from the numerical experiments for the dynamic OSC models are absent in the literature. This is due to the exponential instability of the stellar trajectories in such systems (Goodman et al., 1993; Kandrup et al., 1994), as well as a low accuracy of an integration of the stellar motion equations on the sufficiently large time intervals (Komatsu et al., 2008) (usually, an integration uses the difference schemes, the accuracy order of which does not exceed 4 (Goodman et al., 1993; Kandrup et al., 1994; Komatsu et al., 2008)).

The calculations of the phase coordinates of stars (PCS) in the OSC models by Danilov and Dorogavtseva (2008) have been performed by integrating the equations of stellar motion using the difference schemes of the 10th and 11th accuracy orders on the time interval $t \in\left[0, t_{m}\right]$, where $t_{m} \simeq 5.1 \tau_{v r} ; \tau_{v r}$ is the initial time of the violent relaxation obtained according to the formula $\tau_{v r} \simeq 2.6 \bar{t}_{c r}$ (Aarseth, 1974); $\bar{t}_{c r}$ is the average initial crossing time. Let $t_{0}$ be the time interval for the dynamic evolution of the OSC model, during which a statistical criterion for the accuracy of the phase density calculations is satisfied (Danilov, 1997b) (see also Section 16.3). Near the centers of the models of Danilov and Dorogavtseva (2008) the value of $t_{0} / \tau_{v r} \simeq 3.0-3.9$, and at the periphery of the models $t_{0} / \tau_{v r} \simeq 3.6-5.1$. In this case, the maximum relative error in a calculating of the cluster energy reached on the time interval $t_{m}$ in the Danilov and Dorogavtseva (2008) models was $\sim(1-4) \times 10^{-13}$ in modulus, and the accuracy of a calculating of the phase density function (PDF) of the cluster on the time interval $t_{0}$ can be considered as sufficient for the conclusions about the statistical properties of PDF.

It is of interest to estimate the time $\tau_{c}$ and the radius $r_{c}$ of a correlation for the values of $v=|\mathbf{v}|, r=|\mathbf{r}|$; for the star motion energy $\varepsilon$ per unit of the star mass; for the phase density $f=f(\mathbf{r}, \mathbf{v}, t)$ and for the density of the number of stars $n=n(\mathbf{r}, t)$ in the OSC models of Danilov and Dorogavtseva (2008), where $\mathbf{v}$ is the velocity vector, and $\mathbf{r}$ is the radius-vector of the star. These estimates can be used both in 
discussing of the dynamics of the OSC models, and in the theoretical description of the dynamic processes in the OSCs.

Kandrup (1998) proposed a hypothesis on the increase of the overall coherence of the stellar motions (i.e., on the growth of correlations) in the stellar systems far from equilibrium in comparison with the collisionless systems with the ordinary violent relaxation. According to Kandrup (1998), the motion coherence growth and the preservation of a memory about the initial conditions in the stellar systems should be due to the action of the long-range forces, the interactions of the "wave-wave" type, a coupling and an energy transfer between the different modes of the oscillations on a distance scale comparable to the size of the system. In this connection, it is of interest to study the correlations' distribution of $n$ and $f$ by the correlation values in the OSC models, as well as to analyze the changes of these distributions over time (an analysis of the correlation dynamics). At $t \in\left[0, t_{0}\right]$, the accuracy of the obtained correlations is sufficient for the conclusions about the properties of these distributions, see below.

According to Kandrup (1985), a taking into account the long-range forces and inter-particle correlations in the gravitating systems should lead to the transition of a potential energy from the correlations to the kinetic energy of the system (into the energy of the thermal motion of particles). In this connection, it is of interest to study the fluxes of correlations of the phase density values in the models of Danilov and Dorogavtseva (2008), as well as the analysis of the associated kinetic energy fluxes in the OSC models. In this way, it is possible also to estimate the rate of a transfer of the OSC models' oscillation energy into the energy of peculiar stellar motions.

The objectives of this chapter are: 1) to discuss the correlation parameters $\tau_{c}$ and $r_{c}$ for the phase density, the density of the star number, the modules of a velocity vector and of a radius-vector of stars, the specific energies of the star motions in the OSC models of Danilov and Dorogavtseva (2008);2) to discuss the $\tau_{c}$ and $r_{c}$ values and to use $\tau_{c}$ for the construction of the distributions of the two-point correlations for the different time points, as well as to analyze the changes of these distributions over time; 3 ) to discuss the fluxes in the space of the phase density correlations, as well to analyze the related kinetic energy fluxes in the models of Danilov and Dorogavtseva (2008); 4) to apply the reviewed results in order to analyze the OSC dynamics.

\subsection{Times and Radii of Correlations in OSC Models}

Danilov and Dorogavtseva (2008) considered the cluster models consisting of $N=$ 500 stars, moving along a circular orbit of radius $R_{G}=8200 \mathrm{pc}$ in the plane of the Galaxy around its center. The initial parameters of the numerical dynamic OSC models (Danilov and Dorogavtseva, 2008) are given in Table 1 of Danilov and Dorogavtseva (2003); cluster models 1-6 in Danilov and Dorogavtseva (2008) and Danilov and Dorogavtseva (2003) are numbered in a descending order of the degree of the model's non-stationarity in a regular field (for detailed description of the considered OSC models see Section 16.1). 
Following to Klimontovich (1982, p.136), as well as Danilov and Putkov (2012b), in order to determine the correlation time $\tau_{c}^{(r)}$ in the space of $r$, let us consider the two-time correlation:

$$
g_{2}^{(r)}\left(t, t^{\prime}\right)=\overline{r(t) r\left(t^{\prime}\right)}-\overline{r(t)} \cdot \overline{r\left(t^{\prime}\right)}
$$

where the bar denotes an averaging over all cluster stars; $r(t)$ and $r\left(t^{\prime}\right)$ are the $r$ values for the same star in the time points $t$ and $t^{\prime}$. Let $t^{\prime}=t+\tau$. Substituting $t^{\prime}$ in (11.1), averaging the function $g_{2}^{(r)}(t, t+\tau)$ over time points $t \in\left[0, t_{m}-\tau\right]$, we find the $h_{2}^{(r)}(\tau)$ function and its errors. The plot of this function for cluster model 1 is shown in fig. 11.1a. We note that in all figures of our work we present the data obtained by the PCS calculated by the 11th accuracy order, see above. We shall assume that the correlation time $\tau_{c}^{(r)}$ equals $\tau$, at which $h_{2}^{(r)}(\tau)=h_{2}^{(r)}(0) / e$, where $e$ is the base of the natural logarithm. In this case, for cluster model 1 , we find $\tau_{c}^{(r)}=(2.1 \pm 0.2) \tau_{v r}$. The error indicated here for $\tau_{c}^{(r)}$ is due to the error in a calculating of the $h_{2}^{(r)}(\tau)$ function at $\tau=\tau_{c}^{(r)}$. The similar calculations were made for $v$ and $\varepsilon$, where $\varepsilon$ is the energy of the star motion per unit of the star mass. The plots of the functions $h_{2}^{(v)}(\tau)$ and $h_{2}^{(\varepsilon)}(\tau)$ for model 1 are shown in fig. $11.1 \mathrm{~b}$ and $11.1 \mathrm{c}$, respectively. $\tau_{c}^{(v)}$ for model 1 is obtained equal to $\tau_{c}^{(v)}=(1.29 \pm 0.04) \tau_{v r}$, and $\tau_{c}^{(\varepsilon)} \gtrsim 5.1 \tau_{v r}$. Thus, $\tau_{c}^{(v)}<\tau_{c}^{(r)}<\tau_{c}^{(\varepsilon)}$.

During the evolution of the cluster model 1 , the correlations are most rapidly destroyed in the space of $v$ values, and most slowly in the space of $\varepsilon$. We note that these relations between the correlation times of model 1 are quite agreeing with the relations between the relaxation times $\tau_{v}<\tau_{r}<\tau_{\varepsilon}$, obtained from the data on the stellar fluxes in the spaces of $v, r, \varepsilon$ for this model by Danilov and Dorogavtseva (2003). According to Table 2 from Danilov and Dorogavtseva (2003) for cluster model 1, we can write $\tau_{v} \lesssim \tau_{c}^{(v)}, \tau_{r} \simeq \tau_{c}^{(r)}, \tau_{c}^{(\varepsilon)} \simeq \tau_{\varepsilon}=(5.2 \pm 0.8) \tau_{v r}$. The $\tau_{v r}$ $\tau_{v}, \tau_{r}$ values characterize the time of the cluster's transition to a virial equilibrium only in the absence of the conditions for a gravitational instability (Danilov, 2011). According to Danilov and Dorogavtseva (2008), the periodic oscillations of the stellar velocities' dispersions along the coordinate axes in model 1 are still conserved at $t \simeq 5.1 \tau_{v r}$; the synchronization time of a rotation of this cluster model with its orbital motion is $t_{s}=(7.5 \pm 0.2) \tau_{v r}$, and the spherization time of the stellar velocity distribution in model 1 is $t_{\sigma} \simeq(7.6-8.9) \tau_{v r}$. For models $2-6$, the relations $\tau_{c}^{(v)}<\tau_{c}^{(r)}<\tau_{c}^{(\varepsilon)}<t_{s}, t_{\sigma}$ are also fulfilled, see Table 11.1, as well as Danilov and Dorogavtseva (2008). Therefore, the time to smooth out the rapid random changes in models 1-6 when calculating the correlation radii in the spaces of $v, r, \varepsilon$ in Danilov and Putkov (2012b) was chosen equal to the corresponding correlation time, see also Klimontovich (1982, p.139).

We note a slight increase of $h_{2}^{(v)}(\tau)$ at $\tau / \tau_{v r} \in[3.3,4.8]$ in fig. 11.1b, as well as increase of $h_{2}^{(v)}(\tau)$ at $\tau / \tau_{v r} \in[0.5,1.5]$ in fig. 11.1d. Perhaps, for the space of the $v$ values in the OSC models, there are two or more correlation times, which is a sign of a non-stationarity of such systems (Klimontovich, 1982, p.137,143). 

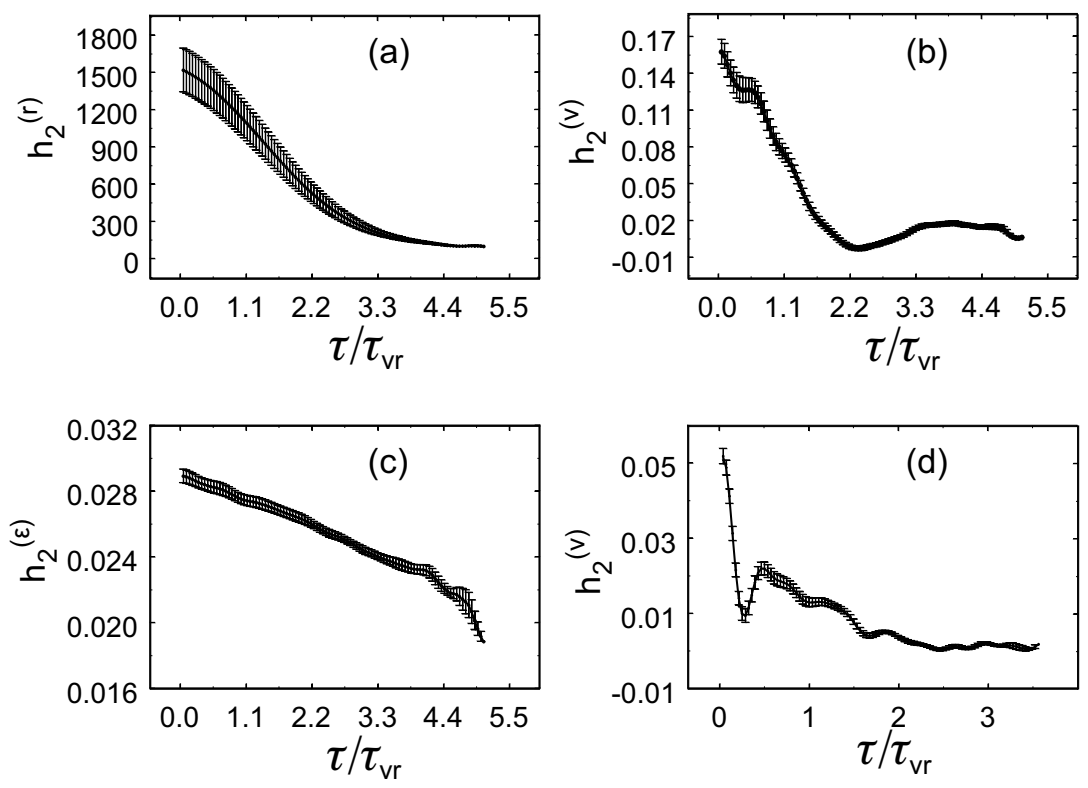

Fig. 11.1 The dependencies of $h_{2}^{(r)}, h_{2}^{(v)}, h_{2}^{(\varepsilon)}$ on $\tau$ for the OSC model 1 (a-c); the dependency of $h_{2}^{(v)}$ on $\tau$ for the OSC model 5 (d)

Following to Klimontovich (1982); Danilov and Putkov (2012b), we consider a two-point correlation for $r$ :

$$
k_{2}^{(r)}(t, i, j)=\left\langle r_{i}\left(t^{\prime}\right) r_{j}\left(t^{\prime}\right)\right\rangle-\left\langle r_{i}\left(t^{\prime}\right)\right\rangle\left\langle r_{j}\left(t^{\prime}\right)\right\rangle, \quad i, j=1, \ldots, N
$$

where $i, j$ are the numbers of the stars; brackets $\langle\ldots\rangle$ mean the averaging over time $t^{\prime} \in\left[t, t+\tau_{c}^{(r)}\right]$ of the values indicated under the sign of the brackets, at $t \leq t_{m}-\tau_{c}^{(r)}$. Let $r_{i j}(t)=\left\langle\left|\mathbf{r}_{i}\left(t^{\prime}\right)-\mathbf{r}_{j}\left(t^{\prime}\right)\right|\right\rangle$, where an averaging over $t^{\prime}$ was performed in the same interval as for $k_{2}^{(r)}(t, i, j)$. The value of $k_{2}^{(r)}(t, i, i)$ characterizes the correlation between positions of the $i$-th star in the space of $r$ at different times $t^{\prime}$ and is equal to the dispersion of the $r_{i}$ deviations from $\left\langle r_{i}\right\rangle$ on the time interval $t^{\prime} \in\left[t, t+\tau_{c}^{(r)}\right]$. Danilov and Putkov (2012b) calculated $k_{2}^{(r)}(t, i, j)$ and $r_{i j}(t)$ for the time points $t \in\left[0, t_{m}-\tau_{c}^{(r)}\right]$. Fig. 11.2a shows the distribution of $k_{2}^{(r)}(t, i, j)$ by $r_{i j}(t)$ on the interval $r_{i j}(t) \leq 2 R_{t}$, averaged over $t \in\left[0, t_{m}-\tau_{c}^{(r)}\right]$, where $R_{t}$ is the tidal radius of the cluster model 1 obtained according to King (1962). The mean over $t$ values of $k_{2}^{(r)}(t, i, j)$ in fig. 11.2a are denoted as $m_{2}^{(r)}$ and are given in $\mathrm{pc}^{2}$ depending on $r_{i j}$. The vertical bars in fig. 11.2a indicate the errors of $m_{2}^{(r)}$.

We shall assume that the correlation radius $r_{c}^{(r)}$ is the value of $r_{i j}$, at which $m_{2}^{(r)}=\left.e^{-1} m_{2}^{(r)}\right|_{r_{i j}=0}$. In this case, for model 1 , we find $r_{c}^{(r)}=0.74 \pm 0.03 \mathrm{pc}$. The 

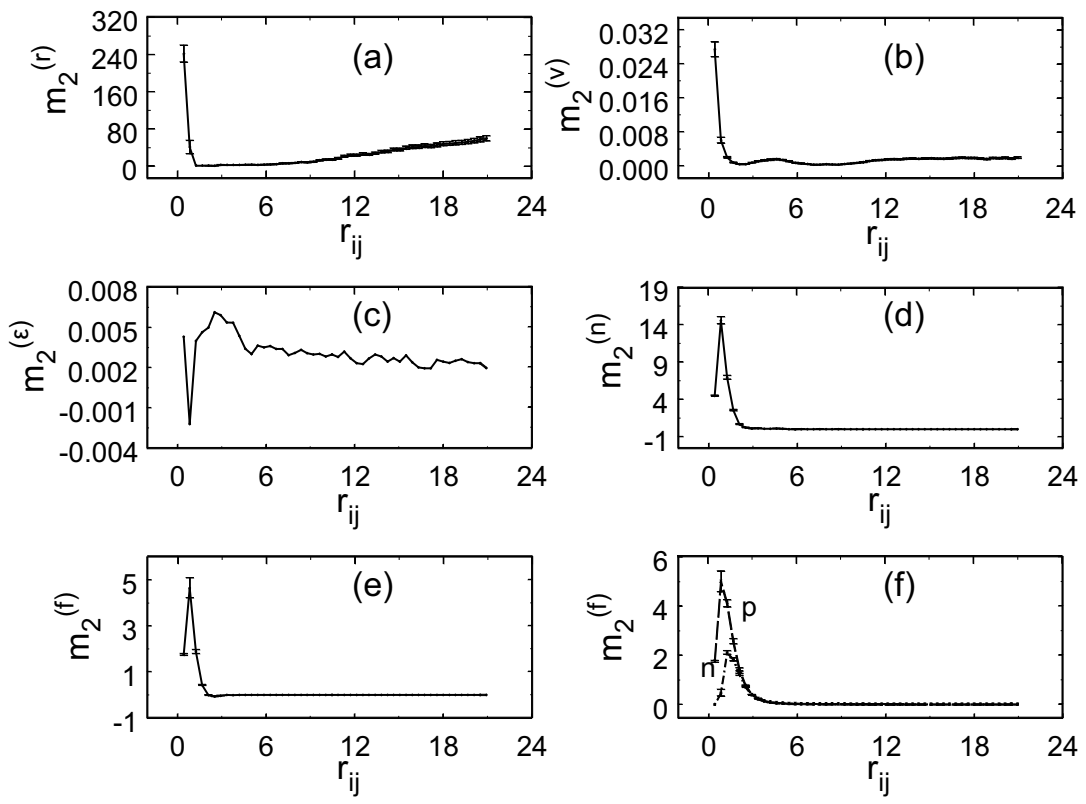

Fig. 11.2 The dependencies of $m_{2}^{(r)}, m_{2}^{(v)}, m_{2}^{(\varepsilon)}, m_{2}^{(n)}, m_{2}^{(f)}$ on $r_{i j}$ in the OSC model 1

error of $r_{c}^{(r)}$ indicated here is due to the error in the calculation of the $m_{2}^{(r)}$ function of $r_{i j}$ at $r_{i j}=r_{c}^{(r)}$. The similar calculations were performed for $v, \varepsilon, n$ and $f$, where estimates of the number density of stars $n$ and phase density $f$ were obtained in the vicinity of each star considering the data on phase coordinates of this star and the five stars closest to it. The correlation disruption in the space of $v$ in model 1 occurs at the distances $r_{c}^{(v)}=0.76 \pm 0.02 \mathrm{pc}$, see fig. $11.2 \mathrm{~b}$, where $m_{2}^{(v)}$ values are given in (pc/Myr) $)^{2}$. Thus, $r_{c}^{(r)} \simeq r_{c}^{(v)}$. At $t=1.6 \tau_{v r}$, the number of stars inside the sphere of this radius changes from $N_{p h}=20.1 \pm 7.2$ in the center of model 1 to $N_{p h}=1.6 \pm 0.2$ at the average values of the core density of this cluster model (see Table 2 from Danilov (2010)). In this case, an average distance $r_{m} \sim n^{-1 / 3}$ between the stars changes from $r_{m}=0.44 \pm 0.06 \mathrm{pc}$ to $r_{m}=1.04 \pm 0.03 \mathrm{pc}$, respectively. Thus, $r_{c}^{(r)} \simeq r_{c}^{(v)} \simeq r_{m}$.

According to fig. 11.2a the $m_{2}^{(r)}$ values increase with growing $r_{i j}$ at $r_{i j}>1.5$ pc. The degree of correlation of the $r$ values in model 1 continues to increase with increasing $r_{i j}$ also at $r_{i j}>2 R_{t}$. In this case, the cluster stars with $r_{i}>R_{t}$ form a group, the motion of which is largely determined by the force field of the Galaxy, which leads to a sufficiently coordinated motion of these stars in the Galaxy and to a formation of the large values of $\left|m_{2}^{(r)}\right|$ (see also fig. 11.3a). A certain role in increasing the correlations at $r_{i j} \rightarrow \infty$ belongs to transfer of the correlations to larger distances from the cluster center as the outer regions expand as a result of the escape 
of a part of the stars from the cluster (see Saslaw (1985, p.197) on a propagation of the correlations in the problem of a clustering of galaxies in the expanding universe).

In fig. 11.2c the values of $m_{2}^{(\varepsilon)}$ values are given in (pc/Myr) . According to fig. $11.2 \mathrm{c}$, with increasing $r_{i j}$ at $r_{i j}<1 \mathrm{pc}, m_{2}^{(\varepsilon)}$ rapidly decreases reaching $m_{2}^{(\varepsilon)}<0$. $r_{c}^{(\varepsilon)}$ is obtained equal to $r_{c}^{(\varepsilon)} \simeq 0.6 \pm 0.01 \mathrm{pc}$ from the conditions $\left.m_{2}^{(\varepsilon)}\right|_{r_{i j}=r_{c}^{(\varepsilon)}}=$ $\left.e^{-1} m_{2}^{(\varepsilon)}\right|_{r_{i j}=0}$ and $m_{2}^{(\varepsilon)}=k_{2}^{(\varepsilon)}(0, i, j)$, since $\tau_{c}^{(\varepsilon)}>t_{m}$, and here for the estimation of $r_{c}^{(\varepsilon)}$, we accept $\tau_{c}^{(\varepsilon)}=t_{m}$. The negative $m_{2}^{(\varepsilon)}$ values at $r_{i j} \in[0.71,0.97] \mathrm{pc}$ indicate the influence of stellar encounters (the exchange of energies of the stars participating in encounters) in the formation of the distribution $m_{2}^{(\varepsilon)}$ by $r_{i j}$ at such $r_{i j}$ (see Saslaw (1985, p.173) on the relationship between the sign of the correlations and the direction of changes in the fluctuating values). According to fig. $11.2 \mathrm{c}$, the stellar energies are most correlated in the range of $r_{i j} \in[2.2,3.1] \mathrm{pc}$, which indicates the existence in the model of the radial waves of a potential and a force field with a wavelength $\lambda^{(\varepsilon)}=2.51 \pm 0.21 \mathrm{pc}$ (since in the models of Danilov and Dorogavtseva (2008) the radial density and potential oscillations are mainly observed). A reduction of the degree of randomness of the stellar motions and changes in their energies at $r_{i j} \simeq \lambda^{(\varepsilon)}$ may well lead to a decrease in the production of an entropy and to self-organization in the cluster model (for open systems, see Klimontovich (1995, p.45,49)). At $r_{i j}>\lambda^{(\varepsilon)}$, the value of $m_{2}^{(\varepsilon)}$ slowly decreases with increasing $r_{i j}$, which indicates the disruption of such correlations. At $r_{i j}=r_{c, e}^{(\varepsilon)}=16.25 \pm 0.13$ pc, $m_{2}^{(\varepsilon)}$ decreases in $e$ times as compared to $\left.m_{2}^{(\varepsilon)}\right|_{r_{i j}=\lambda^{(\varepsilon)}}$. Thus, in model 1, the oscillations in $\varepsilon$ with the wavelengths of $\lambda \in\left[\lambda^{(\varepsilon)}, r_{c, e}^{(\varepsilon)}\right]$ are formed with a different probability. Apparently, in this wavelength interval, the effect of the force field oscillations of model 1 on the stellar energy can be considered as significant.

In fig. 11.2d, the $m_{2}^{(n)}$ values are given in $\mathrm{pc}^{-6}$. The distribution of $m_{2}^{(n)}$ by $r_{i j}$ reaches its maximum close to $r_{i j}=\lambda^{(n)} \simeq 0.84 \pm 0.21 \mathrm{pc}$. At $r_{i j}>\lambda^{(n)}, m_{2}^{(n)}$ decreases with increasing $r_{i j}$ and reaches $\left.e^{-1} m_{2}^{(n)}\right|_{r_{i j}=\lambda^{(n)}}$ at $r_{i j}=r_{c}^{(n)}=1.41 \pm 0.02$ pc. In order to calculate $m_{2}^{(n)}$ and $m_{2}^{(f)}$, we used the $\tau_{c}^{(r)}$ value with the averaging by $t$ given above, since $\tau_{c}^{(r)}>\tau_{c}^{(v)}$.

We note that the distributions of $m_{2}^{(n)}$ and $m_{2}^{(f)}$ by $r_{i j}$ are very similar each other and reach their peaks near $r_{i j}=0.84 \pm 0.21 \mathrm{pc}$, see fig. $11.2 \mathrm{e}$, where $m_{2}^{(f)}$ are given in $\left(\mathrm{Myr}^{6} / \mathrm{pc}^{12}\right)$. Consequently, in model 1 , the radial density and phase density waves with a wavelength $\lambda^{(n)}$ exist and, possibly, with the wavelengths $\lambda=i_{n} \lambda^{(n)}$, where $i_{n}$ is an integer $\left(i_{n}=2,3,4\right.$, see below). The reduction of the degree of a randomness of the changes in $n$ and $f$ (and, consequently, in the stellar motions) at $r_{i j} \simeq \lambda^{(n)}$ may well lead to a decrease in the production of an entropy and a self-organization in the cluster model (Klimontovich, 1995). The value of $r_{i j}=r_{c}^{(f)}$ corresponding to the condition $\left.m_{2}^{(f)}\right|_{r_{i j}}=\left.e^{-1} m_{2}^{(f)}\right|_{\left.\lambda^{(n)}\right)}$ is obtained equal to $r_{c}^{(f)}=1.30 \pm 0.02 \mathrm{pc}$. Fig. 11.2f shows the distributions of $m_{2}^{(f)}$ for the positive and negative $k_{2}^{(f)}(t, i, j)$ values marked by the letters $p$ and $n$, respectively (in the case $k_{2}^{(f)}(t, i, j)<0$ in fig. 
$11.2 \mathrm{f}$ the $\left|m_{2}^{(f)}\right|$ values are given). At $k_{2}^{(f)}(t, i, j)>0$, the largest values of $m_{2}^{(f)}$ are reached at $r_{i j}=\lambda_{p}^{(f)}=0.84 \pm 0.21 \mathrm{pc}$, and the values $m_{2}^{(f)}=\left.e^{-1} m_{2}^{(f)}\right|_{r_{i j}=\lambda_{p}^{(f)}}$ are reached at $r_{i j}=r_{c, p}^{(f)}=2.00 \pm 0.03$ pc. At $k_{2}^{(f)}(t, i, j) \leq 0$, the largest $\left|m_{2}^{(f)}\right|$ values are reached at $\lambda_{n}^{(f)} \simeq 1.26 \pm 0.21 \mathrm{pc}$, and values $m_{2}^{(f)}=\left.m_{2}^{(f)}\right|_{\lambda_{n}^{(f)}}$ are reached at $r_{i j}=r_{c, n}^{(f)}=2.50 \pm 0.02 \mathrm{pc}$. In the space of $r_{i j}$, the wavelengths $\lambda_{p}^{(f)}$ and $\lambda_{n}^{(f)}$ correspond to the sizes of the phase density fluctuations with the largest correlations values modulo. The wavelengths of the corresponding $f$ oscillations in model 1 are enclosed in the intervals $\lambda \in\left[\lambda_{p}^{(f)}, r_{c, p}^{(f)}\right]$ and $\lambda \in\left[\lambda_{n}^{(f)}, r_{c, n}^{(f)}\right]$.

Fig. 11.3 shows the values of $y_{r}=k_{2}^{(r)}(0, i, j)$ depending on $r_{i j}$ for model 1 . When calculating $y_{r}$, we used $\tau_{c}^{(r)}$. According to fig. 11.3, at $\left|y_{r}\right|<50 \mathrm{pc}^{2}$ and $r_{i j}<2 R_{t} \simeq 21 \mathrm{pc}$, the regions with the positive and negative correlations are closely intermixed in the space of values $r_{i j}$. Consequently, the processes of an increasing and a decreasing of $r$ for the stars with numbers $i$ and $j$ included in the pair $(i, j)$, occur in the same regions of the space of $r_{i j}$. However, for close $r_{i j}$ values, the values $y_{r}>0$ reach several times higher magnitudes than $\left|y_{r}\right|$ at $y_{r}<0$, which indicates the prevalence of changes in $r$ with the same sign of $\dot{r}=\frac{d r}{d t}$. Apparently, the pairs of stars with larger $y_{r}>0$ participate in the collective radial stellar motions in the cluster model 1. Probably, the pairs of stars with $y_{r}<0$ and $r_{i j}<(3-4) \mathrm{pc}$ are involved in the chaotic motion to a greater extent. In this case, the negative values of $y_{r}$ can be due to the effect of the stellar encounters.

We note that at $r_{i j}<2 R_{t}$ and $y_{r}<0$, the sign of $y_{r}$ can also be due to the participation of a pair of stars in a collective motion (for example, in the radial density waves with a wavelength $\lambda$, if $r_{i j} \simeq\left(2 k_{\lambda}+1\right) \lambda / 2$ at $\left.k_{\lambda}=0,1,2, \ldots\right)$. Fig. $11.3 \mathrm{~b}$ shows a fragment of the region given in fig. 11.3a. At $r_{i j}>12 \mathrm{pc}$ in fig. 11.3b, the vertical areas of an increased density are seen as the condensations of points near the certain values of $r_{i j}$. For brevity, let us call them as $C_{r}$-condensations. Such condensations contain points with the different values of the correlation moduli (also including the rather large values). The presence of $C_{r}$-condensations indicates the existence of the radial density waves at the cluster periphery (see below).

Fig 11.4a shows the values $y_{v}=k_{2}^{(v)}(0, i, j)$ depending on $r_{i j}$ for model 1. When calculating $y_{v}$, we used the value $\tau_{c}^{(v)}\left(\tau_{c}^{(v)}<\tau_{c}^{(r)}\right)$. Therefore, the largest $r_{i j}$ values in fig. 11.4a are smaller than in fig. 11.3a (in time $\tau_{c}^{(r)}$, stars with the energies above the critical value manage to leave the cluster for a longer distance than in time $\left.\tau_{c}^{(v)}\right)$. In fig. 11.4a, as in fig. 11.3a, the positive correlations dominate, and most points are located in the region $\left|y_{v}\right|<0.03(\mathrm{pc} / \mathrm{Myr})^{2}$. Probably, in this case, a significant part of the correlations in fig. 11.4a is due to the action of the collective processes on the positions of stars in the phase space. Perhaps, a small (in size) cloud of points in the region $r_{i j}<4.4 \mathrm{pc}$ is due to the action of the stellar encounters, an influence of which in fig. 11.4a is more noticeable than in fig. 11.3a.

Fig. 11.4b shows the values $y_{\varepsilon}=k_{2}^{(\varepsilon)}(0, i, j)$ depending on $r_{i j}$ for model 1. In order to estimate $y_{\varepsilon}$, we adopted the value $\tau_{c}^{(\varepsilon)}=5.1 \tau_{v r}$. In the region of $r_{i j}<25$ pc, occupied mainly by the stars with the energies below a critical one, in fig. 

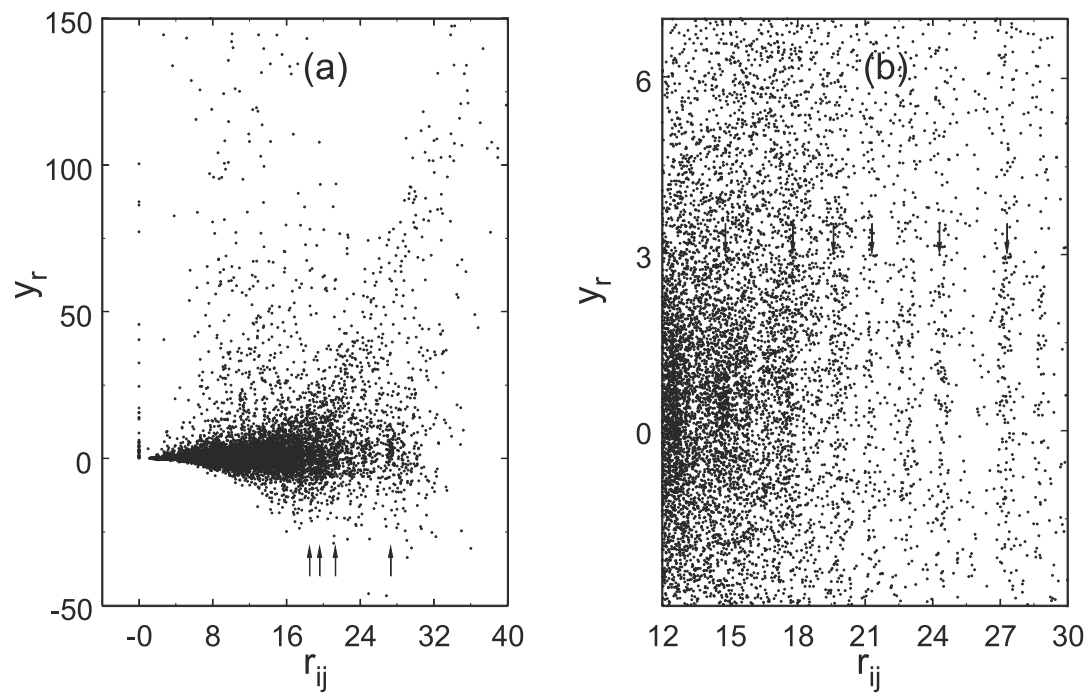

Fig. 11.3 The diagrams $\left(y_{r}, r_{i j}\right)$ for the OSC model 1. The arrows show some of the most visible $C_{r}$-condensations

$11.4 \mathrm{~b}, C_{r}$-condensations of the points near certain $r_{i j}$ values are well visible. Such condensations of points can be caused by the presence in the model 1 of radial waves of density and potential with the corresponding wavelengths $\lambda \simeq r_{i j}$. At $r_{i j} \rightarrow \infty$, the maximum $\left|y_{\varepsilon}\right| \rightarrow 0$. In this case, one of the stars of the pair $(i, j)$ is located near the cluster center and experiences the random changes in the time interval $\tau_{c}^{(\varepsilon)}$, and the second star is located at large distances from the cluster and moves away from it. Over time, the changes of stellar energies in such pairs are becoming less and less correlated. When $r_{i j}<12 \mathrm{pc}$ and $r_{i j} \rightarrow 0$, both a dispersion of the $y_{\varepsilon}$ values and the contribution of stellar encounters to the changes of energies $\varepsilon$ increase (the main part of the fig. $11.4 \mathrm{~b}$ is composed by the points with $\left|y_{\varepsilon}\right|<0.005$ (pc/Myr) ${ }^{4}$, which indicates the chaotic changes of $\varepsilon$ ). Probably, a significant role in the changes of the energy of stars at $r_{i j} \gg 25$ pc near certain $r_{i j}$ values, corresponding to the $C_{r}$-condensations of points, can be played by the external field of the Galaxy and the resonances between the frequencies of motion of these stars relative to the cluster and the frequency of the orbital motion of the cluster relative to the center of the Galaxy.

Danilov and Putkov (2012b) calculated the values of $y_{n}=k_{2}^{(n)}(t, i, j)$ and $y_{f}=$ $k_{2}^{(f)}(t, i, j)$ for a number of the time points $t \in\left[0, t_{m}-\tau_{c}^{(r)}\right]$ (when calculating $y_{n}$ and $y_{f}$, we used $\left.\tau_{c}^{(r)}\right)$. Fig. $11.4 \mathrm{c}$ shows the values of $y_{n}=k_{2}^{(n)}(0, i, j)$ depending on $r_{i j}$ for model 1 . The diagram $\left(y_{f}, r_{i j}\right)$ has a form similar to that shown in fig. $11.4 \mathrm{c}$, and, therefore, is not given here. As in the case of fig. 11.3a, on figs. 11.4a,b the positive correlations of $y_{n}$ and $y_{f}$ predominate over negative in their number and absolute magnitude. The causes and mechanisms considered above also take part 

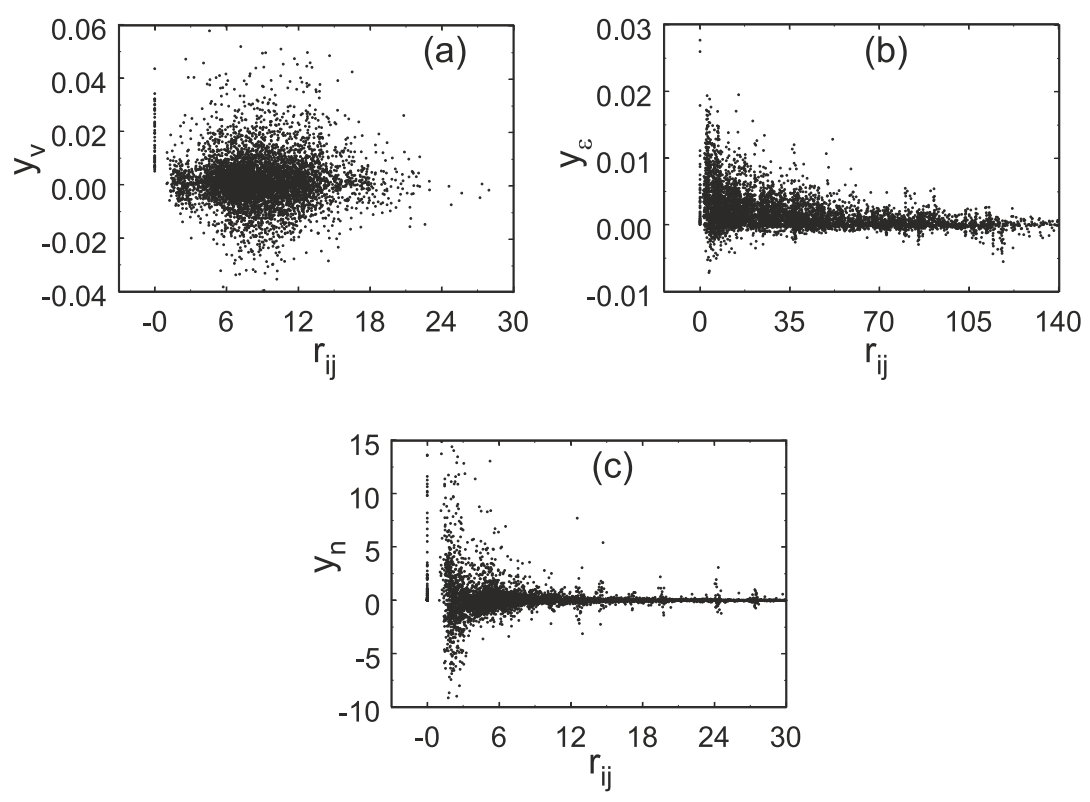

Fig. 11.4 The diagrams $\left(y_{v}, r_{i j}\right),\left(y_{\varepsilon}, r_{i j}\right),\left(y_{n}, r_{i j}\right)$ for the OSC model 1

in the formation of peculiarities of the points' location in fig. 11.4c. In the interval of distances $r_{i j} \in[10,28] \mathrm{pc}$ in fig. $11.4 \mathrm{c}$ the $C_{r}$-condensations of the points are visible located near some values of $r_{i j}$, which indicates the existence of the radial waves of the density and the phase density at the cluster periphery. Distances $\Delta r_{i j}$ between the $C_{r}$-condensations of the points nearest to each other are $\lambda \sim 1.7,2.5,3.4$ pc, which approximately $2,3,4$ times exceeds the value $\lambda^{(n)} \simeq 0.84 \mathrm{pc}$, see above. The $C_{r}$-condensations of the points in figs. $11.4 \mathrm{c}, 11.3 \mathrm{~b}$ and on the diagram $\left(y_{f}, r_{i j}\right)$ are located near almost identical values of $r_{i j}$. Near the same $r_{i j}$ values, the $C_{r}$ condensations of points are also detected in figs. 11.4a,b when they are examined with a larger resolution.

Comparison of the diagrams $\left(y_{n}, r_{i j}\right)$ obtained using the PCS of the 10th and 11th accuracy orders, showed that the typical errors (the standard deviations from the mean) of $y_{n}$ and $r_{i j}$ are $0.2 \times 10^{-4} \mathrm{pc}^{-6}$ and $0.7 \times 10^{-7} \mathrm{pc}$, respectively, and the largest errors are approximately 3 times larger than the indicated values, which is much smaller than the size of the points in fig. 11.4c. Thus, there is a detailed agreement between the diagrams $\left(y_{n}, r_{i j}\right)$, obtained using the PCS of the 10th and 11th accuracy orders (in these diagrams, both the positions of the $C_{r}$ condensations and the number of points in these condensations coincide). The diagrams in fig. 11.3, 11.4a have the same characteristics.

Comparison of the diagrams $\left(y_{\varepsilon}, r_{i j}\right)$ obtained using the PCS of the 10th and 11th accuracy orders showed that the typical errors of $y_{\varepsilon}$ and $r_{i j}$ in fig. $11.4 \mathrm{~b}$ are $0.11 \times 10^{-2}(\mathrm{pc} / \mathrm{Myr})^{2}$ and $0.49 \mathrm{pc}$, respectively. The noticeably larger errors of $y_{\varepsilon}$, 
$r_{i j}$ in fig. $11.4 \mathrm{~b}$ compared with the errors of $y_{n}, r_{i j}$ in fig. $11.4 \mathrm{c}$, are due to the fact that in calculating $y_{\varepsilon}$ and $r_{i j}$ for fig. 11.4b the averaging of the values of $\varepsilon_{i}, \varepsilon_{j}$, $\varepsilon_{i} \varepsilon_{j}$ and $r_{i j}$ are given over time points $t, \sim 40 \%$ of which satisfies the inequality $t>t_{0}$. Despite this, the distributions of $y_{\varepsilon}, r_{i j}$, the position and density of the $C_{r}$-condensations in diagrams $\left(y_{\varepsilon}, r_{i j}\right)$ obtained using the PCS of the 10th and 11th accuracy orders are largely consistent with each other. The correlation coefficient $k_{r}$ of the $r_{i j}$ values, obtained with the 10th and 11th accuracy orders, is $k_{r} \simeq 0.99991$. The correlation coefficient $k_{\varepsilon}$ of the $y_{\varepsilon}$ values, obtained with the 10th and 11th accuracy orders, is $k_{\varepsilon} \simeq 0.92 . k_{r}$ and $k_{\varepsilon}$ are close to unity; for model $1, t_{0} \simeq 3.1 \tau_{v r}$, see above.

Danilov and Leskov (2005) carried out a Fourier analysis of the stellar trajectories at the periphery of cluster model 1 . In the distribution of the stellar trajectories over their periods, "peaks" were found, with periods commensurate with the oscillation period of the system's regular field. According to Danilov and Leskov (2005) and Danilov (2005), the formation of small groups of the halo stars' trajectories with such periods indicates that a set of distances from the cluster center is formed in the system, near which the halo stars preferentially move in the presence of the regular field oscillations. Probably, the formation of many $C_{r}$-condensations of points in figs. $11.3,11.4$ is due to the formation in the cluster model of such groups of trajectories, as well as of the polarization clouds (Gilbert, 1968, 1970), the gravitational excitations, which are a close analog of plasmons in plasma (Saslaw, 1985, p.92), and also are the response of the system to the motion of stars near the distances from the cluster center mentioned above. The $C_{r}$-condensations of points are also observed in the diagrams $\left(y_{\mathbf{x}}, r_{i j}\right)$ for the OSC models 2-6 (here, $\left.\mathbf{x}=(r, v, \varepsilon, n, f)\right)$. We note that such condensations are not formed in the diagrams $\left(y_{\mathbf{x}}, v_{i j}\right)$ in the OSC models 1-6, which indicates the existence of an effective mechanism that destroys such condensations in the space of values $v_{i j}=\left\langle\left|\mathbf{v}_{i}\left(t^{\prime}\right)-\mathbf{v}_{j}\left(t^{\prime}\right)\right|\right\rangle$ in these models $\left(\tau_{v}<\tau_{r}\right.$ (Danilov and Dorogavtseva, 2003)). An analysis of the distributions of $m_{2}^{(\mathbf{x})}$ by $v_{i j}$ indicates the existence of the waves of the coordinates' values of the vector $\mathbf{x}$ in the space of $v_{i j}$ with the wavelengths $v_{i j}=\lambda_{v}^{(\mathbf{x})}(\mathrm{pc} / \mathrm{Myr})$ in the OSC models 1-6 (Table 11.1).

Estimates of the correlation parameters for the OSC models 1-6 are given in the Table 11.1 (the $\lambda_{v}^{(\mathbf{x})}$ values are given in the columns of Table 11.1 for each model from top to bottom in an order of decreasing of the corresponding values of $m_{2}^{(\mathbf{x})}>0$ ). According to Table 11.1, $r_{c}^{(v)}$ and $r_{c, n}^{(f)}$ grow with increasing mass and size of the core of the OSC model; the $r_{c}^{(\varepsilon)}$ values grow, and the $r_{c}^{(f)}$ values decrease with increasing density of the cluster model. We note that, as the model number increases, in the time dependencies of $h_{2}^{(v)}$, a noticeable local maximum appears (see, for example, fig. 11.1d for model 5), which corresponds to the oscillations with a period $P^{(v)}$ in the space of $v$. The $P^{(v)}$ values for models 3-6 are also given in Table 11.1 and are in a good agreement with the periods of the oscillations of these models' virial coefficient (see column 2 of Table 2 in Danilov and Leskov (2005)). 
Table 11.1 Parameters of OSC models

\begin{tabular}{|c|c|c|c|c|c|c|}
\hline № of model & 1 & 2 & 3 & 4 & 5 & 6 \\
\hline$\tau_{c}^{(r)} / \tau_{v r}$ & $2.1 \pm 0.2$ & $2.0 \pm 0.2$ & $1.7 \pm 0.2$ & $1.9 \pm 0.3$ & $1.8 \pm 0.2$ & $1.7 \pm 0.2$ \\
\hline$\tau_{c}^{(v)} / \tau_{v r}$ & $1.29 \pm 0.04$ & $1.39 \pm 0.06$ & $1.0 \pm 0.2$ & $0.35 \pm 0.04$ & $0.19 \pm 0.01$ & $0.22 \pm 0.01$ \\
\hline$P^{(v)} / \tau_{v r}$ & - & - & $0.62 \pm 0.02$ & $0.76 \pm 0.03$ & $0.50 \pm 0.02$ & $0.58 \pm 0.02$ \\
\hline$\tau_{c}^{(\varepsilon)} / \tau_{v r}$ & $>5.1$ & $>5.1$ & $>4.1$ & $>5.1$ & $>3.6$ & $>3.9$ \\
\hline$r_{c}^{(r)}, \mathrm{pc}$ & $0.74 \pm 0.03$ & $0.68 \pm 0.02$ & $0.69 \pm 0.02$ & $0.69 \pm 0.02$ & $0.70 \pm 0.01$ & $0.73 \pm 0.01$ \\
\hline$r_{c}^{(v)}, \mathrm{pc}$ & $0.76 \pm 0.02$ & $0.73 \pm 0.01$ & $0.88 \pm 0.07$ & $0.75 \pm 0.01$ & $1.36 \pm 0.09$ & $2.41 \pm 0.09$ \\
\hline$r_{c}^{(\varepsilon)}, \mathrm{pc}$ & $0.60 \pm 0.01$ & $0.68 \pm 0.21$ & $0.68 \pm 0.06$ & $0.78 \pm 0.09$ & $0.68 \pm 0.21$ & $0.87 \pm 0.24$ \\
\hline$r_{c, e}^{(\varepsilon)}, \mathrm{pc}$ & $16.25 \pm 0.13$ & $2.80 \pm 0.46$ & $4.80 \pm 0.05$ & $3.87 \pm 0.10$ & $2.47 \pm 0.10$ & $2.78 \pm 0.77$ \\
\hline$\lambda^{(\varepsilon)}, \mathrm{pc}$ & $2.51 \pm 0.21$ & $1.26 \pm 0.22$ & $1.67 \pm 0.22$ & $2.09 \pm 0.21$ & $1.26 \pm 0.21$ & $1.67 \pm 0.21$ \\
\hline$r_{c}^{(n)}, \mathrm{pc}$ & $1.41 \pm 0.02$ & $1.29 \pm 0.03$ & $1.25 \pm 0.01$ & $1.29 \pm 0.02$ & $1.33 \pm 0.02$ & $1.46 \pm 0.03$ \\
\hline$r_{c}^{(f)}, \mathrm{pc}$ & $1.30 \pm 0.02$ & $1.29 \pm 0.02$ & $1.21 \pm 0.02$ & $1.17 \pm 0.01$ & $1.53 \pm 0.01$ & $1.15 \pm 0.03$ \\
\hline$r_{c, p}^{(f)}, \mathrm{pc}$ & $2.00 \pm 0.03$ & $1.35 \pm 0.03$ & $1.46 \pm 0.03$ & $1.54 \pm 0.02$ & $1.90 \pm 0.01$ & $1.15 \pm 0.03$ \\
\hline$r_{c, n}^{(f)}, \mathrm{pc}$ & $2.50 \pm 0.02$ & $1.25 \pm 0.02$ & $2.59 \pm 0.01$ & $1.96 \pm 0.02$ & $3.31 \pm 0.01$ & $5.825 \pm 0.03$ \\
\hline$\lambda^{(f)}, \mathrm{pc}$ & $0.84 \pm 0.21$ & $0.84 \pm 0.21$ & $0.84 \pm 0.21$ & $0.84 \pm 0.21$ & $0.84 \pm 0.21$ & $0.84 \pm 0.21$ \\
\hline$\lambda_{v}^{(r)}$, & $0.09 \pm 0.02$ & $0.07 \pm 0.01$ & $0.07 \pm 0.01$ & $0.66 \pm 0.01$ & $0.07 \pm 0.01$ & $0.88 \pm 0.01$ \\
$\mathrm{pc} / \mathrm{Myr}$ & - & - & - & - & $0.74 \pm 0.01$ & - \\
\hline$\lambda_{v}^{(v)}$, & $0.10 \pm 0.01$ & $0.12 \pm 0.01$ & $0,16 \pm 0.01$ & $0.16 \pm 0.05$ & $0.18 \pm 0.01$ & $0.13 \pm 0.01$ \\
$\mathrm{pc} / \mathrm{Myr}$ & - & - & - & - & $0.14 \pm 0.01$ & - \\
\hline$\lambda_{v}^{(\varepsilon)}$, & $0.47 \pm 0.02$ & $0.46 \pm 0.02$ & $0.18 \pm 0.02$ & $0.20 \pm 0.02$ & $0.27 \pm 0.02$ & $0.24 \pm 0.02$ \\
$\mathrm{pc} / \mathrm{Myr}$ & $0.16 \pm 0.02$ & $0.25 \pm 0.02$ & $0.27 \pm 0.02$ & $0.44 \pm 0.02$ & - & - \\
\hline$\lambda_{v}^{(n)}$, & $0.19 \pm 0.02$ & $0.15 \pm 0.01$ & $0.17 \pm 0.01$ & $0.07 \pm 0.01$ & $0.19 \pm 0.01$ & $0.13 \pm 0.01$ \\
$\mathrm{pc/Myr}$ & - & $0.27 \pm 0.01$ & - & $0.17 \pm 0.01$ & $0.10 \pm 0.01$ & - \\
\hline$\lambda_{v}^{(f)}$, & $0.15 \pm 0.02$ & $0.27 \pm 0.01$ & $0.10 \pm 0.01$ & $0.17 \pm 0.01$ & $0.19 \pm 0.01$ & $0.08 \pm 0.01$ \\
$\mathrm{pc} / \mathrm{Myr}$ & - & $0.15 \pm 0.01$ & $0.24 \pm 0.01$ & $0.07 \pm 0.01$ & - & - \\
\hline$\tau_{f} / \tau_{v r}$ & $0.51 \pm 0.01$ & $0.52 \pm 0.01$ & $0.47 \pm 0.01$ & $0.52 \pm 0.01$ & $0.56 \pm 0.01$ & $0.67 \pm 0.02$ \\
\hline$v_{v^{2}}$ & $0.22 \pm 0.02$ & $0.14 \pm 0.01$ & $0.027 \pm 0.004$ & $0.017 \pm 0.003$ & $0.012 \pm 0.002$ & $0.011 \pm 0.002$ \\
\hline$\tau_{v r} / T$ & $1.56 \pm 0.23$ & $0.50 \pm 0.09$ & $0.17 \pm 0.07$ & $0.14 \pm 0.05$ & $0.10 \pm 0.08$ & $0.095 \pm 0.094$ \\
\hline$\Delta t / \tau_{v r}$ & 0.0507 & 0.1014 & 0.082 & 0.1014 & 0.0712 & 0.0772 \\
\hline
\end{tabular}

\subsection{Distributions of Correlations}

Let $N_{k}\left(y_{n}\right)$ be the number of correlations $y_{n}$ in a certain interval of the $y_{n}$ values. In order to discuss the dynamics of the correlations in the cluster model 1 , let us consider the distributions of the correlation numbers $N_{k}\left(y_{n}\right)$ and $N_{k}\left(y_{f}\right)$ over $y_{n}$ and $y_{f}$ for several time points. Fig. 11.5a,b show the histograms of the distribution of $N_{k}\left(y_{n}\right)$ and $N_{k}\left(y_{f}\right)$ for the time points $t / \tau_{v r}=1.06,2.54$ (in fig. 11.5a,b corresponding histograms are marked with numbers 1 and 2 ). According to fig. 11.5a,b the correlations with small $\left|y_{n}\right|$ and $\left|y_{f}\right|$ significantly dominate in their number and density of distribution over the correlations with large $\left|y_{n}\right|$ and $\left|y_{f}\right|$. Over time, the 

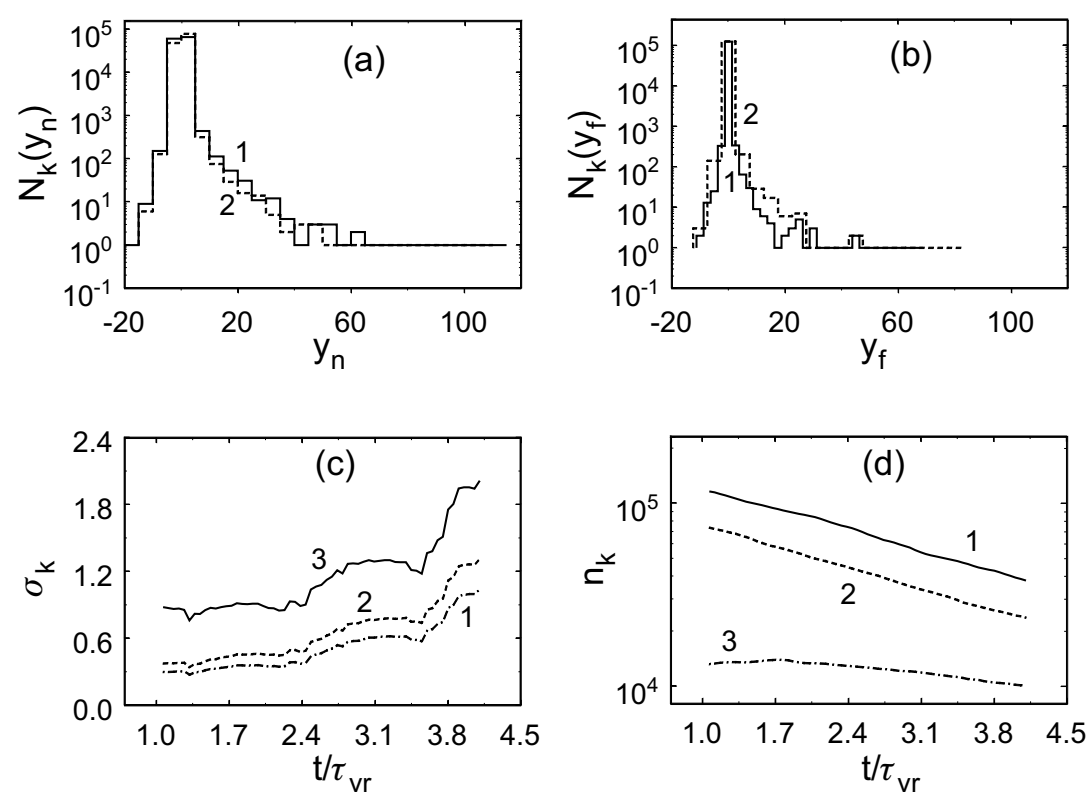

Fig. 11.5 The histograms of the distribution of $N_{k}\left(y_{n}\right), N_{k}\left(y_{f}\right)(\mathrm{a}, \mathrm{b})$ and the time dependencies of $\sigma_{k}, n_{k}$ for the OSC model $1(\mathrm{c}, \mathrm{d})$

distribution $N_{k}\left(y_{f}\right)$ is more noticeably expanded along the abscissa axis than the distribution $N_{k}\left(y_{n}\right)$. According to diagrams $\left(y_{n}, r_{i j}\right),\left(y_{f}, r_{i j}\right)$, see fig. $11.4 \mathrm{c}$, small values of $\left|y_{n}\right|$ and $\left|y_{f}\right|$ are generally reached in the region of large $r_{i j}$.

Fig. $11.5 \mathrm{c}$ shows the time dependencies of standard deviations $\sigma_{k}$ of $y_{f}$ from the mean $y_{f}$ for a given $t$ in the cases $r_{i j} \leq 0.5 R_{t}, R_{t}, 2 R_{t}$ (in fig. $11.5 \mathrm{c}$ the curves corresponding to these cases are marked with numbers $1,2,3$, respectively; $y_{f}$ values are given in $\left.\left(\mathrm{Myr}^{6} / \mathrm{pc}^{12}\right)\right)$.

Fig. $11.5 \mathrm{~d}$ shows the time dependencies of the number $n_{k}$ of the star pairs $(i, j)$ for the cases $r_{i j} \leq 0.5 R_{t}, R_{t}, 2 R_{t}$ (the curves corresponding to these cases are marked in fig. $11.5 \mathrm{~d}$ with numbers $1,2,3$, respectively). Letter $t$ in figs. $11.5 \mathrm{c}, \mathrm{d}$ marks the value $\left\langle t^{\prime}\right\rangle=t+0.5 \tau_{c}^{(r)}$ equal to the average value of the time points $t^{\prime}$ used to calculate the correlations $y_{f}$, see explanations to formula (11.2). According to fig. $11.5 \mathrm{c}, \mathrm{d}$, the values of $\sigma_{k}$ mainly grow, and the $n_{k}$ values mainly decrease with increasing $t$, which indicates an increase over time of the correlation of the phase density fluctuations in the cluster model 1 , which is especially noticeable in the region of small $r_{i j}$ (i.e., mainly in the cluster core), as well as a systematic decrease with time of the number of stars and the number of correlations in the cluster core. Thus, the hypothesis of Kandrup (1998) on the growth of the correlations (and, consequently, a coherence of the stellar motions) in such systems is confirmed.

Danilov (2002b) showed that an entropy of the star system of the cluster model 1 decreases with the distances from its center $r \leq R_{t}^{-}(t)$ during the evolution $\left(R_{t}^{-}(t)\right.$ 
is the tidal radius of the cluster obtained by Danilov (2002b) from the data on the phase coordinates of the stars with the retrograde trajectories in the cluster). According to Danilov (2002b), a decrease of an entropy is due to a decrease in the number of stars with $r \leq R_{t}^{-}(t)$ in the cluster. However, the second reason for a decrease in an entropy may be the growth of correlations in the cluster. During the evolution, model 1 approaches the state of stable equilibrium, and at $t \simeq 2.7 \tau_{v r}$ this model is already sufficiently close to it (see fig. 11.2 from Danilov (2011), where the position of the boundary of the unstable oscillations' region for model 1 is determined by the auxiliary collisionless homogeneous ellipsoidal cluster model (Danilov, 2008)). According to fig. 11.5c, in the range of values $t / \tau_{v r} \in[2.85,3.55]$, the growth of correlations in the core of model 1 is slowed down, and $\sigma_{k}$ even begins to decrease. If at $t \simeq(3.1-3.2) \tau_{v r}$ the cluster model 1 crosses the boundary of the region of the unstable oscillations of the phase density $f$, then the oscillations inside the region of the stable oscillations cease to be supported by the action of the gravitational instability and begin to decay, which should lead model 1 to a stable equilibrium, followed by an increase of entropy and a core compression due to the stellar encounters and a dissipation of the high-energy stars. In this case, according to fig. 2 from Danilov (2011), model 1 returns to the region of the unstable oscillations, and the growth of correlations continues.

Probably, in some cases the increase of $\sigma=\left(\sigma_{k}\right)_{r_{i j} \leq 0.5 R_{t}}$ over $t$ can be used as one of the empirical signs of a self-organisation of such systems (in addition to the criteria for a self-organisation considered by Klimontovich (1995, p.49,487), associated with a minimum of an entropy production in the system). When constructing the criterion for a self-organisation of the OSCs and their models, it is necessary to take into account the presence of the complex-conjugate roots of the dispersion equation (31) from Danilov (2008) in the problem of the natural frequencies of the small phasedensity oscillations at the cluster center. Thus, the effect of a gravitational instability in the OSC models significantly changes the classical scenario of the evolution of the systems of this type to the state of a stable equilibrium (with an increasing entropy) described by Klimontovich (1995). We note that the rapid loss of the stars by the clusters can also lead to an increase in $\sigma$ over time $t$ (in this case, the stars leaving the cluster are weakly connected with the other stars, they take away many correlations with the small $\left|y_{f}\right|$, and the proportion of the correlations with the large $\left|y_{f}\right|$ increase in the cluster). Model 4 is more free of this effect due to its larger density, see fig. 11.6c. In model 4, it is convenient to observe the change of the stages of cooling and heating of the core as a result of the formation and destruction of the correlations with large $\left|y_{f}\right|$.

A comparison of the $\sigma$ values obtained in the framework of the 10th $\left(\sigma_{10}\right)$ and 11th $\left(\sigma_{11}\right)$ accuracy orders for model 1 , showed that when $t \leq 3.7 \tau_{v r}$, the values of $\left|\sigma_{10}-\sigma_{11}\right| / \sigma_{11} \leq 0.22$ and $\sigma_{10}<\sigma_{11}$. At $t>3.7 \tau_{v r}$, the value of $\left|\sigma_{10}-\sigma_{11}\right| / \sigma_{11}$ rapidly grows with increasing $t$. Apparently, the results of the calculations of $\sigma$ for the cluster model 1 should not be used when $t>3.7 \tau_{v r}$. We note that at $t>2.1 \tau_{v r}$, the maximum values of $t^{\prime}$ used for calculating $y_{f}$, begin to satisfy a condition $t^{\prime}>t_{0} \simeq 3.1 \tau_{v r}$ near the center of the cluster model 1 , which leads to the appearance of the small differences in the estimates of $\sigma_{10}$ and $\sigma_{11}$ at $t>2.1 \tau_{v r}$. 

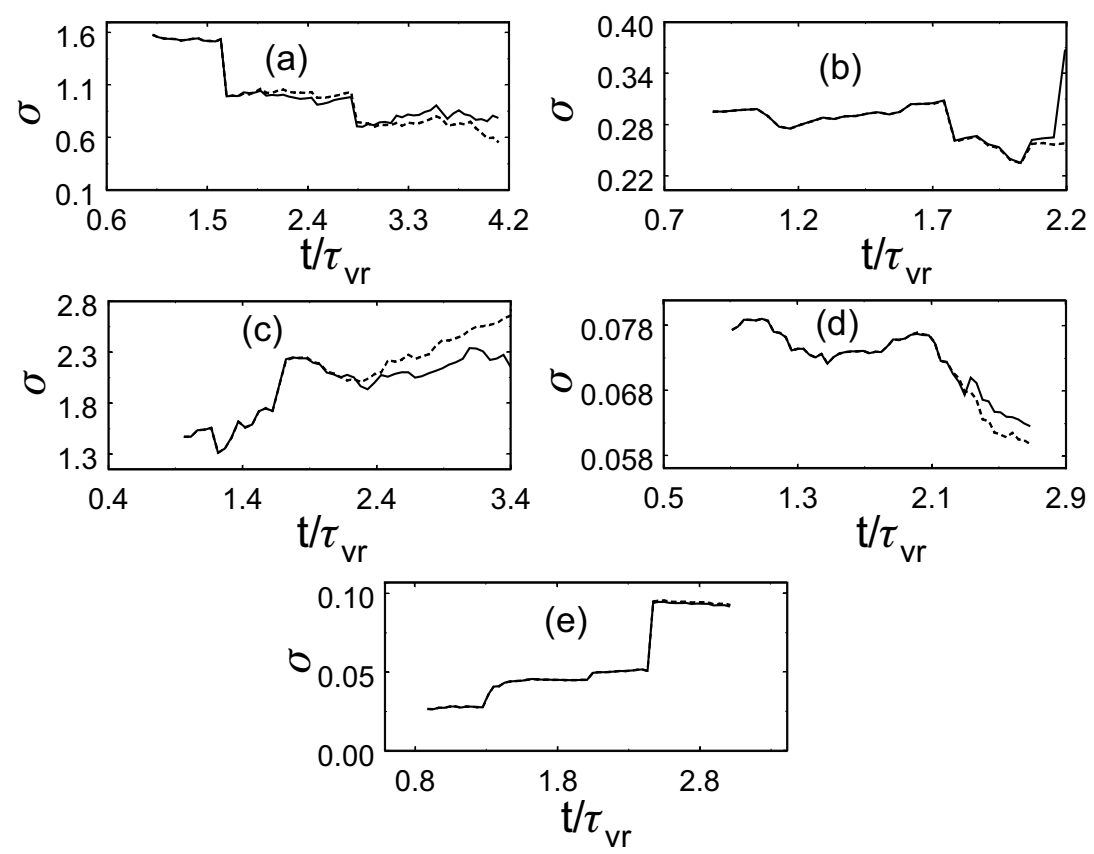

Fig. 11.6 The time dependencies of $\sigma$ for the OSC models 2-6 respectively (a-e)

Fig. 11.6 shows the time dependencies of $\sigma$ for the OSC models 2-6. The solid and dashed lines indicate the dependencies $\sigma=\sigma_{11}(t)$ and $\sigma=\sigma_{10}(t)$, respectively. In contrast to model 1 (see curve 3 in fig. 11.5c), in model 2, $\sigma$ decreases with time, which is due to the greater density of model 2 , smaller loss of stars in comparison with model 1, and a destruction of the correlations due to the stellar encounters. The saltatory changes in $\sigma$ are caused by the escape of one or several stars from the region of the large $\left|y_{f}\right|$ (according to the estimates of Section 11.4 of this book, each of these stars participates in the creation of $\sim 20-40$ correlations with the large $\left.\left|y_{f}\right|\right)$. In model 3 , the radius and the mass of the core, the dispersion of the residual stellar velocities, and the influence of the tidal forces of the Galaxy are greater than in model 2 (see fig. 1 of Danilov (2011), and also column 2 in Table 3 from Danilov (2011)). A large dispersion of the stellar velocities in model 3 leads to a weakening of the correlations between the stars in a comparison with the correlations in model 2 (see fig. 11.6a,b), and to a decrease of $\sigma$ over time $t$ due to the tidal heating of the cluster. Model 5 also evolves in a similar way, see fig. 11.6d. In model $6, \sigma$ abruptly increases over time $t$, which is caused by the formation of the correlations with the large $\left|y_{f}\right|$. A definite contribution to the formation of the correlations in models 3-6 can be made by the oscillations with the periods $P^{(v)}$ mentioned in Section 11.3 of this book (see Table 16.1 of Section 16.1) and by the collective stellar motions associated with these oscillations. 


\subsection{Fluxes of Correlations in the Space of $y_{f}$}

Following to Danilov (2002b), we will divide all $K=N(N+1) / 2=125250$ of the values of $y_{f}$ of the cluster model at some time point $t$ into groups of $k=12525$ values in the order of an augmentation of $y_{f}$. Then the values $y_{f}^{\prime} \in$ $\left(\left(y_{f}\right)_{k \times(i-1)+1},\left(y_{f}\right)_{k \times i}\right] \equiv\left(Y_{f}\right)_{i}(t)$ set in the space of $y_{f}$ the value of the interval $\Delta\left(y_{f}\right)_{i}=\left(y_{f}\right)_{k \times i}-\left(y_{f}\right)_{k \times(i-1)+1}$, where $i=2, \ldots, 10$. When $i=1$, it is necessary to consider the interval of the values of $y_{f}^{\prime} \in\left[\left(y_{f}\right)_{k \times(i-1)+1},\left(y_{f}\right)_{k \times i}\right] \equiv\left(Y_{f}\right)_{1}(t)$, in order to include also the correlation with the smallest $\left(y_{f}\right)_{1}$ in the consideration. Let $n^{\left(y_{f}\right)}(i, j)$ be the number of correlations that pass in time $\Delta t$ in the space of $y_{f}$ from the interval $i$ to the interval $j$. This number can be obtained from the data on the phase coordinates of the stars (PCS) of the cluster model at times $t$ and $t+\Delta t$ :

$$
n^{\left(y_{f}\right)}(i, j)=\sum_{p=1}^{K} \delta_{p}
$$

where $\delta_{p}=1, \quad$ if $\quad\left(y_{f}\right)_{p}(t) \in\left(Y_{f}\right)_{i}(t), \quad\left(y_{f}\right)_{p}(t+\Delta t) \in\left(Y_{f}\right)_{j}(t+\Delta t)$; $\delta_{p}=0$ in all other cases.

Values $\Delta\left(y_{f}\right)_{i}$ at the time points $t$ and $t+\Delta t$ are usually not equal to each other for any value $i=1, \ldots, 10$. The value of $n^{\left(y_{f}\right)}(i, j) / \Delta t$ is equal to the flow of correlations from the interval $\left(Y_{f}\right)_{i}(t)$ to the interval $\left(Y_{f}\right)_{j}(t+\Delta t)$ in the space of $y_{f}$. In the framework of the methods used to set the $\Delta\left(y_{f}\right)_{j}$ values, we find a total number of the transitions of the correlations in the time $\Delta t$ to the interval $\Delta\left(y_{f}\right)_{j}$ :

$$
\sum_{i=1}^{10} n^{\left(y_{f}\right)}(i, j)=k, j=1, \ldots, 10 .
$$

Formulas (11.4) are convenient to use in order to check a correctness of the computations of the matrix $n^{\left(y_{f}\right)}(i, j)$. In the case of an equilibrium in the space of $y_{f}$ (a balance of the correlation flows), the matrix $n^{\left(y_{f}\right)}(i, j)$ should be symmetrical (Danilov, 2002b). Following to Danilov (2002b), the average number of the transitions of the correlations in time $\Delta t$ on $j$ intervals in the space of $y_{f}$ can be written in the following form:

$$
v^{\left(y_{f}\right)}(j)=\left\{\begin{array}{l}
\frac{1}{10+j} \sum_{i=1-j}^{10} n^{\left(y_{f}\right)}(i, i+j), j=-9, \ldots,-1 \\
\frac{1}{10-j} \sum_{i=1}^{10-j} n^{\left(y_{f}\right)}(i, i+j), j=0,1, \ldots, 9 .
\end{array}\right.
$$

In formulas (11.5), the numbers of all possible transitions of correlations to $j$ intervals are averaged in the space $y_{f}$. The values $j>0(<0)$ correspond to an increase (decrease) of the $y_{f}$ value as a result of such transition; $v^{\left(y_{f}\right)}=v^{\left(y_{f}\right)}(j)$ is the distribution of the mean transition numbers by the $j$ values in the space of $y_{f}$ in time $\Delta t$. A symmetry of this distribution with respect to $j=0$ indicates a 

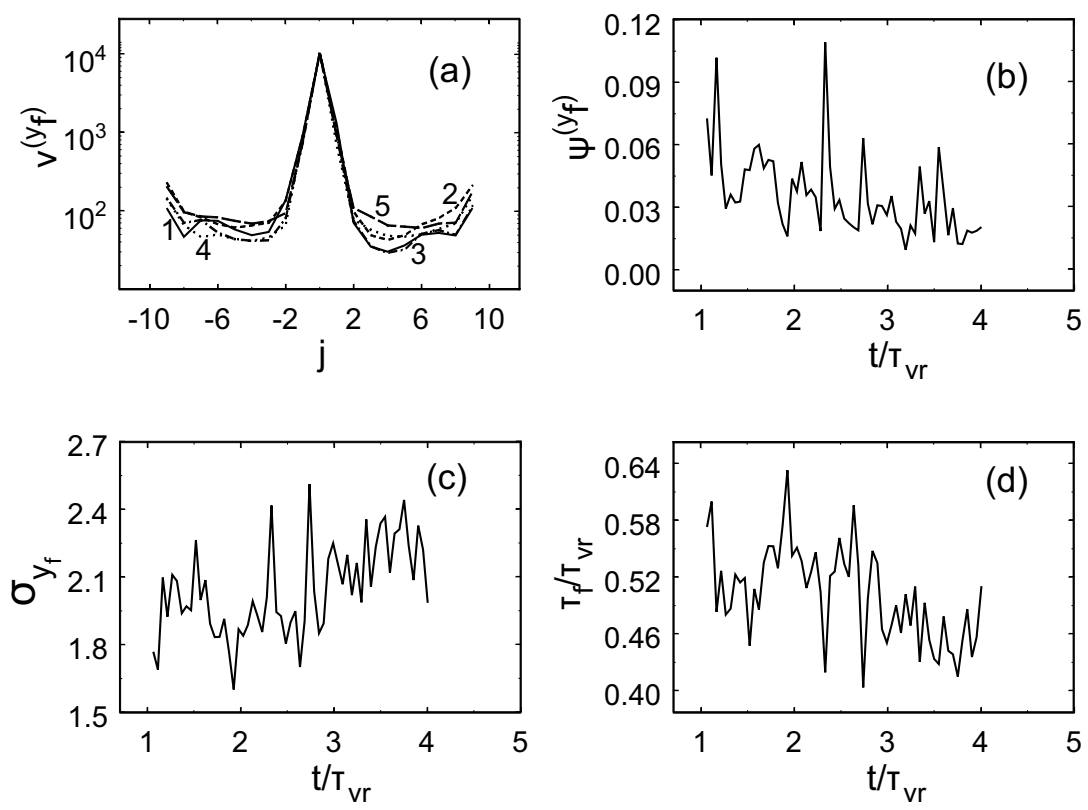

Fig. 11.7 The distributions of $v^{\left(y_{f}\right)}=v^{\left(y_{f}\right)}(j)$ (a) and the time dependencies of $\left.\psi^{\left(y_{f}\right)}, \sigma_{(} y_{f}\right)$, $\tau_{f}$ for the OSC model $1(\mathrm{~b}-\mathrm{d})$

balance in the transitions of the correlations in the space of $y_{f}$. According to Danilov (2002b), the degree of non-symmetry of the matrix $n^{\left(y_{f}\right)}(i, j)$ can be expressed in one number:

$$
\psi^{\left(y_{f}\right)}=\frac{\sum_{j=1}^{10} \sum_{i=1}^{10}\left|n^{\left(y_{f}\right)}(i, j)-n^{\left(y_{f}\right)}(j, i)\right|}{\frac{1}{2} \sum_{j=1}^{10} \sum_{i=1}^{10}\left(n^{\left(y_{f}\right)}(i, j)+n^{\left(y_{f}\right)}(j, i)\right)} .
$$

Let $\sigma_{y_{f}}^{2}$ be a dispersion of the distribution $v^{\left(y_{f}\right)}$. The number of intervals $\Delta y_{f}$ "traversed" by the correlation $y_{f}$ in the time $\Delta t$ towards the increasing (or decreasing) is equal on average to $v_{y_{f}}=0.5 \sigma_{y_{f}} / \Delta t$. Since the cluster size in the space of $y_{f}$ is equal to ten intervals $\Delta y_{f}$ (see the explanations to the formula (11.3), then a relaxation time of the cluster in the space of $y_{f}$ is $\tau_{f}=10 / v_{y_{f}}=20 \Delta t / \sigma_{y_{f}}$ (in model 1 , the value $\Delta t=0.0507 \tau_{v r}$ was used, so that $\left.\tau_{f}=1.014 \tau_{v r} / \sigma_{y_{f}}\right)$. Thus, $\tau_{f}$ is the mean time of the change of the correlation $y_{f}$ under the action of all the forces and mechanisms present in the cluster by the value of the order of the cluster size in the space of $y_{f}$.

The results of the calculations using the formulas (11.3), (11.5), (11.6) for model 1 are shown in fig. 11.7. In fig. $11.7 \mathrm{a}$, for five time points $t_{i} / \tau_{v r}=$ $1.06,1.52,2.03,2.54,3.04$, the plots of the distributions $v^{\left(y_{f}\right)}=v^{\left(y_{f}\right)}(j)$ are given, 
the numbers $i$ near the curves correspond to the time points $t_{i}$. For all the considered time points, the matrices $n^{\left(y_{f}\right)}(i, j)$ are largely symmetric, the diagonal elements of these matrices are much larger than the other ones; the distribution $v^{\left(y_{f}\right)}=v^{\left(y_{f}\right)}(j)$ are quite symmetric with respect to $j=0$. Therefore, in model 1 , there is a state close to the balance of the transitions in the $y_{f}$ space over the entire considered time interval. The model is dominated by the transitions of the correlations $y_{f}$ in $j$ to $\Delta j=0,1,2$. According to fig. $11.7 \mathrm{~b}$, the degree of an asymmetry of the matrix $n^{\left(y_{f}\right)}(i, j)$ decreases slightly with time, on average, and the dispersion $\sigma_{y_{f}}^{2}$ of the distribution $v^{\left(y_{f}\right)}$, according to fig. $11.7 \mathrm{c}$, weakly grows with an increase of $t$ on average. Thus, during the evolution of model 1 , the balance of the correlation transitions in the space of $y_{f}$ is fulfilled more and more accurately, and the role of the correlation transitions in $j$ to a greater number of intervals $\Delta j$ somewhat increases.

According to fig. $11.7 \mathrm{~d}$, the $\tau_{f}$ value on average changes a little with time and is of about $\tau_{f}=(0.50 \pm 0.01) \tau_{v r}$ at $t \leq 4.01 \tau_{v r}$ and $\tau_{f}=(0.52 \pm 0.01) \tau_{v r}$ at $t \leq 3.04 \tau_{v r}$. This value is in a good agreement with the time $t_{c}^{(1)}$ of the development of a PDF instability with respect to the small initial perturbations in the core of model 1, see Table 2 of Danilov and Dorogavtseva (2003). In the paper of Danilov and Dorogavtseva (2003), $t_{c}^{(1)} \simeq 0.5 \tau_{v r}$ is considered as an estimate of the local relaxation time of the core of the cluster model 1 .

According to Danilov and Dorogavtseva (2003), in the compared versions a and b of model 1 (with small differences in the initial PCS), at the time $t \simeq t_{c}^{(1)}$, under the conditions of an exponential instability of the trajectories, the stars already occupy, with a nonzero density, all the regions of the phase space available for motion, and the PDF in the versions $\mathbf{a}$ and $\mathbf{b}$ of the OSC models begin to change approximately in the same way with time. According to Danilov (2008), at $t \simeq t_{c}^{(1)}$ in model 1 , a balance is formed between a development and a decay of the PDF perturbations, which is achieved in the system as a result of the combined effect of a gravitational instability and a violent relaxation.

For brevity, we denote $z_{i j}=n^{\left(y_{f}\right)}(i, j)$. Let us consider the correlation flows between the regions with the large and small $\left|y_{f}\right|$. According to fig. $11.5 \mathrm{~b}$, the transitions of $y_{f}$ between the intervals $\left(Y_{f}\right)_{m}, m=4,5,6$, and $\left(Y_{f}\right)_{n}, n=1,2,8,9,10$, correspond to such flows. The mean value of $y_{f}$ for model 1 at $t<(3.6-3.7) \tau_{v r}$ is in the interval $\left(Y_{f}\right)_{5}$. The number of the transitions of $y_{f}$ in time $\Delta t$ from the region of large $\left|y_{f}\right|$ to the region of small $\left|y_{f}\right|$ is

$$
N_{1}=\sum_{j=4}^{6}\left(\sum_{i=1}^{2}+\sum_{i=8}^{9}\right) z_{i j} .
$$

The number of the reverse transitions is

$$
N_{2}=\sum_{i=4}^{6}\left(\sum_{j=1}^{2}+\sum_{j=8}^{9}\right) z_{i j}
$$


( $N_{1}$ and $N_{2}$ include the largest elements of the matrix $z_{i j}$ for the considered transitions of the correlations; the $z_{i j}$ values at $i=10, j=4,5,6$ or at $i=4,5,6, j=10$ are very small in comparison with $z_{i j}$ indicated in the formulas for $N_{1}$ and $N_{2}$ ). $N_{3}=N_{1}-N_{2}=0$ in the case of a balance of the considered transitions.

At $t<3.7 \tau_{v r}$ in model 1 , the values of $N_{1}, N_{2}, N_{3}$ on average decrease with increasing $t$ and $N_{1}>N_{2}>N_{3}\left(N_{1}: N_{2}: N_{3} \sim 539: 396: 143\right.$ for values of $N_{i}$ averaged over $t$ ). Thus, in model 1, the transitions of the type "1" are dominated when $y_{f}$ pass from the region of large $\left|y_{f}\right|$ to the region of small $\left|y_{f}\right|$. The mean values of $v, r$, the dispersions $\sigma_{v}$ and $\sigma_{r}$ of $v$ and $r$ of the stars participating in the correlation flows between regions with the large and small $\left|y_{f}\right|$, on average grow slowly over time $t$. We denote as $\mu_{r}=r_{1} / r_{2}$ the average over time $t$ ratio of $r_{1}(t)$ and $r_{2}(t)$ at $t<3.7 \tau_{v r}$, where $r_{1}(t)$ is the mean $r(t)$ over the number of correlations $y_{f}$ for the stars $(i, j)$ participating in transitions of the type "1"; $r_{2}(t)$ is the same for the reverse transitions of type "2". The values $\mu_{v}=v_{1} / v_{2}, \mu_{\sigma_{r}}=\sigma_{r_{1}} / \sigma_{r 2}$, $\mu_{\sigma_{v}}=\sigma_{v 1} / \sigma_{v 2}, \mu_{v^{2}}=v^{2}{ }_{1} / v^{2} 2$ are introduced similarly. In the case of model 1, we find $\mu_{r}=1.33 \pm 0.03, \mu_{v}=1.09 \pm 0.01, \mu_{\sigma_{r}}=1.34 \pm 0.04, \mu_{\sigma_{v}}=1.24 \pm 0.03$, $\mu_{v^{2}}=1.22 \pm 0.02$. Thus, the transitions of the $y_{f}$ correlations of the type "1" dominating in model 1 lead to the formation of a stream of the stars towards the cluster center $\left(\mu_{r}>1\right)$. These stars also transfer a kinetic energy to the cluster center (since $\mu_{v}^{2}>1$ and $\mu_{v^{2}}>1$ ). Consequently, the core and the central parts of the cluster are "heated", which leads to their expansion and "cooling" (Danilov, 2011).

The values $v_{v}=\mu_{v}^{2}-1=\left(v_{1}^{2}-v_{2}^{2}\right) / v_{2}^{2}$ and $v_{v^{2}}=\mu_{v^{2}}-1$ can be regarded as the estimates of the ratio of the kinetic energy of the collective stellar motions in cluster model 1 (the radial and other ones) to the kinetic energy of the peculiar ("thermal") motions of these stars. For model 1, we find $v_{v}=0.18 \pm 0.01<1$, $v_{v^{2}}=0.22 \pm 0.02<1$. We note that Danilov (2002b) revealed a flux of the star energy $\varepsilon$ directed towards the center of model 1 due to the oscillations of the regular force field of the cluster (for the stars moving towards the cluster center, the energies $\varepsilon$ increase, and for the stars moving from the center, they decrease). In this case, a kinetic energy $\delta T>0$ is transferred to the cluster center (since $\varepsilon<0, \varepsilon \neq$ const, and the kinetic energies of stars on average increase with the increasing $r$, see fig. 9.1 for model 1). This energy $\delta T$ partially goes to the "heating" of the core (it is redistributed among the stars of the core), and partially turns into the energy of the cluster oscillations.

Figs. 11.8a,b show the histograms of the distribution $F(v)$ of the velocities of the stars $i$ and $j$, participating in the transitions of the types "1" and "2" of the correlations $y_{f}$ in time $\Delta t$ at $t=1.521 \tau_{v r}$. The indices 1 and 2 for $i$ and $j$ denote the type ("1" or "2") of the correlation transitions. The solid lines in figs. 11.8a,b indicate the distributions $F(v)$ for the stars participating in the transitions type "1". The dot-and-dash lines show the distributions $F(v)$ for the stars participating in the transitions type "2" (we will call them "thermal" for brevity, since they correspond to the stars from the regions of the phase space with the minimum values of $\left|y_{f}\right|$ ). When constructing the histograms in fig. 11.8a,b the choice of the interval $\Delta v$ was made in the following way. Starting from the small $\Delta v$ values, the interval $\Delta v$ was 

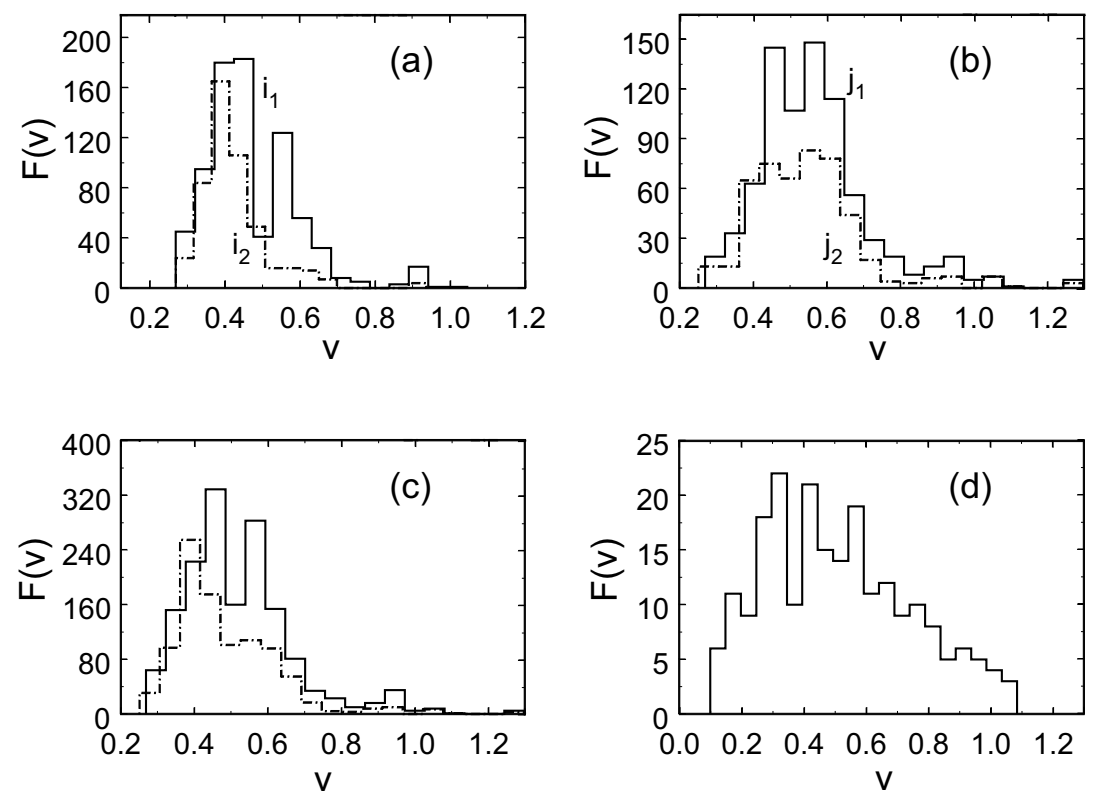

Fig. 11.8 The histograms of the distribution of $F(v)$ for the OSC model 1 at $t=1.521 \tau_{v r}$

increased until the "thermal" distributions of $F(v)$ were smoothed out. At this $\Delta v$, the histograms in fig. $11.8 \mathrm{c}, \mathrm{d}$ were built. A further increase of $\Delta v$ leads to the loss of information and to roughening of the $F(v)$ distributions. Distributions of the stars $i_{1}$ and $j_{1}$ in fig. 11.8a,b have the well-defined "peaks" with the local maxima near the values of $v=v_{a} \simeq 0.45 \mathrm{pc} / \mathrm{Myr}$ and $v=v_{b} \simeq 0.55 \mathrm{pc} / \mathrm{Myr}(1 \mathrm{~km} / \mathrm{s} \simeq 1 \mathrm{pc} / \mathrm{Myr})$. Within each such "peak" the same star can be detected several times if its phase coordinates strongly correlate with the phase coordinates of several stars participating in the type "1" transitions. Fig. 11.8c shows the total "thermal" distribution of stars $i_{2}$ and $j_{2}$ (the dot-and-dash line) and the total distribution of stars $i_{1}$ and $j_{1}$ (the solid line). In the total distribution of stars $i_{1}$ and $j_{1}$, the local maxima also near the values of $v=v_{a}, v_{b}$ are seen.

Fig. 11.8d shows the histogram of the distribution $F(v)$ of the stars with $r<0.5 R_{t}$ for cluster model 1 at $t=1.521 \tau_{v r}$. In fig. 11.8d three "peaks" are quite noticeable in the region of $v \in[0.25,0.60] \mathrm{pc} / \mathrm{Myr}$. These "peaks" are significantly distinguished above the level of the "thermal" part of the distribution $F(v)$ passing in this $v$ region near the points of the local minima of the distribution $F(v)$, or below these points judging from figs. $11.8 \mathrm{a}-\mathrm{c}$. Two of these "peaks" are located near the values of $v=v_{a}, v_{b}$ and are due to the impact of the transitions of the types "1" and "2" on the distribution $F(v)$ of the stars with $r<0.5 R_{t}$. The third (highest) "peak" in fig. $11.8 \mathrm{~d}$, is apparently formed by the transitions of the correlations $y_{f}$ between the intervals $\left(Y_{f}\right)_{k}$, which are closer than in the transitions of the types "1" and "2", and have the intermediate values of $\left|y_{f}\right|$. We note that only $24 \%$ of all elements of 
the matrix $z_{i j}$ correspond to the transitions of the types "1" and "2"; the transitions between the close intervals $\left(Y_{f}\right)_{k}$ occur much more often than the transitions of the type "1" and "2" see fig. 11.5b, so they can form even higher "peaks on the distribution $F(v)$ in fig. 11.8d, than transitions of the types "1" and "2".

The presence of the "peaks" on the distribution $F(v)$ in fig. $11.8 \mathrm{~d}$ in the region $v \in[0.25,0.60] \mathrm{pc} / \mathrm{Myr}$, as well as a gravitational instability of the core of model 1 are signs of a turbulence in stellar motions in this cluster model. Among the signs of the turbulence of the stellar motions in model 1, we can also can mention a large number of the wave motions which form in this cluster model, see Section 11.2, and the transfer of energy from the region of the large $\left|y_{f}\right|$ to the region of the small $\left|y_{f}\right|$ (the dominant transitions of the type "1"), as well as the chaotization of the phases of the stellar motion trajectories in model 1, noted by Danilov and Leskov (2005) (see the conclusion of the paper of Danilov and Leskov (2005) on the proximity of the distribution of the dependency phases of $r(t)$ to the uniform distribution for the stars of model 1). One of the reasons for the chaotisation of the phases of the stellar trajectories can be a chaotisation of the phases of the wave motions and the action of the non-thermal fluctuations of the density $n$ and the force field on the stellar trajectories in the cluster model. According to Genkin (1971), the possibility of a turbulence in the space of velocities in the stellar systems is determined by the oscillations of the regular field and by the presence of the density waves. Such waves and oscillations of the force field are a characteristic feature of the numerical dynamic OSC models of Danilov and Dorogavtseva (2003, 2008); Danilov and Leskov (2005); Danilov (2002b, 2005, 2008). Perhaps a turbulence accelerates the "heating" of the core by the regular field oscillations in model 1 , and increases the frequency of the encounters and interactions of the stars. It also increases the rate of the transfer of an energy of the collective stellar motions to the peculiar ones (on the turbulent heating in the plasma see in Tsytovich (1971)).

The distribution $F(v)$ of the stars with $r<R_{t}$ for the cluster model 1 at $t=$ $1.521 \tau_{v r}$ does not contain the well-marked local maxima near the values $v=v_{a}, v_{b}$. Probably, a turbulence mainly develops in the core of model 1.

Let $F_{c}(v)$ and $F_{d}(v)$ are the values of $F(v)$ in fig. $11.8 \mathrm{c}$ (the solid line) and $11.8 \mathrm{~d}$, respectively, and $q$ is the mean $F_{c}(v) / F_{d}(v)$ value over two local maxima of the distributions $F(v)$ near $v \simeq 0.45 \mathrm{pc} / \mathrm{Myr}$ and $v \simeq 0.55 \mathrm{pc} / \mathrm{Myr}$. For the stars of model 1 with $r<0.5 R_{t}$ we find $q=15.3 \pm 1.4$. Let $T$ be the total kinetic energy of the stars of the cluster model 1 in the region $r<0.5 R_{t}$. Taking into account the flow of the kinetic energy from the collective stellar motions to the "thermal" motions due to transitions of the types "1" and "2", we find

$$
\dot{T} \simeq\left(N_{1} \mu_{v^{2}}-N_{2}\right) m_{\odot} \overline{v_{2}^{2}} /(2 q \Delta t),
$$

where $\dot{T}=\frac{d T}{d t}, \overline{v_{2}^{2}}$ is the mean square of the velocities of the stars with $r<0.5 R_{t} ; m_{\odot}$ is the mass of the Sun. In this case, for model 1, we obtain $\dot{T}=(1.56 \pm 0.23) T / \tau_{v r}$. The rate of the $T$ change under the action of the stellar encounters $\dot{T}_{s t}=0.5 T / \tau_{s t}$ is easily obtained from equation (2.359) of Chandrasekhar (1942). Here, $\tau_{s t}$ is the relaxation time of the system under the action of stellar encounters. According to 
Table 3 of Danilov (2010) for model 1, we find $\tau_{s t}=(2.6 \pm 0.3) \tau_{v r}$. Consequently, $\dot{T}_{s t}=(0.19 \pm 0.02) T / \tau_{v r}$ and $\dot{T} / \dot{T}_{s t}=8.1 \pm 1.5$ for this cluster model. Thus, $\dot{T}_{s t}<\dot{T}$, and transitions of the types "1" and "2" in the space of correlations $y_{f}$ make the main contribution to the heating of the core and the central parts of the cluster model 1 . Since $v_{v}<1, v_{v^{2}}<1$, and the value $\dot{T}_{s t}$ is not many orders of magnitude less than $\dot{T}$, then we can consider a turbulence in the stellar motions, which forms in the cluster model 1 as a weak one (Tsytovich, 1971, p.41,49).

The estimates of $\dot{T}$ caused by the transitions of the types "1" and "2", as well as the estimates of $v_{v^{2}}$ and the time intervals $\Delta t$ (in the units of time $\tau_{v r}$ ), used in (11.3) when calculating of these flows, are given in Table 11.1 for the OSC models $1-6$. In order to estimate $\dot{T}$ and $v_{v^{2}}$, we used the values $t \leq t_{\text {max }}$, at which $\left|\sigma_{10}-\sigma_{11}\right| / \sigma_{11} \leq 0.22$. According to Table 11.1, with an increase of the number of the cluster model (and with a decrease of the degree of its non-stationarity), $\dot{T}$ and $v_{v^{2}}$ decrease. Probably, in models 2-6, the main source of heating of the cluster core is associated with the fairly slow changes in $y_{f}$ and more frequent transitions in a smaller number of intervals in the space of $y_{f}$ over time $\Delta t$ comparing with the transitions of the types "1" and "2".

\subsection{Conclusions}

1. In this chapter, we have considered the estimates of the correlation time $\tau_{c}$ in the OSC models in the spaces of $r, v, \varepsilon$. During the evolution of the OSC models, the correlations are most rapidly destroyed in the space of the $v$ values, and most slowly in the space of $\varepsilon$. According to the data on the correlations $h_{2}^{(v)}$ for models 3-6, we have noted the oscillations of $v$ over time, and defined the periods $P^{(v)}$ of these oscillations, which are in a good agreement with the periods of the oscillations of the values of the virial coefficient of these models.

2. We have considered the estimates of the radii of the correlations $r_{c}$ in the spaces of the values of $\mathbf{x}=(r, v, \varepsilon, n, f)$. The $r_{c}$ values in these spaces quite agree with the average distance between the stars. The values of $\varepsilon, n, f$ have the largest twopoint correlations in the region of $r_{i j}=\lambda^{(\varepsilon)}, \lambda^{(n)}, \lambda^{(f)}$, respectively. It indicates the existence in the models of the radial waves of a potential and a force field with the wavelength $\lambda^{(\varepsilon)}$, as well as the density and the phase density waves with wavelengths $\lambda^{(n)}$ and $\lambda^{(f)}$. We have noted the $C_{r}$-condensations of the points at the diagrams $\left(y_{\mathbf{x}}, r_{i j}\right)$, which also indicates the existence of the radial density waves in the spaces of $\mathbf{x}$ at the periphery of the models and allows us to determine the lengths of these waves. In the space of the moduli of the relative stellar velocities $v_{i j}$, similar condensations were not found (Danilov and Putkov, 2012b), which is due to their active destruction and the short relaxation times $\tau_{v}$ in comparison with $\tau_{r}$ in the considered OSC models.

3. We have discussed the distributions of the correlations of the $n$ and $f$ values in the OSC models. The very high maxima of these distributions in the region of the small moduli of correlations are noted. Evolution of $50 \%$ of the considered OSC 
models proceeds with an increase of the dispersion of such distributions and with a decrease of the number of the correlations with $r_{i j}<0.5 R_{t}, R_{t}, 2 R_{t}$. We have confirmed the hypothesis of Kandrup (1998) on the possibility of the increasing correlations (and coherence) of the motions in such systems over time.

4. We have considered the flows of the correlations in the space of $y_{f}$. The distributions of the mean numbers of the transitions of the correlations in time $\Delta t$ in $j$ intervals in the space of $y_{f}$ have a very high maximum at $j=0$. These distributions are very symmetric with respect to $j=0$. The degree of asymmetry of such distributions decreases, and their dispersion, on average, weakly grows or conserves during the evolution of the OSC models. It indicates a proximity to the balance of the transitions of the correlations in the space of $y_{f}$ in the OSC models. The relaxation time of a cluster in the space of $y_{f}$ agrees well with the time of the development of the instability of the phase density function with respect to the small initial perturbations of the stellar phase coordinates.

5. We have considered the correlation flows between the regions with the large and small values of $\left|y_{f}\right|$ (the transitions of the types "1" and "2"). We have noted the excess of the correlation flow from the region of the large $\left|y_{f}\right|$ to the region of the small $\left|y_{f}\right|$ over the reverse flow. We have viewed the estimates of the ratios of the kinetic energies of the collective and thermal stellar motions $\left(v_{v}<1, v_{v^{2}}<1\right)$. We have noted the signs of a weak turbulence in the stellar motions of cluster model 1. The arguments in favor of the existence of a turbulence in the cores of the OSC models have been listed. We have considered the estimates of the heating rate $\dot{T}$ of the central areas of the OSC models due to the transitions of the types "1" and "2" in the cluster. For model 1, the $\dot{T}$ value is noticeably larger than the heating rate of these regions due to the stellar encounters $\dot{T}_{s t}$, and the transitions of the types "1" and "2" make the main contribution to the heating of the central regions of this cluster model. With a decrease in the degree of non-stationarity of the cluster, $\dot{T}$ and

$v_{v^{2}}$ decrease. Probably, in models 2-6, the main source of a heating of the cluster core is associated with fairly slow changes of $y_{f}$ and with more frequent transitions in the space of $y_{f}$ over time $\Delta t$ in a smaller number of intervals comparing to the transitions of the types "1" and "2".

\section{References}

Aarseth, S.J.: Dynamical evolution of simulated star clusters. I - Isolated models. Astron. Astrophys. 35, 237-250 (1974).

Chandrasekhar, S.: Principles of Stellar Dynamics. Univ. Chicago Press, Chicago, Ill (1942). LCCN: 43-5608 (BKS1)

Danilov, V.M.: Statistical analysis of dynamical open-cluster models with small differences in their initial stellar phase coordinates. Astron. Letters, 23, 322-326 (1997b).

Danilov, V.M.: Stellar Fluxes in Numerical Dynamical Models of Open Clusters. Astron. Reports 46, 887-899 (2002b). doi:10.1134/1.1522077 
Danilov, V.M.: The Motion of Halo Stars in Dynamical Numerical Models of Open Clusters. Astron. Reports 49, 3604-610 (2005). doi:10.1134/1.2010649

Danilov, V.M.: Analysis of density fluctuations in models of open clusters. Astron. Reports 52, 888-899 (2008). doi:10.1134/S1063772908110036

Danilov, V.M.: Phase-Density Fluctuations at the Centers of Six Open Clusters. Astron. Reports 54, 514-527 (2010). doi:10.1134/S1063772910060053

Danilov, V.M.: On the dynamics of open clusters, Astron. Reports 55, 473-486 (2011). doi:10.1134/S1063772911060035

Danilov, V.M., Dorogavtseva, L.V.: Estimates of Relaxation Times in Numerical Dynamical Models of Open Star Clusters. Astron. Reports 47, 483-491 (2003). doi: $10.1134 / 1.1583775$

Danilov, V.M., Dorogavtseva, L.V.: Timescales for mechanisms for the dynamical evolution of open star clusters. Astron. Reports 52, 467-478 (2008). doi:10.1134/S1063772908060048

Danilov, V.M., Leskov, E.V.: Properties of Stellar Trajectories in Numerical Dynamical Models of Open Star Clusters. Astron. Reports 49, 190-200 (2005). doi:10.1134/1.1882777

Danilov, V.M., Putkov, S.I.: The dynamics of correlations in open-star cluster models. Astron. Reports 56, 623-637 (2012b). doi:10.1134/S1063772912080021

Genkin, I.L.: Relaxation in a regular field. Reports of the USSR Academy of Sciences, Series in Mathematics and Physics 197, 1042-1044 (1971). (In Russian).

Gilbert, I.H.: Collisional Relaxation in Stellar Systems. Astrophys. J. 152, 1043-1056 (1968). doi:10.1086/149616

Gilbert, I.H.: Gravitational Polarization in Spherical Stellar Systems. Astrophys. J. 159, 239-246 (1970). doi:10.1086/150306

Goodman, J., Heggie, D.C., Hut, P.: On the Exponential Instability of N-Body Systems. Astrophys. J. 415, 715-733 (1993). doi:10.1086/173196

Kandrup H.E.: Should a self-gravitating system relax towards an isothermal distribution? Astrophys. Space Sci. 112, 215-223, (1985). doi:10.1007/BF00653505

Kandrup H.E.: Collisionless Relaxation in Galactic Dynamics and the Evolution of Long Range Order. Annales of the New York Academy of Sciences. 858, 28-47 (1998).

Kandrup, H.E., Magon, M.E., Smith, H.C.: On the sensitivity of the N-body problem toward small changes in initial conditions. 4. Astrophys. J. 428, 458-465 (1994). doi:10.1086/174259

King, I.R.: The structure of star clusters. I. An empirical density law. Astron. J. 67, 471-485 (1962). doi:10.1086/108756

Klimontovich, Yu.L.: Statistical Physics. Nauka, Moscow (1982). (In Russian).

Klimontovich, Yu.L.: Statistical Theory of Open Systems. Yanus-K, Moscow (1995). (In Russian).

Komatsu, N., Kiwata, T., Kimura, S.: Numerical irreversibility in self-gravitating small N-body systems. Physica A: Statistical Mechanics and its Applications. 387, 2267-2278 (2008). doi:10.1016/j.physa.2007.12.012

Prigogine, I.: Non-Equilibrium Statistical Mechanics. Interscience Publishers, New York, London (1962). 
Prigogine, I., Severne, G.: On the statistical mechanics of gravitational plasmas. Physica 32, 1376-1396 (1966).

Prigogine, I., Stengers, I.: Time, Chaos, Quantum: Towards the Resolution of the Paradox of Time. Progress, Moscow (1994). (In Russian).

Saslaw, W.C.: Gravitational Physics of Stellar and Galactic Systems. Cambridge University Press, Cambridge (1985). doi:10.1017/CBO9780511564239 ISBN:0 $52123431 \mathrm{X}$

Severne, G., Haggerty, M.J.: Kinetic theory for finite inhomogeneous gravitational systems. Astrophys. Space Sci. 45, 287-302 (1976). doi:10.1007/BF00642666

Tsytovich, V.N.: Theory of turbulent plasma. Atomizdat, Moscow (1971). (In Russian). 



\title{
Chapter 12 \\ Correlations, Spectra, and Instability of Phase-Density Oscillations in Models of Open Star Clusters
}

\begin{abstract}
In this chapter, we consider the two-time correlation and the cross correlation functions for the phase density fluctuations of the OSC models. We give the estimates of the correlation time of the phase density oscillations (from 0.1 to 1 of the violent relaxation time of the model $\tau_{v r}$ ) and the estimates of the mean phase velocities of a propagation of such oscillations in the cluster models. These velocities are 2-20 times less than the mean square velocities of the stars in the cluster core. We discuss the application of the Fourier transform of the cross-correlation functions for the calculation of the power spectra and the dispersion curves of the phase density oscillations. We confirm a presence of known unstable oscillations of the phase density associated with the homological oscillations of the cluster cores. A number of new unstable oscillations of a phase density in these models are detected (up to 32-41 pairs of the oscillations with the different complex conjugate frequencies in each model; the rise time of the amplitudes of such oscillations in $e$ times is $(0.4-10) \tau_{v r}$, the phases of such oscillations are fairly uniformly distributed). Astrophysical applications of the results are discussed (an irregular structure of the open star clusters; a formation and a decay of the quasi-stationary states in such clusters).
\end{abstract}

\subsection{Introduction}

The theoretical estimates of the phase density fluctuations and the correlations corresponding to them for the spatially homogeneous and inhomogeneous systems with long-range interactions (including the self-gravitating systems) have recently been performed by Chavanis (2006a, 2008a,b). For such estimates, the kinetic equations written with a number of the simplifying assumptions were used in these papers. The expressions obtained by Chavanis (2006a, 2008a,b) for the correlation functions have a rather complicated form, which makes it difficult to use them for the analysis of the dynamic processes in such systems. In our opinion, it would be more productive to calculate directly the correlation functions from the data on the numerical 
integration of the equations of the gravitating particles' motion in the problems of the galactic clustering and the evolution of the Universe (see, for example, Baertschiger and Labini (2004); Bottaccio et al. $(2003,2002)$ ), as well as on the modeling of the OSC dynamics (Danilov and Putkov, 2012b).

Danilov and Putkov (2012b) calculated the two-time correlations for the values of $r=|\mathbf{r}|, v=|\mathbf{v}|$ and for the stellar energy $\varepsilon$ per unit of the star mass as well as the two-particle correlations of $r, v, \varepsilon$, of the star number density $n=n(\mathbf{r}, t)$ and the phase density $f=f(\mathbf{r}, \mathbf{v}, t)$ of the OSC models in the vicinity of these stars (here, $\mathbf{r}$ is a radius vector and $\mathbf{v}$ is a star velocity vector). According to the data on correlations, Danilov and Putkov (2012b) determined the time $\tau_{c}$ and the radius $r_{c}$ of the correlation in the spaces of the indicated parameters; they defined the parameters of the density, potential and phase density waves in the models of the star clusters; also, they noted the signs of the formation of the polarization clouds when certain distances between stars were reached; in addition, they found a dominant flow of the correlations of the phase density values from the region of strong correlations to the region of weak correlations. Such a flow leads to the onset of a kinetic energy $T$ flow to the cluster center (from the region of the large-scale $f$ oscillations to the region of the small-scale ones (Danilov, 2002b) with the smaller amplitudes and the higher frequencies). According to Danilov and Putkov (2012b), the heating rates of the cores of the cluster models by this flow are $\dot{T} \sim(0.1-1.6) T / \tau_{v r}$, where $T$ is a total kinetic energy of stars with the distances $r \leq 0.5 R_{t}$ from the the cluster center; $\tau_{v r}$ is the initial time of a violent relaxation of the cluster model taken equal to $\tau_{v r} \simeq 2.6 \bar{t}_{c r}$ according to the estimates of Aarseth (1974); $\bar{t}_{c r}$ is the average crossing time; $R_{t}$ is a tidal radius of the cluster (King, 1962), the $\tau_{v r}$ and $t_{c r}$ values were obtained from the data on the initial parameters of the OSC models. Danilov and Putkov (2012b) found the signs of a weak turbulence in the motions of the stars of the core of model 1 which has the highest $\dot{T}$ and the greatest degree of a non-stationarity in a regular field. For this model, $\dot{T} \simeq(1.6 \pm 0.2) T / \tau_{v r}$, which is $8.1 \pm 1.5$ times faster than the rate of heating of the core by the stellar encounters. The correlation functions were obtained by Danilov and Putkov (2012b) from the data of the numerical experiments for the dynamic OSC models (Danilov and Dorogavtseva, 2008).

It is of interest to study theoretically the turbulence of the stellar motions in the OSCs (and in their models). However, such a study should be preceded by a study of the full spectrum of oscillations of such systems and an analysis of the instability of these oscillations, the identification of all (or most) unstable oscillations in the OSC models. For oscillations in the plasma, see Volkov et al. (1983); Bernar et al. (1973); according to Volkov et al. (1983), the main criterion of a plasma turbulence is the broadening of the oscillation spectrum, the chaotization of their amplitudes and phases, and the presence of an energy flow across the spectrum. At present, so complete information on the dynamics of the OSC models is missing. We note that in the theoretical works (Chavanis, 2008b; Severne and Haggerty, 1976; Gilbert, 1968, 1970; Nardini et al., 2012), the differential equations for the single-particle distribution functions and the two-particle correlation functions are used in the study of the spatially inhomogeneous stellar systems. When writing such equations, the different simplifying assumptions are usually used. Except for the Danilov and Putkov 
(2012b), there are no studies featuring a calculation of the correlation functions from the data of the numerical experiments for the dynamic OSC models. In our opinion, this is due to the exponential instability of the stellar trajectories in such systems (Goodman et al., 1993; Kandrup et al., 1994) and the low accuracy of an integration of the equations of a stellar motion over the sufficiently large time intervals (Komatsu et al., 2008) (usually, the difference schemes with the accuracy order not more than 4 are used for such integration (Goodman et al., 1993; Kandrup et al., 1994; Komatsu et al., 2008)).

Danilov and Dorogavtseva (2008) performed the calculations of the PCS for the OSC models by integrating the equations of stellar motion using the difference schemes of the 10th and 11th accuracy orders on the time interval $t \in\left[0, t_{m}\right]$, where $t_{m} \simeq 5.1 \tau_{v r}$. Let $t_{0}$ be the time interval of the dynamic evolution of the OSC model, during which a statistical criterion for the accuracy of the phase density calculations is fulfilled (Danilov, 1997b). Near the centers of the OSC models of Danilov and Dorogavtseva (2008), $t_{0} / \tau_{v r}$ is equal to $t_{0} / \tau_{v r} \simeq 3.0-3.9$, and at the periphery of the models, it is equal to $t_{0} / \tau_{v r} \simeq 3.6-5.1$. The maximum relative error in the computation of the cluster energy reached over a period of time $t_{m}$ in the models of Danilov and Dorogavtseva (2008) in modulo was $(1-4) \times 10^{-13}$, and the accuracy of the calculation of the cluster's PDF over a time interval $t_{0}$ can be considered sufficient for the conclusions about the statistical properties of the PDF. When $t \in\left[0, t_{0}\right]$, the accuracy of the distribution of the correlations obtained by Danilov and Putkov (2012b) is sufficient for the conclusions about the properties of these distributions. According to Danilov and Putkov (2012b), a comparison of the investigated distributions or dependencies (for example, the oscillation spectra or the dispersion curves) obtained by the methods of the 10th and 11th accuracy orders also gives an effective method for the monitoring of the results of calculations, see below.

It is of interest to consider the estimates of the correlation time $\tau_{c}=\tau_{c}^{(f)}$ for $f$ in the OSC models of Danilov and Dorogavtseva (2008). Such estimates were not performed by Danilov and Putkov (2012b), since the two-time correlations of $f$ were not calculated. The $\tau_{c}^{(f)}$ values can be used to calculate the average phase velocity $v_{f}$ of a propagation of the $f$ oscillations in the OSC models (see, for example, Volkov et al. (1983); Bernar et al. (1973)). Also, it is of interest to determine the average phase velocities $v_{r}, v_{v}$ of the oscillations in the spaces of $r, v$ in the OSC models according to the data on $\tau_{c}$ and $r_{c}$ of Danilov and Putkov (2012b). Such estimates for the OSC models have not been carried out previously.

Beginning from the work of Miller (1964), an analysis of the instability of the motions in the many-body problem was carried out in a number of papers (see, for example, Kandrup $(1989,1990 a, b))$. For the systems close to the state of a stable dynamic equilibrium (Goodman et al., 1993), the exponential instability of the stellar trajectories leads to an increase of the entropy of the system (Zaslavsky and Sagdeev, 1988, p.105), (Klimontovich, 1995, p.506).

The divergence between the close trajectories in non-stationary stellar systems and the influence of the collective effects on the stellar trajectories were studied by various methods in the papers of Kandrup (1989, 1990a,b); Gurzadyan and Savvidy 
(1986); Boccaletti et al. (1991); Danilov and Leskov (2005). In the papers of Kandrup $(1989,1990 \mathrm{a}, b)$; Boccaletti et al. (1991) within the framework of the "geometric" approach for the elliptical galaxies, the estimates of the time $\tau_{v r} \sim \bar{t}_{c r}$ were obtained. In Boccaletti et al. (1991) the virial theorem and the assumption that such systems are close to the state of a stable equilibrium were used in order to obtain such estimates. The theoretical estimates of Kandrup (1989, 1990a,b); Boccaletti et al. (1991) of $\tau_{v r} \sim \bar{t}_{c r}$ were confirmed using the numerical experiments by Kandrup and Smith (1991); Kandrup et al. (1992), etc. Danilov and Leskov (2005) investigated the maximum Lyapunov characteristic exponent $\lambda$ of the stellar trajectories of the OSC models with the different degrees of non-stationarity. It was shown that the $\lambda$ values averaged by all stellar trajectories of the models decrease during the transition from the models with an average degree of non-stationarity to more non-stationary models. It indicates a decrease of the stochasticity of the stellar motions in the strongly non-stationary systems.

The dynamics of the collisionless gravitating systems near the time-dependent state of an unstable equilibrium with the distribution of the phase density $f_{0}$ was discussed theoretically by Kandrup (1998). In such systems, there should be the oscillations $\delta f$ of the phase density $f$ with respect to a distribution $f_{0}$ which corresponds to a saddle point associated with the local energy extremum $H\left(f_{0}\right)$ of the mean field of the system. In this case, $H(f)-H\left(f_{0}\right)$ increases for some $\delta f$ and decreases for other $\delta f$, see the analysis of formula (18) from Kandrup (1998).

In the OSC models which are to a different degree close to the unstable equilibrium state (Danilov, 2011), the correlations in the positions of stars in the phase space can increase with time, and the entropy in these cases decreases (Danilov and Putkov, 2012b). In the OSC models, due to their low density and the action of the external field of the Galaxy, the numerous little-studied instabilities of the collective stellar motions exist and dominate, see below. Among such instabilities, at the present time, only one of them, connected with the homologous oscillations of the OSC cores (Danilov, 2008), has been theoretically studied. For such oscillations, the mass density $\rho$ in a spherical stellar system can be represented in the form $\rho(r, t)=$ $\rho_{1}(r) \rho_{2}(t)$. The study of the oscillations' instabilities in the OSCs and their models will make it possible in the future to reveal the mechanisms for the development of these instabilities, to classify them (like Jeans, non-Jeans, beam, gradient ones etc. (Polyachenko and Fridman, 1976), in some cases similar to plasma ones). The instabilities of the collective stellar motions determine both the irregular structure of the OSCs (more irregular than in the globular clusters) and the dynamic evolution of OSCs (the development of non-stationarity in a regular field, the development of a turbulence in the stellar motions due to the interaction of the different modes of the oscillations, the formation and growth of the polarization clouds in the OSC models, etc.). In this connection, it is of interest to study the oscillations of the phase density in the OSCs and to analyze the instability of these oscillations. According to Volkov et al. (1983); Bernar et al. (1973), the cross-correlation functions of the fluctuations of a number of the plasma characteristics at two points at a distance $r$ from each other can be used to construct the dispersion curves and analyze the instabilities of the oscillations in a plasma. It is of interest to use such a method to construct the 
frequency spectra (power spectra) and to investigate a number of the instabilities of the $f$ oscillations in the OSC models. Such studies of the OSC models were first performed by Danilov and Putkov (2013b). The results of such study can be used both in the discussion of the dynamics of the OSC models, and in the theoretical description of the dynamic processes in the OSCs.

The objectives of this chapter are: 1) to discuss the values of $\tau_{c}^{(f)}, v_{f}, v_{r}, v_{v}$ for the OSC models of Danilov and Dorogavtseva (2008); 2) to discuss the two-time and cross-correlation functions for the phase-density $f$ fluctuations in the OSC models; 3 ) to consider the frequency spectra and the dispersion curves of the $f$ oscillations in the OSC models; 4) to apply the obtained results to the analysis of the OSC dynamics.

\subsection{Estimates of $\tau_{c}^{(f)}, v_{f}, v_{r}, v_{v}$ in the OSC Models}

Description of the OSC models of Danilov and Dorogavtseva (2008) see in Section 16.1.

Following to Klimontovich (1982, p.136), as well as to Danilov and Putkov (2013b) and the notation of Danilov and Putkov (2012b), to determine the correlation time $\tau_{c}^{(f)}$ in the space of $f$ values, let us consider a two-time correlation

$$
g_{2}^{(f)}\left(t, t^{\prime}\right)=\overline{f(t) f\left(t^{\prime}\right)}-\overline{f(t)} \cdot \overline{f\left(t^{\prime}\right)},
$$

where the bar on top denotes averaging over all cluster stars; $f(t)$ and $f\left(t^{\prime}\right)$ are the $f$ values in the vicinity of the same star at time points $t$ and $t^{\prime}$. Other arguments $(\mathbf{r}$, v) of the functions $f$ and $g_{2}^{(f)}$ are not written for a sake of brevity. Estimates of the phase density $f$ were obtained here in the neighborhoods of each star, taking into account the data on the phase coordinates of this star and the five stars closest to this star, as by Danilov and Putkov (2012b). Let $t^{\prime}=t+\tau$ and $\tau>0$. Substituting $t^{\prime}$ in (12.1), averaging the function $g_{2}^{(f)}(t, t+\tau)$ over the time points $t \in\left[0, t_{m}-\tau\right]$, we find the function $h_{2}^{(f)}(\tau)$ and its errors (at $\tau<0$, it is necessary to average the function $g_{2}^{(f)}(t, t+\tau)$ over time points $\left.t \in\left[-\tau, t_{m}\right]\right)$. The plot of function $h_{2}^{(f)}(\tau)$ for the cluster model 1 at $\tau>0$ is shown in fig. 12.1. This function is even: $h_{2}^{(f)}(\tau)=h_{2}^{(f)}(-\tau)$, see also in the book of Bendat and Piersol (1980), therefore its plot at $\tau<0$ is not given.

As in Danilov and Putkov (2012b), we assume the correlation time $\tau_{c}^{(f)}$ is equal to the $\tau$ value, at which $h_{2}^{(f)}(\tau)=h_{2}^{(f)}(0) / e$, where $e$ is the base of the natural logarithm. In this case, for the cluster model 1 , we find $\tau_{c, 11}^{(f)}=(0.98 \pm 0.14) \tau_{v r}$ using the PCS obtained by the method with the 11th accuracy order, and $\tau_{c, 10}^{(f)}=$ $(1.09 \pm 0.16) \tau_{v r}$ using the PCS of the 10th accuracy order. The error of $\tau_{c}^{(f)}$ indicated here is due to the error in calculating the function $h_{2}^{(f)}(\tau)$ at $\tau=\tau_{c}^{(f)}$. The estimates 


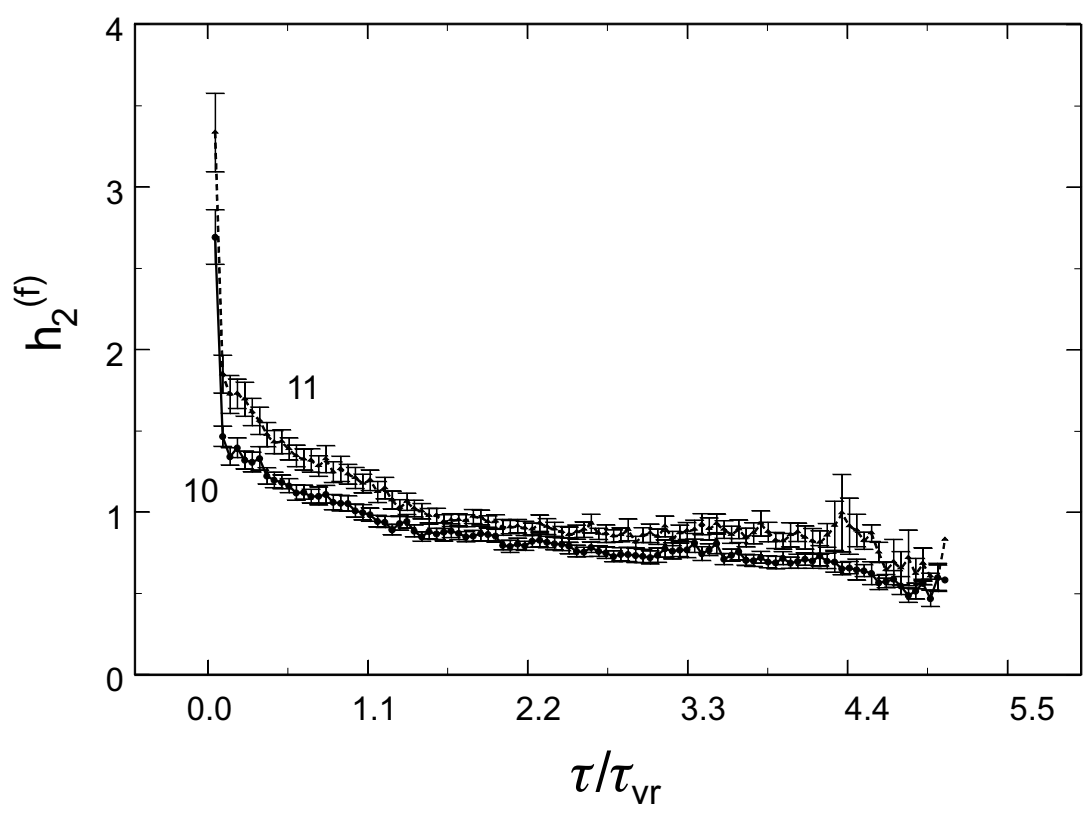

Fig. 12.1 The dependencies of $h_{2}^{(f)}$ on $\tau$ for the OSC model 1 at $\tau>0$. Numbers 10 and 11 indicate the accuracy order of the PCS used for the calculation of these curves

of $\tau_{c, 11}^{(f)}$ and $\tau_{c, 10}^{(f)}$ do not differ statistically. For the OSC models 1-6, Table 12.1 gives the estimates of $\tau_{c}^{(f)}$ obtained using the PCS of the 11th order of accuracy. According to Table 12.1 and Table 1 from the paper of Danilov and Dorogavtseva (2008), the values of $\tau_{c}^{(f)} / \tau_{v r}$ and $\tau_{c}^{(f)}$ decrease with an increase of the model number and with a decrease in the degree of non-stationarity of the cluster model. Thus, the correlations in the space of the $f$ values is most rapidly destroyed during the evolution of model 6. Taking into account the data of Danilov and Putkov (2012b) on $\tau_{c}^{(r)}$ (the correlation time in the space of $r$ ), we find $\tau_{c}^{(f)}<\tau_{c}^{(r)}$ in the OSC models 1-6. Relation $\tau_{c}^{(r)} / \tau_{c}^{(f)}=q$ increases from $q \simeq 2.1$ in model 1 to $q \simeq 17.0$ in model 6.

According to Volkov et al. (1983, p.27), the averaged estimate of the phase velocity of the $f$ oscillations can be obtained from the relation of the time and the radius of a correlation $v_{f} \simeq r_{c}^{(f)} / \tau_{c}^{(f)}$ (in the spaces of $r$ and $v$, the average phase velocities $v_{r}$ and $v_{v}$ are defined similarly). Table 12.1 gives the values $v_{f}, v_{v}$, and $v_{r}$ (in $\mathrm{pc} / \mathrm{Myr}$; $1 \mathrm{pc} / \mathrm{Myr} \simeq 1 \mathrm{~km} / \mathrm{s}$ ), obtained using the PCS of the 11th order of accuracy and taking into account the data on the correlation parameters in models 1-6 of Danilov and Putkov (2012b). According to Table 12.1, the $v_{f}$ and $v_{v}$ values in models 1-4 are significantly (5-20 times) smaller than $v_{f}$ and $v_{v}$ in models 5, 6. In the case of the values $v_{r}$, such large differences between the models are not observed. Perhaps, such differences of $v_{f}$ and $v_{v}$ in models $1-4$ and 5, 6 are due to the greater wavelengths 
$\lambda$ of the $f$ and $v$ oscillations in models 5,6 . Let us consider a wave extending radially in the cluster model: $\delta f \propto \exp (-i \varphi)$, where $\varphi=\omega t-k_{r} r ; \omega$ and $k_{r}$ are the frequency of $f$ oscillations and the corresponding wave number, respectively. Assuming $\varphi=$ const, $\omega=2 \pi / P=$ const,$k_{r}=2 \pi / \lambda=$ const, differentiating $\varphi$ over time $t$, we get $v_{f}=\frac{d r}{d t}=\omega / k=\lambda / P$, where $P$ is the oscillation period.

The periods of the radial oscillations of models 1-6 differ a little from each other (see, for example, Table 2 from Danilov and Leskov (2005)). Therefore, the differences in the wavelengths $\lambda$ of the $f$ and $v$ oscillations in models 1-4 and 5, 6 can be one of the reasons for the differences between $v_{f}$ and $v_{v}$ in these models. Perhaps, the large $v_{f}$ and $v_{v}$ values in models 5 and 6 are related to the presence of the more extended and massive cores in these models compared to models 1-4. In addition, in model 6, during the periods of a greatest compression to the Galactic plane, a toroidal structure is formed with an increased density of the number of stars inside the torus with an equatorial plane close to the plane of the Galaxy. In this model, the condensation waves extending along the cluster core from the $\zeta$ axis (passing through the center of a mass of the model and perpendicular to the plane of the Galaxy) are observed with a speed comparable with the average speed of the peculiar stellar motions (Danilov and Leskov, 2005).

We note that the small $v_{f}$ and $v_{v}$ values in models $1-4$ can be the result of the formation and superposition of several counter-extending traveling radial waves in these models (in this case, the average phase velocity of the radial waves in the cluster can be small). Danilov and Ryazanov $(1985,1987)$ performed the numerical integration of the Vlasov equations, they found and investigated the counter-propagating radial flows of stars formed in a non-stationary isolated spherical system with a constant phase density (a water-bag model). Such flows are formed in the system's core during the periods of the system contractions and are caused by the periodic motion of a region with the zero phase density inside the phase region occupied by the system. In this case, near the center of the system, a density perturbation is periodically formed (a region of a reduced density), which then is carried by a stream of stars to the boundary of the system's core. It is possible that such flows of stars are more actively formed in the OSC models 1-4 with a higher degree of non-stationarity than in models 5 and 6 , which affects the estimates of the correlation parameters $r_{c}^{(f)}$ and $\tau_{c}^{(f)}$, as well as the estimates of $v_{f}$ for models 1-4.

Table 12.1 shows the mean square velocities $v_{c}$ of the core stars (in pc/Myr) for the stars with the distances from the center $r \leq 0.5 R_{t}$ obtained by Danilov and Putkov (2012b). According to Table 12.1, the $v_{c}$ values are 10-20 times greater than $v_{f}$ for models $1-4$ and approximately 2 times larger than $v_{f}$ for models 5,6 . Thus, the average phase velocities of the $f, v, r$ oscillations in the OSC models 1-6 are sufficiently small in comparison with the mean square velocities $v_{c}$ of stellar motion in the core. 
Table 12.1 The correlation time $\tau_{c}^{(f)}$, the initial time of a violent relaxation $\tau_{v r}$, the phase velocities of the oscillations' propagation, a mean square velocity $v_{c}$ of the motion of the core stars in the OSC models

\begin{tabular}{|c|c|c|c|c|c|c|}
\hline № & 1 & 2 & 3 & 4 & 5 & 6 \\
\hline$\tau_{c}^{(f)} / \tau_{v r}$ & $0.98 \pm 0.14$ & $0.60 \pm 0.05$ & $0.52 \pm 0.03$ & $0.64 \pm 0.06$ & $0.14 \pm 0.02$ & $0.10 \pm 0.01$ \\
\hline $\begin{array}{c}\tau_{v r}^{1}, \\
\mathrm{Myr}\end{array}$ & 49.93 & 41.84 & 41.84 & 34.25 & 41.84 & 41.84 \\
\hline $\begin{array}{c}v_{f}, \\
\mathrm{pc} / \mathrm{Myr}\end{array}$ & $0.027 \pm 0.001$ & $0.051 \pm 0.004$ & $0.056 \pm 0.003$ & $0.053 \pm 0.005$ & $0.26 \pm 0.04$ & $0.27 \pm 0.03$ \\
\hline $\begin{array}{c}v_{v}, \\
\mathrm{pc} / \mathrm{Myr}\end{array}$ & $0.012 \pm 0.001$ & $0.013 \pm 0.001$ & $0.021 \pm 0.005$ & $0.063 \pm 0.007$ & $0.17 \pm 0.01$ & $0.26 \pm 0.02$ \\
\hline $\begin{array}{c}v_{r}, \\
\mathrm{pc} / \mathrm{Myr}\end{array}$ & $0.007 \pm 0.001$ & $0.008 \pm 0.001$ & $0.001 \pm 0.0001$ & $0.011 \pm 0.002$ & $0.009 \pm 0.002$ & $0.010 \pm 0.001$ \\
\hline $\begin{array}{c}v_{c}, \\
\mathrm{pc} / \mathrm{Myr}\end{array}$ & $0.56 \pm 0.02$ & $0.56 \pm 0.01$ & $0.54 \pm 0.01$ & $0.57 \pm 0.01$ & $0.58 \pm 0.01$ & $0.55 \pm 0.01$ \\
\hline$v_{c} / v_{f}$ & $20.7 \pm 1.0$ & $10.9 \pm 0.9$ & $9.7 \pm 0.6$ & $10.8 \pm 1.0$ & $2.2 \pm 0.3$ & $2.0 \pm 0.2$ \\
\hline
\end{tabular}

Note - 1 - Danilov and Dorogavtseva (2008).

\subsection{Cross-Correlation Function}

Let $X(t, \mathbf{r})=f(t, \mathbf{r})-\overline{f(t, \mathbf{r})}$, where $f$ is obtained by the $n_{s t}$ stars from the vicinity of a point with a coordinate $\mathbf{r}$ in the cluster model, and the bar above denotes averaging over time $t \in\left[0, t_{m}\right]$ (an origin of the coordinate system $\mathbf{r}$ coincides with the cluster's center of a mass, $t_{m}$ is the largest $t$ values used to calculate the correlations). For a brevity, we do not write the argument $\mathbf{v}$ for the functions $f$ and $X . X$ is usually called a deviation or a fluctuation (Volkov et al., 1983; Bernar et al., 1973), and is assumed to be a random variable that forms a stationary random process. Following to Volkov et al. (1983); Bernar et al. (1973), as well as Danilov and Putkov (2013b), we write the autocorrelation function

$$
C_{x x}(\tau, \mathbf{r})=\frac{1}{t_{m}} \int_{0}^{t_{m}} X(t, \mathbf{r}) X(t+\tau, \mathbf{r}) d t
$$

as well as the cross-correlation function of the $f$ fluctuations

$$
C_{x x}(\tau, \mathbf{r}, \Delta \mathbf{r})=\frac{1}{t_{m}} \int_{0}^{t_{m}} X(t, \mathbf{r}) X(t+\tau, \mathbf{r}+\Delta \mathbf{r}) d t
$$

In a general case, these functions can be written in the form of the limits on $t_{m}$ as $t_{m} \rightarrow \infty$ from the expressions in the right-hand sides of the relations (12.2) and (12.3). Here, $\tau$, like in the previous section, is a time delay.

Let $\mathbf{r}=\mathbf{0},|\Delta \mathbf{r}|=r$. In this case, the distances of two points 1 and 2 from the center of the cluster are 0 and $r$, respectively. Following to Bernar et al. (1973), we denote the functions (12.2), obtained by the $n_{s t}$ stars closest to each of the points 
1 and 2 , as $C_{11}(\tau)$ and $C_{22}(\tau)$, respectively. In this case, we denote the functions (12.3) as $C_{12}(\tau, r)$. Following the methods of Bernar et al. (1973), we consider the $X$ fluctuation in the form of a wave packet propagating in the radial direction relative to the cluster center:

$$
X(t, r)=\int_{0}^{\infty} A(\omega) \exp [i(r k(\omega)-\omega t)] d \omega
$$

Here, the amplitude $A(\omega)$ and the wave number $k(\omega)$ of the waves composing the packet depend on the frequency of these waves $\omega$. In the case (12.4), a Fourier transform of the function $C_{11}(\tau)$ (or $C_{22}(\tau)$ ) is equal to the function $F(\omega)$, and a Fourier transform of the function $C_{12}(\tau, r)$ is equal to

$$
F_{1}(\omega)=F(\omega) \exp (\operatorname{irk}(\omega))
$$

see also formulas (3.41) or (4.8)-(4.11) from the book of Bendat and Piersol (1980). Thus, considering the Fourier transform of the function $C_{12}(\tau, r)$, we can find $\left|F_{1}(\omega)\right|=F(\omega)$ (a frequency spectrum) and $\frac{1}{r} \operatorname{Arg}\left(F_{1}(\omega)\right)=k(\omega)$ (a dispersion relation); $r k(\omega) \in[-\pi / 2, \pi / 2]$ is an oscillation phase $f$ with the frequency $\omega$.

Danilov and Putkov (2013b) considered a set of the positions of the point 2 on the sphere of a radius $r$ with the center at the mass center of the cluster. To do this, the coordinates of these points were specified in a spherical coordinate system $(r, \theta, \phi)$ with a step $\Delta \theta=\Delta \phi=\pi /(4 k)$ at $k=1,2,4$. Then, the transition was performed to the right Cartesian rectangular coordinate system $(\xi, \eta, \zeta)$ with the origin at the mass center of the cluster ( $\zeta$ axis is directed perpendicular to the Galactic plane, $\xi$ axis is directed from the center of the Galaxy in the Galactic plane, and $\eta$ axis is aimed in the direction of the cluster motion in the Galactic plane). Together with point 1 , the total number of points $n_{p}$, in the vicinity of which $X$ fluctuations are considered, equals $n_{p}=27,115,483$ at $k=1,2,4$, respectively. The plots of the mean values of the $C_{12}(\tau, r)$ function in model 1 at $r=1 \mathrm{pc}, n_{s t}=30, n_{p}=27$ in case $t_{m}=t_{0}$, where $t_{0} \simeq 3 \tau_{v r}$, are shown in fig. 12.2. An averaging of the $C_{12}(\tau, r)$ values was performed by Danilov and Putkov (2013b) over 26 values $C_{12}(\tau, r)$, corresponding to the different positions of point 2 on a sphere of the radius $r=1 \mathrm{pc}$. The vertical bars in fig. 12.2 indicate errors of the corresponding mean values of $C_{12}(\tau, r)$. When constructing fig.12.2(a) and fig. 12.2(b), the PCS of the 11th and 10th accuracy orders, respectively, were used in Danilov and Putkov (2013b). The differences of the curves $C_{12}(\tau, r)$ obtained in the 11th and 10th accuracy orders in fig. 12.2 are noticeably fewer than the differences of the curves $h_{2}^{(f)}$ in fig. 12.1, which is due to the use of the smaller $t_{m}$ than for fig. 12.1. When calculating the curves in fig. 12.2 in Danilov and Putkov (2013b), only the PCS at $t \leq t_{0}$ were used; an averaging by $t$ of $f$ and $X(t, \mathbf{r}) X(t+\tau, \mathbf{r}+\Delta \mathbf{r})$ in (12.3) was carried out over the intervals $t \in\left[0, t_{0}-\tau\right]$ at $\tau>0$ and $t \in\left[-\tau, t_{0}\right]$ at $\tau<0$. The differences of the curves $C_{12}(\tau, r)$, obtained with the 11th and 10th accuracy orders, are generally smaller or much smaller than the errors of $C_{12}(\tau, r)$ indicated in fig. 12.2, and these differences decrease with the decreasing $|\tau|$. We note a significant asymmetry of $C_{12}(\tau, r)$ function with respect to $\tau=0$. Therefore, the frequency spectrum is specified not only by the real part of the 

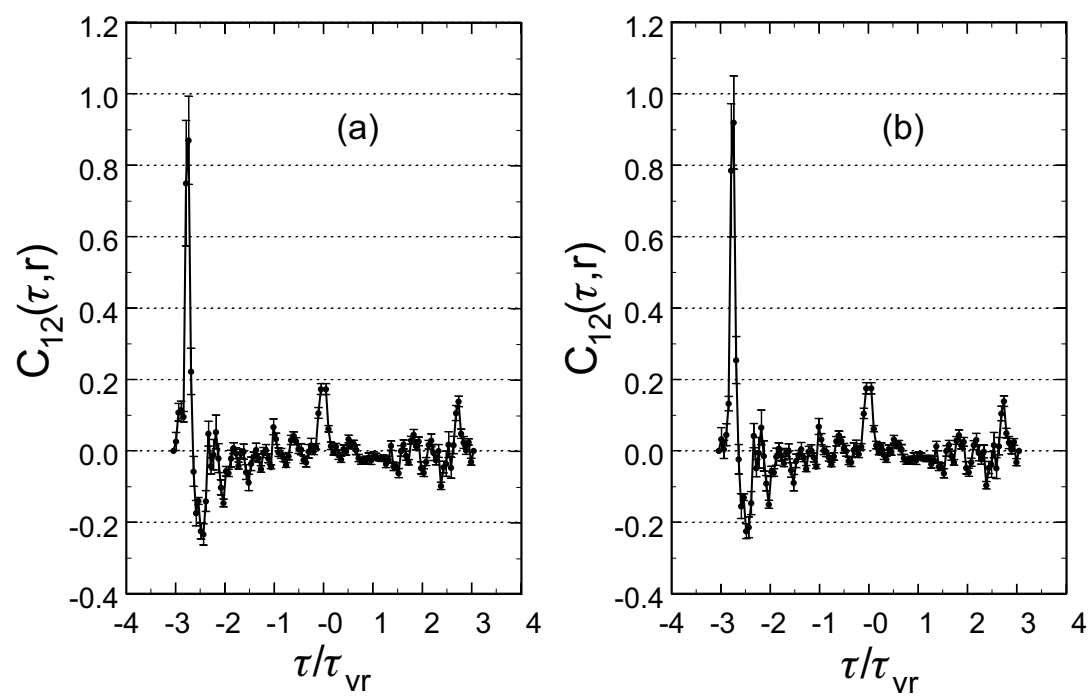

Fig. 12.2 The cross-correlation function in the OSC model $1 ; r=1 \mathrm{pc}, n_{s t}=30, n_{p}=27$ (fig. a and $b$ are built with the use of the PCS of the 11th and the 10th accuracy orders, respectively)

Fourier transform for the $C_{12}(\tau, r)$ function (Bendat and Piersol, 1980). In fig. 12.2, we detect a number of the local extremums of the $C_{12}(\tau, r)$ function that go beyond of the errors of $C_{12}(\tau, r)$ in the points $\tau_{j}=P_{j}$, which indicates the existence of the increased cross correlations (with the different signs) between the $f$ oscillations with the periods $P_{j}$ in the vicinity of points 1 and 2 .

\subsection{Frequency Spectra and Dispersion Curves}

In order to calculate the frequency spectra and the dispersion curves of the $f$ fluctuations in the OSC models 1-6, Danilov and Putkov (2013b) used the Fast Fourier Transform (FFT) program from the book of Gray (1976). Here, instead of the circular frequencies $\omega$, it is more convenient to use the cyclic frequencies $v=\omega /(2 \pi)$. In this case, the complex conjugate pairs of roots $v_{j}=v_{r e, j} \pm i v_{i m, j}$ of the equation $k_{v}^{(0)}=0$ allow us to determine the period $P_{j}=1 / v_{r e, j}$ and time $t_{j}=1 /\left(2 \pi v_{i m, j}\right)$ of the instability increase of the $f$ oscillations. Danilov and Putkov (2013b) computed separately the Fourier transforms $\mathcal{F}_{1}(v)$ and $\sigma_{\mathcal{F}}(v)$ for the mean $C_{12}(\tau, r)$ and their errors $\sigma_{C}(\tau, r)$. Then the relative phases (the wave numbers) of the $f$ fluctuations were calculated:

$$
k_{\nu}^{(0)}=\frac{1}{r} \operatorname{Arg}\left(\mathcal{F}_{1}(v)\right) \quad \text { and } \quad k_{v}^{(1,2)}=\frac{1}{r} \operatorname{Arg}\left(\mathcal{F}_{1}(v) \pm \sigma_{\mathcal{F}}(v)\right) .
$$


The functions $k_{v}^{(0,1,2)}$ were used to estimate the frequencies $v$ and their errors.

Fig. 12.3 shows the plot of the function $S_{v}=\left|\mathcal{F}_{1}(v)\right| / \tau_{v r}$ obtained by Danilov and Putkov (2013b) for model 1 from the mean values of the function $C_{12}(\tau, r)$ and the errors of these mean values. $S_{v}$ values in fig. 12.3 are given in $\left(\mathrm{Myr}^{6} / \mathrm{pc}^{12}\right)$ ). Here and below, $v$ is taken equal to the number of the $f$ oscillations in time $\tau_{v r}$. A number of the local maxima of the $S_{v}$ function that go beyond the errors of $S_{v}$ and are related to the local extremums of the $C_{12}(\tau, r)$ function can be seen on the $S_{v}$ dependency on $v$. At $v \in[0.5,2.4]$ (the low-frequency region), $S_{v}$ values are 2-3 times larger than at $v \in[2.6,5.2]$ (the medium frequencies). At larger $v$, the $S_{v}$ values decrease. According to fig. 12.3, the low frequencies $v \in[0.5,2.4]$ corresponding to the oscillations of the core and the entire cluster, make the largest contribution to the average power of the $f$ oscillations (see, for example, Volkov et al. (1983)). The frequencies of the local maxima of $S_{v}$ from the region of the medium frequencies are approximately 2.0 times (sometimes 3.0 times) higher than the frequencies of the corresponding local maxima of $S_{v}$ from the low-frequency region. It indicates the occurrence of the resonances between the $f$ oscillations with the different frequencies $v$ onto the formation of $S_{v}$ in model 1 (it is possible that the transfer of the energy of the $f$ oscillations from low to high frequencies, to the region of the oscillations with small amplitudes, has a resonant character). The local maximum points of $S_{v}$ correspond to both the stable oscillations (for example, $v \simeq$ $1.85,3.35$ in figs. $12.3,12.4$ ) and the unstable $f$ oscillations (see, for example, $v \simeq$ $1.13,1.36,4.41$ in figs. $12.3,12.4)$.

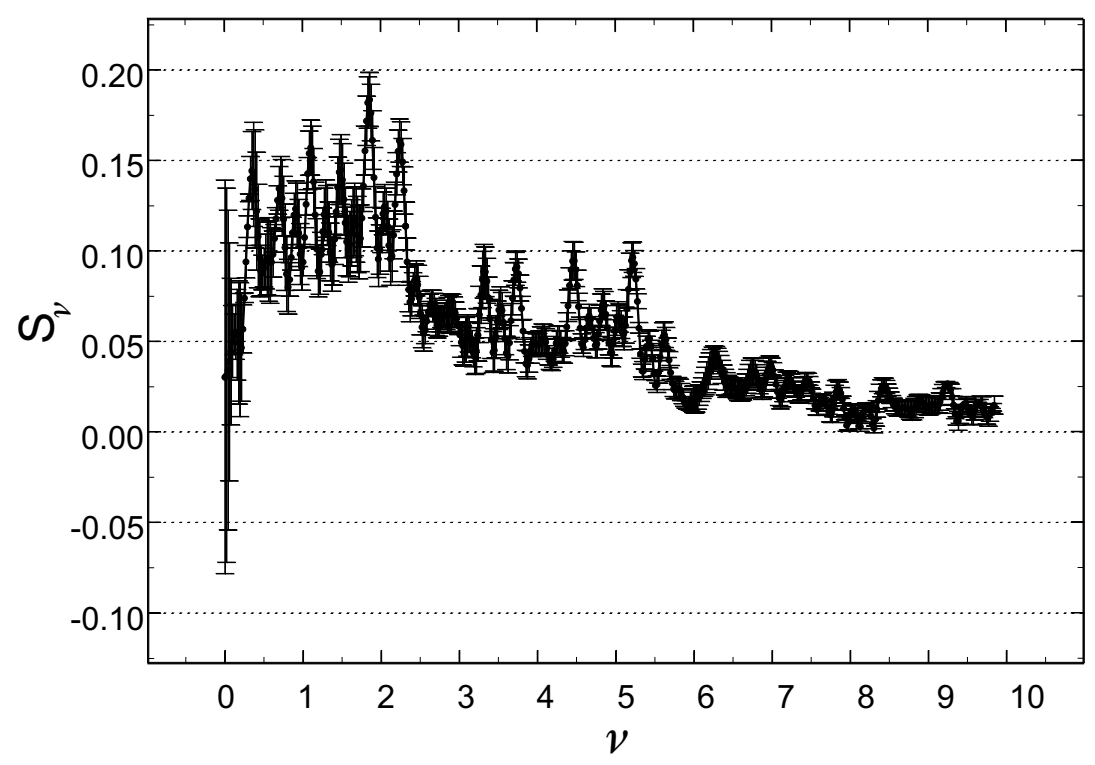

Fig. 12.3 The dependency of $S_{v}$ on $v$ for the OSC model $1 ; r=1 \mathrm{pc}, n_{s t}=30, n_{p}=27$ 
The presence of the local maxima on the curve $S_{v}$ indicates the increased power and intensity of an excitation of the $f$ oscillations with frequencies from the neighborhoods of points of these local maxima in model 1. The curves $S_{v}=S_{v}^{(10)}$ and $S_{v}=S_{v}^{(11)}$, obtained by Danilov and Putkov (2013b) at $t_{m}=t_{0}$ for the OSC models according to the data on the PCS of the 10th and 11th accuracy orders, respectively, practically do not differ each other. Frequencies $v$ of the corresponding local maxima of $S_{v}^{(10)}$ and $S_{v}^{(11)}$ coincide, and the values $\left|S_{v}^{(10)}-S_{v}^{(11)}\right| / S_{v}^{(11)}$ averaged over all $v$ values are approximately 1.9 times less than the values $\sigma\left(S_{v}\right) / S_{v}^{(11)}$ averaged over all $v$, where $\sigma\left(S_{v}\right)$ are the errors of $S_{v}^{(11)}$, indicated by the vertical bars in fig. 12.3. Therefore, the accuracy of the PCS used by Danilov and Putkov (2013b) can be considered as sufficient for the construction and analysis of curves $S_{v}$.

Let $\bar{S}_{v}$ be the mean $S_{v}^{(11)}$ in the region of small $v$, corresponding to the oscillation frequencies of the cluster core, and $\Delta S_{v}$ is the average difference between the maximum and minimum $S_{v}^{(11)}$ in this region of $v$.

The initial ratios of the radii of the core and the halo in the cluster models 1, 2, and 4 are the same; the number of stars in the cores of these models is $N_{1}=100$, and the density of these models increases from the first to the fourth model (the initial numbers of stars in the models are the same, and the initial radii of models $1,2,4$ decrease with an increasing model number). In cluster models $2,3,5,6$, the initial number of stars in the core and the core size increase from model 2 to model $6\left(N_{1}=400\right)$ with the identical initial radii of the halo (see, for example, Table 1 of Danilov and Dorogavtseva (2003)). The values $\bar{S}_{v}^{\prime}=\bar{S}_{\nu} \tau_{v . r}$ and $\Delta S_{v}^{\prime}=\Delta S_{\nu} \tau_{v r}$ (in $\mathrm{Myr}^{7} / \mathrm{pc}^{12}$ ) for models $1,2,4$ are obtained equal to $\bar{S}_{v}^{\prime}=5.8 \pm 0.4,2.3 \pm 0.7,3.3 \pm 0.7$ and $\Delta S_{v}^{\prime}=2.9 \pm 0.3,2.0 \pm 0.6,2.3 \pm 0.6$, respectively. With the increase of the size and the mass of the core in models $2,3,5,6$, the values $\bar{S}_{v}^{\prime}$ and $\Delta S_{v}^{\prime}$ decrease approximately in 15 and 14 times, respectively (when going from model 2 to model 6 ). Thus, a change of the initial density of the cluster model has a relatively weak effect on $\bar{S}_{v}^{\prime}$ and $\Delta S_{v}^{\prime}$, and an increase of the dimensions and the mass of the cluster core significantly reduces $\bar{S}_{v}^{\prime}$ and $\Delta S_{v}^{\prime}$. Let $\overline{\Delta v}_{i}$ be the average distance between the points of the neighbour local maxima of the function $S_{v}^{(11)}$ in the region of small $v$, corresponding to the largest values of $S_{v}^{(11)}$ and of the oscillation frequencies of the core of the $i$-th cluster model. The values $\overline{\Delta v}_{i}$ in models 1, 2, 4 increase with an increasing cluster density:

$$
\overline{\Delta v}_{2} / \overline{\Delta v}_{1}=1.71 \pm 0.13, \quad \overline{\Delta v}_{4} / \overline{\Delta v}_{1}=2.80 \pm 0.65 .
$$

The number of the local maxima of the functions $S_{v}^{(11)}$ in the low-frequency regions of the spectra with the large $S_{v}^{(11)}$ values decreases (in 2 times) with an increasing density of the OSC models (when going from model 1 to model 4). It is probable that the greater degree of a non-stationarity of model 1 in comparison with other models is ensured by the larger $\bar{S}_{v}^{\prime}$ values, by a greater number of the local maxima of the function $S_{v}^{(11)}$, and by a higher density of their location in the low-frequency region of the spectrum. 
Fig. 12.4 shows the dependencies of $k_{v}=k_{v}^{(0)}$ on $v$ for model 1 obtained for the function $C_{12}(\tau, r)$ given in fig. 12.2a. The values of $n_{p}=27,483$ were used. Widely changing the $n_{s t}$ and $n_{p}$, we can weaken the influence of a random noise on the form of dependency of $k_{v}$ on $v$ in the different frequency intervals of $v$ (see, for example, the almost vertical lines near $v \simeq 2.5$ in figs. 12.4a,b). With this, the shape and the position of the "sinusoidal" regions of the dependencies of $k_{v}$ on $v$, connected with the instabilities of the $f$ oscillations, practically do not change. The further such areas are from the line $k_{v}=0$, the greater the growth increments of such instabilities are (in these cases, the roots of the equation $k_{v}=0$ are the complex conjugate ones). For example, two pairs of the complex conjugate frequencies $v$ correspond to the "sinusoidal" section of the curve $k_{v}$ near $v \simeq 1.1-1.5$. The oscillation periods $P_{5}, P_{6}$ and the instability growth times $t_{5}, t_{6}$ for these roots are given in Table 12.2. A pair of the complex conjugate frequencies corresponding to $P_{6}, t_{6}$, for model 1 was found in the framework of a linear analysis of the instability of the $f$ oscillations in the cluster core (Danilov, 2008). The intersection of the curve of $k_{v}$ dependency on $v$ and the line $k_{v}=0$ corresponds to the real frequencies $v$ of the $f$ oscillations (if there is no an abrupt change of the phase $r k_{v}$ from $\mp \pi / 2$ to $\pm \pi / 2$; in this case, the real part of the Fourier transform of the function $C_{12}(\tau, r)$ reaches the zero value in the neighborhood of the corresponding $v$; such phase changes can be caused both by regular (not random) changes of $C_{12}(\tau, r)$ with an increasing $v$, and by a random noise in the estimates of $\left.C_{12}(\tau, r)\right)$.

Table 12.2 shows the $P_{j}$ and $t_{j}$ values (in the units of the time $\tau_{v r}$ ) for the $f$ oscillations with the largest increments of the instability growth in the considered ranges of $v$ in the OSC models $1-6$. The values $P_{j}$ and $t_{j}$ indicated in Table 12.2 of our work are obtained using the PCS of the 11th accuracy order. For model 1, the data on 17 pairs of such roots are given for $r=1 \mathrm{pc}$. The total number $n_{c}$ of such pairs of roots in this model for $r=1 \mathrm{pc}$ reaches 41-42. With an increasing $r, n_{c}$ in model 1 change. At $r=2 \mathrm{pc}$, we find $n_{c}=32-33$; at $r=3 \mathrm{pc}, n_{c}=42$; at $r=5 \mathrm{pc}, n_{c}=32$, and at $r=9 \mathrm{pc}$, we find $n_{c}=22$. The $P_{j}$ and $t_{j}$ values indicated for models 1-6 in Table 12.2 were determined by an approximation of the section of the dispersion curve corresponding to a given root $v_{j}$ by polynomials of 3-7th orders. Then the roots of this polynomial were found numerically, and the complex-conjugate pairs of roots were used for the calculation of $P_{j}$ and $t_{j}$.

It is possible that the degree of an instability of the OSC models at different distances from the cluster center is related to the number of the complex-conjugate pairs of roots of the equation $k_{v}=0$. In this case, a certain role in the formation of the instability regions at the different distances from the center of the cluster model can be played by the resonances between the frequency of the orbital motion of the cluster in the Galaxy and the frequencies of the natural oscillations of the phase density in the cluster models (see also Danilov (2011)).

The following notations were used when writing the $P_{j}$ and $t_{j}$ values in Table 12.2. If $P_{j}$ obtained from the data on $k_{\nu}^{(0)}$ is at the boundary of the $P_{j}$ range obtained from the data on $k_{v}^{(0,1,2)}$, then in Table 12.2 it is shown in parentheses with a prime on the side of the range where its value is indicated. If $P_{j}$ obtained from the data 

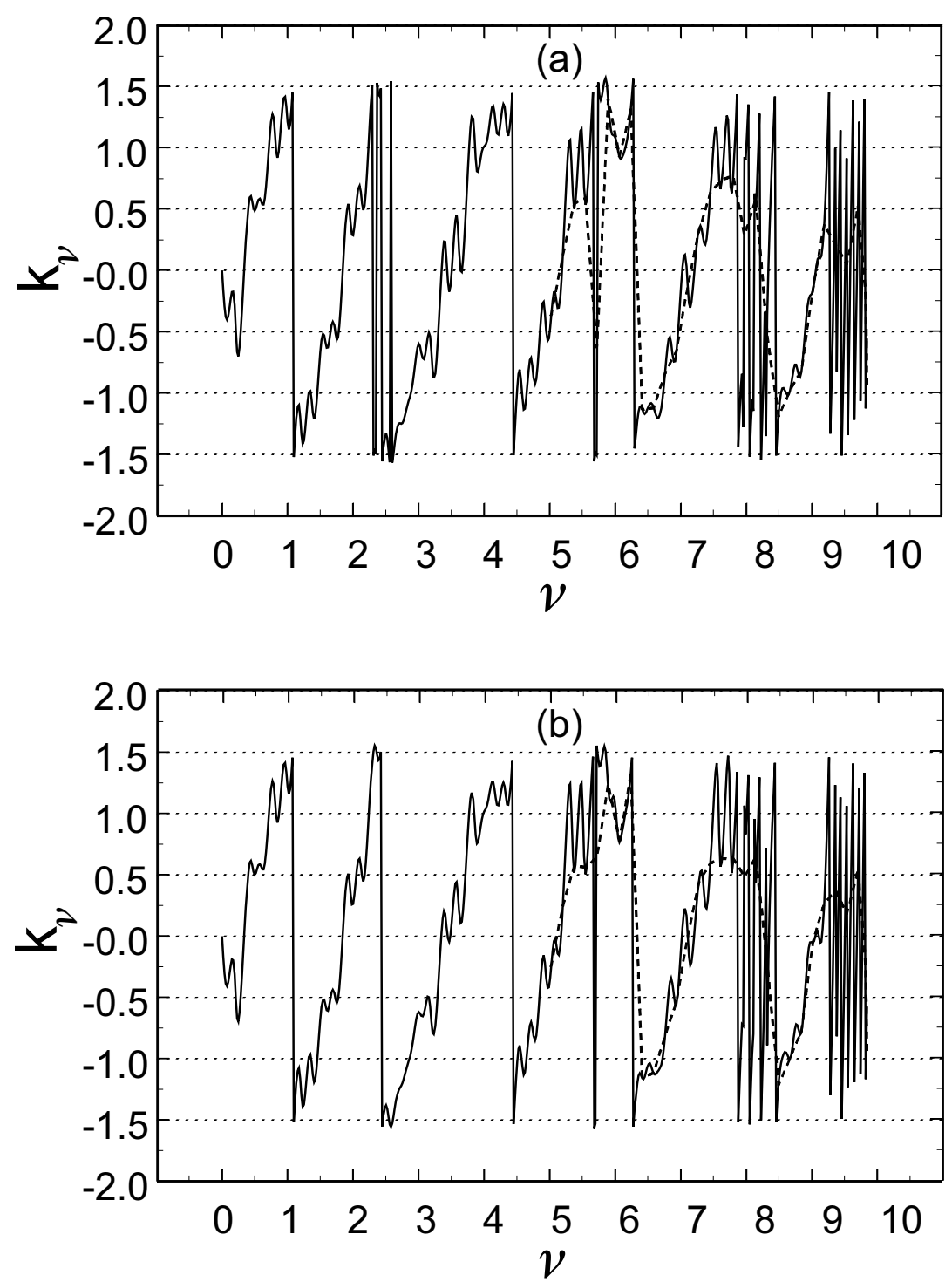

Fig. 12.4 The dispersion curves of the $f$ oscillations for the OSC model 1 at $r=1 \mathrm{pc}, n_{s t}=30$ : (a) - the curve is obtained at $n_{p}=27$; (b) - the same at $n_{p}=483$ 
on $k_{v}^{(0)}$, is inside the range of the $P_{j}$ values obtained from the data on $k_{v}^{(1,2)}$, then in Table 12.2 it is shown as $P_{j} \pm \sigma\left(P_{j}\right)$, where $\sigma\left(P_{j}\right)$ is a mean deviation from $P_{j}$ obtained by the data on $k_{v}^{(1,2)}$. In those regions of $v$, where the influence of a random noise in $k_{v}^{(0)}$, caused by the random oscillations of the mean $C_{12}(\tau, r)$, can not be suppressed by the changes of $n_{p}$ and $n_{s t}$, in order to isolate the signal at the noise level, we use the smoothing of the $k_{v}$ dependencies on $v$ by the method of a locally weighted regression (Cleveland and Devlin, 1988). The curves $k_{v}$ smoothed in this way at $v>5$ are shown by the dotted lines in fig. 12.4. The $P_{j}$ and $t_{j}$ values obtained with the help of such smoothed curves $k_{v}$, are indicated by the sign ":" in Table 12.2.

Dispersion curves obtained for the OSC models at $t_{m}=t_{0}$ from the data on the PCS of the 10th accuracy order practically do not differ from the dispersion curves of the 11th accuracy order. $P_{j}$ and $t_{j}$, obtained by the dispersion curves of the 10th and 11th accuracy orders $\left(P_{j}^{(10)}, t_{j}^{(10)}\right.$ and $\left.P_{j}^{(11)}, t_{j}^{(11)}\right)$, are in a good agreement with each other. In order to compare the values $P_{j}^{(10)}$ and $P_{j}^{(11)}, t_{j}^{(10)}$ and $t_{j}^{(11)}$ for model 1 in our work, we selected several frequencies $v_{j}$ of the unstable $f$ oscillations from the different parts of the range of the $v$ frequencies considered in fig. 12.4 (the accepted values $j=5,6,10,14,16$ correspond to the values of $P_{j}, t_{j}$ from Table 12.2 for model 1). Let $\Delta P=\overline{\Delta P_{j}}$ and $\Delta t=\overline{\Delta t_{j}}$ be the average values of $\Delta P_{j}=\left|P_{j}^{(11)}-P_{j}^{(10)}\right|$ and $\Delta t_{j}=\left|t_{j}^{(11)}-t_{j}^{(10)}\right|$. Let $\sigma_{P}$ and $\sigma_{t}$ be the average (by these $j$ ) errors $\sigma\left(P_{j}\right)$ and $\sigma\left(t_{j}\right)$ for the $P_{j}$ and $t_{j}$ values from Table 12.2. Then, $\Delta P \simeq 0.68 \sigma_{P}, \Delta t \simeq 0.85 \sigma_{t}$, and $\overline{\left(\Delta P_{j}\right) / P_{j}} \simeq 0.002, \overline{\left(\Delta t_{j}\right) / t_{j}} \simeq 0.04$. Thus, the estimates of $P_{j}$ and $t_{j}$ obtained with the methods of the 11th and the 10th accuracy orders, agree well with each other, and an accuracy of the used PCS can be considered sufficient for the construction and analysis of the dispersion curves.

According to Table 12.2, for model $1, P_{6}=(36.4-36.9)^{\prime}$ Myr and $t_{6}=81.3 \pm 2.5$ Myr (here, we used $\tau_{v r}=49.9 \mathrm{Myr}$, according to Danilov and Dorogavtseva (2008)). The period $P_{\gamma}$ and the time $t_{\lambda}$ of the development of the $f$ oscillations' instability, obtained in the linear collisionless approximation in the analysis of the $f$ oscillations associated with the homological oscillations of the cluster core for this model are $P_{\gamma}=37.4 \pm 1.8 \mathrm{Myr}$ and $t_{\lambda}=53.7 \pm 2.9 \mathrm{Myr}$, see columns 5, 6 of Table 12.2. Thus, the estimates of $P_{6}$ and $P_{\gamma}$ are in a good agreement with each other. The value of $t_{6}$ is approximately 1.5 times larger than $t_{\lambda}$, which is probably due to the nonlinearity of the $f$ oscillations and due to the impact of the stellar encounters on the $f$ oscillations in the core of model 1 . In time $\Delta t \simeq t_{\lambda}, t_{6}$, the stellar encounters manage to "heat up", to some extent, the region of a development of the instability, which reduces its rate of development. The initial relaxation time of the cluster model due to the stellar encounters is $\tau_{s t}=121.9 \pm 12.2 \mathrm{Myr}$ and $\tau_{s t}=72.6 \pm 7.2 \mathrm{Myr}$ for models 1 and 6, respectively ( see Danilov (2010), Table 3). Therefore, $\Delta t<\tau_{s t}$ for model 1. In Table 12.2, the pairs of values $P_{j}$ and $t_{j}$ which are the closest to the theoretical estimates of $P_{\gamma}$ and $t_{\lambda}$ for models 1-6 are marked with "*" (see Table 12.2, columns $4,5)$. On average for models $1-6$, for the values $P_{j}$ and $t_{j}$ marked with " ${ }_{*}$ ", we find $P_{j}=(1.09 \pm 0.06) P_{\gamma}$ and $t_{j}=(1.56 \pm 0.18) t_{\lambda}$. 
Table 12.2 The parameters of the unstable $f$ oscillations in the OSC models $\left(P_{j}\right.$ and $t_{j}$ are given in the units of the time $\tau_{v r}$ )

\begin{tabular}{|c|c|c|c|}
\hline № & 1 & 2 & 3 \\
\hline$P_{1}$ & ${ }^{\prime}(6.5-7.3)$ & $1.7 \pm 0.1$ & $1.19 \pm 0.03$ \\
\hline$t_{1}$ & '(4.0-5.7) & $1.7 \pm 0.1$ & $1.7 \pm 0.3$ \\
\hline$\overline{P_{2}}$ & ${ }^{\prime}(3.0-3.5)$ & $1.6 \pm 0.1$ & $* 0.93 \pm 0.03$ \\
\hline$t_{2}$ & $(4.0-10.0)^{\prime}$ & $1.8 \pm 0.2$ & $1.7 \pm 0.5$ \\
\hline$P_{3}$ & $1.19 \pm 0.01$ & $* 1.00 \pm 0.02$ & $0.830 \pm 0.007$ \\
\hline$t_{3}$ & $1.75 \pm 0.25$ & $2.2 \pm 0.4$ & $(1.7-1.9)^{\prime}$ \\
\hline$P_{4}$ & $0.94 \pm 0.01$ & $0.594 \pm 0.004$ & (0.69-0.71) \\
\hline$t_{4}$ & $(2.0-2.6)^{\prime}$ & $1.13 \pm 0.09$ & $(1.1-2.4)^{\prime}$ \\
\hline$P_{5}$ & $0.89 \pm 0.01$ & $0.541 \pm 0.003$ & $0.65 \pm 0.01$ \\
\hline$t_{5}$ & $(2.51-2.57)^{\prime}$ & $2.9 \pm 0.9$ & $'(1.17-1.30)$ \\
\hline$\overline{P_{6}}$ & $*(0.73-0.74)^{\prime}$ & $0.496 \pm 0.002$ & $(0.562-0.563)^{\prime}$ \\
\hline$t_{6}$ & $1.63 \pm 0.05$ & $2.4 \pm 0.5$ & $1.8 \pm 0.5$ \\
\hline$\overline{P_{7}}$ & ${ }^{\prime}(0.410-0.411)$ & $0.2738 \pm 0.0004$ & $0.484 \pm 0.005$ \\
\hline$t_{7}$ & $1.43 \pm 0.07$ & $2.0 \pm 1.5$ & $1.8 \pm 0.5$ \\
\hline$P_{8}$ & '(0.4078-0.4082) & $0.213 \pm 0.004$ & $0.449 \pm 0.001$ \\
\hline$t_{8}$ & $1.14 \pm 0.07$ & $1.8 \pm 0.5$ & $1.4 \pm 0.1$ \\
\hline$\overline{P_{9}}$ & $0.2271 \pm 0.0002$ & $0.1631 \pm 0.0001$ & $0.32764 \pm 0.00002$ \\
\hline$t_{9}$ & $(1.55-1.61)^{\prime}$ & $2.2 \pm 0.3$ & $1.5368 \pm 0.0003$ \\
\hline$\overline{P_{10}}$ & ${ }^{\prime}(0.2214-0.2215)$ & $0.1429 \pm 0.0003$ & $0.2643 \pm 0.0001$ \\
\hline$t_{10}$ & $2.14 \pm 0.14$ & $2.3 \pm 0.5$ & $1.41 \pm 0.01$ \\
\hline$\overline{P_{11}}$ & $0.17923 \pm 0.00004$ & $0.1182 \pm 0.0004$ & $0.25128 \pm 0.00005$ \\
\hline$t_{11}$ & $2.49 \pm 0.07$ & '(1.4-4.1) & $3.0 \pm 0.1$ \\
\hline$\overline{P_{12}}$ & $(0.170-0.175)^{\prime}$ & $0.1067 \pm 0.0001$ & $0.198 \pm 0.002$ \\
\hline$t_{12}$ & $1.52 \pm 0.03$ & $1.9 \pm 0.2$ & $1.4 \pm 0.2$ \\
\hline$\overline{P_{13}}$ & $0.1631 \pm 0.0001$ & $0.1049 \pm 0.0003$ & $0.19135 \pm 0.00004$ \\
\hline$t_{13}$ & $0.85 \pm 0.01$ & $2.4 \pm 0.9$ & $1.45 \pm 0.02$ \\
\hline$\overline{P_{14}}$ & $0.155 \pm 0.001$ & & $0.180929 \pm 0.000001$ \\
\hline$t_{14}$ & $0.76 \pm 0.09$ & & $4.0 \pm 0.2$ \\
\hline$P P_{15}$ & $0.124 \pm 0.004:$ & & $0.17296 \pm 0.00001$ \\
\hline$t_{15}$ & $(0.44-0.98)^{\prime}:$ & & $2.20 \pm 0.03$ \\
\hline$P_{16}$ & $0.1174 \pm 0.0004$ & & $0.13773 \pm 0.00002$ \\
\hline$t_{16}$ & $1.01 \pm 0.32$ & & $1.13 \pm 0.04$ \\
\hline$P P_{17}$ & $(0.105-0.106)^{\prime}:$ & & $0.13324 \pm 0.00003$ \\
\hline$t_{17}$ & $(1.15-1.79)^{\prime}:$ & & $1.26 \pm 0.02$ \\
\hline$P P_{18}$ & & & $0.12984 \pm 0.00005$ \\
\hline$t_{18}$ & & & $1.53 \pm 0.02$ \\
\hline$P P_{19}$ & & & $0.1189 \pm 0.0002$ \\
\hline$t_{19}$ & & & $0.787 \pm 0.004$ \\
\hline$P_{20}$ & & & $0.11582 \pm 0.00005$ \\
\hline$t_{20}$ & & & $1.06 \pm 0.01$ \\
\hline \begin{tabular}{|l|}
$P_{21}$ \\
$t_{21}$
\end{tabular} & & & $\begin{array}{c}0.09016 \pm 0.00001 \\
(1.82-1.84)^{\prime}\end{array}$ \\
\hline $\begin{array}{l}P_{22} \\
t_{22}\end{array}$ & & & $\begin{array}{c}0.0871 \pm 0.0001 \\
1.0 \pm 0.2\end{array}$ \\
\hline
\end{tabular}


End of table 12.2

\begin{tabular}{|c|c|c|c|}
\hline$№$ & 4 & 5 & 6 \\
\hline$P_{1}$ & $5.0 \pm 0.2$ & $(1.26-1.36)$ & $* 0.895 \pm 0.007$ \\
$t_{1}$ & $2.1 \pm 0.7$ & $1.5 \pm 0.5$ & $3.2 \pm 1.3$ \\
\hline$P_{2}$ & $(2.548-2.550)$ & $1.01 \pm 0.03$ & $0.749 \pm 0.003$ \\
$t_{2}$ & $1.5 \pm 0.3$ & $1.3 \pm 0.4$ & $2.5 \pm 0.3$ \\
\hline$P_{3}$ & $1.90 \pm 0.09$ & $* 0.849 \pm 0.001$ & $0.6614 \pm 0.0003$ \\
$t_{3}$ & $3.5 \pm 0.3$ & $2.4 \pm 0.6$ & $2.28 \pm 0.09$ \\
\hline$P_{4}$ & $* 1.47 \pm 0.08$ & $0.72 \pm 0.01$ & $0.5741 \pm 0.0002$ \\
$t_{4}$ & $2.25 \pm 0.13$ & $2.1 \pm 0.3$ & $2.9 \pm 0.1$ \\
\hline$P_{5}$ & $0.905 \pm 0.003$ & $0.612 \pm 0.003$ & $(0.285-0.294)$ \\
$t_{5}$ & $1.32 \pm 0.06$ & $3.1 \pm 0.2$ & $1.6 \pm 0.3$ \\
\hline$P_{6}$ & $0.578 \pm 0.014$ & $0.529 \pm 0.003$ & $(0.268-0.271)^{\prime}$ \\
$t_{6}$ & $1.7 \pm 0.9$ & $2.3 \pm 0.3$ & $(1.55-1.77)^{\prime}$ \\
\hline$P_{7}$ & $0.500 \pm 0.001$ & $0.417 \pm 0.001$ & $(0.258-0.259)^{\prime}$ \\
$t_{7}$ & $1.27 \pm 0.03$ & $1.84 \pm 0.11$ & $(1.0-1.3)$ \\
\hline$P_{8}$ & $0.21942 \pm 0.00003$ & $0.1984 \pm 0.0003$ & $(0.244-0.245)^{\prime}$ \\
$t_{8}$ & $2.22 \pm 0.01$ & $1.36 \pm 0.02$ & $(1.3-1.5)$ \\
\hline$P_{9}$ & $0.20594 \pm 0.00005$ & $0.13572 \pm 0.00002$ & $(0.1681-0.1683)$ \\
$t_{9}$ & $3.7 \pm 0.3$ & $1.82 \pm 0.08$ & $2.7 \pm 0.8$ \\
\hline$P_{10}$ & $0.3024 \pm 0.0004$ & $0.13129 \pm 0.00002$ & $0.1634 \pm 0.0002$ \\
$t_{10}$ & $1.01 \pm 0.04$ & $3.3 \pm 0.6$ & $2.4 \pm 0.2$ \\
\hline$P_{11}$ & $0.1574 \pm 0.0001$ & $0.12342 \pm 0.00007$ & $(0.145-0.146)^{\prime}$ \\
$t_{11}$ & $1.22 \pm 0.03$ & $1.8 \pm 0.2$ & $(1.5-1.9)^{\prime}$ \\
\hline$P_{12}$ & $(0.12156-0.12158)^{\prime}$ & $0.1200 \pm 0.0006$ & $0.098683 \pm 0.000003$ \\
$t_{12}$ & $(1.89-1.92)$ & $2.4 \pm 1.0$ & $2.5 \pm 0.1$ \\
\hline$P_{13}$ & $0.11538 \pm 0.00005$ & $0.1148 \pm 0.0002$ & $0.09734 \pm 0.00001$ \\
$t_{13}$ & $2.26 \pm 0.06$ & $2.3 \pm 0.5$ & $(1.29-1.38)^{\prime}$ \\
\hline$P_{14}$ & & $0.1014 \pm 0.0006$ & $0.08963 \pm 0.00001$ \\
$t_{14}$ & & $2.3 \pm 1.4$ & $2.40 \pm 0.03$ \\
\hline$P_{15}$ & & $2.1 \pm 0.3$ & $1.5 \pm 0.1$ \\
$t_{15}$ & & & \\
\hline & & & \\
& & & \\
\hline
\end{tabular}

According to Table 12.2, the periods of the unstable oscillations in model 1 are from $P_{1} \simeq 345 \mathrm{Myr}$ to $P_{17} \simeq 5.3 \mathrm{Myr} . P_{1}>P_{G} \simeq 222.1 \mathrm{Myr}$ where $P_{G}$ is the period of a revolution of the cluster model around the Galactic center (see, for example, Table 3 for models 1, 6 from Danilov (2010)). Probably, in the development of the oscillations of model 1 with periods $P_{1}$ and $P_{2}$, an important role is played by the external force field of the Galaxy. In the development of the large-scale oscillations with the periods $P_{3}-P_{6}$, a larger role is played by the regular force field of the cluster. The oscillations with the periods $P_{7}-P_{8}$ may be due to the non-homological nature of the oscillations of model 1 and its core (see estimates of the frequencies of the homological and non-homological oscillations of the ellipsoidal halo models in Danilov (2008)). The oscillations with the small amplitudes and the periods $P_{9}-P_{17}$ may be due to the formation of the polarisation clouds that arise as a response of the system to the passage of a star in it, as well as due to the occurrence of the resonances 
between the $f$ oscillations at different frequencies (see above) or a turbulence in the stellar motions in the cluster model 1 (Danilov and Putkov, 2012b).

We note that the phases $r k_{\nu}$ of the unstable $f$ oscillations in fig. 12.4 are distributed fairly evenly along the axis $k_{v}$. The multiple changes of a sign of $k_{v}$ in the considered frequency range in fig. 12.4 indicate the formation in model 1 of a number of the counter-propagating running radial waves of the $f$ oscillations. The similar changes of a sign of $k_{v}$ are also observed on the dispersion curves of the cluster models 2-6.

Figs. 12.5a,b show the histograms of the distributions $N\left(k_{v}\right)$ of the values $k_{v}$, used in the construction of fig. 12.4a,b, respectively. The dashed lines in fig. 12.5a,b indicate the histograms in the case $v \leq 7.72$ (in this case, we exclude from a consideration the sections of the dispersion curves that are strongly distorted by the influence of a random noise in $k_{v}^{(0)}$, caused by the random oscillations of the mean $C_{12}(\tau, r)$ values). The histograms $N\left(k_{v}\right)$ for the OSC models 1-6 show a sufficiently uniform distribution of the phases of the $f$ oscillations, which also indicates the formation of a number of the counter-propagating running radial waves of the $f$ oscillations in the models, and provides the small mean values of the phase velocities of such waves in OSC models. The presence of a large number of
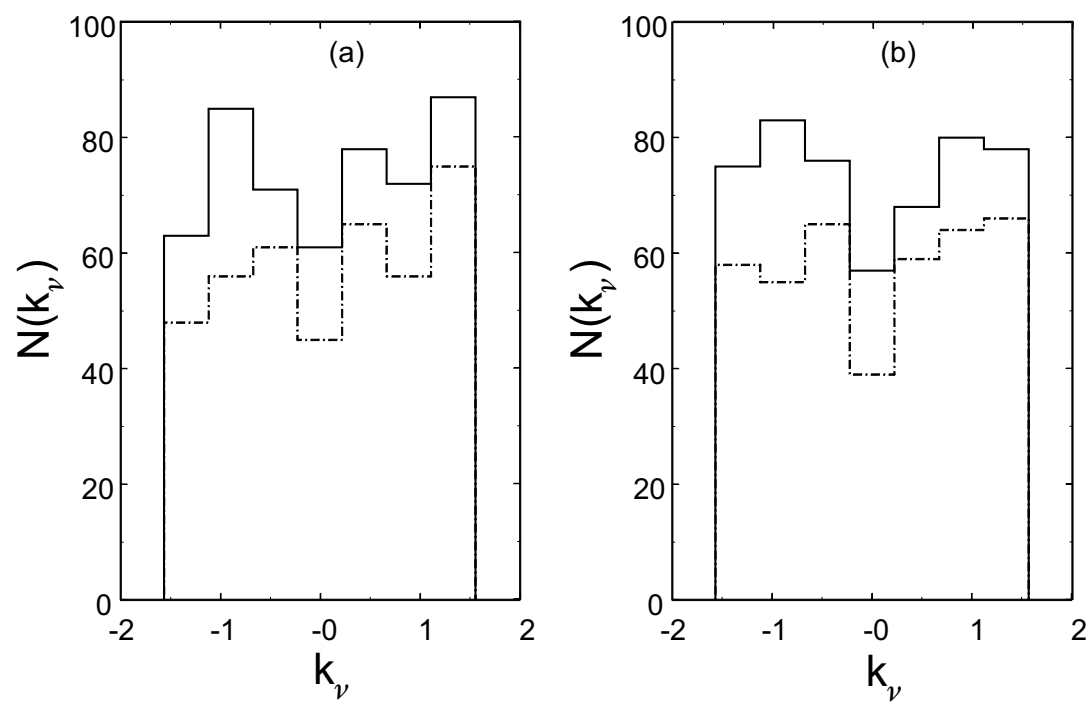

Fig. 12.5 The histograms of the distribution $N\left(k_{v}\right)$ for the OSC model 1 at $r=1 \mathrm{pc}, n_{s t}=30$ : (a) was obtained at $n_{p}=27$; (b) was obtained at $n_{p}=483$. The dot-and-dash lines indicate the histograms at $v \leq 7.72$

the unstable oscillations and the increased power and intensity of an excitation of such oscillations in model 1 (see above, as well as in Volkov et al. (1983)) can be considered as arguments in a favor of a turbulence developing in the core of this cluster model. 
The collective motions in the OSCs and their instabilities determine the irregularity of the structure of such clusters. A number of features of the OSC structure indicating the non-stationary nature of these clusters in a regular field are well known (an irregular shape of the cores, which does not correspond to the equilibrium state of the OSCs; a presence of the step-like structures in the radial density profiles, etc. (Barkhatova, 1956; Seleznev, 1994; Binney and Tremaine, 2008; Danilov and Putkov, 2012a)). The instabilities of the collective motions in the OSCs make it possible to explain the structures and the dynamic processes observed in the OSCs and their models (see the discussion of the dynamic evolution of such clusters in Danilov and Putkov (2012b); Danilov (2011); Danilov and Putkov (2012a)).

In the last few years, the phenomena of an incomplete violent relaxation and a meta-stable equilibrium (the so called quasi-stationary states, QSS) observed in the models of the systems with the long-range interactions are actively discussed (Chavanis, 2008a,b, 2006b; Bouchet et al., 2010). According to these papers, the reasons for the occurrence of such states in these systems are not completely clear. In the case of the OSCs, one of the possible causes for the formation of the metastable equilibrium states is a temporary suppression of the instability of the radial oscillations in the core of the system due to its "heating" by the oscillations of the regular cluster field. The following temporary expansion of the core and of the whole cluster (after the decay of the collective oscillations and the transition of the kinetic energy of the cluster oscillations to the thermal energy of a stellar motion) leads to a cooling of the core and the development of a gravitational instability in time $t \leq \tau_{s t}$, where $\tau_{s t}$ is the relaxation time of the cluster due to the stellar encounters (see Danilov (2011) on the stages of a temporary virilization during up to $10^{8}$ years in the OSC models with a subsequent development of the oscillations of the cluster core). The existence and instability of such oscillations in the OSC models have been theoretically considered by Danilov (2011) and Danilov (2008), see an introduction to this chapter, and are confirmed by one more method in this part of the book.

\subsection{Conclusions}

1. In this chapter, we have considered the estimates of the correlation time $\tau_{c}^{(f)}$ in the space of the $f$ values in the OSC models 1-6. Correlations in the space of the $f$ values are most rapidly destroyed during the evolution of model 6. Comparison with the results of Danilov and Putkov (2012b) on $\tau_{c}^{(r)}$ (a correlation time in the space of $r$ ) showed that $\tau_{c}^{(f)}<\tau_{c}^{(r)}$ in the OSC models 1-6. The ratio $\tau_{c}^{(r)} / \tau_{c}^{(f)}=q$ increases from $q \simeq 2.1$ in model 1 to $q \simeq 17.0$ in model 6 .

2. We have considered the estimates of the mean phase velocities $v_{f}, v_{r}, v_{v}$ of the oscillations of $f, r, v$ in the OSC models 1-6. The values $v_{f}$ and $v_{v}$ in models 1-4 are 5-20 times smaller than $v_{f}$ and $v_{v}$ in models 5, 6. Possibly, it is due to the greater wavelengths of the oscillations of $f$ and $v$ in models 5,6 . The small values of $v_{f}$ and $v_{v}$ in models 1-4 can be the result of the formation and superposition of the several counter-propagating running radial waves in these models. The values 
$v_{f}$ are 10-20 times smaller than the mean square velocities $v_{c}$ of a stellar motion in the cores of models 1-4 and approximately 2 times smaller than $v_{c}$ in model 5, 6 .

3 . We have discussed the cross-correlation functions of the phase-density $f$ oscillations in the OSC models. We have noted a number of the local extremums of the function $C_{12}(\tau, r)$, exceeding the limits of the errors of the $C_{12}(\tau, r)$ values at the points $\tau_{j}=P_{j}$. It indicates the existence of the increased cross correlations (with the different signs) between the $f$ oscillations with periods $P_{j}$ in the cluster center and at the distance $r$ from its center. We have also considered the frequency spectrum of the $f$ oscillations corresponding to the function $C_{12}(\tau, r)$. The largest contribution to the average power of the $f$ oscillations is made by low frequencies $v \in[0.5,2.4]$, corresponding to the oscillations of the core and of the entire cluster. A number of the local maxima of the frequency spectrum that go beyond the limits of the errors of the calculation of the spectrum are noted. It indicates the increased power and intensity of an excitation of the $f$ oscillations with the frequencies $v$ from the neighborhoods of the local maxima points of $S_{v}$. The possible role of the resonances in the formation of the different parts of the frequency spectrum have been noted. A comparative analysis of the spectra of models 1-6 allows us to assume that the greater degree of a non-stationarity of model 1 in comparison with other models is provided by the larger values $\bar{S}_{v}^{\prime}$, by a greater number of the local maxima of the function $S_{v}$, and by the greater density of the maxima location in the low-frequency region of the spectrum.

4. We have considered the dispersion curves for the phase-density $f$ oscillations in the numerical dynamic OSC models. We have confirmed the presence of the known unstable phase-density oscillations in the cores of the cluster models associated with the homologous oscillations of the cores. A few dozens of the new unstable phase-density oscillations have been detected in each of these models. The number of such oscillations decrease not monotonically with the increasing distance $r$ from the cluster center. The possible mechanisms for the development of such oscillations in the OSC models have been discussed. The high-frequency unstable $f$ oscillations may well be because of the development of the polarisation clouds due to the passage of stars in the OSC models, the effect of resonances between the $f$ oscillations at the different frequencies or the turbulence in the stellar motions in the cluster model 1.

5. The phases $r k_{v}$ of the unstable $f$ oscillations are fairly evenly distributed along the $k_{v}$ axis. The histograms $N\left(k_{v}\right)$ also show a fairly uniform distribution of $k_{v}$. The multiple changes of the $k_{v}$ sign in the considered frequency range on the dispersion curves indicate a formation of a number of the counter-propagating running radial waves of the $f$ oscillations in models 1-6.

6 . The presence of a large number of the unstable $f$ oscillations, as well as the increased power and, consequently, the intensity of an excitation of such oscillations in model 1 are considered as arguments in favor of the turbulence developing in the cluster model 1.

7. An investigation of the velocities of the stellar motion in a number of the OSCs from the list of Danilov and Seleznev (1994) can provide the additional data (to the data obtained in Danilov and Putkov $(2012 \mathrm{a}, \mathrm{c})$ ) on the non-stationarity of these clusters, on the instability of the $f$ oscillations in the clusters, the parameters of which 
are indicated in Table 12.2 by the sign "*". To do this, the radial dependencies of the velocity dispersion of the cluster stars, the dispersion of the tangential velocities obtained from the data on the proper motion of stars, the dispersion of the radial stellar velocities in the wide vicinities of these clusters can be used. An increase of the dispersion of the stellar velocities with a distance from the cluster center is an important kinematic sign of the OSC non-stationarity (Danilov, 2011).

\section{References}

Aarseth, S.J.: Dynamical evolution of simulated star clusters. I - Isolated models. Astron. Astrophys. 35, 237-250 (1974).

Baertschiger, T., Labini, F.S.: Growth of correlations in gravitational N-body simulations. Physical Rev. D. 69, 123001 (2004). doi:10.1103/PhysRevD.69.123001

Barkhatova, K.A.: An open star cluster NGC 7086. Astronomicheskii Zhurnal, 34, 556-562 (1956) (In Russian).

Bendat, J.S., Piersol, A.G.: Engineering Applications of Correlation and Spectral Analysis. Wiley-Interscience, New York (1980).

Bernar, M., Briffo, J., Bussac, J., et al.: Correlation methods of analysis of plasma experiments. In: Diagnostics of plasma, Isssue 3. Atomizdat, Moscow (1973), 449-460.

Binney, J., Tremaine, S.: Galactic Dynamics, Second edition. Princeton University Press, Princeton, Oxford (2008). ISBN: 9780691130279

Boccaletti, D., Pucacco, G., Ruffini, R.: Multiple relaxation time-scales in stellar dynamics. Astron. Astrophys. 244, 48-51 (1991).

Bottaccio, M., Montuori, M., Pietronero, L., et al.: N-body simulations for structure formation from random initial conditions. Memorie della Societá Astronomica Italiana Suppl. 1, 120-129 (2003).

Bottaccio, M., Pietronero, L., Amici, A. et al.: Clustering in N-body gravitating systems. Physica A. 305, 247-252 (2002). doi:10.1016/S0378-4371(01)00670-7

Bouchet, F., Gupta, S., Mukamel, D.: Thermodynamics and dynamics of systems with long-range interactions. Physica A. 389, 4389-4405 (2010). doi:10.1016/j.physa.2010.02.024

Chavanis, P.H.: Hamiltonian and Brownian systems with long-range interactions: II. Kinetic equations and stability analysis. Physica A. 361, 81-123 (2006a). doi:10.1016/j.physa.2005.06.088

Chavanis, P.H.: Quasi-stationary states and incomplete violent relaxation in systems with long-range interactions. Physica A. 365, 102-107 (2006b). doi:10.1016/j.physa.2006.01.006

Chavanis, P.H.: Hamiltonian and Brownian systems with long-range interactions: III. The BBGKY hierarchy for spatially inhomogeneous systems. Physica A. 387, 787-805 (2008a). doi:10.1016/j.physa.2007.10.026 
Chavanis, P.H.: Hamiltonian and Brownian systems with long-range interactions: IV. General kinetic equations from the quasilinear theory. Physica A. 387, 1504-1528 (2008b). doi:10.1016/j.physa.2007.10.034

Cleveland, W.S., Devlin, S.J.: Locally weighted regression: an approach to regression analysis by local fitting. Journal of the American Statistical Association. 83, 596610 (1988). doi:10.1080/01621459.1988.10478639

Danilov, V.M.: Statistical analysis of dynamical open-cluster models with small differences in their initial stellar phase coordinates. Astron. Letters, 23, 322-326 (1997b).

Danilov, V.M.: Stellar Fluxes in Numerical Dynamical Models of Open Clusters. Astron. Reports 46, 887-899 (2002b). doi:10.1134/1.1522077

Danilov, V.M.: Analysis of density fluctuations in models of open clusters. Astron. Reports 52, 888-899 (2008). doi:10.1134/S1063772908110036

Danilov, V.M.: Phase-Density Fluctuations at the Centers of Six Open Clusters. Astron. Reports 54, 514-527 (2010). doi:10.1134/S1063772910060053

Danilov, V.M.: On the dynamics of open clusters, Astron. Reports 55, 473-486 (2011). doi:10.1134/S1063772911060035

Danilov, V.M., Dorogavtseva, L.V.: Estimates of Relaxation Times in Numerical Dynamical Models of Open Star Clusters. Astron. Reports 47, 483-491 (2003). doi:10.1134/1.1583775

Danilov, V.M., Dorogavtseva, L.V.: Timescales for mechanisms for the dynamical evolution of open star clusters. Astron. Reports 52, 467-478 (2008). doi:10.1134/S1063772908060048

Danilov, V.M., Leskov, E.V.: Properties of Stellar Trajectories in Numerical Dynamical Models of Open Star Clusters. Astron. Reports 49, 190-200 (2005). doi:10.1134/1.1882777

Danilov, V.M., Putkov, S.I.: Non-stationarity parameters of open clusters. Astron. Reports 56, 609-622 (2012a). doi:10.1134/S106377291208001X

Danilov, V.M., Putkov, S.I.: The dynamics of correlations in open-star cluster models. Astron. Reports 56, 623-637 (2012b). doi:10.1134/S1063772912080021

Danilov, V.M., Putkov, S.I.: VizieR Online Data Catalog: Dynamical parameters of open clusters (Danilov+,2012) (2012c).

Danilov, V.M., Putkov, S.I.: Correlations, spectra and instability of phase-space density fluctuations in open-cluster models. Astrophys. Bull. 68, 154-168 (2013b). doi:10.1134/S199034131302003X

Danilov, V.M., Ryazanov, A.P.: On modelling spherical instationary collisionless stellar systems. In: Barkahatova, K.A. (ed.) Astronomical-geodetical investigations. Ural State Univ. Publ., Sverdlovsk (1985), 19-47 (In Russian).

Danilov, V.M., Ryazanov, A.P.: On a dynamics of spherical non-stationary stellar systems. Astronomicheskii Tsirkulyar. No.1487, 3-4 (1987). (In Russian).

Danilov, V.M., Seleznev, A.F.: The catalogue of structural and dynamical characteristics of $103 \mathrm{OCl}$ and the first results of its investigations. Astron. Astrophys. Trans. 6, 85-156 (1994). doi:10.1080/10556799408232061

Gilbert, I.H.: Collisional Relaxation in Stellar Systems. Astrophys. J. 152, 1043-1056 (1968). doi:10.1086/149616 
Gilbert, I.H.: Gravitational Polarization in Spherical Stellar Systems. Astrophys. J. 159, 239-246 (1970). doi:10.1086/150306

Goodman, J., Heggie, D.C., Hut, P.: On the Exponential Instability of N-Body Systems. Astrophys. J. 415, 715-733 (1993). doi:10.1086/173196

Gray, D.F.: The observation and analysis of stellar photospheres. Wiley, New York (1976). ISBN:04713238029780471323808

Gurzadyan, V.G., Savvidy G.K.: Collective relaxation of stellar systems. Astron. Astrophys. 160, 203-210 (1986).

Kandrup H.E.: The time scale for "mixing" in a stellar dynamical system. Phys. Lett. A. 140 97-100 (1989). doi:10.1016/0375-9601(89)90497-0

Kandrup H.E.: How fast can a galaxy "mix"? Physica A. 169, 73-94, (1990a). doi:10.1016/0378-4371(90)90217-G

Kandrup H.E.: Divergence of nearby trajectories for the gravitational N-body problem. Astrophys. J. 364, 420-425 (1990b). doi:10.1086/169425

Kandrup H.E.: Violent Relaxation, Phase Mixing, and Gravitational Landau Damping. Astrophys. J. 500, 120-128 (1998). doi:10.1086/305721

Kandrup, H.E., Magon, M.E., Smith, H.C.: On the sensitivity of the N-body problem toward small changes in initial conditions. 4. Astrophys. J. 428, 458-465 (1994). doi:10.1086/174259

Kandrup, H.E., Smith, H.Jr.: On the sensitivity of the N-body problem to small changes in initial conditions. Astrophys. J. 374, 255-265 (1991). doi:10.1086/170114

Kandrup, H.E., Smith, H.Jr., Willmes, D.E.: On the sensitivity of the N-body problem to small changes in initial conditions. III. Astrophys. J. 399, 627-633 (1992). doi:10.1086/171954

King, I.R.: The structure of star clusters. I. An empirical density law. Astron. J. 67, 471-485 (1962). doi:10.1086/108756

Klimontovich, Yu.L.: Statistical Physics. Nauka, Moscow (1982). (In Russian).

Klimontovich, Yu.L.: Statistical Theory of Open Systems. Yanus-K, Moscow (1995). (In Russian).

Komatsu, N., Kiwata, T., Kimura, S.: Numerical irreversibility in self-gravitating small N-body systems. Physica A: Statistical Mechanics and its Applications. 387, 2267-2278 (2008). doi:10.1016/j.physa.2007.12.012

Miller R.H.: Irreversibility in Small Stellar Dynamical Systems. Astrophys. J. 140, 250-256 (1964). doi:10.1086/147911

Nardini, C., Gupta, S., Ruffo, S., et al.: Kinetic theory of nonequilibrium stochastic long-range systems: phase transition and bistability. J. Stat. Mech.: Theory and Experiment 12, P12010 (2012). doi:10.1088/1742-5468/2012/12/P12010

Polyachenko, V.L., Fridman, A.M.: Equilibrium and stability of gravitating systems. Nauka, Moscow (1976). (In Russian).

Seleznev, A.F.: Stellar surface density distributions in ten open clusters. Astron. Astrophys. Trans. 4, 167-177 (1994). doi:10.1080/10556799408205373

Severne, G., Haggerty, M.J.: Kinetic theory for finite inhomogeneous gravitational systems. Astrophys. Space Sci. 45, 287-302 (1976). doi:10.1007/BF00642666 
Volkov, Ya.F., Dyatlov V.G., Mitina, R.I.: Diagnostics of turbulent plasma. Naukova Dumka, Kiev (1983). (In Russian).

Zaslavsky, G.M., Sagdeev R.Z.: Introduction to non-linear physics: from pendulum to turbulence and chaos. Gl.Red.Fiz.Mat.Lit., Moscow (1988). (In Russian). 


\title{
Chapter 13 \\ Smoothing of Force Functions and Oscillation Spectra of Open Star Cluster Model
}

\begin{abstract}
In this chapter, we consider the cross-correlation functions for the fluctuations of the potential $U$ and the phase density $f$ of the OSC model for different distances from its center at the different values of the smoothing parameter $\epsilon$ of the force functions in the equations of the cluster stars' motion. We discuss the power spectra and the dispersion curves of $U$ and $f$. The spectrum of the $U$ oscillations has a simpler form in comparison with the spectrum of the $f$ oscillations. The most powerful $U$ oscillations are related to the $f$ oscillations and are located at the low frequencies $v<3 / \tau_{v r}$; at the medium and high frequencies $\left(v>3 / \tau_{v r}\right)$, the contribution of the $U$ oscillations to the formation of the $f$ oscillations is small or equal to zero (here, $\tau_{v r}$ is the time of a violent relaxation of the cluster). A number of the unstable $U$ oscillations in the core of the cluster model are noted (up to 30 pairs of the oscillations with the different complex conjugate frequencies). The dependencies of the spectra and the dispersion curves of the $f$ and $U$ oscillations on $\epsilon$ are discussed. We note a "repeatability" (a significant correlation) of the spectra for certain values of $\epsilon$ and the instability of the shape of the dispersion curve to small changes of $\epsilon$. The astrophysical applications of the results are discussed (the decay of the wave of the $f$ oscillations running from the periphery of the cluster to its center into several waves with the frequencies commensurate with the frequency of the external (tidal) impact; radiation and reflection of the waves of the $f$ and $U$ oscillations near the boundary of the cluster core; a possible discreteness of the wavelengths and phases of the $f$ and $U$ oscillations in the cluster model).
\end{abstract}

\subsection{Introduction}

A smoothing of the force functions in the equations of the stellar motion is often used in modeling the dynamics of the gravitating systems, see, for example, Binney and Tremaine (2008); Kandrup and Haywood (1991, 1992); Kandrup et al. (1992); El-Zant (1998); Goodman et al. (1993); Danilov (1997a). Such an approach greatly facilitates the numerical integration of the equations of a stellar motion, but does not 
consider the closest encounters of stars with the mutual distances $r_{i j}<\epsilon$, and the potential of the system is most often considered in the form

$$
U(\mathbf{r})=\sum_{j=1}^{N} G m_{j} / \sqrt{\left(\mathbf{r}-\mathbf{r}_{j}\right)^{2}+\epsilon^{2}}
$$

(here, $\epsilon$ is a smoothing parameter, $\mathbf{r}$ is the radius vector of the point, $N$ is the star number, $m_{j}$ is the mass of the star with number $j \in[1, N]$; in this case, the point potential of an individual star is replaced by the potential of the Plummer sphere). As a justification for such smoothing, the following argument is usually used: for a sufficiently small $\epsilon$, the close encounters of the stars with the mutual distances $r_{i j}<\epsilon$ are quite rare, and the estimates of the contribution of such encounters to the relaxation of the system due to the pair encounters are negligible (see, for example, Dehnen (2001)). Using a smoothing of the force functions, the exponential instability of the $N$-body problem was studied in the papers of Kandrup and Haywood (1991, 1992); Kandrup et al. (1992); El-Zant (1998); Goodman et al. (1993) (for the first time, the exponential instability of such systems was recognized in the work of Miller (1964)). According to Kandrup and Haywood (1991, 1992); Kandrup et al. (1992); El-Zant (1998), the time $t_{e}$ of an increase of the initial perturbations of the stellar trajectories in $e$-times is comparable with the crossing time $t_{c r}$ (the time of the star's intersection of the system) or slightly less than $t_{c r}$. The value $t_{e} / t_{c r}$ weakly decreases with the increasing $N$, if smoothing is sufficiently small. According to the estimates of Goodman et al. (1993); Hemsendorf and Merritt (2002), performed at $N<10^{3}$ and at $N \lesssim 10^{5}, t_{e} / t_{c r} \sim 1 / \ln (N)$ or $\sim 1 / \ln (\ln (N))$. An increase of $\epsilon$ leads to the increasing $t_{e} / t_{c r}$ and decreasing rate of growth of perturbations in the system (Kandrup et al., 1992; El-Zant, 1998; Goodman et al., 1993).

Dehnen (2001); Merritt (1996); Athanassoula et al. (2000); Hernquist and Katz (1989); Rodionov and Sotnikova (2005) discussed and applied the optimal values of $\epsilon=\epsilon_{\text {opt }}$ (first obtained by Merritt (1996) from the condition of the minimum of the mean irregular force impact on the star) in order to model the collisionless stellar systems. According to Merritt (1996), at large $\epsilon>\epsilon_{\text {opt }}$, the potential of the system is significantly distorted and the spatial resolution and a "graininess" of the potential are reduced. When $\epsilon<\epsilon_{o p t}$, the fluctuations of the forces in the system increase, and it ceases to be collisionless. According to Merritt (1996); Athanassoula et al. (2000), $\epsilon_{o p t}$ depends on the density distribution in the system model and decreases with an increasing $N$ in the system. Athanassoula et al. (2000) considered three different models of the mass density distribution in the system and showed that in the systems with a higher mass concentration to the center, $\epsilon_{\text {opt }}$ can be reduced. Athanassoula et al. (2000) also discuss two different types of smoothing of the point mass potential via a Plummer's generalized potential (see formula (11) from Athanassoula et al. (2000)) and a cubic spline proposed by Hernquist and Katz (1989). According to Athanassoula et al. (2000), these types of smoothing can in some cases give a better representation for the forces in the system than a smoothing according to the potential of the Plummer's standard sphere. 
Dehnen (2001), when modeling the collisionless systems, proposed to use an adaptively varying $\epsilon$ depending on the average distance between the stars at a given point in the system (this approach allows us to construct a much better approximation of the many-body systems to the models of the collisionless systems obtained with the help of the corresponding Boltzmann equation (Dehnen, 2001)). According to Rodionov and Sotnikova (2005), this approach in the numerical experiments leads to a change of the total energy of the many-body systems with the time $t$. Rodionov and Sotnikova (2005), for the spherically symmetric equilibrium stable models of gravitating systems, proposed to obtain $\epsilon_{\text {opt }}$ from the analysis of the time evolution of the distribution functions of the many-body systems for the various $\epsilon$. Price and Monaghan (2007) considered the changes in the Lagrange function of the system leading to a conservation of an energy (and an angular momentum) in the many-body problem. In order to do this, Price and Monaghan (2007) proposed to introduce a small addition to the interaction force of two stars, related to the gradient of the smoothing parameter with respect to the spatial coordinates (a similar idea was also considered by Hernquist and Barnes (1990)). According to the calculations performed by Price and Monaghan (2007) (see fig. 13.7, 13.6 from Price and Monaghan (2007)), this approach leads to the changes in the density distribution and in the energy constant of the system in comparison with the case of the non-adaptive $\epsilon=$ const .

It is of interest to study the dependencies on $\epsilon$ of the parameters of the OSC models of Danilov and Dorogavtseva (2008) with the significantly lower values of $\epsilon=0.012 \bar{r}_{i j}$ used by Danilov (1997a); Danilov and Dorogavtseva (2008). Here, $r_{i j}$ is the distance between two stars with the numbers $i$ and $j$ in the cluster, $i, j \in[1, N]$, $\overline{r_{i j}}$ is the the initial mean $r_{i j}$ for all pairs of the stars in the cluster model. In this case, the value of $\epsilon$ averaged by six cluster models of Danilov and Dorogavtseva (2008) is $\epsilon \simeq 0.084 \pm 0.005 \mathrm{pc}$, which is much less than the average impact distance $p_{2} \simeq R / N^{1 / 2} \simeq 0.45 \mathrm{pc}$ of the dominant pair encounter of the stars, and the average distance between the stars $p_{1} \simeq R / N^{1 / 3} \simeq 1.32 \mathrm{pc}$ (here, $R$ is the cluster radius). According to the estimates of Binney and Tremaine (2008); Goodman et al. (1993), the conditions $p_{1}>\epsilon>p_{2}$ allow to smooth the forces of the pair interactions between the stars without an appreciable weakening of the spatial resolution of the potential and the exponential instability (Miller, 1964) in the many-body system.

According to Table 2 from Danilov (2010), it is possible to obtain the average (over a period of the regular field oscillations) distance $\delta_{r}$ between the stars located near the cluster model center. On average over the OSC models of Danilov and Dorogavtseva (2008), $\delta_{r} \simeq 1.11 \pm 0.10 \mathrm{pc}$. In this case, $\delta_{r} / \epsilon \simeq 13.2 \pm 1.4$. Thus, $\epsilon$ in the OSC models of Danilov and Dorogavtseva (2008) is significantly smaller than $p_{i}$ and $\delta_{r}$, which indicates a quite "collisional" character of these models (only the fairly rare close encounters of the stars with $r_{i j}<\epsilon$ in the OSC models of Danilov and Dorogavtseva (2008) are not considered). We note that Price and Monaghan (2007) considered only the changes in the radial distributions of a density and in a total energy of an isolated gravitating system under the changes of $\epsilon$. It is also of interest to analyze the effect of the changes in $\epsilon$ on the degree of non-stationarity of the OSC model, the density of the star number, and the kinetic energy of the cluster core in the presence of the external field of the Galaxy. These cluster characteristics 
can influence the formation of the oscillation spectra of the phase density $f$ and the potential $U$ of the cluster, as well as the dispersion curves of these oscillations and the estimates of the parameters of such oscillations. An analysis of the changes in the spectra and the dispersion curves of the $f$ and $U$ oscillations as a function of the $\epsilon$ changes for the OSC models has not been carried out previously. Such an analysis is of considerable interest, since it gives an additional information on the character, parameters, and reasons for the formation of the standing and running waves of the $f$ and $U$ oscillations in the OSC models with the different $\epsilon$.

The spectra and the dispersion curves of the $f$ oscillations in the framework of the numerical experiments on the modeling of an OSC dynamics were first obtained by Danilov and Putkov (2013b,a). For this purpose, the methods of a diagnostics of a turbulent plasma considered by Volkov et al. (1983); Bernar et al. (1973) were used. According to Volkov et al. (1983); Bernar et al. (1973), the cross-correlation functions of the fluctuations of a number of the plasma characteristics at two points at a distance $r$ from each other can be used to construct the dispersion curves and to analyze the instabilities of the oscillations in a plasma. The use of such a method by Danilov and Putkov (2013b,a) for the construction of the frequency spectra (power spectra) and the study of a number of the $f$ oscillation instabilities in the OSC models of Danilov and Dorogavtseva (2008) made it possible to detect a number of the new unstable phase density oscillations in these models (up to 32-41 pairs of the oscillations with the different complex conjugate frequencies in each model) and to estimate the parameters of these oscillations. For model 1, the most complicated $f$ oscillation spectrum was obtained, which, according to Danilov and Putkov $(2013 \mathrm{~b}, \mathrm{a})$, is one of the signs of a weak turbulence in the stellar motions in this cluster model. Since $\epsilon$ appears in the equations of the stellar motion due to the potential $U$ (see above) it is also of interest to analyze the $U$ oscillations in this cluster model. Comparison of the spectra of the $f$ and $U$ oscillations in the OSC model 1 of Danilov and Dorogavtseva (2008) allows us to study the question of the degree of coupling between the $f$ and $U$ oscillations with the different cyclic frequencies $v$ (in the following, the frequencies $v$ are given in the units of $\tau_{v r}^{-1}$ and are equal to the number of the $f$ (or $U$ ) oscillations during the violent relaxation time $\tau_{v r}$ of the cluster model).

According to Danilov and Putkov (2013b), the smallest phase-density $f$ oscillations (and closest to them) near the centers of the OSC models are not equal to the frequency $v_{G}$ of the OSC rotation around the Galactic center, but are approximately in a rational ratio with it. For example, in model 1, the frequencies of three such oscillations are approximately $3 / 5,20 / 13$ and $12 / 5$ of $v_{G} \simeq 0.225$. A similar situation exists in other OSC models of Danilov and Dorogavtseva (2008). Therefore, Danilov and Putkov $(2013 \mathrm{~b}$,a) made a conclusion about a possible influence of the external field of the Galaxy onto this part of the $f$ oscillations spectrum. $v_{G}$ to a greater extent characterizes the frequency of the tide, i.e. it is associated with the changes in the density at the periphery of the OSC models. The frequencies of the phase-density oscillations corresponding to the external effect on the cluster at the different distances from the model centers may differ from $v_{G}$. The waves of the $f$ oscillations with a frequency $v_{G}$ or close to it, which run towards the cluster center 
( $k_{v}<0, k_{v}$ is a wave number), are observed at all distances $r$ from the cluster center considered in Danilov and Putkov (2013b). Apparently, when such waves pass to the cluster center, the characteristics of the waves change, the wave decays into several components with the commensurate frequencies. In model 1, the oscillations with the frequency $v_{G}$ are stable at $r=3-9 \mathrm{pc}$ and, probably, at $r>9 \mathrm{pc}$. The instability, and then the decay of such oscillations, into the frequency-commensurate unstable oscillations takes place when $r<3 \mathrm{pc}$.

In the OSC models of Danilov and Dorogavtseva (2008), the motion of the waves of the $f$ oscillations with certain frequencies $v$ close to certain distances $r$ from the cluster center has an unusual character. For example, in model 1 near the distance $r$ $=3 \mathrm{pc}$ (approximately at the core boundary) at frequencies close to $v=0.34,1.81$, the standing waves of the phase density oscillations are observed $\left(k_{v}=0\right)$. At $r$ $=2.7 \mathrm{pc}$, the waves at frequencies $v=0.34,1.81$ become to be running toward the cluster center, see fig.13.1a. At $r>3.3 \mathrm{pc}$, at these frequencies, the waves running from the cluster center are formed (fig. 13.1b, $k_{v}>0$ ). A similar phenomenon can be observed near the distances from the center $r=3.3-3.4 \mathrm{pc}$ at the frequency $v \simeq 3.6$, see fig. $13.1 \mathrm{~b}$.
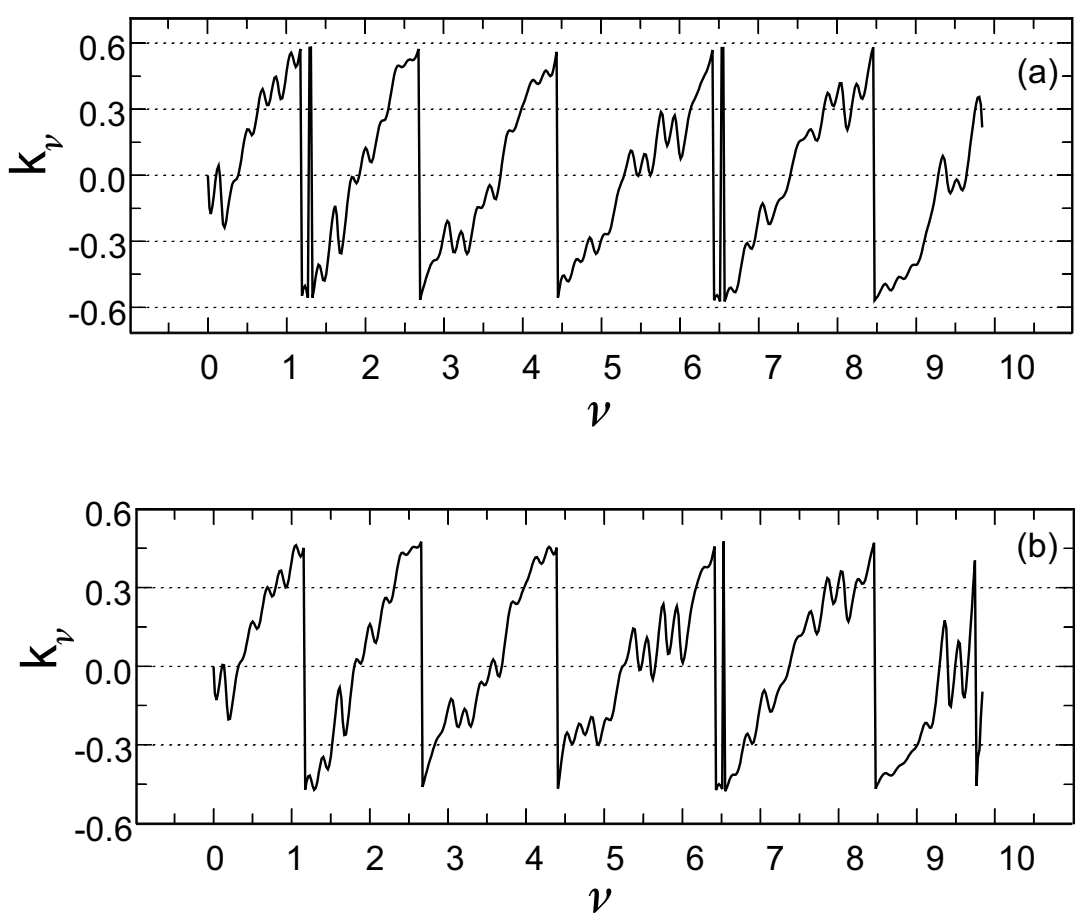

Fig. 13.1 The dependencies of $k_{v}$ on $v$ for the $f$ oscillations in the OSC model 1 at $n_{p}=483$, $n_{s t}=30$ : (a) the curve is obtained at $r=2.7 \mathrm{pc}$; (b) the same at $r=3.3 \mathrm{pc}$ 
Perhaps, there is a reflection of the running waves from the boundary of the cluster center, with three standing waves form one running wave. The mean (by the frequency $v$ ) standing wave at $r=3 \mathrm{pc}$ is located in a region with an anomalous dispersion (where $k_{v}$ decreases with an increasing $v$; the value $k_{v}$ is obtained using the programs of the calculating of the dispersion curves $k_{v}=k(v)$ from Danilov and Putkov (2013b)). We note that the running waves can sometimes be represented as a superposition of the standing waves with the same wavelength as in the running wave (Crawford, 1968).

A probable reason for such changes in the characteristics of the phase-density waves near $r=3 \mathrm{pc}$ in model 1 , also, can be a radiation of such waves in the opposite by $r$ directions from the boundary of the cluster core experiencing the radial oscillations and determining the degree of the model's non-stationarity (Danilov, 2008; Danilov and Putkov, 2012a).

Probably, in the OSC models, both reflection and emission of the waves at the frequencies $v$ corresponding to these waves at the certain distances $r$ from the cluster center can exist. This suggests a possible quantisation of such waves and the existence of certain relationships between the dimensions of the clusters and possible wavelengths $\lambda$ of the standing waves in the cluster, since in the finite-size systems (with respect to $r$ ) only an integer number of the values of $\lambda / 2$ can be placed, see, for example, Crawford (1968). It is of interest to study the observed manifestations of the discreteness of the emerging wavelengths and phases of the $f$ oscillations in the OSC models with the different $\epsilon$ values.

The objectives of this part of the work are: 1) to consider a number of the structural and dynamic characteristics of the core of the OSC model 1 of Danilov and Dorogavtseva (2008) for the different values of the smoothing parameter $\epsilon ; 2$ ) to discuss the cross-correlation functions, the frequency spectra and the dispersion curves for the phase-density $f$ fluctuations and the potential $U$ fluctuations in this cluster model for the different $\epsilon$ values; 3 ) to use the reviewed results in order to analyse the dynamics of the OSCs and their models.

\subsection{On a Connection Between the Parameters of the OSC Model and $\epsilon$}

Danilov and Dorogavtseva (2008) consider a cluster model consisting of $N=500$ stars with the masses equal to the solar one and moving along a circular orbit with the radius of $R_{G}=8200 \mathrm{pc}$ in the plane of the Galaxy around its center (the Galaxy potential model of Kutuzov and Osipkov (1980) was used). The nitial parameters of these numerical dynamic OSC models are given in Table 1 from Danilov and Dorogavtseva (2003); a description of the cluster models 1-6 (Danilov and Dorogavtseva, 2008, 2003) is given in Section 16.1. One can find there the discussion of the difference schemes of the 10th and 11th accuracy orders, the statistical criterion for the accuracy of the calculation of the phase density (Danilov, 1997b) used for the construction of the OSC models, and an introduction of the time 
interval $t_{0}$ of the dynamic evolution of the OSC model, during which the statistical accuracy criterion is fulfilled. At $t \in\left[0, t_{0}\right]$, an accuracy of the distributions of the correlations obtained in Danilov and Putkov (2012b) proves to be sufficient for the conclusions about the properties of these distributions. According to Danilov and Putkov (2013b), a comparison of the cross-correlation functions, the oscillation spectra or the dispersion curves obtained by the 10th and 11th accuracy orders also provides an effective method for a controlling of the results of the calculations.

Following to Danilov and Putkov (2014), we consider the effect of a smoothing of the force functions at the right-hand sides of the equations of a stellar motion on the evolution of the OSC model 1 (Danilov and Dorogavtseva, 2008). Let $\epsilon=$ $\epsilon_{1}=0.012 \overline{r_{i j}}$ (the OSC models in are obtained by Danilov and Dorogavtseva (2008) using the values of $\epsilon=\epsilon_{1}$ ). Let $\alpha=2 E / W$ be a virial coefficient of the cluster model at the time $t$, where $E$ and $W$ are the total and potential energy of the cluster (the influence of the external field of the Galaxy on the cluster is not taken into account in the formulas for $E$ and $W$ ). In what follows, we shall use the value $\alpha$, obtained by the stars with the distances $r \leq R_{t}$ from the cluster center, where $R_{t}$ is a tidal radius of the cluster in the field of the Galaxy (Hemsendorf and Merritt, 2002). Let us consider the evolution of the cluster model at $\epsilon=\epsilon_{1}$ and at $\epsilon=2 \epsilon_{1}=\epsilon_{2}$ for a period of time $t \in\left[0,3 \tau_{v r}\right]$. The values of $\alpha$ averaged over $t$ are: $\alpha_{\epsilon_{1}}=0.410 \pm 0.002$ and $\alpha_{\epsilon_{2}}=0.401 \pm 0.002$ for $\epsilon_{1}$ and $\epsilon_{2}$, respectively.

Let $\sigma^{\prime}\left(\alpha_{\epsilon}\right)$ be a standard deviation of $\alpha$ from the average values of $\alpha$ (over $\left.t \in\left[t^{\prime}, t^{\prime}+P_{r}\right]\right)$ for a given $\epsilon$, where $t^{\prime} \in\left[0,\left(3 \tau_{v r}-P_{r}\right)\right] ; P_{r}$ is a period of the oscillations of a regular field in the cluster model. The average (over all $t^{\prime}$ ) values of $\sigma^{\prime}\left(\alpha_{\epsilon}\right)$ are: $\sigma\left(\alpha_{\epsilon_{1}}\right)=0.122 \pm 0.026$ and $\sigma\left(\alpha_{\epsilon_{2}}\right)=0.116 \pm 0.035$ for $\epsilon_{1}$ and $\epsilon_{2}$, respectively. Considering $\sigma\left(\alpha_{\epsilon}\right)$ as an estimate of $\delta \alpha$, which is an amplitude of the $\alpha$ oscillations, we find $\delta \alpha_{\epsilon_{1}} / \delta \alpha_{\epsilon_{2}}=1.06 \pm 0.39$. The values of $\delta \alpha$ in the OSC models of Danilov and Dorogavtseva (2008) characterize the degree of a non-stationarity of the models in a regular field. Thus, an increase of $\epsilon$ by a factor of two does not lead to a statistically significant change in the degree of non-stationarity of the cluster model 1 .

Let $T_{i, \epsilon}$ be an average (by $t \in\left[0,3 \tau_{v r}\right]$ ) square of the star's velocity obtained from stars with the distances $r \leq i$ pc from the cluster center at $i=1,2,5$ for a given $\epsilon$. According to the data on the phase coordinates of the stars of the cluster model at $t \in\left[0,3 \tau_{v r}\right]$, we find $T_{1, \epsilon_{1}} / T_{1, \epsilon_{2}}=1.13 \pm 0.06, T_{2, \epsilon_{1}} / T_{2, \epsilon_{2}}=1.07 \pm 0.02$, $T_{5, \epsilon_{1}} / T_{5, \epsilon_{2}}=1.05 \pm 0.01$. Consequently, with a two-time increase in $\epsilon, T_{i, \epsilon}$ decreases significantly (by $\sim 13-7 \%$ ) in the cluster core (at $i=1,2)$.

Let $N_{i, \epsilon}, \rho_{i, \epsilon}, R_{i, \epsilon}$ be the average (by $t \in\left[0,3 \tau_{v r}\right]$ ) number of stars, concentration, and mean radius of a group of stars with the distances $r \leq i \mathrm{pc}$ from the center of the cluster model for a given $\epsilon$. According to the stellar coordinates of the cluster model at $t \in\left[0,3 \tau_{v r}\right]$, we find $N_{1, \epsilon_{1}} / N_{1, \epsilon_{2}}=1.40 \pm 0.14, N_{2, \epsilon_{1}} / N_{2, \epsilon_{2}}=1.12 \pm 0.03$, $N_{5, \epsilon_{1}} / N_{5, \epsilon_{2}}=1.013 \pm 0.005$. Thus, when $\epsilon$ increases by two times, the number of stars $N_{i, \epsilon}$ decreases significantly (by $\sim 40-12 \%$ ) in the cluster core. At the same time, the average radius $R_{i, \epsilon}$ varies very little (in the core of the cluster model, the changes of $R_{i, \epsilon}$ are within the errors of $R_{i, \epsilon}$ ). Moreover, $\rho_{1, \epsilon_{1}} / \rho_{1, \epsilon_{2}}=1.35 \pm 0.16$, $\rho_{2, \epsilon_{1}} / \rho_{2, \epsilon_{2}}=1.14 \pm 0.04, \rho_{5, \epsilon_{1}} / \rho_{5, \epsilon_{2}}=1.08 \pm 0.02$. Consequently, as $\epsilon$ increases 
by two times, the concentration of stars $\rho_{i, \epsilon}$ in the cluster core also decreases significantly (by $\sim 35-14 \%$ ); part of the stars moves from the core to the cluster periphery.

Let $K_{i, \epsilon}$ be a total kinetic energy of stars with distances $r \leq i \mathrm{pc}$ from the center of the cluster model for a given $\epsilon$. During the evolution of the cluster model, the values $K_{i, \epsilon}$ oscillate around its average (by $t$ ) values $\bar{K}_{i, \epsilon}$. According to the data on the phase coordinates of stars, we find $\bar{K}_{1, \epsilon_{1}} / \bar{K}_{1, \epsilon_{2}}=1.8 \pm 0.3, \bar{K}_{2, \epsilon_{1}} / \bar{K}_{2, \epsilon_{2}}=1.21 \pm 0.04$, $\bar{K}_{5, \epsilon_{1}} / \bar{K}_{5, \epsilon_{2}}=1.07 \pm 0.01$. Thus, a total kinetic energy of the stellar motion in the core of the cluster model decreases noticeably with the two-fold increase of $\epsilon$.

Let $\sigma\left(K_{i, \epsilon}\right)$ be a standard deviation of $K_{i, \epsilon}$ from $\bar{K}_{i, \epsilon}$ at $t \in\left[0,3 \tau_{v r}\right]$. The value of $\sigma\left(K_{i, \epsilon}\right)$ can be considered as an estimate of the oscillation amplitude of $K_{i, \epsilon}$ at the interval $t \in\left[0,3 \tau_{v r}\right]$. Let $z_{i}=\left(\sigma\left(K_{i, \epsilon_{2}}\right) / \bar{K}_{i, \epsilon_{2}}\right) /\left(\sigma\left(K_{i \epsilon_{1}}\right) / \bar{K}_{i, \epsilon_{1}}\right)$. From the data on the phase coordinates of the stars, we find $z_{1}=1.1 \pm 0.5, z_{2}=1.3 \pm 0.7$, $z_{5}=1.1 \pm 0.7$. Probably, with a two-fold increase in $\epsilon$, the values of $\sigma\left(K_{i, \epsilon}\right) / \bar{K}_{i, \epsilon}$ on average increase in the cluster core. However, the errors of $z_{i}$ are large enough, which does not allow us to reliably isolate this effect.

Thus, with a two-fold increase in $\epsilon$, the degree of non-stationarity of the cluster model 1 (Danilov and Dorogavtseva, 2008) practically does not change. In the core of the model, there is a significant decrease in a total kinetic energy of the stars $K_{i, \epsilon}$, a star number $N_{i, \epsilon}$, a stellar concentration $\rho_{i, \epsilon}$, and the mean square of the stellar velocity $T_{i, \epsilon}$; the mean radii of stellar groups $R_{i, \epsilon}$ and the $\sigma\left(K_{i, \epsilon}\right) / \bar{K}_{i, \epsilon}$ values vary only within the limits of the errors. Consequently, a two-fold change in $\epsilon$ significantly changes the structure and some important dynamic parameters of the cluster model. Therefore, such a change in $\epsilon$ for this OSC model can be considered significant.

\subsection{Cross-Correlation Functions}

Let $\mathbf{X}(t, \mathbf{r})=\left(X_{1}, X_{2}\right)=\mathbf{y}(t, \mathbf{r})-\overline{\mathbf{y}(t, \mathbf{r})}$, where $\mathbf{y}=\left(y_{1}, y_{2}\right)=(f, U)$, the value of $f$ is obtained by $n_{s t}$ stars from the neighborhoods of a point with the coordinate $\mathbf{r}$ in the cluster model, and the bar above denotes an averaging over time $t \in\left[0, t_{m}\right]$ (an origin of the coordinate system $\mathbf{r}$ coincides with the mass center of the cluster, $t_{m}$ is the largest value of $t$ used to calculate the correlations). For the sake of a brevity, the argument $\mathbf{v}$ of the functions $f$ and $X_{1}$ is not written here. $\mathbf{X}$ is usually called the deviation or fluctuation of $\mathbf{y}$ (Volkov et al., 1983; Bernar et al., 1973; Danilov and Putkov, 2013b) and is assumed to be a random variable that forms a stationary random process. Unlike to $f$ and $X_{1}$, the $U$ and $X_{2}$ values at the point $\mathbf{r}$ are determined by all cluster stars. Following to Volkov et al. (1983); Bernar et al. (1973); Danilov and Putkov (2013b), we denote the cross-correlation function of the $\mathbf{y}$ fluctuations as $\mathbf{C}(\tau, \mathbf{r}, \Delta \mathbf{r})$, where the components of the vector $\mathbf{C}$ have the following form:

$$
C_{j}(\tau, \mathbf{r}, \Delta \mathbf{r})=\frac{1}{t_{m}} \int_{0}^{t_{m}} X_{j}(t, \mathbf{r}) X_{j}(t+\tau, \mathbf{r}+\Delta \mathbf{r}) d t, \quad j=1,2 .
$$


Here, $\tau$ is a time delay. Let $\mathbf{r}_{1}=\mathbf{r}$ and $\mathbf{r}_{2}=\mathbf{r}+\Delta \mathbf{r}$ be the coordinates of the points 1 and 2 , in which the fluctuations $X_{j}$ are considered. In a general case, the functions $C_{j}(\tau, \mathbf{r}, \Delta \mathbf{r})$ can be written in the form of the limits on $t_{m}$ as $t_{m} \rightarrow \infty$ from the expressions in the right-hand sides of the relations (13.1).

Let $\mathbf{r}=\mathbf{0},|\Delta \mathbf{r}|=r$. In this case, the distances of two points 1 and 2 from the cluster center are equal to 0 and $r$, respectively. Following to Bernar et al. (1973), we denote the functions (13.1) as $C_{j, 12}(\tau, r)$ and consider the fluctuation $X_{j}$ in the form of a wave packet propagating in a radial direction relative to the cluster center:

$$
X_{j}(t, r)=\int_{0}^{\infty} A_{j}(\omega) \exp \left[i\left(r k_{j}(\omega)-\omega t\right)\right] d \omega, \quad j=1,2 .
$$

Here, the amplitude $A_{j}(\omega)$ and the wave number $k_{j}(\omega)$ of the waves composing the packet depend on the frequency of these waves $\omega, i=\sqrt{-1}$. In case of (13.2), a Fourier transform of the function $C_{j, 12}(\tau, r)$ can be written in the following form:

$$
F_{1, j}(\omega)=F_{j}(\omega) \exp \left(\operatorname{irk}_{j}(\omega)\right),
$$

see also formulas (3.41) or (4.8) -(4.11) from the book of Bendat and Piersol (1980); $F_{j}(\omega)$ is a Fourier transform for the autocorrelation function of the fluctuations $X_{j}$ at point 1 (or 2), written with the help of (13.1) at $\Delta \mathbf{r}=\mathbf{0}$ (see (2) from Danilov and Putkov (2013b)). Thus, considering the Fourier transformation of the function $C_{j, 12}(\tau, r)$, we can find $\left|F_{1, j}(\omega)\right|=F_{j}(\omega)$ (a frequency spectrum) and $\frac{1}{r} \operatorname{Arg}\left(F_{1, j}(\omega)\right)=k_{j}(\omega)$ (a dispersion relation); $r k_{j}(\omega) \in[-\pi / 2, \pi / 2]$ is the phase of the oscillation $y_{j}$ with the frequency $\omega$.

Danilov and Putkov (2014) considered a number of the positions of point 2 on a sphere of radius $r$ with the center at the mass center of the cluster. For this, in a spherical coordinate system $(r, \theta, \phi)$ with the step $\Delta \theta=\Delta \phi=\pi /(4 k)$, the coordinates of these points at $k=1,2,4$ were set. Then, the transition to the right Cartesian rectangular coordinate system $(\xi, \eta, \zeta)$ with the origin at the mass center of the cluster was performed ( $\zeta$ axis is perpendicular to the plane of the Galaxy, $\xi$ axis is directed from the center of the Galaxy in the plane of the Galaxy, and axis $\eta$ is directed towards the cluster motion in the plane of the Galaxy). Together with point 1, the total number of points $n_{p}$ (in the vicinity of which the fluctuations $\mathbf{X}$ are considered) is $n_{p}=27,115,483$ at $k=1,2,4$, respectively.

The plot of the mean values of the function $C_{12}^{(U)}=C_{2,12}(\tau, r)$ built by Danilov and Putkov (2014) for model 1 at $r=1 \mathrm{pc}, n_{p}=483$ in the case $t_{m}=t_{0}$, where $t_{0} \simeq 3 \tau_{v r}$, is given in fig. 13.2. The values $C_{12}^{(U)}$ were averaged over 482 values of $C_{12}^{(U)}$, corresponding to different positions of point 2 on the sphere of the radius $r=1 \mathrm{pc}$. The vertical bars in fig. 13.2 show the errors of the corresponding mean $C_{12}^{(U)}$ values. When constructing fig. 13.2, the PCS of the 11th accuracy order were used. In the calculation of the curve in fig. 13.2 only the PCS obtained at $t \leq t_{0}$ were used; the $U$ and $X_{2}$ values were averaged over $t$ on the intervals $t \in\left[0, t_{0}-\tau\right]$ at $\tau>0$ and $t \in\left[-\tau, t_{0}\right]$ at $\tau<0$. We should note the asymmetry of the function $C_{12}^{(U)}$ with respect to $\tau=0$; therefore, the frequency spectrum is specified not only 
by the real part of the Fourier transform of the function $C_{12}^{(U)}$ (Bendat and Piersol, 1980). In comparison with $C_{12}^{(f)}=C_{1,12}(\tau, r)$, see fig. 2a from Danilov and Putkov (2013b), the function $C_{12}^{(U)}$ is noticeably more symmetric, which leads to a much larger contribution of the real part of the Fourier transform of the $C_{12}^{(U)}$ function to the spectrum of the $U$ oscillations.

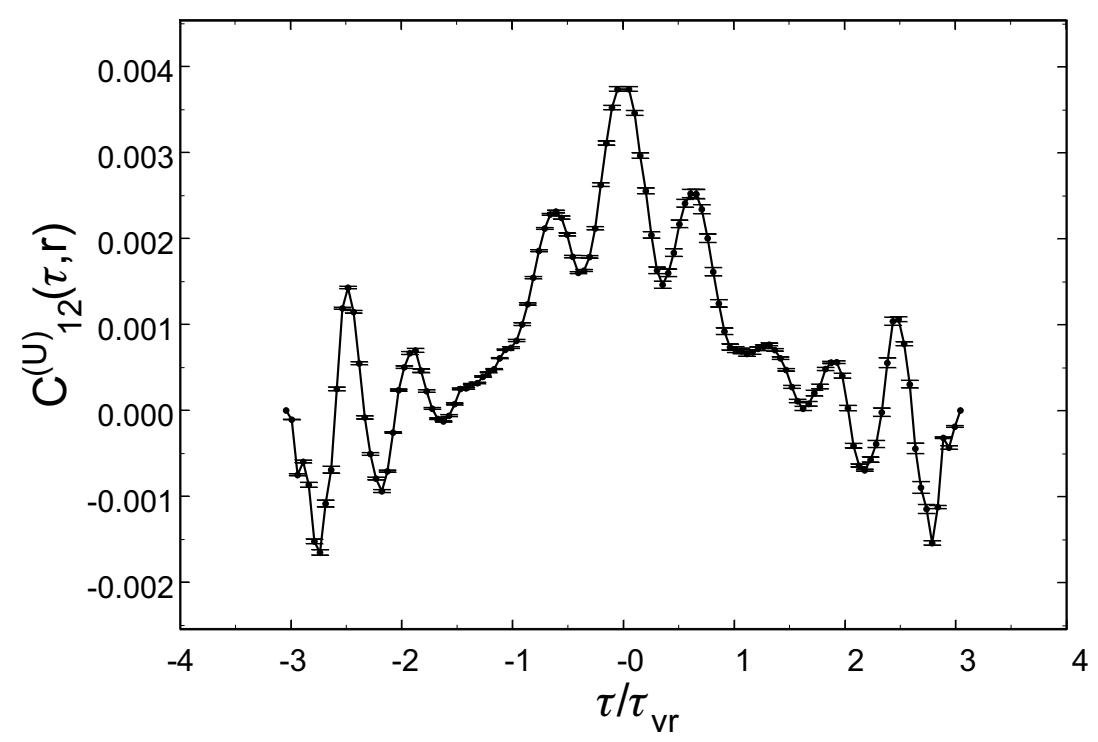

Fig. 13.2 The cross-correlation function of the $U$ potential oscillations for the OSC model 1 at $r=1 \mathrm{pc}, n_{p}=483$

In fig. 13.2, we note a number of the local extremums of the $C_{12}^{(U)}$ function that go beyond the limits of the errors of $C_{12}^{(U)}$ at points $\tau_{j}=P_{j}$. It indicates the existence of the increased cross correlations (with the different signs) between the $U$ oscillations with the periods $P_{j}$ in the vicinity of points 1 and 2 .

\subsection{Frequency Spectra and Dispersion Curves}

In order to calculate the frequency spectra and the dispersion curves of the $\mathbf{y}$ fluctuations in the OSC model 1, we used the fast Fourier transform (FFT) program from the book of Gray (1976). Here, instead of the circular frequencies $\omega$, it is more convenient to use the cyclic frequencies $v=\omega /(2 \pi)$. The Fourier transforms $\mathcal{F}_{1, j}(v)$ and $\sigma_{\mathcal{F}, j}(v)$ for the average values of $C_{j, 12}(\tau, r)$ and their errors $\sigma_{C, j}(\tau, r)$ were calculated separately. Then the relative phases (the wave numbers) of the $y_{j}$ fluctuations were calculated: 


$$
k_{v, j}^{(0)}=\frac{1}{r} \operatorname{Arg}\left(\mathcal{F}_{1, j}(v)\right) \quad \text { and } \quad k_{v, j}^{(1,2)}=\frac{1}{r} \operatorname{Arg}\left(\mathcal{F}_{1, j}(v) \pm \sigma_{\mathcal{F}, j}(v)\right)
$$

at $\mathrm{j}=1,2$. The complex conjugate pairs of the roots $v_{l}=v_{r e, l} \pm i v_{i m, l}$ of the equation $k_{v, l}^{(0)}=0$ allow us to determine the period $P_{l}=1 / v_{r e, l}$ and the time $t_{l}=1 /\left(2 \pi v_{i m, l}\right)$ of a growth of the instability of the $y_{j}$ oscillations. The functions $k_{v, j}^{(0,1,2)}$ were used to estimate the frequencies $v$ and their errors.

Fig. 13.3a shows the plot of the function $S_{v}=S_{v}^{(U)}=\left|\mathcal{F}_{1,2}(v)\right| / \tau_{v r}$, obtained for model 1 from the data on the mean values of the function $C_{12}^{(U)}$ and the errors of these mean values. For a brevity, in the names of the ordinates in figs.13.3, 13.4, 13.7 the superscript $((U)$ or $(f))$ is omitted when the functions $S_{v}$ are denoted. Also, in figs. 13.3, 13.7, the second subscript $j$ is omitted when denoting the dispersion curves. However, the figure captions indicate which oscillations $(U$ or $f$ ) correspond to the functions $S_{v}$ and $k_{v}$ shown in the figures. The values $S_{v}^{(U)}$ in fig. 13.3 are given in $(\mathrm{pc} / \mathrm{Myr})^{2}$. A number of the local maxima of the function $S_{v}^{(U)}$ that go beyond the limits of the errors of $S_{v}^{(U)}$ and are connected with the local extremums of the function $C_{12}^{(U)}$ can be seen on the dependency of $S_{v}^{(U)}$ on $v$.
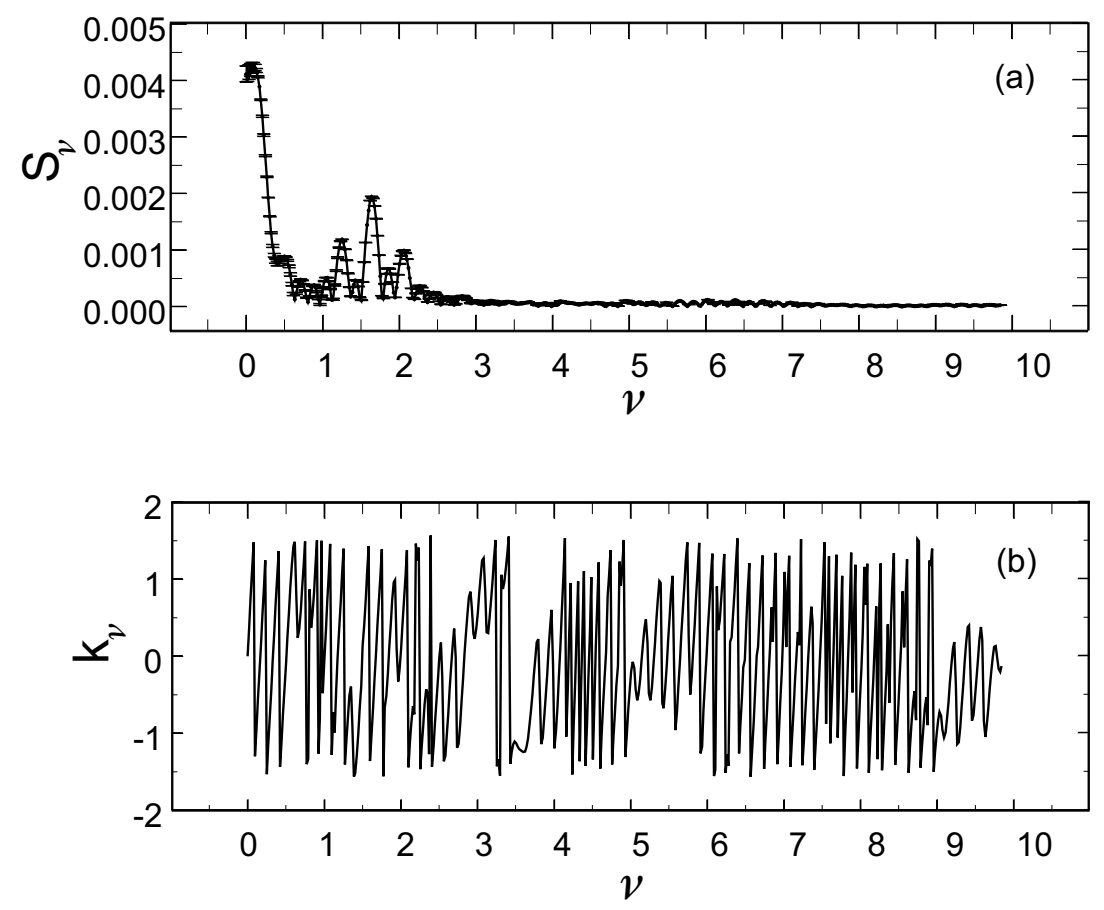

Fig. 13.3 The spectrum $S_{v}$ (a) and the dispersion curve $k_{v}$ of the $U$ oscillations for the OSC model 1 at $r=1 \mathrm{pc}, n_{p}=483$ (b) 
At $v<2.5$ (a low frequency region), the values of $S_{v}^{(U)}$ are noticeably larger than at $v>3$. At $v \in[5,7.5]$, the values of $S_{v}^{(U)}$ slightly increase in comparison with $S_{v}^{(U)}$ at $v \in[3,5]$ or $v>7.5$. As in the case of the $f$ oscillations (see fig. 3 from Danilov and Putkov (2013b)), the largest contribution to the average power of the $U$ oscillations is made by the low frequencies corresponding to the oscillations of the core and the entire cluster.

Fig. 13.3b shows the dispersion curve $k_{v}=k_{v, 2}$ of the $U$ oscillations for the OSC model 1 at $r=1 \mathrm{pc}, n_{p}=483, n_{s t}=30, \epsilon=\epsilon_{1}$. According to this curve at distances $r \leq 1 \mathrm{pc}$ from the cluster center, it is easy to note up to 30 unstable $U$ oscillations, which is $(71-75) \%$ of the number of the unstable $f$ oscillations in this model (Danilov and Putkov, 2013b). Among these 30 unstable oscillations, only 10 ones have the frequencies $v<3$ and, consequently, the largest powers. Thus, in the central parts of the OSC model 1, the amount of the unstable $U$ oscillations is less than $\sim 3 / 4$ of the number of the unstable $f$ oscillations. In this case, the fraction of the unstable $U$ oscillations with the sufficiently large values of $S_{v}^{(U)}$ is only approximately $24 \%$ of the total number of the unstable $U$ oscillations.

The dependencies of $S_{v}$ on $v$, for a brevity, we will call as spectra. To compare the spectra $S_{v}$ of the $U$ and $f$ oscillations in the OSC model 1 in fig. 13.4, we give the spectrum $S_{v}^{(U)}$ (solid line) and the spectrum $S_{v}^{(f)}$ of the $f$ oscillations multiplied by the constant value of $\beta=1 / 80\left(\mathrm{pc}^{14} / \mathrm{Myr}^{8}\right)$ for the convenience of comparison (the dependency of $\beta S_{v}^{(f)}$ on $v$ is shown in fig. 13.4 by dotted line). The spectra shown in fig. 13.4 were calculated for $r=1 \mathrm{pc}, n_{p}=483$; the spectrum $S_{v}^{(f)}$ was calculated by Danilov and Putkov (2013b) at $n_{s t}=30$. According to fig. 13.4, the frequencies of the $U$ and $f$ oscillations corresponding to the local maxima of $S_{v}^{(U)}$ and $S_{v}^{(f)}$, are basically in a good agreement with each other at $v \lesssim 2.5$, and only slightly agree (or disagree) at $v>2.5$. Moreover, at $v>2.5$, the relative contribution of the $U$ oscillations to the average (over the entire spectrum $S_{v}^{(U)}$ ) power of the $U$ oscillations is noticeably smaller than in the case of the $f$ oscillations. Apparently, the low-frequency $f$ oscillations are closely related to the $U$ oscillations, and at $v>2.5$, the contribution of the $U$ oscillations to the formation of the $f$ oscillations can be small or zero. In this case, the high-frequency $f$ oscillations can be more the result of the instability development only in the space of velocities, rather than coordinates (for example, the development of a weak turbulence in the stellar motions of the OSC model 1 (Danilov and Putkov, 2012b)).

It is convenient to estimate the "slope" of the $y_{i}$ oscillation spectrum to the axis $v$ by means of the difference ratio $q=\left(S_{v, b}-S_{v, a}\right) /\left(v_{b}-v_{a}\right)$, where $v_{a}=1.5$ and $v_{b}=7.5$ are the mean $v$ values on the intervals $v \in[1,2] \equiv \mathcal{N}_{a}$ and $v \in[7,8] \equiv \mathcal{N}_{b}$, respectively; $S_{v, a}$ and $S_{v, b}$ are the mean $S_{v}$ values at the intervals $\mathcal{N}_{a}$ and $\mathcal{N}_{b}$, respectively. The oscillations with the frequencies from the interval $\mathcal{N}_{a}$ to a greater extent characterize the oscillations of the cluster core, and have a greater power. The high-frequency oscillations with $v \in \mathcal{N}_{b}$ and $v>8$ have a small power, which varies a little with the increasing $v$ in the $U$ and $f$ oscillation spectra, see fig. 13.4. The parameter $q$ describes quite well the average "slopes" of the $y_{i}$ oscillation spectra. 


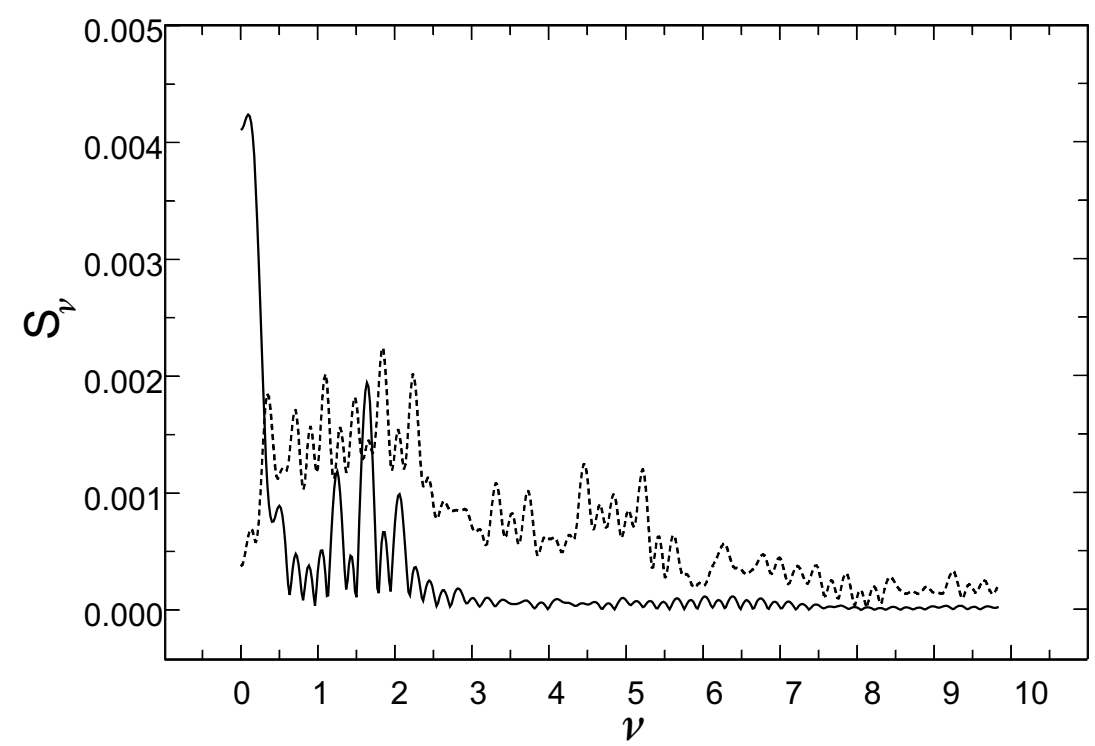

Fig. 13.4 The spectra $S_{v}$ of the $U$ and $f$ oscillations for the OSC model 1 at $r=1 \mathrm{pc}, n_{p}=483$. The spectrum $S_{v}^{(U)}$ is shown by a solid line, $\beta S_{v}^{(f)}$ is shown by a dotted line $(\beta=$ const, the spectrum $S_{v}^{(f)}$ was obtained by Danilov and Putkov (2013b) with $n_{s t}=30$ )

The calculations of the parameter $q$ by Danilov and Putkov (2014) were performed for a number of the $\epsilon$ values from the interval $\left.\epsilon / \epsilon_{1} \in[0.8,2.2]\right)$. In the calculation of the spectra and $q$, the PCS of the 11th accuracy order and values $r=1,3,5 \mathrm{pc}$, $n_{p}=483$ were used. Fig. 13.5a shows the dependency of $q$ on $\epsilon$ for the $f$ oscillations in the cluster model 1 at $r=1 \mathrm{pc}, n_{s t}=30$. The vertical bars in fig. 13.5a indicate the errors of $q$, caused by the errors of the spectra $S_{v}^{(f)}$. According to fig. 13.5a for most values of $\epsilon$, the values of $q$ are close to zero, which corresponds to the small slopes of the $f$ oscillation spectra. However, several local minima are seen on the plot of the $q$ dependency on $\epsilon$, the deepest of which is the $q$ minimum at $\epsilon=\epsilon_{1}$. Such local minima are due to the changes in the form of the spectra $S_{v}^{(f)}$ in the interval of the frequencies $v \in \mathcal{N}_{a}$ for small changes of $\epsilon$ near the points of the local minima of the $q$ dependency on $\epsilon$. To a greater extent, the values of $S_{v}^{(f)}$ change at the points of the local maxima (the frequencies of the local maxima of the spectra $S_{v}^{(f)}$ change not so significantly for the small changes in $\epsilon$ ). In addition, we observe a number of the $\epsilon$ values, for which the shape of the spectra, the frequencies, and the values of the $S_{v}^{(f)}$ local maxima, and sometimes the slope of the spectra, are in a good agreement with these characteristics of the spectrum $S_{v}^{(f)}$ at $\epsilon=\epsilon_{1}$. Apparently, there is a "repeatability" or a proximity (a matching) of the spectra shape for some fixed $\epsilon$ values, for example, $\epsilon / \epsilon_{1}=1.0,1.5,2.0$ (in this sequence of spectra, the $q$ slope decreases with the increasing $\epsilon$ ) or $\epsilon / \epsilon_{1}=1.15,1.25,1.55,1.7,1.8,2.2$, in this case, $q$ varies much less than at $\epsilon / \epsilon_{1}=1.0,1.5,2.0$. 

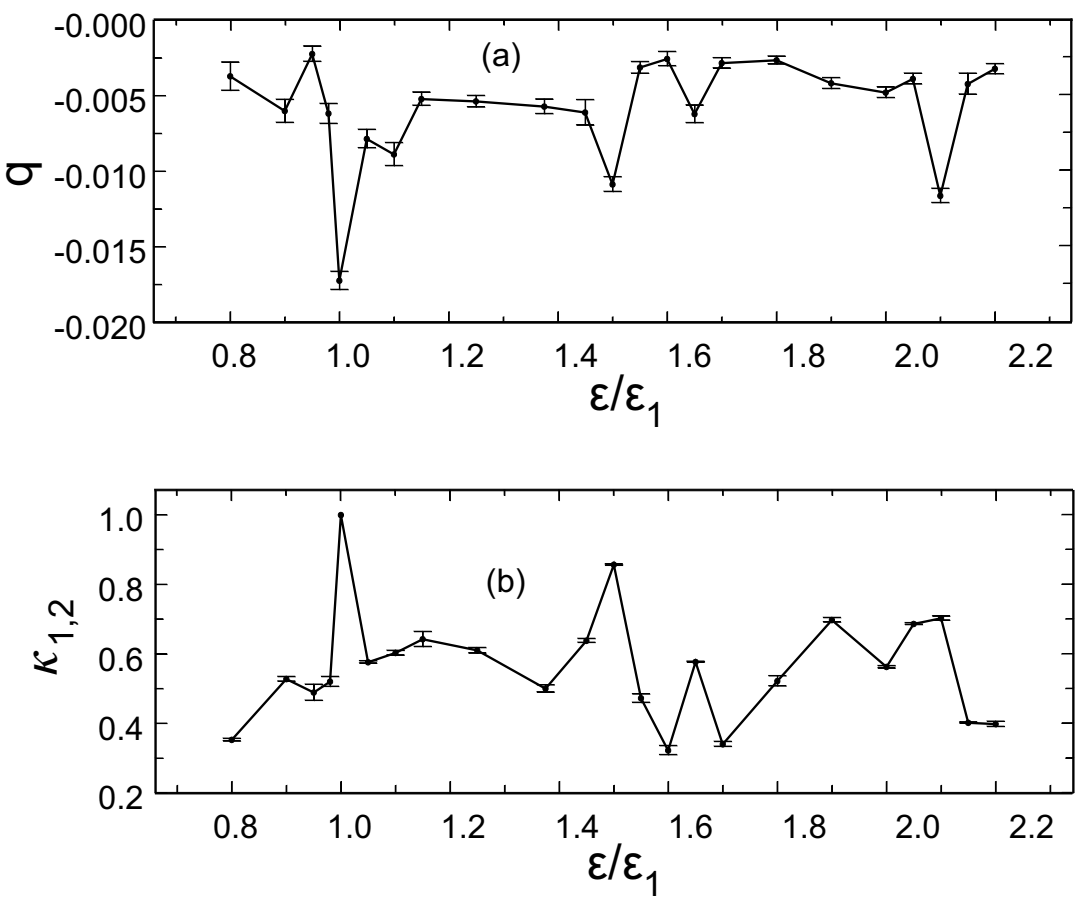

Fig. 13.5 The dependencies on $\epsilon$ of $q$ (a) and $\kappa_{1,2}$ (b) for the $f$ oscillations in the OSC model 1 at $r=1 \mathrm{pc}, n_{p}=483, n_{s t}=30$

Fig. 13.5b shows the dependency on $\epsilon$ of the correlation coefficient $\kappa_{1,2}$ of the spectrum $S_{v}^{(f)}$ obtained at $\epsilon=\epsilon_{1}$, and the spectrum $S_{v}^{(f)}$ obtained at an arbitrary $\epsilon$. It is easy to see that the points of the deeper local minima on the dependency $q=q(\epsilon)$ coincide (or agree well) with the points of the corresponding local maxima on the dependency $\kappa_{1,2}=\kappa_{1,2}(\epsilon)$. The presence of the local maxima on the dependency $\kappa_{1,2}=\kappa_{1,2}(\epsilon)$ also indicates the "repeatability" of the spectra $S_{v}^{(f)}$ for some fixed values of $\epsilon$. A decrease in $\kappa_{1,2}(\epsilon)$ at $\epsilon / \epsilon_{1}<1$ and $\epsilon / \epsilon_{1} \rightarrow 0.8$ is due to an increase in the differences between the spectra $S_{v}^{(f)}$, obtained by the PCS of the 11th and 10th accuracy orders at $\epsilon / \epsilon_{1}<1$, see below.

We should note that the total number of the unstable $f$ oscillations in the OSC model 1 decreases on average with the increasing $\epsilon$. For example, at $r=1 \mathrm{pc}$, a number of pairs $n_{c}$ of the $f$ oscillations with the complex conjugate frequencies varies from 41-42 at $\epsilon=\epsilon_{1}$ to $n_{c}=12$ at $\epsilon=2.2 \epsilon_{1}$, which indicates a decrease of the degree of an instability of the $f$ oscillations with the increasing $\epsilon$. However, near the values $\epsilon / \epsilon_{1}=1.0,1.05,1.25,1.5,2.0$, the value of $n_{c}$ is on average close to 40 and is $n_{c}=41-42,42,40-41,40,38-39$, respectively. Near the values of $\epsilon / \epsilon_{1}=1.15,1.55,1.7,1.8$, the value of $n_{c}$ is on average close to 30 and varies in the interval $n_{c} \in[24,34]$. 
The total number of the pairs of the unstable $f$ oscillations at $r=1 \mathrm{pc}$ in the OSC model 1 is sufficiently large $\left(n_{c}=34-42\right)$ for the values of $\epsilon$ close to $\epsilon_{1}$.

Fig. 13.6a shows the dependencies $q=q(\epsilon)$ obtained from the spectra $S_{v}^{(f)}$ of the OSC model 1 at $r=3 \mathrm{pc}$ (a solid line) and $r=5 \mathrm{pc}$ (a dotted line). The dependencies $q=q(\epsilon)$ in fig. 13.6a have generally the same form as in fig. 13.5a. According to fig. 13.6a with the increasing $r$, the values of $q$ approach zero, and the slope of the spectra $S_{v}^{(f)}$ decreases.
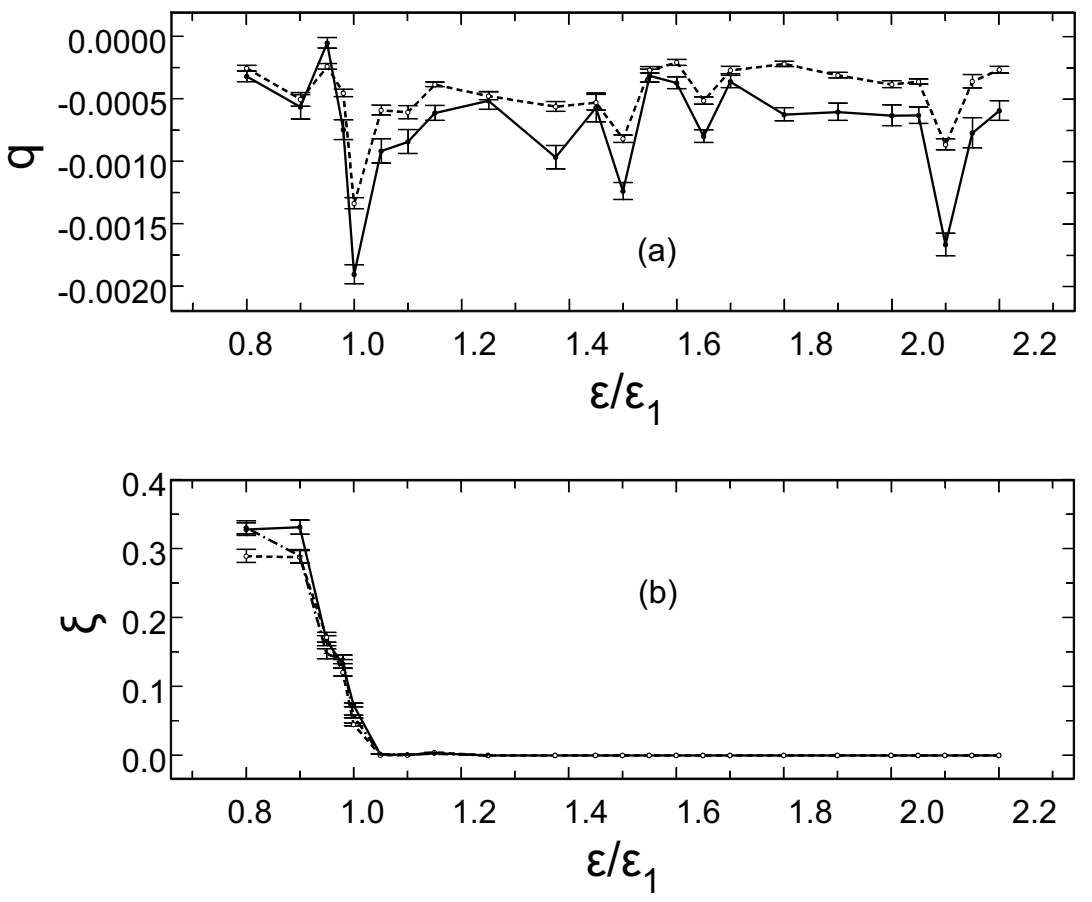

Fig. 13.6 The dependencies on $\varepsilon$ of $q$ (a) and $\xi$ (b) for the $f$ oscillations in the OSC model 1 at $n_{p}=483, n_{s t}=30$; the curves at $r=3$ pc are indicated by solid line and by dotted line at $r=5$ pc, the curve at $r=1 \mathrm{pc}(\mathrm{b})$ is shown with the dot-and-dash line

Let

$$
\xi=\left|S_{v, 1}^{(10)}-S_{v, 1}^{(11)}\right| /\left(S_{v, 1}^{(10)}+S_{v, 1}^{(11)}\right) .
$$

Fig. 13.6b shows the dependencies $\xi=\xi(\epsilon)$ obtained by Danilov and Putkov (2014) from the spectra $S_{v}^{(f)}$ of the OSC model 1 at $r=1 \mathrm{pc}$ (the dot-and-dash line), $r=3$ pc (the solid line) and $r=5 \mathrm{pc}$ (the dotted line). The values of $S_{v, 1}^{(10)}$ and $S_{v, 1}^{(11)}$ were obtained using the PCS of the 10th and 11th accuracy orders, respectively. It is easy to see that the use of $\epsilon<\epsilon_{1}$ when calculating the spectra $S_{v}^{(f)}$ is unacceptable, since it leads to the significant changes in the spectra due to the errors in the computation of the PCS. 
We should note that the smallest values of $q$ (and the large slopes) in the spectra of the OSC model 1 with the different $\epsilon$ values are realised when all running waves of the $f$ oscillations in the frequency interval $v \in \mathcal{N}_{a}$ have the same sign of the wave number $k_{v, j}$ and, therefore, move in the same direction along $r$ (in this case, the total power of the oscillations in the frequency interval $v \in \mathcal{N}_{a}$ and the slope of the spectrum are the largest), see figs. 13.7a,c. If two or more counter-propagating running waves of a comparable total power are observed in this frequency interval, then the total power of all oscillations with the frequencies $v \in \mathcal{N}_{a}$ becomes small, and close to zero $q$ values and small slopes of the spectrum are generated, see figs. $13.7 \mathrm{~b}$,d. The same result can be easily obtained for the mean (over a sufficiently large time interval) amplitude of the sum of two sinusoidal waves with the close wavelengths and frequencies, but with the markedly different amplitudes and the different directions of the motion along $r$ (with a counter motion of two sinusoidal waves, the time-averaged amplitude of the combined wave is the smallest; when these waves goes in the same direction, the average amplitude increases).
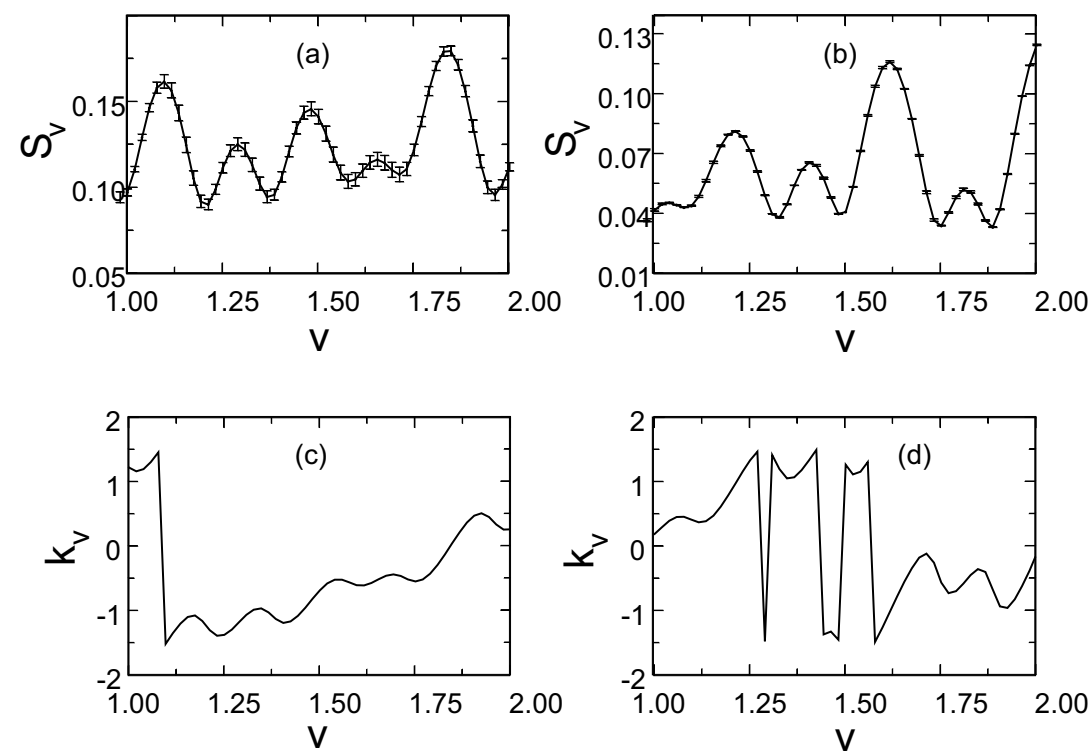

Fig. 13.7 The spectra and the dispersion curves of the $f$ oscillations for the OSC model 1 at $r=1$ pc, $n_{s t}=30, n_{p}=483, v \in \mathcal{N}_{a}:(\mathrm{a}, \mathrm{c})$ are obtained at $\epsilon=\epsilon_{1} ;(\mathrm{b}, \mathrm{d})$ are obtained at $\epsilon=1.05 \epsilon_{1}$

The $q$ values for the spectra $S_{v}^{(U)}$ are also due to the presence or absence of the counter-propagating running waves of the oscillations of a potential $U$ in the frequency interval $v \in \mathcal{N}_{a}$. The dependencies $q=q(\epsilon)$ for the spectra $S_{v}^{(U)}$ have a similar nature with $q=q(\epsilon)$ for the spectra $S_{v}^{(f)}$ (fig. 13.5a and 13.6a) and, therefore, are not given here. 
Evidently, not all running (and standing) waves can form in the OSC models with the close values of $\epsilon$, but only those satisfying to the certain relations between the wavelength and the size of the system in the direction of the wave propagation. A standing wave can be easily represented as the sum of two counter-propagating waves with the equal amplitudes and frequencies running in the radial direction (Crawford, 1968). It puts into a correlation the length of these waves and the size of the system in the radial direction. If the wavelengths in the OSC model 1 are subject to the quantization conditions, this also applies to the phases $r k_{v, j}$ of the oscillations with such wavelengths. Since $r k_{v, j} \in[-\pi / 2, \pi / 2]$, then even the small phase changes near the values $r k_{v, j}=0, \pm \pi / 2$ can change the direction of a motion of the waves and the form of the dispersion curves for the small changes in $\epsilon$. In our opinion, the quantum nature of the phase density and potential waves formed in the OSC models may well be the reason that the spectra of the $f$ and $U$ oscillations in the models with different $\epsilon$ can be noticeably different even with the slightly different $\epsilon$.

\subsection{Conclusions}

1. In this part of the study, we have considered the results of the correlation and spectral analysis of the phase density and potential oscillations in the OSC model 1 of Danilov and Dorogavtseva (2008) for different distances $r$ from the cluster center and for different values of the smoothing parameter $\epsilon$ of the force functions in the equations of the cluster stars' motion.

2. In the case of $\epsilon=\epsilon_{1}$, we have noted a passage of the $f$ oscillation waves with the frequency of an external influence (a tide) $v_{G}$ from the periphery to the center of a cluster. At the same time, the parameters of such wave change, the wave decays into several components with the frequencies commensurate with $v_{G}$. In model 1 , the oscillations with the frequency $v_{G}$ are unstable at $r \in[3,9] \mathrm{pc}$, and, probably, at $r>9 \mathrm{pc}$. The instability and, then, the decay of such oscillations into the frequency-commensurate unstable oscillations takes place at $r<3 \mathrm{pc}$.

3. In the case of $\epsilon=\epsilon_{1}$ at the distances $r \simeq 3 \mathrm{pc}$ from the cluster center (near the boundary of the cluster core) in the OSC model 1, the waves of the $f$ oscillations at certain frequencies (for example, $v=0.34,1.81$ ) move either toward the cluster center (at $r<3 \mathrm{pc}$ ), or from the center of the cluster (at $r>3 \mathrm{pc}$ ). It is possible in the presence of the reflections of the waves from the core boundary or when waves radiate from the core boundary in the directions opposite relative to $r$ (according to Danilov and Putkov (2012a), the radial oscillations of the core determine the degree of a non-stationarity of the cluster model).

4. We have noted that with a two-fold increase in $\epsilon$ in comparison with $\epsilon_{1}$, the degree of non-stationarity of the cluster model 1 (Danilov and Dorogavtseva, 2008) practically does not change. In the core of the model, there is a significant decrease of the total kinetic energy of stars $K_{i, \epsilon}$ (in 1.2-1.8 times), of the star number $N_{i, \epsilon}$ (in 1.1-1.4 times), of the star concentration $\rho_{i, \epsilon}$ (in 1.14-1.35 times) and of the mean square of the stellar velocity $T_{i, \epsilon}$ (in 1.07-1.13 times). The mean radii of the 
star groups $R_{i, \epsilon}$ and the values of $\sigma\left(K_{i, \epsilon}\right) / \bar{K}_{i, \epsilon}$ vary only within the limits of their errors.

5. We have discussed the cross-correlation function $C_{12}^{(U)}$, the frequency spectrum $S_{v}^{(U)}$ and a dispersion curve for the $U$ oscillations in the cluster model. It has been shown that the function $C_{12}^{(U)}(\tau, r)$ is significantly more symmetrical with respect to $\tau=0$, than $C_{12}^{(f)}(\tau, r)$. The spectrum $S_{\nu}^{(U)}$ is much simpler in comparison with $S_{v}^{(f)}$ (the power-most-significant $U$ oscillations are located in the low-frequency region $v<2.5$ ). In the core of the cluster model 1, up to 30 unstable $U$ oscillations are found, which is $(71-75) \%$ of the number of the unstable $f$ oscillations. The low-frequency $U$ and $f$ oscillations in the core of the cluster model have the local maxima at the coinciding frequencies and are, probably, closely related each other. The contribution of the $U$ oscillations to the formation of the $f$ oscillations at $v>3$ can be small or zero.

6. We have discussed the "slope" of the $q$ spectrum and its dependency on $\epsilon$, as well as the correlation coefficients between the spectra of the $f$ oscillations in the cluster model 1 with different $\epsilon$. The main reason for the sharp changes of $q$ at the small changes in $\epsilon$ is the change of the counter-propagating running waves of the $f$ (and $U$ ) oscillations in the frequency interval $v \in[1,2]$ to the waves with the frequencies from the same interval but running in the same direction along $r$ (it indicates the instability of the shape of the dispersion curve to the small changes of $\epsilon$ ). The "repeatability" of spectra has been noted (at some fixed values of $\epsilon$, the spectra are significantly correlated). The features of the spectra and the dispersion curves of the $f$ oscillations noted here (and in point 3 of this Section) can be related to the existence of certain relations between the dimensions of the cluster and the wavelengths of the standing and running waves in the cluster, and be determined by the discreteness of the wavelengths (and the oscillation phases in the waves) formed in the cluster with the finite dimensions in the direction of the wave propagation.

7. A detailed study of the radial profiles of the stellar density and the stellar velocities of a number of OSCs from the list of Danilov and Seleznev (1994) is able to provide the necessary data on the wavelengths of a density and a phase density in these clusters, and will also allow us to estimate the relationships between the lengths of these waves and the cluster sizes (and sizes of the cluster cores). A comparison of these estimates with the results of the numerical modelling of the OSC dynamics will make it possible to draw the conclusions about the possibility of a quantisation of the density and the phase density waves in the observed star clusters.

\section{References}

Athanassoula, E., Fady, E., Lambert, J.C., et al.: Optimal softening for force calculations in collisionless N-body simulations. Mon. Not. R. Astron. Soc. 314, 475-488 (2000). doi:10.1046/j.1365-8711.2000.03316.x 
Bendat, J.S., Piersol, A.G.: Engineering Applications of Correlation and Spectral Analysis. Wiley-Interscience, New York (1980).

Bernar, M., Briffo, J., Bussac, J., et al.: Correlation methods of analysis of plasma experiments. In: Diagnostics of plasma, Isssue 3. Atomizdat, Moscow (1973), 449-460.

Binney, J., Tremaine, S.: Galactic Dynamics, Second edition. Princeton University Press, Princeton, Oxford (2008). ISBN: 9780691130279

Crawford, F.S.Jr.: Waves (Berkeley Physics Course, Volume 3). McGraw-Hill, New York (1968). ISBN 10: 0070048606 ISBN 13: 9780070048607

Danilov, V.M.: Numerical experiments simulating the dynamics of open clusters in the Galactic field. Astron. Reports 41, 163-173 (1997a).

Danilov, V.M.: Statistical analysis of dynamical open-cluster models with small differences in their initial stellar phase coordinates. Astron. Letters, 23, 322-326 (1997b).

Danilov, V.M.: Analysis of density fluctuations in models of open clusters. Astron. Reports 52, 888-899 (2008). doi:10.1134/S1063772908110036

Danilov, V.M.: Phase-Density Fluctuations at the Centers of Six Open Clusters. Astron. Reports 54, 514-527 (2010). doi:10.1134/S1063772910060053

Danilov, V.M., Dorogavtseva, L.V.: Estimates of Relaxation Times in Numerical Dynamical Models of Open Star Clusters. Astron. Reports 47, 483-491 (2003). doi: $10.1134 / 1.1583775$

Danilov, V.M., Dorogavtseva, L.V.: Timescales for mechanisms for the dynamical evolution of open star clusters. Astron. Reports 52, 467-478 (2008). doi:10.1134/S1063772908060048

Danilov, V.M., Putkov, S.I.: Non-stationarity parameters of open clusters. Astron. Reports 56, 609-622 (2012a). doi:10.1134/S106377291208001X

Danilov, V.M., Putkov, S.I.: The dynamics of correlations in open-star cluster models. Astron. Reports 56, 623-637 (2012b). doi:10.1134/S1063772912080021

Danilov, V.M., Putkov, S.I.: Correlations and instabilities of the phase density oscillations in the open star cluster models. Publ. Udmurt. Univ. Mathematics, Physics, Computer Sciences. Issue 2, 65-73 (2013a) (In Russian). ISSN: 19949197, eISSN: 2076-5959

Danilov, V.M., Putkov, S.I.: Correlations, spectra and instability of phase-space density fluctuations in open-cluster models. Astrophys. Bull. 68, 154-168 (2013b). doi:10.1134/S199034131302003X

Danilov, V.M., Putkov, S.I.: Smoothing of Force Functions and the Fluctuation Spectra of an Open Star Cluster Model. Astrophys. Bull. 69, 27-39, (2014). doi:10.1134/S1990341314010039

Danilov, V.M., Seleznev, A.F.: The catalogue of structural and dynamical characteristics of $103 \mathrm{OCl}$ and the first results of its investigations. Astron. Astrophys. Trans. 6, 85-156 (1994). doi:10.1080/10556799408232061

Dehnen, W.: Towards optimal softening in three-dimensional N-body codes - I. Minimizing the force error. Mon. Not. R. Astron. Soc. 324, 273-291 (2001). doi:10.1046/j.1365-8711.2001.04237.x 
El-Zant, A.A.: On the stability of motion of N-body systems: the effect of the variation of particle number, softening and rotation. Astron. Astrophys. 331, 782792 (1998).

Goodman, J., Heggie, D.C., Hut, P.: On the Exponential Instability of N-Body Systems. Astrophys. J. 415, 715-733 (1993). doi:10.1086/173196

Gray, D.F.: The observation and analysis of stellar photospheres. Wiley, New York (1976). ISBN:04713238029780471323808

Hemsendorf, M., Merritt, D.: Instability of the Gravitational N-Body Problem in the Large-N Limit. Astrophys J. 580, 606-609 (2002). doi:10.1086/343027

Hernquist, L., Barnes, J.E.: Are some N-body algorithms intrinsically less collisional than others? Astrophys. J. 349, 562-569 (1990). doi:10.1086/168343

Hernquist, L., Katz, N.: TREESPH - A unification of SPH with the hierarchical tree method. Astrophys. J. Suppl. Ser. 70, 419-446 (1989). doi:10.1086/191344

Kandrup, H.E., Haywood, S.Jr.: On the sensitivity of the N-body problem to small changes in initial conditions. Astrophys. J. 374, 255-265 (1991). doi:10.1086/170114

Kandrup, H.E., Haywood, S.Jr.: On the sensitivity of the N-body problem to small changes in initial conditions. II. Astrophys J. 386, 635-645 (1992). doi:10.1086/171045

Kandrup, H.E., Haywood, S.Jr., Willmes, D.E.: On the sensitivity of the N-body problem to small changes in initial conditions. III. Astrophys J. 399, 627-633 (1992). doi:10.1086/171954

Kutuzov, S.A., Osipkov, L.P.: A Generalized Model for the Three-Dimensional Gravitational Potential of Stellar Systems. Soviet Astron. 24, 17-22 (1980).

Merritt D.: Optimal Smoothing for N-Body Codes. Astron. J. 111, 2462-2464 (1996). doi:10.1086/117980

Miller R.H.: Irreversibility in Small Stellar Dynamical Systems. Astrophys. J. 140, 250-256 (1964). doi:10.1086/147911

Price, D.J., Monaghan, J.J.: An energy-conserving formalism for adaptive gravitational force softening in smoothed particle hydrodynamics and N-body codes. Mon. Not. R. Astron. Soc. 374, 1347-1358 (2007). doi:10.1111/j.13652966.2006.11241.x

Rodionov, S.A., Sotnikova, N.Ya.: Optimal Choice of the Softening Length and Time Step in N-body Simulations. Astron. Reports 49, 470-476 (2005). doi:10.1134/1.1941489

Volkov, Ya.F., Dyatlov V.G., Mitina, R.I.: Diagnostics of turbulent plasma. Naukova Dumka, Kiev (1983). (In Russian). 


\title{
Chapter 14 Spectra of Wave Numbers of Oscillations in OSC Models
}

\begin{abstract}
In this chapter, we discuss the cross-correlation functions for the phase density $f$ fluctuations and the velocity moduli $v$ of the stars in six OSC models (for cluster model 1, we consider the different values of the smoothing parameter $\epsilon$ of the force functions in the equations of a stellar motion). We discuss the use of the spatial Fourier transform of the cross-correlation functions with a zero time shift in order to calculate the spectra of the wave numbers of the $f$ and $v$ oscillations. The spectrum of the wave numbers of the $f$ oscillations is simpler in comparison with the spectrum of the wave numbers of the $v$ oscillations. The most powerful $f$ and $v$ oscillations are located in the region of the small values of the wave number $k$ (and the large wavelengths $\lambda>(0.91-1.25) \mathrm{pc})$. A significant contribution to the average power of the $f$ and $v$ oscillations is made by the homologous oscillations of the clusters. The dependencies of the spectra of the wave numbers of the $f$ and $v$ oscillations on $\epsilon$ in the cluster model 1 are discussed. Such spectra change noticeably less when $\epsilon$ is changed than the frequency spectra of the $f$ oscillations. With an increasing $\epsilon$, the slope of the wave number spectrum on average increases, which is caused by a change in the structure of the cluster model along the distance from its center. The astrophysical applications of the obtained results are discussed (the difference of the obtained spectra from the Kolmogorov spectrum; the discreteness of the wavelengths of the $v$ oscillations in five cluster models; the widths of the spectra of the powermost-significant oscillations of the models; the estimates of the tidal radii of the clusters, etc.).
\end{abstract}

\subsection{Introduction}

Louis and Gerhard (1988); Mathur (1990); Weinberg (1994); Vandervoort (2003), as well as Binney and Tremaine (2008) in chapter 5 of their book discuss the features of the oscillation spectra formation in the models of the spherical collisionless isolated star systems, as well as the features of the distributions of the phase density and the energy of the stars in such models. Louis and Gerhard (1988) constructed 
a self-consistent model of an isochronous spherical system undergoing the radial oscillations of the small finite amplitudes (Henon, 1959) (in the isochronous model, the orbital period of the star's motion depends only on the star's energy and does not depend on the angular momentum of the star's motion). In order to describe and analyze the oscillations of such a model, an extended (8-dimensional) phase space was used by Louis and Gerhard (1988), in which such a system is stationary (Lichtenberg and Lieberman, 1983); the invariants of the stellar motion in a system with an extended phase space are determined; the Poincaré sections (Lichtenberg and Lieberman, 1983) for a number of stellar trajectories are constructed; a Fourier analysis of these trajectories is carried out, and the frequency spectra of these trajectories are constructed. With the help of the Poincare sections, for the oscillation amplitude of the model considered by Louis and Gerhard (1988), the resonance orbits of the stars with the real frequencies $\omega_{n}$ of the oscillations of the values of the phase coordinates of the stars have been found. Wherein, $\Omega: \omega_{n}=n: 1$, where $\Omega$ is a frequency of the radial oscillations of the system model; $n$ is an integer. A large number of the stellar trajectories in this model have been captured in the resonant families with the frequencies close to or equal to $\omega_{n}$.

According to Louis and Gerhard (1988), a self-consistent distribution of the phase density by energies has the local minima ("hollows") at the energies of stars with trajectories located near the stable closed resonant orbits (according to Louis and Gerhard (1988), the stellar motions along such orbits contribute to a damping of the model's oscillations), as well as the local maxima near the positions of the nonresonant and some resonant unstable closed orbits that "support" the oscillations of the system model. In the "gaps" of the phase density distribution by energy, the phase density perturbation caused by the oscillations of the system, is comparable in magnitude with the value of the unperturbed equilibrium phase density (Louis and Gerhard, 1988).

Mathur (1990), using the Vlasov and Poisson equations for a spherical collisionless isolated system, wrote a linearized equation for a small perturbation $\varepsilon f$ of the phase density function $f$, which contains on the right-hand side the sum $M+N$ of operators $M$ and $N$ (see formulas (1.3a) and (1.3b) from Mathur (1990)), acting on $f$ function. The $M$ operator is used by Mathur (1990) to describe changes in the $f$ function as a result of the motion of the stars along their phase trajectories in the equilibrium unperturbed system. The action of this operator in the equation for the perturbations of the $f$ function ensures damping of the oscillations in the system and its tendency to an equilibrium as a result of a phase mixing. The $N$ operator takes into account the self-consistency of the system and the action on it of the force field produced by a small perturbation of the phase density. The spectrum of eigenvalues $\omega$ of the $M$ operator is continuous, but there may be the the gaps, caused by the joint action of the $M$ and $N$ operators; here, $\omega$ is the eigenfrequencies of the $f$ oscillations in the system. According to Mathur (1990), in these gaps, there might exist one or more modes of the stable oscillations with the real frequencies, which prevents the damping of oscillations in the system and its tendency to equilibrium.

According to Weinberg (1994), even the weakly damped density (and phase density) oscillations exist and can persist for a long time in the models of the 
spherical collisionless isolated star systems. In order to show this, Weinberg (1994) developed and applied to the King's models (King, 1966) the method for an analysis of the dispersion relation (see references, section 2 and appendix B in Weinberg (1994)). For four models of star clusters (King, 1966) with the different values of the concentration parameter and the potential at the center, the estimates of a damping time were obtained by Weinberg (1994). This damping time exceeds 20 average times of a crossing by the star of the distance equal to the radius of the sphere (with the center at the cluster mass center) containing a half of the cluster mass.

Vandervoort (2003) carried out a general investigation of the stationary oscillations of the collisionless systems with a constant frequency and amplitude, and with a continuous spectrum of the real frequencies. The only significant limitation in Vandervoort (2003) was the assumption of the integrability of the stellar motion equations in the force field of the unperturbed potential of the system with the stationary oscillations. However, the results obtained by Vandervoort (2003) can be used to describe the oscillations of the spherical isolated systems. Such steadystate oscillation waves are the gravitational analogue of the Van Kampen waves in a plasma. It is a particle stream (modulated, for example, by density) moving with a velocity equal to the phase velocity of the wave and accompanied by the polarization cloud resulting from the action of the beam on the plasma electrons; see a review of Kadomtsev (1968) and in the case of the gravitating systems see Binney and Tremaine (2008, p. 413-415)). According to Vandervoort (2003), the isolated frequencies in the oscillation spectrum of the considered systems can also be complex.

Binney and Tremaine (2008) (see chapter 5, p. 438) discussed the oscillations of the spherical collisionless isolated systems with a continuous resonant frequency spectrum of the damping oscillations containing the gaps in the vicinity of the real frequencies of non-damping oscillations. The resonant spectrum in Binney and Tremaine (2008) is determined by the stars, the radial velocities of which coincide with the phase velocity of the wave of a potential, and satisfy to the resonance relation (5.149) between the real frequencies $\omega$ of the radial oscillations of the potential and $n \Omega_{r}$, where $n$ is an integer; $\Omega_{r}$ is a frequency of the oscillations of the star's orbit radius.

We note that Louis and Gerhard (1988); Mathur (1990); Weinberg (1994); Vandervoort (2003); Binney and Tremaine (2008) call a set of the oscillation frequencies that satisfy the certain conditions and relationships as the frequency spectrum of a system (powers of these oscillations, as a rule, are not calculated). In addition, the models of the stellar systems considered by Louis and Gerhard (1988); Mathur (1990); Weinberg (1994); Vandervoort (2003); Binney and Tremaine (2008) are too simple in comparison with the observed OSCs, in which the matter densities are sufficiently small, and the influence of the external force field of the Galaxy is very significant, which leads to the development of the numerous unstable oscillations and to the maintenance of a non-stationarity in such clusters in time intervals comparable with their average lifetime (Wielen, 1971). At the same time, the certain features of the OSC oscillation spectra quite can be explained in the framework of 
the mechanisms considered by Louis and Gerhard (1988); Mathur (1990); Weinberg (1994); Vandervoort (2003); Binney and Tremaine (2008).

A study of the frequency spectra of the oscillations of the numerical dynamic OSC models (Danilov and Dorogavtseva, 2008) was carried out in the recent papers of Danilov and Putkov (2013b,a); Putkov (2013); Danilov and Putkov (2014). Danilov and Putkov $(2013 \mathrm{~b}, \mathrm{a})$ considered the phase density $f$ oscillations in six OSC models at the different distances $r$ from the cluster center. The spectra of the $f$ oscillation were obtained by Danilov and Putkov (2013b,a); Putkov (2013); Danilov and Putkov (2014) using the cross-correlation functions corresponding to these oscillations and the Fourier transforms of these functions. Danilov and Putkov (2013b) confirmed the existence of the known unstable $f$ oscillations associated with the homologous oscillations of the cluster cores (Danilov, 2008, 2010) in the OSC models and a number of the new unstable oscillations (up to 32-41 pairs of the $f$ oscillations with the different complex conjugate frequencies in each cluster model); they obtained the estimates of the rise time of the amplitudes of such oscillations in $e$ times to be $(0.4-10) \tau_{v r}$, where $\tau_{v r}$ is the time of a violent relaxation of the cluster model. Danilov and Putkov (2013b) shows the plots of the cross-correlation function and the frequency spectrum of the oscillations in the OSC model 1, which has the lowest density among the six models considered by Danilov and Dorogavtseva (2008). Danilov and Putkov (2013a) gave the frequency spectra of six OSC models.

Putkov (2013) investigated the 5th and 6th models, which have the most extensive massive cores among all models considered by Danilov and Dorogavtseva (2008). The initial parameters of the OSC models are given in Table 1 of Danilov and Dorogavtseva (2008). The every cluster model consists of 500 stars with the masses equal to the solar mass; the cluster moves along a circular orbit of the radius $R_{G}=$ $8200 \mathrm{pc}$ in the plane of the Galaxy around its center in the force field of the potential of Kutuzov and Osipkov (1980); at the initial moment, the every OSC model consists of two homogeneous spherical subsystems (core and halo) with the coinciding mass centers; the OSC models of Danilov and Dorogavtseva (2008) are numbered in an order of the decreasing cluster non-stationarity. Putkov (2013) considered the waves of the $f$ oscillations propagating from the central regions of the cluster along its equatorial plane coinciding with the Galactic plane. According to Putkov (2013), the $f$ oscillation frequencies in these models are in the rational relations with each other (the points of the neighboring local maxima at the oscillation spectra are at the equal distances from one another by a frequency). Perhaps, such an arrangement of the local maxima at the spectra of the $f$ oscillations is due to the action of the resonances in these models.

Danilov and Putkov (2014) studied the oscillations of the phase density and the potential $U$ in the cluster model 1 for the different values of the smoothing parameter $\epsilon$ of the force functions in the equations of a stellar motion. According to Danilov and Putkov (2014), the power-most-significant $U$ oscillations are related to the $f$ oscillations and are located in the spectrum at the low frequencies $v<3 / \tau_{v r}$. Danilov and Putkov (2014) detected a number of the unstable $U$ oscillations in the core of cluster model 1 (up to 30 pairs of the oscillations with the different complex conjugate frequencies). They noted the passage of the radial waves of the 
$f$ oscillations with the frequency of an external influence (tide) from the periphery to the cluster center, as well as the decay of such waves into several components with the commensurate frequencies in the cluster core. Danilov and Putkov (2014) discussed the possibilities of a reflection and emission of the $f$ oscillation waves from the cluster core boundary. The analysis of the changes in the spectra of the $f$ and $U$ oscillations with the change of the smoothing parameter $\epsilon$ performed by Danilov and Putkov (2014) leads to the conclusion that the spectra are "repetitive" for some fixed $\epsilon$ values. This point, according to Danilov and Putkov (2014), may be due to the existence of certain relationships between the size of the cluster and the wavelengths in the cluster and, possibly, due to the discreteness of the oscillation wavelengths and phases in the waves.

According to Bernar et al. (1973); Volkov et al. (1983), the spatial Fourier transform of the cross-correlation functions of the fluctuations of a number of the plasma characteristics with a zero time shift can be used to construct the spectra of the wave numbers of the oscillations in a plasma. It is of interest to use this method for constructing the spectra of the wave numbers of the oscillations in the OSC models of Danilov and Dorogavtseva (2008), and to study in details the wavelengths and the power of the oscillations formed in such models.

Danilov and Putkov (2012b) noted a weak turbulence in the stellar motions in the core of the OSC model 1. In order to discuss the dynamic state of the cluster models and the turbulence parameters in this model, it is necessary to investigate the oscillations of the moduli of the stellar velocity $v$ and the $f$ values (since $f$ at each time depends not only on the coordinates but also on the velocities of the stars). We note that the conclusion made by Danilov and Putkov (2012b) about a turbulence in the OSC model 1 was obtained in the study of the dynamics of the pair correlations in the $f$-space, but was illustrated using the distributions of the velocity moduli of the cluster core stars, as well as the velocity moduli of the stars participating in the formation of such correlations. In this case, the phase density $f$ was calculated by the stellar groups with a small number of stars $n_{s t}=6$ closest to any arbitrarily point in the cluster. Such values of $n_{s t}$ correspond more to the local and random character of the $v$ and $f$ oscillations. Oscillations of such groups can be used in analyzing the effect of the stellar encounters (in the case of small relative velocities of the stars comprising the group) or in describing a turbulence (in the case of larger relative velocities of the stars in the group). Danilov and Putkov (2013b,a); Putkov (2013); Danilov and Putkov (2014) used the value $n_{s t}=30$ for a computation of the cross-correlation functions. In this case, the dispersion curves of the wave number dependencies on the frequency are released from a random noise; the oscillations of such groups become more large-scale, they remarkably repeat the oscillations of the entire cluster, and can be used with an analyzing of the degree of a non-stationarity of the system. Since the cases $n_{s t}=6$ and $n_{s t}=30$ can describe the different dynamic processes, it is of interest to use both values when plotting the wave number spectra for the value of $n_{s t}$.

According to Danilov and Putkov (2014), the change of $\epsilon$ results in the change of some structural and dynamic characteristics of the cluster model and in the changes in the frequency spectra. Therefore, the changes of $\epsilon$ can also affect the formation of 
the wave number spectra. In this connection, it is of interest to construct the spectra of the wave numbers of the oscillations in the OSC models for the different values of the smoothing parameter $\epsilon$.

The objectives of this chapter are: 1) to discuss the spatial cross-correlation functions (with a zero time shift) and the spectra of the wave numbers of the oscillations of the phase-density $f$ and the modulus of the stellar velocity $v$ in the OSC models of Danilov and Dorogavtseva (2008); 2) to discuss such correlation functions and spectra for the $f$ and $v$ oscillations in the cluster model 1 for the different values of $\epsilon ; 3)$ to apply the obtained spectra to the analysis of the dynamics of the OSCs and their models.

\subsection{Cross-Correlation Functions}

Let $\mathbf{X}(t, \mathbf{r})=\left(X_{1}, X_{2}\right)=\mathbf{y}(t, \mathbf{r})-\overline{\mathbf{y}(t, \mathbf{r})}$, where $\mathbf{y}=\left(y_{1}, y_{2}\right)=(v, f) ; v=|\mathbf{v}| ; \mathbf{v}$ is a vector of the star speed; the $v$ and $f$ values are obtained by $n_{s t}$ stars from the neighborhoods of the point with coordinate $\mathbf{r}$ in the cluster model, and the bar above denotes an averaging over time $t \in\left[0, t_{m}\right]$ (the origin of the coordinate system $\mathbf{r}$ coincides with the cluster mass center, $t_{m}$ is the largest $t$ values used for calculation of the correlations). For a brevity, the argument $\mathbf{v}$ for the functions $f$ and $X_{2}$ is not written here. The value $\mathbf{X}$ is usually called the deviation or fluctuation of $\mathbf{y}$ (Danilov and Putkov, 2014; Bernar et al., 1973; Volkov et al., 1983) and is assumed to be a random variable that forms a stationary random process. Following to Danilov and Putkov (2014); Bernar et al. (1973); Volkov et al. (1983), we denote the crosscorrelation function of the $\mathbf{y}$ fluctuations as $\mathbf{C}(\tau, \mathbf{r}, \Delta \mathbf{r})$, where the coordinates of the vector $\mathbf{C}$ have the form

$$
C_{j}(\tau, \mathbf{r}, \Delta \mathbf{r})=\frac{1}{t_{m}} \int_{0}^{t_{m}} X_{j}(t, \mathbf{r}) X_{j}(t+\tau, \mathbf{r}+\Delta \mathbf{r}) d t, \quad j=1,2 .
$$

Here, $\tau$ is a temporal delay. Let $\mathbf{r}_{1}=\mathbf{r}$ and $\mathbf{r}_{2}=\mathbf{r}+\Delta \mathbf{r}$ be the coordinates of points 1 and 2 , in which fluctuations $X_{j}$ are considered. In a general case, the functions $C_{j}(\tau, \mathbf{r}, \Delta \mathbf{r})$ can be written in the form of the limits on $t_{m}$ as $t_{m} \rightarrow \infty$ from the expressions in the right-hand sides of the relations similar to (14.1).

Let $\tau=0, \mathbf{r}=\mathbf{0},|\Delta \mathbf{r}|=r$. In this case, the distances of two points 1 and 2 from the cluster center are 0 and $r$, respectively. Averaging $C_{j}$ from (14.1) by two angular coordinates of the vector $\Delta \mathbf{r}$, we find the functions $K_{j}(r)=\overline{C_{j}(0, \mathbf{0}, \Delta \mathbf{r})}$ of a spatial cross-correlation (Bernar et al., 1973; Volkov et al., 1983), which are symmetric relatively to the permutations of points 1 and 2 with each other (here, the upper line means the averaging of $C_{j}$ over the angular coordinates of the vector $\left.\Delta \mathbf{r}\right)$. The properties of the cross-correlation functions are described in Volkov et al. (1983, p. 23) and in Bendat and Piersol (1980), formula (3.28).

Danilov and Putkov (2015) considered a number of positions of point 2 on a sphere of radius $r$ with a center at the cluster mass center. In order to do so, in a spherical 
coordinate system $(r, \theta, \phi)$ with the step $\Delta \theta=\Delta \phi=\pi /(4 s)$, the coordinates of these points were specified with $s=1,2,4$. Then the transition to the right Cartesian rectangular coordinate system $(\xi, \eta, \zeta)$ with the beginning at the cluster mass center was performed. $\zeta$ axis is perpendicular to the plane of the Galaxy, $\xi$ axis is directed from the center of the Galaxy in the Galactic plane, and axis $\eta$ is aimed in the direction of the cluster motion in the Galactic plane. Together with point 1, the total number of points $n_{p}$ in the vicinity of which the fluctuations $\mathbf{X}$ are considered, is $n_{p}=27,115,483$ at $s=1,2,4$, respectively.
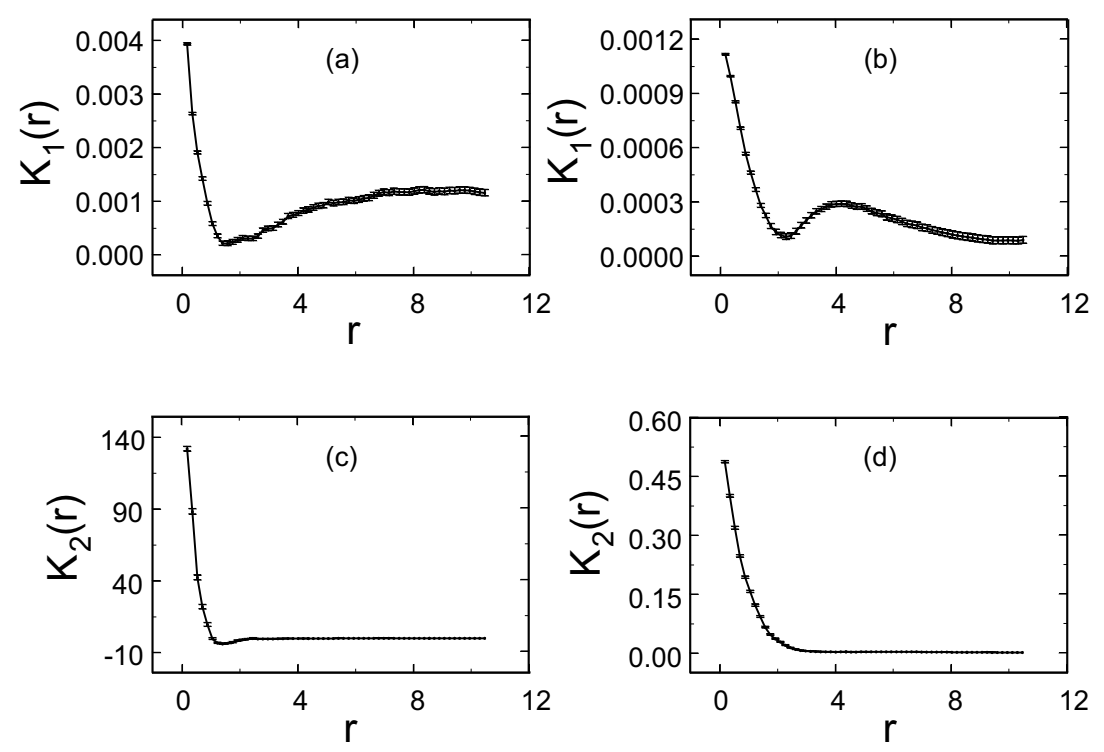

Fig. 14.1 The plots of the cross-correlation functions of the $v(\mathrm{a}, \mathrm{b})$ and $f(\mathrm{c}, \mathrm{d})$ oscillations in the OSC model 1 at $n_{s t}=6(\mathrm{a}, \mathrm{c})$ and $n_{s t}=30(\mathrm{~b}, \mathrm{~d})$

The plots of the $K_{j}(r)$ functions from the paper of Danilov and Putkov (2015) for model 1 at $n_{p}=483$ in the case $t_{m}=3 \tau_{v r}$ are shown in fig. 14.1. The $r, K_{1}(r)$ and $K_{2}(r)$ values in fig. 14.1 are given in $\mathrm{pc},(\mathrm{pc} / \mathrm{Myr})^{2}$ and $\left(\mathrm{Myr}^{3} / \mathrm{pc}^{6}\right)$, respectively. An averaging of $C_{j}$ in Danilov and Putkov (2015) was performed over 482 values of $C_{j}$ corresponding to different positions of point 2 on the spheres of radius $r$ with $r \leq R_{t}$, where $R_{t}$ is a tidal radius of the cluster (King, 1962). The vertical bars in fig. 14.1 show the errors of the corresponding mean values of $C_{j}$. When constructing fig. 14.1, the phase coordinates of stars (PCS) of the 11th accuracy order were used (Danilov and Dorogavtseva, 2008). According to fig. 14.1, the dependencies on $r$ of the $K_{1}(r)$ values have a more complex form in comparison with $K_{2}(r)$, which leads to the more complex spectra of the wave numbers of the $v$ oscillations in comparison with the spectra of the wave numbers of the $f$ oscillations, see below. In fig. 14.1b the correlations of the oscillations at $r$ close to $4 \mathrm{pc}$ stand out noticeably. Also, in fig. 14.1a we can observe a noticeable increase of the correlations with an increase 
of $r$, which indicates an increasing role of the oscillations with such wavelengths in the dynamic evolution of the cluster model 1. For the OSC models 2-6, the dependencies $K_{j}=K_{j}(r)$ have qualitatively the same character as in fig. 14.1.

\subsection{Spectra of Wave Numbers}

In order to calculate the spectra of the wave numbers of the $\mathbf{y}$ fluctuations in the OSC models, Danilov and Putkov (2015) used the Fast Fourier Transform (FFT) program from Gray (1976). The value of $k=1 / \lambda$ was considered as the wave number, where $\lambda$ is the wavelength of the $v$ and $f$ oscillations. The Fourier transforms of $\mathcal{F}_{j}(k)$ and $\sigma_{\mathcal{F}_{j}}(k)$ were calculated separately for the values of $K_{j}(r)$ and their errors $\sigma_{K_{j}}(r)$.
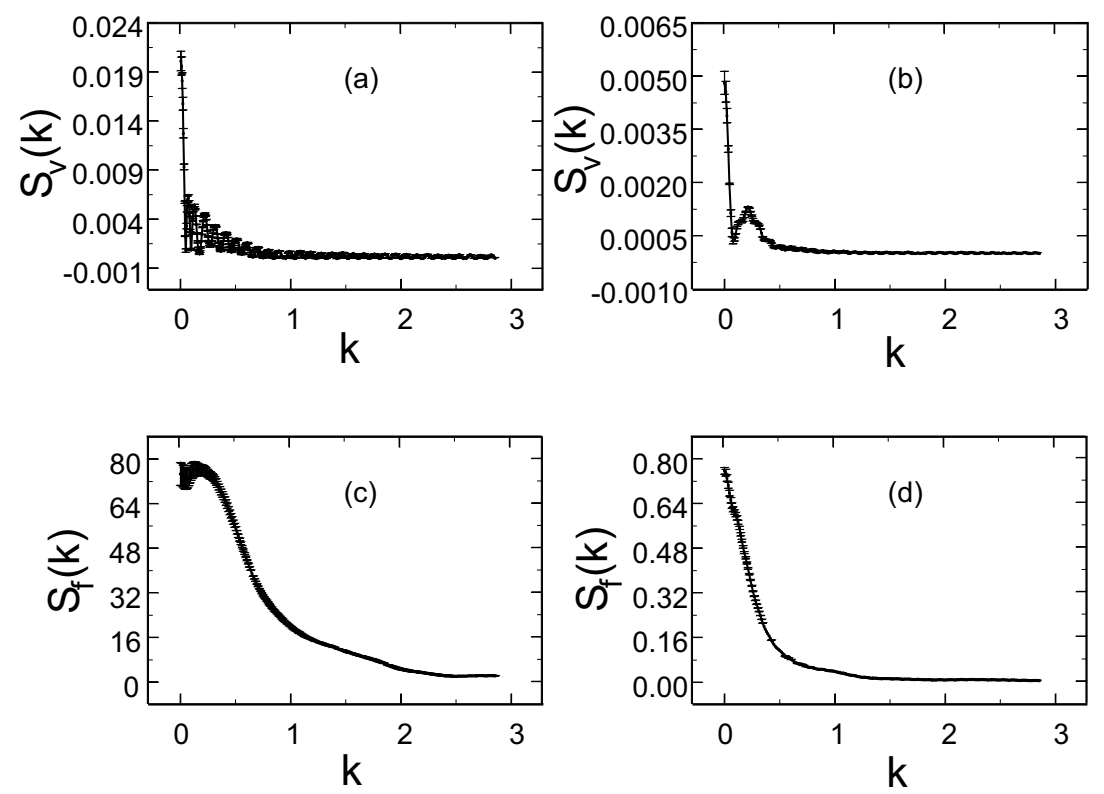

Fig. 14.2 The spectra of the wave numbers of the $v(\mathrm{a}, \mathrm{b})$ and $f(\mathrm{c}, \mathrm{d})$ oscillations for the OSC model 1 at $n_{s t}=6(\mathrm{a}, \mathrm{c})$ and $n_{s t}=30(\mathrm{~b}, \mathrm{~d})$

Fig. 14.2a,b show the plots of the spectra of the wave numbers $S_{v}(k)=\left|\mathcal{F}_{1}(k)\right|$ of the $v$ oscillations obtained by Danilov and Putkov (2015) for model 1 at $n_{p}=483$ by the data on the values of the function $K_{1}(r)$ and its errors $\sigma_{K_{1}}(r)$ at $n_{s t}=6$ and $n_{s t}=30$. The values $k$ and $S_{v}(k)$ in fig. 14.2 are given in $\mathrm{pc}^{-1}$ and $\left(\mathrm{pc}^{3} / \mathrm{Myr}^{2}\right)$, respectively. The plots of the spectra of the wave numbers $S_{f}(k)=\left|\mathcal{F}_{2}(k)\right|$ of the $f$ oscillations obtained for model 1 at $n_{p}=483$ by the data on the $K_{2}(r)$ function and its errors $\sigma_{K_{2}}(r)$ at $n_{s t}=6$ and $n_{s t}=30$, are shown in fig. $14.2 \mathrm{c}$,d. The values of $S_{f}(k)$ are given in $\left(\mathrm{Myr}^{3} / \mathrm{pc}^{5}\right)$. 
At all the spectra of fig. 14.2, the local maxima are observed at $k \leq 0.0056$ $\mathrm{pc}^{-1}$, which corresponds to the wavelengths $\lambda \geq 178.7 \mathrm{pc}$ and the oscillations in the cluster close to the homological ones (in this case, the $v$ and $f$ oscillations at different distances $r \leq R_{t} \simeq 10.468 \mathrm{pc}$ from the center occur in the same phase). The power of such oscillations is large, their contribution to the average power of the oscillations is considerable. We can note the local maxima $S_{f}(k)$ in fig. 14.2 near the values $k \simeq 0.134,0190 \mathrm{pc}^{-1}$ which corresponds to the wavelengths $\lambda \simeq$ $7.44,9.17 \mathrm{pc}<R_{t}$. At the $v$ oscillation spectra, we can note the numerous local maxima that are repeating with an equal step by $k$ till the values $k>1 \mathrm{pc}^{-1}(\lambda<1$ pc), see fig. 14.2a,b. We denote the distance between the points of two adjacent local maxima as $\Delta k$. In this case, the wavelengths for such maxima are related to each other by the ratio $\Delta k=1 / \lambda_{i+1}-1 / \lambda_{i}=$ const. These relations can be easily generalized to the case of the local maxima separated from each other by the value $j \Delta k: \lambda_{i+j}=\lambda_{i} /\left(1+j \Delta k \lambda_{i}\right), j=0,1,2, \ldots, n$.

It should be noted that most often, in the spectra of all considered OSC models, except for the fourth one (which is the densest at $t=0$ ), the same $\Delta k$ values are observed, such that $R_{t} \Delta k \simeq 1$, see Table 14.1. The values $R_{t} \Delta k \simeq 3 / 5,2$ are significantly less frequent in the OSC models (mainly in the region of small $k \simeq 0.062,0.056$; for a brevity, here and below, we do not give the dimension of $\mathrm{pc}^{-1}$ for $k$ ). Let $R_{t} \Delta k=1, c<1, c=$ const,$\lambda_{i}=c R_{t}$. Then, $\lambda_{i+j}=c R_{t} /(1+j c)$, and the wavelengths of a sufficiently large number of the oscillations in the OSC models are related to the dimensions of the cluster; it agrees with the conclusion of Danilov and Putkov (2014) on the discreteness of the wavelengths of the oscillations in the OSC models and on the relationship of these wavelengths to the cluster sizes.

We note that the spectrum of the $v$ oscillations obtained for the OSC model 1 differs strongly from the Kolmogorov spectrum $\left(S_{v}(k)=A k^{-5 / 3}, A=\right.$ const $)$ by the presence of a large number of the local maxima. Varying the value of $A$, we can approximate the upper envelope of some sections of the spectrum observed in model 1 (fig. 14.2a) with the function $A k^{-5 / 3}$ at $k>0.1$. In figs. 14.2a,b, we see the spectra of much younger (in the dynamic aspect) system than in the case of $S_{v}(k)=A k^{-5 / 3}$, that corresponds to the locally isotropic, homogeneous and stationary turbulence with a sustained flow of an energy from small $k$ to large ones (see Tsytovich (1971, p. 9, 16) and Fortov (2000, section I, subsection 7.1, p. 215)). In the case of fig. 14.2a, the action of the instabilities of the $v$ oscillations on the spectrum is large, the energy flux across the spectrum is non-stationary, and the interaction of the vortices has not yet had time to bring the spectrum to a monotonically decreasing form. The reason for the formation of the numerous local maxima on the spectra of fig. 14.2a,b may well be the resonances between the oscillation frequencies with the corresponding wavelengths to such maxima in the cluster model 1.

The shape of the spectra of the $f$ oscillations varies a little during the transition from one model to another. Therefore, we do not give the plots of such spectra for the models 2-6 here.

Figs. 14.3, 14.4 show the plots of the spectra $S_{v}(k)$ of the $v$ oscillations obtained by Danilov and Putkov (2015) for models $2-6$ at $n_{p}=483$ and $k<1$ by the data on the $K_{1}(r)$ values and its errors $\sigma_{K_{1}}(r)$ at $n_{s t}=6$ and $n_{s t}=30$. The spectra $S_{v}(k)$ of 

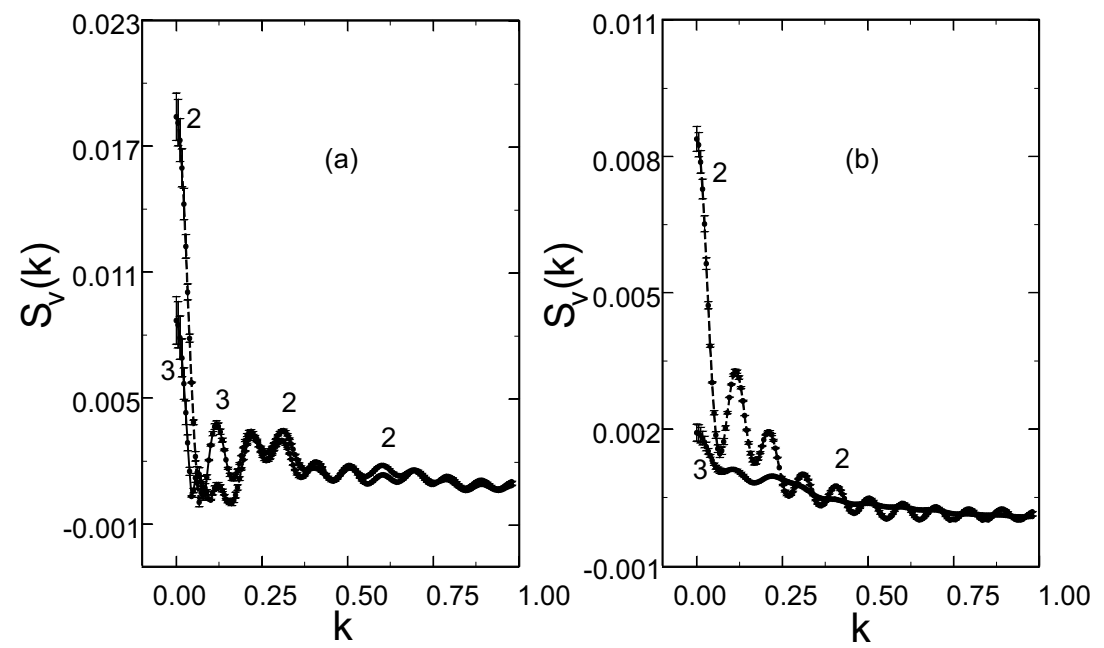

Fig. 14.3 The spectra of the wave numbers of the $v$ oscillations for the OSC models 2 and 3 at $n_{s t}=6$ (a) and $n_{s t}=30$ (b). Numbers 2 and 3 on the plots indicate the spectra of models 2 and 3 , respectively

these models at $k \geq 1$ differ a little from each other and from the spectrum of model 1 , see fig. 14.2a, and, therefore, are not given here.

According to fig. 14.3, in models 2 and 3, the homologous oscillations of model 2 are most powerful ones (model 2 has a higher degree of non-stationarity than model 3 (Danilov and Dorogavtseva, 2008)). In the spectra of models 2 and 3 (as in the spectrum of model 1), we observe a series of the $v$ oscillations with the local maxima equidistant by $k$. At $n_{s t}=30$, the power of the $v$ oscillations in model 2 in the region of values $k<0.25$ is greater than in model 3, see fig. $14.3 \mathrm{~b}$.

At $n_{s t}=6$, the power of the $v$ oscillations in model 3 near the values of $k \simeq 0.118$ is even greater than in model 2, see fig. 14.3b. However, the contribution of the homologous (and close to homologous) cluster oscillations to the mean power of the $v$ oscillations (and to a non-stationarity) of model 2 is greater than in model 3 at $k \simeq 0,0.118$.

According to fig. 14.4, only the oscillation spectrum of the densest model 4 does not contain the oscillations of the $v$ values that are noticeable by the power with the local maxima equidistant by $k$, which indicates the suppression of the mechanisms of the unstable $v$ oscillations' development in the clusters with such a density (an initial radius $\mathrm{R}(0)$ of this model is equal to $0.7 R_{t}$ (Danilov and Dorogavtseva, 2008)). At $n_{s t}=30$, the homologous (and close to them) oscillations in models 6 and 4 are of the greatest power. At $n_{s t}=6$, such oscillations in models 5 and 4 have the greatest power.

When discussing the general characteristics of spectra $S_{v}(k)$ and $S_{f}(k)$ of the $v$ and $f$ oscillations, for a brevity, the spectra are further denoted as $S(k)$. As a characteristic of the spectrum section widths with the most powerful $f$ and $v$ 

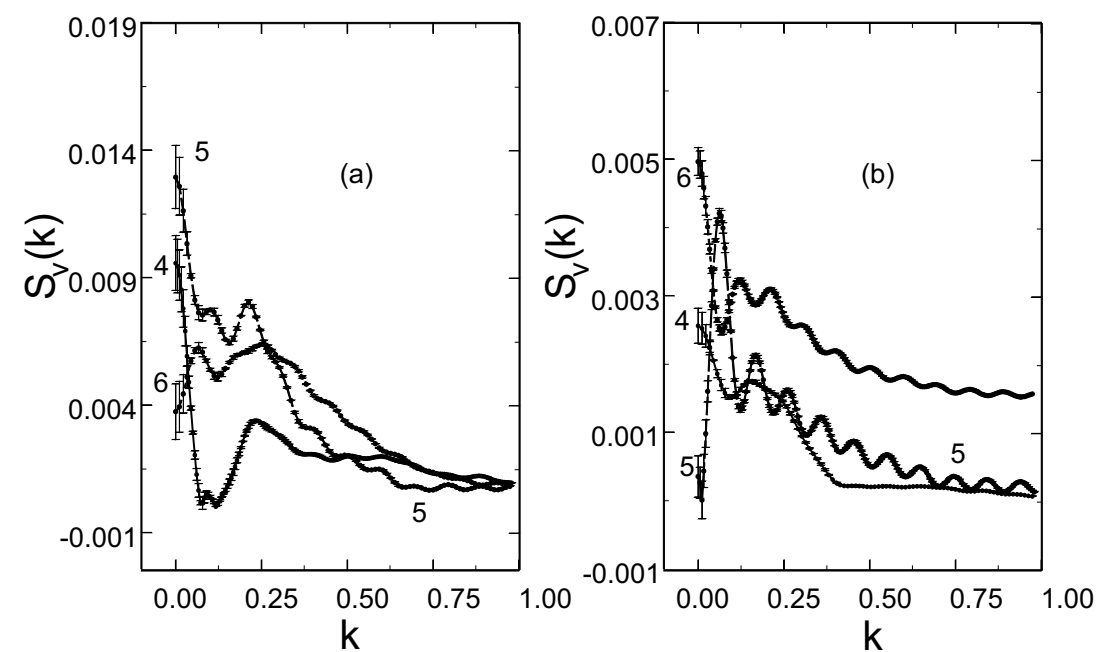

Fig. 14.4 The spectra of the wave numbers of the $v$ oscillations for the OSC models $4,5,6$ at $n_{s t}=6$ (a) and $n_{s t}=30$ (b). Numbers $4-6$ on plots indicate the spectra of models $4-6$, respectively. The spectrum of model 6 is shifted upwards along the ordinate axis by the value 0.0015

oscillations, we use the value of $k=k_{e}$, which is determined from the conditions $S\left(k_{e}\right)=e^{-1} S\left(k_{m}\right)$ and $k_{e}>k_{m}$, where $e$ is the base of a natural logarithm; $S\left(k_{m}\right)$ is the largest of the values of the $S(k)$ spectrum at the points of the local maxima at $k>0.1$. In this case, when estimating the spectral width, the highest local maximum in the spectrum for $k$ close to zero is often not taken into account. This maximum characterizes to a greater extent the initial conditions and the homology of the oscillations at the stage of the first compression of the cluster model. The nonhomologous oscillations of the clusters at $k>0.1$ and the parameters of the resulting "final" spectrum generated by the initial conditions from Danilov and Dorogavtseva (2008) are of greater interest. However, in the cases when at $k>0.1$, the local maximum close in height to the highest one is absent in the spectrum (for example, in the case of $S_{f}(k)$ at $n_{s t}=30$ in models 1,3-6), the point of the highest local maximum at $k<0.1$ is used as $k_{m}$. If in the neighborhood of the point $k=k_{e}$ two local maxima of the spectrum at the points $k_{1}<k_{e}$ and $k_{2}>k_{e}$ are observed, then, to estimate $k=k_{e}$, we use a straight line close to an upper envelope and connecting the "vertices" of these local maxima (the points with coordinates $\left(k_{l}, S\left(k_{l}\right)\right), l=1,2$ ) and the intersection of this line with the line $S(k)=S\left(k_{e}\right)=$ const. The $k_{e}$ and $k_{m}$ values for the every OSC model are listed in Tables 14.1 and 14.2. For spectra $S_{v}(k)$ in Table 14.1, the values $k=k_{\text {in }}$ are listed, which correspond to the point of the first local maximum in a series of such maxima located in the $k \geq k_{\text {in }}$ region, with the constant distance $\Delta k$ between the points of the neighbour local maxima.

According to Table 14.1, in models 1-4, 6, the values of $k_{e}$ decrease when moving from $n_{s t}=6$ to $n_{s t}=30$. Consequently, the spectrum of the wave numbers of the $v$ oscillations with $S_{v}(k) \geq S_{v}\left(k_{e}\right)$ becomes narrower with this transition. In model 
Table 14.1 The parameters of the waves of the velocity modulus $v$ oscillations in the OSC models, $\mathcal{N}$ is the number of the cluster model

\begin{tabular}{|c|c|c|c|c|}
\hline \multirow{2}{*}{} & \multicolumn{2}{|c|}{$k_{i n}, \mathrm{pc}^{-1}$} & \multicolumn{2}{c|}{$k_{m}, \mathrm{pc}^{-1}$} \\
\cline { 2 - 5 } & $n_{s t}=6$ & $n_{s t}=30$ & $n_{s t}=6$ & $n_{s t}=30$ \\
\hline 1 & $0.123 \pm 0.003$ & $0.123 \pm 0.003$ & $0.123 \pm 0.003$ & $0.213 \pm 0.003$ \\
\hline 2 & $0.224 \pm 0.003$ & $0.112 \pm 0.003$ & $0.313 \pm 0.003$ & $0.112 \pm 0.003$ \\
\hline 3 & $0.118 \pm 0.003$ & $0.106 \pm 0.003$ & $0.118 \pm 0.03$ & $0.106 \pm 0.003$ \\
\hline 4 & - & - & $0.235 \pm 0.003$ & $0.343 \pm 0.003$ \\
\hline 5 & $0.498 \pm 0.003$ & $0.168 \pm 0.003$ & $0.213 \pm 0.003$ & $0.168 \pm 0.003$ \\
\hline 6 & $0.252 \pm 0.005$ & $0.123 \pm 0.003$ & $0.252 \pm 0.003$ & $0.123 \pm 0.003$ \\
\hline
\end{tabular}

\begin{tabular}{|c|c|c|c|c|}
\hline \multirow{2}{*}{} & \multicolumn{2}{|c|}{$k_{e}, \mathrm{pc}^{-1}$} & \multicolumn{2}{c|}{$R_{t} \Delta k$} \\
\cline { 2 - 5 } & $n_{s t}=6$ & $n_{s t}=30$ & $n_{s t}=6$ & $n_{s t}=30$ \\
\hline 1 & $0.51 \pm 0.02$ & $0.38 \pm 0.04$ & $1.01 \pm 0.02$ & $1.00 \pm 0.02$ \\
\hline 2 & $0.759 \pm 0.008$ & $0.288 \pm 0.003$ & $0.98 \pm 0.03$ & $1.03 \pm 0.01$ \\
\hline 3 & $0.60 \pm 0.02$ & $0.45 \pm 0.01$ & $1.006 \pm 0.024$ & $1.03 \pm 0.04$ \\
\hline 4 & $0.83 \pm 0.03$ & $0.343 \pm 0.003$ & - & - \\
\hline 5 & $0.414 \pm 0.005$ & $0.496 \pm 0.007$ & $1.02 \pm 0.03$ & $1.02 \pm 0.02$ \\
\hline 6 & $0.615 \pm 0.003$ & $0.421 \pm 0.005$ & $1.00 \pm 0.07$ & $1.00 \pm 0.02$ \\
\hline
\end{tabular}

Table 14.2 Parameters of oscillation wave of phase density $f$ in OSC models, $\mathcal{N}$ is the number of cluster model

\begin{tabular}{|c|c|c|c|c|}
\hline \multirow{2}{*}{} & \multicolumn{2}{|c|}{$k_{m}, \mathrm{pc}^{-1}$} & \multicolumn{2}{c|}{$k_{e}, \mathrm{pc}^{-1}$} \\
\cline { 2 - 5 } & $n_{s t}=6$ & $n_{s t}=30$ & $n_{s t}=6$ & $n_{s t}=30$ \\
\hline 1 & $0.134 \pm 0.003$ & $0.000 \pm 0.003$ & $0.80 \pm 0.02$ & $0.292 \pm 0.005$ \\
\hline 2 & $0.134 \pm 0.003$ & $0.101 \pm 0.003$ & $1.01 \pm 0.03$ & $0.310 \pm 0.003$ \\
\hline 3 & $0.235 \pm 0.003$ & $0.000 \pm 0.003$ & $0.922 \pm 0.005$ & $0.371 \pm 0.006$ \\
\hline 4 & $0.302 \pm 0.003$ & $0.000 \pm 0.003$ & $1.129 \pm 0.008$ & $0.45 \pm 0.01$ \\
\hline 5 & $0.146 \pm 0.003$ & $0.000 \pm 0.003$ & $0.73 \pm 0.01$ & $0.290 \pm 0.003$ \\
\hline 6 & $0.090 \pm 0.003$ & $0.000 \pm 0.003$ & $0.67 \pm 0.01$ & $0.199 \pm 0.003$ \\
\hline
\end{tabular}

5 , with this transition, the spectrum of $k$ becomes wider. The $k_{i n}$ values do not increase with this transition (in models $2-5$, the $k_{\text {in }}$ values decrease). Consequently, the beginning of a series of the local maxima with $\Delta k=$ const at such transition basically comes nearer to $k=0$ (to the large $\lambda$ values). According to Table 14.2, for the spectra of the $f$ oscillations in the OSC models 1-6 at the transition from $n_{s t}=6$ to $n_{s t}=30$, the values of $k_{e}$ and $k_{m}$ decrease. In this case, the spectrum of the $f$ oscillations with $S_{f}(k) \geq S_{f}\left(k_{e}\right)$ becomes narrower with respect to $k$. 
Thus, the larger $n_{s t}$ values more often allow us to study the oscillations of a larger scale with the larger wavelengths in the OSC models (Danilov and Dorogavtseva, 2008).

In the case $n_{s t}=6$ in models $1,2,4$, with an increasing density of the cluster model, $k_{e}$ increases for the $v$ and $f$ oscillations. The spectra of such oscillations become wider by $k$. In models $2,3,5,6$, with the increasing mass and size of the cluster core, $k_{e}$ decreases for the $f$ oscillations (in the case of the $v$ oscillations, the $k_{e}$ values decrease in the sequence of models $2,3,5$, and then significantly increase in model 6 with the largest number of stars in the cluster core). In the case $n_{s t}=30$, in models 1, 2, 4, with an increasing density of the cluster model, $k_{e}$ grows for the $f$ oscillations and decreases for the $v$ oscillations with the transition from model 1 to model 2 , and then increases with the transition from model 2 to model 4 . In models $2,3,5,6$, with an increase of the mass and size of the core, $k_{e}$ increases for the $f$ oscillations with a transition from model 2 to model 3, and then decreases in the sequence of models 3, 5, 6 (in the case of the $v$ oscillations, the values of $k_{e}$ increase in the sequence of models $2,3,5$, and then decrease with a transition to model 6 with the largest number of stars in the cluster core).

Thus, the following most simple dependencies of the $k_{e}$ values on the parameters of the OSC models are observed at $n_{s t}=6$ :

- 1) $k_{e}$ increases with an increasing density of the cluster model for the $v$ and $f$ oscillations;

- 2) $k_{e}$ decreases with the increasing size and mass of the core of the model for the $f$ oscillations;

- 3) at $n_{s t}=30, k_{e}$ increases with an increase of the density of the cluster model for the $f$ oscillations.

We note that the small $k_{e}$ values in the low-density OSC models correspond to the larger wavelengths $\lambda_{e}=1 / k_{e}$ and to the larger scale of the oscillations. Such oscillations in time usually occur at lower frequencies, which agrees with a decrease in the frequency of the radial oscillations with the decreasing cluster density, see, for example, formula (9) from Lynden-Bell (1967). The smaller values of $k_{e}$ in the OSC models with the more massive and extended cores correspond to the larger wavelengths $\lambda_{e}$, which is quite in an agreement with the increase of the scale of the oscillations, since the non-stationarity of the OSCs is mainly provided by the oscillations of the cluster cores (Danilov and Putkov, 2012b).

Table 14.3 lists the average (over $k \lesssim 2.86$ ) values of

$$
\chi=\left|S^{(10)}(k)-S^{(11)}(k)\right| /\left(S^{(10)}(k)+S^{(11)}(k)\right),
$$

for six OSC models (Danilov and Dorogavtseva, 2008), where $S^{(10)}(k)$ and $S^{(11)}(k)$ are spectra of the wave numbers of the oscillations in the OSC models obtained by Danilov and Putkov (2015) from the data on the PCS of the 10th and 11th accuracy orders at $n_{s t}=6,30\left(\chi=\chi_{v}\right.$ and $\chi=\chi_{f}$ in the case of the $v$ and $f$ oscillations, respectively). The $\chi$ values characterize the mean (with respect to $k$ ) relative error of the computation of the $S(k)$ spectra in the considered OSC models. According to Table 14.3, an increase of the $n_{s t}$ values from 6 to 30 often leads to 
Table 14.3 The errors $\chi$ of the $S(k)$ spectra in the OSC models, $\mathcal{N}$ is the number of the cluster model

\begin{tabular}{|c|c|c|}
\hline \multirow{2}{*}{} & \multicolumn{2}{|c|}{$\chi_{v}$} \\
\cline { 2 - 3 } & $n_{s t}=6$ & $n_{s t}=30$ \\
\hline 1 & $0.036 \pm 0.004$ & $0.149 \pm 0.010$ \\
\hline 2 & $0.060 \pm 0.005$ & $0.042 \pm 0.004$ \\
\hline 3 & $0.00060 \pm 0.00004$ & $0.000183 \pm 0.000009$ \\
\hline 4 & $0.031 \pm 0.002$ & $0.0100 \pm 0.0004$ \\
\hline 5 & $0.000157 \pm 0.000007$ & $0.00018 \pm 0.00001$ \\
\hline 6 & $0.000 \pm 0.000$ & $0.000007 \pm 0.000001$ \\
\hline $\mathcal{2}$ & \multicolumn{2}{|c|}{$\chi_{f}$} \\
\cline { 2 - 3 } & $n_{s t}=6$ & $n_{s t}=30$ \\
\hline 1 & $0.0030 \pm 0.0001$ & $0.0161 \pm 0.0006$ \\
\hline 2 & $0.00140 \pm 0.00003$ & $0.0104 \pm 0.0005$ \\
\hline 3 & $0.000046 \pm 0.000002$ & $0.000023 \pm 0.000001$ \\
\hline 4 & $0.0067 \pm 0.0003$ & $0.0035 \pm 0.0002$ \\
\hline 5 & $0.0000025 \pm 0.0000003$ & $0.000067 \pm 0.000003$ \\
\hline 6 & $0.000 \pm 0.000$ & $0.0000006 \pm 0.0000001$ \\
\hline
\end{tabular}

an increase of $\chi$. The largest values of $\chi$ are reached in the case $\chi=\chi_{v}$ for the OSC model $1\left(\chi_{v}=0.149 \pm 0.010\right)$, which is caused mainly by the errors of the calculations of $S_{v}(k)$ at $k>1$ (in the case $k \leq 1$, at $n_{s t}=30$ for model 1 , we find $\left.\chi_{v}=0.051 \pm 0.006\right)$. According to Table 14.3, $\chi_{v}>\chi_{f}$.

Thus, the accuracy of the PCS from Danilov and Dorogavtseva (2008) used in the calculation of the $S(k)$ spectra in this work is quite sufficient for the conclusions about the parameters and properties of the $S(k)$ spectra.

\subsection{Dependencies of $S(k)$ Spectra of OSC Model 1 on $\epsilon$}

According to Danilov and Putkov (2014, 2015), the estimate of the "slope" of the $S(k)$ spectra of the wave numbers of the $v$ and $f$ oscillations in the cluster model 1 is convenient to carry out using the difference ratio

$$
q=\left({ }^{b} S(k)-{ }^{a} S(k)\right) /\left(k_{b}-k_{a}\right),
$$

where $k_{a}=1.55 ; k_{b}=1.95 ;{ }^{a} S(k)$ and ${ }^{b} S(k)$ are the mean $S(k)$ values in the following intervals of $k$, respectively: $k \in[0.1,1.0] \equiv \mathcal{N}_{a}$ and $k \in[1.5,2.4] \equiv \mathcal{N}_{b}$. The wave numbers $k$ from the interval $\mathcal{N}_{a}$ correspond to the wavelengths $\lambda$ from 1 to $10 \mathrm{pc}$ (such an interval of the $v$ and $f$ oscillations is comparable with the distance interval from the average distance between the stars near the center of the cluster model (Danilov and Putkov, 2014) to a distance close to the tidal radius $R_{t}$ of the 
cluster). Such oscillations, on average, have a greater power than the oscillations with $k \in \mathcal{N}_{b}$, corresponding to the wavelengths $\lambda$ from 0.42 to $0.67 \mathrm{pc}$.
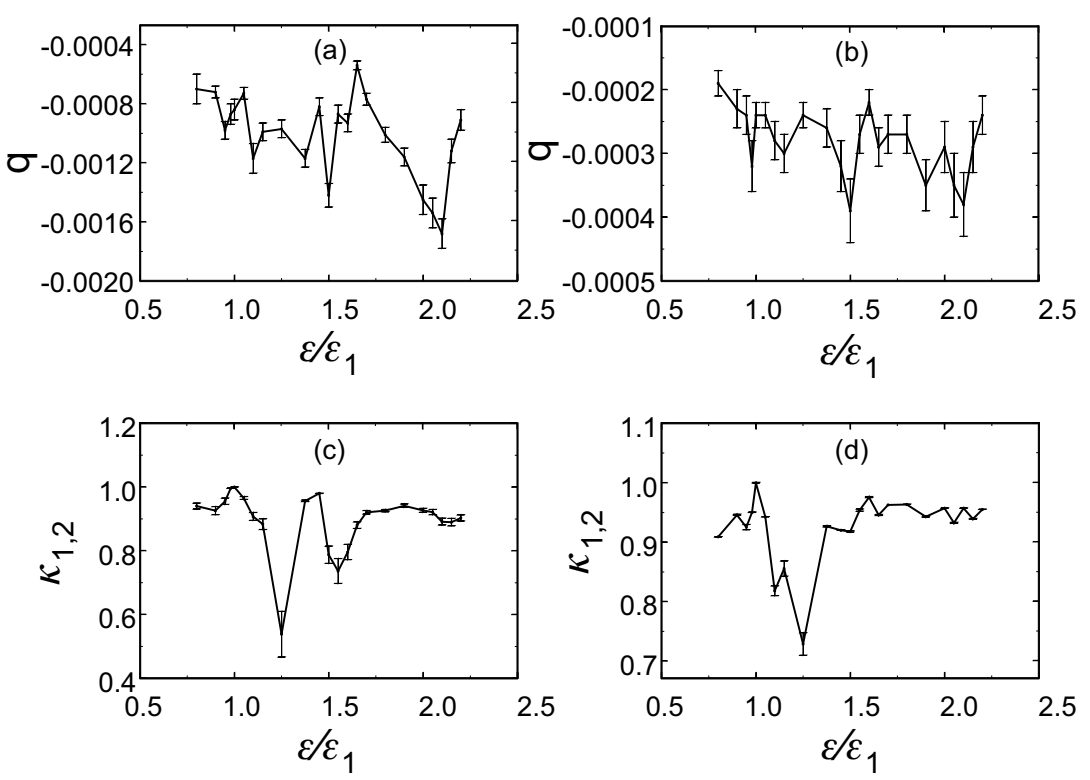

Fig. 14.5 The dependencies on $\epsilon$ of $q$ and $\kappa_{1,2}$ for the $v$ oscillations in the OSC model 1 at $n_{s t}=6$ $(\mathrm{a}, \mathrm{c})$ and $n_{s t}=30(\mathrm{~b}, \mathrm{~d})$

Figs. 14.5a,b and figs. 14.6a,b show the plots of the dependencies of $q=q(\epsilon)$ for the spectra $S_{v}(k)$ and $S_{f}(k)$ at $n_{s t}=6,30$. As in Danilov and Putkov (2014), the $\epsilon_{1}$ value is assumed equal to $\epsilon_{1}=0.012 \bar{r}_{i j}\left(\bar{r}_{i j}\right.$ is the initial distance between the stars in the cluster model 1, averaged over all different pairs of stars in the cluster). According to fig. 14.5a,b, the values of $q(\epsilon)$ on average decrease slightly with an increasing $\epsilon$. Consequently, the slopes of the spectra $S_{v}(k)$ on average increase with an increasing $\epsilon$. At $n_{s t}=30$, such an increase in the slope is quite small (comparable with the largest of the errors of $q$ shown in fig. 14.5b). At $n_{s t}=6$, such an increase in the slope of $S_{v}(k)$ with an increasing $\epsilon$ is more noticeable. We can mark the values of $\epsilon / \epsilon_{1}=1.1,1.5,2.1$, at which the dependencies $q=q(\epsilon)$ have the local maxima (at such $\epsilon$ values, the slopes of $S_{v}(k)$ noticeably increase in comparison with the slope of the $S_{v}(k)$ spectrum at $\left.\epsilon=\epsilon_{1}\right)$. According to fig. 14.6a,b, the values of $q(\epsilon)$ on average increase slightly with an increasing $\epsilon$. Consequently, the slopes of the $S_{f}(k)$ spectra on average decrease with an increasing $\epsilon$.

We note that an increase of the slope of the $S_{v}(k)$ spectrum is due to an increase of the $v$ oscillation power with small $k \in \mathcal{N}_{a}$ (the large $\lambda$ ) in comparison with the power of the $v$ oscillations with the larger $k \in \mathcal{N}_{b}$ in the cluster model. According to Danilov and Putkov (2014), with an increasing $\epsilon / \epsilon_{1}$ from 1 to 2 , a part of the core stars moves to the halo (to the cluster periphery); the total kinetic energy of the 

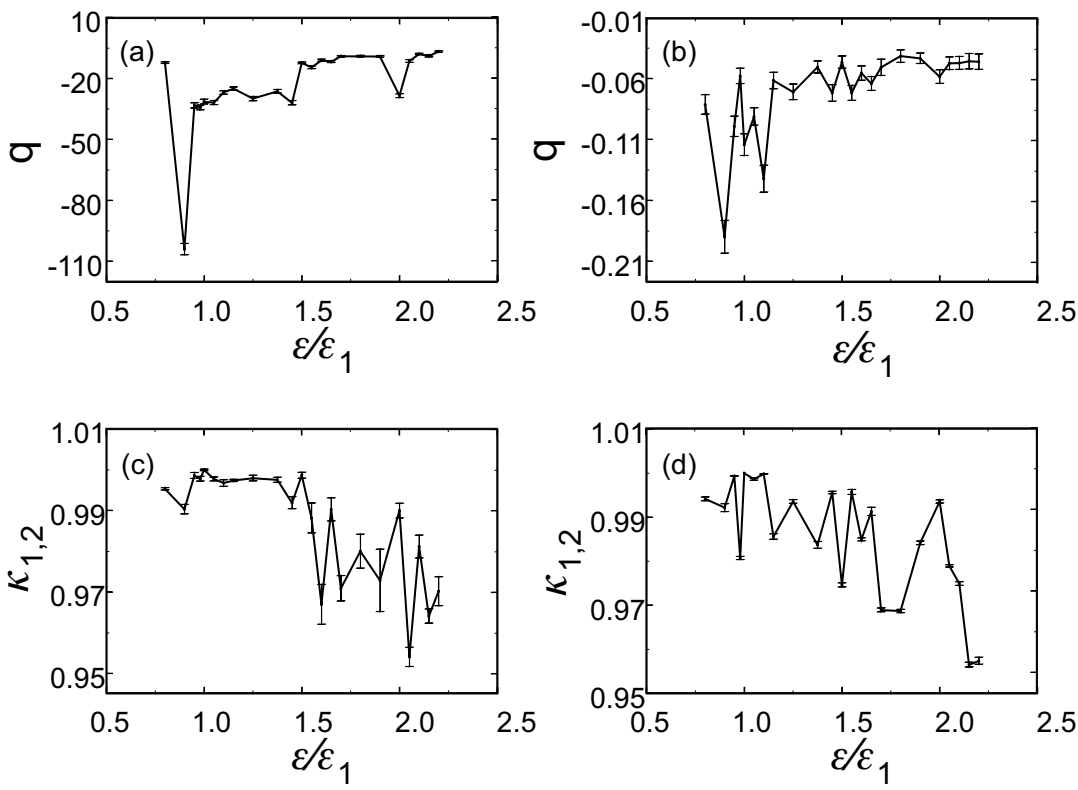

Fig. 14.6 The dependencies on $\epsilon$ of $q$ and $\kappa_{1,2}$ for the $f$ oscillations in the OSC model 1 at $n_{s t}=6$ $(\mathrm{a}, \mathrm{c})$ and $n_{s t}=30(\mathrm{~b}, \mathrm{~d})$

motion of the core stars decreases noticeably with the practically unchanged degree of non-stationarity of the cluster model. Consequently, in this case, the role (and power) of the halo oscillations with the larger scales and wavelengths increases in the dynamics of the cluster model.

Figs. 14.5c,d and figs. 14.6c,d show the dependencies on $\epsilon$ of the correlation coefficient $\kappa_{1,2}(\epsilon)$ between the $S(k)$ spectrum obtained at $\epsilon=\epsilon_{1}$, and the $S(k)$ spectrum obtained at an arbitrary value of $\epsilon$. The dependencies $\kappa_{1,2}=\kappa_{1,2}(\epsilon)$ in fig. $14.5 \mathrm{c}, \mathrm{d}$ were obtained by comparing the spectra $S_{v}(k)$ with each other. According to fig. $14.5 \mathrm{c}$, at $n_{s t}=6$, the dependency $\kappa_{1,2}=\kappa_{1,2}(\epsilon)$ has two local minima at $\epsilon / \epsilon_{1}=1.25,1.55$. At $n_{s t}=30$, (fig. 14.5d) the dependency $\kappa_{1,2}=\kappa_{1,2}(\epsilon)$ has two most prominent local minima at $\epsilon / \epsilon_{1}=1.1,1.25$. At $\epsilon<\epsilon_{1}$, the values $\kappa_{1,2}(\epsilon)$ decrease in comparison with $\kappa_{1,2}\left(\epsilon_{1}\right)$, which is associated with an increase of the errors of the PCS used for the calculation of the $S(k)$ spectra in this work (see also Danilov and Putkov (2014)). According to fig. 14.6c,d, the values of $\kappa_{1,2}(\epsilon)$ obtained by comparing of the $S_{f}(k)$ spectra with each other at $\epsilon=\epsilon_{1}$ and $\epsilon \neq \epsilon_{1}$ are close to 1 and, on average, decrease weakly with an increasing $\epsilon$ at $\epsilon>\epsilon_{1}$. Consequently, on average, with an increasing $\epsilon$, the differences in the $S_{f}(k)$ spectra obtained at $\epsilon=\epsilon_{1}$ and $\epsilon>\epsilon_{1}$ increase, which, apparently, is due to the marked above changes in the mean slopes of the $S_{f}(k)$ spectra at an increasing $\epsilon / \epsilon_{1}$ from 1 to 2. Comparison between the dependencies $\kappa_{1,2}=\kappa_{1,2}(\epsilon)$ in figs. 14.6c,d of this work and fig. 5(b) from Danilov and Putkov (2014) indicates that the spectra of the wave numbers of the $f$ (and $v$ ) oscillations at $\epsilon=\epsilon_{1}$ and $\epsilon \neq \epsilon_{1}$ differ significantly less than the spectra 
of the $f$ oscillations. In the case of the $S_{v}(k)$ spectra, the notable differences of $\kappa_{1,2}$ from the unity are attained only near the marked above points of the local minima of the dependencies $\kappa_{1,2}=\kappa_{1,2}(\epsilon)$ in figs. $14.5 \mathrm{c}$,d of this work.

\subsection{Conclusions}

1. In this part of the work, we have considered the results of the correlation and spectral analysis of the oscillations of the phase density and the velocity moduli of stars in six OSC models of Danilov and Dorogavtseva (2008).

2. We have discussed the spatial cross-correlation functions for the fluctuations of the phase-density $f$ and the modulus of the stellar velocity $v$ of the OSC models, as well as the spectra of the wave numbers of the $f$ and $v$ oscillations in the clusters models. In order to calculate the spectra, we used the spatial Fourier transform of the cross-correlation functions with a zero time shift.

3. The spectrum of the wave numbers of the $f$ oscillations turned out to be simpler in comparison with the spectrum of the wave numbers of the $v$ oscillations. The power-most $f$ and $v$ oscillations in the considered OSC models are located in the region of the small values of the wave number $k$ (and the large wavelengths $\lambda>(0.91-1.25) \mathrm{pc})$. A significant contribution to the average power of the $f$ and $v$ oscillations is made by the homologous oscillations of the clusters. We have defined the widths $k_{e}$ and the points $k=k_{m}$ of the highest local maxima of the considered spectra for each OSC model (see Tables 14.1 and 14.2). We have discussed the dependency of the spectra width on the parameters of the cluster models and on the number of stars $n_{s t}$, from which the $f$ and $v$ values were determined.

4. In five cluster models, we have noted the $v$ oscillations repetitive with an equal step over $k$. We have defined the points $k=k_{\text {in }}$ of the 1 st local maximum in a series of the local maxima points equidistant relative to $k$ in the spectra of the $v$ oscillations (Table 14.1). We have written the formula that relates the wavelengths of the $v$ oscillations, which are most common in the wave numbers' spectra of the OSC models. The assumption of Danilov and Putkov (2014) on the discrete nature of the wavelengths of the oscillations formed in the OSC models and the relationship of these wavelengths with the dimensions of the models has been substantiated. The spectrum of the wave numbers of the $v$ oscillations in the OSC model 1 corresponds to the spectrum of the system, which is much younger dynamically than the system with the Kolmogorov spectrum. Some of the waves of the $f$ and $v$ oscillations found in the OSC models in this work and in the works of Danilov and Putkov (2013b); Putkov (2013) may well be a gravitational analog of the Van Kampen waves (Van Kampen, 1955) in plasma.

5. For the cluster model 1, we have considered the different values of the smoothing parameter $\epsilon$ of the force functions in the equations of a stellar motion. We have calculated the "slopes" $q$ of the wave numbers' spectra of the $f$ and $v$ oscillations and their dependency on $\epsilon$, as well as the coefficients of a correlation between the spectra of the $f$ and $v$ oscillations in the cluster model 1 with the different $\epsilon$. The spectra 
of the wave numbers of the $f$ and $v$ oscillations in this model change significantly less with the changing $\epsilon$ than the frequency spectrum of the $f$ oscillations obtained for this model by Danilov and Putkov (2014). An increase of the slope of the $S_{v}(k)$ spectrum with an increasing $\epsilon$ is due to a change in the structure of the OSC model (since in this case the part of the core stars move to the halo, and the total kinetic energy of the core stars decreases with the practically unchanged degree of a nonstationarity of the cluster model (Danilov and Putkov, 2014)). An increasing $\epsilon$ affects the $S_{f}(k)$ spectra less than the $S_{v}(k)$ spectra.

6. An investigation (the Fourier analysis) of the autocorrelation functions of the radial dependencies of the average velocity moduli (and the moduli of the average values of the velocity vector components) of the stellar motion in the series of the OSCs from the list of Danilov and Seleznev (1994) can allow us to obtain the necessary data on the wavelengths of the stellar velocity oscillations in the clusters, as well as to estimate a relationship between the lengths of these waves and the tidal radii of the clusters. A comparison of these estimates with the results of the numerical modeling of the OSC dynamics will make it possible to draw the conclusions about the dynamic state and the parameters of the observed stellar clusters. Since $R_{t} \Delta k \simeq 1$ (see Table 14.1 and Section 14.3) the wavelengths of two or more neighbor (with respect to $k$ ) oscillations in the clusters can be used to estimate $R_{t}$ and the total masses of the OSCs.

\section{References}

Bendat, J.S., Piersol, A.G.: Engineering Applications of Correlation and Spectral Analysis. Wiley-Interscience, New York (1980).

Bernar, M., Briffo, J., Bussac, J., et al.: Correlation methods of analysis of plasma experiments. In: Diagnostics of plasma, Isssue 3. Atomizdat, Moscow (1973), 449-460.

Binney, J., Tremaine, S.: Galactic Dynamics, Second edition. Princeton University Press, Princeton, Oxford (2008). ISBN: 9780691130279

Danilov, V.M.: Analysis of density fluctuations in models of open clusters. Astron. Reports 52, 888-899 (2008). doi:10.1134/S1063772908110036

Danilov, V.M.: Phase-Density Fluctuations at the Centers of Six Open Clusters. Astron. Reports 54, 514-527 (2010). doi:10.1134/S1063772910060053

Danilov, V.M., Dorogavtseva, L.V.: Timescales for mechanisms for the dynamical evolution of open star clusters. Astron. Reports 52, 467-478 (2008). doi:10.1134/S1063772908060048

Danilov, V.M., Putkov, S.I.: The dynamics of correlations in open-star cluster models. Astron. Reports 56, 623-637 (2012b). doi:10.1134/S1063772912080021

Danilov, V.M., Putkov, S.I.: Correlations and instabilities of the phase density oscillations in the open star cluster models. Publ. Udmurt. Univ. Mathematics, Physics, Computer Sciences. Issue 2, 65-73 (2013a) (In Russian). ISSN: 19949197, eISSN: 2076-5959 
Danilov, V.M., Putkov, S.I.: Correlations, spectra and instability of phase-space density fluctuations in open-cluster models. Astrophys. Bull. 68, 154-168 (2013b). doi:10.1134/S199034131302003X

Danilov, V.M., Putkov, S.I.: Smoothing of Force Functions and the Fluctuation Spectra of an Open Star Cluster Model. Astrophys. Bull. 69, 27-39, (2014). doi:10.1134/S1990341314010039

Danilov, V.M., Putkov, S.I.: Wavenumbers Spectra of Oscillations of Open Star Cluster Models. Astrophys. Bull. 70, 71-82 (2015). doi:10.1134/S1990341315010083

Danilov, V.M., Seleznev, A.F.: The catalogue of structural and dynamical characteristics of $103 \mathrm{OCl}$ and the first results of its investigations. Astron. Astrophys. Trans. 6, 85-156 (1994). doi:10.1080/10556799408232061

Fortov, V.E. (ed.): Encyclopaedia of low-temperature plasma. Introductory volume I. Nauka, Moscow (2000). (In Russian).

Gray, D.F.: The observation and analysis of stellar photospheres. Wiley, New York (1976). ISBN:04713238029780471323808

Henon, M.: Lámas isochrone: I. Annales d'Astrophysique. 22, 126-139 (1959).

Kadomtsev, B.B.: Landau damping and echo in plasma. Uspekhi Fiz. Nauk 95, 111-129 (1968). (In Russian).

King, I.R.: The structure of star clusters. I. An empirical density law. Astron. J. 67, 471-485 (1962). doi:10.1086/108756

King, I.R.: The structure of star clusters. III. Some simple dynamical model. Astron.J. 71, 64-75 (1966). doi:10.1086/109857

Kutuzov, S.A., Osipkov, L.P.: A Generalized Model for the Three-Dimensional Gravitational Potential of Stellar Systems. Soviet Astron. 24, 17-22 (1980).

Lichtenberg, A.J., Lieberman, M.A.: Regular and Chaotic Dynamics. SpringerVerlag, New York, NY (1983).

Louis, P.D., Gerhard, O.E.: Can galaxies oscillate? - A self-consistent model of a non-stationary stellar system. Mon. Not. R. Astron. Soc. 233, 337-365 (1988). doi:10.1093/mnras/233.2.337

Lynden-Bell, D.: Statistical mechanics of violent relaxation in stellar systems. Mon. Not. R. Astron. Soc. 136, 101-121 (1967). doi:10.1093/mnras/136.1.101

Mathur, S.D.: Existence of oscillation modes in collisionless gravitating systems. Mon. Not. R. Astron. Soc. 243, 529-536 (1990).

Putkov, S.I.: On phase density fluctuations in two open cluster models. Astronomicheskii Tsirkulyar No. 1604, 1-3 (2013).

Tsytovich, V.N.: Theory of turbulent plasma. Atomizdat, Moscow (1971). (In Russian).

Van Kampen, N.G.: On the theory of stationary waves in plasmas. Physica 21, 949-963 (1955).

Vandervoort, P.O.: On stationary oscillations of galaxies. Mon. Not. R. Astron. Soc. 339, 537-555 (2003). doi:10.1046/j.1365-8711.2003.06196.x

Volkov, Ya.F., Dyatlov V.G., Mitina, R.I.: Diagnostics of turbulent plasma. Naukova Dumka, Kiev (1983). (In Russian).

Weinberg, M.D.: Weakly damped modes in star clusters and galaxies. Astrophys. J. 421, 481-490 (1994). doi:10.1086/173665 
Wielen, R.: On the Lifetimes of Galactic Clusters. Astrophys. Space Sci. 13, 300-308 (1971). doi:10.1007/BF00649160 


\title{
Chapter 15 \\ Dynamics of the Coronas of Open Star Clusters
}

\begin{abstract}
In this chapter, we consider the method of the corona separation in the OSC models. The method uses the stellar trajectories that do not escape the limits of the coronas at the time intervals $t$, comparable to the average lifetime $\tau$ of such clusters. For six numerical cluster models, we consider the corona models and discuss the direction and nature of their dynamic evolution. The retrograde stellar motions dominate in the coronas. In the interval of the stellar distances from the cluster center from 1 to 3 cluster tidal radii, a formation of the density and the phase density distributions close to the equilibrium ones is noted. We approximate the phase density of the corona and the cluster by the distributions depending on three arguments. This temporal equilibrium of the corona is due to the balance of the number of stars that move to the corona from the central regions of the cluster and escape to the corona periphery or beyond it. We note the signs of the gravitational coupling of the corona stars up to the distances of four cluster tidal radii from the cluster center (the presence of a large number of the corona stars with the retrograde mean motion close to the periodic one in the Galactic plane; (91-99) \% of the corona stars at the time intervals of the cluster lifetime satisfy the criterion of a gravitational coupling). We estimates the rate of the corona stars' dissipation from 0.03 to 0.23 of the number of the corona stars during the time of a violent relaxation of the cluster at $t \geq \tau$.
\end{abstract}

\subsection{Introduction}

A density increase in the number of stars in the wide vicinity of the OSCs was first noted by Shapley and Trumpler (Kholopov, 1981, p. 314). In more detail, the structure of a number of OSCs and globular clusters (core, corona, intermediate region) was studied in the works of Kholopov and Artyukhina (Kholopov, 1981). According to Kholopov (1981), the extended regions of an increased density of the number of stars (coronas) and the denser cluster cores are the characteristic structural features of any cluster. More certain and reliably, the parameters of the OSC coronas 
can be obtained after an identifying the stars which can possibly belong to clusters, taking into account the data on the proper motions of these stars (Kholopov, 1981). The conclusions about the presence of the extended halos or coronas around the well-studied OSCs formed by the stars located at the large distances from the cluster center, including the stars beyond the King's tidal radius $R_{t}$ (King, 1962), can be found in the papers of Pels et al. (1975); Van Leeuwen (1985). According to the estimates of Kholopov (1981), the coronas of the clusters considered by Kholopov (1981) are dynamically stable in the gravitational field of the Galaxy.

The radial distributions of the apparent density of stars and other associated distributions for a number of OSCs at sufficiently large distances $r$ from the centers of these clusters have been studied in a number of papers (see, for example, Oort (1979); Raboud and Mermilliod (1998a,b); Adams et al. (2001, 2002); Chen et al. (2004); Kharchenko et al. (2009),etc.). In these papers, the changes of the shape of the OSCs (flattening, ellipticity) with an increasing $r$ are discussed. Danilov et al. (1985) proposed a method for an estimating the sizes and numbers of stars in the OSCs based on the comparison of the number of stars $N(r)$ in a circle of the radius $r$ in the projection onto the tangent plane in the region of the cluster $\left(N_{0}(r)\right)$ and in several neighbor fields $\left(N_{i}(r), i=1, \ldots, k, k=4-6\right)$ surrounding the cluster. Comparison of the $N_{0}(r)$ value and the mean number of field stars $\bar{N}(r)$ averaged over all $i \in[1, k]$ allows us to determine more accurately the cluster parameters than with the method of the apparent densities of stars $F(r)$. The use of the normalized deviations of $N_{0}(r)$ from $\bar{N}(r)$ makes it possible to isolate statistically the coronal areas of the cluster against the surrounding stellar field at significantly larger distances from the cluster center than using the $F(r)$ curves, and to confirm the conclusions of Kholopov (1981) about the existence of the extended coronas with an extremely low stellar density in the OSCs (Danilov et al., 1985).

Stone investigated young open star clusters NGC 654 (Stone, 1980) and NGC 6823 (Stone, 1988). He found the outer regions in these clusters inhabited by the cluster member stars with the radius of these regions of about four cluster core radius. Herewith, the corona stars of NGC 6823 are younger than the cluster core stars. Nilakshi (2002) investigated 38 rich OSCs and concluded that all clusters, including the youngest ones, have a corona. Corona is the outer region of the cluster which exists since its formation. Despite the low density of stars in the corona region, it contains $\sim 75 \%$ cluster members (Nilakshi, 2002). Röser et al. (2011) investigated the structure of the Hyades cluster by the data on the proper motions of stars up to the distance of $30 \mathrm{pc}$ from the cluster center. They found that the interval of distances from $R_{t}$ to $30 \mathrm{pc}$ contains $37 \%$ members of the cluster (by a mass). Davenport and Sandquist (2010); Balaguer-Nùñez et al. (2013) studied the structure of the M67 cluster by the photometric data (Davenport and Sandquist, 2010) and by the data on the proper motion of stars (Balaguer-Nùñez et al., 2013); they found an extended elongated halo of the probable member stars up to the distance of about one degree from the cluster center.

Fellhauer and Heggie (2005) constructed and investigated an equilibrium ellipsoidal model of a gravitationally unbound star cluster in the tidal field of the Galaxy. They used the following considerations. Star clusters are gradually destroyed when 
they move in the gravitational field of the Galaxy. As soon as the cluster density becomes lower than the critical one, it is often assumed that the cluster evolution has completed with this. However, the remnants of such system continue the motion along the similar trajectories, which allows Fellhauer and Heggie (2005) to construct a density-homogeneous model in a state of unstable equilibrium. At the initial time point, the stars in this model move according to the epicyclic theory, corrected taking into account the self-gravitation of the system; the initial dispersions of the velocities of the stars along $x$ and $y$ axes (in the Galactic plane) are zero at each point $(x, y)$ of the system, the motion of all stars is retrograde. The numerical experiments performed by Fellhauer and Heggie (2005) show that the systems with a low density ( $\sim 1 \%$ from the field density) and an initial value of the major semiaxis $a$ of the system ellipsoid $a=50 \mathrm{pc}$ moving along a circular orbit with a radius of $10 \mathrm{kpc}$ in the Galaxy can survive during $\sim 20$ revolutions around the Galactic center (in this case, the decay time is estimated as the time of an increase by $50 \%$ of the radius $r_{h}$ of the sphere containing a half of the mass of the system). Danilov et al. (2014) considered the numerical dynamic OSC models with the corona radii less than $40 \mathrm{pc}$. Therefore, Danilov et al. (2014) constructed the corona models close to the equilibrium ones for the sufficiently large time intervals comparable to the lifetime $\tau$ of the OSCs. According to Danilov et al. (2014), it is of interest to use the more realistic initial conditions for the stellar motion in the cluster corona in comparison with those used by Fellhauer and Heggie (2005).

At present, various methods for estimating the tidal dimensions of OSCs in the field of forces of the Galaxy are known and used. King (1962); von Hoerner (1957); Innanen (1979); Innanen et al. (1983); Merritt (1984); Allen and Richstone (1988) determine the tidal radius $R_{t}$ of the cluster from the condition of the balance of the forces acting on the test cluster star along the line connecting the mass centers of the cluster and the Galaxy. Often the $R_{t}$ value is defined as the radius of the stability region of the orbits of the cluster stars in the field of the external forces. In this case, an analysis of the stability of the solutions of the stellar motion equation system is performed (Chandrasekhar, 1942; Angeletti et al., 1983; Angeletti and Giannone, 1983, 1984; Angeletti et al., 1984), or a qualitative analysis of the first integral of this system (if it is known) is performed without a preliminary integrating of the equations of a stellar motion (Rein, 1936). In the second case, the region of the possible motion of the star is determined, where the square of the relative velocity of the star in the coordinate system associated with the mass center of the cluster is positive. Among the early works on the stability of the clusters moving along the circular orbits around the Galactic center, we can mention the works of Bok (1934) and Mineur (1939). The earlier studies on this problem are discussed in the review of Rein (1936). The later works of Angeletti et al. (1983); Angeletti and Giannone (1983, 1984); Angeletti et al. (1984) can be attributed to this direction of a research. They use the calculation of the multipliers of the matrix of the fundamental solution of the equations of motion of the test star in the framework of Floquet theory. Keenan et al. (1973); Jefferys (1976); Keenan (1981a,b), with the different methods that use the numerical integration of the test star motion in the field of forces of the cluster and the Galaxy, showed that the stars with the retrograde trajectories in 
the cluster $\left(\operatorname{sign}\left(l_{z}\right)=-\operatorname{sign}\left(L_{Z}\right)\right)$ are weakly influenced by the Galaxy field to the greater distances from the cluster center than the stars with "prograde" trajectories $\left(\operatorname{sign}\left(l_{z}\right)=\operatorname{sign}\left(L_{Z}\right)\right)$. Here, $l_{z}$ and $L_{Z}$ are the $z$ and $Z$ components of the vectors $\mathbf{I}$ and $\mathbf{L}$ of the angular momenta of the star motion relative to the cluster center and of the cluster motion relative to the center of the Galaxy, respectively; $z$ and $Z$ axes are perpendicular to the Galactic plane. Jefferys (1976) ascribed this property of the trajectories to the existence of a second quasi-integral of motion complementary to the Jacobi integral. Jefferys (1976); Fukushige and Heggie (2000) used the secant surface method for an approximate analysis of the cluster stability.

For the system of equations of a stellar motion in the cluster model with a plane circular orbit in the linearised force field of the Galaxy, Aarseth (1973) introduced the integral $C_{i}$ per unit of the star mass, analogous to the Jacobi constant (see (35) from Aarseth (1973)). The critical value $C_{\infty}<0$ of $C_{i}$ for a star that is immobile in the Lagrangian equilibrium points is often used as a criterion for the dissipation of a star from a cluster, although the condition $C_{i}>C_{\infty}$ does not guarantee a fast dissipation of the star (Aarseth, 1973). According to Terlevich (1987), the tidal field of the Galaxy reduces the energy barrier for the dissipation of stars from 0 to $C_{L}=C_{\infty}<0$ and, therefore, accelerates the evolution of the cluster in the direction of a decay.

Fukushige and Heggie (2000) performed a theoretical analysis of the motion of a dissipating star in the vicinity of the Lagrangian points and estimated the dissipation time as a function of the energy of the stellar motion (formula (9) from Fukushige and Heggie (2000)). The smoothed potential of the cluster was used. For a number of cluster models, Fukushige and Heggie (2000) showed by the numerical experiments that stars with energies above the critical one can remain gravitationally bound in the cluster for a very long time (much longer than the dynamic time).

Takahashi and Baumgardt (2012) used the energy and the apo-centric criteria to describe the dissipation of stars in the globular cluster models (see (17), (18) from Takahashi and Baumgardt (2012); in this case, the star energy is above the critical one and the apo-centric distance of the star is larger than $2 / 3$ of the tidal radius $R_{t}$ ). For stars that satisfy these criteria (the "potential dissipators", see the term in Takahashi and Baumgardt (2012)), it was shown that such stars quite can be captured again by the cluster due to the stellar encounters. In an earlier paper Ross et al. (1997), for the cluster model in the form of a point mass moving along a circular orbit in the linearised force field of the Galaxy, obtained the conditions for the star's dissipation (the conditions of a removing of the star to an infinite distance from the cluster). It is of interest to use such conditions to study the dissipation of stars from the OSC coronas.

The results of such study can be used in a discussing the dynamics of the OSCs and their models. It is also of interest to discuss the applicability of some convenient phase-density models for the cluster and its corona in the case of the more realistic initial conditions for the phase coordinates of stars than in Fellhauer and Heggie (2005). Such phase density models can be useful for an estimation of the masses of the OSCs and their coronas from observations (by the data on the coordinates and velocities of stars in the OSCs and in the cluster coronas). In addition, such 
phase-density models can be used to compare the observed and the model velocity distributions of stars in the core and at the periphery of the OSCs, when discussing the mechanisms of the formation of the stellar velocity distributions in the OSCs.

The objectives of this part of the work are: 1) to describe the method for separation of the coronas in the OSC models; 2 ) to discuss the models of the coronas for six numerical cluster models; to describe the parameters of the coronas; to discuss the kinematics of the stellar motions in the coronas; to analyse the dynamic evolution of the coronas; 3 ) to discuss the signs of the gravitational coupling of the corona stars at the distances of several tidal radii $R_{t}$ from the cluster center; 4) to analyse the rate of the corona stars' dissipation using the criterion of Ross et al. (1997) of the gravitational coupling of the cluster stars.

\subsection{Constructing Coronas of OSC models}

Following to Danilov et al. (2014), we consider two ways of constructing the coronas of the OSC models. If we regard the corona as a long-lived object, then the rapidly disappearing cluster stars are not members of the corona. Energy is not a very reliable indicator of the star's belonging to the corona (see above). In the nonstationary systems, all the parameters of a star change with time (in the OSC models of Danilov and Dorogavtseva (2008), only the number of the star conserves). The main parameters that really characterize the belonging of the star to the corona (and, consequently, the composition and structure of the corona) are the distance $r$ of the star from the cluster center and the time interval $\tau$ during which the value $r$ does not exceed the radius of the corona $r_{2}$. Let $\tau=3 \tau_{v r}$, that for the OSC models of Danilov and Dorogavtseva (2008) approximately corresponds to $\tau \simeq 1.5 \times 10^{8}$ years and is comparable with the average lifetime of the OSCs (Wielen, 1987), and $r_{1}$ is a radius of the sphere containing some auxiliary group of stars (stars of the core and the intermediate region of increased density (Kholopov, 1981)), $r_{1}<r_{2}, r_{1}=5$ $\mathrm{pc} \simeq 0.5 R_{t}$. For a brevity, we will further call the subsystem corresponding to this group of stars as the core. Let $\mathcal{N}_{1}$ and $\mathcal{N}_{2}$ be a sets of numbers of stars $i$ with the distances from the center $r<r_{1}$ and $r<r_{2}$, respectively, at $t \leq \tau$. In this case, $\mathcal{N}_{1} \subset \mathcal{N}_{2}$. Eliminating from the set $\mathcal{N}_{2}$ all the numbers of the stars from $\mathcal{N}_{1}$, we find the set of numbers of the corona stars $\mathcal{N}_{h}$. Using these numbers and the results of the numerical integration of the equations of a stellar motion, it is possible to construct all the necessary characteristics of the corona for the different time points. We denote this model of the corona as model 1 with the constant numbers of stars. This model can be improved if we use a smaller value for $\tau$ (for example, $\tau_{v r}=\tau / 3$, which is 2.6 times greater than $\bar{t}_{c r}$, see above) and define the set $\mathcal{N}_{h}$ at the times $t^{\prime} \in\left[t, t+\tau_{v r}\right]$. It is the second model of the corona with the variable numbers of stars; in this case, the set $\mathcal{N}_{1}$ will differ from $\mathcal{N}_{1}$ for the first model of a corona. Let $r_{2}=40 \mathrm{pc} \simeq 4 R_{t}$ (for the OSC models of Danilov and Dorogavtseva (2008)). According to the criterion of the gravitational coupling of stars in the cluster (Ross et al., 1997) and the calculation performed by Danilov et al. (2014), at this value of 
$r_{2}$, the number of the corona stars in the OSC models varies only by a few percent over the time $\tau$, see below.

\subsection{Parameters of Coronas of the OSC Models. Mechanisms of Corona Formation and Rate of Dissipation of Stars from Coronas of the OSC Models}

Let $s_{z}=\langle|\zeta|\rangle, s_{r}=\langle r\rangle$ and $s_{z, r}=\langle|\zeta| / r\rangle$ be the average values of $|\zeta|, r$ and $|\zeta| / r$, respectively. In order to study the dynamic evolution of the OSC coronas and cores, Danilov et al. (2014) constructs the time $t$ dependencies of $s_{z}, s_{r}, s_{z, r}$ for the corona and core stars of the OSC models $1-6$ at $t \in[0, \tau]$. The corona models with the constant and variable numbers of stars were considered. Fig. 15.1 shows the dependencies obtained by Danilov et al. (2014) for the OSC model 1 in the case of the cluster subsystem (corona and core) models with the constant numbers of stars. The $s_{z}$ and $s_{r}$ values are given in parsecs, the vertical bars in fig.15.1 show the errors of the mean values; here and further in the figures, the letters $h$ and $c$ mark the corona and the core curves, respectively. For other OSC models, these dependencies have a similar form, and, therefore, are not shown here. These dependencies for the corona contain oscillations that damp with time with a period close to the period of the oscillations of the regular field of this model $P_{r} \simeq 0.6 \tau_{v r}$ (Danilov and Dorogavtseva, 2008). The corona expands with an acceleration along the plane $(\xi, \eta)$ and, at $t \simeq \tau$, forms the "ellipsoid" flattened along the $\zeta$ axis with an average ratio $|\zeta| / r$ equal to $s_{z, r} \simeq 0.24 \pm 0.02$.

At $t / \tau_{v r} \in[2.5,3.0]$, the average $\zeta^{2} / \xi^{2}$ value for the corona is $\left\langle\zeta^{2} / \xi^{2}\right\rangle_{h}=$ $0.200 \pm 0.023$, and for the core, it is $\left\langle\zeta^{2} / \xi^{2}\right\rangle_{c}=0.929 \pm 0.060$. At $t / \tau_{v r} \in[2.5,3.0]$, both the corona and the core of the cluster model 1 have a shape elongated along the $\eta$ axis with the ratio of the semiaxes of the "ellipsoid" $6.8: 5.0: 1.0$ for the corona and $1.21: 1.08: 1.00$ for the core, respectively (the shape of the core differs a little from the spherical). The results obtained by Danilov et al. (2014) are quite in agreement with the results of Chumak et al. (2010), according to which the ellipticities $\left(e=1-\left\langle\zeta^{2} / \xi^{2}\right\rangle\right)$ of the cluster shape in the space $(\xi, \eta, \zeta)$ are small in the central regions of the clusters, and increase with the distance from the center up to 2-3 tidal radii $R_{t}$.

According to fig. $15.1 \mathrm{c}$, the average $|\zeta| / r$ value for the core changes a little with time (in comparison with the corona) near the characteristic value of $s_{z, r} \simeq 0.5$. The values of $s_{x}=\langle|\xi|\rangle$ and $s_{y}=\langle|\eta|\rangle$ for the corona stars in fig. 15.2a,b are given in parsecs and mostly grow with time (most rapidly at $t \simeq 3 \tau_{v r}$ ). According to fig. $15.2 \mathrm{c}$, the relation $s=s_{y} / s_{x}$ changes from $0.82 \pm 0.05$ to $1.23 \pm 0.10$, which is due to the rotation of the corona's "ellipsoid" in the coordinate system $(\xi, \eta, \zeta)$ in the direction opposite to the cluster motion around the Galactic center with a period of $2.54 \tau_{v r} \simeq 126.6 \mathrm{Myr}$. In this work, the rotation of the corona was studied in two ways: 1) using the two-dimensional maps of the surface density distribution of the cluster model constructed for a number of close time points; 2) with the help of 

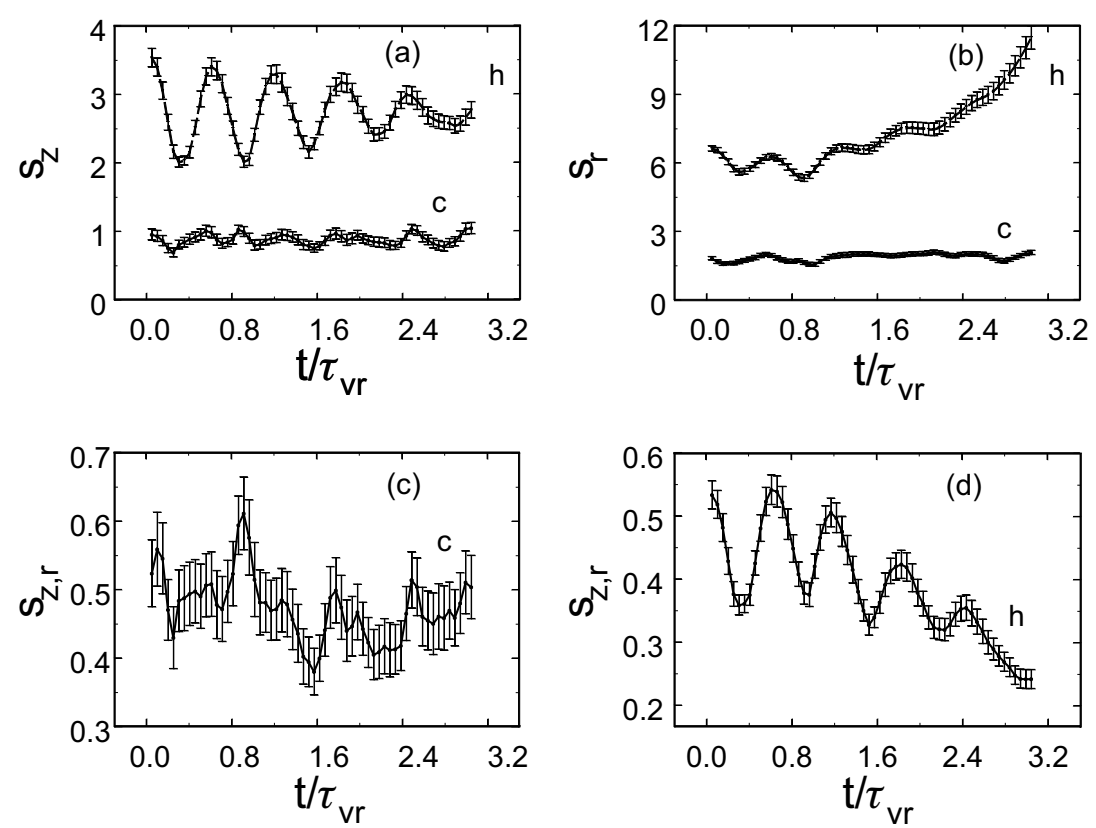

Fig. 15.1 The dependencies on a time $t$ of $s_{z}, s_{r}, s_{z, r}$ for the corona and core stars of the OSC model 1. Letters $h$ and $c$ indicate the curves for the corona and the core, respectively

the time dependencies of the mean tangential velocities $v_{t}$ of the core stars and the corona stars (see below).

1. For the stars composing the coronas of the cluster models, Danilov et al. (2014) plotted the maps of the distribution of the surface density in the projection onto the Galactic plane and the surface density profiles. Herewith, the kernel density estimator (KDE) method was used (Silverman, 1986) and the development of the corona structure of the OSC models in the projection onto the Galactic plane was analyzed. During the evolution of the OSC model, the corona expands and the inhomogeneous spatial structure is formed. The stars go to the periphery approximately at an angle $q \simeq 45^{\circ}$ to the $\xi$ axis (Chumak et al. (2010) detected the values $q \simeq(30-40)^{\circ}$ for their OSC models,). The outer parts of the corona have a specific curved shape, which was noted when studying the formation of the tidal tails of the cluster (see, for example, Chumak et al. (2010)). The tidal tails stretch along the orbit of the cluster (see, for example, Odenkirchen et al. (2003)). A near-periodic change in the elongation direction of the central denser part of the corona has been observed on the density maps with a higher resolution (Danilov et al., 2014).

2. Danilov et al. (2014) plotted the dependencies on time $t$ of the average values of $v, \varepsilon$ and $v_{t}$ for the stars of the corona and the core of the OSC models 1-6 at $t \in[0, \tau]$. Here, $v$ is the stellar velocity module; 

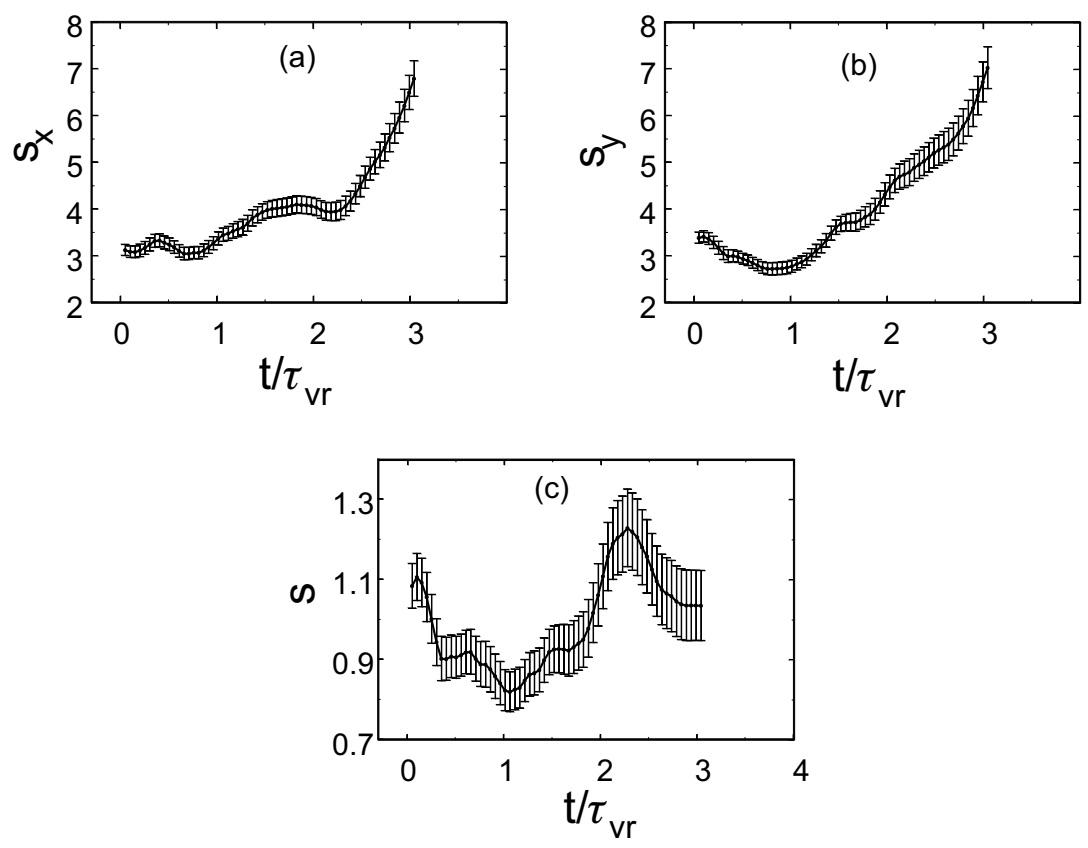

Fig. 15.2 The dependencies on time $t$ of $s_{x}, s_{y}$, and $s=s_{y} / s_{x}$ for the corona stars of the OSC model 1

$$
\varepsilon=\left(v^{2}+\alpha_{1} \xi^{2}+\alpha_{3} \zeta^{2}\right) / 2-U
$$

is the "energy" per unit of the star mass. $\alpha_{1}$ and $\alpha_{3}$ are the constants characterizing the force field of the Galaxy in the neighborhood of the circular orbit of the cluster; the numerical values of these constants were defined by Danilov and Dorogavtseva (2008) using the Galactic potential model of Kutuzov and Osipkov (1980); $\alpha_{1}<0$ and $\alpha_{3}>0 . U=U(\xi, \eta, \zeta)$ is the cluster gravitational potential at the point $(\xi, \eta, \zeta)$.

$$
v_{t}=(\xi \dot{\eta}-\eta \dot{\xi}) / R
$$

is a projection of the tangential velocity of the star onto the plane $(\xi, \eta), R=$ $\sqrt{\xi^{2}+\eta^{2}}$ ). Fig. 15.3 shows the dependencies on $t$ of the values $s_{v}=\langle v\rangle, s_{\varepsilon}=\langle\varepsilon\rangle$, and $s_{v_{t}}=\left\langle v_{t}\right\rangle$ in the case of the subsystems of the cluster model with the constant numbers of stars (fig. 15.3a-c) and of the $s_{v_{t}}$ values in the case of the subsystems with the variable numbers of stars (fig. 15.3d) for the cluster model 1. The values $s_{v}$ and $s_{v_{t}}$ in fig. 15.3 are given in $\mathrm{pc} / \mathrm{Myr}(1 \mathrm{pc} / \mathrm{Myr} \simeq 1 \mathrm{~km} / \mathrm{s})$, the values $s_{\varepsilon}$ are given in $(\mathrm{pc} / \mathrm{Myr})^{2}$.

According to fig. 15.3, the dependencies on time of $s_{v}$ for the corona and $s_{\varepsilon}$ for the corona and the core of the cluster model contain the oscillations with a period close to the period of the oscillations of the regular field of this model $P_{r}$, see 

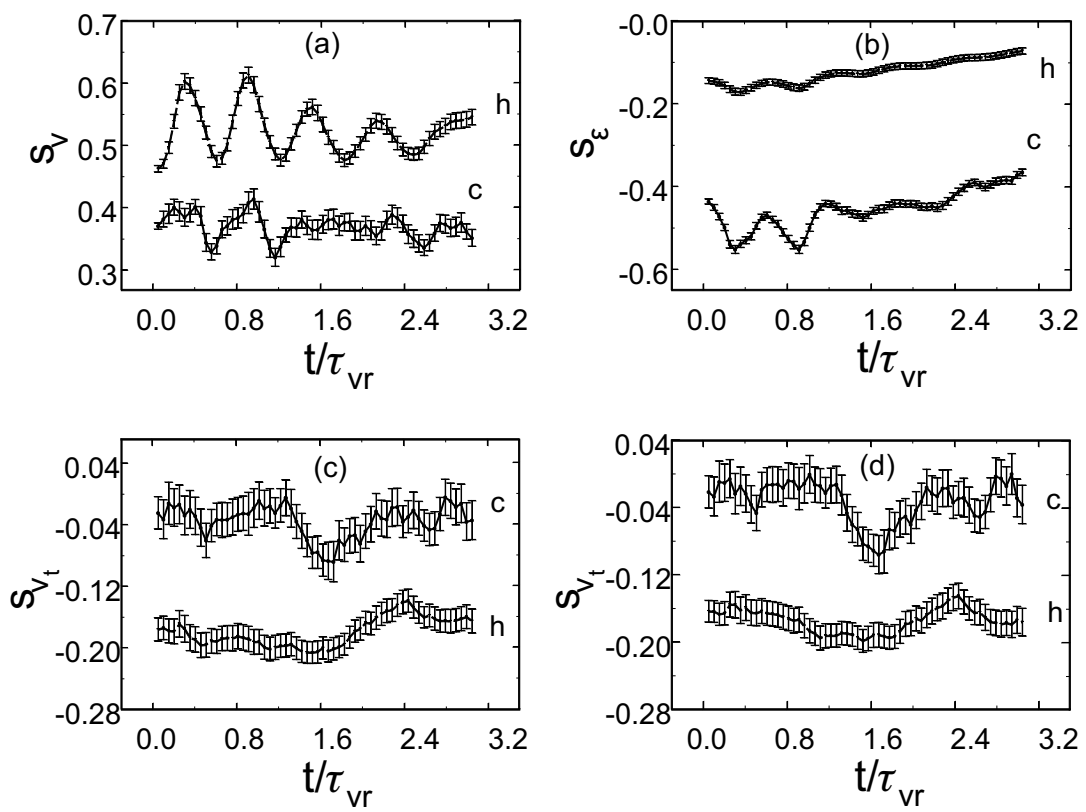

Fig. 15.3 The dependencies of $s_{v}, s_{\varepsilon}$, and $s_{v_{t}}$ on time $t$ for the stars of the corona and the core of the OSC model 1. Letters $h$ and $c$ indicate the curves for the corona and the core, respectively. Fig. $\mathrm{c}$ and $\mathrm{d}$ were constructed for the corona models with the constant and variable number of stars, respectively

above. The values of $\langle v\rangle_{c}$ in fig. 15.3a decrease slightly with an increasing $t$, which indicates a slight expansion of the core, which also is weakly noticeable in fig. $15.1 \mathrm{~b}$ (the time-dependent $\langle r\rangle_{c}$ in fig. $15.1 \mathrm{~b}$ is easily approximated by a linear function of a time $\langle r\rangle_{c} \simeq \gamma_{0}+\gamma_{1} t / \tau_{v r}$, where $\left.\gamma_{0}=1.735 \pm 0.033 \mathrm{pc}, \gamma_{1}=0.0931 \pm 0.0187 \mathrm{pc}\right)$. For all considered points of time, $\langle v\rangle_{c}<\langle v\rangle_{h},\left\langle v_{t}\right\rangle_{c}<\left\langle v_{t}\right\rangle_{h}$ and $\langle\varepsilon\rangle_{c}<\langle\varepsilon\rangle_{h}$. The values of $\langle\varepsilon\rangle_{c}$ and $\langle\varepsilon\rangle_{h}$ on average increase with an increasing $t$, while the value of $\langle\varepsilon\rangle_{h}$ is close to zero, which indicates the presence of a large number of the corona stars with the energies $\varepsilon>0$. Fig. 15.3c,d show the time dependencies of the values $\left\langle v_{t}\right\rangle$ for the corona (and the core) models with the constant and variable numbers of stars. The dependencies for an every subsystem shown in fig. 15.3c,d differ only slightly. We note that other time dependencies of the parameters of the subsystems with the constant and variable numbers of stars differ even less. Therefore, we will use further a simpler model of the subsystems with the constant numbers of stars. According to fig. $15.3 \mathrm{c}, \mathrm{d}$, the retrograde stellar motions predominate in the corona. This result quite agrees with the results of a comparative analysis of the stability of the "prograde" and "retrograde" stellar motions in the cluster models performed by Keenan et al. (1973); Jefferys (1976); Keenan (1981a,b).

Fig. 15.4 shows the time dependencies of the number of stars $N_{c}$ in the core and $N_{h}$ in the corona of model 1 for the case of the subsystems with the variable numbers 

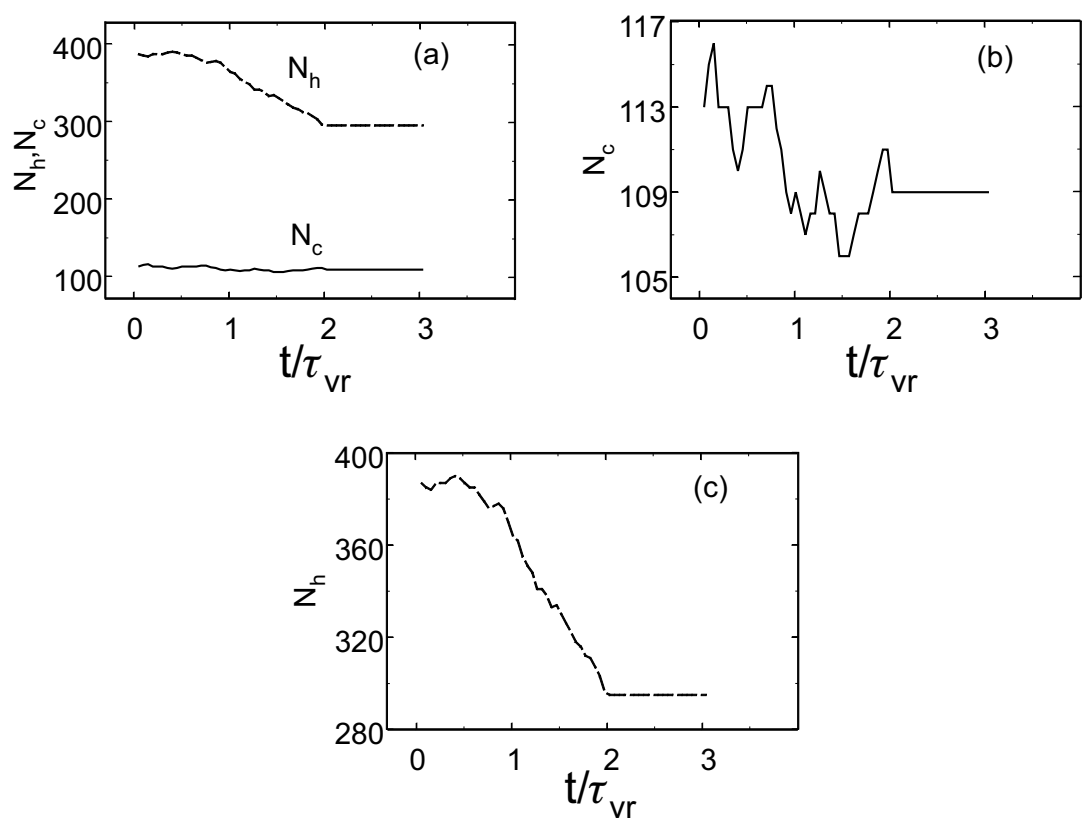

Fig. 15.4 The dependencies of $N_{h}$ and $N_{c}$ on time $t$ for the stars of the corona and the core of the OSC model 1 (the corona model with the variable number of stars)

of stars. Fig. 15.4a shows the time dependency of the number of stars in the core and the corona. Figs. 15.4b,c show these dependencies separately with an increased scale along the ordinate axis. By the time $t=2 \tau_{v r}$, the values $N_{c}$ and $N_{h}$ are obtained equal to $N_{c}=109$ and $N_{h}=295$ (in the model of the subsystems with the constant numbers of stars $N_{c}=93$ and $N_{h}=307 ; 100$ stars of model 1 of the cluster in time $\tau$ move away from its center for a distance greater than $r_{2}=40 \mathrm{pc}$; these stars form the tidal tails of the cluster).

Fig. 15.5 shows the logarithms of the spatial density profiles in the corona of model 1 for several time points $t_{i}=i \times \tau_{v r}(i=0,1,2,3)$; the numbers $i$ in fig. 15.5 indicate the curves corresponding to the time points $t_{i}, \rho=\sqrt{\xi^{2}+\eta^{2}}$. With an increasing $t$, there is an increase of the sizes of the $r, \rho$ and $|\zeta|$ regions, where the density profiles coincide or are close to each other (including the curve with the number 3). Although, the largest values of $r, \rho$ and $|\zeta|$ at the boundaries of these regions already correspond to the points located far beyond the surface of the zero velocities corresponding to the critical value of the Jacobi constant. Best of all this can be seen on a motion picture or with the density profiles plotted for the time points with a small step by $t$. The dimensions of the region with the small changes in the corona density profiles with an increasing $t$ reach the values of $r \simeq 3 R_{t}$.

Danilov et al. (2014) performed an estimation of the phase density $F$ of the cluster in the neighbourhoods of each star, taking into account the data on the phase 

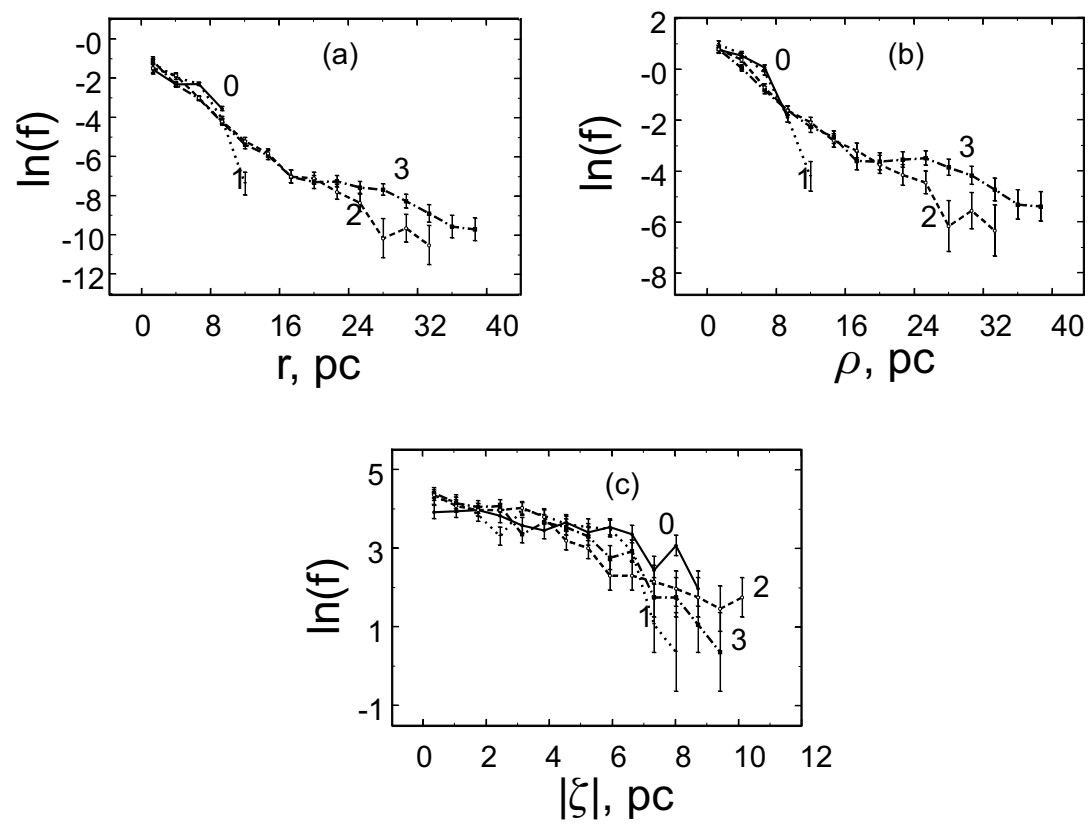

Fig. 15.5 The dependencies of $\ln (f)$ on $r, \rho,|\zeta|$ ( $f$ is the spatial density of the number of the corona stars in the OSC model 1)

coordinates of this star and the five cluster stars closest to this one. The same estimates of the phase density of the cluster corona were obtained by Danilov et al. (2014) for the vicinity of the corona stars. Fig. 15.6 shows the logarithms of the mean values of $F$ as a function of the specific energies $\varepsilon$ of the stars for the neighbourhoods of which the values $F$ were obtained, for two time points $t_{1}=2.5 \tau_{v r}$ and $t_{2}=3 \tau_{v r}$. The vertical bars in fig. 15.6 indicate the errors of the logarithms of the mean $F$ values; the numbers 1 and 2 indicate the curves corresponding to the time points $t_{1}$ and $t_{2}$. According to fig. 15.6, the curves of the dependencies of $\ln (F)$ on $\varepsilon$ for the time points $t_{1}$ and $t_{2}$ are close to each other in a wide range of the $\varepsilon$ values both for the cluster (fig. 15.6a), and its corona (fig. 15.6b).

Thus, despite the indications of a dynamical instability of the corona (small densities in comparison with the critical one, $r_{2} \simeq 4 R_{t}$, and an accelerated expansion of the corona), in the interval of the distances from the center $r / R_{t} \in[1,3]$, we can note the formation of the density and the phase density distributions close to an equilibrium in the OSC models 1-6. This temporal equilibrium of the corona is due to the balance between the number of stars that come to the corona from the central regions of the cluster and escape to the corona periphery or beyond it.

Fig 15.7a shows the dependencies of the phase density logarithms for the corona and the cluster on $\varepsilon$. The upper broken line corresponds to the phase density function of the cluster, the lower one corresponds to the cluster corona PDF. The dot-and-dash 

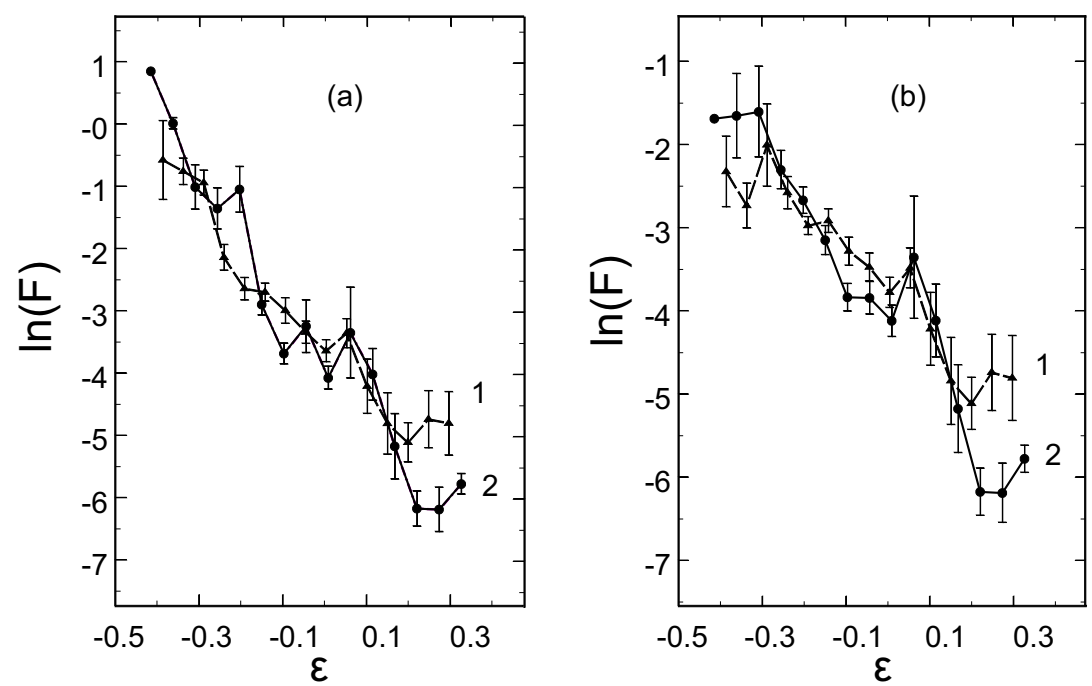

Fig. 15.6 The dependencies of $\ln (F)$ on $\varepsilon$ for the OSC model 1. $F$ is the phase density of the number of stars in the cluster $(a)$ and the cluster corona $(b)$. Numbers 1 and 2 indicate the curves for the time points $t=2.5 \tau_{v r}$ and $t=3 \tau_{v r}$, respectively
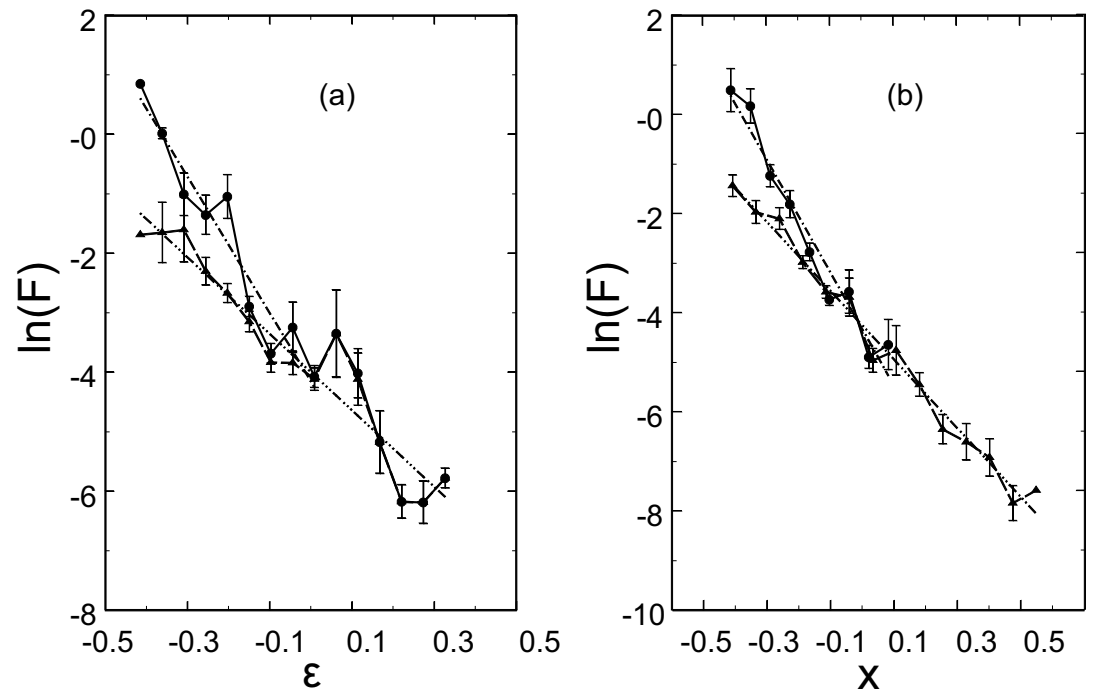

Fig. 15.7 The dependencies of $\ln (F)$ on $\varepsilon$ and $x$ at $t=3 \tau_{v r}$ ( $F$ is the phase density of the number of stars in the OSC model 1) 
Table 15.1 The values $\beta_{k}$ at $k=1,2,3$ and $C=-1 / \beta_{4}$ in the OSC models at $t=3 \tau_{v r}$

\begin{tabular}{|c|c|c|c|c|c|}
\hline $\begin{array}{c}\text { № } \\
\text { of the model }\end{array}$ & Object & $\beta_{1}$ & $\begin{array}{c}\beta_{2} \\
(\mathrm{Myr} / \mathrm{pc})^{2}\end{array}$ & $\begin{array}{c}\beta_{3} \\
(1 / \mathrm{Myr})\end{array}$ & $C$ \\
\hline 1 & Cluster & $-4.94 \pm 0.12$ & $-9.98 \pm 0.60$ & $0.013 \pm 0.001$ & $2.59 \pm 0.33$ \\
& Corona & $-4.85 \pm 0.11$ & $-7.63 \pm 0.54$ & $0.017 \pm 0.002$ & $3.12 \pm 0.56$ \\
\hline 2 & Cluster & $-4.92 \pm 0.11$ & $-8.87 \pm 0.47$ & $0.012 \pm 0.001$ & $4.65 \pm 0.81$ \\
& Corona & $-4.83 \pm 0.10$ & $-7.34 \pm 0.43$ & $0.015 \pm 0.002$ & $7.10 \pm 2.13$ \\
\hline 3 & Cluster & $-5.73 \pm 0.15$ & $-10.10 \pm 0.57$ & $0.019 \pm 0.002$ & $3.26 \pm 0.36$ \\
& Corona & $-5.21 \pm 0.13$ & $-5.81 \pm 0.52$ & $0.031 \pm 0.004$ & $4.89 \pm 1.33$ \\
\hline 4 & Cluster & $-4.67 \pm 0.12$ & $-7.52 \pm 0.43$ & $0.030 \pm 0.002$ & $6.10 \pm 1.66$ \\
& Corona & $-4.19 \pm 0.12$ & $-4.79 \pm 0.42$ & $0.044 \pm 0.005$ & $68.4 \pm 333.9$ \\
& Cluster & $-4.43 \pm 0.10$ & $-7.07 \pm 0.41$ & $0.032 \pm 0.003$ & $\infty$ \\
& Corona & $-4.18 \pm 0.10$ & $-4.76 \pm 0.40$ & $0.044 \pm 0.005$ & $\infty$ \\
\hline 5 & Cluster & $-5.62 \pm 0.14$ & $-10.13 \pm 0.50$ & $0.021 \pm 0.002$ & $3.77 \pm 0.55$ \\
& Corona & $-5.35 \pm 0.12$ & $-6.56 \pm 0.42$ & $0.031 \pm 0.003$ & $10.61 \pm 5.66$ \\
& Cluster & $-5.11 \pm 0.13$ & $-9.27 \pm 0.53$ & $0.024 \pm 0.002$ & $\infty$ \\
& Corona & $-5.23 \pm 0.10$ & $-6.37 \pm 0.41$ & $0.032 \pm 0.003$ & $\infty$ \\
\hline 6 & Cluster & $-5.05 \pm 0.15$ & $-9.53 \pm 0.45$ & $0.029 \pm 0.002$ & $5.35 \pm 0.93$ \\
& Corona & $-4.84 \pm 0.15$ & $-7.86 \pm 0.46$ & $0.033 \pm 0.003$ & $10.23 \pm 4.27$ \\
& Cluster & $-4.60 \pm 0.13$ & $-8.68 \pm 0.44$ & $0.032 \pm 0.003$ & $\infty$ \\
& Corona & $-4.65 \pm 0.13$ & $-7.50 \pm 0.43$ & $0.035 \pm 0.003$ & $\infty$ \\
\hline
\end{tabular}

lines show a linear regression for each of the broken lines. According to fig. 15.7a, the curves of the $\ln (F)$ dependencies on $\varepsilon$ for the cluster and the corona approach each other with an increasing $\varepsilon$ and practically coincide at $\varepsilon>0$. The distribution of the stellar velocities in the case of the PDF shown in fig. 15.7a is spherically symmetric.

Let us consider the function $\ln (F)$ in the following form:

$$
\ln (F)=\beta_{1}+\beta_{2} \cdot x, \quad x=\varepsilon+\beta_{3} \cdot l_{\zeta}+\beta_{4} \cdot \dot{\zeta}^{2},
$$

where $\beta_{i}$ are the constant values $(i=1, \ldots, 4)$;

$$
l_{\zeta}=\xi \dot{\eta}-\eta \dot{\xi}+\omega\left(\xi^{2}+\eta^{2}\right)
$$

is the specific angular momentum of a stellar motion relative to the $\zeta$ axis.

Such a model for the PDF of the cluster was obtained by Danilov (2000) in the collision-less approach with the maximisation of the cluster entropy and with some constraints (a constancy of the mass, the energy, and the angular momentum with respect to $\zeta$ axis and of the kinetic energy of the motion of the cluster stars along $\zeta$ coordinate in the process of a dynamic evolution of the OSC in the gravitational field of the Galaxy). Fig. 15.7b shows the dependencies of the logarithms of the cluster and the corona phase density on $x$ for the OSC model 1 at $t=3 \tau_{v r}$. The coefficients $\beta_{i}$ were determined by the Marquardt method (Marquardt, 1963) approximating the value of $\ln (F)$ by the function (15.1). Table 15.1 gives the values of $\beta_{k}$ at $k=1,2,3$ 
and $C=-1 / \beta_{4}$ for the OSC models $1-6$ at $t=3 \tau_{v r}\left(\beta_{2}\right.$ are given in $(\mathrm{Myr} / \mathrm{pc})^{2}, \beta_{3}$ in (1/Myr), $\beta_{1}, \beta_{4}$ and $C$ are dimensionless values). According to Table 15.1, the function (15.1) describes well enough the structure of the cluster and the corona of the OSC models $1-3$ in the phase space, and also the cluster structure in models 4-6 (the error of the coefficients $\beta_{k}$ and $C$ in these cases are small in comparison with the values of these coefficients). However, when describing the structure of the corona of the OSC models 4-6, the values of $C$ and their errors increase noticeably (especially in model 4), which can be caused either by the spherisation of the velocity distribution of the corona stars, or by the non-conservation with time of the total kinetic energy $T_{\zeta}$ of the corona stars motion along $\zeta$-coordinate in these models (Danilov, 2000). Therefore, for the OSC models 4-6, we also used the approximation of $\ln (F)$ by the function (15.1) with $\beta_{4}=0$ (in this case, $C=\infty$ ). The corresponding values $\beta_{i}$ are also listed in Table 15.1. According to Table 15.1, at $\beta_{4}=0$, other coefficients $\beta_{k}$ $(k=1,2,3)$ for the OSC models $4-6$ change a little in a comparison with the case $\beta_{4} \neq 0$ and, apparently, depend weakly on $\beta_{4}$. The values of $\beta_{k}$ and $C$ for the cluster $\left(\beta_{k}^{(c)}\right.$ and $\left.C^{(c)}\right)$ and its corona $\left(\beta_{k}^{(h)}\right.$ and $\left.C^{(h)}\right)$ satisfy the following inequalities: $\left|\beta_{1}^{(c)}\right| \gtrsim\left|\beta_{1}^{(h)}\right|,\left|\beta_{2}^{(c)}\right|>\left|\beta_{2}^{(h)}\right|, \beta_{3}^{(c)}<\beta_{3}^{(h)}, C^{(c)}<C^{(h)}$. The numerical values of $\beta_{k}^{(c)} / \beta_{k}^{(h)}$ and $C^{(c)} / C^{(h)}$ for the OSC models can be obtained easily from Table 15.1 and used as approximate values of $\beta_{k}^{(c)} / \beta_{k}^{(h)}$ and $C^{(c)} / C^{(h)}$ for construction of the phase OSC's models (15.1) by the data on the phase coordinates of stars obtained from observations. Here, we should note that the regression lines of the $\ln (F)$ dependencies on $\varepsilon$ for the corona and the cluster of the OSC models 1-6 usually intersect near the value of $\varepsilon \simeq 0$, and for the $\ln (F)$ dependencies on $x$ they intersect near $x \simeq 0$ (for model 1 , see fig. 15.7), which can also be used in the construction of the phase OSC models from the observational data.

There are several ways to determine the coefficients $\beta_{i}(i=1, \ldots, 4)$ for the PDF of the observed OSC:

- 1) if the data on the phase coordinates of a sufficiently large number of cluster stars $(N \simeq 100-1000)$ are known, in order to estimate $\ln (F)$ in the vicinity of these stars, one can find the coefficients $\beta_{i}$ as in this work (by approximating the data on $\ln (F)$ by the functions (15.1));

- 2) an integration of the $F(x)$ function by the stellar velocities makes it possible to obtain a formula for the spatial density distribution of the number of stars that depends on the coefficients $\beta_{i}$. After the transition from the spatial densities to the apparent one, the coefficients $\beta_{i}$ can be found by an approximation of the distributions of the apparent densities obtained from the observational data by the theoretical distributions of apparent densities obtained using (15.1);

- 3) an integration of the $F(x)$ function by the stellar coordinates makes it possible to obtain a formula for the stellar velocity distribution that depends on the coefficients $\beta_{i}$. After the transition from the spatial stellar velocities to the radial velocities and the proper motions of stars, and after the approximation of the radial velocity and the proper motion distributions obtained from the observational data by the theoretical ones according to (15.1), one can find the coefficients $\beta_{i}$. 
We should note that the OSC models 5, 6 have the most massive and extended cores at $t=0$, and model 4 is the densest among the models considered here. The general velocity distribution of corona stars in models $4-6$ at $t=3 \tau_{v r}$ is somewhat closer to spherical one than in the models 1-3. Let $d_{1}=\sigma_{v_{\zeta}} / \sigma_{v_{\xi}}$ and $d_{2}=\sigma_{v_{\zeta}} / \sigma_{v_{\eta}}$, where $\sigma_{v_{\xi}}, \sigma_{v_{\eta}}, \sigma_{v_{\zeta}}$ are the sample standard deviations of the velocities $v_{\xi}, v_{\eta}, v_{\zeta}$ of the corona stars from their mean values, respectively. By the time $t=3 \tau_{v r}$, the values of $d_{i}(i=1,2)$ in models $4-6$ are the following: $d_{1} \simeq 1.12-1.17, d_{2} \simeq 0.95-1.15$. In the case of models $1-3$, we find $d_{1} \simeq 1.19-1.29, d_{2} \simeq 0.90-1.05$, see Table 15.2. Danilov and Dorogavtseva (2008) also detected in models 1-6 a tendency to the formation of a spherical distribution of the stellar velocities; at $R_{c}<r \leq R_{t}$, they obtained the estimates of the spherisation time of the stellar velocity distributions for the cluster halos $t_{\sigma} \simeq(9-25) \tau_{v r}$, see Table 3 from Danilov and Dorogavtseva (2008). The radius of the core $R_{c}$ was determined by Danilov and Dorogavtseva (2008) for each time point by the radial density profile of the cluster model (as the position of the outer by $r$ boundary of the region of the maximum modulus of the density gradient, $R_{c} \lesssim 0.5 R_{t}$ ). According to Table 15.1 , the $\beta_{3}$ value is positive, and is much less than unity for all models. In addition, the $|\varepsilon|$ and $\left|\beta_{3} l_{\zeta}\right|$ values for the corona stars are basically comparable to each other. It indicates a comparable contribution of the angular momenta $\zeta$ and the energy $\varepsilon$ to the value $x$ from (15.1) at $t=3 \tau_{v r}$. Apparently, in the OSC models 1-6, there is a gradual transition from the dependency $F=F(x)$ to $F=F(\varepsilon)$ (at $t=3 \tau_{v r}$ it is more noticeable in the coronas of the OSC models 4-6).

We note that the distribution of the stellar velocities in the case of the PDF $F=F(x)$ to a greater extent corresponds to that observed in the numerical dynamic OSC models of Danilov and Dorogavtseva (2008), than in the case of the PDF $F=F(\varepsilon)$ (for model 1, see fig. 15.7). The corona stars in the OSC models 1-6 at $t=3 \tau_{v r}$ are characterised by the motions from the cluster center along the $\xi$ axis near the points $\xi= \pm R_{t}, \eta=\zeta=0$, as well as by the stellar motions corresponding to the rotation of the corona in the direction opposite to the motion of the cluster relative to the Galactic center. Such motions are most noticeable for the corona stars located near the $\eta$ axis in the vicinity of points $\xi=\zeta=0, \eta= \pm 0.5 R_{t}$, and gradually decrease with a distance from the cluster center along $\eta$ axis. The average velocities of such motions are $\sim(0.1-0.2) \mathrm{pc} / \mathrm{Myr}$. The sample standard deviations of the velocities of the corona stars from their mean values are $\sim(0.2-0.3) \mathrm{pc} / \mathrm{Myr}$, and slightly change (decrease) with a distance from the cluster center. The velocity distributions of the corona stars near the cluster center have a symmetry close to circular in the plane $\left(v_{\xi}, v_{\eta}\right)$ and are stretched along the $v_{\zeta}$ axis $\left(d_{i} \simeq 1.26-1.48\right)$. The values $d_{i}$ tend to unity with a distance from the cluster center along the $\xi$ axis. For example, for the corona of model 1 in the vicinity of points $\xi= \pm R_{t}, \eta=\zeta=0$, we find $d_{i} \simeq 1.03-1.08(i=1,2)$. When moving away from the cluster center along the $\eta$ axis, $d_{1}$ is slightly changed and $d_{2}$ decreases reaching the values of $d_{2}=1.06 \pm 0.10$ at $\xi=\zeta=0, \eta= \pm R_{t}$ in model 1 .

We can ascribe the presence of a large number of the corona stars with the retrograde mean motion close to the periodic one in the Galactic plane $(\xi, \eta)$ to the number of signs of the gravitational coupling of the OSC corona stars up to the 

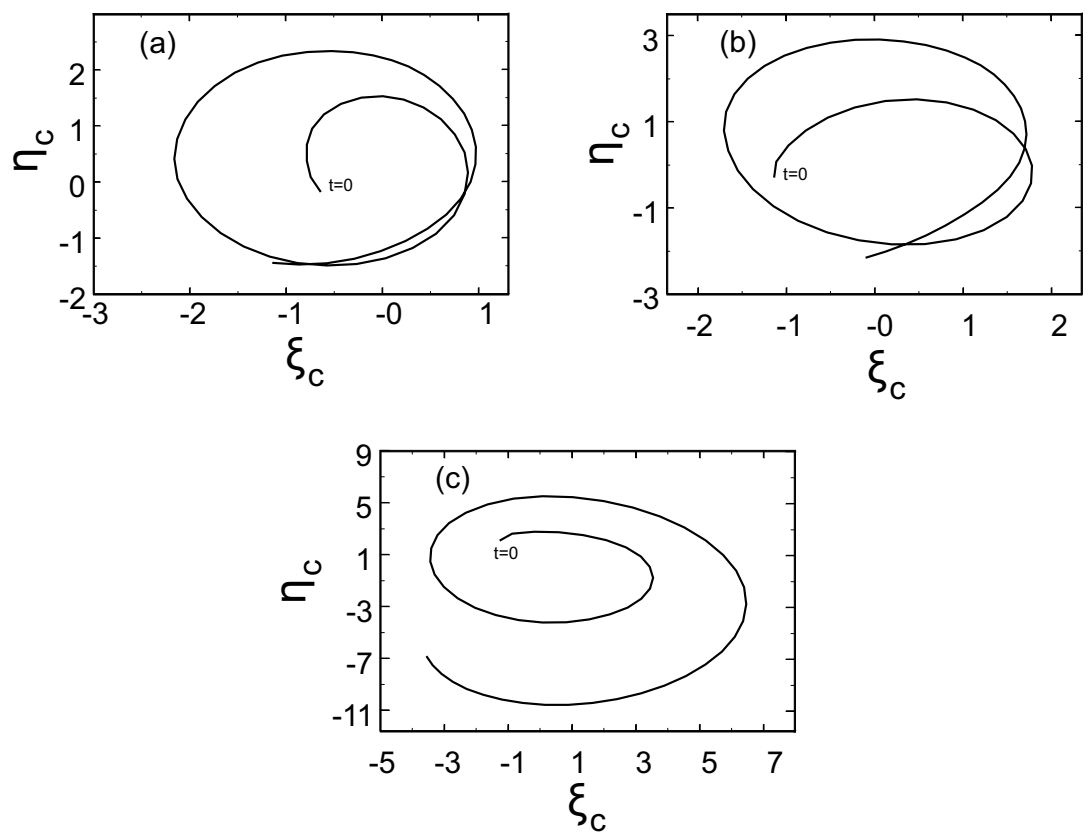

Fig. 15.8 The average trajectories of three groups of the corona stars in the OSC model 1: (a) $N_{s}=36, r \in[3.5,30] \mathrm{pc}$; (b) $N_{s}=27, r \in[4,40] \mathrm{pc}$; (c) $N_{s}=6, r \in[5,40] \mathrm{pc}$

distances of $\sim 4 R_{t}$ from the cluster center. Fig. 15.8 shows the average trajectories of three groups of the corona stars for model 1: a) $\left.N_{s}=36, r \in[3.5,30] \mathrm{pc} ; \mathrm{b}\right)$ $\left.N_{s}=27, r \in[4,40] \mathrm{pc} ; \mathrm{c}\right) N_{s}=6, r \in[5,40] \mathrm{pc}$, where $N_{s}$ is the number of stars in the group. $\xi_{c}$ and $\eta_{c}$ are the average coordinates $\xi$ and $\eta$ of stars in the groups in parsecs. It is clearly seen that the retrograde stellar motions dominate in the corona, in agreement with the results of the studies of the trajectories of the cluster stars by Keenan et al. (1973); Jefferys (1976); Keenan (1981a,b); Takahashi and Baumgardt (2012); Ross et al. (1997).

Danilov et al. (2014) used the conditions of the dissipation of stars found by Ross et al. (1997) for the analysis of the gravitational coupling of the corona of the OSC models $1-6$ on the time intervals $\tau$. Using these conditions, the cluster mass for each star at the time $t$ was assumed equal to the total mass of all stars located at distances $r$, which are smaller than the distance of the given star from the cluster center. For an every star, the time interval $\Delta t$ was calculated during which the conditions for the dissipation of a star from the cluster (Ross et al., 1997) are satisfied (they usually are satisfied for the large $t$ at the end of the considered interval of the dynamic cluster evolution). For the OSC models $1-6$, the mean value of $\Delta t / \tau$ for the corona stars is in the range from $\overline{\Delta t} / \tau=0.0004 \pm 0.0003$ in model 4 to $\overline{\Delta t} / \tau=0.0017 \pm 0.0006$ in model 1. For the $e$-stars moving away in time $\tau$ beyond the distance $r>r_{2}$ from the cluster center, in models $1-6, \overline{\Delta t} / \tau$ is in the range from $\overline{\Delta t} / \tau=0.0933 \pm 0.0251 \mathrm{in}$ 
Table 15.2 The values $N_{h}, N_{3}, N_{5}, \kappa, d, d_{1}$ and $d_{2}$ in the OSC models

\begin{tabular}{|c|c|c|c|c|c|c|c|}
\hline $\begin{array}{c}\text { № } \\
\text { model }\end{array}$ & $\begin{array}{c}N_{h}, \\
t \leq 3 \tau_{v r}\end{array}$ & $\begin{array}{c}N_{3}, \\
t \leq 3 \tau_{v r}\end{array}$ & $\begin{array}{c}N_{5}, \\
t \leq 5 \tau_{v r}\end{array}$ & $\begin{array}{c}\kappa, \\
t / \tau_{v r} \in[3,5]\end{array}$ & $\begin{array}{c}d, \\
\%\end{array}$ & $\begin{array}{c}d_{1}, \\
t=3 \tau_{v r}\end{array}$ & $\begin{array}{c}d_{2}, \\
t=3 \tau_{v r}\end{array}$ \\
\hline 1 & 307 & $11(0)$ & $142(132)$ & $0.23(0.21)$ & 96.4 & $1.19 \pm 0.02$ & $0.90 \pm 0.02$ \\
\hline 2 & 357 & $31(0)$ & $99(90)$ & $0.14(0.13)$ & 91.3 & $1.29 \pm 0.12$ & $0.94 \pm 0.10$ \\
\hline 3 & 291 & $15(0)$ & $47(43)$ & $0.10(0.09)$ & 94.8 & $1.25 \pm 0.06$ & $1.05 \pm 0.06$ \\
\hline 4 & 374 & $5(0)$ & $44(41)$ & $0.06(0.05)$ & 98.7 & $1.12 \pm 0.04$ & $0.95 \pm 0.01$ \\
\hline 5 & 245 & $7(0)$ & $26(23)$ & $0.075(0.066)$ & 97.1 & $1.15 \pm 0.01$ & $1.00 \pm 0.14$ \\
\hline 6 & 369 & $5(0)$ & $15(14)$ & $0.026(0.025)$ & 98.6 & $1.17 \pm 0.14$ & $1.15 \pm 0.13$ \\
\hline
\end{tabular}

model 4 to $\overline{\Delta / \tau}=0.283 \pm 0.015$ in model 1 . Thus, $\overline{\Delta t} / \tau$ increases sharply with the transition from the corona stars to the $e$-stars, which can be considered as one of the arguments in favor of the gravitational coupling of the coronas of the OSC models $1-6$.

Table 15.2 lists for OSC models 1-6 the following parameters: $N_{h}$ is the number of stars in the corona; $N_{3}$ and $N_{5}$ are the numbers of the corona stars satisfying the conditions of Ross et al. (1997) of a dissipation from the cluster at $t \leq 3 \tau_{v r}$ and $t \leq 5 \tau_{v r}$, respectively. The values of $N_{3}$ and $N_{5}$ indicated in the parentheses correspond to the stars that escape to a distance $r>r_{2}$ from the cluster center at $t \leq 3 \tau_{v r}$ and $t \leq 5 \tau_{v r}$, respectively. Let $q=N_{5} / N_{h}$ be a fraction of the dissipating corona stars in time $2 \tau_{v r}$ at $t / \tau_{v r} \in[3,5]$ (since the number of the corona stars with distances $r<r_{2}$ from the cluster center is $N_{h}$ at $t=3 \tau_{v r}$ ). Then we can be regard $\dot{N}_{h} \simeq-\kappa N_{h} / \tau_{v r}$ as an estimate of the average rate of the dissipation of stars from the corona at $t / \tau_{v r} \in[3,5]$, where $\kappa=q / 2$. Table 15.2 lists the value of $\kappa$ for the coronas of the OSC models 1-6, obtained using the criterion of Ross et al. (1997). The values $\kappa$ indicated in parentheses are determined by the stars that escape to a distance $r>r_{2}$ from the cluster center at $t / \tau_{v r} \in[3,5]$. According to Table 15.2, the values $N_{5}$ and $\kappa$ obtained using the criterion of a gravitational coupling of stars (Ross et al., 1997) and the criterion $\left(r<r_{2}\right.$ at $\left.t<\tau\right)$ considered in our work, are in a good agreement with each other. According to Table 15.2, the values of $N_{5}$ and $\kappa$ decrease with an increasing number of the cluster model and with a decrease of the degree of the model's non-stationarity. Consequently, the stability of the corona of the OSC models 1-6 to the decay in the Galactic force field increases with an increasing number of the cluster model and a decreasing degree of its non-stationarity. We note that the same dependency was obtained for these cluster models by Danilov and Dorogavtseva (2008) (the number $n$ of the stars with the distances $r_{n}<R_{t}$ from the cluster center by the moment $t \simeq \tau$ increases with the growing number of the model, see Table 3 from Danilov and Dorogavtseva (2008)).

According to Table 15.2, the estimates of the dissipation rate of the corona stars range from 0.03 to 0.23 of the number of corona stars during the cluster's violent relaxation time at $t / \tau_{v r} \in[3,5]$. The value of $d=\left(N_{h}-N_{3}\right) / N_{h}$ (the proportion of corona stars that are gravitationally related to the cluster according to the criterion 
of Ross et al. (1997) at $t \leq \tau$ ) in percent for models $1-6$ is also given in Table 15.2. According to this table, the value $d \simeq(91-99) \%$ and slightly changes from one model to another, which also confirms the gravitational coupling of the corona of the OSC models considered in this work. The reason for the slow disintegration of the cluster coronas at $r>R_{t}$ and $t \in[0, \tau]$ can be an existence in the clusters of conditions for the formation of a large number of periodic orbits and the stellar trajectories close to the periodic ones with the retrograde motion and the long periods $P$ of a revolution around the cluster center $P \gtrsim \tau$ at the energies $\varepsilon \gtrsim 0$.

In the case of the two-point OSC model (Danilov and Chernova, 2008), such periodic orbits can be easily detected. In this model, one point corresponds to a star with the mass $m_{1}=1 m_{\odot}$, and the second point corresponds to the cluster with the mass $m_{2}=499 m_{\odot}$; the mass center of such a model moves along a circular orbit with the radius $R_{G}=8200 \mathrm{pc}$ around the Galactic center, as well as the OSC models $1-6$. The region of the possible stellar motions in the phase space for the model of Danilov and Chernova (2008) is limited by the Jacobi integral $(\varepsilon=$ const $)$ and, apparently, by an additional quasi-integral that ensures the existence of such orbits (see also Jefferys (1976)). Fig. 15.9 shows three periodic orbits of stars with the specific energies (per unit of the star mass) $\varepsilon=0.1$ (pc/Myr) ${ }^{2}$ (this is much greater than the critical energy $\varepsilon_{t}$, which for the model of Danilov and Chernova (2008) is $\left.\varepsilon_{t} \simeq-0.3236327\right)$. The arrows in fig. 15.9 indicate the initial direction of the stellar motion; $\xi$ and $\eta$ values are given in fig. 15.9 in parsecs.
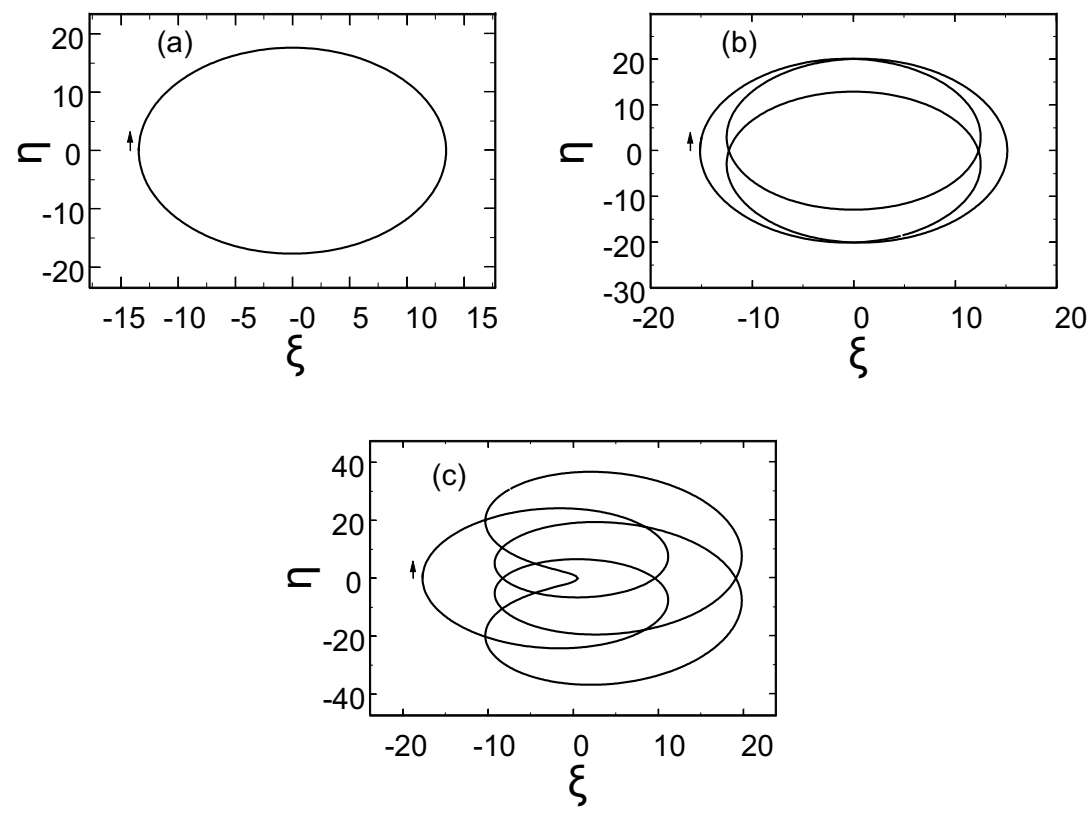

Fig. 15.9 Three periodic orbits of the star with $\varepsilon=0.1$ in the two-point OSC model: (a) $\xi_{0}=$ $-1.28635 R_{t} ;$ (b) $\xi_{0}=-1.448 R_{t}$; (c) $\xi_{0}=-1.6925398 R_{t}$ 
For all three orbits, the initial velocities $\left(\dot{\xi}_{0}, \dot{\eta}_{0}\right)$ of the stellar motion satisfy the following conditions: $\dot{\xi}_{0}=0, \dot{\eta}_{0}>0\left(\dot{\eta}_{0}\right.$ is obtained from the condition $\varepsilon=$ const, see Danilov and Chernova (2008)). The initial coordinates $\left(\xi_{0}, \eta_{0}\right)$ of the star are assumed by Danilov et al. (2014) equal: for the first orbit $\xi_{0}=-1.28635 R_{t}$, for the second orbit $\xi_{0}=-1.448 R_{t}$, and for the third orbit $\xi_{0}=-1.6925398 R_{t} ; \eta_{0}=0$ for all three orbits. The equations of a stellar motion in the model of Danilov and Chernova (2008) were integrated by the Runge-Kutta method of the 4th accuracy order; the maximum relative errors $\sigma_{\varepsilon}$ of $\varepsilon$ for orbits 1 and 2 did not exceed $\sigma_{\varepsilon}=0.19 \times 10^{-12}$, and for the third orbit $\sigma_{\varepsilon}=0.46 \times 10^{-7}$. Such error for orbit 3 was realized only near one point corresponding to the closest encounter of the masses $m_{1}$ and $m_{2}$ (fig. 15.9), the characteristic values of $\sigma_{\varepsilon}$ for third orbit are $\sigma_{\varepsilon}=(0.1-0.6) \times 10^{-12}$.

Thus, according to Danilov et al. (2014), the periodic orbits at the distances $r>R_{t}$ form the mass center of the cluster do exist; the same was noted by Ross et al. (1997). The conditions of a dissipation of stars (Ross et al., 1997) on orbits 1-3 are not fulfilled (the star in these orbits can be considered gravitationally related in the model of Danilov and Chernova (2008)).

The period of the first orbit is $P=120.9105662 \mathrm{Myr}$ (the Lyapunov time $t_{\lambda}=\lambda^{-1}$ for this orbit $t_{\lambda}=132.079 \mathrm{Myr}$ ); for the second orbit, $P=361.6663869 \mathrm{Myr}$ $\left(t_{\lambda}=355.379 \mathrm{Myr}\right)$; for the third orbit, $P=558.1218966 \mathrm{Myr}\left(t_{\lambda}=70.952 \mathrm{Myr}\right)$; $\lambda$ is the maximum Lyapunov characteristic exponent. The $\lambda$ values were obtained by Danilov et al. (2014) within the framework of the Floquet theory from the data on the periods $P$ of the orbits and on the corresponding to these orbits multipliers of the monodromy matrix (see formula (2.22) from Yakubovich and Starzhinsky (1972, p. 87) and Demidovich (1967, p.189)) of a system of the linearized equations in variations written by Danilov and Chernova (2008) for the equations of a stellar motion in the joint force field of the cluster and the Galaxy. $P$ and $t_{\lambda}$ for the orbits shown in fig. 15.9 are quite comparable with $\tau$ for OSCs. In the vicinity of these orbits, a large number of the non-closed trajectories quite may exist with a retrograde motion and with the $t_{\lambda}$ values close to those found here. The time interval of a stellar motion with such trajectories near the cluster quite may be comparable with the periods $P$ for the periodic orbits found here. In the OSC models 1-6, the impact of the stars with such trajectories on the cluster can be significant. Such stars forming the OSC corona can influence the stability of the cluster, increasing the time of its decay in the Galactic force field.

\subsection{Conclusions}

In this chapter, we have discussed the following main results on the structure and the dynamic evolution of the models of the OSC coronas.

1. We have considered the method for the corona separation in the OSC models from the data on the stellar trajectories in these models in the life-time intervals $\tau$ of such clusters. The cluster corona includes the stars satisfying the condition $r<r_{2}$ at $t \leq \tau$; the corona does not contain the stars of the cluster core, which satisfy the 
condition $r<r_{1}$ at $t \leq \tau$, where $r$ is the star's distance from the cluster center; $r_{1}$ and $r_{2}$ are the radii of the core and the corona, respectively (in our calculations, $r_{1} \simeq 0.5 R_{t}$ and $r_{2} \simeq 4 R_{t}$ ).

2. For six numerical OSC models of Danilov and Dorogavtseva (2008), the corona models have been presented; the parameters of the coronas, the direction and a nature of their dynamic evolution have been discussed. The retrograde stellar motions prevail in the corona, which is quite in an agreement with the results of studies on the cluster stars' trajectories of Keenan et al. (1973); Jefferys (1976); Keenan (1981a,b); Takahashi and Baumgardt (2012); Ross et al. (1997).

3 . In the interval of the distances from the center $r / R_{t} \in[1,3]$ in the OSC models, we have found that the close-to-equilibrium distributions of the density and the phase density are formed. We have considered the approximations of the phase density of the corona and the cluster by distributions that depend on the linear combination of an energy, an angular momentum with respect to the $\zeta$ axis, and a kinetic energy in the $\zeta$ coordinate. The temporal equilibrium of the corona is due to the balance of the number of stars coming into the corona from the central regions of the cluster and escaping to the corona periphery or beyond it.

4. We have detected the signs of the gravitational coupling of the corona stars up to the distances $\sim 4 R_{t}$ from the cluster center (the presence of a large number of the corona stars with the retrograde mean motion close to the periodic one in the Galactic plane $(\xi, \eta) ;(91-99) \%$ of the corona stars at the time intervals $\tau$ satisfy the criterion of the gravitational coupling of Ross et al. (1997)).

5. For six OSC models, we have considered the estimates of the corona stars' dissipation rate: $\dot{N}_{h}=-(0.03-0.23) N_{h} / \tau_{v r}$ at $t>\tau$. The estimates of $\dot{N}_{h}$ obtained by Danilov et al. (2014) using the conditions for the dissipation of a star from the cluster of Ross et al. (1997) and the conditions for the star to belong to the corona from point 1 (see above), are in a good agreement with each other.

6. For the two-point OSC model of Danilov and Chernova (2008), we have considered three unstable periodic orbits of the stars with the energies $\varepsilon=0.1(\mathrm{pc} / \mathrm{Myr})^{2}>$ 0 , the periods $P$ and the Lyapunov times $t_{\lambda}$ comparable to the lifetime of the OSC $\tau$. The formation of such orbits and a large number of the retrograde non-closed stellar trajectories close to this orbits in the OSCs and in the OSC models of Danilov and Dorogavtseva (2008) may well be the reason for the formation of the coronas in such systems.

\section{References}

Aarseth, S.J.: Computer simulations of star cluster dynamics. Vistas in Astronomy. 15, 13-37 (1973). doi:10.1016/0083-6656(73)90003-2

Adams, J.D., Stauffer, J.R., Monet, D.G., et al.: The Mass and Structure of the Pleiades Star Cluster from 2MASS. Astron. J. 121, 2053-2064 (2001). doi:10.1086/319965 
Adams, J.D., Stauffer, J.R., Skrutskie, M.F., et al.: Structure of the Praesepe Star Cluster. Astron. Journ. 124, 1570-1584 (2002). doi:10.1086/342016

Allen, A.J., Richstone, D.O.: Tidal limitation of stellar systems on both circular and elongated orbits. Astrophys. J. 325, 583-595 (1988). doi:10.1086/166029

Angeletti, L., Capuzzo-Dolcetta, R., Giannone, P.: Stability of star clusters as galactic satellites. I - Motion in the cluster orbital plane. Astron. and Astrophys. 121, 183187 (1983).

Angeletti, L., Capuzzo-Dolcetta, R., Giannone, P.: Stability of star clusters as galactic satellites. IV Logarithmic galactic potential. Astron. and Astrophys. 138, 404-407 (1984).

Angeletti, L., Giannone, P.: Stability of star clusters as galactic satellites. II - Motion perpendicular to the cluster orbital plane. Astron. and Astrophys. 121, 188-189 (1983).

Angeletti, L., Giannone, P.: Stability of star clusters as galactic satellites. III Preliminary applications to the system of the globular clusters. Astron. and Astrophys. 138, 396-403 (1984).

Balaguer-Nùñez, L., Jordi, C., Muiños, J. L., et al.: Studies on the corona of open clusters. In: Guirado, J.C., Lara, L.M., Quilis, V., and Gorgas, J. (eds.) High. Span. Astrophys VII. Proc. X Sci. Meet. Span. Astron. Soc. (2013) 644-644.

Bok, B.J.: The Stability of Moving Clusters. Harvard Observ. Circ. 384, 1-41 (1934).

Chandrasekhar, S.: Principles of Stellar Dynamics. Univ. Chicago Press, Chicago, Ill (1942). LCCN: 43-5608 (BKS1)

Chen, W.P., Chen, C.W., Shu, C.G.: Morphology of Galactic Open Clusters. Astron. J. 128, 2306-2315 (2004). doi:10.1086/424855

Chumak, Y.O., Platais, I., McLaughlin, D.E., et al.: Numerical simulations of tidal tails for the open cluster NGC 188. Mon. Not. R. Astron. Soc. 402, 1841-1853 (2010). doi:10.1111/j.1365-2966.2009.16021.x

Danilov, V.M.: Equilibrium Phase-Space Density Distribution in Numerical Dynamical Models of Open Clusters. Astron. Reports 44, 298-308 (2000). doi:10.1134/1.163853

Danilov, V.M., Chernova, O.A.: Analysis of stellar trajectories in an open cluster model. Astron. Reports 52, 27-39 (2008). doi:10.1134/S1063772908010046

Danilov, V.M., Dorogavtseva, L.V.: Timescales for mechanisms for the dynamical evolution of open star clusters. Astron. Reports 52, 467-478 (2008). doi:10.1134/S1063772908060048

Danilov, V.M., Matkin, N.V., Pylskaya, O.P.: Statistical Boundaries for Open Clusters. Soviet Astron. 29, 621-625 (1985).

Danilov, V.M., Putkov, S.I., Seleznev, A.F.: Dynamics of the coronas of open star clusters. Astron. Reports 58, 906-921 (2014). doi:10.1134/S1063772914120038

Davenport, J.R.A., Sandquist, E.L.: Death of a Cluster: The Destruction of M67 as Seen by the Sloan Digital Sky Survey. Astrophys. J. 711, 559-572 (2010). doi:10.1088/0004-637X/711/2/559

Demidovich, B.P.: Lectures on the mathematical theory of stability. Nauka, Moscow (1967) (In Russian). 
Fellhauer, M., Heggie, D.C.: An exact equilibrium model of an unbound stellar system in a tidal field. Astron. Astrophys. 435, 875-881 (2005). doi:10.1051/00046361:20042595

Fukushige, T., Heggie, D.C.: The time-scale of escape from star clusters. Mon. Not. R. Astron. Soc. 318, 753-761 (2000). doi:10.1046/j.1365-8711.2000.03811.x

von Hoerner S.: Internal structure of globular clusters. Astrophys. J. 125, 451-469 (1957). doi:10.1086/146321

Innanen, K.A.: The limiting radii of direct and retrograde satellite orbits, with applications to the solar system and to stellar systems. Astron. J. 84, 960-963 (1979). doi:10.1086/112499

Innanen, K.A., Harris, W.E., Webbink, R.F.: Globular cluster orbits and the galactic mass distribution. Astron. J. 88, 338-360 (1983). doi:10.1086/113320

Jefferys, W.H.: On the dynamics and shapes of star clusters. Astron. J. 81, 983-992 (1976). doi:10.1086/111979

Keenan, D.W.: Galactical tidal limits on star clusters. I - Stability of stellar orbits and the zero velocity surfaces. Astron. and Astrophys. 95, 334-339 (1981).

Keenan, D.W.: Galactical tidal limits on star clusters. II - Tidal radius and outer dynamical structure. Astron. and Astrophys. 95, 340-348 (1981).

Keenan, D.W., Innanen, K.A., House, F.C.: Galactic orbits and tidal radii of the Clusters M67, NGC 188 and W Centauri. Astron. and Astrophys. 78, 173-179 (1973).

Kharchenko, N.V., Berczik, P., Petrov, M.I., et al.: Shape parameters of Galactic open clusters. Astron. Astrophys. 495, 807-818 (2009). doi:10.1051/00046361/200810407

Kholopov, P.N.: Star clusters. Nauka, Moscow (1981) (In Russian).

King, I.R.: The structure of star clusters. I. An empirical density law. Astron. J. 67, 471-485 (1962). doi:10.1086/108756

Kutuzov, S.A., Osipkov, L.P.: A Generalized Model for the Three-Dimensional Gravitational Potential of Stellar Systems. Soviet Astron. 24, 17-22 (1980).

Marquardt, D.W.: An algoritm for least-squares estimation of nonlinear parameters. J. Soc. Indust. Appl. Math. 11, 431-441 (1963). doi:10.1137/0111030

Merritt, D.: Relaxation and tidal stripping in rich clusters of galaxies. II. Evolution of the luminosity distribution. Astrophys. J. 276, 26-37 (1984). doi:10.1086/161590

Mineur, H.: Équilibre des nuages galactiques et des amas ouverts dans la Voie Lactée. Évolution des amas. Ann. d'Astropys. 2, 1-244 (1939).

Nilakshi, Sagar, R., Pandey, A.K., et al.: A study of spatial structure of galactic open star clusters. Astron. and Astrophys. 383, 153-162 (2002). doi:10.1051/00046361:20011719

Odenkirchen, M., Grebel, E.K., Dehnen, W., et al.: The Extended Tails of Palomar 5: A $10^{\circ}$ Arc of Globular Cluster Tidal Debris. Astron. J. 126, 2385-2407 (2003). doi: $10.1086 / 378601$

Oort J.H.: Luminosity distribution and shape of the Hyades cluster. Astron. Astrophys. 78, 312-317 (1979).

Pels, G., Oort, J.H., Pels-Kluyver, H.A.: New members of the Hyades cluster and a discussion of its structure. Astron. and Astrophys. 43, 423-441 (1975). 
Raboud, D., Mermilliod, J.-C.: Investigation of the Pleiades cluster. IV. The radial structure. Astron. Astrophys. 329, 101-114 (1998).

Raboud, D., Mermilliod, J.-C.: Evolution of mass segregation in open clusters: some observational evidences. Astron. Astrophys. 333, 897-909 (1998).

Rein, N.F.: Kriticheskii obzor po teorii ustoichivosti meteornykh skoplenii i zvezdnykh kuch. Publications of the Sternberg State Astronomical Institute 9, 191-217 (1936). (In Russian).

Röser, S., Schilbach, E., Piskunov, A.E, et al.: A deep all-sky census of the Hyades. Astron. and Astrophys. 531, 92-106 (2011). doi:10.1051/0004-6361/201116948

Ross, D.J., Mennim, A., Heggie, D.C.: Escape from a tidally limited star cluster. Mon. Not. R. Astron. Soc. 284, 811-814 (1997). doi:10.1093/mnras/284.4.811

Silverman, B.W.: Density Estimation for Statistics and Data Analysis. Monographs on Statistics and Applied Probability. Chapman and Hall, London (1986). ISBN: 9780412246203

Stone, R.C.: A UBV photometric study of the open cluster NGC 654. Publ. Astron. Soc. Pacif. 92, 426-431 (1980). doi:10.1086/130690

Stone, R.C.: The inner and outer membership regions of the young open cluster NGC 6823. Astron. J. 96, 1389-1393 (1988). doi:10.1086/114888

Takahashi, K., Baumgardt, H.: Tidal mass loss in star clusters and treatment of escapers in Fokker-Planck models. Mon. Not. R. Astron. Soc. 420, 1799-1808 (2012). doi:10.1111/j.1365-2966.2011.20183.x

Terlevich, E.: Evolution of n-body clusters. Mon. Not. R. Astron. Soc. 224, 193-225 (1987). doi:10.1093/mnras/224.1.193

Van Leeuwen, F.: A proper motion study of the Pleiades cluster. In: Goodman J., Hut P. (eds.) Dynamics of star clusters. IAU Symposium No. 113, Reidel, Dordrecht (1985), 477-480.

Wielen, R.: Dissolution of star clusters in galaxies. In: Grindlay, J.E., Philip, A.G.D. (eds.), Globular Cluster Systems in Galaxies. IAU Symposium No. 126. Kluwer, Dordrecht (1987) 393-408.

Yakubovich, V.A., Starzhinsky, V.M.: Linear differential equations with periodical coefficients and its applications. Nauka, Moscow (1972) (In Russian). 



\section{Chapter 16 Appendix}

\subsection{Description of OSC Models}

Danilov and Dorogavtseva (2008) considered the cluster models consisting of $N=$ 500 stars, moving along a circular orbit of radius $R_{G}=8200 \mathrm{pc}$ in the plane of the Galaxy around its center. We used the model for the potential of the Galaxy of Kutuzov and Osipkov (1980), as well as the rotating coordinate system $(\xi, \eta, \zeta)$ with the origin at the mass center of the cluster; the axis $\xi$ is directed from the center of the Galaxy, the axis $\eta$ is aimed along the direction of the cluster motion, the axis $\zeta$ is perpendicular to the plane of the Galaxy. The masses of the stars $m_{i}$ were taken each equal to one solar mass $\left(m_{i}=1 m_{\odot}, i=1, \ldots, 500\right)$. At the initial point of time, the cluster was modeled as two homogeneous balls with the coinciding centers of mass, representing the core and the halo of the cluster (the initial halo radius $R_{2}<R_{t}, R_{t}$ is the tidal radius of the cluster model). The initial parameters $R_{1} / R_{2}$ and $N_{1} / N_{2}$ in all considered OSC models satisfy the relation $R_{1} / R_{2} \simeq 0.39 \times\left(N_{1} / N_{2}\right)^{0.35}$ obtained from the observational data by Danilov and Seleznev (1994), where $R_{1}$ and $R_{2}$ are radii of the cluster's core and halo; $N_{1}$ and $N_{2}$ are the numbers of stars of the cluster's core and halo.

The initial positions and velocities of the stars in the OSC models have been specified by a random number generator, so that at $t=0$, the cluster does not rotate with respect to the external galaxies, the initial density of the number of stars at different points of the subsystems (in the halo and in the core) of the cluster in the coordinate space $(\xi, \eta, \zeta)$ are approximately constant; the distribution of stellar velocities is spherically symmetric. The moduli of the initial velocities of the stars of the $\mathrm{j}$-th cluster subsystem were calculated by formulas $v_{j}=\sqrt{C_{j} U(\mathbf{r})}, \quad j=$ 1,2 , where $\mathbf{r}=(\xi, \eta, \zeta)$ is the radius vector of the star in the cluster; $U(\mathbf{r})$ is the gravitational potential of the cluster; indices $j=1,2$ correspond to the core and the halo of the cluster, respectively. The constants $C_{j}$ were chosen so that at $t=0$ the virial equilibrium conditions for the cluster and its subsystems were fulfilled without the taking into account the influence of the force field of the Galaxy (Danilov, 1988). The closest encounters of the stars were excluded from consideration. For this, the 
potential $U$ in the equations of motion of stars was considered in the form

$$
U(\mathbf{r})=\sum_{i=1}^{N} G m_{i} / \sqrt{\left(\mathbf{r}-\mathbf{r}_{i}\right)^{2}+\epsilon^{2}}
$$

(here, $\epsilon=0.012 \bar{r}_{i k}$ is the smoothing parameter, $r_{i k}$ is the distance between two stars with numbers $i$ and $k$ in the cluster, $\overline{r_{i k}}$ is the mean initial value of $r_{i k}$ for the cluster model averaged by all pairs of stars).

In the calculations, the following system of units was used: pc, Myr, $M_{\odot}$. The

Table 16.1 The parameters of the OSC models

\begin{tabular}{|c|c|c|c|c|c|c|}
\hline $\mathcal{N}$ & $R_{1} / R_{2}$ & $N_{1} / N_{2}$ & $N_{1}$ & $R_{2} / R_{t}$ & $\langle R\rangle / R_{t}$ & $\langle\delta \alpha / \alpha\rangle$ \\
\hline 1 & 0.24 & 0.25 & 100 & 0.9 & 0.57 & $0.53 \pm 0.09$ \\
\hline 2 & 0.24 & 0.25 & 100 & 0.8 & 0.51 & $0.28 \pm 0.03$ \\
\hline 3 & 0.34 & 0.67 & 200 & 0.8 & 0.44 & $0.15 \pm 0.02$ \\
\hline 4 & 0.24 & 0.25 & 100 & 0.7 & 0.45 & $0.14 \pm 0.02$ \\
\hline 5 & 0.45 & 1.50 & 300 & 0.8 & 0.40 & $0.07 \pm 0.03$ \\
\hline 6 & 0.63 & 4.00 & 400 & 0.8 & 0.42 & $0.06 \pm 0.03$ \\
\hline
\end{tabular}

initial parameters of these OSC models are given in Table 16.1 and Table 1 from Danilov and Dorogavtseva (2008). The OSC models 1-6 are numbered in Danilov and Dorogavtseva (2008) in the order of the decreasing non-stationarity of the model in a regular field. Models $1,2,4$ have the same parameter values $R_{1} / R_{2}$ and $N_{1} / N_{2}$, but the sequentially decreasing radii $R_{2}$. Models $2,3,5,6$ have the same values of $R_{2}$, but the sequentially increasing numbers of stars $N_{1}$ and core radii $R_{1}$. The values $R_{2}$ and the average distance of the star from the center of the model $<R>$ in Table 16.1 are given in the units of the tidal radius $R_{t}$ of the cluster model. The degree of a non-stationarity of the OSC models in a regular field is determined by the amplitude of the oscillations of the virial coefficient $\delta \alpha$, where the virial coefficient $\alpha=2 E / W ; E$ and $W$ are the total and the potential cluster energies obtained without taking into account the influence of the force field of the Galaxy on the cluster. The mean values of the ratio of the amplitudes $\delta \alpha$ of the $\alpha$ oscillations to the mean value of $\alpha$ (averaged over the period $P_{r}$ of the regular field oscillations) are given in column 7 of Table 16.1 .

The phase coordinates of the OSC model stars for the different time points were obtained by Danilov and Dorogavtseva (2008) by integrating the equations of the stellar motion using the difference schemes of the 10th and 11th accuracy orders on the time interval $t \in\left[0, t_{m}\right]$, where $t_{m} \simeq 5.1 \tau_{v r} . \tau_{v r}$ is the time of a violent relaxation of the cluster model. It was taken equal to $\tau_{v r} \simeq 2.6 \bar{t}_{c r}$ according to the estimates of Aarseth (1974). $\bar{t}_{c r}$ is the average crossing time. The maximum relative error in the computation of the cluster energy reached over the period of time $t_{m}$ in the models of Danilov and Dorogavtseva (2008), was modulo (1-4) $\times 10^{-13}$. Let $t_{0}$ be the 
time interval of the dynamic evolution of the OSC model, during which a statistical criterion for the accuracy of the phase density calculations is fulfilled (Danilov, 1997b). At $t \leq t_{0}$, the accuracy of calculating the phase density function (PDF) of the cluster over time interval $t_{0}$ can be considered sufficient for conclusions about the statistical properties of the PDF (Danilov, 1997b) (the empirical distribution functions for the phase coordinates of stars obtained with the 10th and 11th orders with the probability $P \geq 0.9$ by the Kolmogorov criterion are the samples from the same general population). Near the centers of the OSC models (Danilov and Dorogavtseva, 2008), the value of $t_{0} / \tau_{v r}$ is $t_{0} / \tau_{v r} \simeq 3.0-3.9$, and at the periphery of the models, $t_{0} / \tau_{v r} \simeq 3.6-5.1$. The specified values of $t_{0}$ were obtained in the calculations with $\sim 15-16$ decimals.

The initial PCS of the considered OSC models imitate the conditions close to those that arise in the remaining gravitationally bound part of the cluster in the Galaxy force field after the expansion of the initial cluster caused by the loss of the gas from the cluster-cloud system due to the light pressure and the stellar wind of the brightest O-B stars at the earlier stages of evolution (see, for example, Lada et al. (1984); Tutukov (1978)). Moreover, Danilov and Seleznev (1994) noted that the composite "halo-core" OSC model better describes the spatial distribution of stars in the OSC than, for example, the King model (especially at the periphery). This was also noted by Danilov and Putkov (2012a), who showed that the use of the King distribution on average leads to an underestimated number of stars in the cluster in $0.62 \pm 0.02$ times compared with the observations. In the modeling of the OSC dynamics, Danilov and Putkov (2012a) proposed to introduce a homogeneous sphere (halo) in addition to the King density distribution in order to take into account these stars.

According to the estimates of Danilov and Seleznev (1994), the decay time of the OSCs due to the interactions with the standard HI clouds is approximately $30-50$ times greater than the time $(3-5) \tau_{v r}$, at which the OSC models in this book are usually considered, see also Danilov et al. (2014). Thus, the standard HI clouds have a little effect on the dynamic evolution of the OSC models discussed here. According to the estimates of Danilov and Seleznev (1994), the decay time of the OSCs by the action of the giant molecular clouds (GMC) and the Galaxy is 3.4-5.6 times greater than (3-5) $\tau_{v r}$, see also Danilov et al. (2014). Therefore, the close encounters between the OSCs and GMC are quite rare and may well not occur at the time intervals $(3-5) \tau_{v r}$. This is indicated by the observational data of Kharchenko (2009) on the kinematic dimensions of the OSCs of the order of $(2-3) R_{t}$ and on the presence of the coronas in the vicinity of OSCs, see also chapter 15 of this book. Thus, an analysis of the dynamics of the OSCs and their models over time intervals $(3-5) \tau_{v r}$ quite can be carried out without taking into account the interactions between the OSCs and GMC. 


\subsection{Statistical comparison of OSC models}

In order to control the accuracy of the obtained solutions, as well as to analyze the stability of the statistical distributions of a number of characteristics obtained in the simulation of the dynamic evolution of OSCs, a detailed statistical comparison of the cluster models (Danilov, 1997c,b) can be used. In the process of an integrating the equations of the cluster stars' motion for the different time points, we can compare the versions $a$ and $b$ of the cluster model obtained by the schemes of the different accuracy orders. To do this, we divide all stars of the cluster model $a$ at the time point $t$ into 10 groups (50 stars each) in the order of the increasing distances from the cluster mass center. In this way, we set the dimensions of the phase cells in the coordinate space $(\xi, \eta, \zeta)$ and the corresponding intervals $\Delta r_{j}$, of the distances $r$ from the cluster mass center (the $\zeta$ axis is perpendicular to the plane of the Galaxy; the plane $(\xi, \eta)$ lies in the Galactic plane; $j=1, \ldots, 10)$.

We divide the region of the space $(\dot{\xi}, \dot{\eta}, \dot{\zeta})$ occupied by the stars of the $a$ and $b$ versions of the cluster models, by the cells (rectangular parallelepipeds), dimensions of which are so much smaller than the minimum distance between two stars in the space $(\dot{\xi}, \dot{\eta}, \dot{\zeta})$ for any of versions $a$ and $b$, that each cell in the velocity space contains no more than one star in any of the versions $a$ and $b$ of the cluster models. We enumerate these cells in a velocity field in a certain order, after which we write down a simple relation between the number of the cell $i$ and the coordinates of the star in the space $(\dot{\xi}, \dot{\eta}, \dot{\zeta})$. A comparison of the integral stellar distributions $v_{a}(i)$ and $v_{b}(i)$ (which correspond to the $a$ and $b$ OSC model versions) by the numbers $i$ of cells containing these stars, for an each interval $\Delta r_{j}$ of distances from the center, is convenient to carry out using the Kolmogorov criterion:

$$
\lambda=D \sqrt{\frac{n_{a} n_{b}}{n_{a}+n_{b}}},
$$

where $D$ is the modulus of the maximum deviation of two empirical integral functions of the distribution of stars in the velocity space: $v_{a}=v_{a}(i)$ and $v_{b}=v_{b}(i) ; n_{a}=50$ and $n_{b}$ are the sample sizes.

Using the limit distribution of $\lambda$ (see, for example, Bolshev and Smirnov (1983)) we can calculate the probability $P_{j}$ of the fact that the considered PCS samples for an each interval $\Delta r_{j}$ of the star's distance from the cluster center are samples from the same general populations (the criterion of a homogeneity of the PCS value distribution). Thus, $P_{j}$ is the probability that the differences between $v_{a}=v_{a}(i)$ and $v_{b}=v_{b}(i)$ have a random character, while the PCS of the $a$ and $b$ versions of the cluster models have the same distribution functions. A comparison of the sets of the phase coordinates for the OSC models by the Kolmogorov criterion $\lambda$ (Danilov, 1997b), unlike a comparison by thew Pearson criterion $\chi^{2}$ used by Danilov (1997c), does not require a fairly arbitrary partition of the stars into the groups in the velocity space, leading to the loss of a part of the information available in the original PCS. In addition, Danilov (1997b) used one component of the stellar velocity vectors more than Danilov (1997c), which allows to take into account an information about the 
stellar motions in the cluster along a $\zeta$-coordinate and makes a comparison of the stellar phase coordinates in the OSC models more objective.

A statistical comparison of the $a$ and $b$ versions of the cluster models obtained by Danilov (1997b) according to the schemes, accuracy order of which differs by one, showed that in the time interval $t$ from 0 to a certain moment $t_{0}-0.1 \times \tau_{v r}^{(0)}, P_{j}$ is equal to $0.999-1$ for all values of $j$. At $\left(t_{0}-0.1 \times \tau_{v r}^{(0)}<t<t_{0}\right.$ for the central regions of the cluster model $(j=1,2)$, the possible estimates could be $P_{j} \simeq 0.93-0.98$ for the several steps of an integration over time, after which $P_{j}$ again becomes equal to one. Thus, at $t \leq t_{0}$, the accuracy of a calculation of the PCS distribution in the cluster model can be considered sufficient for the conclusions about the physical properties of the model, and the PCS distributions obtained by Danilov (1997b) with the neighbor accuracy orders can be considered statistically indistinguishable. For $t>t_{0}$, at $j=1,2$, the estimates of $P_{j}<0.9$ appear with the subsequent oscillations of the $P_{j}$ values from one to the minimum achieved values. With an increase of $t$ up to $t_{0}+(0.1-0.2) \times \tau_{v r}^{(0)}$, the region of these manifestations of the instability of the numerical solutions for the PCS distribution expands to the core boundary, the minimum values of $P_{j}$ decrease, the amplitude of the $P_{j}$ oscillations increases. With a further increase of $t$, the similar manifestations of the instability of the numerical solutions for the PCS distribution become significant both in the core and near the tidal boundary of the cluster (Danilov, 1997b). The value of $t_{0}$ increases with an increase of the accuracy order of the used difference schemes and with an increase of the number of the decimal digits in the PCS representation, and also with a decrease of the integration time step. However, a significant decrease in the integration time step for the fixed values of the accuracy order of the used difference schemes and the number of the decimal digits in the calculations may lead to a decrease in $t_{0}$ due to the increasing rounding errors during a calculation.

The $t_{0}$ value may vary by $\pm 0.1 \times \tau_{v r}^{(0)}$ with the small changes in the initial PCS (within $\pm 1 \%$ of the PCS values). Therefore, in order to analyze the manifestations of the OSC models' instability to the small initial PCS perturbations, it is necessary: a) to find $t_{0, \min }$, the smallest $t_{0}$ value in the compared versions of the cluster model with the small differences in the initial PCS and to compare the versions of the OSC models at $t<t_{0, \min }$; b) at $t>t_{0, \min }$, to exclude from a consideration the central cluster regions, where the PCS distributions can be distorted by the accumulated computational errors.

A statistical criterion for the integration accuracy of the equations of a stellar motion in the cluster $\left(0.9<P_{j}<1\right.$, see above), which is used simultaneously with the dynamic criterion ( $E=$ const $)$, allows to control the accuracy of the PCS distribution calculation. It makes it possible (using the finite-difference schemes of a sufficiently high accuracy order) to divide by a time the moments of the beginning of the significant manifestations of the actions of the initial PCS perturbations and the calculation rounding onto the PCS distribution when studying the stability of the numerical dynamic OSC models. 


\subsection{Statistical Control of Calculations}

It is convenient to use the calculation technique of Danilov (1999) to study the properties of the PDF in the OSC models and the features of the statistical method of the accuracy monitoring of the PDF calculation. We consider two versions ( $a$ and $b$ ) of the cluster model. As in the previous Section, we divide all stars of the $a$ version at the time point $t$ into 10 groups (by 50 stars in each) in the order of the increasing distances from the cluster mass center. Let $\Delta r_{j}$ be an interval of the distances $r$ from the cluster mass center, within which the $j$-th group of stars is located $(j=1, \ldots, 10)$. We divide the region occupied by the cluster stars in the velocity space $\left(v_{x}, v_{y}, v_{z}\right)$, into $k$ similar cells in the form of the rectangular parallelepipeds, the opposite faces of which are pairwise parallel to one of the coordinate planes in the system $\left(v_{x}, v_{y}, v_{z}\right)$. Let $l_{x}, l_{y}, l_{z}$ be the dimensions of such a cell along the axes $v_{x}, v_{y}, v_{z}$, respectively. Then the number of cells in the region occupied by the cluster stars along the axis $v_{x}$ is

$$
n_{x}=\frac{\max \left(v_{x}\right)-\min \left(v_{x}\right)}{l_{x}},
$$

where $\max _{v_{x}}$ and $\min _{v_{x}}$ (i) are the largest and the smallest values of $v_{x}$ for 500 cluster stars. Let $k_{x}$ be a cell number in the velocity space containing the star with a given value of $v_{x} ; k_{x}=1, \ldots, n_{x}$ :

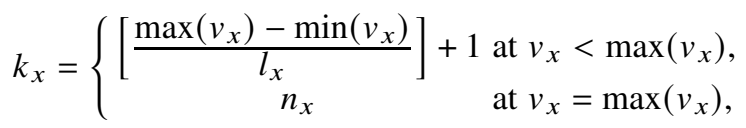

where the square brackets denotes the integer part of the number in brackets. The expressions similar to (16.1) and (16.2) can be written for $n_{y}, n_{z}$ and $k_{y}, k_{z}$.

Let $i$ be the number of the cell containing the star with the coordinates $v_{x}, v_{y}, v_{z}$. Considering (16.1) and (16.2), we find

$$
\left.i=\left(k_{x}-1\right) n_{y}+k_{y}+\left(k_{z}-1\right) n_{x} n_{y}\right),
$$

where $i=1, \ldots, k, k_{x}=1, \ldots, n_{x}, k_{y}=1, \ldots, n_{y}, k_{z}=1, \ldots, n_{z}$. A numbering of cells $i=i\left(k_{x}, k_{y}, k_{z}\right)$ is performed so that, as $i$ increases, the $k_{x}$ values grow faster than $k_{y}$, and the $k_{y}$ values grow faster than $k_{z}$.

Such a partition of the velocity space $\left(v_{x} v_{y}, v_{z}\right)$ for the OSC models also was carried out in the previous section, as well as by Danilov (1997b) when constructing a statistical method for the accuracy of calculations control. However, in this study, the cell sizes are so small that each cell contains not more than one star in any versions $a$ and $b$ of the cluster model. According to (16.1), the volume of the cell in the velocity space is equal to $q=l_{x} l_{y} l_{z}$; the corresponding phase volume of the cell containing stars from the interval $\Delta r_{j}$, is

$$
Q(j)=q \frac{4}{3} \pi\left(r_{j}^{3}-r_{j-1}^{3}\right)
$$


$j=1, \ldots, 10, r_{0}=0$. Let $n^{(a)}(i, j)$ be the number of stars from the $i$-th cell of the velocity space of the OSC model $a$, which fall to the $j$-th interval of the distances from the cluster center. Taking into account the adopted notation, the value of the "coarse-grained" PDF of the OSC model $a$ (corresponding to the intervals $i, j$ in the spaces of the velocities $\mathbf{v}$ and the distances $\mathbf{r}$ from the cluster center) is $n^{(a)}(i, j) / Q(j)$. The average relative difference of the PDFs of the $a$ and $b$ OSC model versions in the interval $\Delta r_{j}$ is:

$$
\Psi_{j}=\frac{\sum_{i=1}^{k}\left|n^{(a)}(i, j)-n^{(b)}(i, j)\right|}{\frac{1}{2} \sum_{i=1}^{k}\left|n^{(a)}(i, j)+n^{(b)}(i, j)\right|}, \quad j=1, \ldots, 10 .
$$

We have reduced the numerator and denominator of the formula (16.4) by the same factor $Q(j)$. Following to Kandrup et al. (1994), the difference between the OSC models $a$ and $b$ can be estimated from the formulas

$$
\Delta R=\frac{1}{2} \ln \sum_{l=1}^{N}\left|\delta \mathbf{r}_{l}\right|^{2}, \quad \Delta V=\frac{1}{2} \ln \sum_{l=1}^{N}\left|\delta \mathbf{v}_{l}\right|^{2},
$$

where $\delta \mathbf{r}_{l}$ and $\delta \mathbf{v}_{l}$ are the differences of the radius vector $\mathbf{r}_{l}$ and the velocity vector $\mathbf{v}_{l}$ of the star with the number $l$ in the OSC models $a$ and $b$. The values $\delta \mathbf{r}_{l}$ and $\delta \mathbf{v}_{l}$ in formulas (16.5) are reduced to a dimensionless form by dividing them by the distance unit $(1 \mathrm{pc})$ and the speed unit $(1 \mathrm{pc} / \mathrm{Myr})$ used in our work, respectively.

Danilov (1999) carried out the calculations of $\Delta R, \Delta V$ and $\Psi_{j}$ at $j=1, \ldots, 10$ for the stars from the intervals $\Delta r_{j}$ at the different time points $t$ in the interval $0<t / \tau_{v r}^{(0)} \leq 1$.7. Danilov (1999) determined the values of $\Delta R, \Delta V$ and $\Psi_{j}$ by comparing the cluster models $a$ and $b$ with the identical initial PCS obtained by the difference schemes of the 6th and 7th accuracy order (see (2) from the paper of Danilov (1997b)), as well as by comparing the $a$ and $b$ OSC models with the slightly different initial PCS obtained by the difference scheme of the 7th accuracy order. In this model for the period of time $0<t / \tau_{v r}^{(0)} \leq 1.7$, the control of the cluster energy is $\left|\delta E / E_{0}\right|<0.0000058$ (where $E_{0}$ is the initial value of $E$ ) and the statistical control of the PDF calculation accuracy is $0.9<P_{j}<1.0$. Here, $P_{j}$ is the probability that the samples of the PCS from all considered intervals $\Delta r_{j}(j=1, \ldots, 10)$ of distances from the cluster center in the $a$ and $b$ cluster models, obtained by the schemes of the 7th and 6th accuracy orders, are the samples from the same general populations of the PCS, i.e. the differences between the PCS distributions in the versions $a$ and $b$ are random. According to Danilov (1997b) in this case, the accuracy of the PCS calculation can be considered sufficient for conclusions about the physical properties of the cluster model's PDF. If, at $t=0$, the cluster model versions $a$ and $b$ coincide, and, at $t>0$, the difference schemes of the 7th and 6th accuracy order are used to calculate the PCS in the model versions $a$ and $b$, then the comparison of the $a$ and $b$ versions for the time points $t$ from the interval $0<t<1.7 \tau_{v r}^{(0)}$ shows that $P_{j} \simeq 1$ 
at $j=1, \ldots, 10$ (Danilov, 1997b), and the values of $\Psi_{j}$ at $j=2, \ldots, 10$ are within the interval $0<\Psi_{j} \leq 0.04$.

In the central region of the cluster $\left(j=1, r \leq \Delta r_{1}\right), \Psi_{1}$ begins to increase systematically with $t>t_{1} \simeq(1.4-1.5) \tau_{v r}^{(0)}$ reaching $\Psi_{1} \simeq 0.38-0.40$ at $t=1.7 \tau_{v r}^{(0)}$. In this interval of the $t$ values, the stellar velocity distribution in the region $r \leq \Delta r_{1}$ can be represented as $\varphi_{0}(\mathbf{v})+\varphi_{1}(\mathbf{v})$, where $\varphi_{0}(\mathbf{v})$ is the smoothed velocity distribution and $\varphi_{1}(\mathbf{v})$ is a perturbation. This perturbation has the form of a sum of the amplifying (with a growing $t$ ) peaks (at different $\mathbf{v}$ ) located approximately in an antiphase in the versions $a$ and $b$ of this cluster model. Thus, the errors of the difference scheme of the 6th accuracy order begin to appear at $t>t_{1} \simeq(1.4-1.5) \tau_{v r}^{(0)}$ in the form of a phase change of the perturbations $\varphi_{1}(\mathbf{v})$ of the stellar velocity distribution in the region $r \leq \Delta r_{1}$. Herewith, the smoothed velocity distribution $\varphi_{0}(\mathbf{v})$ of the stars from the region $r \leq \Delta r_{1}$ can be considered as the same up to the values of $t=1.7 \tau_{v r}^{(0)}$ (with a probability $P_{1} \simeq 1$ ) in the calculations according to the schemes of the 6th and 7 th accuracy orders.

Let us consider the case when the PCS in the $a$ and $b$ cluster model versions at $t=0$ differ a little (within $\pm 1 \%$ of the PCS values), and to calculate the PCS at $t>0$ in the versions $a$ and $b$ the difference schemes of the 7th accuracy order are used. Fig.16.1 shows the plots of the time dependencies of $\Psi_{j}$ at $j=1,9,10$ obtained by Danilov (1999) when comparing the versions $a$ and $b$ of the OSC model.

At $k=125,1000$, on the time interval from 0 to $t_{2} \simeq(0.5-1.1) \tau_{v r}^{(0)}$, the $\Psi_{j}$ values increase with the time both near the center and on the cluster periphery. During this period, the rearrangement of the cluster takes place due to its relaxation toward the more equilibrium state. Near the cluster center, the local relaxation time (as well as $t_{2}$ ) is smaller than on the periphery (where $t_{2} \simeq 1.1 \tau_{v r}^{(0)}$ ). Therefore, the rearrangement of the PDF in the central region completes earlier than at the cluster periphery.

At $t_{2} \leq t \leq 1.7 \tau_{v r}^{(0)}$, the $\Psi_{j}$ values experience the small oscillations around some constant equilibrium values $\bar{\Psi}_{j}$ both at $k=125$, and at $k=1000$. The established values of $\bar{\Psi}_{j}$ at $k=125$ are 1.7-2.0 times smaller than at $k=1000$.

During the period $0<t<t_{2}$, the exponential divergence of the orbits of stars with the same numbers $l$ in the versions $a$ and $b$ of the cluster model with the small differences in the initial PCS leads to an increase in the differences in the PDFs of these versions until the corresponding change in the number of stars in the considered cells of the phase space does not begin to be compensated (at $t>t_{2}$ ) due to other stars coming from the neighbor cells of the phase space. This occurs after the formation of an "equilibrium" PDF in the given region of the cluster, when the relaxation transition to the equilibrium state in this region is completed to the extent possible in these system (the oscillations of the regular field and, apparently, the PDF acquire a steady-state character). This is possible, for example, if the cluster PDF $f(\mathbf{r}, \mathbf{v}, t)$ is approximately periodic with respect to the $t$ values (at fixed $\mathbf{r}$ and v) and changes quite little when passing through $t$ from one oscillation period $P_{r}$ of the regular field of the system to another; $P_{r} \simeq 0.7 \tau_{v r}^{(0)}$ in this cluster model. In this case, the instability of the OSC models to the small initial perturbations of the PDF 


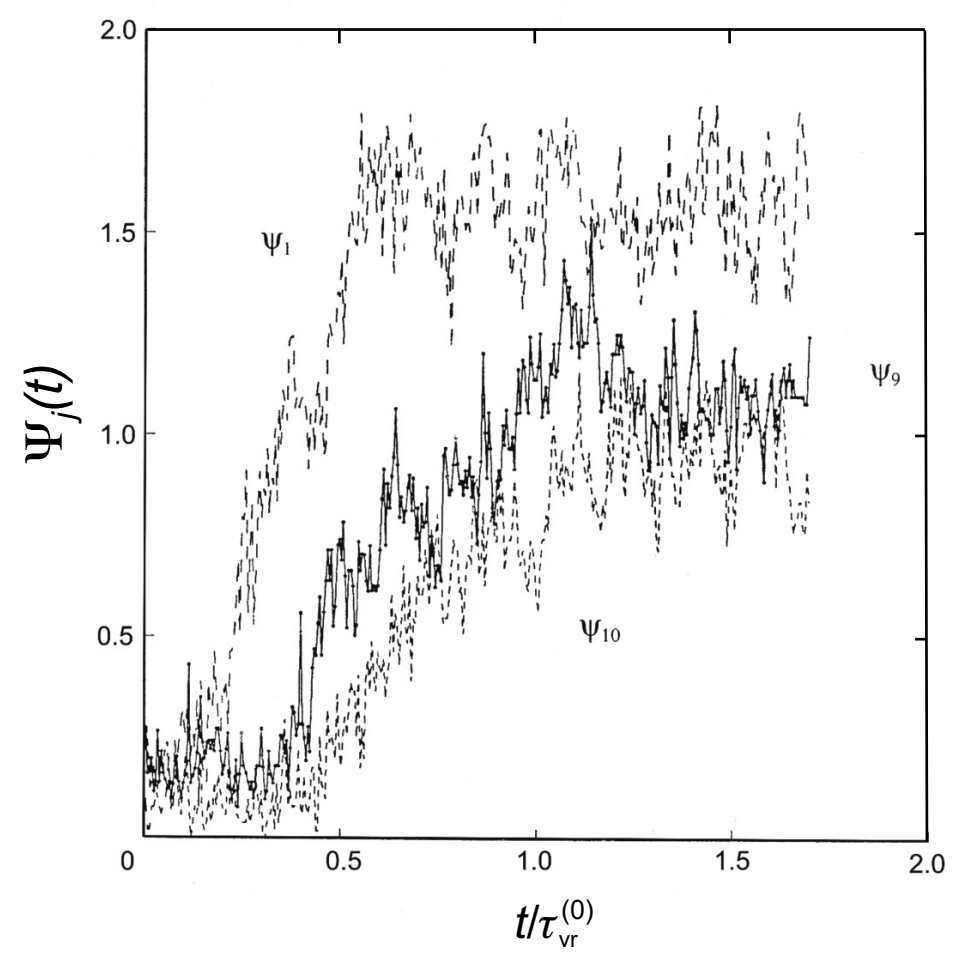

Fig.16.1 The plots of the time dependencies of $\Psi_{j}$ at $j=1,9,10$ in the considered model of star cluster

in an every considered interval of $\Delta r_{j}$ of distances from the cluster center manifests itself as the differences in the velocity distributions $\varphi_{a}(\mathbf{v})$ and $\varphi_{b}(\mathbf{v})$ in the versions $a$ and $b$ of the cluster models of Danilov (1997b) emerging and disappearing with a period of $(0.05-0.15) \tau_{v r}^{(0)}$.

Since the denominator of (16.4) at $t>t_{2}$ differs a little from 50 (usually,

$$
\frac{1}{2} \sum_{i=1}^{k}\left(n^{(a)}(i, j)+n^{(b)}(i, j)\right)=C_{j} \simeq 50 \pm(1-2),
$$

$(j=1, \ldots, 10))$, then the numerator in (16.4) also varies a little over time at $t>t_{2}$. The growing differences between $\varphi_{a}(\mathbf{v})$ and $\varphi_{b}(\mathbf{v})$ at some values of $\mathbf{v}$ are compensated by the disappearing of these differences at other values of $\mathbf{v}$. The values of $\varphi_{a}(\mathbf{v})$ and $\varphi_{b}(\mathbf{v})$ experience such oscillations with respect to $\bar{\varphi}(\mathbf{v}, t)=$ $\frac{1}{2}\left(\varphi_{a}(\mathbf{v})+\varphi_{b}(\mathbf{v})\right)$ that the value $C_{j} \Psi_{j}$ changes a little with time at $t>t_{2}$ (this corresponds to the constant in time amplitude of the oscillations of $\varphi_{a}(\mathbf{v})$ relative to $\bar{\varphi}(\mathbf{v}, t)$ averaged by a velocity for each $j)$. At $t>t_{2}$, the smaller $\bar{\Psi}_{j}$ at the cluster 
periphery in comparison with $\bar{\Psi}_{1}$ are apparently caused by the action of the stellar encounters less tight than in the cluster core, and also by the larger dimensions of the considered phase cells at the cluster periphery than in its core. Even a large DSO (the divergence of stellar orbits) at the cluster periphery may not lead to a change of the coarse-grained PDF if the DSO dimensions do not exceed the dimensions of the considered cells of the phase space).

In the central regions of the cluster model, the density of the number of stars and the amplitude of the oscillations of the regular field are the greatest, so the established values of $\bar{\Psi}_{j}$ can be due to both the action of the stellar encounters in the cluster and the interaction of stars with the changing regular cluster field. The difference between the compared versions $a$ and $b$ of the OSC model at the cluster periphery can also be due to the difference of the orbits of stars formed in the versions $a$ and $b$ of the cluster model under the action of the mechanisms mentioned above. At $0<t \leq t_{2}$, the rate of an increase of $\Psi_{1}$ with time in this cluster model are 2 times larger than the rate of an increase of $\Psi_{9}$ and $\Psi_{10}$; the value $t_{2}$ in the cluster core is 2 times smaller than at the cluster periphery (Fig.16.1). Apparently, the rate of the DSO in the cluster core is greater than at its periphery. We note that a high rate of the DSO in the cluster core is accompanied by the shorter time of the formation of the "equilibrium" PDF in the cluster core $\left(t \simeq t_{2}\right)$ in comparison with its external areas. This is an argument in favor of the existence of a connection between the exponential instability of the orbits of stars and the relaxation processes in the system. The characteristics of the PDF instability with respect to the small initial perturbations of the PDF can serve as an indicator of the dynamic state of the cluster model. At $t \simeq t_{2}$, there is a sharp change in the properties of this instability of the PDF (see above, as well as Fig.16.1), caused by the formation of some "equilibrium" PDF in the cluster models. The value of $t_{2}$ can be regarded as an estimate of the local relaxation time in the cluster model to the indicated "equilibrium" state. However, the virial equilibrium and a stationarity in the field of the regular forces in such systems can not be achieved, since the irregular forces dominate over the regular ones in a significant part of the volume of the non-isolated open clusters (Danilov, 1997a, 1987) and support a significant non-stationarity in the regular field in the observed OSCs (Danilov and Seleznev, 1994). It is found in the OSC models in the form of the oscillations of the values of the virial coefficient $2(T+\Omega) / \Omega$ with the practically constant amplitude and period (here, $T$ is the total kinetic energy of the peculiar motions of the cluster stars, $\Omega$ is the potential energy of the cluster). After the formation of the indicated "equilibrium" state during the time $t_{2}$, the further evolution of the cluster PDF probably does not pass so violently and rapidly (as at $t<t_{2}$ ) as a result of the gradual development of the "halo-core" structure of the cluster due to the stellar encounters. The evolution of the cluster at $t>t_{2}$ is also accompanied by the appearance of the new groups of the high-energy stars at the cluster periphery after the periodic compressions of the cluster. The formation of such groups is due to the interaction of the stars with the changing regular cluster field (Danilov and Ryazanov, 1985).

The values of $\Delta R$ and $\Delta V$ in the considered cluster model linearly increase with the time in the period from $t \simeq(0.1-0.15) \tau_{v r}^{(0)}$ to $t \simeq 1 \bar{t}_{c r} \simeq 0.4 \tau_{v r}^{(0)}$; it corresponds to an increase of $|\delta r|$ and $|\delta v|$ with time by the law $\sim \exp \left(t / t_{*}\right)$, with 
$t_{*} \simeq 0.16 \tau_{v r}^{(0)} \simeq 0.42 t_{c r},|\delta r|=\sum_{l=1}^{N}\left|\delta \mathbf{r}_{l}\right|,|\delta v|=\sum_{l=1}^{N}\left|\delta \mathbf{v}_{l}\right|$. Thus, the exponential instability of orbits also occurs in the clusters models, the calculation of which is controlled by means of a statistical criterion of accuracy. The value $t_{*}$ quite agrees with the data of Kandrup and Haywood (1992) obtained for the clusters with a smoothing of the force functions in the right-hand parts of the equations of a stellar motion. The values $\Delta R$ and $\Delta V$ at $t>2 t_{c r}$ continue to grow linearly, albeit at a slower rate than at $t<1 t_{c r}$ (the corresponding values are $t_{*, r} \simeq 0.7 \tau_{v r}^{(0)} \simeq 1.8 \bar{t}_{c r}$ for $|\delta r|$ and $t_{*, v} \simeq 1.8 \tau_{v r}^{(0)} \simeq 4.6 \bar{t}_{c r}$ for $\left.|\delta v|\right)$. Such increase of $|\delta r|$ and $|\delta v|$ with time is due to the expansion in the phase space of the subsystem of stars that have the velocities greater than the critical ones and which have left the cluster and leave to the field of the Galaxy.

In the considered versions of the OSC model in the time $1.7 \tau_{v r}^{(0)}$, the phase volumes $Q(j)$ increase several times at $j=1$, and $\sim 2000$ times at $j=10$ (the greater $j$ the greater an increase of $Q(j)$ with time). Thus, the dynamic evolution of the OSC results in a constant decrease in the values of the PDF (the most significant at the cluster periphery). At $t>t_{2}$, the differences in the PDF of the versions $a$ and $b$ of the cluster model (on average over the stellar velocities) turn out to be proportional to the values of the PDF (also averaged by the velocities) for each interval $\Delta r_{j}$ of the distances from the cluster center. Since the values of $\Psi_{j} \simeq \Psi_{j}$ are relatively constant at $t>t_{2}$, with a decrease of the PDF at $t>t_{2}$, the differences in the PDFs of the versions of the OSC model with a little difference in the initial PCS also decrease proportionally. Increase of the phase volumes $Q(j)$ occurs mainly due to an increase in time the intervals $\Delta r_{j}$; the values of $l_{x}, l_{y}, l_{z}$ increase only in $\sim 1.2-2.0$ times over the time $1.7 \tau_{v r}^{(0)}$.

Let us mention the following main results of this part of the work.

1. In the considered OSC model, the star orbits exhibit an exponential instability to small perturbations of the initial PCS. The characteristic time of the corresponding DSO is $t_{*} \simeq 0.4 \bar{t}_{c r}$, which agrees with the estimates of $t_{*}$ by Kandrup and Haywood (1992).

2. At $0<t<t_{2}$, the instability of the PDF of the cluster model to small perturbations of the initial PCS leads to an increase of the mean (over the velocity space) relative changes of the PDF with time, i.e., of the $\Psi_{j}$ values in the considered intervals $\Delta r_{j}$ of the distances from the cluster center. The values of $\Psi_{j}$ are stabilizing at $t>t_{2}$. The reason for this stabilization, apparently, is the formation of the "equilibrium" PDF of the cluster and the relaxation transition to a state close to an equilibrium as far as it is possible in the presence of the steady-state oscillations of the regular field of the cluster model. The instability of the PDF of the cluster model at $t>t_{2}$ has the following features. The growing differences between the "unperturbed" $\varphi_{a}(\mathbf{v}, t)$ and "perturbed" $\varphi_{b}(\mathbf{v}, t)$ velocity distributions in the intervals of time $\sim 0.1 \tau_{v r}^{(0)}$ at some values of $\mathbf{v}$ are compensated by the disappearance of these differences at other values of $\mathbf{v}$. At the same time, the average (by the stellar velocities) oscillation amplitude of the values of $\varphi_{a}(\mathbf{v}, t)$ and $\varphi_{b}(\mathbf{v}, t)$ with respect to $\bar{\varphi}(\mathbf{v}, t)=$ $\frac{1}{2}\left(\varphi_{a}(\mathbf{v})+\varphi_{b}(\mathbf{v})\right)$ in each of the considered intervals $\Delta r_{j}$ of the distances from the cluster center is approximately constant in time. 
3. The local time $t_{2}$ of the relaxation of the cluster to the considered "equilibrium" state in the central regions of the cluster is smaller than at the cluster periphery, where $t_{2}$ practically coincides with the time of a violent relaxation of the cluster $\left.t_{2} \simeq 1.1 \tau_{v r}^{(0)}\right)$. At $t<t_{2}$, the DSO leads to an increase of $\Psi_{1}$ near the cluster center which is $\sim 2$ times greater than the increase of $\Psi_{j}$ at the cluster periphery.

4. At $t<t_{2}$, the DSO leads to an increase of $\Psi_{j}$ in all considered intervals $\Delta r_{j}$ of the distances from the cluster center. The rate of the increase of $\Psi_{j}$ with time in the cluster core is $\sim 2$ times greater than at the cluster periphery. Apparently, the rate of the DSO in the cluster core is greater than at the periphery. The higher rate of the DSO in the cluster core is accompanied by shorter time of the formation of an "equilibrium" PDF in the core in comparison with the values of $t_{2}$ at the cluster periphery. This is an argument in favor of the existence of a connection between the exponential instability of the orbits of stars and the relaxation processes in the system.

5. When using the statistical criterion for the accuracy of the computation of the cluster PDF $\left(P_{j} \simeq 1\right.$ for $j=1, \ldots, 10$ at $\left.0<t \leq 1.7 \tau_{v r}^{(0)}\right)$, the values of $\Psi_{j}$ obtained by comparing the PCS calculated by the difference schemes of the 6th and 7th accuracy orders do not exceed 0.04 for all the considered intervals $\Delta r_{j}$ with the exception of the central region of the cluster $\left(r \leq \Delta r_{1}\right)$. Near the cluster center, the errors of calculation of the PCS according to the scheme of the 6th accuracy order become noticeable even at $t \geq(1.4-1.5) \tau_{v r}^{(0)}$ and manifest themselves as a change in the phases of the perturbations $\varphi_{1}(\mathbf{v})$ of the velocity distribution $\varphi(\mathbf{v})=\varphi_{0}(\mathbf{v})+\varphi_{1}(\mathbf{v})$ in comparison with the phases $\varphi_{1}(\mathbf{v})$ in the distribution $\varphi(\mathbf{v})$ obtained by the calculations of the PCS according to the scheme of the 7th accuracy order $\left(\varphi_{0}(\mathbf{v})\right.$ is the smoothed velocity distribution). The velocity distributions $\varphi_{0}(\mathbf{v})$ in the PCS calculations according to the schemes of the 6th and 7th accuracy orders with probability $P_{1} \simeq 1$ are not statistically different from each other until the time point $t=1.7 \tau_{v r}^{(0)}$.

\section{References}

Aarseth, S.J.: Dynamical evolution of simulated star clusters. I - Isolated models. Astron. Astrophys. 35, 237-250 (1974).

Bolshev, L.N., Smirnov, N.V.: Tables of mathematical statistics. Nauka, Moscow (1983). (In Russian).

Danilov, V.M.: On the Nonstationarity / Motion / of Stellar Clusters in the Galaxy. Astronomicheskii Tsirkulyar No. 1522/DEC, 2-3 (1987). (In Russian).

Danilov, V.M.: Dynamics of Core / Halo Structures in Spherical Stellar Clusters. Sov. Astron. 32, 374-379 (1988).

Danilov, V.M.: Numerical experiments simulating the dynamics of open clusters in the Galactic field. Astron. Reports 41, 163-173 (1997a). 
Danilov, V.M.: Statistical analysis of dynamical open-cluster models with small differences in their initial stellar phase coordinates. Astron. Letters, 23, 322-326 (1997b).

Danilov, V.M.: A statistical comparison of numerical dynamical models for open clusters. Astron. Letters, 23, 79-83 (1997c).

Danilov, V.M.: Some properties of the phase-space density function in numerical dynamical models of open star clusters. Astron. Reports 43, 74-79 (1999).

Danilov, V.M., Dorogavtseva, L.V.: Timescales for mechanisms for the dynamical evolution of open star clusters. Astron. Reports 52, 467-478 (2008). doi:10.1134/S1063772908060048

Danilov, V.M., Putkov, S.I.: Non-stationarity parameters of open clusters. Astron. Reports 56, 609-622 (2012a). doi:10.1134/S106377291208001X

Danilov, V.M., Putkov, S.I., Seleznev, A.F.: Dynamics of the coronas of open star clusters. Astron. Reports 58, 906-921 (2014). doi:10.1134/S1063772914120038

Danilov, V.M., Ryazanov, A.P.: On modelling spherical instationary collisionless stellar systems. In: Barkahatova, K.A. (ed.) Astronomical-geodetical investigations. Ural State Univ. Publ., Sverdlovsk (1985), 19-47. (In Russian).

Danilov, V.M., Seleznev, A.F.: The catalogue of structural and dynamical characteristics of $103 \mathrm{OCl}$ and the first results of its investigations. Astron. Astrophys. Trans. 6, 85-156 (1994). doi:10.1080/105567994082320611

Kandrup, H.E., Haywood, S.Jr.: On the sensitivity of the N-body problem to small changes in initial conditions. II. Astrophys J. 386, 635-645 (1992). doi:10.1086/171045

Kandrup, H.E., Magon, M.E., Smith, H.C.: On the sensitivity of the N-body problem toward small changes in initial conditions. 4. Astrophys. J. 428, 458-465 (1994). doi:10.1086/174259

Kharchenko, N.V., Berczik, P., Petrov, M.I., et al.: Shape parameters of Galactic open clusters. Astron. Astrophys. 495, 807-818 (2009). doi:10.1051/00046361/200810407

Kutuzov, S.A., Osipkov, L.P.: A Generalized Model for the Three-Dimensional Gravitational Potential of Stellar Systems. Soviet Astron. 24, 17-22 (1980).

Lada, C.J., Margulis, M., Dearborn, D.D.: The formation and early dynamical evolution of bound stellar systems. Astrophys. J. 285, 141-152 (1984). doi:10.1086/162485

Tutukov, A.V.: Early Stages of Dynamical Evolution of Star Cluster Models. Astron. Astrophys. 70, 57-61 (1978). 


\author{
Научное издание \\ Данилов Владимир Михайлович \\ НЕСТАЦИОНАРНОСТЬ РАССЕЯННЫХ ЗВЕЗДНЫХ \\ СКОПЛЕНИЙ \\ Монография
}

Scientific Publication

Danilov Vladimir Mikhailovich

NON-STATIONARITY OF OPEN STAR CLUSTERS

Monograph
Электронное сетевое издание размещено в архиве УрФУ
http://elar.urfu.ru

Подписано в печать 22.01.2021.

Формат $60 * 84$ 1/16. Уч.-изд. л. 22,8. Объем данных 10,2 Мб.

Гарнитура Times Roman.

Издательство Уральского университета

620000, Екатеринбург-83, ул. Тургенева, 4.

Тел.: +7(343) 358-93-06, 371-54-48

Факс: +7(343) 358-93-06

E-mail: press-urfu@mail.ru

http://print.urfu.ru 



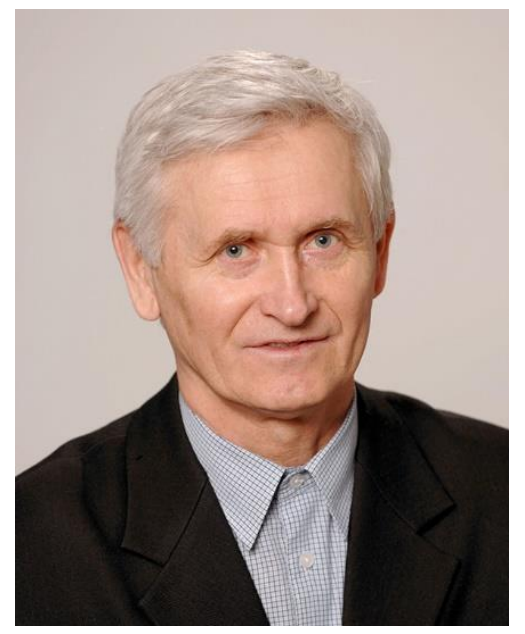

Vladimir M. Danilov was born in 1948 in Chelyabinsk (USSR). He had been graduated from the Ural State University in Sverdlovsk (now the Ural Federal University, Ekaterinburg) in 1971, got the Doctor degree in 1978 and the Doctor of Science degree in 1993; Professor since 2004. Vladimir M. Danilov has been working in the Ural Federal University since 1970, now as Chief Researcher.

Research interests of Vladimir M. Danilov are stellar dynamics and astrophysics. He belongs to the scientific school of stellar astronomy, created by Professor K. A. Barkhatova. His general results concern the dynamics of open star clusters from both theoretical and numerical points of view. V.M. Danilov investigated different aspects of the interaction of clusters with external gravitational fields (such as the Galaxy and gas-star complexes), the instabilities of clusters, proper oscillations of the cluster potential and their phase density, among other topics. 